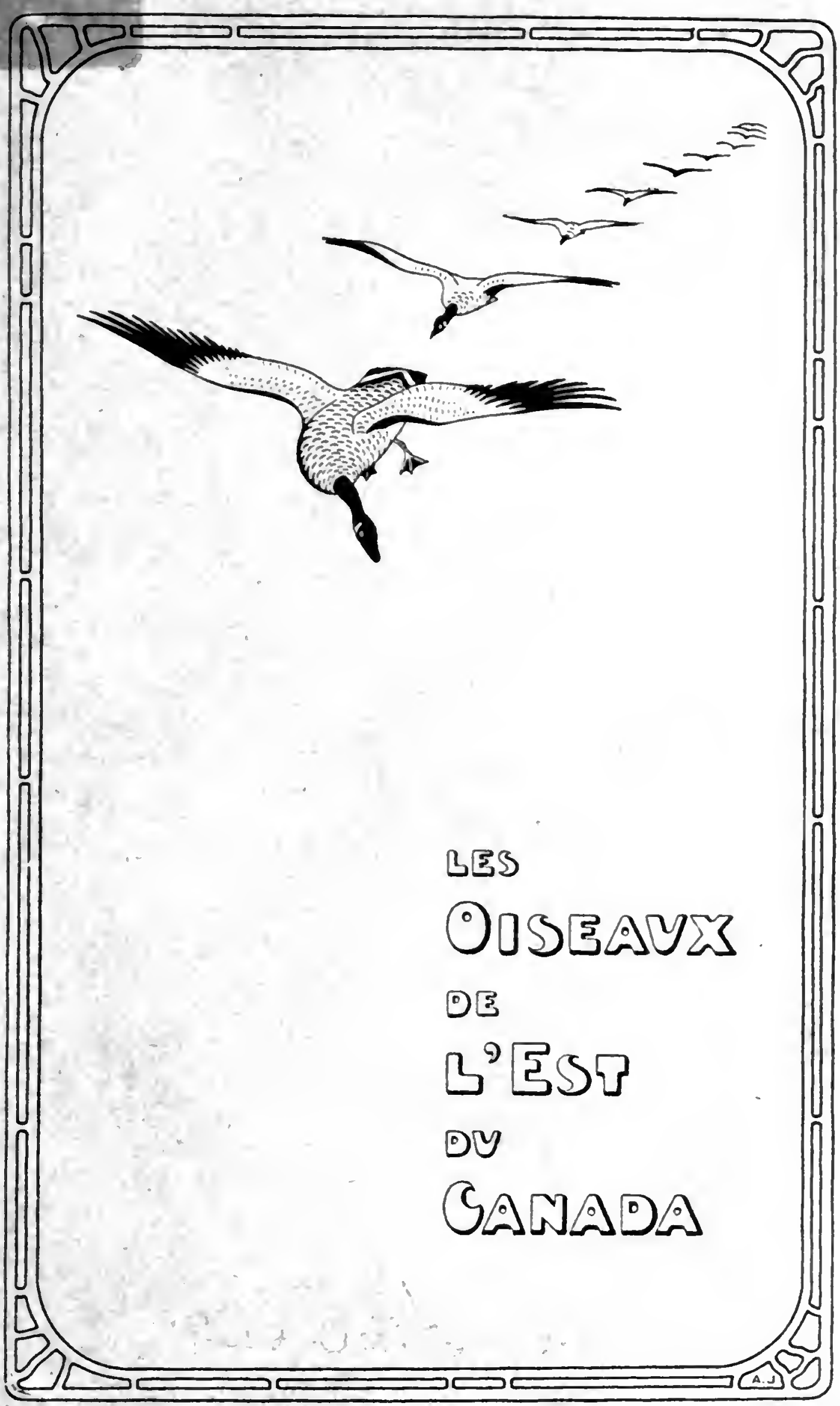




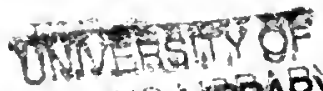 \\ ILLINOIS LIBRARY \\ OT URBANA-CHAMPAIGN \\ BIOLOGY}

ANG 15 贫9? 


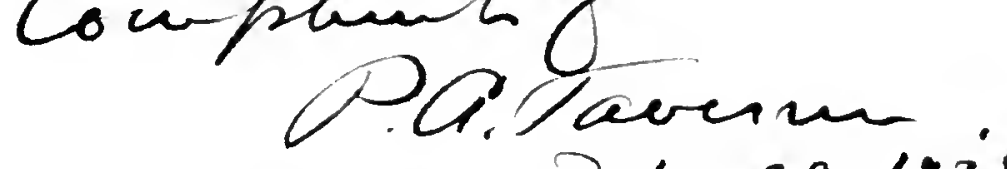

CANADA

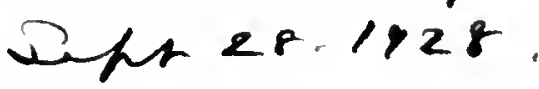

MINISTERE DES MINES

L'hon. Charles Stewart, Ministre; Charles Camsell, Sous-Ministre

\section{COMMISSION GÉOLOGIQUE}

W. H. Collins, Directeur

MÉMOIRE 104

$N^{\circ} 3$, Série bIOLOGIQue

\section{Les Oiseaux de l'Est du Canada}

(DEUXIEME RDITION)

par

P. A. Taverner

Traduit par le personnel attitré du ministère

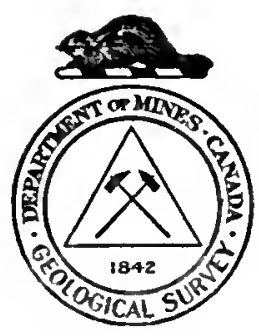

OTTAWA

F. A. ACLAND

MPRIMEUR DE BA TRES EXCELLENTE MAJESTE LE ROI 1922 



\section{TABLE DES MATIÊRES}

Introduction...... . . . . . . . . . . .

Remerciements. . . . . . . . . . . .

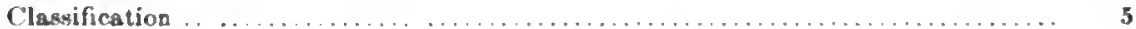

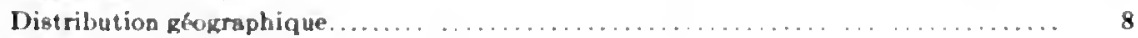

Migration . . . . . . . . . . . . . .

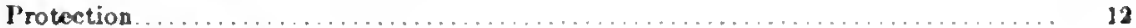

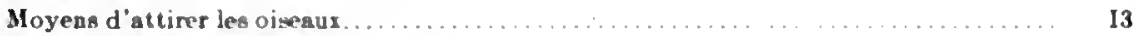

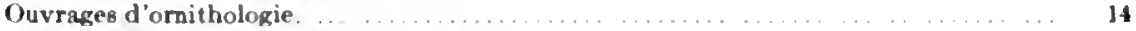

Clel pour les oiveaux de l'eat du Canada .............................. 18

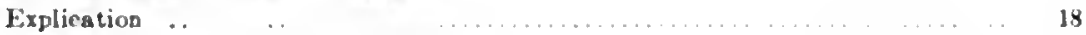

Clel ...................... 10

Table aystemstique... _ . . . . . . 31

Ornitholokie descriptive (notr Index) $\ldots \ldots \ldots \ldots \ldots \ldots \ldots \ldots \ldots$

Index $\quad \ldots \ldots \ldots \ldots \ldots \ldots$

\section{Illustrations}

Planifue I A. Le Grèle au beo bigarte.

B. Le Plongeon a collier. Le Huard....

II A. Le Gokland argents. . 234

B. I a Sterne commune 254

111. A. Ie Harle \& poitrine rous

B. Jo Cunard ordinair. 255

IV. A. Je Canard noir..... 256

13. Is Sarcelle a ailes bleues 256

I. A. Le Canard huppe..

B. La Bernache du Canala. L'Outarde. $\quad \ldots \ldots \ldots \quad 257$

VI. A. Le IButor d'Amérique 259

B. Le Grand Héron bleu... . . $\quad 258$

V11. A. Lo Rale de la Caroline.... . . . . . . . . . . 259

13. La Berase d'Amérique..... . . . . . 259

VIII. A. La Berassine de Wileon .... ...

B. La Maubehe tachete. ... . . 260

IX. A. Le Pluvier Kildir ..... . . 261

B. Le Caille d'Amérique. ......... 201

X. A. Lo Tétras du Canada ....... . . . . . 262

B. La Gelinotte À Iraise...................... 262

XI. A. Lo Pigeon voyageur et la Tourterelle de la Caroline.... 263

B. Le Busard des marais ............................ . 263

XII. A. L'Epervier brun... . . . . . . . . . . . . . . . . . . 264

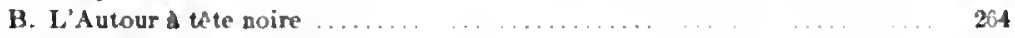

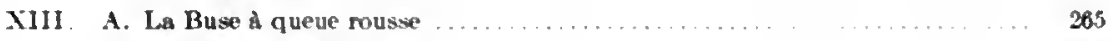

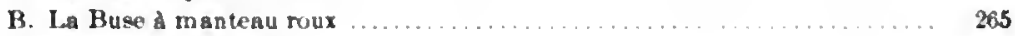

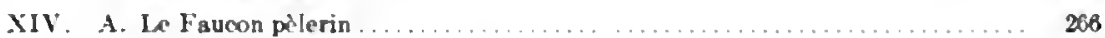

B. Le Faucon épervier. . . . . . . . . . . . . . . . . . . . . . 266

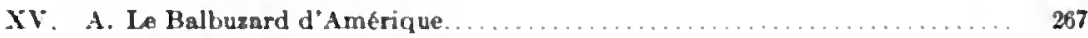

B. La Chouette du Carada.............................. 267

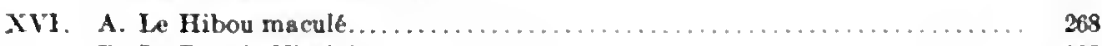

B. Le Due de Vinginie................................. 268

$28588-1\}$ 
Planche XVII. A. Le Coucou à bec noir. Le Coucou à bec jaune.............. 269

B. Le Martin-pêcheur............................ 269

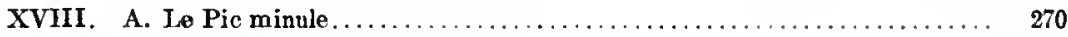

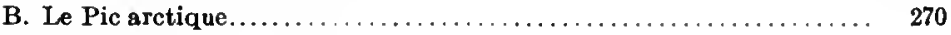

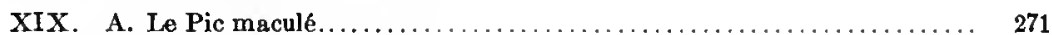

B. Le Pic a huppe écarlate.............................. 271

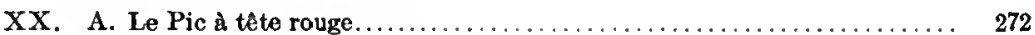

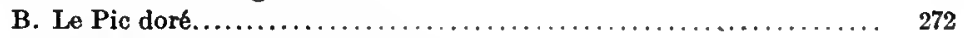

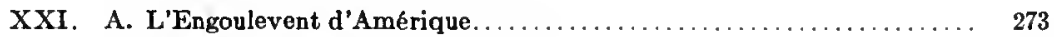

B. Le Martinet des cheminées.......................... 273

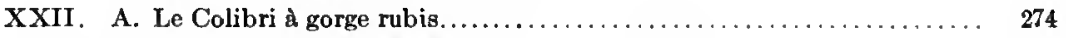

B. Le Moucherolle de la Caroline.......................... 274

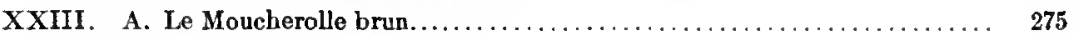

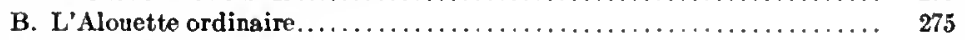

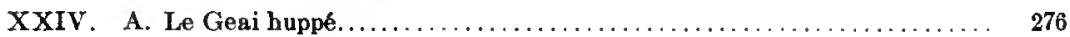

B. Le Geai du Canada............................... 276

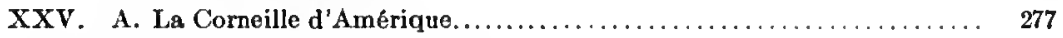

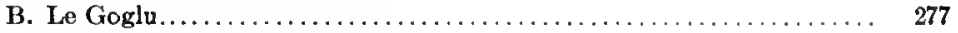

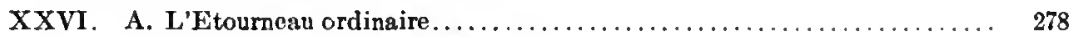

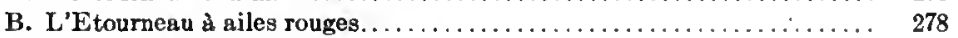

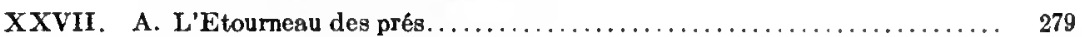

B. Le Loriot de Baltimore............................ 279

XXVIII. A. Le Mainate bronze............................... 280

B. Le Gros-bec des pins.............................. 280

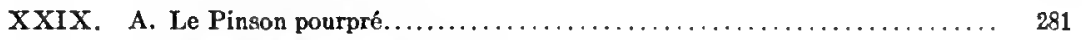

B. Le Moineau domestique............................ 281

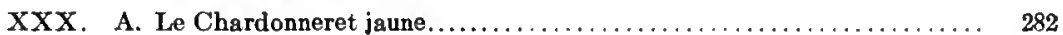

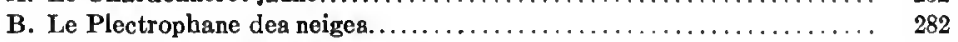

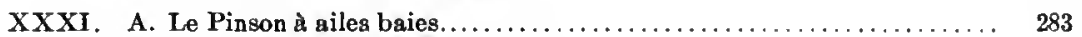

B. Le Pinson à couronne blanche......................... 283

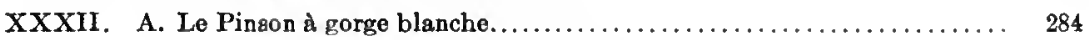

B. Le Pinson de montagne............................ 284

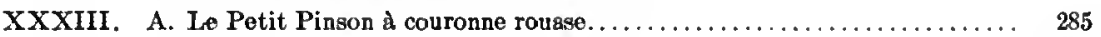

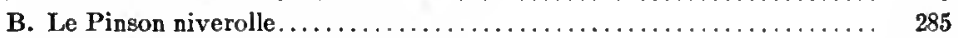

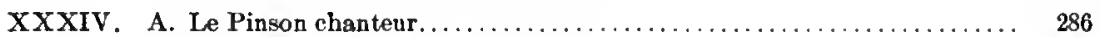

B. Le Gros-bec à poitrine rose........................... 286

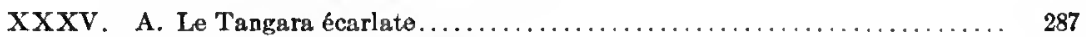

B. L'Hirondelle pourprée.......................... 287

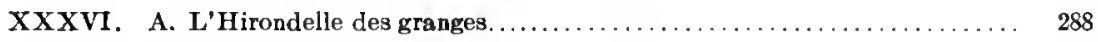

B. L'Hirondelle bicolore............................. 288

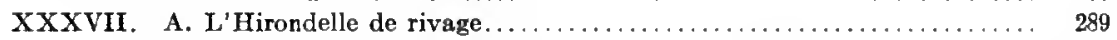

B. Le Jaseur du cèdre.............................. 289

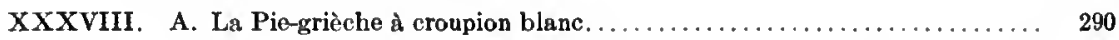

B. Le Vireo aux yeux rouges........................... 290

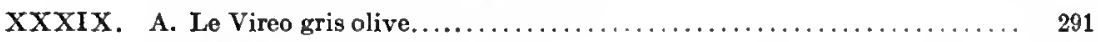

B. La Fauvette noire et blanche........................... 291

XL. A. La Fauvette jaune.............................. 292

B. La Fauvette bleue à gorge noire...................... 292

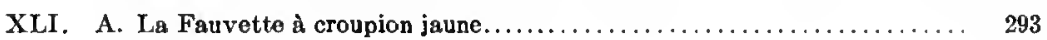

B. La Fauvette à tête cendrée.......................... 293 
Planche XliI. A. La Fauvette de Blackburn.

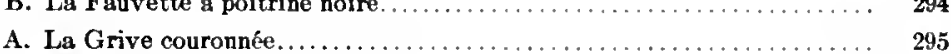

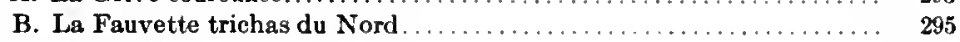

XLIV. A. La Fauvette à queue rousse $\ldots \ldots \ldots \ldots \ldots \ldots \ldots \ldots \ldots \ldots \ldots \ldots \ldots$

B. La Grive de la Caroline............................. 296

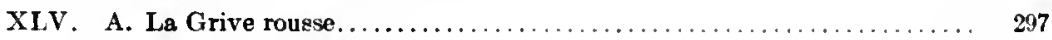

B. Le Troglodyte aédon . . . . . . . . . . . . . . . . . . . . . . . 297

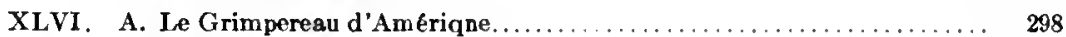

B. La Sittelle de la Caroline.......................... 298

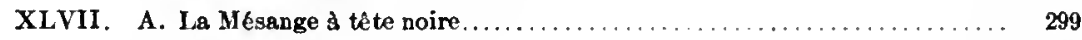

B. Le Roitelet huppe............................... 299

Le Roitelet à couronne rubis......................... 299

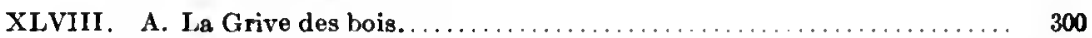

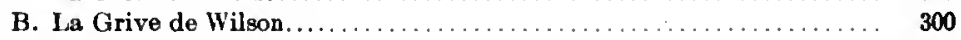

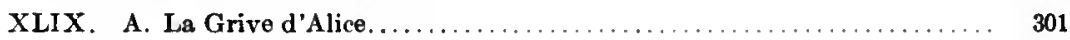

La Grive de Swainson.............................. 301

B. La Grive solitaire................................. 301

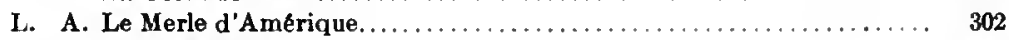

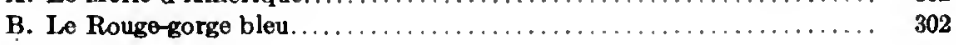

Figures 1 a 68 . Illustrations de la clef... $\ldots \ldots \ldots \ldots \ldots \ldots \ldots \ldots \ldots \ldots \ldots \ldots \ldots \ldots$ 



\section{Les Oiseaux de l'Est du Canada}

\section{INTRODUCTION}

\section{OAJET DE CET OUVRAGE}

Il s'est fait, en ces dernières années, un sérieux réveil de l'intérêt que le public apporte aux sujets d'histoire naturelle. De plus en plus le monde commence à se rendre compte du plaisir et des avantages qu'on peut retirer de l'observation des choses ordinaires de la création. Dans ce champ toujours plus vaste des études de la nature, il est peu de sujets qui aient autant sollicité l'attention générale que celui des oiseaux, et il est peu de formes de la vie qui éveillent au même point le sens esthétique. Ces formes sont magnifiques; elles excitent la curiosité; leurs manifestations fugitives piquent l'imagination; et parce qu'elles se présentent constamment sous de nouveaux aspects, elles ne sont jamais banales.

L'ornithologie est un côté par lequel les problèmes de la nature peuvent s'attaquer avec succès sous toute sorte de points de vue et par tant de chemins qu'il y a la pour beaucoup de gens un travail d'un grand intérêt et d'une grande valeur qui pourra se faire selon le gout d'un chacun et l'oceasion qui s'offrira. Ceux qui ont un penchant pour un travail systématique peuvent fendre leurs définitions au point de les rendre aussi ténues que le permettra la puissance d'observation propre à l'homme. Le psychologue du monde animal peut étendre ses problèmes aussi loin que s'y prêteront les méthodes d'expérimentation dont s'avisera son génie. L'admirateur ordinaire de la nature fera ses observations et ses notes aussi laborieusement que le permettra l'occasion; il pourra donner des renseignements d'un intérêt scientifique aussi bien que populaire; se complaire à observer des beautés passagères, exercer ses dons d'observation, et acquérir un savoir qui accroitrera considérablement la faculté qu'il a de gooter les choses de la nature. Même l'homme qui ne voit que le côté pratique des choses et n'entend rien à celui du sentiment, qui n'éprouve aucune sympathie pour la beauté abștraite, voit son attention éveillée par la valeur marchande des oiseaux.

L'ouvrage sur les "Oiseaux de l'Est du Canada " a été écrit pour éveiller l'intérêt, pour le surexciter là où il existe déjà, qu'il soit esthétique ou pratique, en faveur de l'étude des oiseaux canadiens; il a aussi pour buts: $1^{\circ}$ de faire voir l'importance de cette étude sous le rapport du sentiment, de la science et du commerce; $2^{\circ}$ d'aider à reconnaitre les races indigènes; $3^{\circ}$ de fournir à l'économiste des moyens faciles de distinguer les oiseaux nos amis de ceux qui sont nos ennemis, de manière à en user intelligemment à leur égard et au mieux des intérêts de l'homme et du pays entier; $4^{\circ}$ de présenter sous une forme facilement accessible des données dignes de confiance pour servir de base à des mesures de législation protectrices; $5^{\circ}$ de montrer quelques-uns des pièges où se sont pris dans le passé des gens sans expérience; enfin, $6^{\circ}$ d'indiquer des méthodes propres à éviter ces pièges. 


\section{LE CADRE DE CET OUVRAGE.}

Cet ouvrage comprend l'ensemble des oiseaux que l'observateur ordinaire a chance de rencontrer entre la côte de l'Atlantique et les prairies situées au nord de la frontière internationale. Cette région forme une aire zoologique naturelle (voir Distribution p. 8) comprenant ce qu'on peut appeler les terres forestières du Canada, section assez homogène au triple point de vue physique, géographique et zoologique. Les prairies sont absolument différentes de caractères et, par conséquent, offrent un aspect totalement différent de la vie des oiseaux. Les oiseaux de plein air sont naturellement autres que ceux des terres de forêts; de là vient que le Manitoba a été adopté comme frontière occidentale du territoire zoologique dont traite le présent ouvrage.

Bien que n'étant pas à proprement parler une liste complète des oiseaux du Canada oriental, ce livre s'en rapproche cependant beaucoup. On n'a pas tenu compte d'un petit nombre d'espèces dont le status canadien est douteux, qui se présentent ou rarement ou seulement par accident. On a usé à cet égard de la plus grande liberté, et certaines espèces ont été admises parce que cela était utile; quelques-unes comme pouvant probablement se rencontrer en cherchant bien, quelques autres comme faisant ressortir un cas généralement intéressant de façon plus piquante que ne feraient des espèces indigènes ordinaires, quelques-unes enfin parce qu'on les a confondues avec des formes plus communes.

\section{LE PLAN DE L'ouvrage.}

L'arrangement systématique (voir Classification p. 5 et la nomenclature p. 8) dont on s'est servi est celui du catalogue complet de l'Union des Ornithologistes américains, 3ème édition, 1910, et des éditions supplémentaires de 1912 et de 1920 . Bien qu'il soit reconnu que cette classification est quelque peu imparfaite et que les détails en sont donnés à titre d'essai, c'est cependant celle sur laquelle est basée la plus grande partie de la littérature américaine récemment parue au sujet des oiseaux et qui est d'un usage général dans l'Amérique du Nord.

En traitant le sujet des sous-espèces, on s'est départi de la méthode ordinaire pour en adopter une que l'auteur croit plus strictement conforme aux faits de la nature et aux vues modernes. Les espèces ont été traitées comme des assemblages de sous-espèces, chacune de rang et d'importance identiques, et non plus, ainsi que c'est la coutume, comme des espèces avec des sous-espèces subordonnées .qui dépendent des premières. L'espèce est d'abord donnée comme un tout, comprenant ses races sous-spécifiques, et sous un sous-titre il est fait mention des sous-espèces spéciales qui se présentent dans le cadre géographique de l'ouvrage. Il n'est résulté de cela aucune confusion, aucun changement, sauf dans l'emploi des noms dans la langue du pays, où le lecteur trouvera quelques innovations apportées aux noms donnés et autorisés par l'Union des Ornithologistes américains. Dans la nomenclature scientifique, la véritable importance des espèces relativement aux sous-espèces a été exprimée; mais les noms ordinaires n'ont pas, jusqu'ici, rendu cette idée de subordination, et c'est ce fait qui, dans bien des cas, a entraîné l'usage de termes sous-spécifiques définis alors quc, selon la nature du cas, il était impossible d'en déterminer l'exactitude, ou malavisé de les recomnaitre. C'est ainsi qu'il y a eu une tendance dans l'opinion tant popu- 
laire que scientifique à attacher une importance indue à ces petites définitions. Pour corriger cet état de choses, certaines adaptations de noms vulgaires ont été nécessaires, mais on s'est écarté alors le moins possible de l'usage reçu. D'anciens termes ont été rétablis là où c'était possible, mais comme les noms courants étaient aussi donnés, aucune confusion ne pouvait se produire. Dans quelques cas il a été nécessaire d'appliquer le nom sousspécifique reconnu du type a l'espèce entière et d'en adopter un nouveau pour la forme ainsi dépouillée. En procédant de la sorte, il était bon qu'il se fit dans l'usage courant un changement aussi petit que possible et compatible avec le but en vue. C'est pour cela que, sauf quand de bonnes raisons s'y opposaient, le nouveau nom sous-spécifique se formait en faisant précéder d'un adjectif, le terme spécifique employé jusque-là. Chaque changement apporté à l'usage en cours a été adopté d'après ses mérites propres. Quoiqu'il ne soit pas douteux que le principe de cette réforme ait sa raison d'être, la manière de l'appliquer a donné lieu à beaucoup de raisonnement, à de nombreuses communications avec d'autres gens, et à quelques hésitations dans des cas particuliers.

L'alouette ordinaire est un exemple de ce problème. La sous-espèce type Otocoris alpestris alpestris, a généralement été connue comme étant l'alouette ordinaire, en dépit du fait que l'une quelconque des quatorze autres races géographiques a autant de droit à ce nom et que c'est le seul nom pour l'espèce entière. La manière la plus simple c'est d'appeler la sous-espèce Otocoris alpestris alpestris (bien que décrite la première, elle n'a pas de supériorité, par la classification, sur d'autres formes) l'alouette. ordinaire de l'Est, et d'appliquer le nom d'alouette ordinaire à la collection entière des sous-espèces du même ordre, en le faisant synonyme du nom scientifique à deux termes Otocoris alpestris.

La pie-grièche migratrice offrait d'autres difficultés. La manière logique de procéder consisterait à dénommer l'espèce entière la pie-grièche de Louisiane, d'après son nom scientifique de ludovicianus. Mais ce serait introduire un nom peu familier que peu de personnes reconnaitraient. L'espèce a donc été appelée ici la pie-grièehe, et l'oiseau du Canada oriental, le "loggerhead" migrateur, en admettant que l'oiseau appelé jusque-là «loggerhead" puisse logiquement être dénommé le aloggerhead" méridional.

Ce serait espérer beaucoup trop que de croire que ce résultat satisfera tout le monde; le présent auteur espère cependant qu'il sera accepté jusqu'au jour où le comité de l'Union des Ornithologistes américains sera saisi de la question et prendra des décisions qui feront autorité.

Dans les pages suivantes, le numéro et le nom, dans la langue du pays aussi peu modifiée que possible, ont été empruntés pour l'édition anglaise, a la nomenclature de l'Union des Ornithologistes américains. Pour la traduction française de ces noms, qui se présentent d'abord en caractères gras comme en-têtes spécifiques, on s'est guidé principalement sur l'excellent ouvrage de Dionne: "Les oiseaux de la province de Québec." Le terme correspondant en anglais est alors indiqué, entre parenthèses, puis vient le nom spécifique latin en lettres italiques. Plusieurs des noms français ont été fournis par le Dr C. W. Townsend qui a des connaissances très grandes dans l'ornithologie des provinces de l'Est. Là où nous n'avons pas pu trouver de noms français correspondant à l'anglais, nous avons cru devoir donner entre guillemets la traduction littérale soit du nom anglais soit du nom latin. 
rang identique ou d'un rang subordonné. La classe Aves ou Oiseaux peut être représentée par un arbre, la hauteur duquel représenterait le temps en âges géologiques depuis le plus ancien, tout en bas, jusqu'à l'âge actuel, près du sommet. Le tronc sera montré comme étant double à sa base; une branche serait une souche morte, courte, et représenterait les oiseaux fossiles, à dents, race qui devint éteinte avant les temps géologiques actuels; l'autre branche, grosse et prospère, représenterait les formes modernes sans dents. Cette branche, à son tour, se partagerait en deux autres, à petite distance de la base, et représenterait les deux sous-classes, les oiseaux sans bréchet et les oiseaux à bréchet. Les premiers seraient représentés par la branche de beaucoup la plus petite; les derniers se diviseraient et se subdiviseraient en branches représentant d'abord des ordres, ensuite des familles, puis des genres et enfin des espèces.

La valeur de ces divisions, c'est-à-dire, la somme de différenciation nécessaire pour élever un groupe de genres au rang d'une famille, ou une collection de familles au rang d'un ordre, est quelque chose qui dépend de l'expérience et de la décision du savant, vu qu'il n'y a sur ce point aucune règle faisant autorité. Néanmoins il s'est créé peu à peu une entente approximative sur ce sujet, bien que la tendance constante parmi les spécialités a été de faire des distinctions de plus en plus subtiles et de multiplier le nombre des différents groupes.

La plus petite division, généralement adoptée, est celle de l'espèce. Bien que chacun de nous se fasse une idée plus ou moins exacte de ce qu'est une espece, qu'on l'appelle de ce nom ou de tout autre, aucune définition satisfaisante n'a jamais été établie pour l'expliquer. C'est ce qu'on appelle communément une "sorte d'animal". Ainsi le cheval est une différente "sorte" ou espèce qu'un âne, le rouge-gorge bleu qu'un rouge-gorge. Ils ont des caractères différents bien tranchés, nichent régulièrement avec des oiseaux de même espèce seulement, et produisent des petits de même espèce. Des espèces différentes ne nichent pas communément ensemble, mais quand cela arrive il se forme des croisements ou des produits hybrides qui sont, en général, stériles. Jusqu'à un temps relativement récent on ne reconnaissait aucune division plus petite, mais grâce à une étude serrée du sujet il est devenu évident aux yeux des ornithologistes que, au sein de l'espèce, il y a des variations considérables, soit quant aux individus soit quant aux localités.

La variation quant aux individus est la différence naturelle qui peut se présenter à un moment quelconque entre ceux que relie une parenté commune, comme par exemple, entre frères et sœurs du même sang. De même que tout individu provient de son semblable, on peut dire qu'un individu ne reproduit pas toujours son semblable, car, dans certaines limites, de deux créatures, l'une n'est jamais le pur double de l'autre. Il s'agit ici de la variation individuelle, ordinairement faible et irrégulière par l'apparence et la direction, mais qui quelquefois persiste progressivement de génération en génération dans une seule direction pour former la base par laquelle nos évolutionnistes expliquent l'origine des espèces. La variation des individus, toutefois, est négligée dans la classification, à moins qu'elle n'ait fait assez de progrès pour produire une différenciation évidente et constante d'avec le groupe naturel et définissable d'une espèce.

La variation au point de vue géographique peut être considérée comme le résultat d'une tendance ordinaire de la variation individuelle agissant dans une société entière d'individus dirigée vers un but commun; elle passe pour être motivée et guidée par des conditions climatologiques locales et 
d'autres encore. C'est ainsi que nous trouvons souvent que parmi une espèce très disséminée, tous les individus qui habitent certaines localités ont certains caractères qui les séparent des individus des régions environnantes. Les individus d'une contrée déserte et aride sont volontiers plus petits et de couleur plus claire, tandis que ceux d'une contrée chaude et humide sont d'habitude plus gros et plus foncés. Ces différences sont quelquefois manifestes et très visibles; d'autres fois elles sont si légères qu'on ne les remarque qu'en comparant un très grand nombre de spécimens, et qu'on ne les découvre qu'en recourant à des moyennes. C'est ainsi qu'il y a tous les degrés d'une différenciation due à l'habitat géographique, depuis des différences prononcées qui ont presque une importance spécifique, jusqu'aux plus fines nuances d'une différenciation que seuls des spécialistes exercés peuvent distinguer et que l'œil ordinaire est incapable d'apprécier. Toutefois, le fait saillant qui empêche la variation géographique la plus patente d'être reconnue comme complètement spécifique, c'est que ces formes de moindre importance passent l'une dans l'autre et que dans des localités intermédiaires, il peut se rencontrer l'une quelconque des nuances de différenciation qui existent entre l'un des extrêmes et l'autre. De ce mélange graduel des signes caractéristiques entre eux la science n'est pas supposée tenir compte, et quelque subtiles que puissent être les distinctions, les divisions doivent être tranchées et précisées. Quant à nous, nous reconnaissons ces variations qui s'entremêlent et sont dues à une distribution géographique, en tant que race, variétés ou sous-espèces géographiques, ce dernier terme étant aujourd'hui d'un usage courant, et nous les considérons comme des espèces en voie de formation avant que les phases qui les rattachent à la souche originelle aient disparu, à cause de la stérilité croissante entre les variantes extrêmes. Sauf dans les cas rares d'isolement physique, tel que celui d'un habitat insulaire océanique qui s'oppose à un habitat continu, nous considérons, dans la pratique, l'existence des entremêlements comme la preuve d'un status sous-spécifique. En sus de ces divisions d'importance pour la classification il y a quelques autres variantes, qui par suite de leur survenance irrégulière, ne peuvent pas être reconnues dans notre classification. Ce sont les "Albinos", les "Melanos", et les "Formes dichromatiques".

Chaque oiseau de l'Amérique du Nord porte un nom vulgaire ou de la langue du pays, autorisé par l'usage et reconnu par les ornithologistes de renom; il est rarement nécessaire, dans ce cas, d'employer la nomenclature scientifique. Toutefois, il est bon que ceux qui s'intéressent aux oiseaux se familiarisent avec le plus grand nombre possible de noms scientifiques, car ils sont non seulement nécessaires dans les travaux plus avancés, mais ils sont d'un usage pratique pour bien comprendre les rapports généraux qui existent entre les différentes espèces.

Le présent système de nomenclature avec deux termes fut introduit par Linné, le grand botaniste suédois, et employé dans son "Système de la Nature ", dixième édition, 1758, qui est l'autorité acceptée par les ornithologistes américains. Dans ce système chaque espèce a deux noms, le premier est celui du genre auquel elle appartient, le second est celui de l'espèce. Les noms génériques ne sont pas donnés en doutble dans la sphère de la zoologie, ni ceux de l'espèce à propos du genre. C'est ainsi que le rouge-gorge américain s'appelle Planesticus migratorius, c'est-à-dire que l'espèce du genre Planesticus est nommée migratorius. D'autres espèces du Planesticus ont d'autres noms spéciflques. 
rang identique ou d'un rang subordonné. La classe Aves ou Oiseaux peut être représentée par un arbre, la hauteur duquel représenterait le temps en âges géologiques depuis le plus ancien, tout en bas, jusqu'à l'âge actuel, près du sommet. Le tronc sera montré comme étant double à sa base; une branche serait une souche morte, courte, et représenterait les oiseaux fossiles, à dents, race qui devint éteinte avant les temps géologiques actuels; l'autre branche, grosse et prospère, représenterait les formes modernes sans dents. Cette branche, à son tour, se partagerait en deux autres, à petite distance de la base, et représenterait les deux sous-classes, les oiseaux sans bréchet et les oiseaux à bréchet. Les premiers seraient représentés par la branche de beaucoup la plus petite; les derniers se diviseraient et se subdiviseraient en branches représentant d'abord des ordres, ensuite des familles, puis des genres et enfin des espèces.

La valeur de ces divisions, c'est-à-dire, la somme de différenciation nécessaire pour élever un groupe de genres au rang d'une famille, ou une collection de familles au rang d'un ordre, est quelque chose qui dépend de l'expérience et de la décision du savant, vu qu'il n'y a sur ce point aucune règle faisant autorité. Néanmoins il s'est créé peu à peu une entente approximative sur ce sujet, bien que la tendance constante parmi les spécialités a été de faire des distinctions de plus en plus subtiles et de multiplier le nombre des différents groupes.

La plus petite division, généralement adoptée, est celle de l'espèce. Bien que chacun de nous se fasse une idée plus ou moins exacte de ce qu'est une espèce, qu'on l'appelle de ce nom ou de tout autre, aucune définition satisfaisante n'a jamais été établie pour l'expliquer. C'est ce qu'on appelle communément une "sorte d'animal». Ainsi le cheval est une différente "sorte" ou espèce qu'un âne, le rouge-gorge bleu qu'un rouge-gorge. Ils ont des caractères différents bien tranchés, nichent régulièrement avec des oiseaux de même espèce seulement, et produisent des petits de même espèce. Des espèces différentes ne nichent pas communément ensemble, mais quand cela arrive il se forme des croisements ou des produits hybrides qui sont, en général, stériles. Jusqu'à un temps relativement récent on ne reconnaissait aucune division plus petite, mais grâce à une étude serrée du sujet il est devenu évident aux yeux des ornithologistes que, au sein de l'espèce, il y a des variations considérables, soit quant aux individus soit quant aux localités.

La variation quant aux individus est la différence naturelle qui peut se présenter à un moment quelconque entre ceux que relie une parenté commune, comme par exemple, entre frères et sœurs du même sang. De même que tout individu provient de son semblable, on peut dire qu'un individu ne reproduit pas toujours son semblable, car, dans certaines limites, de deux créatures, l'une n'est jamais le pur double de l'autre. Il s'agit ici de la variation individuelle, ordinairement faible et irrégulière par l'apparence et la direction, mais qui quelquefois persiste progressivement de génération en génération dans une seule direction pour former la base par laquelle nos évolutionnistes expliquent l'origine des espèces. La variation des individus, toutefois, est négligée dans la classification, à moins qu'elle n'ait fait assez de progrès pour produire une différenciation évidente et constante d'avec le groupe naturel et définissable d'une espèce.

La variation au point de vue géographique peut être considérée comme le résultat d'une tendance ordinaire de la variation individuelle agissant dans une société entière d'individus dirigée vers un but commun; elle passe pour être motivée et guidée par des conditions climatologiques locales et 
d'autres encore. C'est ainsi que nous trouvons souvent que parmi une espèce très disséminée, tous les individus qui habitent certaines localités ont certains caractères qui les séparent des individus des régions environnantes. Les individus d'une contrée déserte et aride sont volontiers plus petits et de couleur plus claire, tandis que ceux d'une contrée chaude et humide sont d'habitude plus gros et plus foncés. Ces différences sont quelquefois manifestes et très visibles; d'autres fois elles sont si légères qu'on ne les remarque qu'en comparant un très grand nombre de spécimens, et qu'on ne les découvre qu'en recourant à des moyennes. C'est ainsi qu'il y a tous les degrés d'une différenciation due à l'habitat géographique, depuis des différences prononcées qui ont presque unc importance spécifique, jusqu'aux plus fines nuances d'une différenciation que seuls des spécialistes exercés peuvent distinguer et que l'œil ordinaire est incapable d'apprécier. Toutefois, le fait saillant qui empêche la variation géographique la plus patente d'être reconnue comme complètement spécifique, c'est que ces formes de moindre importance passent l'une dans l'autre et que dans des localités intermédiaires, il peut se rencontrer l'une quelconque des nuances de différenciation qui existent entre l'un des extrêmes et l'autre. De ce mélange graduel des signes caractéristiques entre eux la science n'est pas supposée tenir compte, et quelque subtiles que puissent être les distinctions, les divisions doivent être tranchées et précisées. Quant a nous, nous reconnaissons ces variations qui s'entremêlent et sont dues à une distribution géographique, en tant que race, variétés ou sous-espèces géographiques, ce dernier terme étant aujourd'hui d'un usage courant, et nous les considérons comme des espèces en voie de formation avant que les phases qui les rattachent a la souche originelle aient disparu, a cause de la stérilité croissante entre les variantes extrêmes. Sauf dans les cas rares d'isolement physique, tel que celui d'un habitat insulaire océanique qui s'oppose à un habitat continu, nous considérons, dans la pratique, l'existence des entremêlements comme la preuve d'un status sous-spécifique. En sus de ces divisions d'importance pour la classification il y a quelques autres variantes, qui par suite de leur survenance irrégulière, ne peuvent pas être reconnues dans notre classification. Ce sont les "Albinos", les "Melanos", et les "Formes dichromatiques".

Chaque oiseau de l'Amérique du Nord porte un nom vulgaire ou de la langue du pays, autorisé par l'usage et reconnu par les ornithologistes de renom; il est rarement nécessaire, dans ce cas, d'employer la nomenclature scientifique. Toutefois, il est bon que ceux qui s'intéressent aux oiseaux se familiarisent avec le plus grand nombre possible de noms scientifiques, car ils sont non seulement nécessaires dans les travaux plus avancés, mais ils sont d'un usage pratique pour bien comprendre les rapports généraux qui existent entre les différentes espèces.

Le présent système de nomenclature avec deux termes fut introduit par Linné, le grand botaniste suédois, et employé dans son "Système de la Nature ", dixième édition, 1758, qui est l'autorité acceptée par les ornithologistes américains. Dans ce système chaque espèce a deux noms, le premier est celui du genre auquel elle appartient, le second est celui de l'espèce. Lcs noms génériques ne sont pas donnés en doưble dans la sphère de la zoologie, ni ceux de l'espèce à propos du genre. C'est ainsi que le rouge-gorge américain s'appelle Planesticus migratorius, c'est-à-dire que l'espèce du genre Planesticus est nommée migratorius. D'autres espèces du Planesticus ont d'autres noms spéciflques. 
Les trois buts de toute nomenclature scientifique sont l'exactitude, l'universalité ct la permanence. A cette fin la terminologie des sujets du monde zoologique est sujette à des lois rigoureuses dont les principes sont universellement acceptés et employés, selon des règles très strictes. En vertu de ces lois le nom scientifique d'une espèce n'est pas une affaire de préférence personnelle, mais est invariable, de telle sorte que personne ne peut le mettre en question et qu'aucun changement ne peut s'introduire dans la nomenclature scientifique sauf ceux qui sont nécessaires pour corriger des erreurs courantes dans l'application des lois de ce code. A mesure que les connaissances avançaient il est devenu nécessaire de s'éloigner un peu de la lettre sinon de l'esprit du système à deux termes de Linné, et en ajoutant un troisième terme pour désigner la sous-espèce, d'en faire un nom à trois termes. Partout où un nom à trois termes est en usage, c'est le nom d'une sousespèce de la forme primitive à deux noms. Le premier spécimen décrit, ou le premier spécimen auquel un nom a été attaché, est considéré comme la forme appelée "type". C'est pourquoi en divisant une espèce en sousespèces on donne tout naturellement la première place à la forme qui la première reçut le nom de l'espèce, et son nom sous-spécifique est formé par la répétition de son nom spécifique. C'est ainsi que le rouge-gorge américain qui fut pour la première fois décrit et spécifiquement dénommé par Linné, en 1776, comme migratorius, quand il est mentionné sous-spécifiquement pour le distinguer du rouge-gorge méridional ou de l'occidental, devient Planesticus migratorius migratorius. Le rouge-gorge de l'Ouest qui fut distingué de celui-ci pour la première fois par Ridgway, en 1887, fut nommé par lui Planesticus migratorius propinquus, et le rouge-gorge du Sud, par Batchelder, en 1900, s'appelle Planesticus migratorius achrusterus. Dans la pratique là où les noms génériques ou spécifiques sont donnés par le contexte, il est d'usage de les indiquer par l'initiale comme $P$. migratorius, ou $P$. m. migratorius.

Les variétés sous-spécifiques sont des divisions de l'espèce de moindre importance, sauf dans certains ordres de travaux où une exactitude particulière est nécessaire. Comme ces sous-espèces sont aussi souvent basées sur des circonstances que seuls des observateurs très expérimentés peuvent apprécier, elles restent en dehors des choses qui sollicitent l'intérêt de l'observateur ordinaire.

\section{DISTRIBUTION GÉOGRAPHIQUE.}

Les faits les plus généraux de la distribution géographique des êtres animés sur la planète sautent aux yeux de l'observateur même le moins attentif. Les divisions fondamentales de cette distribution par les tropiques, les zones tempérées et arctiques, sont des plus manifestes, mais une étude serrée de plus près montre qu'au sein de ces larges divisions il s'en trouve de plus étroites et de moins évidentes. En Amérique, au nord du golfe du Mexique, il y a trois régions de la vie animale, lesquelles se conforment grosso modo aux régions susdites et appelées tropicale, australe et boréale. Celles-ci sont subdivisées en zones de vie, caractérisées chacune, par des assemblages particuliers de plantes et d'animaux.

La zone tropicale est suffisamment caractérisée par le nom qu'elle porte et il suffit de la mentionner.

La région australe correspond, de façon générale, à l'idée qu'on se fait en géographie de la zone tempérée. Ellc est divisée en trois zones de vie: 
l'australe inférieure, l'australe supérieure, et la zone de transition. L'australe inférieure pourrait être désignée comme la sous-tropicale, et s'étend au nord comprenant les états du golfe du Mexique et de l'Atlantique méridional; elle ne s'étend pas du tout jusqu'au Canada. La zone australe supérieure est la première qui nous intéresse directement dans l'est du Canada; elle ne fait que traverser la frontière sur le rivage du lac Erié et comprend la fameuse zone à fruits dite du Niagara. La présence fréquente ou régulière de nombreuses espèces méridionales à Pointe Pelée dans le comté d'Essex (Ontario) indique le développement le plus fort de cette zone dans le Dominion. Elle frise encore une fois notre frontière méridionale dans la Saskatchewan et peut-être quelques-unes des vallées les plus chaudes qui aboutissent dans la Colombie britannique méridionale. La zone australe la plus au nord, ou zone tempérée, forme la zone de transition qui comprend la majeure partie des régions les plus cultivées du Canada. Elle occupe les rivages de la baie de Fundy, le cours supérieur du Saint-Laurent, le Québec et l'Ontario méridionaux, les sections inférieures des provinces des prairies et une bande du rivage maritime dans la Colombie britannique; elle indique la limite de la culture extensive.

La région boréale est divisée en zones: canadienne, hudsonienne, et arctique. La zone canadienne comprend le reste des terres forestières, au nord de la zone de transition; elle est surtout conifère et se continue à travers le continent jusque vers la limite nord des terres généralement cultivées. La zone hudsonienne se trouve dans la région plus au nord, celle des petits arbustes ou des arbres rabougris, contrée impropre à l'agriculture; et la zone arctique s'étend à travers les terres stériles jusqu'au pôle.

Ces zones de vie basées sur la température, et qui suivent approximativement les lignes de latitude, sont cependant détournées de leur direction vers l'est et vers l'ouest par des circonstances locales assez variables comme le voisinage des courants marins ou froids ou chauds, la présence de grandes étendues d'eau, l'altitude au-dessus de la mer, l'influence des vents froids ou chauds, des barrières de montagnes, et d'autres causes encore. En sorte qu'au lieu de zones égales, on les trouve irrégulières et elles ne suivent qu'à peu près les parallèles de latitude.

L'altitude est un facteur important dans la distribution de la vie qui dépend de la température. Sous les tropiques lorsqu'on fait l'ascension d'une haute montagne on peut rencontrer la température de chaque zone, depuis celle de la plaine environnante jusqu'à la zone arctique du sommet couvert de neige, et l'on rencontrera aussi les assemblages correspondants d'espèces habitant chacune de ces zones. La jonction des zones arctique et hudsonienne au détroit de Belle-Isle, sous la même latitude que Lands-End en Angleterre, est une illustration de l'énorme influence du courant arctique qui descend du détroit de Davis et contraste avec l'influence du courant - chaud du Gulf-Stream qui s'en va mourir sur les rivages d'Angleterre.

Nous pouvons également observer des groupements plus petits qui sont basés sur des conditions autres que la température et ce sont les variations d'humidité qui sont les facteurs décisifs en ce cas. C'est ainsi que la vie dans les forêts de l'Est diffère manifestement de celle des plaines plus arides des provinces des prairies et toutes deux diffèrent de façon frappante de la vie sur les pentes humides du Pacifique.

Si l'on prend les formes de l'Est comme typiques, dans l'acception ordinaire du mot, les oiseaux de prairie que l'on peut comparer sont légère- 
ment plus petits et beaucoup plus pâles de couleur, tandis que sur la côte humide du Pacifique ils sont plus gros et beaucoup plus foncés de couleur. Grâce à ces influences nous trouverons donc dans l'Ouest beaucoup de sous-cspèces des oiseaux de l'Est. Des espèces relativement peu nombreuses habitent sans modifications tout le continent; plusieurs sont représentées à l'Est et à l'Ouest par deux sous-espèces, ou même davantage qui laissent voir plus ou moins de différenciation et, en d'autres cas, elles sont remplacées par des espèces étroitement apparentées, ou ne le sont pas du tout.

En observant ces divisions de la faune il faut cependant se rappeler que, pour ce qui regarde les oiseaux, ces associations doivent dépendre entièrement des sujets reproducteurs. Les oiseaux voyagent si loin et par tant de détours dans leurs migrations qu'ils peuvent traverser mainte région de faune spéciale au printemps et en automne bien qu'ils ne nichent que dans une seule. De là vient que, en déterminant l'habitat auquel il faut rapporter une certaine région, il n'y a pas à tenir compte de ces oiseaux passagers.

Bien que les aires de distribution indiquées sous les titres spécifiques qui suivent, soient plutôt vagues, il y en a plusieurs qui suivent des lignes générales assez semblables. C'est ainsi que quelques-unes sont appelées: "La région des grands lacs inférieurs," ce sont probablement les formes australes supérieures. (L'Ontario et le Québec-Sud» se rapporte à une espèce d'une zone de transition, tandis que "Au delà de la colonisation populeuse ou à la limite des champs cultivés» se rapporte naturellement à des espèces de la zone canadienne.

Les espèces suivantes sont données pour indiquer quels oiseaux doivent se trouver dans chaque zone:

Zone australe superieure
Cardinal
Loriot des vergers
Roitelet de la Caroline
Pinson des sauterelles
Gobe-mouches gris bleuâtre
Dickcissel
Zone de transition ${ }^{1}$
Goglu
Grive des bois
Vireo a front jaune
Loriot de Baltimore
Pinson aux yeux rouges
Coucou
Pinson des champs
Rouge-gorge bleu
Grive de la Caroline

Zone canadienne Mésange de la baie d'Hudson Sittelle du Canada Grive de Swainson Pic d'Amérique Grive solitaire Pinson a gorge blanche Geai du Canada Grive d'Alice Pinson niverolle

Zone hudsonienne

Buse pattue Pinson fauve Pie-grieche boréale Pinson à couronne blanche Gros-bec des pins Farlouse d'Amérique

Zone arctique
Lagopede
Harfang
Plectrophane des neiges
Faucon
Plectrophane

Le arctique

Harfang

Plectrophane

\section{MIGRATION.}

La migration des oiseaux, leur apparition et leur disparition périodiques et saisonnières constituent un des phénomènes les plus visibles de la nature. Le fait que beaucoup d'oiseaux disparaissent en hiver est chose universellement connue et qui attira de tout temps l'attention. Bicn qu'on vît là autrefois un fait mystérieux et qu'il ne soit pas encore parfaitement compris dans tous ses détails, on commence à s'en étonner moins et à l'admirer

\footnotetext{
1 La plupart des especes de cette zone ga rencontrent aussi dans la partie supérieure du continent austral, mais atteignent lá leur limita septentrionale. La préaence de ees especes, en méme tempa que l'abseneo de cellea des zonea extremea, sont les caracteres lea plus marqués de la zone de transition.
} 
davantage à mesure que des connaissances exactes prennent la place des vagues imaginations de jadis. Aujourd'hui on sait où nos espèces du Nord passent l'hiver et l'on connait la plupart des routes qu'elles suivent quand elles viennent et s'en vont. On sait qu'à tout prendre elles sont guidées par des sens, par des influences ordinaires et quotidiennes assez connues, probablement très développées et non par les puissances et les instincts mystérieux qu'on leur supposait autrefois.

La cause fondamentale de la migration est évidemment la croissante ou décroissante masse de leurs subsistances. Les oiseaux quittent les pays du nord où ils sont nés parce que c'est là le seul moyen de ne pas mourir de faim. Plusieurs espèces peuvent résister au froid les plus rigoureux, mais aucune ne peut vivre longtemps sans nourriture, et quoique certaine nourriture d'oiseau persiste pendant l'hiver au Canada, la quantité en est petite et ne suffit qu'à une population peu nombreuse, et cette provision même diminue rapidement, ou, dans le Nord, est ensevelie sous la neige. La raison qui, en automne, les fait émigrer vers le Sud, est assez évidente, mais pour quelle raison un oiseau quitterait-il un climat doux et une nourriture abondante, au midi, pour affronter un voyage dangereux et se trouver finalement dans une contrée où l'hiver finit à peine et où il court le danger de mourir de faim. Bien des explications ingénieuses ont été avancées pour expliquer la chose: le désir de retrouver la contrée où il est né, en deux mots, le mal du pays; les souvenirs héréditaires d'une ancienne patrie, se transmettant à travers des âges géologiques; la recherche d'une nourriture spéciale pour couver; et l'insuffisance de places favorables à la nidification dans les contrées méridionales, voilà les raisons qui ont été alléguées comme pouvant expliquer le fait en question. Toutefois il n'est pas nécessaire d'avancer une explication compliquée ou embrouillée, alors qu'il y en a une simple et claire. Si nous nous rappelons que, dans la saison de la couvaison, la population des oiseaux est considérablement accrue par la naissance des petits; que si, en hiver, il y a place pour un nombre considérable d'oiseaux dans les localités méridionales l'accroissement de cette population, au printemps, dépasse les capacités alimentaires du pays; que, vers la même époque critique la région tempérée, au nord, est toute entière ouverte aux nouveaux arrivants avec une grande abondance de nourriture, le sujet cesse d'être mystérieux. En fait, ce n'est que par la migration qu'il est possible de tirer parti des moyens de sustentation que possèdent les régions tempérées à moins que les oiseaux ne jeûnent ou ne soient hivernants pendant les hivers, deux états à chacun desquels la nature des oiseaux répugne tout à fait.

Bien que la quantité disponible des vivres soit la cause fondamentale ou première de la migration, il nous faut chercher ailleurs encore pour expliquer à quelles sollicitations immédiates ses méthodes obéissent. Forcés qu'ils étaient à l'origine, par la faim, dans un sens et dans l'autre, ces déplacements annuels maintenant sont devenus instinctifs et ont lieu avant que la situation ne soit à l'état aigu, que les angoisses de la faim ne se soient fait sentir, ou que l'organisme ne soit affaibli par la privation de nourriture.

Le champ des migrations varie selon les espèces. Il y a un très petit nombre d'espèces qui, au sens propre de ce mot, n'émigrent pas du tout. Pour d'autres espèces les individus vivant plus au nord sont les seuls à quitter leurs cantonnements, ceux qui vivent au sud demeurent, en fait, stationnaires, bien que pour la majorité des espèces canadiennes, la communauté entière se transporte au sud. L'oiseau dont le champ de migration est le plus étendu c'est sans conteste la sterne arctique, oiseau qui niche 28588-2 
depuis le golfe du Saint-Laurent jusqu'aux régions polaires et qui hiverne, au sud, jusqu'au continent antarctique.

Les méthodes de migration sont presque aussi variées que le champ parcouru. Certaines espèces avancent le long du jour d'un sommet d'arbre $\grave{a}$ un autre, de bosquet en bosquet, trouvant leur route dans la direction voulue. D'autres font de longs vols, les uns très haut, les autres plus bas, dans l'air. Certains oiseaux ne volent que pendant le jour; d'autres voyagent de nuit, et nous ne sommes instruits de leur passage que par des circonstances accidentelles, soit que leur voix affaiblie nous arrive de plus haut dans l'obscurité, soit qu'ils se montrent soudain à nous vers le matin. Ils voyagent en troupes d'une seule ou de plusieurs espèces mélangées, en groupes épars, ou comme individus isolés.

Un bon nombre d'espèces, sinon toutes, suivent des routes plus ou moins définies tant pour partir de leurs stations de couvaison que pour y retourner; d'autres vont et reviennent par des routes tout à fait différentes. Il arrive aussi que des nappes d'eau relativement petites suffisent à détourner quelques espèces de leur route; d'autres, au contraire, traversent sans hésiter de vastes étendues de mer, sans se soucier des passages rapprochés et commodes sur terre ferme, que choisissent des espèces très proches parentes. Chez quelques espèces ce sont les oiseaux plus âgés qui vont en avant; chez d'autres les mâles précèdent les femelles.

Quant à la manière dont les oiseaux trouvent leur route c'est un point encore vaguement compris et les individus qui se sont éloignés beaucoup de leur habitat et de leur route habituelle laissent voir qu'ils sont aussi parfaitement perdus que le serait tout autre animal sur un terrain à lui inconnu. Il est sûr que l'expérience joue ici un grand rôle et il est hors de doute que les jeunes oiseaux sont en grande partie guidés par les mouvements de leurs aînés lesquels, grâce à une expérience acquise, connaissent la route et peuvent servir de guides. On peut comprendre comment les oiseaux savent suivre en voyage de vastes points de repère tels que de grands systèmes de cours d'eau, des chaines de montagnes, des rivages maritimes, mais aucun sens qui nous soit familier n'explique comment certaines espèces retournent sans se tromper à des îles océaniques par dessus d'immenses étendues de mer toujours de même apparence. Il se peut qu'elles aient un sens spécial qui les aide à s'orienter.

\section{PROTECTION.}

Pour ce qui regarde leur nourriture accoutumée les oiseaux sont très accommodants; les graines, les plantes, les fruits, les insectes, la viande ou le poisson, tout est bon à différentes espèces, et par conséquent, presque toutes les régions ont leur quote-part d'oiseaux qui leur sont propres. L'oiseau a une vie intense; son cœur bat rapidement plus que celui d'autres animaux; la température de son sang est plus élevée, et il consomme une quantité énorme de force vitale lorsqu'il vole. La chaleur un peu fiévreuse et les efforts continus exigent une quantité correspondante de nourriture; conséquemment l'oiseau, en tant que facteur économique, demande à être pris au sérieux. Bien qu'il y ait peut-être quelque exagération à dire, comme ont fait quelques auteurs, que l'équilibre entier de la nature dépend des oiseaux et que sans eux le pays serait un stérile désert peuplé par des insectes seulement, il faut cependant reconnaître que le nombre des oiseaux ne pourrait être réduit d'une quantité sérieuse sans les plus graves conséquences pour les contrées. La destruction de tonnes de semences 
d'ivraie et de millions d'insectes doit inévitablement avoir une grande influence sur le bien-être de la société, et la négligence à tenir compte de ce fait doit réagir très sérieusement sur la population qui n'accorde pas à ses oiseaux la protection nécessaire.

Toutefois le problème du "status" des espèces individuelles d'oiseaux n'est pas aussi simple qu'il semble au premier abord. Il y faut plus qu'un examen superficiel et bien des choses doivent être prises en considération si l'on se propose de connaître la vérité des choses. Quelquefois les oiseaux travaillent à l'unisson du bien de l'homme, et quelquefois à l'encontre de ce bien. Ils peuvent faire du bien dans une saison et du mal dans une autre; ou bien encore leur influence indirecte peut nuire à la somme de leurs effets immédiats d'une façon surprenante.

Il faut donc distinguer soigneusement entre les impressions générales qu'on retire quant au bien ou au mal que fait un oiseau. Une observation simplement accidentelle dans la vie n'est jamais suffisante pour déterminer même sa provision de nourriture. La façon de faire actuellement en usage fondc ses conclusions presque entièrement sur l'examen du contcnu de l'estomac d'oiseaux sauvages pris pendant l'année, ce qui constitue la seule preuve non sujette à caution. Pour ce travail la Commission biologique des Etats-Unis a examiné et décidé les cas de plusieurs milliers d'estomac d'oiseaux; les résultats de ses recherches sont à la disposition de ceux qui tiennent à les consulter et a s'en servir.

En tant que l'un des facteurs de ce délicat équilibre des phénomènes naturels les oiseaux doivent être respectés.

Il y a certains oiseaux qui, à cause de leur taille, de leurs habitudes et de leur chair sont regardés comme gibier légitime. La chasse de ces oiseaux est un exercice fortifiant et propre à donner une bonne santé à celui qui s'y livre, le dressant à la vie des bois, aux courageuses aventures, à la vie en plein air et à tirer avec adresse. Le véritable sportsman a un code de morale à lui, fondé sur des principes économiques, autant qu'humanitaires. Il ne tire jamais sans donner à sa victime une chance égale et tire peu si le gibier n'est pas bon à manger. Il a aussi soin de ne pas épuiser la quantité de gibier d'où dépend son sport à venir. Toutefois, le véritable art cynégétique n'a pas été universel et son absence malheureusement trop commune a eu pour effet une rareté de plus en plus constante du gibier. Des mesures restrictives ont à la vérité été décrétées mais elles ont d'habitude plutôt suivi que précédé les résultats qu'on en attendait; les mesures prises aujourd'hui auraient dû l'être hier, d'où il est résulté que dans une grande partie de ce pays, le gibier est déjà chose du passé.

\section{MOYENS D'ATTIRER LES OISEAUX.}

Quiconque s'intéresse aux oiseaux trouve un très grand plaisir à les avoir autour de sa maison et de son jardin où il peut les observer à loisir. Un petit bout de jardin peut être rendu attrayant à plusieurs espèces par des méthodes appropriées. Les effets d'une protection complète sont bien démontrés dans quelques-uns des plus grands parcs où les plus timides oiseaux d'étang trouvant qu'il n'y a là rien à craindre de la part des hommes deviennent presque aussi confiants que la volaille de basse-cour. C'est ce qui arrive aussi aux plus petites espèces de jardin. En même temps que l'absence du trouble causé par les gens, il faut encore aux oiseaux d'être protégés contre le chat de là maison. 
Il est aussi important de procurer aux oiseaux de la nourriture en hiver. Les bosquets qui portent du fruit, de la graisse pendue aux arbres, du grain, des noix cassées; de petits morceaux de viande séchée à l'abri de la neige, ne manquent jamais d'attirer les oiseaux en hiver.

En été, quand les provisions données par la nature sont abondantes, il est rarement nécessaire de procurer de la nourriture aux oiseaux, bien qu'une rangée de tournesols mûrs, ou les fleurs de jardin montées en graines compensent bien la peine qu'elles donnent. Une mare peu profonde d'eau claire ne manque jamais d'être une source de plaisir pour les oiseaux ordinaires de nos jardins. Ils s'y baignent et s'y désaltèrent; par une journée de grande chaleur il n'est pas rare de voir plusieurs oiseaux qui attendent leur tour pour jouir de cette bienfaisante fraîcheur. La plus simple forme de baignoire pour oiseaux est une poêle peu profonde, placée au large et à bonne distance d'un abri afin que l'oiseau soit protégé contre les ehats. Dans les villes où l'on prend soin des arbres, où le bois mort est vite enlevé, eertaines espèces d'oiseaux ont toujours bien de la peine à trouver des places favorables pour la nidification. Il y a au moins une demi-douzaine d'espèces qui font leurs nids très naturellement dans des branches creuses; qui reviennent d'elles-mêmes se mettre dans des boîtes de diverses sortes préparées à leur intention; beaucoup d'autres oiseaux peuvent, à l'occasion, se décider à en faire autant. Des boîtes propres à cet ouvrage sont décrites dans quelques-uns des livres indiqués à la page 17. Dans nombre d'écoles, où l'on apprend aux garçons certains travaux manuels, on les encourage à fabriquer des boites à oiseaux. On donne ainsi de l'essor à leur faculté naturelle d'invention et en même temps ils apprennent à s'intéresser aux oiseaux qui fréquentent leurs maisons.

\section{OUVRAGES D'ORNITHOLOGIE.}

La littérature de l'ornithologie dans l'Amérique du Nord est considérable en même temps que variée; il se peut même qu'aucune science naturelle ne puisse autant que celle des oiseaux s'étudier avec l'aide d'ouvrages soit pour les commençants soit pour les naturalistes plus avancés. Il se trouve toujours quelque ouvrage qui s'adapte à tous les dégrés de la connaissance du sujet, en même temps qu'à toutes les bourses. La liste suivante des ouvrages sur ce sujet peut se recommander; les premiers cités répondent spécialement aux besoins des commençants.

Bird Guides, par Chester K. Reed: Partie I, "Water and game birds east of the Rockies (including the hawks and owls "; Partie 2, eLand birds east of the Rockies". Doubleday, Page and Company, Garden City, N.-Y., prix \$1, relié en toile, \$1.25 relié en cuir.

Ce sont de petites éditions, presque de poche de gilets, avec reliures flexibles, de $3 \frac{1}{4}$ pouces sur $4 \frac{1}{2}$, très commodes à porter dans la poche sur le terrain. Elles renferment des illustrations coloriées petites et faciles à reconnaitre, de tous les oiseaux des deux sexes, et de courtes descriptions.

Colour Key, North American Birds, par Frank M. Chapman et Chester K. Reed; 8 vo., Doubleday, Page, N.-Y., prix, \$2.50. tructif.

Semblable, quant au plan, a l'ouvrage précédent, mais plus détaillé et plus ins-

A Guide to the Birds of New England and Eastern New York, par Ralph Hoffman: 8 vo., Houghton, Mifflin \& Co., prix \$1.50.

Ouvrage éminemment désirable; quoique ne traitant pas d'une région très limitée il comprend la plupart des oiseaux de l'est du Canada. Il donne les clefs pour les oiseaux de chaque saison basées sur la couleur; des descriptions détaillées, et aussi beaucoup d'illustrations en noir montrant des détails spécifiques; il donne de copieux renseignements de diverses sortes. 
Birds of Ontario, ps Thomas Mcllwraith: 2e édition, 1894, 8 vo., Wm. Briggs, Toronto.

Une liste annotée de tous les oiseaux dont l'auteur savait l'éxistence dans l'Ontario, a cette époque, avec des descriptions et beaucoup de renseignements touchant leurs habitudes, etc. Malheureusement l'ouvrage est épuisé et on ne peut se le procurer que par l'entremise des libraires de livres d'occasion et qui ont la spécialité des ouvrages d'ornithologie.

Handbook of the Birds of Eastern North America, par Frank M. Chapman: in-12, D. Appleton \& Co., prix, \$3.50.

C'est un manuel presque complet sur les oiseaux de l'Amérique du Nord orientale, et qui est inestimable pour les naturalistes avancés autant que pour les commençants. Il renferme des descriptions détaillées et exactes de tous les plumages, des mesurages et des dates de migration, en méme temps qu'une somme considérable de détails intéressants et utiles avec une excellente introduction sur les oiseaux et l'étude des oiseaux. Aussitôt qu'un commencant s'est familiarisé avec les rudiments de l'ornithologie, il devrait se procurer ce manuel.

Key to the Birds of North A merica, par Elliot Coues: 5e édition, 1903, vols. 2, large $8 \mathrm{v}$., Dana Estes \& Co., prix, \$12.50.

C'est ici, peut-être, l'ouvrage dont l'autorité est le plus généralement acceptée sur le sujet des oiseaux de l'Amérique. Il s'adresse avant tout au naturaliste avancé, mais il renferme une masse de renseignements qui ne peuvent se trouver nulle part ailleurs, et c'est pour nos ornithologistes l'autorité qui décide en dernier ressort sur les questions discutces.

Le Catalogue des Oiseaux du Canada, par John et James M. Macoun: 8 vo., publié par le ministère des Mines, Commission géologique, Ottawa, 1909.

C'est une liste complète de toutes les espèces et sous-espèces d'oiserux qu'on rencontre au Canada, au Groenland et a Terre-Neuve avec leurs habitats soit de couvaison soit de migration, aussi rigoureusement exposés que le permettait l'etat de cette science à l'époque de la publication du livre. Cette liste est bascé en grande partie sur les explorations et les expériences des auteurs, complét́́es par des connaissances tirées de toutes les sources à leur disposition; elle donne beaucoup de renseignements touchant les habitudes de couvaison, mais pas grand'chose de plus qui intéresse le public. Les éditions anglaise et française sont actuellement épuisées.

Birds of North and Middle America, par Robt. Ridgway: Bulletin $n^{\circ} 50,8$ vols, United States National Museum.

C'est un ouvrage monumental qui devait comprendre huit volumes, mais qu'on a étendu à dix et même davantage, dont sept sont en vente, les autres devant suivre aussi rapidement que le travail pourra se faire. C'est le plus récent, le plus détaillé et le plus savant ouvrage sur le sujet, mais ne contient nen comme faits vivants ni récits populaires ayant quelque rapport avec le sujet principal. On ne le trouve pas communément en vente, mais on peut se le procurer de rencontre ou par l'entremise du Département des Documents publies à Washington.

A part ces ouvrages généraux ceux qui suivent et qui traitent de sujets spéciaux d'ornithologie, sont recommandés:

The Warblers of North A merica, par Frank M. Chapman: D. Appleton \& Company, prix, \$3. The Water-fowl Family, par Sanford, Bishop et Van Dyke: Macmillan Co., prix, \$2.

North American Land Birds par Baird, Brewer et Ridgway: Little, Brown \& Co., 3 vols. Réimprimé de l'original. La partie traitant des oiseaux aquatiques de la série fut publiée a l'origine dans les Mémoires du Museum of Comparative Zoology, Harvard College, 1884, mais est actuellement épuisée et très difficile à se procurer.

\section{Comme ouvrages de lecture, les suivants sont à recommander:}

How to Study Birds, par Herbert K. Job: Outing Publishing Co., prix, \$1.50.

The Sport of Bird Study, par Herbert K. Job: Outing Publishing Co., prix, \$2.

Wild Wings, par Herbert K. Job: Outing Publishing Co., prix, \$3.

Ce sont tous des ouvrages extrêmement intéressants et qui renferment une admirable collection de photographies d'oiseaux d'après nature.

Bird Craft, par Mable Osgood Wright: Macmillan Co., prix, \$2.50.

Nombreuses illustrations hors texte. 
Rambles of a Canadian Naturalist, par S.-T. Wood: Illustrés, J.-M. Dent \& Sons, Ltd., London et Toronto, 1916, prix, $\$ 1.50$.

C'est une série de courtes esquisses et d'observations sur les choses de la nature autour de Toronto. Elles sont bien écrites et avec sympathie, la plupart, mais non toutes, se rapportant aux oiseaux.

The Bird, its Form and Function, par C. William Beebe: Henry Holt \& Co., N.Y.

Cette liste pourrait être étenduc indéfiniment, mais il est probable que nous en avons assez donné.

Touchant les sujets d'ornithologie qui intéressent le commerce, l'attention du lecteur est attirée sur l'immense quantité de renseignements utiles publiés par le Biological Survey des Etats-Unis dans ses nombreux bulletins, circulaires et rapports. Bien que cette littérature n'ait eu d'abord en vue que les Etats-Unis, elle s'applique tout aussi bien aux oiseaux du Canada. La liste complète de ces documents, avec les prix, peut s'obtenir chez le Surintendant des Documents publics, à Washington. Les prix ne sont que nominaux.

Parmi les publications canadiennes de cette nature, nous pouvons citer rouvrage suivant:-

The Birds of Ontario in Relation to Agriculture, par Chas. W. Nash: ministère de l'Agriculture de l'Ontario, Toronto, Bulletin 173.

Parmi les travaux pouvant s'appliquer au Canada on n'en citera que quelques-uns, car leur nombre est légion, et ils sont disséminés à travers beaucoup d'ouvrages.

Ontario:

Birds of Ontario, par Thomas McIlwraith, 2e édition, 1894, 8 vols. Wm. Briggs, Toronto.

Birds of Toronto, par J.-H. Fleming; Auk, vol. XXIII, pp. 437-453; vol. XXIV, p. 71-89.

The Natural History of the Toronto Region. Oiseaux dessinés par J. H. Fleming. Publié Québec: par le Canadian Institute, Toronto, 1913, prix, 2 ou $\$ 2.50$.

Les Oiseaux de la province de Québec, par C.-E. Dionne: Dussault et Proulx, 1906.

The Birds of Montreal, par E.-D. Wintle: Drysdale \& Co., Montréal, 1908.

Nouvelle-Ecosse:

Birds of Nava Scotia, par A. Downs, publié par Harry Piers: Proc. and Trans. Nova Scotia Inst., Sc., vol. VII, p. 142-178.

Nouveau-Brunswick:

A Catalogue of the Birds of New Brunsurick, par M.-A. Chamberlain: Bull. Nat. Hist. Soc., Nouveau-Brunswick, No. 1, p. 23-68.

L'ornithologie, comme toutes les autres branches de la science, a ses propres publications périodiques. La plus importante dans l'Amérique du Nord c'est l'Auk, revue trimestrielle qui est l'organe officiel de l'Union des Ornithologistes américains. En sus d'articles purement scientifiques, elle publie des centaines de monographies qui intéressent les Canadiens, y compris les listes locales copieusement annotées avec des remarques historiques venant de toutes les parties du Dominion et des descriptions de mœurs d'oiseaux racontécs de façon populaire. Abonnement, \$3 par an. L'éditeur, depuis 1912, est Witmer Stone, Academy of Science, Philadelphia (Pa.).

Le "Wilson Bulletin,» revue bi-mensuelle, est l'organe officiel du club ornithologique Wilson; il est dévoué aux intérêts de l'Ouest central. Abon- 
nement \$1 par année, publié par Lynds Jones, Oberlin, Ohio. Adresse: The Treasurer P.-B. Coffin, 3232 Groveland Ave., Chicago, Ill. C'est une publication qui a moins de prétentions que l'A $u k$, mais qui contient beaucoup de choses intéressantes pour le commun des lecteurs et qui publie quelques-uns des articles les plus intéressants sur les oiseaux et leurs mœurs.

"Bird-lore" est une revue mensuelle qui s'adresse au grand public et qui se distingue par son luxe et ses illustrations. C'est l'organe officiel des Sociétés nationales Audubon et il est dévoué à une étude populaire et à la protection des oiseaux. Cette revue en est aujourd'hui à son dix-huitième volume; elle est publiée par Frank Chapman. Abonnement, au Canada, \$1.75 par an. Adresse: Bird-lore, Crescent and Mulberry streets, Harrisburg ( $\mathrm{Pa}$.$) .$

Le "Canadian Field Naturalist", qui continue "The Ottawa Naturalist" est une revue mensuelle (neuf numéros par année) publiée par le "Ottawa Field Naturalists Club ), éditeur, D. Jenness, Musée commémoratif Victoria, Ottawa, Ontario. Abonnement \$1.50 par an. Adresse: C.-L. Patch, Sec., Musée commémoratif Victoria, Ottawa, Ontario. Cette publication représente plusieurs sociétés d'histoires naturelles du Dominion et contient une grande quantité de sujets de zoologie, tous intéressants, beaucoup de remarques et d'articles sur les oiseaux du Canada.

A propos de la protection des oiseaux et de la manière de les attirer près des habitations, dans la masse considérable d'ouvrages sur la matière, on peut recommander spécialement les suivants:-

How to Attract and Protect Wild Birds, par Martin Hiesmann: Witherby \& Co., London, 1. s. 6 d.

C'est un récit étendu des méthodes employées par le Baron von Berlepsch, en Allemagne, et qui donne une infinité de méthodes a employer sur les grandes et les petites propriétés.

Wild Bird Guests, par Harold Baynes: E.-P. Dutton \& Co., New-York, 1915, \$2.

Cet ouvrage raconte de façon très intéressante et de manière agréable la méthode employée par l'auteur et ses amis grâce à laquelle ils firent de Meriden (New Hampshire) un véritable village modèle pour les oiseaux, lesquels devinrent là si familiers et gentils qu'on en faisait des oiseaux domestiques, venant quand on les appelait et se posant sans crainte sur les personnes. L'ouvrage est magnifiquement illustré, avec beaucoup de photographies montrant les méthodes et les résultats.

The Domestic Cat, par Edward H. Forbush, Ornithologiste, de l'Etat de Mass.; State Board of Agriculture, Bulletin $n^{\circ} 2,1916$.

Ouvrage qui traite a fond du chat domestique dans ses rapports avec la vie des oiseaux à l'état sauvage.

Bird Houses and How to Build Them, par Ned Dearborn: United States Department of Agriculture, Farmer's Bulletin a ${ }^{\circ} 609$. Adresse: Department of Public Documents, Washington, D.C., Prix, environ dix cents.

Sur le sujet du moineau anglais, en tant que fléau, on peut recommander les ouvrages suivants:--

The English Sparrow in North America, par Walter B. Barrows: Bull. $n^{\circ} 1$, U.S. Dept. of Agriculture, 1889 , p. 405.

How to Destroy the English Sparron, par Ned Dearborn: Farmer's Bulletin $n^{\circ} 383$, U.S. Dept. of Agriculture, 1910.

The English Sparrow as a pest, par Ned Dearborn: Farmer's Bulletin $\mathrm{n}^{\circ} 493$, U.S. Dept. of Agriculture, 1921.

Ces rapports font le procès au moineau anglais, très équitablement, et le condamnent très franchement; ils indiquent divers moyens de limiter le nombre de ces oiseaux. 
CLEF POUR LES OISEAUX DE L'EST DU CANADA.

\section{Explication.}

Dans les descriptions zoologiques une "clef » est un dessin à l'aide duquel un spécimen peut servir à décrire successivement des groupes grands ou petits en en relevant les caractères saillants et l'identité spécifique.

La "clef » que nous donnons ici est la modification de celle établie par MM. Frank M. Chapman et Ernest Thompson Seton et qui fut publiée dans le "Handbook to the Birds of Eastern North America") du premiel. On espère qu'il sera d'un grand secours pour les commençants. Un des avantages de cette clef c'est d'être indépendante des caractères variables d'âge, de sexe ou de saison, et qu'elle servira pour les spécimens jeunes et femelles aussi bien que pour les mâles et adultes.

La méthode pour s'en servir est comme suit: étant donné qu'on a un oiseau d'une espèce inconnue. Pour en déterminer le nom, on le compare d'abord avec le premier titre en caractères gras, numéroté en chiffres romains-I, Pieds toti-palmés. Si eette description ne s'applique pas à l'oiseau, on passe au titre suivant en chiffres romains - II, Pieds semi-palmés, ou III, Pieds sans palmure prononcée. Admettant que cette dernière particularité s'applique bien à l'oiseau en question nous passons aux titres du rang inférieur qui suit, lesquels sont donnés en lettres majuscules alphabétiques, et nous avons les alternatives $F$, jambes longues, et $\mathrm{G}$, jambes courtes. Quelquefois il est difficile de décider si une jambe doit être tenue pour longue ou courte, et alors les détails donnés dans le dessin peuvent aider à décider la question. En ce cas les jambes, dironsnous, ne sont pas extrêmement longues, pas plus longues, par rapport à la taille de l'oiseau, que ne sont les jambes d'un poulet ou d'un moineau; c'est pourquoi, avec la lettre $G$, nous nous référons à un nombre d'alternatives subordonnées distinguées par des lettres initiales minuscules-k, pieds de poulet, forts et serrés pour gratter; 1, pattes à fortes griffes pour tenir la proie; $\mathrm{m}$, pattes petites et faibles; $\mathrm{n}$, pattes petites ou de taille moyenne faites solidement et jambes couvertes d'écailles ou de plaques de corne. Un coup d'œil donné à diverses pattes figurées sous chaque titre facilitera la constatation du sujet. Si nous admettons qu'on se soit décidé en faveur du dernier titre, nous comparerons notre spécimen aux alternatives suivantes, numérotées avec des chiffres arabes-19, deux orteils en avant; 20 , trois orteils en avant. Il ne peut pas y avoir de confusion ici et nous admettons que notre spécimen ayant trois orteils en avant appartient au groupe considérable des oiseaux percheurs. Nous le comparons, par conséquent, avec les dessins aux traits qui suivent pour voir avec lesqucls il s'accorde le mieux. Le bec n'est pas large et plat; donc ce n'est pas un moucherolle; il n'y a pas de touffe aux oreilles, ni de long orteil postérieur, et la narine n'est pas recouverte de touffes de plumes, ce ne peut donc être ni l'alouette ordinaire, ni un corbeau, ni un geai. Le dessin suivant, le bec du goglu arrête nos regards et dans le groupe suivant le bec du moineau. Un coup d'œil sur ce qui reste prouve que notre oiseau doit être un goglu ou l'un des moineaux. La gravure (p. 277) et la description du goglu n'y ressemblent pas du tout; c'est pourquoi nous passons aux moineaux et lisons la description générale du moineau avec les remarques (p. 182) et considérons les gravures. Après avoir regardé de près toutes les illustrations nous trouvons que notre spécimen s'accorde avec celui du pinson chanteur, et 
en relisant les caractères distinctifs, notre opinion s'en trouve confirmée. L'oiseau a la poitrine fortement rayée et ramassée à son centre; il n'a ni la raie jaune au-dessus de l'œil comme le pinson des prés, ni les plumes blanches extérieures de la queue comme le pinson à ailes baies. Nous avons donc l'assurance que, sans avoir pour commencer, d'autre connaissance ornithologique que celle-ci: que le spécimen est un oiseau du Canada de l'Est, nous avons réussi à le situer dans sa propre espèce.

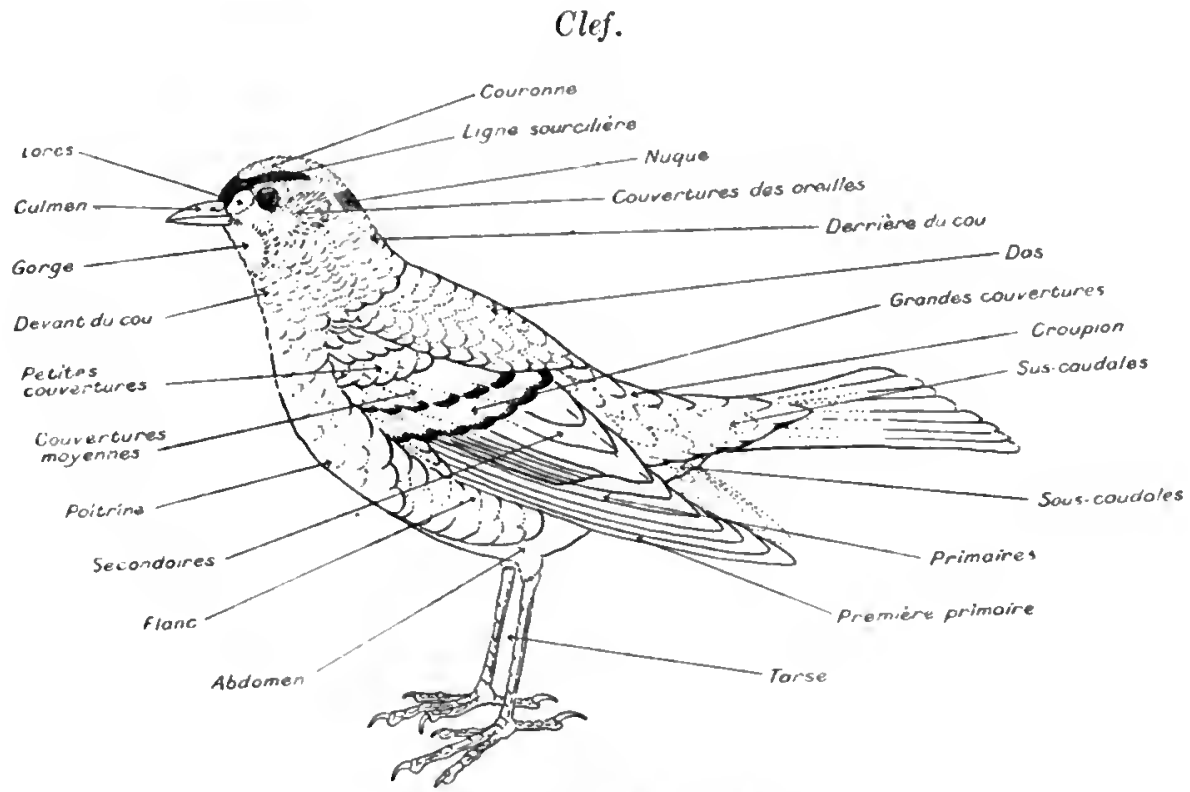

Figure 1-Régions Plumaires D'UN giseau TYPE.

I. Pieds toti-palmés.-Deux ou trois palmures complètes à chaque pied.

A, Quatre doigts,

a, Tarse aplati.

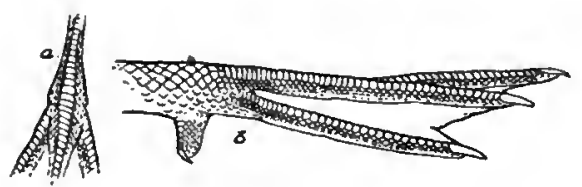

Figure 2-Plongedr

Plongeurs.............................. p. 46 . 


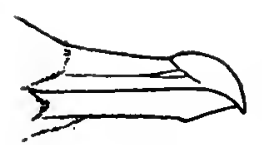

Figure 3-LABBE

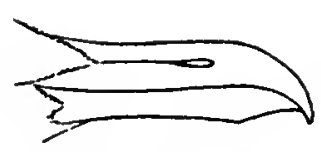

Figure 4-Movet'

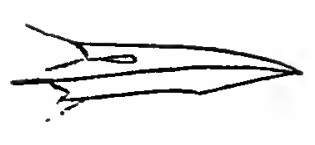

Figure 5-STERNE

b, L'aile fermée est plus longue que la queue sauf chez certains labbes (figure 3) et certaines sternes (figure 5), auxquels cette règle ne s'applique que si l'on ne tient pas compte des plumes rectrices centrales très allongées de ceux-là ou des rectrices externes de celles-ci. Becs tels que représentés.

Palmipèdes aux longues ailes-Mouettes, sternes, labbes......... p. 50.

c, Membranes entre tous les doigts (3 membranes).

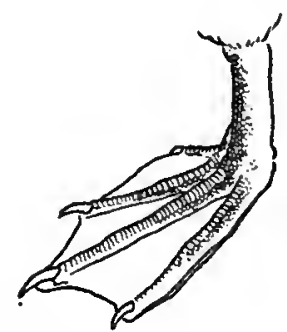

Figure 6-Fou de BAssan

Palmipèdes totipalmés-Fous de Bassan, cormorans, etc.......... p. 64 .

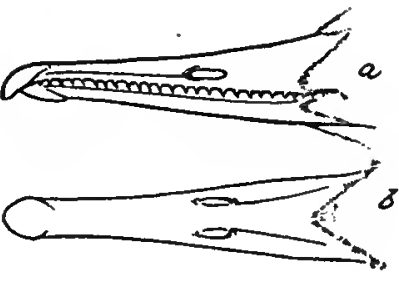

Figure 7-HA RLE

(a Côté de bec

(b) Sommet du bec
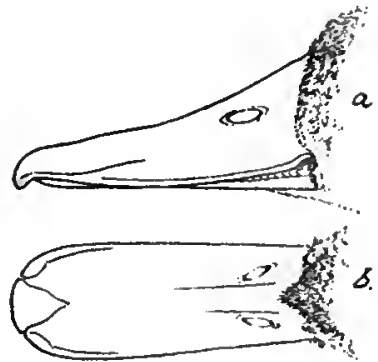

Figure 8-CANaRd

(a) Côté du bec

(b) Sommet du bec

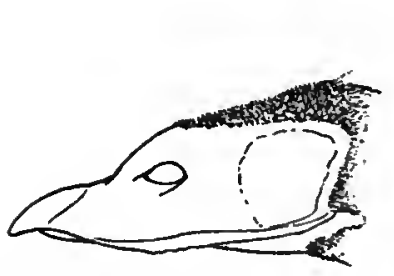

Figure 9-Macreuge ì La roe bec

, Bec denté ou aplati (comme les canards).

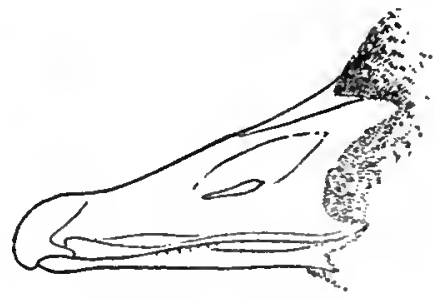

Figure 10-OIs

Palmipèdes au bec en crible-Harles, canards, oies et cygnes p. 67. 
e, Narines tubulaires au sommet du bec.

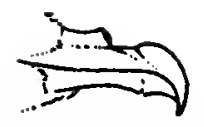

Figure 11-PÉtreL

Palmipèdes d narines tubulaires-Pétrels, etc. p. 60 .

B, Trois doigts (sans gros orteil) excepté la mouette tridactyle (p. 53).

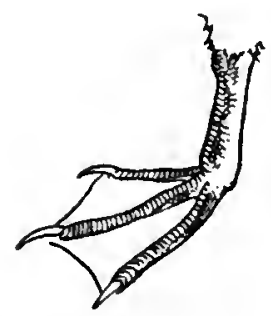

Figure 12-GuTLLE Mor

Pingouins, guillemots, etc.................... 47 .

II. Pieds semi-palmés-Les palmures se réduisent à des festons, des dentelures ou de petites membranes à la base des doigts. Quatre doigts, sauf dans les cas mentionnés.

C, Tarse très aplati, palmures telles que représentées.

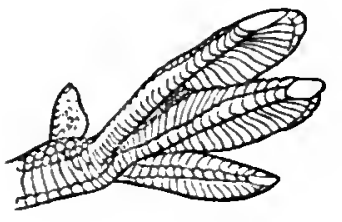

Figure 13-GBìne

Grèbes p. 44.

D, Le bec s'étend sur le front formant une plaque frontale.

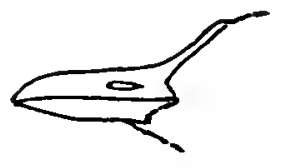

Figure 14-Foulave

La foulque p. 98 .

E, Petits oiseaux; bec long et effilé; trois ou quatre doigts.

f, Bec dépourvu d'élargissement terminal dur; quatre doigts, excepté le sanderling (p. 107).

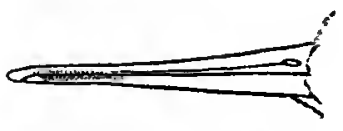

Figure 15-Echassicr

Oiseaux de rivage-Phalaropes, bécassine, maubèches, pluvier...... p. 98. 
g, Bec pourvu d'un élargissement terminal dur; trois doigts, excepté le pluvier à ventre noir (p. 114).

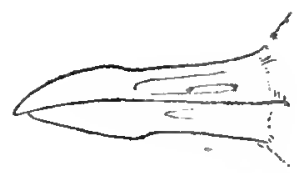

Figure 16-PLuvier KMDIR

Pluvier

p. 115 .

\section{Pieds sans palmure prononcée.}

F, Pattes longues pour marcher dans l'eau ou dans la boue; doigts longs, effilés et flexibles aux jointures.

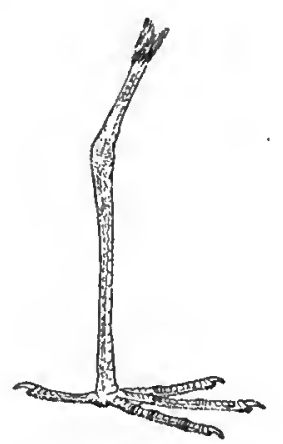

Figure 17-HéroN

h, Bec gros et corné; plage nue autour des yeux.

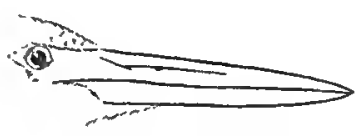

Figure 18-HÉRoN

1, Gros orteil pourvu d'une crête (griffe pectinée).

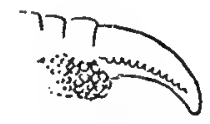

Figure 19-Griffe pectinḱt

Hérons. p. 87 et 89 .

2, Front nu.

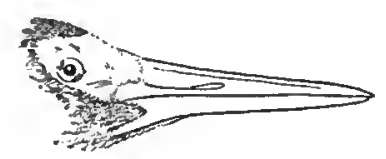

Figure 20-Grue

Grues............................... p. 93 . 
i, Becs longs, flexibles et uniformément effilés.

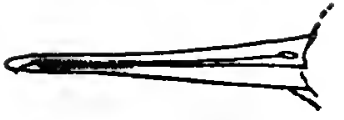

Figure 21-Bécusstive

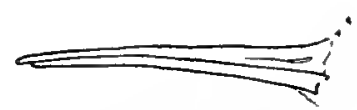

Figure 22-MACB亡̀ch

3, Bec plutôt frêle, pas

beaucoup plus épais à

la base qu'au sommet. Quatre

doigts, excepté le sanderling (p. 107).

Phalaropes, bécasses, maubèches p. 98 .

4, Bec manifestement plus épais à la base qu'au sommet.

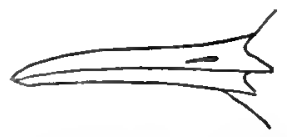

Figure 23-RALES A LONG BEC

Râles-(râle élégant et râle de la Virginie) p. 94,95 .

j, Bees courts.

5 , Bec mou à la base, aboutit à un élargissement terminal dur. Trois doigts, excepté le pluvier à ventre noir (p. 114).

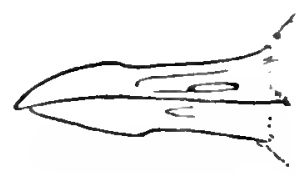

Figure 24-Plevire

Pluvier.

p. 113.

6 , Bec très fort.

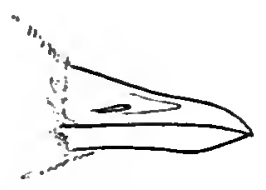

Figure 25-Rítes At BEC coort

Râles (râle de la Caroline et râle jaune)

p. 95 et 96 .

7, Bec fort avec plaque frontale remontant sur le front.

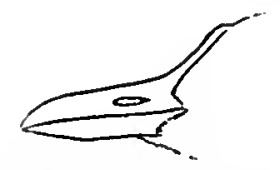

Figure 26-Galunule

Gallinules. p. 96. 
8, Bec corné à la base, affecte la forme d'un coin vu de profil, et semble légèrement tourné en haut.

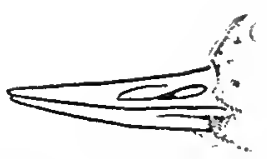

Figure 27-TOURNE-PIERRE

Tourne-pierre............................ 116 .

G, Les pattes sont courtes pour mieux se percher, marcher, grimper et vivre dans les arbres ou sur le sol.

$\mathrm{k}$, Les pieds, comme ceux des poulets, sont forts et compacts; les doigts moins flexibles; ongles forts et obtus pour gratter. Tarse emplumé ou nu. Les doigts avec ou sans appendices en forme de crêtes.

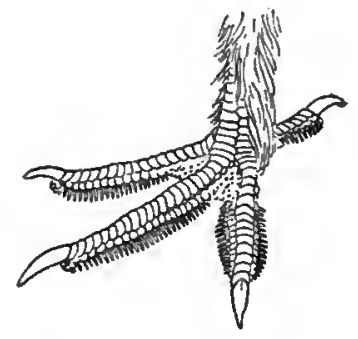

Figure 28-TÉtras

9, Bec plutôt conique, le plúmage s'étend jusqu'à la narine ou à peu près.

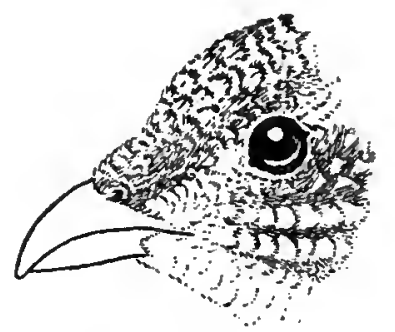

Figure 29-Tetras

Tétras et caille............................. p. 118 .

10, Bec crochu au bout; le cou et la tête sont nus.

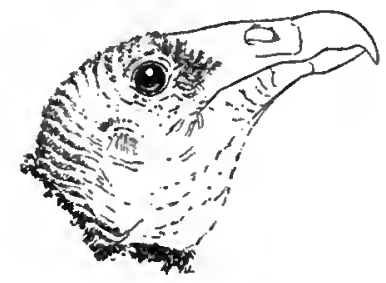

Figure 30-VAUTOUR

Vautours........................... 127, 128 . 
1, Le pied est puissant pour retenir la proie; ongles longs, forts, aigus et recourbés; tarse emplumé ou nu.

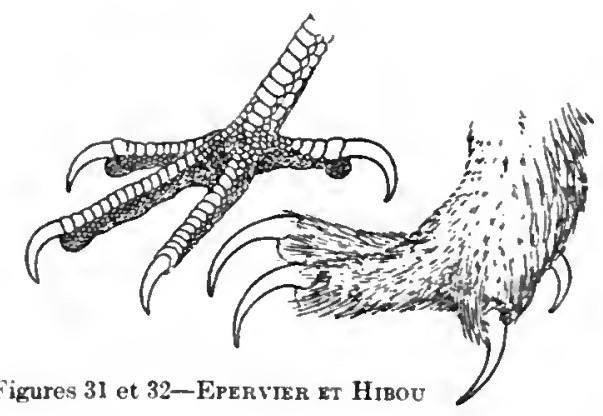

Diseaux de proie........................... p. 126 .

11, La cire est nue à la base du bee, Le tarse est toujours nu (excepté la buse pattue et l'aigle: doré). Les doigts sont toujours nus (figure 31.)
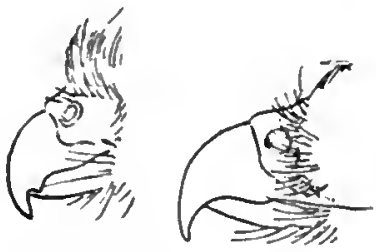

Figure 33

(a) FA CCON

(b) Epervier ou Ajgle

Eperviers et aig'es.

p. 129.

12, La cire est cachée sous le plumage; l'œil est au centre d'un disque de plumes plus ou moins circulaire. Le tarse et les doigts sont emplumés (Figure 32).

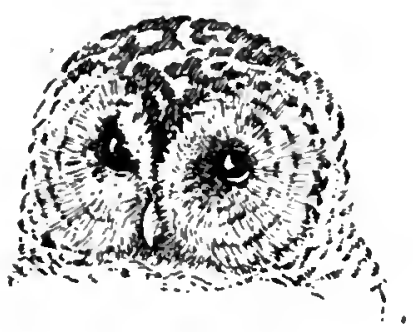

Figure 34-Hinou

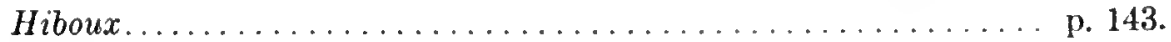

$\mathrm{m}$, Pieds petits et délicats.

13, Les narines s'ouvrent au milieu d'une base molle et gonflée.

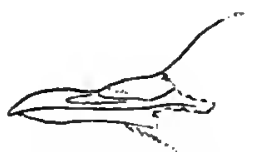

Figure 35-Pigeon

Pigeons. p. 124. 
14, Les deux doigts externes sont reliés ensemble sur la moitié de leur longueur.

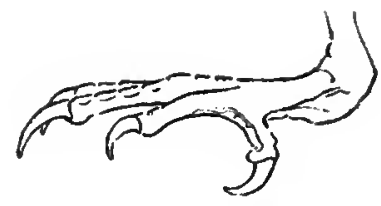

Figure 36-MARTTN-PÊCHEUR

Martins-pêcheurs. p. 150,151 .

15, Deux doigts orientés en avant, et deux en arrière (voir aussi les pics).

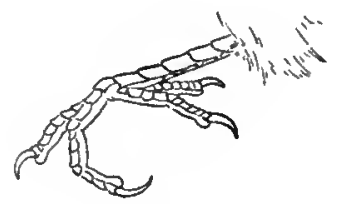

Figure 37--Covcou

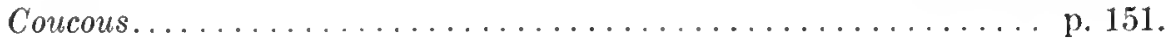

16, Bee très petit; bouche énorme, s'ouvrant jusqu'au-dessous des yeux.

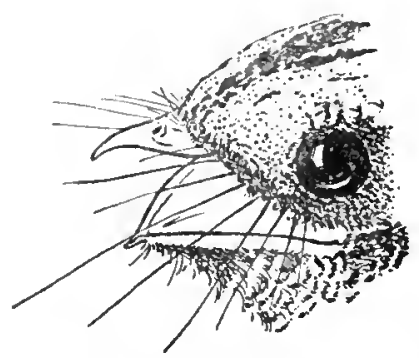

Figure 38-ENGothevent

Engoulevents, engoulevents criards, engoulevents d'Amérique . . . p. 159, 160.

17, Reetrices terminées par des épines pointues.

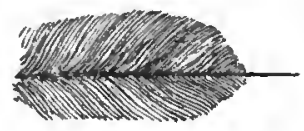

Figure 39-MaRTINET

Martinet des cheminées....................... p. 162 .

18 , Bec très frêle et en forme d'alène. Oiseaux extrêmement petits.

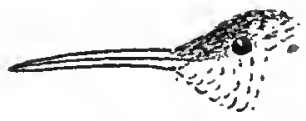

Figure 40-Oiseat-Modche

Oiseau-mouche p. $163,164$. 
$\mathrm{n}$, Pieds de taille moyenne ou petits, mais pas perceptiblement faibles ni flasques ni lâchement articulés. Pattes recouvertes d'écailles ou de plaques.

19, Deux doigts en avant et un ou deux en arrière. Bec en ciseau au bout.

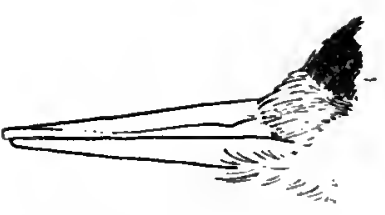

Figure 41-PIC

Pics.

p. 154 .

20, Trois doigts en avant; gros orteil aussi bien développé que le médian; l'ongle du pouce ordinairement aussi long ou plus long que celui du médian.

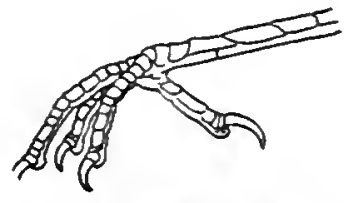

Figure 42-Percheur

Percheurs p. 164.

Pour reconnôtre les détails des percheurs.

Bec légèrement plus large que haut à la base et légèrement crochu au bout.
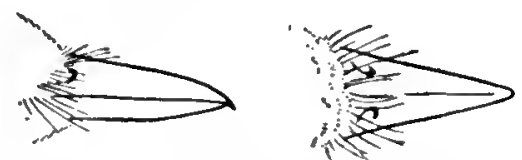

b

Figure 43-MoccherolLE

(a) Côtê du bec

(b) Sommet du bec

Moucherolles

p. 165.

$\AA$ remarquer le toupet aux oreilles et le grand ongle au pouce. Le plectrophane et le pipit sont les seules autres espèces possédant ce dernier trait.

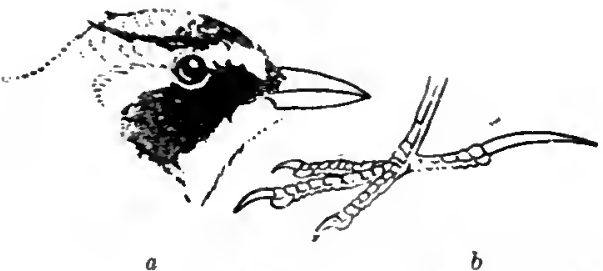

Figure 44-ALOUETTE ORDINAIRE

Alouette ordinaire p. 170 .

Nez fort, narines recouvertes de touffes velues.

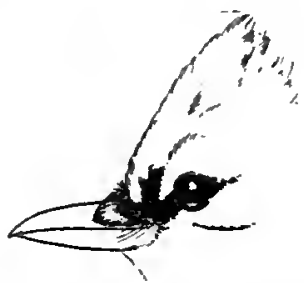

Figure 45-GeAI BLE U H UPP Corneilles, Geais, etc..................... p. 171 . 
Crêtc du bec se continuant ordinairement plus ou moins sur le front.

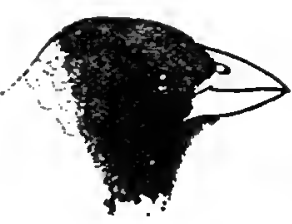

Figure 40-GogLU

L'étourneau et le goglu (figure 46) ont des becs comme celui du moineau, voir cette espèce.

Figure 48-Lortot de BaLtimore

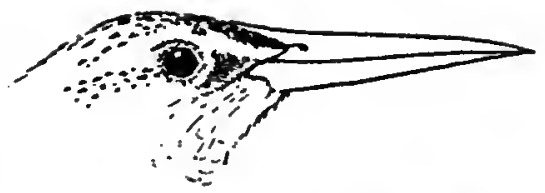

Figure 47-E'TOURNEA D DS PRÉS
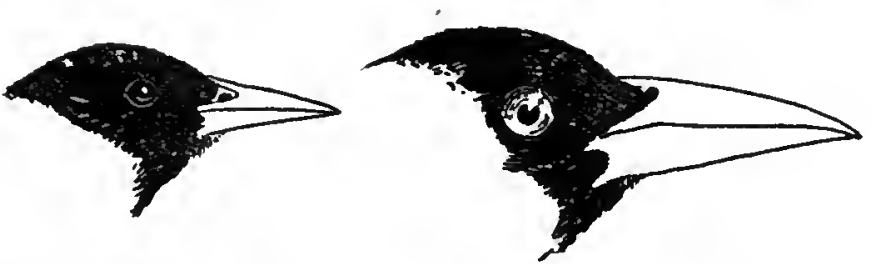

Figure 49-M AINATE BRONZÉ

Etourneaux.............................. 175 .

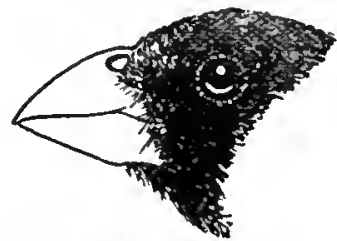

Figure 50-Gros BEC ì POITRINE ROSE

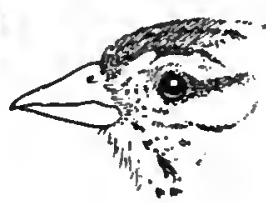

Figure 51-Pinson CHANTEUR

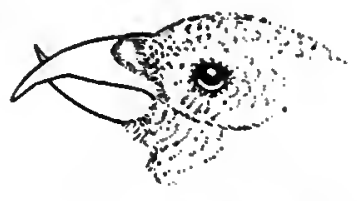

Figure 52-Bre croist ì AILES BLA NCHRS

Bec conique, fort pour croquer les graines. (Les becs du goglu et de l'étourneau ordinaire sont à peu près semblables, voir descriptions).

Moineaux............................ 182 .

Dent faible ou prononcée sur le côté tranchant de la mandibule supérieure.

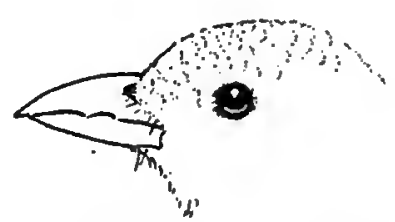

Figure 53-Tangara b́carlate

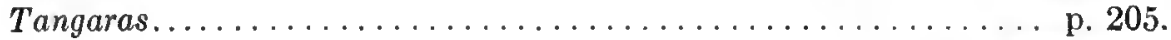

Bec très petit, large et aplati à la base.

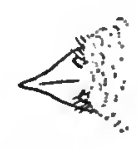

(a)

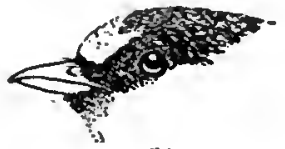

(b)

Figure 54-HiroNd BLLE ì FroNT BLA No

(a) Sommet du bec

(b) Côté du bec

Hirondelles............................... p. 206. 
Crête et bande noire des yeux très en évidence.

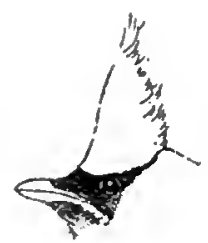

Figure 55-JASEURB

Jaseurs............................. p. 210 .

Une dent près du bout de la mandibule supérieure.

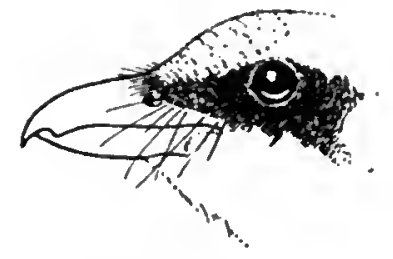

Figure 56-PIE-G RI ìche

Vireos et pies-grièches........................ 211, 213.

Petits oiseaux aux couleurs vives. Les couleurs les plus communes sont peut-être vert olive et jaune; mais les bleus, rouges et autres couleurs ne sont pas rares.

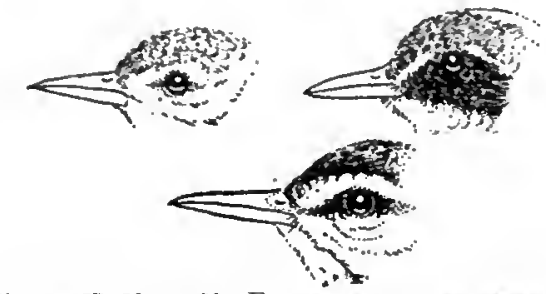

Figures 57,58 et 59 -FAUVETTE DU TRNNESBge FAUVETTE $\dot{I}$ CROUPION JAUNE ET GRIVE

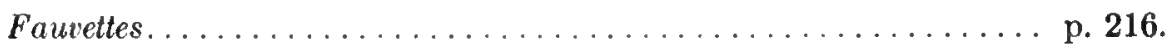

Bec fin et pointu, grand ongle au pouce.

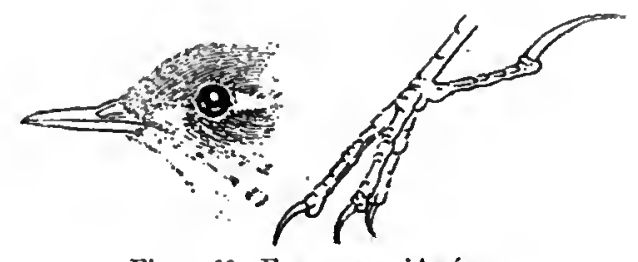

Figure 60-Farlouse d'AmériQue 


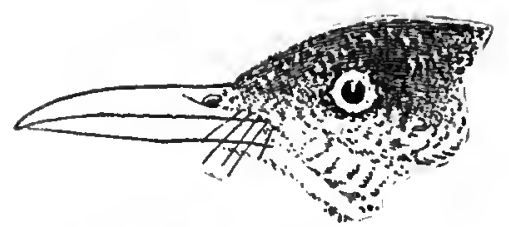

Figure 61-Grive RoUss:

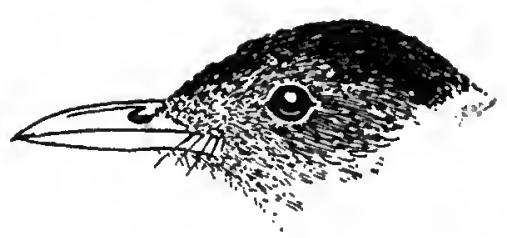

Figure 62-Grive de LA CAROLIN

Becs tels que représentés. Le merle est un oiseau de forte taille brun rougeâtre et blanc; la grive de la Caroline est même d'un gris ardoise.

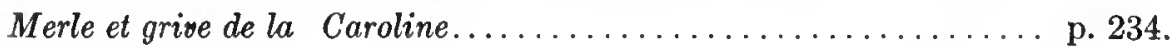

Petits oiseaux aux teintes brun cendré.

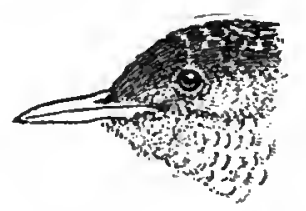

Figure 63-TROGLOdTTE AÉdoN

Troglodytes

p. 236.

Petits oiseaux aux couleurs brun cendré.

Queue longue et raide, pennes pointues aux extrémités.

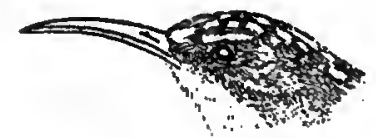

Figure 64-Grimpereat d'A MERIQUe

Grimpereaux.

p. 239.

Bec légèrement tourné en haut.

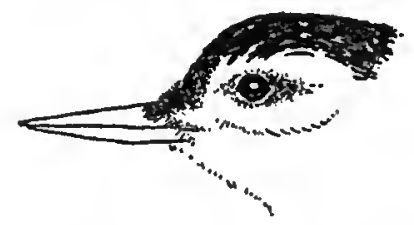

Figure 65-STTELLE DE LA CAROLINR

Sittelles. p. 240 .

Tout petits oiseaux aux couleurs gris, blanc et noir.

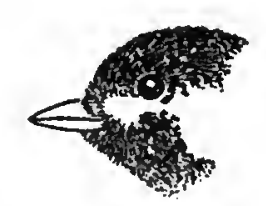

Figure 66-M GSA NGE ì TATE NOIRE

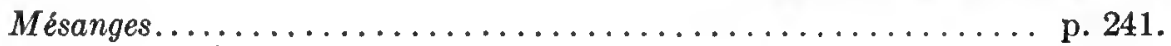


Tout petits oiseaux couleur vert olive. Les mâles ont la couronne tachetée de couleurs vives.

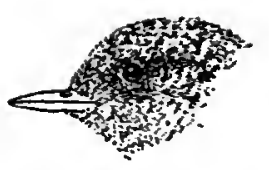

Figure 67-Rotrelet HUPPÉ

Roitelets

p. 243.

Oiseaux de taille moyenne aux couleurs ordinairement brun tendre avec poitrine plus ou moins mouchetée, sauf le merle et le rouge-gorge bleu.

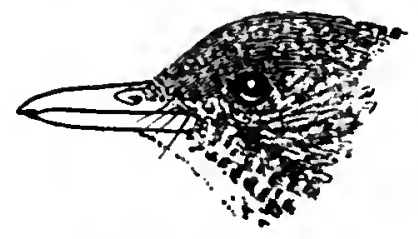

Figure 68-G hIVx DE WILsoN

Grives

p. 245.

\section{TABLE SYSTEMATIQUE.}

Les titres en petites capitales ont des parties ou des paragraphes spéciaux qui leur sont consacrés; ceux en italiques sont incidemment mentionnés dans le texte. Les espèces marquées d'un astérique sont représentées en couleurs.

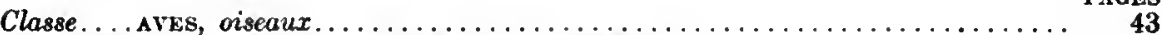

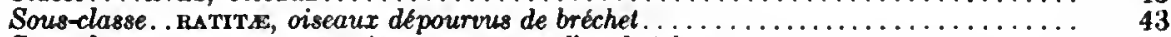

Sous-classe. . . CARINATs, oiseaux pourvus d'un bréchet.................. 43

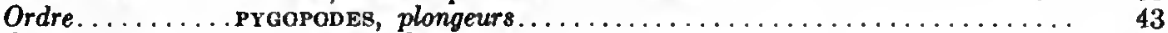

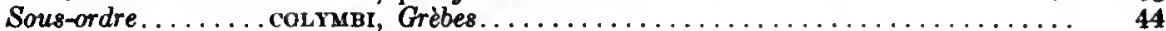

Famille........... colyмвт

Grebe de l'Ouest................ 44

ORkे BE $\lambda$ COU RODGE $\ldots \ldots \ldots \ldots \ldots \ldots$ 44

GREBE CORND.............. 45

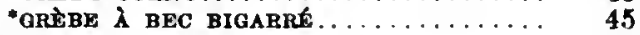

Sous-ordre. . . CEPPE1, Plongeons et Pingouins...................... 46

Famille.............

* plongeon A coldier............. 46

PLONGEON $\lambda$ GORGE ROUSSE......... 47

Famille............ucrow, Pingouins, Guillemots et Macareux.......... 47

MACAREUX ARCTIQUE............ 48

GUILLEMOT NOIR.............. 48

Guillemot de l'Atlantique........ 48

Guillemot de Mandt............. 48

GUILLEMOT ORDINAIRE............ 48

Grand guillemot de l'A tlantigue..... $\quad 49$

GUILLEMOT ג GROS BEO.......... 49

Guillemot de Brürnich........... 49

PINGOUIN COMMUN.............. $\mathbf{4 9}$

GRAND PINQOUIN.............. 5

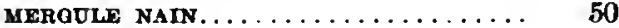


PAGES

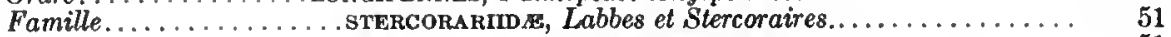

LABBE POMARIN.............. 51

LABBE PARASITE............... 51

LABBE A LONGUE QUEUE.......... 52

Famille...........ARm

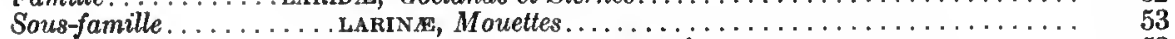

MOUETTE $\lambda$ TROIS DOIGTS........... 53

Mouette de l'Atlantique............ 54

GOELAND A MANTEAT OLAUQUE........ 54

GOÉ LAND A AILES BLANCHES. . . . . . . . . 54

GOÉlLAND À MANTEAU NOIR. . . . . . . . 55

"Gofiland argenté. . . . . . . . . . . 55

Gofland DE DELAWARE........... 56

oofland DE BONAPARTE.......... 56

Goéland de Franklin................. $\quad 56$

Mouette rieuse................... 57

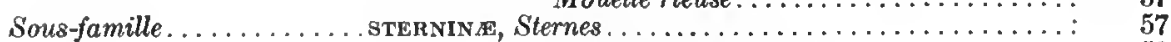

STERNE CASPIENNE............ 58

STERNE DE FORSTER............ 58

"sterne commune............. 59

STERNE ARCTIQUE. . . . . . . . . . 59

STERNE NOIRE................ $\quad 60$

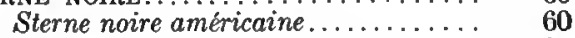

Ordre.......... tubinanes, Palmipedes d narines tubulaires............. $\quad 60$

Famille....................... 61

FULMaR........................... 61

Fulmar de l'A lantique............. 61

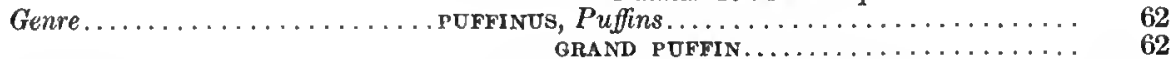

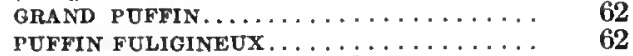

Genres................. ocennodroma n ocennites, Pétrels.......... 63

PETREL DE TIEACH............... 63

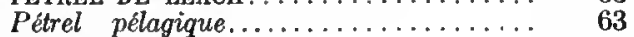

PETREL DE WILSON.............. 63

Ordre.......... steganopones, Palmipèdes toti-palmés.............. 64

Famille. . . . . . . . . . . . suldos, Fous ou Fous de Bassan................. 64

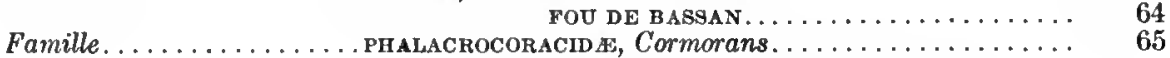

CORMORAN ORDINAIRE............ 65

CORMORAN A AIGRETTES............. 65

Cormoran d aigrettes de l'Est.......... 66

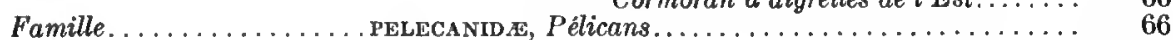

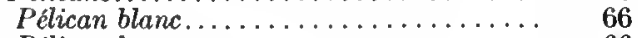

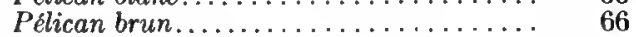

Ordre...........

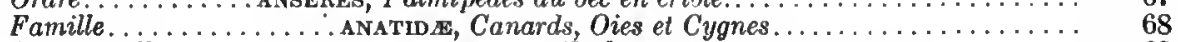

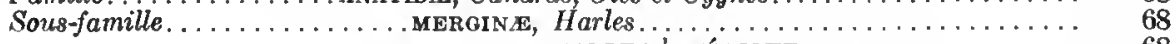

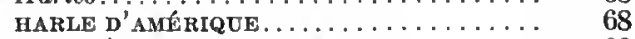

*HARLe A poltrine Rousge.............. 69

PETIT HARLE...................... 69

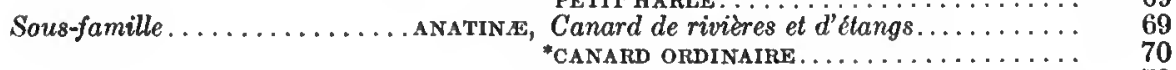

*anamd nolr................. 70

CANARD CHIPEAU.............. 71

CANARD D'AMERIQUE............. 71

SARCELLE A AILES VERTES ........... 71

* Sarcelle $\lambda$ AILES Bleues............ 72

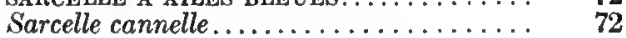

CANARD SODCHET. . . . . . . . . . . 72

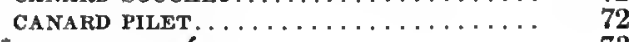

*anard hupte............... 73 
PAOES

Sous-famille........... FULIOULINe, Canards de baies, de mer ou plongeurs.... 74

MILOUIN $\lambda$ TÊTE ROUSSE............ 74

MILOUIN AUX YEUX ROUGES........... 74

MORILLON $A$ TETE NOIRE.......... 75

PETIT MORILLON . . . . . $\ldots \ldots \ldots \ldots \ldots, \quad 75$

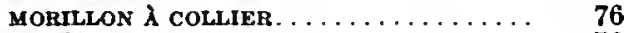

MUCEPHALE.................... 76

Bucéphale d'A mérique........... 76

BUCEPHALE D'ISLANDE............ 76

PETIT BUCEPHALE.............. 77

CANARD $A$ LONGUE QUEUE.......... 77

CANARD HISTRION................ 78

Genres...............somateru et oldemia, Eiders ê Macreuses..... 78

CANARD EIDER ................ 79

Eider du Nord.................. 79

EIDER D'AMÉRIQUE. . . . . . . . . . . . . $\quad \mathbf{7 9}$

EIDER REMARQUABLE............. 80

MACREUEE D'AMERTQUE. . . . . . . . . $\quad \mathbf{8 0}$

MACREUSE VELOUTEE............. 80

MACREUSE $\boldsymbol{\lambda}$ LARGE BEC . . . . . . . . . 81

CANARD ROUX............... 81

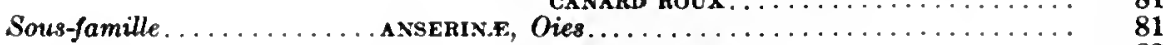

OIE BLANCHE. ................ 82

Petite vie blanche................ 82

Grande oie blanche............ 82

OIE BLEUE. . . . . . . . . . . 82

orE $\lambda$ Front BLANC.............. 82

Oie d front blanc d'A mérique........ 82

* bernache du CaNada............ 83

Bernache de Hutchin.............. 83

BERNACHE COMMUNE ........... 83

Bernache d'A mérique. ............... 83

Bernache commune noire. . . . . . . . . 8

Sous-famille. . . . . . . . crgnive, Cygnes.................... 84

CYONE D'AMERIQUE........... 84

CYGNE TROMPETTE. ............ 85

Ordre. ...... HERodiones, Echassiers d'eau profonde................... 86

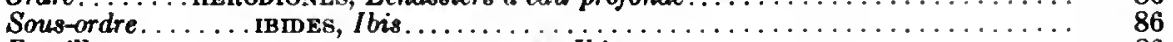

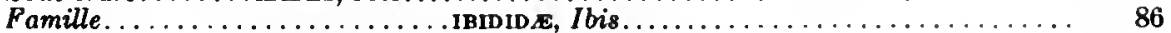

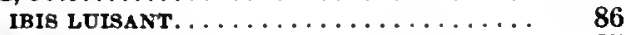

Sous-ordre. . . . . . HERoDII, Echassiers du type Héron. . . . . . . . . . . . . . . 87

Pamille. . . . . . . . Andeid

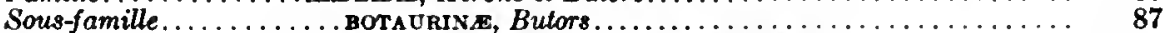

* butor diugrique............ 87

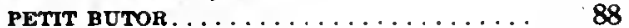

Petut butor de Cory .................. 88

Sous-famille. . . . . . ARDEIN

"oraNd HERON BLEU............ 89

Grand Héron bleu de l'Est......... 89

HERoNs BLANC8............... 90

AIORETTE BLANCHE D' AMÉEIQUE....... 90

PETIT hERON BLEU............. 91

Héron de la Louisiane................ 91

HERON VERT.................. 91

Heron vert $\mathrm{d} u$ Nord $\ldots \ldots \ldots \ldots \ldots \ldots \ldots \ldots, \quad 92$

HERON DE NUIT................ 92

Héron de nuit d'Amerique.............. 92

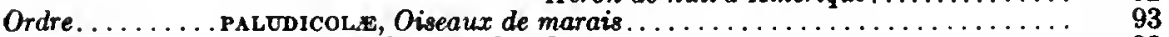

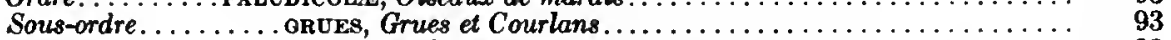

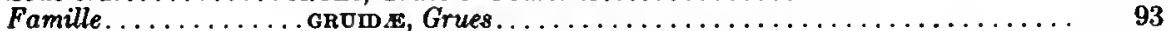

ORUE DU MEXIQUE. . . . . . . . . . 93

Grue du Canada................... 93

Grue d'A mérique.................. 93

Sous-ordre...... RAll, Petils oiseaux de marais. 
Famille.......... RALLID

Sous-famille............ RALLINÁ, Ralles vrais.................. 94

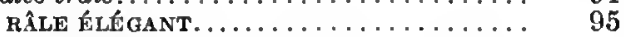

Rale d'eau salée..................... 95

RÂLE DE VIRGINIE. .............. 95

Rale de la Jamarque............... 95

*RÂLE dE LA CAROLINE............. 96

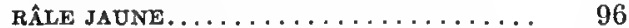

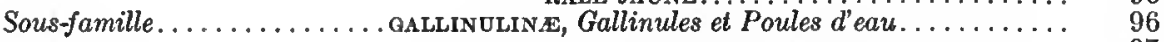

oALLINULE DE LA MARTINIQUE........ 97

GALLINULE DE LA FLORIDE. .......... 97

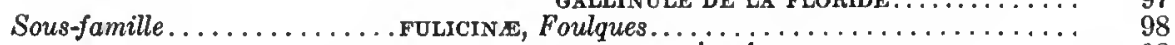

FoULQUE D'AMERIQUE........... 98

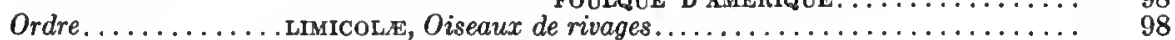

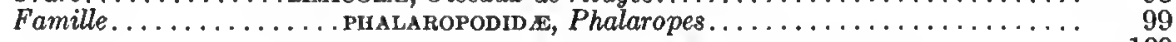

PHALAROPE ROUX................. 100

PHALAROPE HYPERBOREEN . . . . . . 100

PHALAROPE DE WILSON............. 100

Famille........... Recunvirostrid s, Avocettes et échasses.............. 101

AVOCETTE D'AMERIQUE............... 101

Famille............. scolopacid s, Oiseaux du type bécassine............... 101

*BÉCASSE D' AMÉ́ RIQUE.............. 102

*BECASSINE dE WILSON . . . . . . . . . 102

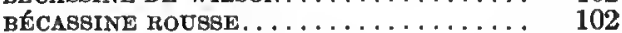

Bécassine d long bec............... 103

MAUBtCHE A LONOS PIEDS............. 103

MAUBECHE A POITRINE ROUSSE......... 104

MAUBECHE POURPREE. . . . . . . . . . . . 104

Maubèche pourpre de l'Est........ 104

MAUBECHE A POITRINE CENDR氏E. . . . . . . 104

MAUBECHE $\lambda$ CROUPION BLANC........ 105

MAUBECHE DE BAIRD .............. 105

MAUBECHE DE WILSON............. 105

PELIDNE.................... 106

Maubeche dे dos roux............... 106

MAUBECHE CoURLIs................ 106

MA UBËCHE SEMI-PALAfE. . . . . . . . . . . 107

Maubeche de l'Ouest. . . . . . . . . . 107

SANDERLING................... 107

BARGE MARBREE. . . . . . . . . . . 108

BARGE DE LA BAIE D'GUDSON. . . . . . . . 108

GRAND CHEVALIER A PIEDS JAUNES. . . . . 108

PETIT CheV ALIER A PIEDS JAUNES. . . . . . 109

CHEVALIER SOLITAIRE ............... 109

Chevalier solitaire de $l^{\prime} E_{s t} \ldots \ldots \ldots \ldots . . . . .109$

CHEVALIER SEMI-PALME.............. 110

Chevalier de l'Est................. 110

Chevalier de l'Ouest.............. 110

MAUBECHE A LONGUE QUEUE.......... 110

MAUBÈCHE $\grave{\lambda}$ POITRINE JAUNÂTRE.......... 111

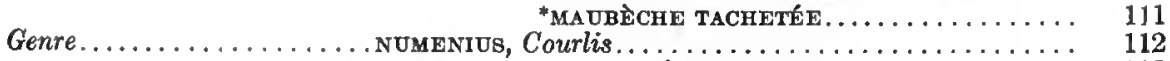

COURLIS A LONG BEC.............. 112

COURLIS DE LA BAIE D'HUDSON........ 113

COURLIS DU NORD ................ 113

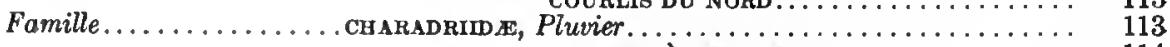

PLUVIER A VENTRE NOIR. . . . . . . . 114

PLUVIER DoRt D'AMt́RIQUE.......... 114

Pluvier doré de l'Est............ 115

* pluvier kildir. . . . . . . . . . . . . 115

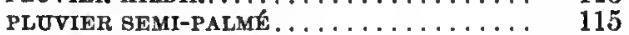

Pluvier rayé d'Europe............. 116

PLUVIER CRIARD ................ 116

Pluvier blanc.................. 116 


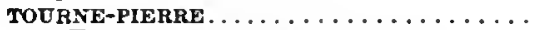

Famille. RAMATOPODID s, Huitriers.

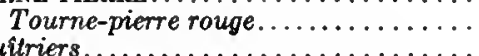

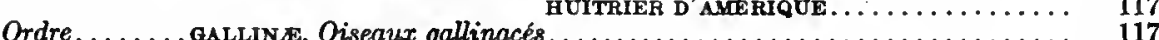

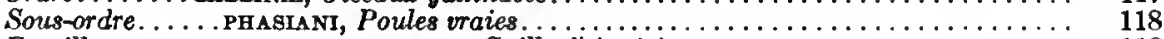

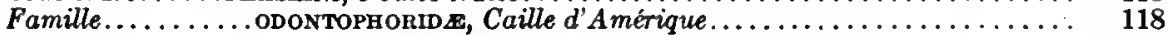

"callle......................... 118

Cailie de la virginie............... 119

Famille.

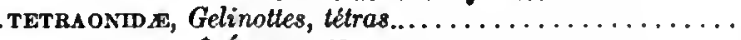

*tétras du canada............... 120

Gelinotte noire d'A mérique........ 120

Tétras du Canada............. 120

* gelinotre a fraise............. 120

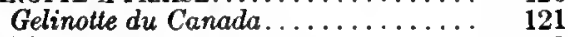

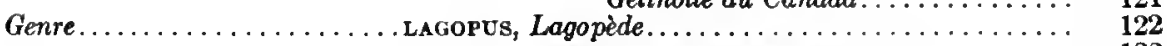

OELINOTTE DEB SAULES. . . . . . . . . . 122

Lagopede des saules............ 122

Lagopede d'Allen............... 122

LAGOPEDE DES ROCHERS. . . . . . . . . 122

Lagopede arctique. . . . . . . . . . . 122

Lagopede de Reinhardt. . . . . . . . . 122

Lagopede de Welsh. . . . . . . . . . . 122

POULE DES PRAIRIES . . . . . . . . . . . . . 122

Poule des prairies du Nord........ 122

OELINOTTE $\lambda$ QUEUE AIGÜ̈.......... 123

Gelinotte dे quewe aigué du Nord.... 122

Gelinotte des prairies d queue aiguë... 123

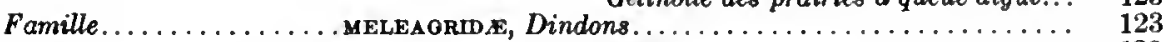

DINDON SAUVAGE............... 123

Dindon sautage du Nord............ 123

Ordre......... columb s, Pigeons et Colombes..................... 124

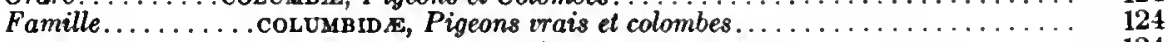

PIGEON voYagedR............... 124

"Tourterelle.............. 125

Tourterelle de la Caroline........... 125

Ordre. . . . . RA PTores, Oiseaux de proie.................... 126

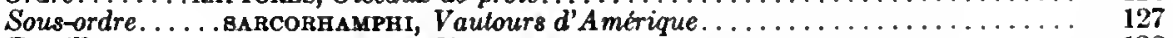

Famille......... cathantid

VAUTOUR AURA............... 128

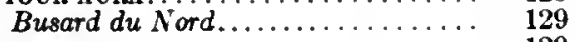

VAUTOUR NOR. .............. 129

Sous-ordre. . . Falcones, Oiseaux de proie diurnes.................... 129

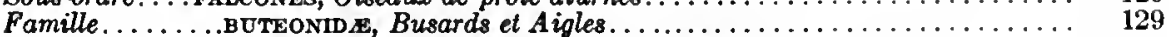

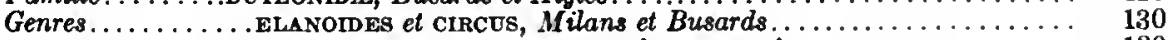

MILAN $\lambda$ QUEUE D'ARONDE.......... 130

- BUSARD des marais. . . . . . . . . . 130

Genres......... Accipiter et Astun, Eperviers aux courtes ailes........... 131

- Epervier brun................ 131

epervie de cooper............ 132

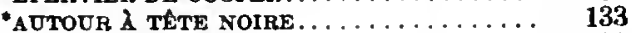

Autout de l'Est. . . . . . . . . . 133

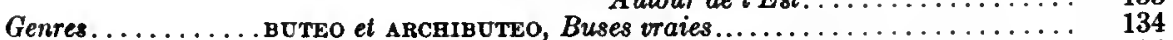

• BUSE $\lambda$ QUEUE ROUSSE............ 134

Buse de l'Est d queue rousse.......... 134

* Buse $\lambda$ mantea foux............ 135

Buse de l'Est d manteau roux....... 135

BUSE DE SWAINSON.............. 136

BUSE DE PENSYLVANIE. . . . . . . . . 136

BUSE PATTUE ................. 136

Buse patlue d'Amérique............ 137

Genres......... aquila et halidetus, Aigles................. 137

AIGLE DORE ................ 137

AIOLE $\lambda$ TÊTE BLANCHE........... 138

Aigle d tete blanche du Nord....... 138 


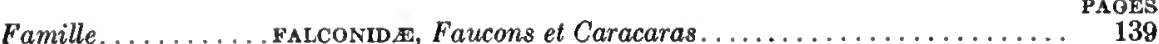

Sous-famille........... falconin

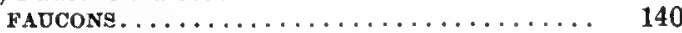

FAUCON BLANC................. 140

FAUCON.................... 140

Faucon gris..................... 140

Faucon noir.................. 140

*FAdCON PÈLERIN.................. 140

Faucon ordinaire.................. 141

FAUCON DES PIGEONS. . . . . . . . . . 141

Faucon des pigeons de l'Est........ 141

* FAdCon EPERVIER................ 142

Faucon épervier de l'Est........... 142

Sous-famille.............. PoLyborine, Caracaras................... 139

Famille............... . . . . . . . . . . . . . . 142

"orfraie d'amerique. . . . . . . . . . . . 143

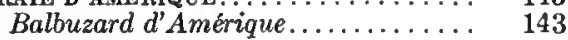

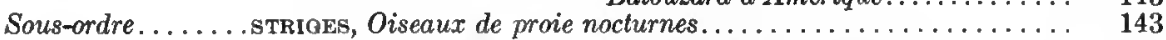

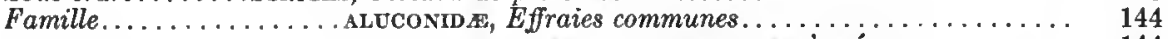

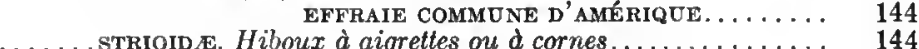

HIBOU $A$ OREILLES LONGUES. . . . . . . . . . 144

HIBOU $\lambda$ OREILLES COURTES. . . . . . . . 145

"Chouette du Canada............... 145

Chouette du Canada de l'Est......... 146

HIBOU CENDRE. .................... 146

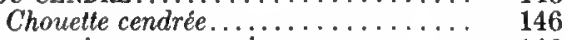

NYCTALE D'ACADIE DE L'ARCTIQUE. . . . . . 146

Nyctale de Richardson............ 146

NYCTALE D'ACADIE. . . . . . . . . . . . . . 148

*hibou maculf................... 148

Hibou maculé de l'Est............. 148

"DUC DE VIRGINIE...... . . . . . . . . . 148

Duc de Virginie de l'Est........... 149

Duc de Virginie de l'Ouest......... 148

Duc de l'A retique.............. 148

Duc du Labrador............... 149

HARFANO................... 149

CHOUETTE EPERVİ̀ RE........... 150

Chouette éperviere d'Amérique....... 150

Ordre......... coccrges, Coucous et Martins-pêcheurs................ 150

Sous-ordre. . . . . . . . cucoli, Coucous. . . . . . . . . . . . . . . . . . . 150

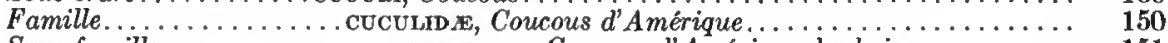

Sous-famille............... coccrzin s, Coucous d'Amérique des bois............. 151

"COUCOU A BEC JAUNE. . . . . . . . . . 151

*COUCOU A BEC NOIR............. 152

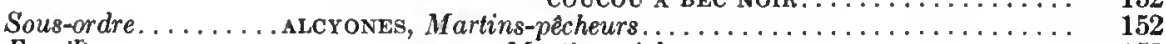

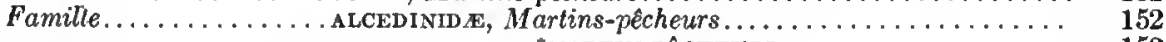

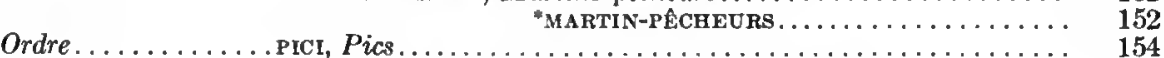

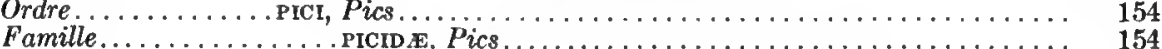

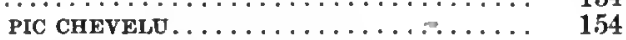

Pic chevelu de l'Est................ 155

Pic chevelu du Nord.............. 155

"pic minule. ................. 155

Pic Minule du Nord.............. 155

- pic arctique................. 155

PIC D'AME RIQUE. . . . . . . . . . . . 156

Pic de l'Est....................... 156

"pic macdré.................. 156

Pic de l'Est................... 156

*PIC A HUPPE ECARIATE............... 157

Pic du Nord d huppe écarlate. . . . . . 157

"PIC A TÊTE Rodoe.............. 158 
Famille..............PICIDE-Suite

PIC DE la CAROLINE........... 158

*PIC DORE.................. 158

Pic doré du Nord..................... 159

Ordre. . . . Macrochines, Engoulevents, Martine's et Oiseaux-mouches............ 159

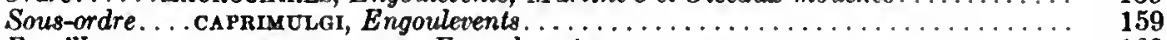

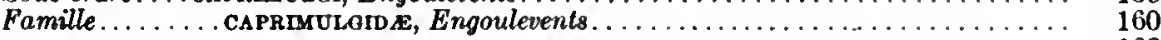

ENGOULEVENT DE LA CAROLINE........ 160

ENGOULEVENT CRIARD. . . . . . . 160

Engoulevent criard de l'Est............ 160

*ENGovLEVENT d'amtriqUE.......... 161

Engoulevent de l'Est............... 161

Sour-ordre. . . . . . CYPsELI, Martinets et congénères............... 162

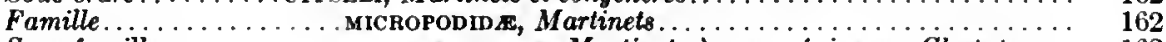

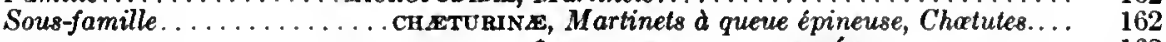

"MARTINET des chemintes........... 162

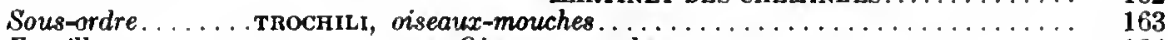

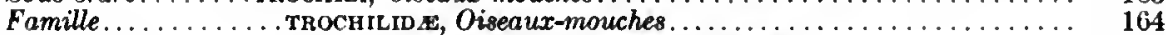

colibri $\lambda$ goroe rubis. . . . . . .

Ordre... . . . . Passeres, Passeteaux, Oiseaux-percheurs ............... 164

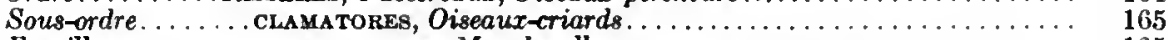

Famille.......... Tyrannto

MOUCHEROLLE A QUEUE EN CISEAUX.... 165

* Motcherolde de LA CAROLINE......... 166

MOUCHEROLLE $\lambda$ HUPPE.............. 166

* MOUCHEROLLE BRUN. . . . . . . . . . . 167

MODCHEROLLE AUX CÖTES OLIVE........ 167

MOVCHEROLLE VERDÂTRE............ 168

MOUCHEROLLE A VENTRE JAUNE........ 169

MOVCHEROLLE D'ACADIE............ 169

MOUCHEROLLE DE TRAILL. . . . . . . . . 169

Moucherolle des aulnes........... 169

Moucherolle des aulnes de louest... 169

PETIT MOUChEROLLE............. 170

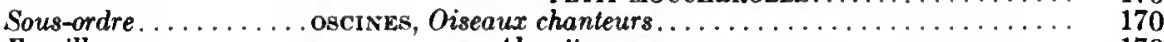

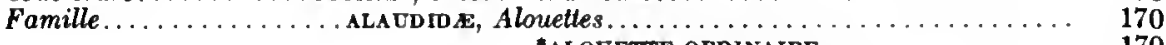

ALOUETTE ORDINAIRE. . . . . .

Alouette de l'Est.................. 171

Alouette des prairies............. 171

Alouette de Hoyt . . . . . . . . . . 171

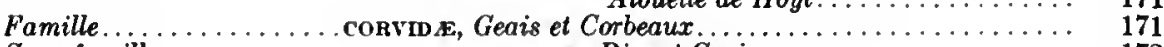

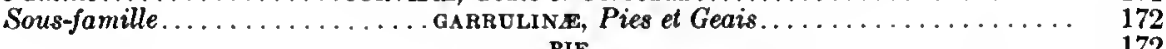

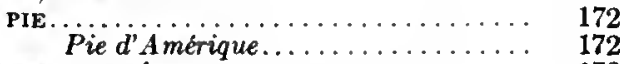

oear huppt.................. 172

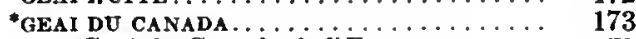

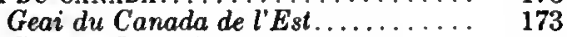

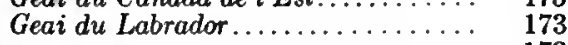

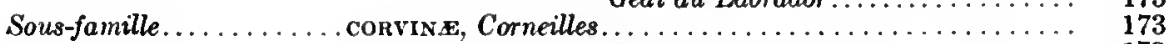

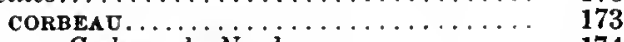

Corbeau du Nord................... 174

* Corneille d' amérique.............. 174

Corneille de l'Est............... 174

Famille............. stuRnides, Vrais étourneaux vulgaires.............. 175

fTOURNEA vULOAIRE................ 175

Famille............. ICteride, Elourneaux d'A métique................. 176

ooole........................ 177

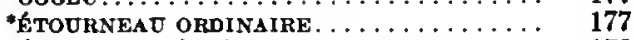

ETOURNEAU $\lambda$ TETE JAUNE. . . . . . . . . 178

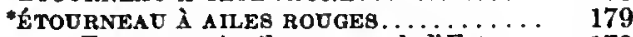

Etourneau d ailes rouges de l'Est.... 179

Etourneau d ailes rouges du Nord.... 179

*ETourneau des PRés............. 179

LORIOT DES VERGERB. . . . . . . . . 180 
Famille.............. ICTERID $\not$-Suite

LORIOT DE BALTIMORE. ............. 180

MAINATE COULEUR DE ROUILLE........ 181

*MAINATE BRONZÉ................ 181

Mainate bronzé...................... 182

Mainate pourpré............. 182

Famille.... . . . . . . . . . FrIngILLID . Moineaux, Linottes, Pinsons ou Bruants... 182

GROS-BEC $\lambda$ COURONNE NOIRE......... 183

Gros-bec d couronne noire de l'Est... 183

* GROS-BEC DES PINS. . . . . . . . . . . . . . 184

Gros-bec des pins du Canada....... 184

*PINSON POURPRE................... 184

Pinson pourpré de l'Est.......... 185

* MOINEAU DOMESTIQUE. . . . . . . . . . . . 185

BEC CRoIst...................... 187

Bec croisé d'A mérique........... 187

BEC CROISÉ $\lambda$ AILES BLANCHES. . . . . . . 188

Genre.................... Acantuis, Sizerins................... 188

SIZERIN DE HORNEMANN............. 188

Sizerin de Groënland........... 188

Sizerin blanc............... 188

LINOTTE D'AMERIQUE............. 189

Sizerin ordinaire............. 189

Sizerin de Holboll. ............. 189

Grand sizerin.............. 189

"CHARDONNERET JAUNE. . .......... 190

Chardonneret de l'Est........... 190

CHARDONNERET DES PING............ 190

* Plectrophane des neiges........... 191

Plectrophane des neiges commun.... 191

PLECTROPHANE DE LAPONIE........... 191

Plectrophane de l'Est ........... 191

*PINSON A AILES BaIEG... . . . . . . . . . . 192

Pinson d ailes baies de $l$ 'Est.......... 192

PINSON D'IPSWICH................ 192

PINSON DES PRÉS. . . . . . . . . . 192

Pinson des prés de l'Est......... 193

PINSON DES SAUTERELLES. . . . ........ 193

Pinson des sauterelles de l'Est...... 193

PINSON DE HENSLOW.................. 194

Pinson de Henslow de l'Est........ 194

PINSON DE LECONTE. . . . . . . . . . . . . . . . . 194

PINGON A QUEUE AIOUË............. 194

Pinson des Prairies................. 195

Pinson d̀ queue aiguë d'Acadie.... . 195

PINSON ORDINAIRE............... 195

Pinson ordinaire de l'Est........... 195

PINSON DE HARRIS. . . . . . . . . . . . . . 195

* PINSON A COURONNE BLANCHE.......... 196

Pinson d couronne blanche de l'Est .. $\quad 196$

* PINSON A GOROE BLANCHE. . . . . . . . . . . 196

"PINSON DE MONTAGNE............... 197

Pinson de montagne de l'Est........ 197

* petit pinson $\lambda$ couronNe rousse...... 197

Petit pinson d couronne rousse de l'Est 197

PINGON COULEUR D'ARGILE............ 198

PINSON DES CHAMPS. . . . . . . . . . . . . . . 198

Pinson des champs de $l$ Est......... 198

*PINSON Niverolle. . . . . . . . . . . . . . . 199

PINSON NIVERolle CoUleut D'AROILE... 199

PINSON CHANTEUR................... 199

Pinson chanteur de $l$ Est............ 199

PINSON DE LINCOLN................ 200

Pinson de Lincoln de $l$ Est........... 200

PINSON DES MARAIS............... 201 
Genre. ACANTHIS-Suite

PINSON FAUVE.

Pinson fauve de $l$ 'Est.............. 201

PINBON AUX YEUX ROUOES............ 202

Pinson aux yeux rouges de $l$ 'Est..... 202

GROS-BEC OU CARDINAL ............... 202

Gros-bec de l'Est............... 203

*aros-B EC $\lambda$ PoItrine rose........... 203

GROS-BEC BLEU................ 204

Gros-bec bleu de $l$ 'Est............... 204

PINSON INDIGo.................. 204

DICKCIBSEL. . . . . . . . . . . . . 204

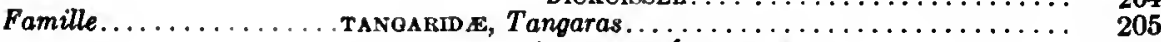

- tangara tcarlate.............. 205

TA NOARA VERMILLON............. 205

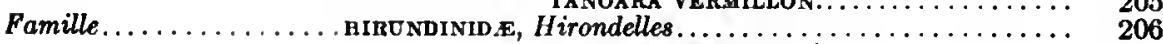

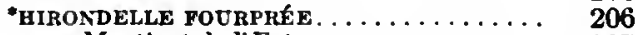

Martinet de l'Est............ 207

HIRONDELLE A FRONT BLANC.......... 207

Hirondelle d front blanc de l'Est.... 208

"HIRONDELLE dEs GRANGES.......... 208

"HIRONDELLE BICOLORE............ 208

"hirondelle de RIVAOE........... 209

HIRONDELLE $\lambda$ AILES HÉRISSEES....... 209

Famille............ вомвчciLLID

JASEUR DE BOHEME............ 210

- JASEUR do cidRE.............. 210

Famille........... Lanim

PIE-GRİ̀CHE BOREALE. . .......... 211

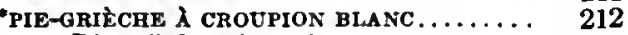

Pie-grieche migratrice........... 212

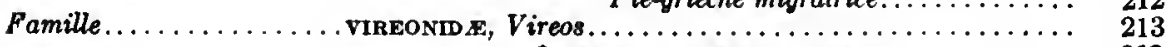

"VIREO AUX YEUX ROUGES . . . . . . . . . . 213

VIREO DE PHILADELPHIE........... 214

"vireo orIs oLrve. . . . . . . . . . . . . 214

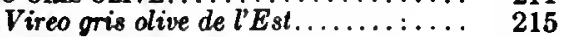

VIREO $\lambda$ FRONT JAUNE. . . . . . . . . . 215

VIREo gOLTTAIRE.............. 215

Vireo d tête bleue................ 215

VIREO A UX YEUX BLANCS........... 216

Vireo aux yeux blancs $d$ 'Amerique.. 216

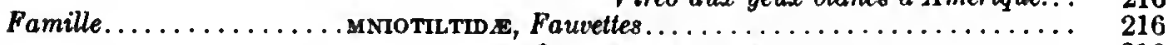

- FaUvetTe NoIRE ET BlaNche........ 216

FAUVETTE PROTONOTAIRE......... 218

Genre ................ vermivora, Fauvettes vermitores............ 218

FAUVETTE VERMIVORE............ 218

FAUVETTE AUX AILES BLEUES ......... 218

FAUVETTE $\lambda$ AILES DOREES . . . . . . 219

FAUVETTE DE NASHVILLE........... 219

Fauvette de Nashville de l'Est....... 220

FA UVETTE $A$ COURONNE ORANGEE...... 220

Fauvette d couronne orangée de l'inté-

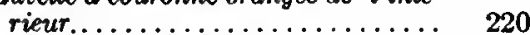

FAUVETTE DU TENNESSEE. . . . . . . . 220

FAUVETTE D'AMERIQUE. ............ 221

Fauvette d'A merique du Nord...... 221

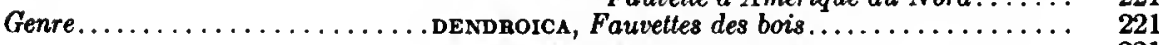

FAUVETTE DU CAP MAT............. 221

- FaUVETte JaUne................ 222

Fauvette jaune de $l$ l'Est............. 222

* FaUVETTE blede $\lambda$ gorae Noire........ 222

Fauvette bleue d gorge noire du Nord.. 222

"PauvetTe $\lambda$ crodpion jaUne........ 222

* FaUVETte $\lambda$ tête cendre.......... 223 
Genre............... DENDroICA-Suite.

FAUVETTE BLEUE.................. 223

FATVETTE DE PENSYLVANIE.

FAUVETTE A POITRINE BAIE............ 224

FAUVETTE RAYEE.............. 225

*FaUVetTe de BlackbURn. . . . . . . . . 225

"FAUVETTE ORISE $\lambda$ GOROE NOIRE....... 225

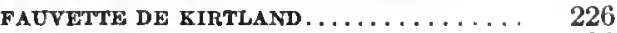

FAUVETTES DES PINS. . . . $\ldots \ldots \ldots \ldots \ldots, 226$

FAUVETTE DES PAlMters. . . . . . . . 227

Fauvette des palmiers de lintérieur. . 227

Fauvette d̀ couronne rousse......... 227

FAUVETTE DES PRÉs................. 227

Genre............... serorus, Fauvettes hochequeues................. 228

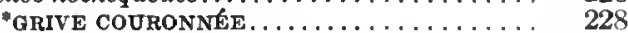

GRIVE DES RUISSEAUX. . . . . . . . . 228

Grive des ruisseaux de l'Est........ 229

Grive des ruisseaux de Grinnell...... 229

GRIVE DE LA LOUISLANE............ 229

Genres............. opononnis et oEOTuLYPIS, Fauvettes terrestres......... 229

FAUVETTE DU KENTUCKY........... 229

FAUVETTE DU CONNECTICUT. . . . . . . 229

FAUVETTE DE PHILADELPHIE............ 230

" pauvette trichas du mary land . . . . . 230

Fauvette trichas du Nora.......... 231

FAUVETTE BABILLARDE.............. 231

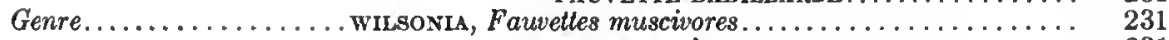

FAUVETTE A CAPUCHON............. 231

FAUVETTE A CALOTTE NOIRE........... 232

Fauvette de Wilson............. 232

FAUVETTE DU CANADA............... 232

*FaUVETte A Queug rousse............ 232

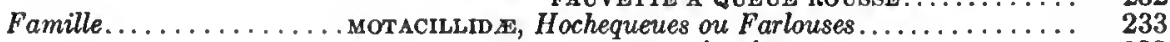

FARLOUSE D'AMÉRIQUE............... 233

Famille.............mimid s, Moqueurs et Merles.................... 234

ORIVE POLYGLOTTE................ 234

Grive polyglotte de l'Est........... 234

* grtve de la caroline. . . . . . . . . . . 234

"GRIVE Rodsse.................... 235

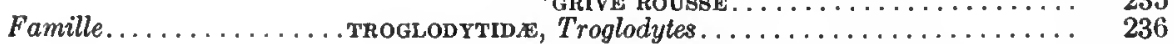

ROITELET DE LA CAROLINE.......... 236

Roitelet de la Caroline du Nord..... 236

ROITELET DE BEWICK............. 237

Roitelet de Bewick de l'Est............ 237

*TROoLODYTE AEDON................ 237

Troglodyte aédon de l'Est............. 237

TROGLODYTE D'HIVER. ............ 238

Troglodyte d'hiver de l'Est......... 238

TROGLODYTE DES MARAIS À BEC COURT... 238

THOGLODYTE DEs MARAIS A LONO BEC.... 238

Roitelet des marais de l'Est........ 238

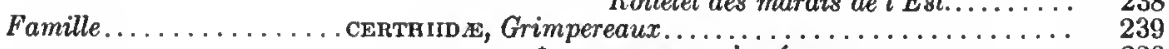

*ormpereau d'anírique. . . . . . . . . . . . 239

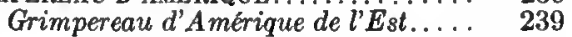

Famille. SITTID 2 , Sittelles

* SITTELLE de la caroline............

Sittelle d poitrine blanche........... 240

SITTELLE DU CANADA............... 241

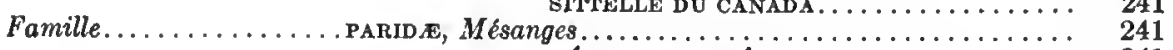

MESANGE HUPPEE................. 241

* mésange $\lambda$ tête noIre............. 242

Mesange d̀ tête noire de l'Est....... 242

Mesange de la Caroline................ 242

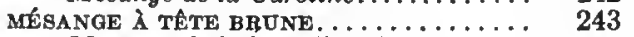

Mésange de la baie d'Hudson.......... 243

Mésange du Canada............. 243 
Famille.

sruviw se, Fauvettes et roitelets de l'ancien monde.

PAOES

Sous-famille REOULIN

"ROITELET HUPPE. . . . . . . . . . . . 244

Roitelet huppe de l'Est......... $\mathbf{2 4 4}$

-ROITELET $\lambda$ COURONNE RUBIS. . . . . . . . 244

Roitelet d couronne rubis de l'Est.... 244

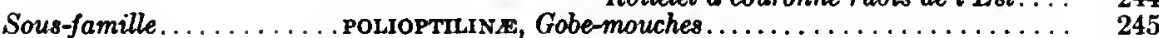

COBE-MOUCHES GRIS BLEU. ......... 245

Gobe-mouches de l'Est.......... 245

Famille.......... Tundwes, Grives et congénères.................. 245

Sous-famille.......... TURDINe, Grives traies.................... 245

"oRIVE dEs BOIS.............. 246

- orIVE de wILson. .............. 246

Grive d'Amerique.............. 246

Grives des sautes............... 246

"GRIVE D' alice................ 247

Grive aux côtes de la tête grisatres.... 247

Grive de Bicknell............ 247

- orive a d dos oltvktre............ 247

Grive de Stainson. . . . . . . . . . 247

-orive solitaire............... 247

Grive solitaire de l'Est............ 248

- MerLe d'auterique. . . . . . . . . . . . 248

Le Merle de l'Est............... 248

TRAQUET MoTteUX............... 248

Traquet du Groenland............ 249

"ROUOE-GORGE BIEU................ 250

Rouge-gorge bleu de l'Est........ 250 


\title{
ORNITHOLOGIE DESGRIPTIVE.
}

\author{
Classe-aVES. OISEAUX.
}

Les oiseaux, en tant que formant une classe, peuvent être divisés en oiseaux avec dents et oiseaux sans dénts, bien que les premiers soient une classe éteinte et connue seulement par des restes fragmentaires conservés comme fossiles. Tous les oiseaux aujourd'hui sont dépourvus de dents. Quelques espèces, comme les harles, sont pourvues de dentelures, dans le bec corné, lesquelles ont une ressemblance superficielle avec les dents (figure, 7, p. 20) mais, à l'examen, on reconnait que ce ne sont pas de vraies dents.

\section{SOUS-CLASSE-CARINAT E. OISEAUX A BRECHET}

Les oiseaux modernes sans dents sont divisés en deux sous-classes: les Ratito, ou oiscaux sans bréchet, et les Carinatoe ou oiseaux a bréchet. Les Ratitoe, comprennent les autruches et les émeus qui sont dépourvus de bréchet au sternum où s'attachent les muscles des ailes, et qui ne volent pas. Il ne s'en trouve pas dans l'Amérique du Nord; c'est pourquoi il n'en est pas question ici, le terme sternum à bréchet se rapporte à la projection élevée, mince, scmblable à la quille d'un bateau qui part du milieu du sternum, et à laquelle s'attachent les muscles puissants de la poitrine et des ailes.

Les oiseaux à bréchet (sous-classe des Carinato) se divisent en plusieurs ordres qui sont traités suivant la succession adoptée par l'Union des Ornithologistes américains.

\section{Ordre-Pygopodes. Oiseaux Plongeurs.}

Description générale. Les plongeurs, ainsi que le nom l'implique sont des oiseaux organisés pour des intentions qui les appellent sous l'eau. L'articulation de la hanche est située très en arrière sur le corps et le mécanisme de la jambe est plus propre à nager qu'à marcher. Le tarse, la partie visible de la jambe, est tres aplati (figure 2, p. 19) et les orteils sont ou partiellement (fig. 13, p. 21) ou complètement (fig. 2 et 6, p. 19 et 20) palmés. Les ailes sont petites en comparaison de la taille du corps. Le bec est droit et généralement effilé, modérément long, mais quelquefois, aplati et profond, comme dans les cas des pingouins et des macareux; dans cette dernière espèce cette spécialisation atteint son plus haut développement dans l'ordre.

Traits distinctifs. Orteils, trois ou quatre, entièrement ou partiellement palmés; tarse aplati, queue peu marquée ou petite.

Indications sur le terrain. Ailes et queue petites; becs droits, étroits, différents du bec de canard. Les plongeurs ressemblent superficiellement aux canards, mais là où les canards prendraient leur vol les plongeurs plongent.

Nid. Dans le voisinage immédiat de l'eau, sur les rives couvertes de roseaux ou les arêtes rocheuses, ou encore dans les crevasses et les trous du sol.

Comme conséquence de la construction particulière de la jambe, le corps est obligé de se tenir très droit quand l'oiseau est sur terre, il marche alors avec difficulté. Bien plus, certaines espèces sont presque sans force sur le sol et ne peuvent s'élever dans l'air à moins d'être sur l'eau, ou sur un terrain escarpé, ou encore contre un vent contraire très fort. Ils nagent et plongent avec facilité, et, bien que les ailes soient petites en comparaison de la taille du corps, une fois qu'ils ont pris leur essor ils volent avec de rapides battements d'ailes, avec vitesse et vigueur, en ligne droite ou par de longues courbes sans évolution ni manœuvres. 
Valeur économique. Les oiseaux plongcurs se nourrissent presque exclusivement d'animaux aquatiques qu'ils prennent et poursuivent sous l'eau. Au point de vue économique ces oiseaux ont peu d'importance. Les oiseaux qui représentent cet ordre au Canada se divisent en trois familles: les Grèbes, Colymbida; les Plongeons, Gaviida; les Pingouins, Guillemots, etc., Alcido.

\section{SOUS-ORDRE-COLYMBI. GRËBES. FAMILLE-COLYMBID瓜. GRÈBES.}

Description générale. Les grèbes et les plongeurs ont des pattes lobées et non tout a fait palmées, et sont sans queue très visible. Au lieu des membranes allant d'un orteil à l'autre, comme dans la plupart des oiseaux nageurs, les doigts sont pourvus d'un rebord dentelê de bouts plats semblables à des lobes, ou d'appendices attachés à l'orteil. Ces doigts constituent d'excellentes palettes pendant la nage, et en se repliant ils offrent un minimum de résistance à l'eau, au retour. Leur extraordinaire habileté à plonger a fait donner à ces oiseaux les sobriquets communs de "diable plongeurs n, "sorciers de l'eau * etc.

Traits distinctifs. Membranes des orteils dentelées (figure $13 \mathrm{p} .21$ ), queue courte, bec pointu, et l'éclat argenté très singulier des plumes des parties de dessous.

Indications sur le terrain. Bec pointu et queue peu visible. Pattes rejetées droites derrière en volant.

Nid. Dans les roseaux et les joncs qui bordent les mares ou les étangs, sur les tas de végétaux ou flottants ou stationnaires.

Habitat. Les grèbes sont répandus sur tout le Canada et jusqu'à la zone arctique. A l'époque de la couvaison on les trouve plutôt sur l'eau douce que sur l'eau salée. Il y a trois espèces de cette famille qui se présentent régulièrement dans l'est du Canada, une quatrième espèce, le grèbe occidental, mentionné incidemment ici, a été signalé plusieurs fois par erreur, car on ne peut certifier qu'un seul cas où on l'ait vu.

Les grèbes sont les habitants par excellence des étangs d'eau douce et des lacs, bien qu'en certaines occasions ils fréquentent la mer en nombre. La couleur de ceux d'âge adulte se présente en larges taches; les plus jeunes font voir des raies bien tranchées, surtout autour de la tête, indiquant par là que la famille descend d'un ancêtre commun rayé. Les sternum du grèbe, si utilisés comme garnitures pour les modistes, proviennent d'oiseaux de cette famille. La grande quantité de ces oiseaux qu'on sacrifie pour cet usage, et la façon dont on les enlève de leurs endroits préférés de couvaison en réduisent naturellement le nombre.

Valeur économique. Comme ces oiseaux se nourrissent presque exclusivement de chair aquatique, ils n'ont presque aucune valeur économique immédiate. On trouve souvent de grande quantités de plumes dans l'estomac des grèbes, mais la raison d'être de leur présence n'est pas encore comprise. Rien ne prouve que ce soient les restes d'oiseaux saisis comme proie.

Genre-Colymbus. Grèbes cornus.

2. Le Grèbe à cou rouge. (HoLbcell's GreBE), Colymbus holbœlli. L, 19. C'est le plus grand de nos grèbes. Les grèbes devenus adultes en été ont les joues et la gorge blanche et un cou d'un rouge chataigne fort riche.

Traits distinctifs. La taille suffit d'habitude a reconnaitre le grèbe. Les oiseaux encore jeunes n'ont généralement qu'un semblant de cou brun rougeâtre.

Indications sur le terrain. Sur le terrain la taille servira aussi à distinguer ce grèbe-là de tous les autres; et la présence d'une plaque blanche sur l'aile, ainsi qu'un dos non tacheté, le distinguera du plongeon avec lequel on risquerait de le confondre. La plaque blanche de la joue est très visible par contraste avec la couronne noire et le cou rouge.

Nid. Sur des compostes de matières végétales, flottantes ou stationnaires, ou sur des llots marécageux près des rives de lacs d'eau douce.

Habitat. A travers le continent; couvaison à l'est, au nord de la colonie actuelle. Dans les provinces des prairies et à l'ouest ils nichent au sud et au dela de la limite des Etats-Unis. 
Cet oiseau, sauf dans l'Ouest, dans la saison de la couvaison, est plus communément vu sur de grandes étendues d'eau que sur de plus petites. Il est relativement rare dans l'est du Canada.

3. Le Grèbe cornu. (HORNED GREBE). Colymbus auritus. L. 13.50. Le grèbe cornu est à peu près de la même taille que le grèbe à bec bigarré mentionné ci-après, mais avec un bec plus aiguisé et plus effilé. L'adulte de l'été a un cou rouge (tout à fait comme celui à cou rouge), des touffes à l'oreille d'un jaune brunátre et proéminentes, et une fraise en forte saillie sur les joues depuis le derrière de la tête jusqu'au cou. En automne et en hiver une tête d'un noir blanc brillant forme constraste avec la coloration plus matte du grèbe à bec bigarré.

Traits distinctifs. Le jeune sujet, oiseau ordinaire d'un noir grisâtre et blanc peut être confondu avec le jeune grèbe à bec bigarré, mais peut être distingué par son front et sa poitrine d'un blanc brillant, son bec effilé et la tache blanche sur l'aile. l'aile.

Indications sur le terrain. Bec mince, aiguisé, avant-cou blanc et plaque planche sur

Nid. Comme le précédent.

Habitat. A travers le continent, couvant par endroits en dedans des limites de la colonisation actuelle, et au nord, mais plus ordinairement à l'ouest qu'à l'est.

Le grèbe cornu qui est migrateur habite les plus larges étendues d'eau et se rencontre moins souvent dans les bourbiers où le grèbe à bec bigarré se rencontre fréquemment.

6. Le Grèbe a bec bigarté. (PIED-BILled GREBE). Podilymbus podiceps. L, 13.50. Planche I A.

Traits distinctifs. Le grèbe à bec bigarré peut être mis à part de tous les autres grèbes canadiens à cause de son bec relativement plus lourd et gros avec sa tache et son culmen plus fortement arqué; on le distingue aussi, du jeune grèbe cornu, de tout plumage, à cause de la couleur foncée, moins brillante de son avant-cou, de sa poitrine et de ses dessous, et aussi de l'absence de la plaque blanche de l'aile.

Indications sur le terrain. Taille et forme du bec, moucheture sur le bec, pas de plaque blanche sur l'aile, et plaque noire sur le cou au printemps.

Nid. Le long des bords marécageux d'étangs et de lacs sur des supports ou stationnaires ou flottants.

Habilat. A travers le continent, la couvaison se faisant depuis nos frontières sud vers le nord; il est probable qu'un grebe trouvé couvant au sud d'une ligne tirée d'Ottawa à Sault-Ste-Marie appartiendra à cette espece.

Nous avons ici le grèbe commun de l'est du Canada où on le trouve dans la saison de la couvaison, ou pendant la migration, sur presque chaque étang ou bourbier. Il fréquente moins que les espèces parentes les eaux claires et dégagées. La virtuosité des grèbes comme plongeurs est bien connue et fort développée dans la présente espèce. Plongeant à l'éclair du coup de feu, le grèbe est souvent en sûreté sous l'eau au moment où le plomb meurtrier touche l'endroit qu'il venait d'occuper. Même les fusils se chargeant par la culasse n'ont pas toujours assez de rapidité pour l'atteindre, bien que la poudre sans fumée ait enlevé au grèbe presque toute sa supériorité. Les grèbes ont la faculté de nager ou très bas ou très haut dans l'eau. En expulsant l'air du plumage épais et doux et en le comprimant dans les cavités de son corps le grèbe peut augmenter son poids spécifique, et s'enfoncer peu à peu dans l'eau jusqu'a ce que le bec seul soit au-dessus de la surface, position dans laquelle il se cachera, et, sauf quelque accident, défiera les yeux les plus perçants.

Valeur économique. Nous n'avons que peu de renseignements précis all sujet de la composition exacte des aliments des grèbes. Le grèbe à bec bigarré, toutefois, se nourrit de petits poissons et d'insectes aquatiques, en y ajoutant plus ou moins de matières végétales. Le poisson, eu égard à l'habitat de l'oiseau, appartient principalement à l'espèce qui hante les eaux $28588-4 \frac{1}{2}$ 
bourbeuses et sans importance économique. Quant aux insectes que mange le grèbe, ce sont surtout les espèces de proie telles que les escargots d'eau qui, parfois, causent quelque dommage au frai de poisson. La matière végétale est sans importanee. En somme, nous pouvons considérer le grèbe a bec bigarré eomme tout à fait inoffensif sauf dans les cas rares où il fait une visite passagère aux mares et aux étangs où s'élèvent des truites ou d'autres poissons de prix.

\section{SOUS-ORDRE-CEPPHI. PLONGEONS ET PINGOUINS}

\section{FAMILLE-GRAVIID A. PLONGEONS.}

Description générale. Les plongeons sont de grands plongeurs avec un bec droit, très pointu et avec des pattes très palmées, (figure 2, p. 19). A l'état adulte ils ont des couleurs dont les dessins forment des contrastes frappants, généralement noirs et blanes.

Traits distinctifs. Plus grands que des canards et le cou plus court que celui des oies. Ces traits-là et le bec si effilé et pointu sont distinctifs. La queue plus en évidence que chez les grebes.

Indications sur le terrain. Taille, longueur du cou et bec. Pendant le vol les pattes sont entraînées derrière la queue.

Nid. Sur les rivages bas, dans le voisinage immédiat de l'eau ou ils peuvent plonger directement dans l'eau depuis leur nid.

Les plongeons sont peut-être des plongeurs supérieurs encore aux grèbes mais ils s'enlèvent moins facilement sur l'eau et, sauf par une bonne brise, qu'ils peuvent affronter; ils doivent battre longtemps l'eau de leurs ailes avant de s'envoler.

Valeur économique. Leur nourriture se compose presque entièrement de poisson, mais, vu le petit nombre de plongeons qui se trouve d'habitude dans une localité donnée, leur importance économique est insignifiante.

\section{Genre-Gavia. Plongeons.}

7. Le Plongeon a collier. LE huARd. (common looN). Gavia immer. L, 32. Planche I B.

Traits distinctifs. Le plongeon à l'état adulte est facile a distinguer des autres plongeons par sa coloration bien marquée, mais les jeunes sont un peu plus difficiles à différencier. La taille et l'absence de tache sur le dos le distingueront du plongeon à cou rouge avec lequel on le confond très facilement.

Indications sur le terrain. Taille et dos non taché des jeunes, comme ci-dessus. La plupart des plongeons qu'on voit dans nos lacs intérieurs appartiennent à cette espèce.

Nid. Tout près de l'eau sur les rives marécageuses ou rocheuses de lacs intérieurs, là où, à la moindre alarme, les plongeons peuvent filer droit sous l'eau. Le nid, plutôt massif, est bâti avec des matières végétales en décomposition.

Habitat. A travers tout le Canada, couvant partout où les conditions s'y prêtent, y séjournant souvent pendant l'hiver jusqu'au moment où la glace ferme les eaux.

La plupart de ceux qui fréquentent nos rivières et nos lacs connaissent bien le rire strident du plongeon. Le plongeon a un autre cri qui commence bas, s'élève haut et tombe subitement avec des notes fortes et perçantes. L'oiseau est souvent bruyant la nuit ou au moment d'un orage, et les oiseaux s'appellent souvent et se répondent d'une rive à l'autre.

A cause de l'envahissement continu de la colonisation et du dérangement qui s'ensuit pour les nids, le plongeon est devenu plus rare en ces dernières années, et, dans plus d'une de ses anciennes retraites, on ne le voit plus guère aujourd'hui que dans lc temps de la migration. Il reste, toutefois, un grand nombre de lacs solitaires dans le nord inhabité où les plongeons peuvent vivre et nicher sans être dérangés et il n'est pas à prévoir 
que cette espèce si pittoresque disparaisse prochainement. Des mesures protectrices encouragées par une opinion publique plus éveillée viendraient sans doute regarnir nos lacs et nos étangs de ces hôtes d'été et augmenter le nombre des hôtes de simple passage.

Valeur économique. Bien que le plongeon soit un gros oiseau, la largeur de son gosier n'admet que des poissons de relativement petite taille. Ce fait mis en rapport avec le petit nombre d'oiseaux des lacs peu étendus et la masse immense de poissons des très grands lacs, rend les ravages que peuvent commettre les plongeons sans importance économique quelconquc. L'espèce ne devrait donc pas être condamné à la destruction.

11. Le Plongeon à gorge rousse. (RED-THROATED LOON). Gavil Siellata. L, 25. C'est un plongeon plus petit que le huard, et qui ne présente pas le contraste intense de la coloration noire et blanche du dos. Sa tête et son cou sont gris, et les adultes en été ont au cou une plaque d'un rouge marron.

Traits distinctifs. Les sujets adultes peuvent se distinguer du plongeon commun par la taille et la coloration, comme ci-dessus. Les jeunes peuvent se reconnaftre à leurs dos finement tachetés qui se distinguent des lignes légèrement grises du plongeon à collier.

Indications sur le terrain. Taille et coloration du dos.

Nid. Habitudes de couvaison semblables à celles de l'espèce précédente.

Habitat. Répandu sur tout le Canada; rare dans l'intérieur, plus commun sur les côtes. Niche dans l'est depuis le nord de l'Ontario, le Québec central et le NouveauBrunswick vers le nord.

Valeur économique. La même que pour le plongeon à collier mais moins importante parce qu'il est moins répandu.

\section{FAMILLE ALCIDE. PINGOUINS, guILLEMOTS ET MACAREUX.}

Description générale. Cette famille se compose d'espèces exclusivement maritimes, qui ne se rencontrent que rarement ou par occasion sur les esux douces. Bien qu'elle soit dans son élément sur l'eau elle se tient droit sur terre ferme et marche avec beaucoup plus d'aisance que le grèbe ou le plongeon. Le bec est sujet à plus de variations que celui des familles précités, puisqu'il va du bec droit, effilé des guillemots jusqu'au bec très comprimé des macareux.

Traits distinctifs. La forme caractéristique du plongeur combinée avec les pattes palmées et l'absence de pouce (figure 12 p. 21 ).

Indications sur le terrain. Ressemblance générale avec les canards, mais leur cou court et leur bec pointu, quelquefois profond et étroit, sont caractéristiques.

Nid. Couvent en nombreuses compagnies, souvent d'especes mixtes, sur des flots rocheux ou sur des falaises battues par la mer. Ne font pas de nid mais déposent leurs cufs sur le sol. Leurs cufs sont extraordinairement gros, vu la taille de l'oisean, et tout à fait en forme de poire, ce qui fait qu'ils roulent en cercles plutôt qu'en droite ligne et ainsi risquent moins de tomber des rebords nus et rocheux qui leur servent de nids.

Habitat. Le long de nos rivages maritimes, des limites méridionales jusqu'au pôle arctique. Ils ne sont qu'occasionnellement sur les eaux douces, bien qu'une espèce ait fait quelquefois des migrations anormales et soudaines vers les grands lacs inférieurs.

Cette famille fréquente la pleine mer, ne venant au rivage que pour nicher. Ces oiseaux diffèrent des autres plongeurs en ce qu'ils se servent d'habitude de leurs ailes sous l'eau tout comme pour voler. Sur la côte du Labrador leurs cufs ont, dans le passé, beaucoup servi aux pécheurs comme nourriture.

Valeur économique. Ne mangeant pas autre chose que les plus petits poissons et les crustacés pris en mer, où les quantités en sont considérables, ces oiseaux ne peuvent faire aucun dommage. 


\section{Sous-famille-Fraterculinæ. Macareux.}

\section{Genre-Fratercula. Macareux.}

13. Le Macareux arctique. Le PERRogdet DE MER. (PUFFIN.) Fratercula arctica. L, 13. Le macareux est un petit plongeur assez grotesque, noir en-dessus, blanc en-dessous et avec une face grise. Il est remarquable par son bec qui est ridiculement profond et aplati, presque aussi haut que long et fort en coulcurs de rouge et de jaune.

Traits distinctifs. Le bec est toujours caractéristique.

Indications sur le terrain. Le bec peut être distingué d'aussi loin que l'oiseau vivant peut s'apercevoir.

Nid. Couve dans les crevasses et les fentes des falaises rocheuses, ou se terre dans le sol d'ilots déserts. Le long de la côte du Labrador il y a plusieurs fles dites "Aux Perroquets 》 a cause du grand nombre de ces oiseaux qui couvent dans ces parages. Malheureusement les dégáts qu'y font les pêcheurs ont lamentablement réduit le nombre de ces oiseaux, et à moins de mesures protectrices en leur faveur ils disparastront bientôt.

Habitat. Les macareux sont répandus le long des rivages maritimes des deux côtés de l'Atlantique depuis le Canada et l'Angleterre jusqu'à la zone arctique.

La vue seule de cet oiseau suffit à reconnaitre la justesse du surnom de perroquet qu'on lui donne. A l'inverse d'autres plongeurs il se tient debout sur ses doigts et montre une grande agilité dans ses mouvements.

\section{Sous-famille- Ethilnæ. Petits PIngouins, Gulllemots nains, Guillemots.}

\section{Genre-Cepphus. Pigeons de mer.}

27. Le Guillemot noir. Le PIgeon De Mer. (BLACK guillemot). Cepphus grylle. L, 13. En été le guillemot noir est un petit plongeur, noir comme du charbon, avec de longues plaques blanches aux ailes et des pieds rouges. En hiver, les parties de dessous sont blanches et les plumes de dessus sont noires mais avec des taches blanches. Les ailes ne changent pas en été.

Traits distinctifs. En été l'espèce ne peut pas se confondre avec une autre. En hiver, la taille et la coloration des ailes sont presque tout aussi caractéristiques.

Indications sur le terrain. Les traits susdits sont des indications sur le terrain, facilement reconnaissables.

Nids. Dans les cavités de rochers ou dans quelques ouvertures de talus raboteux au pied des falaises.

Habitat. Le guillemot de l'Atlantique est commun le long de notre côte de l'Atlantique, mais n'est vu que rarement ou même jamais dans l'intérieur des terres.

SOUS-ESPECES. Il y a deux sous-espèces du guillemot noir au Canada. Le guillemot de l'Atlantique qui est l'oiseau type, et le guillemot Mandt Cepphus grylle mandti, race du nord habitant les régions polaires et qui se distingue par les couvertures entièrement blanches de l'aile, jusqu'à la base, au lieu d'y avoir une barre noire dissimulée. L'Union américaine des Ornithologistes donne dans son catalogue cet oiseau comme formant une espèce propre, mais il conviendrait de n'y voir qu'une sous-espèce.

Le guillemot noir est un des habitants les plus communs de notre rivage maritime où il est reconnu de tous ceux qui le fréquente. Il vit moins en troupes que les autres membres de la famille et niche d'habitude tout seul et non en colonies, quoique parfois ces oiseaux se réunissent dans certaines localités, attirés par une communauté d'intérêts.

\section{Sous-famille-Alcinæ. Pingouins, Gulllemots.}

$$
\text { Genre-Uria. Guillemots. }
$$

30. Le Guillemot ordinaire. ra MORMETTE. IA MARMETTE. (COMMON MURRE). Uria troille. $\mathrm{I}, 16$. Ce guillemot est blanc en dessous pendant l'été, avec la tête et le cou foncés, couleur fumée, brun de phoque. Le dos et les ailes sont noirs. En hiver la gorge est de couleur claire voilée de plus ou moins de gris, et le brun est remplacé sur la tête et le cou par du noir estompé de gris surtout sur la gorge. 
Traits distinctifs. Le guillemot ordinaire ressemble beaucoup au guillemot de Brünnich et au pingouin commun. Il peut être différencié, en été, d'avec le premier par la coloration brun pâle de la tête et du cou, et par le fait que le derrière du cou n'est pas plus foncé que le devant. En toute saison il a un bec un peu plus long et plus faible que celui du guillemot de Brünnich. On peut le distinguer du pingouin par le bec qui n'est pas sensiblement aplati ni profond.

Indications sur le terrain. Taille du bec et coloration du cou, telles sont les seules indications sur le terrain qu'on puisse donner pour distinguer les deux guillemots, et a l'état vivant, ces indications ne peuvent être remarquées que grâce aux plus favorables circonstances. Ces oiseaux nagent, dit-on, avec la queue â fleur d'eau et non relevée comme celle du pingouin commun. La différence entre le bec de ce pingouin et celui du guillemot est très facile à constater en temps ordinaire.

Nid. Ils couvent en nombreuses colonies dans les roches, ne font pas de nids et ne pondent qu'un cuf.

Habitat. Abondent le long de la côte maritime de l'est, ne sont jamais, ou très rarement, trouvés dans l'intérieur des terres sur l'eau douce.

SOUS-ESPECES. Le guillemot ordinaire habite les parages du nord des deux océans, le Pacifique et l'Atlantique, étant représenté dans chacun d'eux par différentes sousespèces. Le guillemot de l'Atlantique offre le specimen type et c'est naturellement celui qu'on rencontre sur nos rivages de l'est.

Le nombre de guillemots qui occupent une falaise à nids est quelquefois extraordinaire. Sur le bord des crêtes rocheuses ils se pressent les uns contre les autres comme des lignes de soldats, et ressemblent à ces files de pingouins dont les images nous sont familières.

31. Le Guillemot de Brünnich. (THICK-BILlen gullemat). Uria lomvia. $L$, 16-50. Ce guillemot est presque exactement semblable à l'espèce précédente.

Traits distinctifs. La tête et le cou sont plus foncés et d'un brun plus riche, sans la teinte fumée du guillemot ordinaire, et le cou est un peu plus foncé derrière que devant. Le bec est un peu plus gros et sensiblement plus court et plus massif que celui du guillemot ordinaire.

Indications sur le terrain. Bec plus court, plus gros; la coloration de la tête et du cou aidera, si la vue en est exceptionellement claire, a distinguer cet oiseau vivant du guillemot ordinaire. Bec tranchant et effilé au lieu d'être émoussé et profond; queue non redressée en nageant; tout cela servira à le distinguer du pingouin commun.

Nid. Comme dans l'espèce précédente.

Habitat. Un peu plus au nord que celui du guillemot ordinaire.

SOUS-ESPECES. Le guillemot de Brünnich se rencontre sur les océans Pacifique et Atlantique mais comme des espèces distinctes. Notre spécimen de l'est est l'oiseau type et s'appelle le guillemot de Brünnich.

Le guillemot de Brünnich est le seul nembre de cette famille qu'on trouve sur les grands lacs. Ces oiseaux sont parfois venus par centaines sur les lacs Ontario, Erié, et sur les eaux tributaires de ces lacs à la fin de l'automne et au commencement de l'hiver; ils étaient tous faméliques et aucun ne semblait devoir survivre ou retourner vers leurs demeures maritimes. Leur présence là est encore inexplicable.

\section{Genre-Alca. Pingouins.}

32. Le Pingouin Commun. (Razor-Billed auk.) Alca torda. L, 16.50. Le pingouin commun a la même apparence générale que les deux dernières espèces.

Traits distinctifs. Le bec est très profond et aplati (quoique pas autant que celui du macareux arctique); il est donc facile a distinguer du guillemot, surtout en été lorsqu'une ligne blanche réunit l'œil et la base du culmen et que le bec est traversé par une bande blanche près du bout. Le bec du jeune oiseau d'hiver est moins caractéristique, mais peut tout de même se distinguer de celui des guillemots.

Indications sur le terrain. Bec plus profond et, quand il nage, sa queue redressée en éventail est un admirable point de mire.

Nid. Semblable à celui des deux espèces précédentes, mais ceux-ci se réunissent moins par groupes.

Habitat. Fréquente nos rivages de l'Atlantique jusqu'au pôle. 
Genre-Plautus. Grand Pingouins.

33. Le Grand Pingouin. (GREAT AUK). Plautus impennis. L, 30. Le grand pingouin était le plus grand des plongeurs américains. Les ailes étaient de dimensions tellement diminuées que tout en pouvant servir de parfaites nageoires, elles étaient autrement fort inutiles, et laissaient l'oiseau incapable de voler. Comme cette espèce est aujourd'hui éteinte il est inutile de la décrire plus au long.

Cet oiseau s'était si bien fait à la vie aquatique que le vol ne lui était plus nécessaire, et c'est ainsi que ses ailes avaient fini par le réduire à des nageoires pareilles à celles des pingouins du pôle antarctique et qu'il ne lui était plus possible de voler. Bien qu'il pût vivre dans la mer aussi bien qu'un poisson ou un animal marin quelconque, la terre lui était tout aussi nécessaire qu'à tout autre oiseau en vue de sa reproduction. Mais même ainsi, s'il avait habité l'extrémité du pôle pour le temps de la couvaison, il aurait pu survivre; mais des rochers solitaires et écartés, des ílots le long des iles britanniques et du côté américain de l'Atlantique, étaient les lieux où il nichait, et il se trouvait être en même temps sur la route des bateaux de hardis pêcheurs qui dès les premiers temps de notre histoire se pressaient sur nos rivages. Pour ces pêcheurs les innombrables milliers de grands oiseaux habitant les llots dans le voisinage immédiat de ces pêcheries et si incapables de se protéger sur terre qu'on pouvait les tuer en masse avec des bâtons et des gourdins, constituaient un irrésistible butin. Les pêcheurs se prévalurent de ces occasions et l'on raconte que pour s'épargner la fatigue de leur métier, ces gens jetaient des planches entre leurs bateaux et le rivage, qu'ils y poussaient ces oiseaux sans défense et les assommaient sur le pont. Il est clair que nulle quantité ne pouvait résister à de semblables massacres, et aujourd'hui le grand pingouin n'est plus qu'un intéressant souvenir et n'a pour le représenter que des spécimens et des restes dans quelques muséums particulièrement favorisés.

\section{Sous-famille-Allina. Mergules. \\ Genre-Alle. Mergule.}

34. Le Mergule nain. (DOVEK1E). Alle alle. L, 8. C'est un très petit plongeur, le plus petit de sa famille dans l'est du Canada. Quand on le trouve sur nos eaux il est généralement noir en dessus et blanc à la gorge, aux joues et en dessous. Canada.

Traits distinctifs. Sa taille suffit à le distinguer de tout autre plongeur dans l'est du

Indications sur le terrain. Sa taille et ses battements d'ailes très rapides constituent les meillcures indications sur le terrain.

Nid. Sur les bords des falaises dans l'extrême nord.

Habitat. En été dans l'extrême nord de la région polaire orientale; en hiver le long des rivages maritimes.

Sur les rivages de notre côte et dans nos ports ce petit oiseau ne doit se chercher qu'en hiver. Il vole avec un très rapide mouvement de l'aile.

\section{Ordre-Longipennes. Palmipèdes longipennes.}

Description générale. Les palmipèdes à longues ailes sont des oiseaux de mer, avec quatre doigts et deux membranes, avec les ailes plus longues que la queue, si l'on excepte la plume de la queue médiane extrêmement allongée chez quelques labbes et les queues d'aronde extérieures, également allongées, chez quelques sternes.

Traits distinctifs. On peut les considérer comme un ordre en raison de leurs longues ailes et de leur bec (figures $3,4,5, \mathrm{p} .20$ ) et ils se distinguent des nageurs à narines tubulaires par la place des narines qui sont sur les côtés du bec et non dans un tube au sommet (voir figure 11, p. 21, comme comparaison). 
Indications sur le terrain. On n'en peut donner aucune pour l'ordre entier, sauf la longueur de l'aile et la façon de voler.

Nid. Couvent d'habitude sur le sol ou sur le bord des falaises, mais il y a peu d'uniformité quant à leurs habitudes à cet égard. Canada.

Habitat. Quelques especes sont plus ou moins communes le long des cours d'eau du

Les palmipèdes à longues ailes sont d'admirables voiliers, ne se fatiguant jamais et déployant une agilité extrême dans l'air. Par habitude ils sont pêcheurs, mangeurs de détritus ou pirates. Il n'y a que deux familles de cet ordre au Canada: les labbes et les mouettes, ces dernières comprenant leurs proches parents les sternes ou hirondelles de mer.

Valeur économique. Etant des oiseaux de mer, le dommage qu'ils font est peu de chose, et quelques-uns sont mệme utiles a l'homme.

\section{FAMILLE-STERCORARIIDA. LABBES ET STERCORAIRES.}

Description générale. Les labbes sont des oiseaux de proie sur mer. Comme couleur ils sont brun foncé, et blancs. La famille fait voir un singulier dichromatisme et toutes les especes du Canada apparaissent avec l'une ou l'autre coloration, soit un brun également foncé, soit un brun foncé ou ardoisé avec la tête, le cou et les dessous blancs ou clairs et un bonnet presque noir.

Traits distinclifs. Les becs des labbes (voir figure 3, p. 20) sont caractéristiques, car il y a un ongle bien indiqué à la pointe qui forme un crochet très marqué, clairement séparable du reste de la cire a la base du bec. Ce caractère les distingue facilement des mouettes, tandis que la présence des narines et de deux au lieu de trois membranes aux doigts (figure 6, p. 20) les distingue des cormorans dont les becs sont semblables quant au profil. Le fait que les narines ne sont pas tubulaires (figure 11, p. 21) les différencie d'avec les pétrels auxquels ils ressemblent par ailleurs.

Indications sur le terrain. Les labbes ont une coloration noire sur le dessus, une bande claire très visible à travers le dessous de l'aile près de la pointe, et ressemblent au faucon quand ils volent. Des trois espèces, deux, à l'état adulte, montrent des plumes de la queue si allongées qu'elles les font bien reconnaitre.

Nid. Sur le sol, dans l'herbe.

Le fait des deux phases de coloration et celui des plumages intermédiaires si nombreux rendent l'identification de quelques-uns des labbes assez difficile. Les labbes sont les pirates de l'air; ils font la chasse aux oiseaux pêcheurs qui tiennent une proie et les forcent à rendre gorge s'ils l'ont avalée; les œufs et les oisillons dans le nid ne sont jamais en sâreté.

Trois labbes se rencontrent dans l'est du Canada, ainsi qu'un stercoraire-ce dernier trop rarement et accidentellement, sauf au large de la côte extérieure de Terreneuve, pour qu'il en soit fait mention autrement.

Valeur économique. Les labbes ne sont pas très nombreux, et sauf dans les localités très éloignées et sauvages, où leur nombre leur donne une importance locale, leur influence économique est peu de chose.

\section{Genre-Stercorarius. Labbes.}

36. Le Labbe pomarin. (POMARINE JAEGER). Stercorarius pomarinus. L, 22. (Queue, 9.25, les plumes du centre se projetant au delà des extérieures de $4 \cdot 25$ ). C'est la le plus grand des labbes. Il ressemble tellement aux deux autres espèces par la coloration qu'il est difficile de les en distinguer excepté par la taille et à l'état adulte. L'espèce se présente en deux phases de coloration et dans tous les états intermédiaires.

Traits distinctifs. Les plumes allongées du milieu de la queue de l'oiseau adulte sont larges et tordues a la pointe de façon a se trouver dans un plan vertical au lieu d'horizontal.

Indications sur le terrain. Les plumes larges, allongés et tordues de la queue sont probablement les meilleures de ces indications.

Nid. Sur le sol, dans l'herbe. 
Habitat. Couve dans les îles et dans l'intérieur des terres au pôle arctique à travers le continent. Se rencontre sur les côtes maritimes plus au sud, seulement en qualité d'émigrant.

37. Le Labbe parasite. (PARAsitic JAEGER). Stercorarius parasiticus. L, 17. (Queue, $8 \cdot 25$, les plumes médianes dépassent les plumes extérieures de $3 \cdot 25$ ). Cette espèce se présente avec deux phases de coloration, une claire et une foncée. Dans la phase foncée, la couleur générale est brun foncé un peu plus claire au-dessous avec bonnet noir. Dans la phase claire les parties de dessous, la poitrine, le cou et la face sont blancs, avec bonnet noir. Des phases intermédiaires de coloration se rencontrent également.

Traits distinctifs. La taille plus petite de cet oiseau devrait le distinguer du précédent dans tous les plumages. A l'état adulte les plumes médianes de la queue dépassent seulement les autres de 3 pouces au lieu de 7 comme dans l'espece suivante et sont minces au lieu de larges et tordues comme dans la dernière espèce. Les oiseaux non développés de l'espèce ì longue-queue, n'ayant pas les longues plumes de la queue sont presque tout à fait semblables à cette espece-ci et peuvent se bien distinguer par la couleur des tuyaux des plumes primaires. Dans le labbe parasite les tuyaux des trois premières plumes sont blanes et les autres de plus en plus foncés à mesure qu'ils se succèdent l'un à l'autre dans l'aile. Dans le labbe à longue queue la couleur des tuyaux devient subitement plus foncée après la troisième primaire. Cependant cette marque de distinction n'est pas infaillible et il se rencontre quelquefois des sujets très difficile à identifier.

Indications sur le terrain. Faible projection des plumes médianes de la queue, comme plus haut, et n'étant pas tordues elles offrent une indication très sare pour les oiseaux adultes.

Nid. Sur le sol, dans les bruyères et les marécages du nord.

Habitat. Couve vers le pôle à travers le continent; est beaucoup plus commun sur la côte, dans ses migrations, que dans l'intérieur des terres où on le trouve très rarement quoique peut-être comme un oiseau vagabond.

Ce labbe est le seul de son espèce qu'on puisse rencontrer dans l'intérieur sur les grands lacs. D'autres espèces ont été signalées, mais à l'examen, sauf dans un seul cas, il s'est trouvé que c'étaient de jeunes oiseaux faussement identifiés, généralement à cause de l'absence de la queue allongée.

38. Le Labbe à longue queue. (LONG-TAILED JAEGER). Stercorarius longicaudus. L, 21. (Queue $13 \cdot 25$, la plume du centre dépasse les plumes extérieures de 8). Très sembiable au précédent par la coloration, mais les plumes longues et fines du milieu de la queue dépassent les autres de 7 à 8 pouces au lieu de 3 .

Traits distinctifs. Le labbe à longue queue, quoique ayant presque les dimensions du labbe pomarin, parce que la très longue queue y est comprise, est cependant un bien plus petit oiseau que l'une ou l'autre des deux autres espèces. C'est sa taille qui devrait le faire reconnaitre. Les jeunes individus sont souvent confondus avec le labbe parasite, mais les caractères de l'aile indiqués pour cette espèce servent à les faire reconnaître.

Indications sur le terrain. On ne peut bien reconnaitre que le labbe adulte, à l'état vif, les longues plumes du milieu de la queue étant le meilleur guide.

Nid. Semblable à celui des autres labbes.

Habitat. Couve dans les régions polaires de l'Europe, de l'Asie et de l'Amérique. Ne se voit que rarement sur la côte de l'Est.

Bien que le labbe à longue queue ait été signalé sur les grands lacs, dans la plupart des cas on a pris pour des labbes les jeunes individus de l'espèce précédente.

\section{FAMILLE-LARIDE. GOELANDS ET STERNES.}

Description générale. Les goélands et les sternes sont des palmipèdes à longues ailes, faciles à distinguer des labbes par la forme et la construction du bec qui montre une seule surface continue sans parties distinctes ni jointures (figures 4 et 5, p. 20). Les couleurs de l'adulte sont ordinairement un blanc pur, avec livrées blanches, gris-perle ou noires, souvent avec des bouts d'ailes noirs et des taches au collet ou à la jone.

Traits distinctifs. Le bec à surface continue avec un angle aigu projetê sur la mandibule inférieure suffit à reconnaître cette famille sous tous les plumages. Les couleurs sont très caractéristiques, quoique certaines espèces pendant leur jeunesse sont complètement foncées, se rapprochant ainsi des phases foncées des labbes. 
Indications sur le terrain. La coloration et le vol très caractéristiques sont les meilleures indications.

Nid. Les mouettes nichent généralement sur le sol, sur les bords rocheux ou les rivages plats, dans des lieux sablonneux, herbeux ou marécageux; rarement sur les arbres.

Habitat. Les mouettes et les sternes sont répandues partout, ordinairement près de vastes étendues d'eau, mais quelquefois au loin dans l'intérieur des terres, comme par exemple dans nos régions des prairies.

\section{Sous-famille-Larinæ. Goélands et Mouettes.}

Description générale. Muni de cette description de leur famille on ne pourra confondre les mouettes qu'avec les sternes (voir p. 57).

Traits distinctifs. Elles peuvent être distinguées, soit des labbes par les caractères du bec et de la coloration; (voir la description de la famille a la page précédente) soit des sternes par leur charpente plus robuste et leur façon de voler. Les becs sont plus gros et plus forts (comparez figures 4 et 5, p. 20). Règle générale, les queues sont carrées sans être fourchues visiblement. Quoique aussi bons voiliers que les sternes, ils ont moins d'agilité et accomplissent moins d'évolutions aériennes. Les jeunes mouettes sont souvent tout à fait brunes et de couleurs foncées, en contraste frappant avec l'individu adulte. Les jeunes sternes sont ordinairement de coloration claire.

Indications sur le terrain. Ce sont de gros oiseaux d'un vol plus lourd que celui des sternes. Queue carrée; ont l'habitude de prendre leur nourriture à la surface de l'eau; ne plongent pas avec les ailes, et portent leur bec horizontalement quand elles volent (voir sternes, p. 57). Ce sont la des indications qui serviront a distinguer les mouettes des sternes leurs proches parents.

Nid. Sur les bords rocheux ou les falaises près de l'eau en diverses localites; cela dépend de l'espèce et de l'endroit.

Habitat. Elles sont presque cosmopolites et il y a peu de contrées sans des individus de cette sous-famille. Au Canada on rencontre environ vingt-cinq espèces, mais quelquesunes ne viennent que par hasard en dedans des limites des régions colonisées, et il n'en est pas question ici.

Quoique les mouettes soient essentiellement des oiseaux de mer on les trouve quelquefois à de grandes distances des vastes étendues d'eau, et des compagnies entières suivent fréquemment le laboureur pour chercher des insectes, dans les sillons fraichement ouverts. Elles trouvent leur nourriture à la surface de l'eau, ne plongent jamais, ou glanent sur les plages, dans les baies ou dans les champs.

Valeur économique. Les mouettes mangent de tout en fait de matières animales, du poisson, des crustacés, des mollusques, des insectes, des déchets, et, quand l'occasion s'en présente, des petits oiseaux et des souris.

La quantité de poissons qu'elles consomment est relativement peu importante, car d'habitude c'est seulement les petites espèces très abondantes se rencontrant en grandes compagnies, qui attirent leur attention; elles ne sont pas de patients pêcheurs comme les hérons. Elles recherchent les plages de marée basse pour les mollusques, faisant preuve de beaucoup de savoir-faire pour casser les dures coquilles et en extraire le contenu. La nourriture que fournissent ces ressources n'a presque aucune valeur marchande. Les mouettes débarrassent la contrée chaque année d'une immense quantité de déchets et d'immondices jetés dans les ports et les cours d'eau; elle fréquentent les terres cultivées pour attraper les insectes et quelques espèces ont servi à arrêter le fléau d'une invasion de souris ou d'autres petits mammifères et de sauterelles. Les mouettes, par conséquent, ont leur utilité et doivent être protégées, lors même que quelquefois elles détruisent des œufs et de jeunes oiseaux.

\section{Genre-Rissa. Mouettes d trois doigts.}

40. La Mouette a trois dolgts.(KITTIWAKE). Rissa tridactyla. L, 16. C'est une mouette petite ou de taille moyenne, de la même coloration que le goéland argenté (Voir p. 55). 
Traits distinctifs. La condition rudimentaire ou l'absence presque totale du pouce est toujours une façon de reconnaitre cet oiseau.

Indications sur le terrain. Elle ressemble d tant d'autres mouettes par la coloration générale qu'on ne peut l'en distinguer à l'état vivant que par une observation attentive des plus petits caractêres et cela dans les conditions les plus favorables. La taille est plus petite que celle du goéland de Delaware, et plus grande que celle du goéland Bonaparte.

Elle diffère du goéland argenté et du goéland de Delaware en ce qu'elle a des pattes noires, au lieu de couleur chair ou jaunâtre, et qu'elle manque de petites taches terminales blanehes sur les extrémités noires des primaires. Le bec est d'un jaune toujours égal chez l'adulte, sans la tache rouge du goéland argenté ou la bande noire transversale $\mathrm{du}$ goéland de Delaware. Les jeunes individus ressemblent beaucoup au goéland de Bonaparte par le fait qu'ils ont un bec noir et le même modèle de coloration, mais le bord de devant de l'aile étendue est surtout noir au lieu d'être d'un blane très marqué. Les jeunes de cette espèce ne passent pas par une phase brune, comme font les goélands argentés, mais ils ressemblent a l'individu adulte par une coloration modifiée quoique semblable.

Nid. Elles font un nid solide et bien construit d'algues marines sur les saillies des falaises à pic.

Habilat. Une espèce marine qui est la mouette à trois doigts de l'Atlantique, ne se rencontre que par hasard sur les eaux douces ou à l'intérieur des terres. Elle habite les deux côtés de l'Atlantique; niche, en Amérique, depuis le golfe Saint-Laurent jusqu'au nord, dans les régions polaires. On ne la signalera sur les grands lacs que sur des preuves absolument indéniables.

SOUS-ESPECES. Cette mouette se rencontre sous forme de sous-espèce dans le nord des deux océans Atlantique et Pacifique. Notre spécimen de l'Est, la mouette à trois doigts de l'Atlantique, est le type et se rencontre en Europe et en Amérique.

Valeur économique. La nourriture de cette mouette est probablement semblable à celle des autres mouettes marines, mais sa taille moindre fait qu'on lui est moins hostile qu'à quelques autres membres de sa famille.

\section{Genre-Larus. Goélands.}

42. Le Goéland a manteau glauque. (aLAvcous gULL). Larus hyperboreus. L, 28. Ce goéland est une des plus grosses mouettes. Chez l'individu adulte, la livrée n'est que légèrement teintée de gris; les plus jeunes spécimens sont presque absolument blancs. C'est le plus grand des goélands aux ailes blanches (ceux qui n'ont pas de noir sur la pointe des ailes). Les oiseaux de l'année n'ont que de légères barres d'un gris cendré ou brunâtre.

Traits distinctifs. Sa coloration presque blanche le distingue du goéland à manteau noir auquel il ressemble quant à la taille.

Indications sur le terrain. La taille, la coloration presque blanche, et l'absence de la pointe noire de l'aile constituent les meilleurs signes pour les reconnałtre à l'état vivant.

Nid. Sur le sol, dans l'extrême nord.

Habitat. Couve le long des côtes circompolaires et des fles des deux hémisphères. Se voit sur nos côtes de l'Atlantique en automne et en hiver alors que quelques individus errent dans l'intérieur jusqu'au lac Ontario et peut-être jusqu'au lac Erié.

Valeur économique. Comme il n'est qu'un hôte d'hiver sur nos côtes maritimes et un hôte d'occasion sur les grands lacs, cet oiseau n'a aucune importance économique.

43. Le Goéland à ailes blanches. (ICELAND GULL). Larus leucopterus. L, 24. Ce goéland est environ de la même taille que le goéland argenté, mais avec un manteau gris très clair ou blane, et les extrémités de l'aile blanches-forme plus petite du goéland à manteau glauque.

Traits distinctifs. La taille et les extrémités des ailes blanches au lieu de noires.

Indications sur les terrain. Taille; extrómités des ailes; coloration blanche en général.

Nid. Sur le sol.

Habitat. Il couve dans les régions polaires de l'Amérique orientale et de l'Europe occidentale. Visite notre côte de l'Atlantique en hiver seulement; vagabonde rarement a l'intérieur jusqu'au lac Ontario. 
Valeur économique. Comme ce n'est qu'un hôte de l'hiver sur nos rivages maritimes et qui ne parait que par occasion sur les grands lacs, il n'a que peu ou peut-être même point du tout d'importance économique.

47. Le Goéland a manteau noir. LE GRos ooflaNd. (GREAT BLACK-BACKED GOLL). Larus marinus. L, 29. Un très gros goéland, l'individu adulte avec une livrée tout à fait noire.

Traits distinctifs. Forte taille et dos noir sont les traits caractéristiques de l'individu adulte. Les jeunes sont bruns au lieu de cendrés ou blancs, comme dans le cas du goéland a manteau glauque, avec plus de raies sur le dos et coloration moins égale que celle du goéland argenté.

Indications sur le terrain. Une forte taille et un dos noir sont de bonnes indications dans le cas de l'adulte. La taille est la seule indication qu'on puisse signaler pour les jeunes à l'état vivant.

Nid. Sur le sol, nid fait d'algues marines ou de fragments végétaux.

Habitat. Couve sur la cote de l'Atlantique depuis la Nouvelle-Ecosse jusqu'au póle nord. Par suite des progrès de la colonisation, les endroits favorables à la couvaison sont restreints aux localités plus isolées. C'est un hôte d'hiver plus ou moins régulier quoique plutót rare, du lac Ontario, mais qui ne s'avance que rarement dans l'intérieur le long des grands lacs.

Valeur économique. Avec les mêmes habitudes dans son régime alimentaire que les autres goélands, la plus grande taille, la force et le nombre des goélands à manteau noir, augmentent sa puissance à faire du bien ou du mal. On ne peut certes pas se fier à cette espèce dans le voisinage des nids sans défense ou de jeunes oiscaux d'autres espèces, car les oufs et les oisillons sont avidement dévorés quand l'occasion s'en présente. Par contre, l'occasion pour ce goéland de devenir un fléau important n'est pas grande, sauf dans quelques localités, car sur cette côte du Labrador où le goéland à manteau noir couve, il se trouve une grande quantité d'eiders et d'autres oiseaux de mer. Le dommage qu'il cause sur la côte du Labrador est petit comparé aux dégâts occasionnés par le gaspillage considérable des pêcheurs. II A.

51. Le Goéland argenté. (herring GULL). Larus argentatus. L, 24. Planche

Traits distinctifs. Sa taille et sa coloration générale suffisent en général à distinguer le goéland argenté de toutes les autres espèces canadiennes, mais comme la taille seule n'est que rarement un critère assez digne de foi quand les espèces que l'on contraste ne s'offrent pas ensemble pour être comparées, les points suivants aideront à une détermination précise.

Le goéland argenté tout jeune est le plus foncé de la famille car il se rapproche de très près des labbes de phase sombre à cet égard, mais la forme du bec et les habitudes du goéland préviendront toute confusion avec le labbe. Chez l'individu adulte les pointes noires de l'aile servent à le distinguer de l'un quelconque des goélands à ailes blanches, et les petites taches blanches terminales le distinguent de la mouette à trois doigts. Mais l'absence d'une raie transversale sur le bec le distingue du goéland de Delaware. A l'état adulte, toutefois, le jeune goéland argenté, à un de ses stages de développement, quand la couleur du bec tourne du noir au jaune de la maturité, fait voir une marque très semblable et la comparaison des tailles est la seule qui les fasse distinguer l'un de l'autre.

Indications sur le terrain. La taille et les marques de coloration susdites sont les meilleurs guides sur le terrain. Il est souvent très difficile, parfois impossible, de distinguer le goéland argenté du goéland de Delaware, à l'état vivant, quand des oiseaux encore jeunes de l'une ou l'autre espèce sont vus séparément.

Nid. Sur le sol, ou sur les saillies rocheuses, ou encore sur le sommet plat de grands rochers isolés, dans les nids faits d'algues marines ou de matières végétales de rebut.

Habitat. Se rencontre à peu près partout au Canada; il est surtout commun dans le voisinage immédiat des grandes étendues d'eaux douces ou salées. Couve soit en tous lieux soit dans certaines localités, sauf dans les régions plus méridionales du Canada.

Le goéland argenté est le plus commun de nos goélands. C'est un oiseau migrateur qu'on voit souvent sur le plus petit de nos lacs, même à 
de longues distances de son lieu de couvaison. Les goélands qui séjournent pendant l'été sur les grands lacs inférieurs sont des oiseaux non reproducteurs. En hiver l'espèce demeure sur les eaux plus étendues jusqu'à ce qu'elles soient gelées d'un bout à l'autre et quelquefois, y séjourne tout l'hiver, volant sur les eaux libres ou perchés sur la glace flottante. Les goélands fréquentent les ports et se réunissent en grand nombre vers l'entrée des égouts pour recueillir les déchets. Ils ont aussi appris que les vaisseaux fournissent des résidus de bon goat. Les stations de pêche ont beaucoup d'attraits pour eux et on est presque sûr de voir une grande multitude de ces oiseaux autour des tables qu'on nettoie sur le rivage. Sur la plage les mollusques sont avidement recherchés à la marée basse et les goélands ont appris le truc de porter leur proie à carapace dans l'air pour la laisser retomber sur les rochers, après quoi ils redescendent et retirent la savoureuse matière de sa boíte brisée en morceaux. Par le très mauvais temps ils se réunissent en grand nombre autour des vagues écumantes qui se brisent aux places tempétueuses pour y saisir la nourriture qui est rejetée à la surface de l'eau. Quelquefois ils suivent les immenses compagnies de petits poissons qui visitent périodiquement nos rivages et prélèvement un impôt sur cette inépuisable provision.

Valeur économique.-On verra d'après le résumé qui précède touchant les habitudes des goélands argentés, que comme mangeurs de déchets ils ont leur importance et doivent être protégés.

54. Le Goéland de Delaware. la mauve. (RING-Billed gull). Larus delawarensis. L, 18.50. Comme le goéland argenté (voir p. 55) mais plus petit; l'individu adulte a le bec traversé, près de la pointe, d'une barre ou d'un cercle.

Traits distinctifs. Sauf quand il est adulte et que le cercle sur le bec est visible, la taille est le meilleur guide pour distinguer cette espèce de celle du goéland argenté, mais le jeune goéland de Delaware n'est jamais aussi foncé que cette espèce dans sa premièrc année. Sur le bord de la mer cette espèce peut être confondue avec la mouette à trois doigts ordinaire qui est plus petite. La présence de taches blanches terminales sur les pointes noires de l'aile sert à distinguer le goéland de Delaware. Dans la jeunesse, la coloration brune du goéland de Delaware contraste avec la coloration plus claire de la jeune mouette à trois doigts. Ce goéland manque de la bande terminale de la queue si caractéristique de ces oiseaux et a les pattes et les jambes couleur chair au lieu de noires. L'absence de la bande effilée, étroite et noire de la queue, de la tache foncée de la joue, et la grande différence dans la taille devraient suffire à distinguer cet oiseau du goéland de Bonaparte dans le plumage jeune ou d'hiver.

Indications sur le terrain. Une étude attentive des caractères susmentionnés fera connaitre les seules indications connues de l'auteur.

Nid. Sur le sol, dans un nid fait d'herbes ou de matières végétales.

Habitat. Se trouve presque partout au Canada. Couve par endroits dans les régions qu'il fréquente au Canada, sauf dans les parties les plus méridionales du Dominion, dans la région inférieure des grands lacs.

Valeur économique.-Te régime d'alimentation du goéland de Delaware est le même, d'une façon générale, que celui du goéland argenté, mais il a moins d'influence à cause de sa plus petite taille.

60. Le Goéland de Bonaparte. (Bonaparte's GULL). Larus philadelphia, L, 14. C'est le plus petit de nos goćlands de l'Est. Sa coloration générale ressemble à celle du goéland argenté ou du goéland de Delaware, mais l'individu adulte a un capuchon noir sur la tête et le cou. Les oiseaux jeunes et d'hiver n'ont pas le capuchon, mais ont à l'oreille une tache foncée ou voilée ou peu distincte.

Traits distinctifs. Le capuchon noir sert à distinguer ce goéland-là de toutes les espèces de l'est dans leur plumage d'été. Dans l'ouest de l'Ontario on rencontre parfois le goéland de Franklin. C'est un oiseau légèrement plus grand avec un bec rouge prononcé, et ses pattes presque noires au lieu d'avoir le bec noir, dos pattes d'un rouge corail, et les pointes des primaires en vue sont presque toutes noires au lieu de blanches avec de 
petites pointes noires. Les jeunes oiseaux peuvent se reconnaître à leur tache noire à l'oreille. Ils se distinguent de la mouette à trois doigts, à laquelle ils ressemblent un peu à cause de la partie antérieure de son aile étendue, soit par la bordure noire postérieure de son aile-comme une bordure de deuil-soit par la bande terminale foncée de la queue. Le goéland de Bonaparte peut quelquefois être confondu avec d'autres sternes, mais sa charpente et son action plus lourdes, et l'absence de la moindre apparence d'une queue fourchue devraient rendre la differenciation relativement facile.

La mouette rieuse qui se rencontre parfois sur nos côtes maritimes du sud ressemble beaucoup à cette espèce, mais les plumes extérieures des ailes sont noires au lieu d'être presque toutes blanches, et elle élève des petits et n'émigre pas en hiver. Mais elle est si rare qu'il faut beaucoup de précautions avant de garantir son identité.

Indications sur le terrain. La taille, le capuchon noir chez les adultes d'été, le blanc sur le bord antérieur des ailes, la couleur du bec et des pattes constituent les meilleurs caractères pour reconnaitre cet oiseau à l'état vivant.

Nid. Sur des trones d'arbre, des buissons ou des arbres, quelquefois à 20 pieds audessus du sol.

Habitat. Les endroits où il couve ne sont pas très bien connus, mais il a été trouvé qu'il nichait dans l'Alaska et le Nord-Ouest. Cet oiseau se rencontre en hiver ou pendant leur migration partout au Canada.

Ce petit goéland est plus souvent aperçu comme troupe de passage ou de visite que les autres espèces. Il fréquente aussi plus que ces espèces, les marécages et les terrains inondés, et se voit rarement comme un oiseau solitaire. Il manifeste des goûts de sociabilité et les troupes semblent être maintenues ensemble plus par leurs dispositions sociables que par cette communauté d'intérêts qui prédomine chez les autres espèces de goéland.

\section{Sous-famille-Sterninæ. Sternes.}

Description générale. Les sternes ou hirondelles de mer, comme on les appelle quelquefois, sont petites de charpente et de tenue plus légères et plus gracieuses que les goélands.

Traits distinctifs. Le bec plus léger et plus effilé que celui des goélands (voir le bec, fig. 5, p. 20), et la queue fourchue sont caractéristiques des sternes et les distingueront habituellement sans difficulté. Toutes les espèces canadiennes ont une queue plus ou moins fourchue et toutes, à une exception près (la sterne noire), ont dans leur plumage adulte d'été, un capuchon noir très nettement défini. La forme fourchue de la queue chez les jeunes oiseaux, bien que commençant à se montrer de bonne heure dans leur développement, n'atteint son maximum qu'au moment du départ pour la saison d'hiver; de la vient que pendant l'été et l'automne beaucoup d'individus auront des fourches beaucoup plus petites que ne l'indiquent les mesurages.

Indications sur le terrain. La plus grande légèreté dans l'action de l'aile et une évolution aérienne constante et rapide; le fait que les sternes plongent du haut de l'air, et l'habitude de tourner le bec droit en bas du côté de l'eau au lieu de le tenir parallèlement au corps sont des faits caractéristiques. Les queues fourchues et les capuchons noirs sont aussi de bons signes pour reconnaitre les individus adultes.

Nid. Tandis que les goélands semblent préférer les rivages rocheux pour y nicher, les sternes, sauf celle de Forster et la sterne noire, préfèrent les plages sablonneuses, déposant leurs ceufs dans un cercle de cailloux sans autre préparation quelconque.

Habitat. Comme formant une sous-famille, les sternes fréquentent le sud plus que ne le font les goélands, bien qu'une espèce, au moins, la sterne arctique, ait été trouvée aussi loin, au nord, que se soit aventuré l'homme. Toutes nos espèces émigrent, aucune ne restant dans l'est du Canada pendant l'hiver.

Il suffit d'observer une troupe de sternes en train de se nourrir pour reconnaitre la justesse du surnom que le populaire leur a donné d'hirondelles de mer. La grâce de leurs mouvements, leurs couleurs délicates comme celles des perles, font la joie de qui aime la nature. Les sternes ont, à tout prendre, moins d'habitudes marines que les goélands et ne s'éloignent pas autant qu'eux de la terre. Elles fréquentent les ports, les rivages, les plages et se nourrissent surtout du fretin qu'elles attrapent près de la surface, par des plongeons rapides et subits du haut de l'air, 
mais elles ne ramassent pas les déchets. Dans ces plongeons les oiseaux piquent une tête et font jaillir une écume blanche dans laquelle ils disparaissent un instant, mais ils ne vont jamais loin sous l'eau comme font les fous de Bassan et les cormorans.

\section{Genre-Sterna. Sternes.}

64. La Sterne caspienne. Le GRAND esterlette. (CASPIAN tern). Sterna caspia. L, 21. (Fourchure de la queue, 1.5).1 La plus grande de nos sternes canadiennes. Très semblable, quant à la couleur, à la sterne commune (voir p. 59).

Traits distinctifs. La forte taille de cette sterne est caractéristique. Il $\mathrm{y}$ a deux autres espèces de même taille, mais elles sont trop rares pour qu'on fasse plus que de les mentionner en passant. La sterne caspienne est anssi grande que quelques-uns des plus petits goélands; mais son bec quoique relativement gros pour une sterne est trop gracieux et pointu pour ressembler à celui du goéland.

Indications sur le terrain. Coloration caractéristique de la sterne, sa tenne et sa taille sont les meilleures indications de ce genre. C'est une grande sterne commune avec une queue moins profondement fourchée. Sa voix est tout à fait différente de celle des espèces plus petites, etant plus forte et moins rauque.

Nid. Sur les plages sablonneuses, sur les îles rocheuses ou à gravier.

Habitat. C'est une espèce presque cosmopolite. Trouvée dans l'ancien comme dans le nonvean monde. Se rencontre assez irrégulièrement dans tout le Canada, mais les localités où elle couve sont rares et disséminées à travers le continent depuis le lac Winnipeg jusqu'au Labrador.

L'habitat singulièrement disséminé et irrégulier de cette mägnifique sterne fait croire que c'est une espèce qui disparaît peu à peu. C'est là un fait tout à fait regrettable puisque c'est un des plus beaux de nos oiseaux de mer, et il faut espérer qu'une surveillance attentive des localités où couve cette sterne sera entreprise de façon que l'oiseau continue à orner nos cours d'eau.

Valeur économique. Bien qu'elle se nourrisse surtout de poisson c'est surtout du fretin qu'elle fait sa proie. La rareté de cette espèce l'empêche de causer un dommage de quelque valeur.

69. La Sterne de Forster. (FORster's TERN). Sterna forsteri. L, 15. (Fourchure de la queue 4$)^{1}$. Cette sterne ressemble de très près à la sterne commune par sa taille et sa coloration. (Voir l'espèce suivante).

Traits distinctifs. La sterne de Forster n'a pas de ces teintes grises si délicates sur les flanes et les parties de dessous, qui caractérisent la sterne commune et les plumes extérieures de la queue ont les barbes foncées à l'intérieur et non à l'extérieur du tuyau.

Indications sur le terrain. Les parties de dessous, d'une blancheur parfaite, sont les indications les plus sûres sur le terrain. La voix est plus rauque et plus profonde que celle de la sterne commune et peut toujours se reconnaitre quand on l'a une fois entendue. L'espèce est si rare à l'est du lac Huron qu'il ne faudrait l'identifier à l'état vivant qu'avec des oiseaux qu'on aurait dans la main.

Nid. Sur de petits monticules, dans les marécages herbeux; le nid est fait de fraginents de végétation de rebut.

Habitat. Oiseau de l'ouest ou de l'intérieur. Autrefois il nichait sur la rivière Détroit, mais il semble avoir déserté cette station, et aujourd'bui il couve plus loin à l'onest.

Ces sternes fréquentent les marécages plutôt que les plages et ne se rassemblent pas en troupes comme la sterne commune. Il faudra les chercher comme individus à part dans des troupes d'oiseaux d'autres espèces.

Valeur économique. Bien que mangeurs de poissons, leur taille et leur habitat les empêchent de faire du mal.

\footnotetext{
${ }^{1}$ Ce meaurage montre la différence de longueur entre les plumes médianes et extérieures de la queue. Une grande partie de la longneur totale de ces oiseaux consiste en ce que les plumes extérieures de la queue sont extre mement allongée, et ces plumes là n'atteignent pas leur maximum chez les jeunea oiseaux. De là vient que nous
} donnong ce chiff re pour corriger le meaurage ordinaire qui est tress souvent extremement trompeur. 
70. La Sterne commune. (COMMon TERN). Sterna hirundo. L, 15. (Fourchure de la queue, $3 \cdot 0) .{ }^{1}$ Planche II $\mathrm{B}$.

Traits distinctifs. Les barbes foncées extérieures au lieu d'intérieures sur les plumes externes et longues de la queue, et la poitrine d'un gris délicat au lieu d'un blanc très pur, ainsi que le sont aussi les parties de dessous, distinguent la sterne commune de la sterne de Forster. Son cri très particulier est ausai facile à reconnas tre (voir l'espèce précédente). Le gris de la poitrine et des dessous est plutôt moins prononcé que dans la sterne arctique; les pattes et les pieds sont certainement plus forts et plus lourds. Le bec est d'habitude de couleur foncée vers la pointe, tandis que celui de la sterne arctique est uniformément rouge. Les jeunes ont une demi-teinte brune en dessus et un bec noirâtre. Chez les individus adultes en hiver le capuchon noir est remplacé par un collet plus ou moins rompu sur le derrière de la tête et la partie supérieure du cou. Avec ce plumage la sterne de Forster a une large raie noire en travers de l'œil.

Indications sur le terrain. Sauf en quelques localites où l'on sait que d'autres sternes se rencontrent, il est toujours bon d'en conclure que c'est là l'espèce qu'on a vue. Les parties de dessous, couleur gris-perle, peuvent quelquefois la distinguer de la sterne de Forster, mais son cri plus aigu la fera toujours reconnas tre; cependant il est difficile de la distinguer de la sterne arctique laquelle, du reste, ne doit pas étre cberchée ailleurs que sur la côte extrême de notre mer de l'Est.

Nid. Dans les creux des plages sablonneuses.

Habitat. Une espèce circompolaire qui voyage jusque dans l'Amérique du Sud et l'Afrique. Plus ou moins commune dans tout le Canada, et niche dans les localités favorables dans tout son habitat canadien.

C'est cette sterne qui abonde le plus dans tout l'est du Canada. Commune autour des rivages sablonneux, moins abondante sur les rivages rocheux, on peut la voir sur n'importe laquelle des grandes étendues d'eau salée ou douce, pendant l'été. La merveilleuse légèreté des ailes, les cercles gracieux qu'elle trace, un moment planant de ses ailes qui battent rapidement, et stationnaire dans l'air en fixant ses yeux sur la proie qu'elle convoite tout au-dessous d'elle, le moment d'après, fondant comme un poids lourd dans une eau écumante, tout cela charme constamment le spectateur qui recherche le beau. Son cri perçant, un peu dur, s'harmonise admirablement avec la houle des vagues et avec tout l'encadrement des effets maritimes.

Valeur économique. Quoique assez nombreuse dans les localités favorables, cette espèce l'est trop peu pour être sérieusement destructrice.

71. La Sterne arctique. (Anctic TERN). Sterna paradisca. L, 15.50. (Fourchure de la queue $4 \cdot 50) .1$ Ressemble de très près à la sterne commune (voir plus baut) par la taille et la couleur.

Traits distinctifs. La sterne arctique a, plus que la sterne commune, la couleur grise un peu plus foncée répandue sur la poitrine et les parties de dessous. Le bec est rouge à la pointe, les pieds et les pattes sont tres petits et faibles. L'étendue de son domaine la distingue de la sterne de Forster, et il n'y a que la sterne commune et la sterne roske avec lesquelles on puisse la confondre, mais cette dernière est trop rare dans les eaux canadiennes pour qu'il en soit question.

Indications sur le terrain. Il n'y a pas d'indication sur le terrain qu'on puisse donner au moyen de laquelle cette espèce puisse être reconnue avec certitude, mais la couleur uniformément rouge du bec est un signe recommandable. Dans l'est du Canada c'est une espèce de la côte maritime et il ne faudrait la mentionner comme vue réellement que là où l'on sait qu'elle se rencontre habituellement.

Nid. Dans les creux des plages sablonneuses.

Habitat. Presque cosmopolite, mais plutốt dans l'Est et les régions polaires du Canada; rarement, peut-étre jamais dans la région des grands lacs. Elle est remarquable en ce qu'elle dispose d'un champ de migration de la plus grande étendue parmi toutes les espèces d'oiseaux. Bien qu'elle vole en été jusque vers le pôle nord, elle hiverne au sud jusqu'au continent antaretique.

${ }^{2}$ Voir la note, page 58.

$28588-5$ 
Les remarques faites à propos de la sterne commune s'appliquent également dans le cas de la sterne arctique, si l'on se rappelle que l'habitat de cette espèce n'est exactement que dans l'Est et au pôle nord.

Valeur économique. La petite taille du fretin dont elle se nourrit, et l'abondance de ce poisson dans la mer fait de cette sterne, une espèce aussi inoffensive que toute autre sterne.

\section{Genre-Hydrochelidon. Sternes noires.}

77. La Sterne nolre. (BLACK TERN). Hydrochelidon nigra. L. 10, (Fourchure de la queue 0.8$)^{1}$. C'est la plus petite de nos sternes communes, le gris ardoisé foncé devenant un noir mat sur la tête, le cou et les parties de dessous.

Traits distinctifs. La description qui précède suffit à distinguer les adultes d'eté. Les oiseaux d'hiver et ceux non développés ont une face d'un blane sale, comme la gorge, le tour du cou et les parties de dessous, et le gris au-dessus est teinté plus ou moins de brun. Cet oiseau est cependant toujours beaucoup plus foncé que les plumages correspondants d'autres espèces. Ce fait et la petite taille de l'oiseau suffiraient à le différencier toujours des autres espèces.

Indications sur le terrain. La taille et la coloration font que cette espèce est facile à reconnałtre à l'etat vivant.

Nid. Sur de petits monticules tels que de vieux nids de rats musqués, ou les débris flottants dans les marécages; le nid est fait de matières végétales.

Habitat. La sterne noire américaine est un oiseau de l'intérieur qui niche depuis les grands lacs jusque vers les régions de l'Ouest.

SOUS-ESPECES. La sterne noire se rencontre à la fois en Europe et en Amérique sous des formes sous-spécifiques alliées desquelles celle d'Europe est le type. La sterne noire d'Amérique Chlidonias nigra surinamensis est la seule sous-espèce qui nous intéresse ici.

C'est un oiseau des marécages de l'intérieur. On le voit rarement sur les grandes étendues d'eau, mais dans l'intérieur de son domaine ordinaire aucune étendue d'eau marécageuse n'est sans cet oiseau. Ses habitudes générales ressemblent beaucoup à celles des autres sternes.

Valeur économique. La quantité d'insectes qui entrent dans la nourriture de cet oiseau est probablement plus grande que celle des autres sternes. Dans le Sud on sait qu'il mange les larves des charançons du cotonnier et suit le laboureur de l'ouest pour happer les vers retournés avec la terre. C'est pourquoi nous pouvons hasarder l'opinion que cette sterne est réellement utile. En tout cas le poisson qu'elle prend vit dans la fange et a peu d'importance économique.

\section{Ordre-Tubinares. Palmipèdes à narines tubulaires.}

Description générale. Infatigables voiliers sur les mers profondes, de tailles diverses depuis le grand albatros jusqu'au petit pétrel. Coloration ordinairement terne et uniforme.

Traits distinctifs. Les narines sont encaissées dans des tubes placés au sommert du bec meme (figure 11, p. 21).

Indications sur le terrain. Habitudes générales quant au vol et coloration uniforme. Il est nécessaire de se familiariser avec les diverses especes afin de pouvoir reconnaftre les membres de cet ordre.

Nid. Sur le sol ou dans les terriers de localités isolées; souvent sur des îlots rocheux au loin dans la mer vers lesquels ils trouvent leur route de quelque façon mystérieuse et inexplicable.

Habitat. Comme famille, ce sont des oiseaux de l'hémisphere sud, car c'est la qu'ils atteignent leur plus complet développement comme quantités d'individus et d'espèces. Toutefois quelques-uns habitent le nord jusqu'aux confins du pole.

Les palmipèdes à narines tubulaires sont essentiellement des oiseaux marins, ne touchant la terre ferme que pour la couvaison. L'océan est

\footnotetext{
JVoir la note au bas de la page 58.
} 
leur habitation et les déchets qu'il ballotte suffisent à tous leurs besoins sauf ceux de l'élève des petits. Ces oiseaux, par conséquent, considérés comme classe ne fréquentent pas les eaux basses et ne sont vus communément que par les marins des eaux profondes, les pêcheurs du large et les voyageurs transatlantiques. Il y a deux familles de cet ordre: les albatros, Diomedeida, et les fulmars, les puffins et les pétrels, Procellariida, qui sont appelés ici, pour la commodité, les Petits nageurs à narines tubulaires à cause de leur moindre taille. Comme il n'y a pas d'ailleurs d'albatros sur notre côte de l'Est nous n'avons affaire ici qu'aux Procellariida.

Valeur économique. Eu égard à leur habitat pélagique ils n'offrent que peu ou point du tout d'intérêt économique.

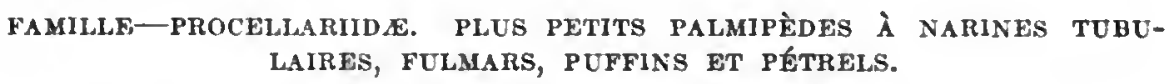

\section{Sous-famille-Fulmarinæ. Fulmars.}

Description générale. Voir la description de l'ordre qui précède.

Traits distinctifs. Les petits nageurs a narines tubulaires sont plus petits que les albatros et sont les seuls membres de cet ordre qu'on trouve ordinairement sur notre obte de l'Est.

Indications sur le terrain. Coloration générale et façons de voler. Ailes étendues avec roideur depuis le corps, puis de longs glissements tranquilles sur des ailes immobiles (fulmars et puffins) ou de petits coups d'ailes à leur surface d'un côté de la vague à l'autre. coté, les pieds quelquefois servant de rames comme si l'oiseau courait sur la surface (pétrels).

Habitat. Cette famille est distribuée sur les océans du globe d'un pole a l'autre. Bien que plusieurs espèces soient régulièrement limitées à l'hémisphère sud ce sont de grands voyageurs et la liste de ceux qui vagabondent sur nos côtes septentrionales eš relativement grande. Il y a beaucoup d'espèces sur le compte desquelles on sait peu de chose et nos connaissances touchant plusieurs d'entre elles se bornent a des spécimens ou uniques ou très peu nombreux qui sont tombés sous la main d'un collectionneur. D'autres espèces que celles indiquées ici peuvent se rencontrer sur nos cótes ou même par occasion sur les grands lacs, mais on ne peut les identifier qu'avec beaucoup de précaution.

Valeur économique. Bien que se nourrissant presque exclusivement de poisson et de déchets leur habitat sur les mers profondes leur enlève toute importance économique.

\section{Genre-Fulmarus. Fulmars.}

86. Le Fulmar. (Folune). Fulmarus glacialis. L, 19. Grand oiseau de la coloration du goéland (phase claire) ou uniformément foncée, gris ardoisé (phase noire) et narines tubulaires.

Traits distinctif8. C'est l'un des plus grands parmi les petits à narines tubulaires, uniformément gris ou blancs et coloration grise comme celle des goélands.

Indications sur le terrain. Façons de voler, ailes étendues avec roideur, longs ghissements, en outre la coloration claire ou grise au lieu de brun foncé comme chez les puffins qui se rapprochent des fulmars par la taille, devraient rendre ordinairement cette espece reconnaissable à l'état vivant.

Le fulmar se rencontre sous la forme sous-spécifique apparentée sur les deux océans Atlantique et Pacifique. Le fulmar de l'Atlantique présente la forme type.

$\mathrm{Nid}$. Niche en nombreuses troupes sur les bords des falaises rocheuses.

Habitat. Le fulmar est un oiseau du nord de l'Atlantique nichant sous les hautes latitudes et qui voyage vers nos côtes méridionales.

Plus fréquemment aperçu par les chasseurs de phoques et de baleines, dont le métier abandonne des déchets qui attirent beaucoup de fulmars. Ils sont aussi aperçus par les voyageurs transatlantiques en plein océan ou près des côtes.

Valeur économique. Sans importance économique. $28588-5 \frac{1}{2}$ 


\title{
Sous-famille-Puffininge. Puffins et Pétrels.
}

\author{
Genre-Puffinus. Puffins.
}

Description générale. Nageurs à narines tubulaires longs de 17 \& 20 pouces. Ies tubes des narines sont apparents, mais bien moins prononcés que chez le fulmar ou le pétrel.

Traits distinctifs. Les deux seules espèces de l'Est canadien que l'observateur ordinaire rencontrera probablement sont ou très foncées ou paraissent de loin presque d'un noir de corbeau.

Indications sur le terrain. Les ailes longues et étroites tenues très raides et normalement au corps; les longs glissements d'un demi-mille ou davantage sur des ailes immobiles, sont des indications caractéristiques. Leur couleur dans les tons bruns devraient les distinguer du fulmar qui est gris mais qui a passablement les mêmes façons de voler.

Nid. On sait très peu de choses des habitudes de couvaison des puffins. Il y en a beaucoup qui nichent dans l'hémisphère sud et qui émigrent vers le nord en été.

Habitat. Sont répandus ou errants sur la plupart des océans du globe. Les endroits ou ils nichent sont de petites îles solitaires presque toutes inconnues.

Les puffins sont d'admirables voiliers, affrontant les tempêtes et les bourrasques avec l'aisance et la grâce des hirondelles. L'observateur ordinaire ne les voit que rarement, à moins que ce ne soit du pont d'un transatlantique.

Valeur économique. Glanant à la surface de la haute mer, prenant le menu fretin ou les déchets que jettent les bateaux pêcheurs, les baleiniers surtout, l'influence économique de ces oiseaux est trop minime pour s'apprécier.

89. Le Grand Puffin. (Greater shenamater). Puffinus gravis. L, 20. Le plus grand de nos puffins ordinaires, plus clair en dessous, avec les plumes sous la queue gris cendré.

Traits distinctif8. La couleur claire des dessous devrait distinguer cet oiseau-ci du puffin fuligineux et sa couleur brune du dos au lieu de la couleur grise, sa coloration panachée le différencient du fulmar.

Indications sur le terrain. Ses façons de voler, ainsi qu'elles ont été décrites plus baut en même temps que sa coloration brune et les dessous blancs le distingueraient, à l'état vivant, soit du fulmar soit de l'espèce suivante.

Nid. On sait très peu, peut-être même rien, des habitudes de couvaison de cette espèce. Elle niche probablement dans l'hémisphère sud sur des îlots déserts qui ne sont pas connus au point de vue ornithologique.

Valeur économique. Sans importance économique.

95. Le Puffin fuliglneux. (sooty shearwater). Puffinus griseus. L, 17. Le plus petit de nos deux puffins les plus communs. Brun très foncé, presque noir, un peu plus clair en dessous.

Traits distinctifs. La taille, quand il est possible de l'estimer ou de la mesurer, et coloration générale foncée.

Indications sur le terrain. Les habitudes de son vol comme elles sont décrites au mot puffin et son aspect à l'état vivant, noir très foncé, presque noir de corbeau-telles sont probablement les meilleures indications.

Nid. Dans des creux du sol. en été.

Habitat. Sur les océans de l'hémisphère sud, ne voyage que rarement vers nos rivages

Les deux puffins et le pétrel de Wilson sont les seules espèces d'oiseaux canadiens dont on sait qu'elles nichent à l'extrémité australe et non boréale de leur champ de migration. Les spécimens qu'on voit ici en été sont des oiseaux migrateurs non de couvage.

Valeur économique. Sans importance économique. 


\section{Genres-Oceanodroma et Oceanites. Pétrels.}

Description générale. L'espèce canadienne de pétrels comprend de petits oiseaux, à peine aussi gros que les rouges-gorges et d'une coloration uniforme d'un brun foncé.

Traits distinctifs. Petite taille; coloration généralement d'un brun fuligineux, le croupion blane.

Indications sur le terrain. Taille, couleur comme ci-dessous et l'habitude de courir de haut en bas des vagues comme s'il marchait sur l'eau sont les indicateurs du pétrel.

L'origine du nom de pétrel vient de Pierre qui marcha sur les eaux de la mer de Galilée. Ces oiseaux sont aussi connus des marins et des voyageurs sous le nom de "Poulets de la mère Carey et leur présence est un présage de tempête, à ce qu'on dit. Malgré leur taille très petite on les rencontre très loin sur la mer et ils ne sont aperçus que rarement par le débardeur sauf dans le voisinage de leurs nids.

Valeur économique. Les pétrels, quoique se nourrissant de poisson, sont très petits et leur habitat est trop éloigné de la civilisation pour avoir une importance économique appréciable.

106. Le Pétrel de Leach. (LEACh's PETREL). Oceanodroma leucorhoa. I 8. Petit oiseau d'un brun fuligineux, un peu plus clair en dessous, avec un croupion blanc. Queue fourchue d'un demi-pouce de profondeur.

Traits distinctifs. Sa queue fourchue, sa coloration un peu plus claire, et ses pieds noirs le distingueront du pétrel de Wilson, le seul qu'on pourrait confondre avec lui.

Indications sur le terrain. La petite dimension de la fourche caudale est peut-étre le signe spécifique le plus sûr a l'état vivant.

Nid. Dans les creux du sol ou sous les pierres.

Habitat. Il habite les deux océans, le Pacifique et l'Atlantique du Nord, couvant sur le côté est du continent depuis le Groenland jusqu'à l'État du Maine.

C'est là le seul pétrel qui couve sur nos côtes. Il peut être très abondant par endroits dans la saison de couvaison et le sol peut être criblé de ses trous sans que l'on puisse même soupçonner sa présence. Il reste dans son trou pendant le jour, mais de nuit il en sort en grande quantité tournoyant dans le voisinage désert, faisant entendre un petit chant sourd, étrange, souvent répété et d'un effet surnaturel.

Valeur économique. Sans importance.

109. Le Pétrel de Wilson. (WILson's PETREL). Oceanites oceanicus. L, 7. Oiseau un peu plus petit que le pétrel de Leach, de la même couleur générale, mais avec une queue un peu plus foncée, moins fourchue, ct avec les palmures des doigts presque toujours jaunes au lieu de tout a fait noires.

Traits distinctifs. De plus petite taille; coloration un peu plus foncée; queue carrée au lieu de fourchue, et les palmures jaunes des doigts distingueront ce pétrel de celui de Leach. Le pétrel pélagique se rencontre aussi, dit-on, au large de la cóte de l'At lantique, mais il est trop rare pour étre décrit ici. Il faudrait une preuve irrécusable de sa prégence. On le reconnait parce que les plumes blanches du croupion ont les bouts noirs.

Indications sur le terrain. I a queue carrée au lieu de légèrement fourchue est le signe probablement le plus digne de confiance, pour identifier ce pétrel a l'état vivant; mais encore faut-il un examen très exact pour faire la distinction.

Nid. Dans des trous du sol ou des crevasses de rochers.

Habitat. Du pôle antarctique au Labrador, et jusqu'aux fles britanniques a travers l'A tlantique.

Le pétrel de Wilson est remarquable parce que c'est une des très rares espèces de l'Amérique du Nord que l'on sait nichant à l'extrémité sud de leur champ de migration. Aussi les individus que nous voyons ici en été sont migrateurs et ne nichent pas. 


\section{Ordre-Steganopodes. Palmipèdes totipalmés.}

Description générale. Oiseaux avec membranes entre les quatre doigts, faisant trois membranes au lieu des deux ordinaires comme dans d'autres (fig. 6, p. 20).

Traits distinctifs. Les caractères des pieds sont toujours des éléments servant à distinguer ces oiseaux.

Les totipalmés canadiens se divisent en trois familles: Sulido, les fous de Bassan; Phalacrocoracida, les cormorans; et Pelecanida, les pélicans. Il y a d'autres familles qu'on a rencontrées ou qu'on peut rencontrer par occasion au Canada, mais qui sont trop rares pour être mentionnées ici.

Valeur économique. Cet ordre-composé de gros oiseaux, se nourrissant tous de poissons et plusieurs fréquentant en grand nombre des eaux soit près des côtes soit à l'intérieur des terres-n'est pas à l'abri de quelque soupçon quant à ses effets économiques. Toutefois il ne faudrait prendre aucune mesure contre aucune espèce avant d'avoir trouvé, par une recherche soigneuse, que la chose fât nécelssaire.

\section{FAMILLE-SULIDA. FOUS OU FOUS DE BASSAN.}

Description générale. Gros oiseaux, la plupart blancs à l'âge adulte; bec aigu et droit sans crochet ni poche prononcée de la gorge ou du gosier.

Traits distinctifs. Les caracteres du bec dans cette famille servent à la distinguer de tous les oiseaux sauf de ceux des tropiques, dont une espèce a été importée de Terreneuve. Mais comme les oiseaux des tropiques ont les plumes médianes de la queue énormément allongées il y a peu de chances de confusion, alors même qu'ils ne seraient pas trop rares dans nos eaux pour mériter une description détaillée.

Les fous de Bassan sont essentiellement des oiseaux de mer qu'on ne voit jamais loin de la mer sauf comme vagabonds. La famille, a tout prendre, est tropicale, et il n'y en a qu'une seule espèce au Canada.

117. Le Fou de Bassan. Le marget. (Gannet). Sula bassana. L, 35. C'est un palmipède à membranes complètes avec un bec droit sans crochet prononcé ni poche remarquable de la gorge ou du gosier.

Traits distinctifs. Les caractères du bec sont distinctifs. L'individu adulte est tout a fait blanc à l'exception des primaires noires et d'une riche couleur crème répandue sur la couronne et le derrière du cou. Le jeune oiseau de l'année est brun grisatre, plus clair en dessous, et chaque plume a un petit $V$ blanc à la pointe qui répand une bigarrure sur le tout. Diverses phases intermédiaires tachetées se présentent parmi ces plumages

Indications sur le terrain. Un corps d'un blanc éblouissant et des bouts d'ailes toutes noires chez l'adulte sont des indications sûres. L'habitude de plonger au vol et de tomber dans l'eau la tête la première comme une flèche pour rester sous l'eau pendant un certain temps est également caractéristique. Les sternes plongent aussi au vol mais elles s'élèvent de l'eau presque immédiatement et rarement elles disparaissent de la vue comme les fous de Bassan. De plus, la grande différence de taille est un témoignage suffisant.

Nid. Niche en bandes nombreuses sur les étages rocheux des falaises maritimes; nids faits d'algues marines.

Habitat. Les deux côtés du nord de l'Atlantique nichant au Canada seulement sur le Bird Rock au large des îles de la Madeleine et sur l'île Bonaventure, comté de Gaspé (Québec), deux endroits situés dans le golfe du Saint-Laurent.

Le fou de Bassan avait autrefois un habitat beaucoup plus étendu et plus ordinaire qu'aujourd'hui; il nichait sur plusieurs ilots rocheux des deux rives du nord de l'Atlantique. N'ayant que peu de valeur comme aliment ou autrement, sauf dans les plus pauvres populations, il a été refoulé d'une station de couvaison à l'autre, si bien que dans le nouveau 
monde il ne reste que deux de ces stations et, à moins que des mesures ne soient bientôt prises, celles-là aussi auront le sort des autres. ${ }^{1}$

Valeur économique. Bien que les fous de Bassan aient été accusés de faire beaucoup de tort aux pêcheries, leurs dégâts ont été fort exagérés. La majeure partie de la vie des fous de Bassan se passe sur la haute mer ou tout près. Quand ils viennent sur le rivage pour nicher, ils ne peuvent faire que peu d'impression sur les immenses bancs de harengs et d'autres poissons qu'ils poursuivent.

FAMILLE-PHALACROCORACIDE. CORMORANS.

Description générale. Grands oiseaux de coloration noire ou très foncée, avec un bec se terminant par un véritable crochet, et avec une petite poche a la gorge ou au gosier.

Traits distinctifs. Bec et petite poche au gosier.

Indications sur le terrain. En vie les cormorans ressemblent un peu aux plongeons mais l'action de leurs ailes est assez caractéristique et peut être plus facilement reconnue que décrite. Quand il vole le cormoran porte son cou tendu et ses pieds cachés sous la longue queue, au lieu de les traíner derrière comme fait le plongeon. Sur l'eau on le reconnait facilement a sa queue très visible et de coloration uniforme.

C'est une famille cosmopolite dont deux espèces seules se trouvent dans l'est du Canada.

\section{Genre-Phalacrocorax. Cormorans.}

119. Le Cormoran ordinaire. (common cormorant). Phalacrocorax carbo. L, 36. Voir la description de la famille, précédemment donnée.

Traits distinctifs. Si la description de la famille est présente à l'esprit, cette espèce peut facilement être distinguée de toutes les autres saul de la suivante. L'individu adulte de cette espèce a un jet de blanc a la basc de la poche du gosier et un autre sur le coté. Quant au reste du plumage sa taille plus grande et quatorze au lieu de douze plumes de la queue sont une indication très claire. L'absence d'aigrette même chez l'adulte n'est pas un témoignage auquel on puisse se fier pour la détermination de l'espèce (voir l'espèce suivante).

Indications sur le terrain. A moins que la taille ou les marques blanches ne soient très visibles il est a peine possible de distinguer les deux cormorans à l'état vivant.

Nid. Semblable a celui de l'espece suivante.

Habitat. Dans les parties nord des trois continents. Au Canada, il est etroitement limité à la côte de l'Est et n'est aperçu que rarement à l'intérieur.

Lors même qu'on l'appelle uordinaire" ce cormoran est le plus rare des cormorans dans l'est du Canada. L'espèce se rencontre sur la côte européenne et y fut appelé "ordinaire" à cause de la quantité qu'il y en a autour des fles britanniques.

Valeur économique. A cause de sa rareté il est de peu d'importance économique au Canada.

120. Le Cormoran à aigrettes. (nodble-Crested conmorant). Phalacrocorax auritus. L, 30. Voir la description de cette famille, précédemment donnée.

Traits distinctifs. Cette espèce peut facilement se confondre avec la précédente seulement. Dans le plumage de l'adulte l'absence d'un blanc quelconque a la base de la poche du gosier et sur les côtes est un signe indicateur. Pour les autres plumages, la taille et la présence de douze au lieu de quatorze plumes à la queue est un trait distinctif sûr. Au commencement du printemps les aigrettes filamenteuses sur les côtés de la tête, au lieu d'une fraise déformée comme dans les espèces précédentes, sont caractéristiques; mais ces traits se perdent de bonne heure dans la saison, avant la couvaison, et par conséquent, sont d'un médiocre secours pour déterminer l'espèce.

1 Depnia que ce paragraphe a été écrit, ces lieux ont été érigés en parages consacrés aux oisenux el une station juqu'ici inconnue a été signalée au dela de la cote du sudest de Terreneuve. 
Indications sur le terrain. Bien que les cormorans se reconnaissent aisément aux caractères donnés dans le titre de la famille, la taille et la présence ou l'absence des taches blanches sont à peu près les seuls traits qui servent à distinguer les deux espèces à l'état vivant.

Nid. Sur le sol, dans les rochers ou sur le bord des falaises; quelquefois dans les buissons ou dans les arbres plutôt bas; dans les assemblages massifs de branches ou de plantes.

Habitat. Le cormoran à aigrettes se rencontre dans le golfe du Saint-Laurent et sur les lacs des provinces des prairies. Il est plutôt rare quoique peut-être migrateur régulier sur les grands lacs.

SOUS-ESPECES. Ceux de l'Amérique du Nord se divisent en quatre sous-espèces dont une seule appartient au champ que couvre cet ouvrage, le cormoran à aigrette de l'Est, le type de cette race.

C'est le cormoran le plus ordinaire dans l'est du Canada. À l'encontre des espèces précédentes il n'est pas strictement marin et se rencontre sur les eaux douces aussi bien que sur les eaux salées. Il s'empare de sa proie en plongeant et en la poursuivant sous l'eau. Au contraire du fou de Bassan il ne plonge pas en volant mais se pose sur la surface et puis pénètre sous l'eau d'un mouvement serpentin, en glissant comme le plongeon. Il ne reste pas sous l'eau plus de quarante secondes en règle générale.

Valeur économique. Le danger qu'il y a à généraliser hâtivement sur la foi d'observations superficielles a été bien illustré par une étude des aliments que prennent ces oiseaux dans le voisinage des rivières à saumon de la Gaspésie ${ }^{1}$. Quoique communément accusés de faire tort aux pêcheries de saumon en dévorant les petits poissons, le menu fretin, un examen très soigneux d'environ trente spécimens a démontré que les centaines d'oiseaux présents mangeaient un poisson qui n'avait aucune valeur économique, et aucun reste de salmonidés ne fut trouvé dans leurs corps. Il est probable que les anguilles, les chabots et autres poissons que prend le cormoran rendent cette espèce plutôt utile que nuisible au saumon et compensent pleinement les quelques saumons qu'il peut prendre à l'occasion. C'est là un bon exemple de la prudence qui est nécessaire avant de condamner aucune espèce d'oiseaux.

\section{FAMILLE-PELECANIDE. PELICANS.}

\section{Genre-Pelicanus. Pélicans.}

Description générale. Gros oiseau avec un bec très long, aplati, une énorme poche de gorge ou de gosier.

Traits distinctifs. Cette énorme poche de la gorge, qui tient un gallon et davantage, et le long bec aplati sont des traits éminemment distinctifs.

Ces oiseaux extraordinaires sont trop rares dans l'est du Canada pour qu'il en soit parlé spécialement. Il y a deux espèces qui ont été prises quelquefois: le pélican blanc, Pelicanus erythrorhynchos, forme qui se rencontre dans l'Ouest, qu'on peut trouver vers la frontière du Manitoba puis dans l'Ouest, mais dans l'Est seulement comme vagabonds; le pélican brun, Pelicanus occidentalis, un oiseau méridional de présence purement fortuite au Canada. Les noms descriptifs sont suffisamment explicatifs pour les différencier. Le pélican blanc est de fait absolument blanc avec une pointe noire à l'aile dans tous les plumages; toute autre couleur prononcée est l'indice d'une autre espèce.

\footnotetext{
1" Ie cormoran a aigrette, Phalecrocoras atritus, et ses rapports svec les industries du asumon dans le golfe Saint-Laurent." Min. des Mines, Com. gél., Csnads, Bull. $n^{\circ} 13$, Série biologique ${ }^{\circ}$ 5, 1915.
} 


\section{Ordre-Anseres. Palmipèdes au bec en crible. Palmipèdes lamellirostres.}

Description génécale. Oiseaux nageurs avec quatre doigts mais seulement deux membranes, ayant un bec plus large vers l'extrémité et bordé de petites projections pareilles à des dents ou a de fines lamelles (figures $7,8,9,10$, p. 20) au moyen desquelles ils filtrent l'eau de leurs aliments.

Traits distinctifs. Tels que ci-dessus.

Indications sur le terrain. Le cou tendu, la queue en évidence, les rapides battements d'ailes des canards et des oies nous sont bien connus. Dans l'eau quelques espèces ont une ressemblace superficielle avec les plongeurs, mais le bec droit, étroit diffère de celui-ci, la queue tout a fait comme celle des canards, et leur disposition à s'envoler plutôt qu'à plonger quand on les dérange, facilite la différenciation.

Nid. Habituellement sur le sol, quelquefois dans des arbres creux, et quelquefois dans les nids abandonnés de corbeaux ou d'autres grands nids; mais rarement loin de l'eau. Les petits sont capables de courir cà et la et de se lancer a l'eau aussitôt après être sortis de l'ouf; mais quant à savoir comment ils sont portés à terre de la hauteur d'un arbrc de 20 à 30 pieds, où est le nid, c'est là un sujet sur lequel il y a des opinions divergentes.

Habitat. Les anséridés ont un habitat répandu sur toutc la terre. En Amérique la majeure partie de l'espèce couve au nord de la frontière internationale. Ils peuvent, par conséquent, être regardés comme des oiseaux d'habitat boréal. En hiver quelques-uns restent au Canada aussi longtemps que les cours d'eau sont ouverts, les autres gagnent le sud, même les eaux plus chaudes des Carolines, le golfe du Mexique et au deld.

L'Ordre des anséridés ne renferme qu'une famille-les anatidés composés des harles, canards, oies et cygnes, et comprend, par conséquent, la grande masse des gros volatiles sauvages auxquels les chasseurs font la guerre. Une des plus grandes sources de confusion, quand il s'arit de distinguer ces diverses espèces c'est le cas qu'on appelle l'éclipse du plumage. Contrairement à la règle qui gouverne les changements dans le plumage, plusieurs de ces espèces jettent à la fois toutes les plumes de leurs ailes, et ne peuvent donc pas voler avant de s'être remplumées. Pendant cette période de relative impuissance causée par la mue des ailes durant la mi-été, beaucoup de mâles revêtent un plumage particulier d'un caractère moins voyant que le plumage normal, qui se rapproche ordinairement de celui de la femelle, et ce qu'on appelle l'éclipse du plumage. Dans l'entre-temps les oiseaux se retirent dans les parties les plus reculées de leur habitat de façon à n'être vus que rarement par le passant. Lorsque s'ouvre le temps de la chasse la plupart de ces oiseaux sont sortis du plumage de l'éclipse, mais le petit nombre de ceux qui en gardent encore les traces est cependant assez grand pour augmenter le chiffre des plumages qu'on peut reconnaitre et pour en compliquer la différenciation. ${ }^{1}$

Valeur économique. L'ordre des anséridés est, au point de vue économique, un des plus importants parmi les oiseaux; non pas comme pour d'autres oiseaux, à cause de leurs habitudes alimentaires, car celles-ci n'ont qu'une influence négative dans les affaires humaines, mais pour d'autres raisons. En effet, aux premiers temps de la colonisation du pays ces oiseaux fournirent des aliments très importants aux habitants dans leur lutte pour la vie; et même aujourd'hui le nombre annuel des oiseaux tués par les chasseurs, constitue un appoint considérable à nos ressources alimentaires.

I Une sutre source assez commune de confusion, c'est l'existence trop fréquente d'une coloration rouge rouille qui se montre souvent sur diverses parties de la tête ou du corps et qui est commune a berucoup d'especes d'ansérides. Cette couleur est due à un depot ferrugineux dans l'ean ou habitent les oiseaux en question, et ne doit pas etre regardé comme la coloration normale dn plumage. 


\section{FAMILLE-ANATIDA. CANARDS, OIES ET CYGNES.}

Description gentrale. Comme cette famille est la seule comprise dans l'ordre des anséridés la description sous le titre antérieur s'applique ici également et ne demande pas à être répétée.

La famille est divisée en cinq sous-espèces: les merginés ou harles; les anatidés ou canards de rivière; les fuligulinés ou canards de mer; les ansérinés ou oies; et les cygninés ou cygnes. Pour les caractères de ces oiseaux, voir sous les titres qui les concernent et qui suivent.

\section{Sous-famille-Merginæ. Harles, Canards pêcheurs, Becs-scie.}

Description générale. Des canards mangeurs de poissons, avec un bec plus cylindrique, en pointe, spatulé ou aplati, moins spatule ou aplati que chez les autres canards (fig. 7, p. 20). Les bords tranchants des mandibules ont une série de dentelures d'où est venu le nom populaire de bec-scie *. L'ongle a la pointe du bec forme un crochet petit mais bien visible. Le doigt postérieur est développé pareil à celui des canards de mer, mais très différent du doigt postérieur des canards de rivière dont il $\nabla$ a être question. Les mâles ont des couleurs brillantes et frappantes, presque toujours blanches et noires. Les femelles ont des couleurs sombres avec la tête et le cou rouges. La plupart des plumages ont des aigrettes. Chez les femelles et les jeunes oiseaux les aigrettes sont ébréchées et sans formes bien distinctes.

Traits distinctifs. Le bec est toujours un signe caractéristique qui distingue facilement les harles des canards de mer qui leur ressemblent par la forme du doigt postérieur.

Indications sur le terrain. Forme de canard très evidente combinée avec le bec long, mince, cylindrique, et légèrement crochu.

Nid. Les harles sont pour la plupart des hôtes de rivières à l'époque de la couvaison, nichant soit sur le sol, entre les roches, soit dans les arbrcs creux.

Habitat. C'est une petite famille dont les membres habitent l'hémisphère nord.

Les harles se nourrissent de poissons et de mollusques saisis sous l'eau et en plongeant; pour cette méthode de s'alimenter leur bec crochu et dentelé est merveilleusement fait et utile. Ces oiseaux fréquentent pendant la période de la couvaison, surtout les eaux douces, bien qu'ils se voient en grand nombre près de la mer. Leur chair n'est pas recherchée quoique de jeunes oiseaux d'automne bien accommodés ne soient pas à dédaigner.

Valeur économique. Les harles mangent le poisson et, dans certaines eaux, comme vers la source des saumons de rivières, ils font des dégâts appréciables. Les cours d'eau à truites sont trop petits pour ces espèces, et comme on n'a point examiné avec soin le contenu des estomacs il ne faudrait pas condamner ces harles sans une connaissance plus exacte du sujet.

\section{Genre-Mergus. Harles.}

129. Le Harle d'Amérique. (AMERICAN MERGANSER, SAW-BILL, goOsaNDEB,) Mergus americanus. $\mathbf{L}, 25$. De la même apparence générale que l'espèce suivante (planche III A) mais les mâles sans aigrettes ni la bande rouge de la poitrine.

Traits distinctifs. Voir l'espece suivante.

Indications sur le terrain. La coloration générale et l'absence des aigrettes ou de la bande pectorale chez le mâle sont distinctives. Quand ils volent, la tête longue, tendue avec le cou, et le dos généralement gris distingueront les femelles de ces deux merginés, d'avec les autres canards qui ont des plaques blanches aux ailes.

Nid. Dans les arbres creux ou dans les cavités des rochers près de l'eau; le nid est fait avec des herbes.

Ilabitat. Il se trouve partout sur le continent; il niche dans tout l'est du Canada sauf dans les parties méridionales.

Le harle d'Amérique est un oiseau des étendues d'eau plutốt petites que grandes, de là vient qu'il est moins commun sur les plus grands lacs 
ou sur la mer que son semblable à poitrine rousse. A cela près les remarques faites sur cette dernière espèce s'appliquent au harle d'Amérique car leurs habitudes sont pareilles.

130. Le Harle a poitrine rousse. LE BEC-SCIE. LE HARLE HUPPÉ. (RED-BREABTED MERGANsen). Mergus serrator. L, 22. (Planche III A).

Traits distinctifs. L'aigrette hérissée et la bande pectorale rougeâtre séparée de la tête par un col blanc très en vue, sont suffisantes pour reconnastre le mâle. La femelle et les jeunes de cette espèce et de la dernière sont très semblables. Le bec est cependant de charpente plus légère et les narines sont un peu plus rapprochées de la base que chez le harle d'Amérique. La tête n'est pas d'habitude d'un brun aussi riche et la gorge supérieure est d'une teinte un peu plus claire de la même couleur et non pas blanche comme dans cette dernière espece.

Indications sur le terrain. L'aigrette et la bande pectorale du mále adulte et l'absence du blanc de la gorge supérieure chez les femelles et les jeunes oiseaux distingueront le harle a poitrine rousse, du harle d'Amérique. L'apparence grise du dos et la longueur de la tête et du cou tendus en avant feront connaistre les becs-scie des autres canards qui ont des plaques blanches aux ailes.

Nid. Sur le sol press de l'eau, quelquefois sur les arbres.

Habitat. Se rencontre plus ou moins dans tout le Canada, nichant partout où on le trouve sauf dans les partics méridionales.

C'est un oiseau des eaux libres de glace, aussi est-il plus souvent aperçu sur les lacs ouverts et sur mer, que ne l'est l'espèce précédente.

Valeur économique. Voir au titre de la sous-famille.

\section{Genre-Lophodytes. Petit Harle.}

131. Le Petit Harle. (hoOded MFronNser). Lophodytes cucullatus. L, 17.50. C'est le plus petit de nos harles. Le male est un oiseau de couleurs blanche et noire tres frappantes avec des cótés d'un brun châtaigne. Son ornement distinctif, le capuchon, est une aigrette plate, pareille a un disque, partant de la base du bec, en forme d'arche sur la couronne, rencontrant le cou a la base de la téte et d'une coloration blanche généralement très pure avec un rebord noir étroit. La femelle est un oiseau de couleur presque sans éclat avec un corps brun sombre, plus clair en dessous, et avec une aigrette hérissée un peu rougeâtre, de forme semblable a celle représentée (planche III A).

Traits distinctifs. Le capuchon du mâle ne ressemble à rien de ce que portent les oiseaux américains. La femelle peut toujours être distinguée des autres harles par sa petite taille.

Indications sur le terrain. Le capuchon du mâle, aigrette rougeftre et la taille de la femelle.

Nid. Dans les arbres creux.

Habitat. A travers tout le continent, mais plus rare dans l'Est que dans l'intérieur. Niche en des endroits choisis partout où on le trouve au Canada.

Le petit harle est un oiseau de l'intérieur, des étangs paisibles et des cours d'eau en pays boisé. C'est le plus comestible des harles; ce fait, ajouté à la déforestation continue et au peu d'empêchements mis à la chasse du gibier, expliquent que cet oiseau disparait de plus en plus.

Valeur économique. Il est douteux qu'on puisse porter de sérieuses accusations contre le petit harle, comme se repaissant d'animaux utiles.

\section{Sous-famille-Anatinæ. Canards de rivières et d'étangs.}

Description générale. Canards typiques avec le bec aplati et en spatule, terminé par un ongle plat, avec des lamelles filtres, le long des rebords intérieurs (fig. 8, p. 20) au lieu de petites projections en forme de dents. Les pieds avec un doigt postérieur petit quoique bien formé, non modifié en un lobe plat ou appendice semblable a une nageoire.

Traits distinctifs. Le bec distinguera les canards de riviere des harles et le doigt postérieur comme c'est dit plus haut, des canards de mer. 
Indications sur le terrain. Quand la vue sera des plus favorables, le bec fera distinguer les anatinés des harles. La plus grande longueur du cou et le corps plus mince sont des indications faibles et plutôt incertaines pour distinguer ces canards-là des canards de mer ou de baie. Les membres de la sous-famille sont plus facilement reconnaissables à l'état vivant comme espèce que comme une classe.

Nid. Sur le sol, à l'exception du canard huppé.

Habitat. Comme formant une classe les canards d'étang et de rivière sont plus abondants dans l'intérieur que sur l'un ou l'autre littoral.

Ainsi que leur nom l'implique les canards de rivière et d'étang fréquentent nos petites étendues d'eau de préférence aux plus grandes. Ils prennent leur nourriture à la surface en enfonçant la moitié antérieure de leur corps et l'atteignant sous l'eau. L'aliment est massé dans le bec et l'eau en est sortie par pression, les lamelles du bec retenant la matière solide.

Valeur économique. Vu que ce sont absolument des oiseaux vivant, sur l'eau, de végétaux et d'insectes, leurs habitudes alimentaires ne constituent aucune menace. On a démontré récemment que les canards se nourrissent beaucoup de larves de moustiques et que le bien qu'ils font ainsi est de surprenante importance. Grâce à une expérience concluante on a vu qu'une paire de canards dans un petit étang a fait plus pour diminuer ce fléau qu'une grande bande de dorades. Comme gibier ils sont très importants, voir p. 13.

\section{Genre-Anas. Canards ordinaires.}

132. Le Canard ordinaire. LE CANARD DE FRANCE. (MALLARD, GREEN-HEAD). Anas platyrhynchos. L, 23. (Planche III B).

Traits distinctifs. Le mâle ne peut être confondu avec aucun autre canard sauvage quoique plusieurs espèces domestiques s'en rapprochent de très près. La femelle est souvent regardée commc appartenant à une autre espèce et est quelquefois appelée Canard gris (Grey duck). Il y a plusieurs autres canards qui s'en rapprochent par la coloration mais le miroir violet avec la raie blanche devant et derrière le miroir servira toujours à la distinguer.

Indications sur le terrain. Tête verte et anneau blanc autour du cou sont des marques très visibles pour le mâle. Le miroir avec ses raies blanches fera reconnaitre la femelle à l'état vivant. Sa queue a aussi une blancheur générale au vol, laquelle est tout à fait caractéristique.

Nid. Sur le sol, habitucllement sous les hautes herbes ou roseaux, non loin de l'eau, quoique parfois à une grande distance dans l'intérieur.

Habitat. Il est de fait répandu d'un bout à l'autre du Canada, mais moins commun dans l'extrémité orientale. Niche partout au Canada, sauf là où il est dérangé par la colonisation.

C'est le "canard sauvage» par excellence, et il est connu sous ce nom du chasseur de l'ancien monde aussi bien que du nouveau. Il est l'ancêtre duquel sont issues nos variétés domestiques, et presque chaque espèce de canards de basse-cour un peu mélangée montrera les têtes vert-noir, le col blanc, ou les couvertures supérieures et recourbées de la queue, indiquant le retour à la forme primitive. Tout en étant un des meilleurs oiseaux pour la table c'est un des canards les plus sauvages, fait qui explique que cet oiseau ne se reproduise qu'en petit nombre dans les localités qui confinent à la civilisation.

133. Le Canard noir. (BLACK pUCK). Anas rubripes. L, 22. Planche IV A.

Traits distinctiff. La coloration généralement sombre ajoutée à la taille rend cette espèce absolument reconnaissable parmi les canards de rivière.

Indications sur le terrain. La taille, la coloration sombre, et l'éclat argenté des ailes de dessous qu'on voit quand il vole. L'absence de la raie blanche qui délimite le miroir le fera distinguer du canard ordinaire.

Nid. Sur le sol, dans l'herbe près de l'eau. 
Habitat. Le canard noir est une espece plus propre aux régions de l'Est que le canard ordinaire et n'est pas trouvé communément à l'ouest des grands lacs. Il niche dans l'est du Canada où on le rencontre.

Aujourd'hui que le canard huppé devient de plus en plus rare, le canard noir est celui qui niche le plus ordinairement dans les parties méridionales du Canada, et le seul qui puisse être appelé un canard nicheur ordinaire dans le voisinage des grands lacs inférieurs. Autrefois presque chaque petit lac ou étang avait sa nichée de canards noirs, mais, par suite du desséchement des marais et des obstacles apportés par l'homme à la couvaison, celle-ci est désormais restreinte aux plus grands marais ou aux endroits les moins accessibles.

C'est un point discuté que celui de savoir s'il y a une ou deux formes du canard noir. Vers la fin de l'automne on prend un bon nombre de très gros oiseaux à pattes rouges. Quant à savoir si c'est une sous-espèce distincte, cela n'est pas décidé de façon concluante.

Valeur économique. Quoique le canard noir fréquente souvent, surtout dans la saison de la couvaison, la lisière des champs cultivés où il glane la nourriture qu'il y peut trouver, il ne fait que peu ou même point de mal. En cette saison il n'y a pas de grandes récoltes à endommager et, alors, comme en tout temps, il fait plus attention à ce qui vit dans l'eau, aussi ne peut-on imputer aucun dégất à cette espèce.

\section{Genre-Chaulelasmus. Canard chipeau.}

135. Le Canard chipeau. (GaDwaLl.) Chaulelasmus streperus. L, 19.50. C'est un canard gris finement mouchetś ou vermiculé des mémes tons que la femelle du canard ordinaire, mais avec les épaules d'un rouge marron et un miroir blanc.

Traits distinctifs. Le miroir blanc est toujours indicatif. Les femelles peuvent, a cela près, étre facilement prises pour de petits canards ordinaires ou des canards d'Amérique.

Indications sur le terrain. Miroir blanc et apparence générale grisátre.

Nid. Sur le sol, dans l'herbe ou sous les buissons.

Habitat. Presque cosmopolite. C'est un des plus rares canards sur les grands lacs inférieurs; presque absent sur la côte de l'est, plus commun à l'ouest où il couve.

$$
\text { Genre-Mareca. Canards siffeurs. }
$$

137. Le Canard d'Amérique. (BALDPate, aMerican widgeon). Mareca americana. L, 19. Canard grisâtre de taille moyenne. Le mâle a les épaules et le bonnet blancs, une raie verte large en travers de l'cil, un miroir noir avec reflets verts, et une légère teinte rose sur la poitrine ct le dos.

Traits distinctifs. Le mâle se distingue facilement; la femelle pourrait aisément se prendre pour la femelle du canard ordinaire ou du canard chipeau, n'était-ce le miroir et les dessous d'un blanc tres pur.

Indications sur le terrain. La couronne blanche et la raie verte de l'ceil sont caractéristiques chez le male; les dessous blancs et la terminaison brusque de la poitrine brune contre les dessous distinguent la femelle a l'etat vivant.

Nid. Sur le sol, dans l'herbe ou sous les buissons. l'ouest.

Habitat. Presque tout le Canada; rare sur la côte de l'Atlantique; plus commun a

Etroitement apparenté au canard siffleur d'Europe qu'on a quelquefois sur nos côtes et auquel il ressemble beaucoup.

\section{Genre-Nettion. Sarcelle.}

139. La Sarcelle à ailes vertes. (GREen-Winged teAL). Nettion carolinense. L, $14 \cdot \vec{j}$. C'est le plus petit de nos canards. Le male a une tête couleur marron, une raie derrière l'cil et le miroir vert irisé; dos finement vermiculeux, poitrine mouchetée; presque blanc en dessous. Ia femelle est un oiseau d'un brun terne beaucoup plus clair en dessous. Elle a le miroir vert caractéristique de l'espèce. 
Traits distinctifs. La taille differenciera toujours ce petit canard de tous les autres, sauf de l'espèce suivante et du petit bucéphale; présence du miroir vert et brillant le distinguera de ce dernier et la complète absence d'un bleu crayeux sur les épaules le distinguera du premier.

Indications sur le terrain. La petite taille en même temps que le miroir vert et l'absence de bleu crayeux sur les épaules sont des signes pour reconnaftre un plumage quelconque.

Nid. Sur le sol près de l'eau.

Habitat. D'un bout à l'autre du continent, mais rare dans l'extrême Est. La race dans l'est du Canada nichait parfois et probablement à l'origine, communément dans la région inférieure des grands lacs.

C'est un des canards de la qualité la plus recherchée. Son habitude de voler en grandes bandes et à toute vitesse le fait bien connaitre des chasseurs. La sarcelle d'Europe vient quelquefois se fourvoyer jusqu'au Canada, mais c'est très rare.

\section{Genre-Querquedula. Sarcelle à ailes bleues et Sarcelle cannelle.}

140. La Sarcelle à ailes bleues. (BLUE-WINGEd TEAL). Querquedula discors. L, 16. Planche IV B.

Traits distinctifs. La taille distinguera la sarcelle à ailes bleues de tout autre canard sauf de la sarcelle à ailes vertes et du petit bucéphale, et le bleu clajr sur les ailes servira dans ces derniers cas. Le canard souchet a aussi un bleu identique sur les ailes, mais la petite taille de la sarcelle à ailes blenes et l'absence du large bec à spatule différencient ces deux espèces.

Indications sur le terrain. Petite taille en méme temps que la marque blanche sur la face, les dessous sombres et le bleu crayeux sur les ailes.

Nid. Sur le sol, au milieu des herbes.

Habitat. D'un bout à l'autre du continent; aujourd'hui plutôt rare dans l'extréme Est. C'est une des rares espèces qui couvent encore régulièrement, quoique de façon éparse dans la région inférieure des grands lacs.

Une sarcelle de l'Ouest, la sarcelle cannelle, est quelquefois signalée dans l'est du Canada, mais la plupart du temps il s'agit de la sarcelle à ailes bleues fortement imprégnée en dessous du fer ${ }^{1}$ qui provient de l'eau.

\section{Genre-Spatula. Canard souchet.}

142. Le Canard souchet. (shoveltier). Spatula clypeata. L, 20. Le mâle a des marques frappantes; la tête d'un noir vert, poitrine blanche et ligne blanche sur les ailes, dessus d'un riche marron ressortant en une ligne effilée contre la poitrine. Les épaules sont d'un bleu crayeux et le miroir vert. Le bec s'élargit à la pointe en une spatule large en forme de cuillère. La femelle ressemble beaucoup à la femelle du canard ordinaire quoique sensiblement plus petite.

Traits distinctifs. Le bec spatulé distingue cette espèce de toutes les autres de quelque plumage que ce soit.

Indications sur le terrain. Poitrine blanche, ventre d'un riche marron, du bleu sur les ailes, et la taille; c'est tout cela qui distingue le mâle. Le bec fera distinguer cet oiseau sous n'importe quel plumage, et quand on peut le voir, il constitue peut-être le meilleur moyen de distinguer la femelle de celle plus grande du canard ordinaire à laquelle elle ressemble.

Nid. Sur le sol, dans l'herbe, pas toujours dans le voisinage immédiat de l'eau.

Habitat. C'est un oiseau de l'Ouest. Il ne se rencontre que rarement sur les grands lacs inférieurs, et ne se voit plus dans l'Est que comme un vagabond.

\section{Genre-Dafila. Canard pilet.}

143. Le Canard pilet. (PINTAIL). Dafila acuta. L, 28. C'est un canard très long et de forme amincie. La tête d'un brun de phoque, l'avant-cou blanc et les parties de dessus de même, le dos finement vermiculeux de ton gris, miroir vert bronze terminé par une ligne d'un marron clair en avant et par une ligne blanche derrière. La queue est graduée par deux plumes médianes très allongées. La femelle ressemble beaucoup à celle du canard ordinaire.

iVoir la note, page 67. 
Traits distinctifs. La longue queue fait reconnaftre le mâle, le canard a longue queue étant le seul canard américain ayant un ornement pareil, à part cela le canard a longue queue est tout \& fait différent, étant un canard de mer, non de rivière. Le miroir vert bronze et ses lignes limites d'un brun rougeâtre et blanches sont caractéristiques d'un plumage quelconque. Les jeunes sarcelles à ailes vertes ont presque les mêmes caractères du miroir, mais la différence de taille fera éviter tout confusion.

Indications sur le terrain. Téte et cou longs et minces, queue a longue pointe; ce sont de bons signes sur le terrain. La tête sombre et l'avant-con et les dessous blancs font reconnaitre le mâle.

Nid. Sur le sol, quelquefois très loin de l'ean.

Habilat. Rare sur la côte de l'Atlantique; devient plus commun vers l'Est. Il niche a partir des provinces des prairies au nord vers le pôle, et jadis a l'est du lac Erié.

\section{Genre-Aix. Canard huppé.}

144. Le Canard huppé. le canard branchu. (wood duck). Aix sponsa. $\mathrm{L}, 18 \cdot 50$. Planche V A.

Traits distinctifs. En se guidant sur les illustrations, il ne devrait y avoir aucune difficulté à reconnaître cette espèce. Le mâle, même dans son plumage sans éclat, conserve toujours une indication des marques de la joue.

Indications sur le terrain. C'est le seul canard d'été commun dans nos contrées méridionales qui ait les parties de dessous absolument blanches. L'anncau ocnlaire tout blanc de la femelle est tres visible.

Nid. Dans les creux des arbres on des branches près des eaux tranquilles.

Habitat. C'est la partie tempérée de l'Amérique du Nord jusqu'au Canada méridional, et d'un océan a l'autre sur ce continent; plus commun dans la région inférieure des grands lacs que sur l'un ou l'autre de nos rivages ou dans les provinces des prairies.

Nous avons là le canard d'Amérique le plus riche en couleur et le plus beau d'Amérique, peut-être bien du monde. La seule espèce qui s'en rapproche c'est le canard mandarin de Chine qu'on trouve souvent enfermé avec lui. Le canard huppé était originairement le "canard d'étée de nos frontières méridionales, presque tous les cours d'eau et les étangs endigués en possédaient au moins une paire; mais, depuis qu'on a déboisé le pays, la canardière du fils de fermier en a fait façon. Ses brillantes couleurs, son nid trop en vue et la facilité avec laquelle on peut le surprendre dans ses retraites plus ou moins boisées en ont fait une proie facile même pour le chasseur encore novice, et le canard court le risque d'être bientôt exterminé. Un canard qui se pose sur des arbres parait paradoxal à la plupart des chasseurs européens, et cependant c'est l'habitude de cette espèce. Ce canard fait son nid sur un arbre creux, à quelque hauteur au-dessus du sol, et du haut duquel il voit les tournants de la mare ou quelque autre eau stagnante. On n'a pas encore résolu la question de savoir comment les petits sont descendus à terre, et il circule à cet égard beaucoup d'opinions divergentes: celle qui veut que les vieux portent les jeunes sur leur dos ou dans leur bec, celle qui pense que les parents poussent leur progéniture hors du nid pour qu'elle arrive de ses ailes rudimentaires jusqu'à terre; toujours est-il que les petits touchent terre de bonne heure et suivent leur mère vers les approches de la rivière ou des eaux dormantes, le mâle restant tout près pour porter secours à sa famille, en cas de danger. Plus tard, ils rechercheront les marécages où ils séjourneront jusqu'à la fin de l'automne, et d'où ils partiront pour le Sud quand l'eau sera devenue froide comme le temps.

Le canard huppé se rend volontiers dans les boites qu'on lui prépare pour nicher, et de cette façon on pourrait probablement l'aider à se multiplier passablement. 
Sous-famille-Fuligulinæ. Canards de baie, de mer, ou plongeurs.

Description générale. Ce sont des canards d'une charpente lourde ou compacte avec un bec aplati ou en forme de spatule, comme celui du canard, quelquefois enflé ou plus haut à la base (fig. 9 , p. 20) mais toujours avec un ongle aplati à la pointe (figures 8 et 9 , p. 20). Le doigt postérieur est changé en une palette plate ou un lobe finement découpé.

Traits distinctifs. Le bec fera distinguer les canards de mer des harles, et le doigt postérieur les différenciera des canards de rivière et d'étang.

Indications sur le terrain. Le bec bien observé, distinguera ces canards des harles, bien qu'ils soient plus facilement reconnus à l'état vivant comme espèce que comme une sous-famille.

Nid. Ordinairement sur le sol, quoique quelquefois sur des arbres.

Habitat. Lors même que certains individus de cette sous-famille se trouvent communément sur tous les cours d'eau navigables du Canada, les espèces et les individus sont plus nombreux sur la côte que dans l'intérieur.

Les canards de baie et de mer, quoique se sentant plus chez eux sur les grandes étendues d'eau, fréquentent souvent les marécages et les eaux peu profondes pour y trouver leur nourriture. Ce sont de bons plongeurs, allant parfois à de surprenantes profondeurs pour y saisir les mollusques ou des matières végétales. Ils comprennent des individus d'une chair exquise pour la table.

Valeur économique. L'importance économique immédiate de leur régime alimentaire se réduit à moins encore que pour les autres canards.

\section{Genre-Marila. Milouins à tete rousse, Milouins aux yeux rouges, et Morillons.}

146. Le Milouin à tête rousse. (REnhead). Marila americana. L, 19. C'est un canard plutôt gros avec la tête et le haut du cou de couleur uniforme, rouge brique; poitrine noire; le dos gris finement vermiculé avec du noir; blanc en dessous. La tête de la femelle est de couleur plus sombre, même d'un gris brun foncé, et le dos couleur de toile remplacé par une teinte uniformément brune, quelquefois sans même un soupçon de vermiculure.

Traits distinctifs. Le mâle ressemble au milouin aux yeux rouges, mais le dos est beaucoup plus sombre et le rouge de la tête ne descend pas aux épaules comme dans cette espèce. Les femelles de ces deux espèces sont même plus semblables, mais la forme du bec est toujours un trait distinctif (voir milouin aux yeux rouges). Les femelles sont aussi un peu comme celles du morillon à tête noire et du bucéphale d'Amérique, mais elles se distinguent par un miroir gris au lieu de noir, ou par la tache a l'aile et par l'absence d'une marque blanche sur la face. Le morillon à collier a un miroir semblable, mais c'est un oiseau beacuoup plus petit et qui a la marque blanche de la face.

Indications sur le terrain. Couleur de la tête et profil de la tête et du bec.

Nid. Ordinairement sur le sol ou peu élevé au-dessus de l'eau, nid fait de roseaux avec plus ou moins de duvet.

Habitat. N'est ordinairement que de passage a l'est des provinces des prairies, bien que dans le passé quelques-uns aient niché par endroits dans la région des grands lacs; rare sur la côte de l'Est.

C'est un de nos plus beaux canards, et lorsqu'on l'a nourri de céleri sauvage sa chair a un goût aussi délicat que celle du milouin aux yeux rouges. Il est moins l'oiseau des vastes étendues d'eau que beaucoup de canards de mer, car on le trouve souvent dans les marais et les étangs.

147. Le Milouin aux yeux rouges. (CANvas-BACK). Marila valisineria. L, 21 . C'est un gros canard; le mâle a la tête et le cou d'un rouge brique sombre; poitrine noire; les dessous sont blancs, le dos est blanc avec de très fines vermiculures foncées qui ressemblent beaucoup à de la toile à laquelle l'espèce doit son nom anglais. Chez la femelle le rouge de la tête et du cou devient du gris brunâtre, et le dos est brunâtre.

Traits distinctifs. Les couleurs sont très semblables à celles des plumages du milouin à tête rousse qu'on peut lui comparer; il se distingue du milouin à tête rousse par la forme 
du bec lequel chez le milouin aux yeux rouges est plus long et plus lourd, naissant au sommet du front et offrant la ligne du culmen presque droite plutôt que concave comme dans le milouin aux yeux rouges. Le mâle est beaucoup plus blanc sur le dos, et le rouge descend jusqu'aux épaules au lieu de s'arrêter à mi-chemin le long du cou. Les jeunes et les femelles peuvent quelquefois être confondus avec les morillons à tête noire, ou à collier, ou avec le bucéphale d'Amérique, mais en être distingués par la taille plus grande ou par l'absence de blanc ou de gris sur l'aile ou de blanc sur la face.

Indications sur le terrain. Téte rouge ou rougestre ou gris-rougeâtre, absence de blanc sur la face ou sur l'aile; en outre forme et profil de la tête et du bec.

Nid. Fait de roseaux, garni de duvet et bati au-dessus de l'esu.

Habilat. Se rencontre régulièrement quoique peu fréquemment sur la partie inférieure des grands lacs à l'est du lac Erié; rare sur la côte de l'Atlantique, mais plus commun dans l'ouest du Canada où il niche.

C'est un des canards les mieux connus et les plus appréciés. Ce milouin évoque dans l'imagination populaire l'idée de la tortue d'eau douce et de la bonne chère. Cette réputation gastronomique est surtout due au céleri sauvage Vallisneria spiralis dont il se nourrit fréquemment et d'où lui est venu son nom spécifique. Toutefois, bien d'autres canards mangent de ce céleri et sont tout aussi bons au goût. Sur les grands lacs l'introduction de la càrpe allemande a occasionné de grands ravages dans les vastes champs de céleri sauvage, et de riz sauvage, deux plantes extrêmement appréciées pour allécher les canards de toute espèce. Une des premières mesures à prendre pour attirer les canards dans les enclos et les étangs réservés devrait être de supprimer toutes les carpes et de planter du céleri sauvage et du riz sauvage Zizania aquatica.

148. Le Morillon à tête noire. (GREATER SCAUP DUCK, AMERICAN SCaUP, BROADBILL, GREATER OR IAKE BLUEBILL). Marila marila. L, 18.50. C'est un canard de taille moy'enne, le mâle, blanc en dessous; la tête, le cou, et la poitrine supérieure sont noirs; le dos blanc et noir, vermiculé et pareil à de la toile. Les femelles n'ont pas ce dos pareil a la toile; les tons noirs sont changés en deg bruns rougeatres de diverses teintes; la marque blanche de la face se trouve a la naissance du bec.

Traits distinctifs. Le morillon à téte noire et le morillon a collier ont presque tout a fait la même coloration, mais ils peuvent en général être distingués par la taille ou par la forme et la dimension de l'ongle terminal sur le bec. Cet ongle est, toute proportion gardée, plus large et assez près d'être circulaire chez le morillon à tête noire. La tête de ce dernier a l'état adulte, a un lustre verdâtre au lieu de passer au violet clair sur la couronne. Une teinte rougeâtre sur la tête des jeunes ct des femelles est quelquefois très distincte, et on peut les prendre pour un milouin aux yeux rouges, ou pour un milouin à tête rousse, ou quelquefois pour un bucéphale d'Amérique, mais une tache blanche a la base du bec et en travers du front, ou des plaques blanches aux ailes sont toujours caractéristiques chez les morillons. Le morillon a collier, qui leur ressemble aussi, a un miroir gris au lieu d'un blanc.

Indications sur le terrain. Il est presque impossible de voir une différence entre les deux morillons a l'ćtat vivant. Les males des deux espèces paraissent noirs sur le tiers antérieur de leur corps, et blanes sur tout le reste excepté la queue, mais y compris les plumes secondaires de l'aile. Une marque blanche sur la face a la base du bec, comme ci-dessus, est la meilleure indication pour la femelle.

Nid. Sur le sol, dans l'herbe près des étangs herbeux.

Habitat. Rarement de passage ou pour nicher sur la cote de l'Atlantique; commun sur les grands lacs.

Le morillon à tête noire est plus que le petit morillon un oiseau des eaux ouvertes, ainsi que cela est indiqué par l'un de ses noms vulgaires, mais il arrive souvent avec d'autres espèces dans les marécages pour y trouver du céleri sauvage et du riz sauvage.

149. Le Petit Morillon. (Lesser scaUP DUCK). Marila affinis. L, 16.5. Presque tout a fait semblable au précédent, mais un peu plus petit. La couronne du mâle adulte a un lustre violacé au lieu de verdâtre de même que sur d'autres parties de la tête.

Traits distinctifs. Voir les espèces précédentes.

$28588-6$ 
Indications sur le terrain. Voir les espèces précédentes, les morillons à l'état vivant ne se distinguent l'un de l'autre que par la taille.

Nid. Sur le sol, près des étangs herbeux.

Habitat. Un peu moins commun que le précédent dans l'est du Canada. Il niche ordinairement dans l'ouest du Canada et, à l'occasion à l'est vers les grands lacs.

Ce canard est l'un des plus communs qu'on reneontre loin de la côte maritime; c'est plus un oiseau de marais et de petits étangs que les précédents.

150. Le Morillon à collier. (R1NG-Necken DUCK). Marila collaris. L, 16.50. Très semblable par la coloration aux deux espèces précédentes, mais sans le dos couleur de toile; avec un petit collier autour de son bec près du bout et un collier d'un brun pâle ou couleur de cuivre autour du cou.

Traits distinctifs. Les jeunes morillons à collier et les femelles peuvent être pris soit pour des morillons à tête noire, des bucéphales d'Amérique, soit pour des milouins à tête rousse ou des milouins aux yeux rouges ${ }^{1}$, mais ils peuvent étre distingués des morillons par le miroir gris au lieu de blanc; la femelle, par le délicat anneau blanc autour de l'œil, et de tous les autres canards par des marques blanches sur la face, généralement reliées en travers du menton.

Indications sur le terrain. Le mâle peut être distingué des morillons par son dos noir et la femelle par son menton blanc et le délicat anneau blane autour de l'œil; tous deux, dans le plumage de l'adulte, par un petit anneau sur le bec.

Nid. Sur le sol, près des étangs herbeux.

Habitat. Oiseau rarement de passage dans l'Est où il nichait autrefois à l'occasion; rare sur les grands lacs.

\section{Genre-Clangula. Bucéphale d'Amérique.}

151. Le Bucéphale d'Amérique. Le plongevr. (oOLden-eye). Clangula clangula. L, 20. C'est un canard plutôt gros, charpente lourde, coloration frappante de blanc et de noir. Le dos et la tête sont noirs; le reste du plumage, y compris une tache circulaire très en vue entrel'oil et le bec, et la plaque sur l'aile sont blanes. La femelle et le jeune mâle sont marqués de taches gris clair et blanches sur le dos, de larges plaques blanches aux ailes, et une tête brun de phoque, le haut du cou est ordinairement en fort contraste avec le cou et la poitrine qui sont ou blancs ou gris.

Traits distinctifs. Dans les plumages des jeunes et des femelles on peut quelquefois confondre le bucéphale d'Amérique avec le milouin à tête rousse ou avec le milouin aux yeux rouges ${ }^{1}$ ou avec les morillons. Dans ces plumages l'absence de la marque blanche sur la face ou sa présence limitée aux côtés de la face, le distinguera des morillons; les plaques blanches aux ailes le différencieront du milouin à téte rousse et du milouin aux yeux rouges.

Indications sur le terrain. La coloration frappante en blanc et noir du mâle; la tête brune et l'absence de marques faciales chez la plupart des jeunes et des femelles. La grosse tête, le cou fort court, le sifflement des ailes pendant le vol sont caractéristiques.

Nid. Sur des branches ou dans le creux des arbres.

Habitat. Les bucéphales habitent la plupart des régions du nord de l'hémisphère boréal. Le bucéphale d'Amérique, qui seul représente cette espèce dans le nouveau monde, est généralement répandu dans presque tout le Canada. C'est ordinairement un oiseau de passage, qui s'établit en hiver dans l'est du Canada, nichant peu dans les provinces de l'Est, mais plus communément dans le Nord-Ouest.

SOUS-ESPECES. Le bucéphale est divisé en deux sous-espèces; celle de l'ancien monde et celle du nouveau monde. Cette dernière, dite bucéphale d'Ámérique, Clangula clangula americana, est la seule qui se rencontre en Amérique.

C'est un des eanards les mieux connus, un oiseau très hardi qui reste sur nos eaux, jusqu'à ce que la glace les ferme.

152. Le Bucéphale d'Islande. (BARRow's golden-eye). Glaucionetta islandica. L, 20. Ressemble au bucéphale d'Amérique, mais le mâle a une tache faciale en forme de croissant au lieu d'être circulaire. La tête a un lustre violet au lieu de vert et le bec est relativement plus élevé à la base.

\footnotetext{
1 Voir a note 1 la page 67.
} 
Traits distinctifs. Les mâles sont facilement distingués des bucéphales d'Amérique, comme c'est dit. plus haut, mais les jeunes oiseaux et les femelles des deux especes sont plus difficiles à reconnastre, et la forme du bec est peut-être le seul trait distinctif auquel on puisse se fier. Les jeunes mâles peuvent être reconnus à une légère excroissance au front, à la base même du bec, qu'on ne peut voir à cause du plumage; mais qu'on sent très bien avec le doigt.

Indications sur le terrain. Des circonstances exceptionnelles peuvent permettre a quelque-uns des faits susdits d'être constatés chez l'oiseau à l'état vivant.

Nid. Sur des branches ou des arbres creux quand c'est possible, sinon, probablement dans des cavités rocheuses ou sur le sol.

Habilat. Très inégal; l'oiseau est commun sur des côtes de l'Est et de l'Ouest, mais absent de presque tout le vaste intérieur; les oiseaux de l'Est nichent probablement dans le nord de Québec.

C'est une espèce qui est du nord plus que la précédente à laquelle elle ressemble par ses mours autant que par son apparence.

\section{Genre-Charitonetta. Petit Bucéphale.}

153. Le Petit Bucéphale. (BUPFLE-HEAD). Charitonetla albeola. L, 14.75. C'est un très petit canard, presque aussi petit que la sarcelle mais dont la coloration présente des contrastes frappants entre le noir et le blanc. Le mâle est blanc en dessous et autour de la base du cou, noiren dessus avec une tête noire irisfe rompue par une large tache blanche triangulaire dont le sommet au-dessous de l'ceil rencontre l'autre partie blanche le long de la nuque. Les plumes des joues sont allongées faisant des bouppes sur les côtés de la face, d'où son nom anglais. La femelle: blanc en dessous et un gris brundtre foncé ailleurs à part une tache blanche indécise sur chaque joue.

Traits distinctifs. Le mâle ne peut être confondu avec aucun autre oiseau; la femelle ressemble plutôt au morillon, mais peut en être distinguée par $8 a$ petite taille et un trait de couleur claire qui s'étend de derrière l'œil vers l'arrière de la couronne au lieu de la plaque blanche à la base du bec. Une tache faciale au lieu de deux et les dessous uniformément clairs la feront distinguer de la femelle du canard histrion à laquelle elle ressemble aussi.

Indications sur le terrain. Taille petite et tacbe blanche a la tête chez le male; la taille, la tache à la joue et la plaque blanche a l'aile chez la femelle.

$N i d$. Sur une branche ou dans le creux d'un arbre.

Habitat. Il est de passage ordinairement depuis la côte de l'Atlantique jusque vers l'ouest; niche au nord et au nord-ouest.

Le nom de "Spirit Duck" fait allusion à sa force comme plongeur et à I extraordinaire facilité qu'il a de disparaitre quand il est blessé.

\section{Genre-Harelda. Canard à longue queue.}

154. Le Ganard à longue queue. Le KAKAwi. (OLD-SQUAw) Hareldahyemalis. $\mathrm{L}$, 21. (Projection de la plume médiane de la queue au delà des autres, 4.50-5.) Un canard de taille moyenne dont le plumage change remarquablement avec les saisons. Le mâle, au printemps, a la poitrine d'un brun de phoque, comme le cou, la tête et le dos; un masque facial presque blanc; des raies couleur d'ocre sur les ailes et a la base postérieure du cou. Le plumage d'hiver est blanc, avec la poitrine et le dos noirs ou brun foncé, une ligne en travers des épaules. Dans l'une et l'autre saison le mâle a deux plumes médianes caudales, très allongées, d'environ 8 pouces de la base a la pointe et dépassant de 4 a 5 pouces les autres plumes caudales. Le plumage de la femelle est intermédiaire entre les deux plumages susdits, montrant surtout du blanc sans ligne bien tranchée entre la couleur foncée de la poitrine et les dessous blanes comme chez les máles.

Traits distinctifs. Les mâles sont caractéristiques; les femelles ne peuvent être confondues qu'avec celles de l'espèce suivante, mais elles sont beaucoup plus claires en dossous et la tête est presque entièrement blanche au lieu d'étre noire.

Indications sur le terrain. La longue queue du male, la tête presque entièrement blanche avec une marque foncée aux joues dans le plumage des jeunes et des femelles.

Nid. Sur le sol, près de l'eau et cacbé sous les buissons ou dans l'herbe.

Habilat. Niche d'un bout à l'autre du continent dans l'extrême nord. Plus commun sur la côte ou les grands lacs que sur de plus petites étendues d'eau. 
Ce canard est chez nous un oiseau d'hiver. Il fréquente nos ports et se trouve souvent avec beaucoup d'autres de son espèce à la bouche des égouts; il reste aussi longtemps que les eaux sont libres, et même durant tout l'hiver. C'est un fort plongeur et mangeur de poissons, mais n'a que très peu de valeur comme chair pour la table. Il plonge à de grandes profondeurs pour sa nourriture et se trouve parfois pris dans les filets de pêcheurs loin de la terre et à des profondeurs étonnantes; on cite un cas où il fut trouvé à 90 pieds.

\section{Genre-Histrionicus. Canards histrions.}

155. Le Canard histrion. LA CANNE dE ROCHE. (HARLEQUiN DUCK). Histrionicus histrionicus. L, 17. C'est un petit canard bien nommé d'apres Arlequin au costume multicolore. La coloration générale du mâle va d'un bleu ardoise à bleu noir, mais sur ce fond il y a une série frappante de croissants, de raies, de taches circulaires, de triangles, et un collier du blanc le plus pur, chacun de ces dessins étant bordé de noir ce qui en relève le contraste frappant; une moucheture brillante d'un riche brun marron décore les flancs et borde les côtés de la couronne. La femelle est très sombre et terne, toute brunâtre avec taches blanches sur le ventre, réunies dans une place qui ne se continue pas, qui méme est médiane. Une tache blanche peu visible devant l'œil et au-dessous et une autre plus caractérisée au-dessus de l'oreille.

Traits distinctifs. In mâle ne peut pas être confondı avec celui d'aucune autre espèce. La femelle ressemble à celle du petit bucéphale par la taille et la coloration, mais son ventre est plus ou moins tacheté au lieu d'avoir une teinte claire uniforme et elle a deux taches faciales au lieu d'une et point de plaque à l'aile.

Indications sur le terrain. Impossible de se tromper quant au mâle. La femelle peut se reconnaître à sa taille et à sa coloration génézalement sombre, à peine plus claire en dessous; absence de la plaque à l'aile; deux plaques claires à la face.

Nid. 'Sur le sol, sous les roches ou dans des amas de bois ou dans des trous creux.

Habitat. Il n'est que de passage dans l'Est, très rare dans l'intérieur quoique commun dans les montagnes de l'Ouest, ou il niche.

C'est un des plus jolis de nos canards; il ne le cède qu'au canard huppé pour la beauté. Sa véritable demeure est sur les bruyants cours d'eau de l'Ouest et du Nord-Ouest où il est bien connu des prospecteurs et des mineurs. Dans l'est du Canada, il fréquente les baies rocheuses et les rivages ou il se nourrit surtout des insectes du sable et des petites crevettes qui remplissent les eaux salées du bord de la mer.

\section{Genre-Somateria. Eiders.}

Quoique ne formant pas une division systématique reconnue des canards ce genre ainsi que le suivant sont singuliers ct font voir des caractères communs très suffisants pour qu'il en soit fait mention ici.

Description généale. Gros oiseaux de forte charpente, les plus gros de nos canards. Les eiders mâles ont de larges masses de couleurs et de teintes délicates qui contrastent cntre elles de façon très tranchée; les macreuses sont presque toutes noires, quelques-unes avec de petites insertions d'un blanc très pur. Les deux espèces ont des becs enflés avec d'étranges excroissances et de brillantes colorations (figure 9, p. 20).

Traits distinctifs. Coloration en général foncée non relevée par les nombreux dessins des macreuses; la brillante coloration en larges masses des eiders mâles, les tons fins et uniformément rayés de divers bruns chez les femelles; la taille, la forme, les enflures et les protubérances du bec dans les deux sexes sont les caractères les plus marqués de la plupart des espèces.

Indications sur le terrain. La coloration générale et le bec.

Nid. Sur le sol près de l'eau, quelquefois a l'abri de rochers on de buissons qui surplombent; le nid est garni d'un duvet que donne la mere. L'édredon qui est dans le commerce provient des nids des eiders.

Habitat. Répandu d'un bout à l'autre du Canada, niche dans le Nord; très commun sur les côtes et sur les étendues d'eau pendant la migration. 
Ce sont des «canards de mer dans la plus pure acception du mot, bâtis pour affronter les plus gros temps et ne venant presque jamais sur les étangs et les marais d'eau peu profonde. Ils se nourrissent de mollusques et de crustacés et de ce qu'ils trouvent en plongeant.

Valeur économique. Leur régime alimentaire a peu d'intérêt pour l'homme, mais dans certains parages, comme au Labrador, ils offrent par leur chair et leurs œufs la principale nourriture animale qui se présente là. Comme leur duvet est un article de commerce de grand prix, les eiders ont une valeur appréciable. Leur nombre va diminuant sans cesse (voir les détails à propos de l'eider d'Amérique) et des mesures énergiques devraient être prises pour conscrver l'espèce.

160. L'Eider du Nord. (EIDER DUCK). Somateria mollissima. L, 23. Le mále: noir en dessous, tranchant fortement sur la blancbeur de la poitrine qui est délicatement teintée d'un rose vineux; blanc en dessus; tête blanche avec une teinte eau-du-nil depuis leo joues jusqu'à la nuque; large raie noire de l'ceil au derrière de la tête. La femelle: coloration uniforme dans un dessin délicat de différents bruns, noirs et d'ocres pâles dispasés en raies brisées autour du corps. Protubérances du bec s'étendant des deux côtés du front en longues langues charnues.

Traits distinctifs. Pas d'erreur possible pour le mâle; la femelle peut se distinguer de celle de l'eider remarquable par le fait que les plumes de la couronne ne s'étendent pas en avant aussi loin que l'extrémité d'arrière de la narine. Cette espèce ressemble beaucoup à l'eider d'Amérique dont on ne peut la distinguer que par la taille et la forme des protubérances du bec sur le front-chez l'eider du Nord elles se terminent en pointe et la pointe n'est pas arrondie - et par la distance entre le point où commencent les plumes sur le côté du bec et la pointe de la protubérance, distance qui est moindre que chez l'eider de l'Amérique.

Indications sur le terrain. Taille et coloration générale.

Nid. Sur le sol, fait entièrement de duvet.

Habitat. L'eider du nord habite les régions du nord de l'Europe et de l'est de l'Amérique. L'eider du nord est le plus septentrional de nos deux eiders semblables (voir l'espèce suvvante). C'est un oiseau strictement de l'Atlantique, qui niche dans les régions polaires de l'est du Canada et dans le Groenland.

SOUS-ESPECES.-L'eider du Nord est représenté en Amérique par une sous-espèce, l'eider du Nord, Somateria millissima borealis. L'eider européen, le type de la race, n'a jamais été signalé en Amérique.

161. L'Eider d'Amérique. Le motac. (AMerican emer). Somatetia dresseri. L, 23. Presque tout à fait semblable à l'espèce précédente.

Traits distinctifs. I a femelle peut être prise pour celle de l'eider remarquable, mais peut en être distinguée par les plumes de la couronne qui ne s'avancent pas plus loin que derrière la narine. Elle peut n'être pas spécifiquement distincte de l'espèce précédento et l'un et l'autre sexe peuvent en être distingués seulement par les bouts arrondis de la protuberance du bec sur le front et par le fait que la distance du plumage sur le côté du bec jusqu'd̀ la pointe des rudiments est plus grande.

Indications sur le terrain. La taille et la coloration générale; il ne peut pas être distingué de l'eider du Nord, à l'état vivant.

Nid. Sur le sol, quelquefois sous des roches ou des buissons qui surplombent; le nid est bâti de duvet arraché de la poitrine de la mère.

Habitat. C'est un oiseau de nos rivages de l'Est, nichant sur le rivage nord du golfe du Saint-Laurent et, par occasion, sur la cốte aussi loin au sud que l'Etat du Maine.

En Scandinavie et en Islande les eiders sont à moitié domestiques et l'édredon tiré de leurs nids est une importante source de revenus. Bien que dans le Labrador et sur le golfe du Saint-Laurent il y ait d'immenses bandes de ces oiseaux, aucune tentative n'a été faite pour en tirer parti, sauf pour la chair; mais cette quantité a été si rapidement diminuée par de stupides tueries qu'il n'en reste plus qu'une petite fraction du nombre primitif. Sur ces rivages froids et désolés où la viande fraiche est rare les eiders devraient 
être épargnés pour servir d'aliments, sinon pour d'autres usages. Des mesures intelligentes et prévisionnelles protégeraient les eiders pour toujours, donneraient une ample quantité de nourriture, soit comme chair soit comme $œ u f s$, et produiraient une riche moisson de duvet qui vaudrait sur le marché bien plus que les carcasses d'oiseaux.

Cette espèce est si étroitement apparentée à la précédente, qu'il ne serait pas étonnant qu'elle fût classée avec l'eider du Nord en qualité de sous-espèce.

162. L'Eider remarquable. (KING EIDER). Somateria spectabilis. L, 23. Le mâle très semblable au précédent, mais le dos est presque tout noir, les joues sont eaudu-nil, le sommet de la tête et le derrière du cou d'un gris-bleu clair, et un $V$ noir sur la gorge; le bec est caractćristique; les rudiments de chair sur le front sont d'un jaune brillant et se rencontrent sur le front ou ils deviennent larges de presque un pouce et forment une bosse relativement énorme. La femelle n'a pas les fortes protubérances au bec et sa coloration présente les mêmes bruns et noirs selon un dessin de raies fines que l'on remarque chez les autres eiders.

Traits distinctifs. Le mâle avec ses protubérances au bec ne peut être pris pour aucune autre espèce. Les femelles ressemblent à celles des autres eiders mais peuvent se distinguer des deux précédentes espèces par le plumage de la couronne qui s'avance jusque derrière les narines.

Indications sur le terrain. Moins de blanc sur le dos et sur les protuberances jaune claire du bec du mâle. Les femelles ne peuvent pas être distinguées avec certitude des autres eiders à l'état vivant.

Nid. Sur le sol, nid garni de duvet.

Habitat. C'est le plus septentrional de nos eiders. Il niche d'un bout à l'autre du continent le long de la côte et des sles du pôle. Il hiverne le long du golfe du Saint-Laurent et des rivages de la Nouvelle-Angleterre. C'est le seul eider qui vagabonde assez régulièrement jusqu'aux contrées basses des grands lacs.

\section{Genre-Oidemia. Macreuses.}

163. La Macreuse d'Amérique. (AMERICan scoter). Oidemia americana. L, 19. Les mâles sont tous noirs; la base du bec est très enflée près du front et d'un jaune brillant. La femelle n'a pas le bec enflé; elle est foncée partout, plus claire en dessous et avec un capuchon visiblement plus sombre qui contraste sur une ligne tranché avec des joues plus claires.

Traits distinctifs. Le mâle est le seul canard canadicn tout noir et que ne relève aucune tache de couleur. Le capuchon foncé de la femelle la distingue d'autres espèces qui lui sont comparables.

Indications sur le terrain. Couleur noire en évidence, bec jaune du mâle; l'absence de taches blanches ou de marques faciales et le capuchon foncé sur la femelle.

Nid. Sur le sol, près de l'eau.

Habitat. Il niche dans l'extrême nord à travers tout le continent; il est commun en hiver sur nos rivages de l'Est et n'est pas inconnu dans les régions basses des grands lacs.

165. La Macreuse veloutée. (white-winged scoter). Oidemia deglandi. L, 22. Brun foncé, presque noir, avec plaques blanches aux ailes et un petit croissant blanc sous l'oil; bec enflé à la base et s'élevant plus brusquement en avant que cela n'est montré dans la figure 9, p. 20 , et la mandibule supérieure est colorée d'un rouge et d'un blanc brillants. La fernelle est d'un brun sombre uniforme. espece

Traits distinctifs. La plaque blanche à l'aile distingue tous les plumages de cette

Indications sur le terrain. Grande taille, couleur sombre générale et uniforme, plaques blanches à l'aile.

Nid. Sur le sol, parmi les buissons ou dessous.

Habitat. Niche dans les hautes latitudes a travers le continent; il abonde sur les côtes pendant le passage, et c'est la plus commune macreuse sur les grands lacs et dans l'intérieur. 
166. La Macreuse à large bec. (surf scoter). Oidemia perspicillata. L, 20. Le mâle est tout noir avec une plaque blanche en travers du front et un triangle de même couleur à la base de la tête; bec très enflé (figure 9, p. 20) et d'une coloration frappante de rouge, de jaune et de noir. La femelle est d'un brun foncé, plus clair en dessous et très semblable à cet égard à la macreuse d'Amérique.

Traits distinctifs. Les plaques blanches de la tête sont propres au mâle. La femelle peut être distinguée de celle de la macreuse veloutée par l'absence des plaques à l'aile, et de la macreuse américaine par l'absence du capuchon et la présence de deux taches claires un peu vagues sur le côté de la face, une à la base du bec et l'autre couvrant l'oreille.

Indications sur le terrain. Du blanc sur la tête du mâle et les deux taches imprécises sur la face de la femelle.

Nid. Dans l'herbe, près de l'eau.

Habitat. C'est à peu près le même que pour la précédente espèce, mais peut-être que cette macreuse est plus commune sur la côte et qu'elle l'est moins sur les grands lacs.

\section{Genre-Erismatura. Canard roux.}

167. Le Canard roux. (RUDDY DuCK). Erismatura jamaicensis. L, 15. C'est un petit canard. Le mâle a une coloration frappante d'un riche brun rougeatre marron sur le cou, les épaules, le dos et les flanes; la couronne et le derrière de la tête presque noirs, les joues et le bas de la face blancs. La femelle est petite, d'un brun grisâtre sombre, plus clair dans les dessous et aux joues, avec une calotte foncée.

Traits distinctifs. Le mâle est le seul canard presque entièrement roux. La femelle peut se reconnaitre à l'éclat argenté, pareil à celui du grèbe, des parties de dessous et, dans tout plumage, aux plumes raides et pointues de la queue.

Indications sur le terrain. La taille, la forme courte et trapue, cou épais. Son habitude de porter quelquefois la queue droite et se dépliant en éventail est aussi un bon guide, quoique certaines macreuses passent pour en faire autant à l'occasion.

Nid. Dans les roseaux au-descus de l'eau.

Habitat. Il niche dans les provinces des prairies vers le Nord; il est plus commun dans ses voyages sur les grands lacs que sur la côte.

\section{Sous-famllle-Anserinæ. Oíes.}

Description générale. Les oies ressemblent aux canards, mais sont plus grandes, avec un corps moins aplati et des pattes relativement plus longues; bec (figure 10, p. 20) plus haut et un peu plus comprimé a la base, plus épais et moins aplati à la pointe, pouvant difficilement être comparé à une spatule, mais ayant l'ongle large à sa pointe, caractéristique de l'ordre.

Indications sur le terrain. Le puissant vol des oies est bien connu. La taille, la coloration et les façons de son vol sont les meilleurs signes pour se guider sur le terrain. Leurs cris rauques et stridents si souveht entendus pendant leur migration sont aussi caractéristiques.

Nid. Sur le sol.

Habitat. Les oies sont de tous les pays. Les espèces américaines nichent plutôt dans les contrées du Nord, voyageant pour s'y rendre à travers l'intérieur aussi bien que le long des côtes.

Les oies sont plus que les canards des oiseaux de terre ferme et herbivores sans être pour cela moins habituées à l'eau. Elles n'ont pas l'habitude de plonger, mais se procurent leur nourriture dans le fond en la picotant et l'attrapant grâce à leur long cou. Les sexes se ressemblent et leur plumage change peu avec les saisons. L'oie est une chaire excellente sur la table et c'est pour cela, aussi bien que pour sa taille, qu'elle est très recherchée des chasseurs. Les oies sont très circonspectes et, bien que leur nombre ait beaucoup diminué, elles ont réussi à se protéger mieux que ne l'ont fait bien d'autres oiseaux recherchés comme gibier.

Valeur économique. Se nourrissant surtout d'herbes et fréquentant dans leurs migrations les régions cultivées plus que ne font d'autres membres de leur espèce, les oies peuvent causer plus de dégâts que les canards, mais on ne le leur a jamais beaucoup reproché. Leur valeur, à d'autres 
égards, est si évidente qu'on s'est bien moins plaint des oies que d'autres espèces également dignes d'être protégées, mais dont l'utilité, quoique réelle, est moins manifeste.

\section{Genre-Chen. Oies sauvages.}

169. L'Oie blanche. (sNow aoose). Chen hyperboreus. L, 23. C'est une oie plutôt petite, d'un blanc parfait avec des primaires noires. Les jeunes ont plus ou moins de gris ou de brun grisâtre répandu par places ou sur tout le corps.

Traits distinclifs. Les bords tranchant des mandibules sont plus ou moins inclinés, écartés l'un de l'autre, montrant des projections serratiformes, pareilles à des dents et donnant à la face l'apparence qui lui a fait donner le nom d'oie rieuse.

Indications sur le terrain. La couleur, le vol et le profil absolument propres aux oies.

Nid. Sur le sol.

Habitat. La petite oie blanche niche sur la côte arctique et dans les fles à l'ouest du golfe de Coronation. Elle voyage vers l'intérieur du continent; la grande oie blanche se rencontre le long de la côte de l'A tlantique.

SOUS-ESPECES. Il y a deux sous-espèces de l'oie blanche au Canada, la petite oie et la grande, qui ne diffèrent que par leur taille, mais comme les deux espèces cohabitent de temps à autre ee n'est pas une distinction à laquelle on puisse se fier. Il y a une différence fondée sur les formes et les tailles relatives des becs, mais il faut, pour s'en rendre compte, des spécimens à comparer. Bien qu'on l'ait souvent signalée inexactement l'oie blanche la plus grande est un oiseau très rare. C'est la forme de l'espèce qu'on trouve dans l'extrême Est et qu'il ne faut chercher que le long de la côte de l'Atlantique et dans le bas du Saint-Laurent.

Le nom de "Wavey" est une corruption du mot indien "Wa-Wa " qui signifie oie sauvage.

169. 1. L'Ole bleue. (BLUE gOOSE). Chen corulescens. L, 26. Corps gris ardoisé, apparence ondulante des plumes les plus claires sur le dos et plus ou moins la méme chose en dessous; la couleur d'ardoise est surtout forte sur les ailes et le croupion; la tête est blanche. Les jeunes sont semblables mais la tête et le cou sont d'un brun grisâtre. Le bec fait voir à un degré un peu réduit les dents serratiformes de l'oie blanche.

Traits distinctifs. La combinaison de la tête blanche en contraste avec les ailes gris bleu se ne voit chez aucune autre oie américaine de l'Est.

Indications sur le terrain. Tête blanche et corps plus foncé sont probablement les indications les plus sûres.

Nid. Sur le sol.

Habitat. Parât être restreint dans la saison de la couvaison à l'est de la baie d'Hudson; le gros de l'émigration se fait à travers le Manitoba; l'oiseau est rarement de passage sur les grands lacs soit qu'il aille vers la vallée du Mississipi où qu'il en revienne.

Pendant longtemps l'oie bleue a passé pour n'être que l'oie blanche avec un plumage d'oison; aujourd'hui on reconnait que c'est une espèce à part.

$$
\text { Genre-Anser. Oie grise. }
$$

171a. L'Oie à front blanc. (WHItE-Fronted goose). Anser albifrons. L, 27. D'un brun grisátre, plus foncé sur la tête et le cou, plus clair en dessous, plaque blanche autour de la base du bec; couleur claire des parties de dessous mouchetées irrégulièrement de noir qui se réunissent à la période de maturité en bandes imprécises et donnent une apparence d'imparfait développement même à des oiseaux d'âge adulte.

Traits distinctifs. Tête brune et face blanche.

Indications sur le terrain. Couleur brune sur tout le corps et face blanche.

Nid. Sur le sol.

Habitat. L'oie à front blane est une espèce presque circompolaire. La sous-espèce américaine niche sur le continent et dans les fles à l'ouest du pôle. Elle voyage à travers l'intérieur du continent et ne se rencontre que rarement à l'est aussi loin que les grands lacs.

SOUS-ESPECES. L'oie de notre pays, l'oie américaine à front blanc, A. a. gambeli, est une sous-espèce de l'oie européenne à front blanc dont elle se distingue par sa taille légèrement plus grande. 


\section{Genre-Branta. Bernache du Canada et Bernache commune.}

172. La Bernache du Canada. L'ottarde'. (CANAdA goose, wild goose). Branta canadensis. L, 35. Planche V B.

Traits distinctifs. Grande taille, tête et cou noirs, gorge blanche, plaque sur la joue.

Indications sur le terrain. Tete et cou noirs, plaque blanche à la gorge. Un V blanc sur la queue qu'on voit très bien quand l'oiseau vole, fera distinguer les membres de cette espèce de tous les plus gros canards.

Nid. Sur le sol, parfois dans de grands nids abandonnés sur des arbres.

Habitat. Niche d'un bout a l'autre du continent depuis la limite nord des arbres jusqu'aux limites de la colonisation.

SOUS-ESPECES. L'oie du Canada comprend plusieurs races géographiquement distinctes. Dans l'Ouest il y a une petite sous-espèce appelée oie de Hutchin, B. c. hutchinsi. Elle passe pour avoir une note différente daus son cri et pour etre facilement reconnaissable a l'état vivant par des chasseurs qui ont quelque expérience. Sa taille plus petite est un critérium incertain, mais c'est la seule différence qu'on puisse indiquer ici. Sa longueur moyenne n'a pas tout a fait 25 pouces. On ne peut s'attendre a la voir que jusqu'au Manitoba, a l'ouest, et avant de l'identifier ailleurs il faut user d'une grande prudence.

La bernache est un oiseau très circonspect et toujours sur l'éveil; elle passe ordinairement la journée en plein air et se rend, le soir ou pendant la nuit, dans les marécages et les champs cultivés pour y chercher sa nourriture; à moins d'être dérangée elle y reste jusqu'au lendemain. Pendant qu'elle est ainsi occupée il y a en toujours une, au moins, qui, son long cou tendu, surveille les environs et guette l'apparence de quelque danger. Il est impossible aux piétons même les plus avisés d'approcher sans être aperçu. C'est pour cette raison que la bernache du Canada a peut-être moins souffert des chasseurs que d'autres membres de son ordre, et avant que les parages où elle niche, ne soient envahis, elle court peu le danger d'être exterminée. A l'origine, cette oie nichait dans les limites de nos régions actucllement peuplées, mais la colonisation l'a chassée de la région la plus méridionale où elle couvait. Néanmoins, elle occupe encore de vastes espaces dans tout le nord du Canada aussi loin que va la limite nord des arbres et, aussi longtemps que tout ce territoire demeurera inhabité, la bernache y trouvera des parages où elle nichera en sécurité. Toutefois, à moins que des méthodes intelligentes de conservation ne finissent par être adoptées, la bernache du Canada disparaitra à mesure que les terrains où elle couve seront envahis par l'homme.

173a. La Bernache commune. (BRsNT). Branta bernicla. L, 26. Très semblable a la bernache du Canada mais plus petite; la tête, le cou et le haut de la poitrine noirs; un collier de blanc étroit et brisé sur le cou.

Traits distinctifs. Une petite oie canadienne foncée sans marque a la face.

Indications sur le terrain. Taille petite, poitrine foncé, absence de marque à la face. Le V blanc sur la queue, visible quand l'oiseau vole bas, distinguera aussi la bernache commune des canards plus gros et noirs, mais non de la bernache du Canada.

Nid. Sur le sol, nid fait d'herbes et garni de duvet.

Habitat. Circompolaire, pour l'espéce. La bernache américaine niche dans l'est de la région polaire, descend dans ses voyages vers les cótes de l'Atlantique, mais rarement dans l'intérieur du continent.

SOUS-ESPECES. La bernache est une espèce circompolaire. L'individu du nouveau monde, la bernache américaine, $B$. $b$. olaucogastra est, comme sous-espèce, distinct de celui de l'ancien monde, lequel n'a jamais été signalé en Amérique. La bernache noire,

1 Une confusion regrettable e'est produite dans l'emploi habituel parmi les Canadiens-francais du nom populaire d'oularde, donne i la bernache du Canada. Pour parler correctement, le nom d'outarde s'applique au "Bustard", espece d'oiseau de ''ancien monde ot qui n'a pas en congénère en Amérique. L'outarde est bien plntôt an oiseau de rivaze qu'une oie, et il n'y a ancune reison de donner eon nom a cette derniere espece. Ise nom francaje qui convient le plus a l'oie du Canoda, et colui-la méme dont l'emploi a été adopte par la Société canadienpe dee oiseaux migrateurs, o'est Bernache du Canada. 
B. nigricans de l'Ouest, est caractérisée par le fait que le noir de la poitrine estrépandu comme teinte sur les parties de dessous; mais elle ne se rencontre dans l'est du Canada que comme un vagabond d'occasion.

La bernache commune est une petite oie. Elle se rencontre sur le bas Saint-Laurent et sur lcs côtes maritimes par bandes de plusieurs centaines, mais se présente rarement ou seulement comme vagabonde dans l'intérieur sur les grands lacs.

\section{Sous-famille-Cygninæ. Cygnes.}

Description générale. Très gros anatidés; blancs; à l'exception peut-être de la grue d'Amérique ou du dindon sauvage, c'est le plus gros des oiseaux d'Amérique.

Traits distinctifs. La taille ajoutée à la couleur suffit à distinguer les deux cygnes. Les lores (espace entre l'œil et le bec) sont dénués de plumes. Le bec commence vers le haut du front, at sa base il est presque rectangulaire en section transversale, la pointe est pourvue d'un ongle plat.

Indications sur le terrain. Taille et couleur, ce sont nos seuls gros oiseaux tout blancs. ${ }^{2}$

Nid. Sur le sol, nid fait avec des herbes et garni d'un duvet pris à la mère.

Habitat. La plupart des cygnes se trouvent dans l'hémisphère boréal, mais n'y sont pas rigoureusement confinés. Én Amérique ils nichent actuellement dans les très hautes latitudes, bien que le cygne-trompette, espèce presque éteinte, nichât dans l'origine aussi vers le Sud que dans l'un des Etats du nord des Etats-Unis.

De temps immémorial les cygnes ont joué un rôle important dans les récits populaires de l'ancien monde ainsi que dans les contes de fées pour enfants, où il est si fréquemment question de ces oiseaux, toutefois c'est un grand sujet de surprise pour bien des gens que d'apprendre que, même de nos jours, les cygnes sauvages sont parfaitement communs au Canada. Les oies sont sauvages et sur leur garde, mais le cygne est encore plus sauvage et plus circonspect. Son cou très long lui permet de pêcher sa nourriture dans des eaux trop profondes pour des oiseaux non plongeurs, et il peut séjourner sur une eau profonde pendant tout le jour, là où il est impossible de l'approcher à l'improviste. Il se rend rarement dans ces marais où l'eau est très basse et où le chasseur peut se cacher, ce qui fait que l'oiseau est rarement pris.

Les épithètes données aux cygnes de l'hémisphère boréal témoignent des ressources extraordinaires de leur organe vocal; c'est ainsi qu'en Amérique nous avons le cygne d'Amérique (siffleur) et le cygne trompette; et qu'en Europe on a le cygne criard et le cygne muet. Des modifications singulières et compliquées de la trachée sous forme de circonvolutions variées dans certains recoins osseux du sternum sont certainement en relation étroite avec la voix, et leur complexité augmente en raison directe de la qualité de la voix telle que l'expriment les qualificatifs susdits; c'est ainsi que le cygne muet n'a aucune circonvolution trachéenne et que la plus haute complexité se rencontre chez le cygne trompette et le cygne criard.

\section{Genre-Olor. Cygnes.}

180. Le Cygne d'Amérique. (whistling swan). Olor columbianus. L, 55. Très gros oiseau, tout blanc.

Traits distinctifs. Le cygne d'Amérique peut être pris seulement pour le cygne trompette. Superficiellement on peut facilement le distinguer par sa plus petite taille seulement. Comme les 'cygnes n'arrivent à leur complet développement qu'au bout de plusieurs années, la taille peut n'être pas toujours en soi une preuve convaincante. Il y a une différence dans la forme du bec, mais elle est trop difficile à caractériser pour être bien décrite ici. L'enroulement de la trachée dans le sternum fournit le meilleur critère de

\footnotetext{
1 Ies autres gros oiseaux du Canada ont tous plus ou moine de noir eur les plumes des ailes.
} 
différenciation. Chez le cygne d'Amérique la trachée fait une seule courbe horizontale sur la surface du sternum, tandis que la trachée du cygne trompette a une courbe perpendiculaire en plus. L'absence d'une tache jaune sur les lores du cygne trompette a été donnée comme une indication, mais cette tache est si souvent absente même chez les cygnes d'Amérique adultes qu'elle est sans utilité comme trait distinctif.

Indications sur le terrain. La taille et l'absolue blancheur. Les deux espèces ne peuvent pas facilement être distinguées l'une de l'autre à l'état vivant, sauf par ceux à qui la voix de chacune d'elles est familière.

Nid. Sur le sol, dans un nid d'herbes, de mousse et garni de duvet.

Habitat. Il niche dans l'extrême Nord après avoir traversé le continent à l'ouest de la baie d'Hudson; il est de passage dans l'intérieur; rare ou absent sur la cóte canadienne de l'Atlantique, mais commun par endroits sur les grandes routes fluviales de la région des grands lacs.

Par le fait de son excessive prudence et parce que la couvaison se fait au loin dans le Nord, le cygne d'Amérique n'a pas vu son nombre sérieusement réduit dans les dernières trente années. La plus grande espèce congénère, celle des cygnes trompettes dont les parages recherchées pour la couvaison et compris dans la région colonisée furent dérangés de bonne heure, est sur le point d'être détruite entièrement.

Les cygnes viennent rarement dans les marécages où l'eau est basse et où le chasseur peut se cacher. Ils sont rarement vus sauf en bandes blanches, serrées, comme des plaques de glace au loin sur l'eau libre ou en l'air volant très haut et hors de la portée des fusils. Leur passage régulier a lieu de nuit, ordinairement dans un grand silence, quelquefois cependant très bruyamment. C'est à de telles mœurs qu'il faut probablement attribuer le fait que peu de nos chasseurs même les plus experts connaissent le cygne à l'état vivant, et qu'un plus petit nombre encore peuvent se vanter de l'avoir pris. Bien que des bandes de plusieurs centaines apparaissent chaque année sur le lac Saint-Clair, on n'en prend pas plus de deux ou trois par année.

L'espèce se rencontre aussi en grand nombre sur la rivière Niagara où, par des nuits obscures ou de brouillard, au printemps, les cygnes sont entrainés avec le courant dans les eaux tumultueuses des rapides et sont emportés par-dessus les chutes sans possibilité d'en réchapper. Pareille catastrophe est arrivée plusieurs fois dans les dernières dix années et des centaines de cygnes ont perdu la vie de cette façon. Chez quelques-uns, tous leurs os sont brisés dans leur corps, d'autres ne sont que faiblement blessés. Les oiseaux morts qui ne sont pas entrainés sous la glace et emportés par le courant sont ramassés. Ceux à moitié morts sont achevés à coup de bâtons et ceux dont les blessures sont seulement légères sont tués d'un coup de fusil. Comme les oiseaux ne sont pas de force, dans les endroits resserrés, à s'élever au-dessus des fentes de la gorge, et répugnent à s'engager sous les ponts jetés par-dessus le bas de la rivière, tous se trouvent confinés dans les eaux étroites au-dessous des chutes, d'où ils ne peuvent s'échapper. La chair du cygne n'est guère bonne à manger et les oiseaux ont peu de valeur pour ceux qui les attrappent à moins que ce ne soit comme une curiosité.

181. Le Cygne trompette. (Trumpeter swan). Olor buccinator. L, 65. Très gros oiseau, tout blanc.

Traits distinctifs. Ne peut se confondre qu'avec le précédent auquel on vous renvoie.

Indications sur le terrain. Taille et blancheur absolue; les deux cygnes ne peuvent pas se distinguer l'un de l'autre avec une entière certitude à l'état vivant.

Nid. Sur le sol, dans un nid d'herbes et de duvet.

Habitat. C'est un oiseau de l'intérieur des terres, qui niche à partir du centre de la rangée nord des Etats jusque vers le nord. 
Tout cygne de plus de 56 pouces de long ou pesant plus de 20 livres appartient probablement à cette espèce. C'était autrefois un oiseau passablement commun sur les grands lacs, mais aujourd'hui il est si rare qu'on peut lè regarder comme une espèce presque ou complètement éteinte. Son territoire de nidification est très au sud de celui du cygne d'Àmérique et au milieu de terres cultivées et très peuplées, voila pourquoi cet oiseau est rapidement détruit.

\section{Ordre-Herodiones. Echassiers d'eau profonde, Hérons, Cigognes et Ibis.}

Description générale. Ce sont d'habitude de grands oiseaux à longues jambes, long cou et long bec, bien bâtis pour marcher dans l'eau et pour y trouver leur nourriture sous la surface dans une eau plus profonde que celle où la trouveraient la plupart des oiseaux aquatiques. Les becs peuvent étre ou droits et aiguisés (figure 18, p. 22) ou modérément recourbés et émoussés comme chez les ibis. Les jambes sont dénudées sur une grande distance au-dessus de l'articulation du talon, et les quatre doigts sont parfaits, bien formés et propres a percher aussi bien qu'à marcher sur un sol mou, avec ou sans membranes rudimentaires (figure $17, \mathrm{p} .22$ ).

Traits distinctifs. D'après cette description les oiseaux de cet ordre peuvent être confondus ou avec les grues ou avec l'un des courlis. Ils peuvent se distinguer des grues par leur front couvert de plumes. L'ibis des bois possède à la fois le front dénudé et le bec recourbé, mais il ne se trouve que rarement au Canada. Ils peuvent se distinguer de l'un quelconque des courlis par l'espace dénudé entre les yeux et la base du bec.

Les individus canadiens de cet ordre se divisent en deux sous-ordres: les Ibides, qui comprennent les spatules et les ibis proprement dits; et les Herodias qui comprennent les hérons, les aigrettes et les butors.

\section{SOUS-ORDRE-IBIDES. LES IBIS.}

Ce sous-ordre comprend deux familles: les spatules qui ne se rencontrent pas au Canada et les ibididés, dont un seul se voit dans le Dominion mais rarement.

\section{FAMILLE-IBIDIDA. LES IBIS.}

Description générale. Oiseau avec un bec long, recourbé, tout à fait émoussé a la pointe et la mandibule supérieure cannelée d'un bout à l'autre.

Traits distinctîfs. Bec recourbé, émoussé et fortement cannele, ce sont là ses caractères. La griffe du doigt du milieu peut être élargie et endurcie sur le bord, mais n'est pas parfaitement pectinée ou pourvue de dents bien formées et en forme de peigne comme chez les échassiers pareils au héron du sous-ordre des hérodias. (Comparez à la figure 19, p. 22).

Il n'y a qu'un seul de ces oiseaux, l'ibis luisant, qu'on puisse trouver au Canada, et cela seulcment comme un vagabond par aventure.

$$
\text { Genre-Plegadis. Ibis luisant. }
$$

186. L'Ibis luisant. (Glossy IBIS, BlaCk curlew). Plegadis autumnalis. L, 24. C'est, en fait, un oiseau tout noir avec des reflets brun marron, verts et violets. Le jeune est brun avec les plumes de la tête légèrement bordées de couleurs claires, avec reflets verdâtres ailleurs.

Traits distinctifs. L'ibis luisant ressemble beaucoup à un gros courlis, mais sa coloration presque noire, ou très sombre, le fera distinguer facilement du courlis. Son bec cannelé (voir plus haut la description de cette famille) le différencie d'autre part.

Nid. Dans des marais a roseaux ou des buissons très bas.

Habitat. Les régions tropicales et sous-tropicales.

L'ibis est assez connu de nom comme l'un des oiseaux sacrés de l'ancienne Egypte. L'ibis luisant est apparenté à l'ibis sacré des bords du Nil et fait voir quelques-uns de ses caractères généraux. Il ne se rencontre qu'occasionnellement au Canada, le long de la frontière méridionale. 
Valeur économique. Trop rare au Canada pour avoir aucune influence économique.

\section{SOUS-ORDRE-HERODII. ECHASSIERS HERODIENS.}

Comme ce sous-ordre est représenté au Canada par une seule famille, les ardéidés, il suffit de les décrire sous ce titre.

\section{FAMILLE-ARDEIDA. HERONS ET BUTORS.}

Description géntrale. Ce sont des oiseaux qui ressemblent au héron, avec le bec droit et très pointu. L'espace devant les yeux est dénudé. Un trait particulier à ce sousordre ce sont les plages de très petites plumes, c'est-d-dire des assemblages de plumes bizarrement formées et qui donnent une poudre sèche dont l'usage est inconnu. Ces plumes se trouvent sur diverses parties du corps et caches sous le plumage apparent. La griffe du doigt du milieu est pectince, c'est-a-dire pourvue d'une rangée de dents bien caractérisées et ressemblant à celles d'un peigne (figure $19, \mathrm{p} .22$ ) et qui ne sont pas seulement des points rugueux, comme chez les ibis.

Traits distinctifs. Les oiseaux de ce sous-ordre pourraient étre pris pour des grues, mais le front garni de plumes est distinctif. La partie de derrière du cou est dénudée, les plumes des cótés se continuant tout autour cachant la dénudation aux yeux de l'observateur distrait. Le doigt postérieur est très long et au niveau des autres doigts au lieu d'étre simplement un peu élevé comme chez les autres échassiers.

Indications sur le terrain. Profil visiblement pareil à celui du héron, avec un cou long et gracieux; bec long et effilé (figure 18, p. 22) et pattes plus longues (figure 17, p. 22). Le cou se replie quand I'oiseau vole, mettant la tête tout près des épaules, les pattes pendant derrière. Les grues, avec lesquelles on peut confondre les ardéidés à l'état vivant, portent le cou étendu.

Il n'y a peut-être pas d'oiseaux aussi bien connus du grand public que ccux-la par leur renommée et par l'observation, cependant nous ne les entendons jamais appeler de leur vrai nom. Les termes de héron, cigogne et grue sont employés à tort et à travers. Il n'y a pas de cigogne au Canada. Les grues ont leur habitat à l'ouest et sont rarement aperçues dans l'est du Canada. Les oiseaux que l'on appelle d'habitudes des grues sont de vrais hérons. La famille est divisée en deux familles: Botaurince, butors, et Ardeina, les hérons vrais et les aigrettes.

\section{Sous-famille-Botaurinæ. Butors.}

Description générale. Habitent les marais, ressemblent aux hérons, d'une charpente plus lourde, d'un corps moins gracieux que les véritables hérons.

Traits distinctifs. Bien que formant une sous-famille bien définie ces oiseaux sont difficiles à caractériser dans une description courte et non technique. Dans les espèces canadiennes la couleur est le meilleur guide et le plus reconnaissable. Si l'on en excepte le petit butor de Cory, qui est très rare (voir à l'article Petit Butor, $n^{\circ} 191$ p. 88) les especes canadiennes ont de fortes quantités de jaune d'ocre st' $r$ leur plumage, couleur qui, en quelque grosse quantité, est absente du corps des véritable bérons.

Les butors fréquentent les marais et les maréca es. On ne les voit pas dans les lieux ouverts qui mènent à ces marais; mais ils se laissent choir dans le milieu ou sur les bords parmi les herbages ou les roseaux, s'approchant de leur proie sans bruit et à l'abri de cette végétation.

\section{Genre-Botaurus. Butors.}

190. Le Butor d'Amérique. (AMErican BITTERN). Botaurus lentiginosus. $L$, 28. Planche VI A.

Traits distinctifs. Avec sa coloration jaune répandue sur tout son corps, la même dans les deux sexes et à tout âge, le butor ne peut se confondre avec aucune autre espèce canadienne. Le petit butor est le seul autre oiseau de même conformation qui montre 
surtout du jaune, mais sa taille est si petite qu'il n'y a aucun risque de les confondre. Il peut y avoir des lignes noires depuis les côtés de la face, ou ne pas y en avoir, quels que soient le sexe, l'âge et la saison.

Indications sur le terrain. Quand l'oiseau s'élève au-dessus des roseaux ou des herbages, son long cou, ses pattes pendantes et sa coloration jaunatre sont faciles à reconnaítre. $\mathrm{Vu}$ d'une certaine distance, sa forme, sa tête rentrée dans le corps, et ses jambes tendues en arrière, ressemblent tellement à celles du héron que, si la lumière ne vient faire distinguer la coloration, la taille seule les fera distinguer l'un de l'autre.

Nid. Sur le sol dans l'herbe, dans les champs de foin ou dans les marais à roseaux, un nid fait avec des herbes ou des roseaux.

Habitat. Cet oiseau est commun dans toutes les parties habitées du Canada, nichant partout où on le trouve.

Des allusions au mugissement solitaire du butor sont fréquentes dans la littérature anglaise. On ne peut guère prétendre que notre butor américain "mugisse", mais son cri est très singulier et unique en son genre parmi les oiseaux américains. Les termes ordinaires de "pompe à tonnerre", "enfonceur de bâton », se rapportent aux bruits étranges qu'émet cet oiseau. Près d'un marécage on peut entendre un bruit pareil à celui que ferait quelqu'un qui enfoncerait à coups de maillet en bois un pieu dans la boue liquide. C'est comme le bruit sourd d'un coup dont le liquide produit par succion l'écho, suivi immédiatement d'un autre coup. D'autres fois on entend des sons comme si quelqu'un manœuvrait une pompe aspirante qui tirerait de l'eau à une certaine hauteur, mais sans pouvoir la faire monter plus haut. Ce sont là des variantes du chant d'un butor, en amour, mais où il est impossible de distinguer des propriétés vocales.

Valeur économique. Le butor fréquente les marais, mange des grenouilles, des écrevisses, des serpents, du fretin, des crustacés, des insectes, et même, probablement, des petits oiseaux et des souris. Il ne mange que peu, même point du tout, de matières végétales. Les butors sont inoffensifs en tant que classe, et peuvent être utiles.

\section{Genre-Ixobrychus. Petit Butor.}

191. Le Petit Butor. (LeAst BitTern) Ixobrychus exilis. L, 13. C'est le plus petit échassier, ressemblant au héron, qu'on trouve au Canada. Sa coloration offre des amas de tons crème, d'ocre, de rouges indiens, avec un dos et une calotte noir ou brun, selon le sexe.

Traits distinctifs. A cause de sa petite taille et de sa coloration frappante, on ne peut le confondre avec aucun autre oiseau du Canada sauf peut-être avec son parent très rapproché et très rare le Petit Butor de Cory, I. neoxenus. Chez ce dernier oiseau les tons crème et ocre sont remplacés par un brun phoque ou rougêtre, et on ne sait encore de façon certaine si c'est ou non une phase de la coloration de l'individu ordinaire.

Indications sur le terrain. Sa petite taille, et ses couleurs frappantes font qu'il est impossible de se méprendre au sujet de cette espèce. Elle est rarement vue, sauf de très près quand la couleur et la taille sont évidentes.

Nid. Généralement au-dessus de l'eau dans un nid placé sur une plate-forme de joncs morts, dans un marais ou un bouquet de roseaux.

Habitat. C'est un oiseau commun mais plutôt confiné dans certaines localités des parties méridionales de l'Ontario et du Québec. Il couve partout où il se rencontre au Canada.

Le petit butor fréquente les marais où l'eau séjourne, où croît la massette et qui, en outre, sont de quelque étendue; il n'est guère vu là que par ceux qui visitent son tranquille territoire. Le butor américain est souvent aperçu volant de marais en marais, mais le petit butor ne s'aventure guère plus loin que son marécage habituel. C'est un oiseau silencieux et qui n'a pas ou très peu de talents vocaux. 


\section{Sous-famille-Ardeinæ. Hérons vrais et Aigrettes.}

Description générale. Ce sont des oiseaux plus sveltes et gracieux que les butors et, en général, de plus grande taille. Les plus petits sont très peu plus petits que les butors d'Amérique et aucun n'est aussi petit que le petit butor. La couleur constitue la plus simple manière de les distinguer.

Traits distinctifs. La couleur des bérons est habituellement le bleu d'ardoise ou les verts foncés, mais quelquefois les hérons sont tout blancs. Les butors, d'autre part, ont une couleur jaune prononcée. Bien des hérons ont de belles longues plumes sur la couronne, le bas de la gorge, ou sur le dos, et toutes les espèces canadiennes, dans les plumages de la couvaison, font voir ces plumes plus ou moins longues sur l'un ou l'autre de ces points, mais elles sont babituellement absentes en automne.

Indications sur le terrain. C'est le profil caractéristique pendant le vol, avec le bec très pointu, la tête rentrée dans les épaules, les jambes pendantes derrière, attitudes communes aux butors et aux bérons, lesquels sont reconnus plutôt comme espèce que comme sous-famille. Toutefois, tout oiseau de ce genre qui n'est pas manifestement un butor est probablement un héron.

Nid. Les bérons bâtissent habituellement leurs nids en société au sommet des arbres de forêts humides, mais quelquefois dans les buissons ou sur le sol.

Les hérons sont pêcheurs, dans des eaux de marais bien ouverts, recherchant les parages herbeux moins que ne font les butors. Au lieu de poursuivre leur proie, ils demeurent immobiles et attendent qu'elle soit à portée.

\section{Genre-Ardea. Grand Héron bleu.}

194. Le Grand Héron bleu. LA GRUE. (GReat bLUE heroN). Ardea herodias. $L, 42$. Planche VI B.

Traits distinctifs. C'est le plus grand héron trouvé au Canada; la grue de l'ouest du Canada est le seul oiseau avec lequel on puisse le confondre. Le front très garni de plumes est un trait bien distinctif. (Comparez les figures 18 et 20, p. 22.)

Indications sur le terrain. Le profil comme celui du héron, la taille et la coloration générale sont les meilleures indications. Bien différent de la grue qui vole le cou tendu, le grand héron bleu, comme les autres hérons, voyage le cou replié et la tête rentrée dans les épaules.

Nid. Il le bâtit généralement en société d'autres bérons dans les forêts humides, comme dans les marécages de tamarack, de frêne et d'orme; c'est un nid de grandes proportions fait de buchettes au sommet des arbres.

Habitat. Le Canada presque en entier; le héron niche partout où on le trouve.

SOUS-ESPECES. Le grand héron bleu comprend plusieurs sous-espèces dont l'individu type, le grand héron bleu de l'Est, Ardea heroidias herodias, est le seul qui se rencontre dans l'est du Canada.

Le grand héron bleu recherche les eaux ouvertes et basses. Il fréquente rarement les marais roseliers très épais, bien qu'on le trouve souvent sur leurs confins ou sur le bord des étangs qui s'y trouvent. Il préfère les abords découverts et peu profonds des rivières, ou ceux un peu plats des marais ou des rivages que recouvre la marée. C'est un chasseur solitaire, qui tantôt avance avec prudence et des mouvements à peu près imperceptibles, tantôt aussi immobile qu'une statue, regarde l'eau jusqu'à ce que sa proie soit à sa portée, alors, avec la rapidité de l'éclair, il la saisit de son bec acéré. Les hérons, qui nichent en nombreuses compagnies sur des arbres humides se sont trouvés spécialement exposés à cette persécution insensée qui paraît s'attaquer à tous nos plus grands oiseaux. Circonspects et soupçonneux d'ordinaire ils perdent dans le voisinage de leurs nids beaucoup de leur prudence habituelle, et dans leurs héronnières haut perchées les hérons peuvent être tués en grand nombre. Les héronnières sont habituellement bien connues de toute la contrée environnante et, dans la saison de la couvaison, souvent visitées par le chasseur rural qui tue les parents et laisse les petits mourir de faim, lors même qu'un propriétaire de campagne a souvent assez à 
cœur l'intérêt général pour protéger les héronnières sur ces terres à lui. Il résulte de là que cet oiseau si pittoresque se fait rare. Les héronnières une fois détruites de cette façon sont rarement, peut-être même jamais repeuplécs et de nouvelles s'établissent difficilement. Les oiseaux qui nichent en société sont rarement poussés vers de nouveaux parages pour leurs amours. Ils demeurent où ils sont jusqu'à ce que les individus qui composent leur bande soient exterminés. Le héron est un oiseau inoffensif qui mériterait d'être protégé de toute façon.

Valeur économique. La nourriture du grand héron bleu est presque entièrement animale, de sa nature, puisqu'elle se compose surtout de grenouilles, de serpents, de fretin, le tout de nulle importance économique. Les grues visitent les champs pour leur nourriture, les hérons jamais. D'aventure le héron peut suivre un cours d'eau que parcourent les truites et dont les méandres sillonnent les prairies, mais ces cas sont rares et ne suffisent pas a condamner l'espèce. Il arrive souvent que les hérons recherchent les rets posés dans les étangs par les pêcheurs, mais la petitesse de leur gosier, les cmpêche de prendre quoi que ce soit de valeur et la suspicion des propriétaires de ces rets est bien gratuite.

Bien que ne formant pas une classe systématiquement reconnue de hérons, il $\mathrm{y}$ en a plusieurs espèces pourvues d'un plumage absolument blanc et qui sont assez distinctes pour justifier qu'on en discute.

Parmi quelques-unes de ces espèces, les aigrettes, le plumage de l'adulte est blanc; en d'autres c'est une apparence dichromatique, c'est-à-dire que l'espèce se présente sous deux phases de couleurs, l'une et l'autre normale, et ne pouvant se rapporter ni a l'albinisme ni au mélanisme, pas plus qu'au sexe, à l'âge ou à la saison. En d'autres espèces encore le blanc appartient à un plumage de jeunesse. Ces plumages blancs étaient une source de grande confusion pour l'identification des espèces aussi longtemps qu'on ne les avait pas soumis à une investigation complète. Tous les hérons blancs appartiennent à un habitat méridional et sont rares au Canada.

\section{Genre-Herodias. Aigrettes.}

196. L'Aigrette blanche d'Amérique. (AMERICAN EGREX). Herodias egretta. $L, 41$. Presque aussi grande que le grand héron bleu, mais toujours d'un blanc très pur. Dans la saison des amours, une manière de cascade de quelque cinquante plumes fines et droites commence au milieu du dos et tombe en festons sur le bas du dos et la queue.

Traits distinctifs. Taille, couleur et profil, manifestement de héron.

Indications sur le terrain. Comme ci-dessus.

Nid. En société, nids faits de branches sur les arbres ou dans des buissons au-dessus de l'eau.

Habitat. Dans les Etats du Sud et du golfe du Mexique; ne se montre au Canada que comme vagabond de hasard.

L'aigrette américaine, avec le héron blanc comme neige et une autre espèce de même nature, constituent l'origine de l'aigrette bien connue, c'està-dire de cette huppe de plumes que vendent les modistes. Comme ces plumes ne poussent que dans la saison des amours, et comme le voisinage immédiat des héronnières est le seul endroit où ces prudents oiseaux peuvent être facilement approchés, il est évident que la récolte de ce magnifique ornement doit s'accompagner de beaucoup de cruauté. On se défend en disant que ces plumes sont ramassées quand elles sont tombées du dos des mâles. C'est un argument contestable, car si quelqu'un veut chercher dans les basses-cours pour y trouver les belles plumes que la volaille a jetées il 
décrouvira vite que les plumes d'aigrette seront rares et de pauvre qualité. L'argument est d'autant plus douteux que les héronnières sont situées dans les marais compacts des régions tropicales où le sol n'est que boue ct eau où la végétation est de nature à empêcher toute recherche minutieuse et méthodique. Le chasseur de plumes se cache d'habitude dans la héronnière et avec un fusil de petit calibre, tire les oiseaux l'un après l'autre jusqu'à ce que la troupe soit toute exterminée. Les plumes sont alors arrachées des corps qu'on laisse pourrir par terre. Les petits qui restent, meurent d'inanition en haut dans les nids. Des lois locales ont été édictées interdisant de tuer ces oiseaux, mais sans résultat. Il y a quelques années les étangs de la Floride et des Etats du golfe avaient encore tout l'embellissement que leur donnait la présence de ces oiseaux immaculés; aujourd'hui ces lieux ont à pcu près perdu un de leurs plus grands attraits par l'imminente destruction de ces oiseaux. Comme on trouva impossible de distinguer les plumes étrangères des indigènes ou d'empêcher le trafic des unes tandis qu'on permettait celui des autres, une loi fédérale a enfin été promulguée aux Etats-Unis qui défend d'importer les plumes d'oiseaux à l'usage des modistes. Des lois semblables ont été depuis votées en Grande-Bretagne et dans ses colonies. Les aigrettes ne sont pas la seule espèce qui ait tant souffert; les sternes et d'autres oiseaux de mer, les oiseaux de Paradis et beaucoup d'espèces insectivores ont été victimes de la vanité et de la cupidité humaines.

\section{Genre-Florida. Petit Héron bleu.}

200. Le Petit Héron bleu. (LitThe blue heron). Florida carulea. L, 22. C'est un petit héron très beau et gracieux. L'individu adulte a la tête et le cou brun marron, le reste du corps d'un bleu d'ardoise foncé. Des plumes finement pointues sur les épaules et sur le devant du bas cou. Le jeune est presque du blanc le plus pur teinté de bleu d'ardoise.

Traits distinctifs. La couleur qui distingue l'oiseau adulte est décrite plus haut. Le jeune oiseau, tout blanc, ressemble beaucoup au petit héron de la Louisiane, mais peut s'en distinguer par les pattes jaune verdâtre et par les pointes bleu d'ardoise des primaires.

Nid. Niche par groupes, nids faits de buchettes dans les buissons ou sur les arbres all-dessus de l'eau.

Habitat. L'Amérique tropicale. Couve dans les Etats du Sud et du Golfe. Sa présence au Canada est purement accidentelle. On voit rarement au Canada des oiseaux ayant le plumage de l'âge adulte. Les jeunes oiseaux sont les seuls qui soient susceptibles de s'égarer dans l'intérieur de nos frontières.

Voleur économique. Trop rares au Canada pour avoir aucune importance économique.

\section{Genre-Butorides. Hérons verts.}

201. Le Héron vert. (GREEN HERON). Butorides virescens. L, 17. C'est le plus petit de nos hérons ordinaires. Le dos est d'un vert grisâtre brillant avec de courtes pennes, pareilles aux plumes et recouvrant les ailes. La face, les cótés du cou, et la gorge, aussi bien que les dessous, sont d'un riche marron. La tête a une calotte noire qui s'allonge en une petite huppe.

Traits distinctifs. La description qui précède peut ressembler à celles de la dernière espèce, mais le vert brillant si visible du dos, l'absence des plumes du cou, la taille plus petite et la charpente plus lourde empêchent de les confondre sérieusement. C'est ici, d'ailleurs, une espèce commune dans son territoire propre, et celle qu'on est assez sûr de rencontrer dans la région des grands lacs. Toute autre espèce qui pourrait lui être comparable est très rare.

Indications sur le terrain. Taille et coloration générale.

Nid. Solitaire et non par groupes; léger et ouvert fait de branches dans des buissons ou sur des arbres d'habitude au-dessus de l'eau. 
Habitat. Pas très commun dans le sud de l'Ontario, mais rare à l'est. Couve partout où on le rencontre au Canada.

SOUS-ESPECE. Le héron vert forme des sous-espèces, mais le type, c'est-à-dire le héron vert du nord, est la seule espèce qu'on rencontre au Canada.

Le héron vert n'est pas aussii porté à s'aventurer en pleine eau que l'est le grand héron bleu, ni dans les marécages herbeux, comme le butor. Les halliers d'aune en terrains inondés, les bords buissonneux de paisibles ruisselets, les mares où s'attardent des courants endormis, les prairies où s'éjournent les castors, sont l'habitat préféré de ces hérons. Ils aiment la solitude plus que ne font les autres hérons au temps de la couvaison et, bien que plusieurs" couples puissent occuper une localité particulièrement favorisée, c'est la communauté d'intérêts et non l'instinct de la sociabilité qui les fait se réunir.

Valeur économique. La nourriture du héron vert se compose d'écrevisses, d'insectes, de grenouilles et de fretin. On l'a accusé d'être nuisible à certains poissons, mais comme l'oiseau est petit et relativement rare, et comme les parages d'eaux dormantes qu'il fréquente d'habitude ne le mettent pas en contact avec beaucoup d'espèces importantes, on ne peut pas le considérer comme une menace bien sérieuse.

\section{Genres-Nycticorax et Nyctanassa. Hérons de nuit.}

202. Le Héron de nuit. LE QUAC. (BLACK-CROWNED NIOHTHERON). Nycticorax nycticorax. L, 24. Plus petit que les grands bleus et plus grand que les herons verts, plus semblable au butor comme taille. Le plumage de l'oiseau adulte est reconnaissable à première vue. Le corps est blanc, à ombres légères avec des teintes de gris clair. Le dos et la couronne sont noirs; de celle-ci tombent une ou deux plumes longues, fines, en forme de pinceau. Le jeune oiseau a une apparence tout à fait différente, il a des raies brun grisâtre sur un fond blanchâtre.

Traits distinctifs. L'individu adulte est caractéristique. Le jeune peut, à un coup d'œil hâtif, paraftre ressembler au butor, mais il manque absolument de toute nuance bien jaune et le dessin très simple de la coloration est très différent, du schéma de couleur compliqué et finement vermicule de cet oiseau.

Nid. Souvent en société avec le grand héron bleu, nid fait ordinairement avec des buchettes sur des arbres, quelquefois sur le sol.

Habitat. Le héron de nuit est un oiseau dont l'habitat est irrégulier et local; on le rencontre dans l'est de l'Ontario, l'ouest de Québec et du Manitoba, en colonies occasionnelles. Dans l'Ontario, depuis Kingston vers l'ouest, il est très rare.

SOUS-ESPECES. Le héron de nuit se rencontre dans les deux hémisphères (est et ouest). L'oiseau du nouveau monde, appelé le héron de nuit d'Amérique, $N$. $n$. noevius, est une sous-espece distincte de celle de l'ancien monde.

C'est un héron d'une charpente plutôt lourde et qui, quoique non sans beauté ni sans grâce, n'a pas les lignes fines et élancées de la plupart des hérons et ressemble aux butors par sa charpente. Ses habitudes sont une combinaison de celles du grand héron et de celles du héron vert.

Le héron de nuit à couronne jaune, Nyctanassa violacea, se reucontre aussi au Canada, mais trop rarement pour qu'il en soit dit davantage. L'individu adulte est en général un oiseau couleur gris d'ardoise, finement rayé de noir sur le dos, et dont la tête est très distinctement noire et blanche. Le jeune héron est si semblable au héron noir qu'on l'en distingue difficilement, mais sa tête est plus foncée que le dos, et il n'y a pas trace de brun rougeâtre sur les primaires, ce qu'une minutieuse inspection fait voir sur le jeune héron de nuit qui est noir. - Les jeunes hérons de nuit jaunes ne devraient être signalés au Canada qu'avec précaution.

Valeur économique. Sa nourriture est semblable à celle du héron vert et son importance économique à peu près indentique. 


\section{Ordre-Paludicolæ. Oiseaux de marais, Grues, Râles, etc.}

Description générale. C'est un ordre assez mal délimité; il comprend un certain nombre de familles d'oiseaux qui marchent habituellement dans l'eau et qui ne peuvent être assignés ni à la famille des hérons ni à celle des oiseaux de rivage, quoique ressemblant superficiellement à l'une et à l'autre. Ce sont des oiseaux qui ont quatre longs doigts bien développés, sans membranes, et avec des pattes bien faites pour marcher dans l'eau. On ne les définit que par la description des sous-familles. L'espèce canadienne se divise en deux sous-ordres; les grues qui comprennent en outre les courlans, etc., et les ralli (rales) qui comprennent en outre les gallinules et les foulques. Du sous-ordre des grues il n'y a au Canada que les gruidés, les grues proprement dites.

\section{SOUS-ORDRE-GRUES. GRUES ET COURLANS.}

Comme le courlan, de la famille des aramidés, ne se rencontre pas au Canada, nous ne pouvons nous intéresser qu'à la seule famille des gruidés, c'est-à-dire aux grues. En ce qui concerne le Canada, ce sous-ordre peut s'appeler les "Grands Oiseaux de marais", expression, toutefois, qui ne se justifie que parce qu'elle est d'un usage commode.

\section{FAMILLE-GRUIDE. GRUES.}

Description générale. Grands oiseaux ressemblant au héron; couleur bleu d'ardoise, terne, avec teinte de rouille, ou blanc, ou pur, avec primaires noires. Toutes les couleurs sont des teintes uniformes, sur tout le corps, et les oiseaux n'ont ni panache ni huppe.

Traits distinctifs. Les grues se distinguent des hérons en ce qu'elles ont le front et l'espace autour de l'oil dénudé ou avec une très petite quantité de plumes bizarrement pareilles à des cheveux, et en ce qu'il leur manque la structure pectinée sur la griffe du milieu (voir figure 19, p. 22); le bec est plus petit, toute proportion gardée, que celui du héron, mais de forme plus lourde, soit comme matière soit comme forme (comparez figures 18 et 20, p. 22 ).

Indications sur le terrain. Les grues volent le cou tendu su lieu d'avoir la tête rentrée dans les épaules comme les hérons et, contrairement aux habitudes des hérons, elles paissent en troupes dans les champs des régions cultivées.

\section{Genre-Grus. Grues.}

206. La Grue du Mexique. (8andhill crave). Grus mexicana. L, 40 . Très semblable au grand héron bleu, mais sans plumes en aucune saison. Une couleur bleu uniforme par tout le corps lavee de rouge rouille ou de brun, surtout forte dans les phases de la jeunesse.

Traits distinctifs. Elle se distingue du grand héron bleu par son front dénudé, etc. comme cela est décrit sous le titre précédent (comparez figures 18 et 20, p. 22). Autrement elle ne peut etre confondue qu'avec la petite grue brune Grus canadensis, grue du Canada, dont elle se distingue uniquement par la taille-la longueur de cette dernière étant d'environ 36 pouces et la grue du Mexique de 40 pouces. Les spécimens intermédiaires, comme talle, n'étant pas rares, la différenciation n'est pas toujours facile a constater, et les deux variétés peuvent se rencontrer dans l'est du Canada. Une autre grue, la grue huante, Grus americana, qu'on trouve dans l'Ouest, s'est rencontrée quelquefois dans l'Est, mais sa couleur d'un blanc très pur, ou lavée de couleur rouille, ses primaires noires, et sa grande taille- 50 pouces-la font facilement reconnaitre.

Indications sur le terrain. Le front dénudé de coloration rouge terne; elle vole le cou tendu au lieu de replié, et a plutôt les habitudes des localités élevées.

Nid. Dans des endroits humides, marécageux, fait de matières végétales de rebut.

Habitat. L'ouest du Canada, nichant a l'intérieur des régions cultivées et vers le Nord.

La grue du Mexique parait avoir été un hôte plus commun de l'est du Canada, de la région des grands lacs tout au moins, dans les premiers temps plus qu'aujourd'bui. Elle n'est actuellement qu'un rare vagabond à l'est des provinces des prairies, bien que quelques individus continuent à nicher dans le sud du Michigan; il n'est pas impossible qu'il y en ait encore quelques-unes dans les parties adjacentes de l'Ontario. 
Valeur économique. Cette grue est trop rare dans l'est du Canada poul' y avoir aucune importance économique. Quoiqu'elle se nourrisse de graminées plus que ne font les hérons, et qu'elle visite, souvent en nombre, les terrains cultivés dans le temps des migrations, excepté lorsqu'elle se concentre en grand nombre dans une région limitée, elle fait peu de dégâts. La quantité d'insectes dont elle s'alimente est considérable.

\section{SOUS-ORDRE RALLI. PETITS OISEAUX DE MARAIS.}

En ce qui concerne le Canada, ce sous-ordre-comprenant les râles, les gallinules et les foulques-peut-être appelé celui des petits oiseaux de marais, par comparaison avec celui des grandes grues. Ces oiseaux n'ont pas la forme du héron ct ne peuvent pas être confondus avec les grues par rapport soit à la forme soit au port et à la taille. De ce sous-ordre il n'y a qu'une seule famille, celle des rallidés, qui se rencontre dans l'est du Canada.

\section{FAMILLE-RALLIDȦ. OISEAUX DU TYPE RÂLE.}

Description générale. Doigts longs et effilés dans le but de couvrir un grand espace de terre molle où le pied se fixe avec peine. Les caractères du pied sont un peu comme ceux des oiseaux de rivage, mais le doigt de derrière est aussi long et bien développé que les autres, et son insertion est au même niveau qu'eux au lieu d'être légèrement plus élevée. A cet egard, ils ressemblent aux hérons, mais peuvent s'en distinguer par leur forme qui n'a rien du héron et par leurs lores emplumés.

Ce sont des oiseaux de marais, et bien typiques, qui se cachent dans les hautes herbes et les roseaux, qui courent rapidement sur des masses peu résistantes de végétation à moitié flottante, qui préfèrent se cacher plutôt que de voler à l'approche du danger. Ils nagent tous, quelques-uns toujours, les autres par occasion. La famille est divisée en trois sous-familles: Rallinc, les râles vrais; Gallinulina, gallinules ou poules d'eau; et les Fulicino, ou foulques.

\section{Sous-famille-Rallinæ. Rales vrais.}

Description générale. Oiseaux à corps très plat, comprimés latéralement, bien faits pour se glisser parmi des roseaux et des herbes qui croissent très serrés; ailes petites, arrondies et relativement faibles; la charpente totale de l'oiseau est lâche, lui donnant la flexibilité par sa tenue et son habitat, mais ne se prêtant pas à un effort considérable et continu.

Traits distinctifs. Très facilement reconnaissable aux caractéristiques négatives: oiseaux semblables au râle, comme décrit plus haut, qui ne sont ni gallinules ni foulques; sans avoir la plaque frontale sur le front comme ces oiseaux (figures.23 et 25 p. 23).

Indications sur le terrain. Les râles se dressent dans l'herbe droit sous vos pieds et partent d'un vol lent et incertain, les jambes pendantes, et le cou tendu. Ils s'enlèvent visiblement avec peine et précipitation, volent sans force à une petite distance au-dessus du marais et puis y retombent subitement.

Les râles sont très habiles à se cacher dans l'herbe. Ils filent le long des étroites pistes entre les bouquets de hautes herbes avec l'adresse et la vitesse des souris. Ils se fient à leur adresse à se cacher bien plus qu'à leurs ailes pour échapper au danger, souvent ils se laisseront prendre dans la main plutôt que de s'envoler. Un râle s'enfuira une fois comme s'il était saisi de panique, puis se laissera choir tranquillement; on ne le forcera que rarement alors à reprendre son vol, et caché dans un petit bouquet il ne sera que peu souvent découvert, sauf par le flair subtil d'un chien. Les râles savent nager, mais ne s'y décident que rarement et seulement à une petite distance comme lorsqu'ils passent d'un bouquet d'herbages à un autre et qu'ils trouvent l'eau trop profonde pour s'y aventurer à pied. 
Les râles font beaucoup de bruit, surtout de nuit et même de jour, un bruit soudain et inattendu fera naitre dans le marais un concert de cris rauques et saccadés, lors même qu'aucun oiseau ne se fait voir.

Nos râles canadiens peuvent se partager en deux divisions: un type à long bec et un à bec court (figures 23 et 25, p. 23). La première division comprend le râle élégant et le râle de la Virginie, deux espèces qui ont une coloration semblable; la seconde comprend le râle de la Caroline et le râle jaune, qui n'ont qu'une ressemblance générale quant à la couleur, mais tous deux ont un bec trapu très semblable.

\section{Genre-Rallus. Râles.}

208. Le Rale élégant. (king ratu). Rallus elegans. L, 15. Bec long; les joues, le cou et la poitrine d'un brun cannelle; le dos noir brunátre, chaque plume ayant une large bordure d'une teinte d'ocre de la couleur de la poitrine; les flancs rayés de blanc et de noir. Les jeunes ont même couleur, mais voilée de noir.

Traits distinctifs. Au Canada, ce râle ne peut être confondu qu'avec celui de Virginie, de méme coloration, mais le rále élégant est beaucoup plus grand.

Indications sur le terrain. Le vol sans force, qui est bien d'un râle comme il s'échappe de l'herbe; la taille, la coloration générale et le long bec brun rouge.

Nid. Dans les marécages humides, dans un nid fait d'herbe.

Habitat. Plutôt méridional, vient régulièrement en dedans de nos frontières le long des régions inférieures des grands lacs, couve partout ou on le trouve au Canada.

Le râle élégant peut être regardé comme le type des râles à long bec (figure 23, p. 23). Il a un cou plus long, une tenue et une conformation plus gracieuses que le type du bec court. Cette série est l'une d'entre plusieurs séries américaines d'oiseaux où des espèces distinctes ne diffèrent l'une de l'autre que par la taille. Avec le râle élégant et le râle de Virginie cette série renferme le râle claquet, oiseau exotique des marais salants des côtes plus mériodionales et qu'on ne rencontre jamais au Canada.

212. Le Rale de la Virginie. (VIrginta RaIL). Rallus virginianus. L, 9.50. Plus petit que le râle élégant, mais autrement, tout semblable à lui.

Traits distinctifs. Il est facile à distinguer du râle élégant par sa plus petite taille, et du râle de la Caroline par son long bec rougeâtre et $\mathrm{sa}$ coloration générale. Les jeunes oiseaux sont fortement lavés de noir et ont souvent été pris à tort pour des ráles noirs. Le râle noir est même plus petit que le râle jaune $(5 \cdot 0)$ et a un bec court; la présence d'aucun râle noir n'a été signalée au Canada sur un témoignage qui ne laissát aucun doute. On peut cependant le chercher dans la région des grands lacs, surtout dans le voisinage des terres basses de Saint-Clair, où il y a d'assez fortes preuves de sa présence, quoique aucun spécimen n'en ait encore été pris.

Indications sur le terrain. La taille, la coloration, le long bec rougeâtre (figure 23, p. 23) et ce vol incertain bien typique du râle qui veut se sortir du marais.

Nid. Dans les marécages humides, dans un nid d'herbe.

Habitat. Le sud du Canada à travers le continent, et au nord des limites actuelles des terres cultivées. Il couve partout où on le rencontre au Canada.

C'est un râle beaucoup plus commun que le râle élégant et qu'on peut s'attendre à trouver dans tout marais ou dans toute prairie mouillée de son territoire. Ses habitudes ne diffèrent pas essentiellement de celles des a!ntres râles.

Valeur économique. Son habitat situé dans les terres où rien ne se récolte exclut l'idée que cet oiseau s'alimente de choses de quelque valeur. Sa nourriture consiste largement en insectes, graines de marais et matières végétables. 
Genre-Porzana. Rale des genêts. VII A.

214. Le Rale de la Carollne. (sora raiL). Porzana carolina. L, 8.50. Planche

Traits distinctifs. Se distingue du râle de Virginie par son bec court et conique (figure 25 , p. 23), sa coloration générale; et du râle jaune par une plus grande taille et par sa coloration.

Indications sur le terrain. A son vol incertain et balancé, quand l'oiseau s'élève au-dessus des herbes, on reconnaît le râle; le bec court, la coloration générale et l'absence de blanc sur les ailes sont caractéristiques de l'espèce.

Nid. Sur le sol dans le marécage mouillé, nid fait d'herbages, etc.

Habitat. Situé beaucoup plus au nord que celui du râle de Virginie, mais s'étendant à travers le continent; couve partout où il se rencontre au Canada.

Dans les premiers temps de la colonisation, le râle de la Caroline constituait un gibier commun; mais l'asséchement des marais, et la facilité qu'avaient les chasseurs à en remplir parfois leur carnassière en ont bien diminué le nombre. Dans les marécages à marée, dans quelques-uns des Etats dits Atlantiques, on chasse régulièrement ce râle à la fin de l'automne. C'est le mieux connu de nos râles, et bien que le nombre en soit réduit comparé à celui que donnent les récits de nos anciens voyageurs, c'est encore un oiseau assez commun. Il ne lui faut pas, comme aux râles élégants et à ceux de Virginie, un habitat très étendu, il suffit parfois du plus petit bourbier au couple et à ses petits pour y passer la saison.

Valeur économique. Pas très différente de celle du râle de Virginie.

\section{Genre-Coturnicops. Râle jaune.}

215. Le Rale jaune. (YELLow RAIL). Coturnicops noveboracensis. L, 7. C'est un râle plus petit, au bec court, un peu comme le râle de la Caroline. La coloration est aussi la même, comme effet général, mais les dessous sont lavés de lignes couleur d'ocre, les plumes de derrière ont quelques lignes blanches très fines transversales au lieu de marginales. L'oiseau a aussi des plaques blanches aux ailes, ce qui est caractéristique de l'espèce.

Traits distinctifs. Se distingue du râle de la Caroline par la taille et la coloration.

Indications sur le terrain. La taille et les plaques blanches des ailes.

$\mathrm{Nid}$. Sur le sol, à la lisière humide des marécages; nid fait avec des herbes.

Habitat. Plus septentrional que celui des autres râles et s'étendant bien au delà des terres actuellement cultivées; niche partout où il se rencontre.

C'est de tous les râles le plus adroit à se dérober aux regards et à se tenir caché. Comme il est presque impossible de faire lever un râle, il se peut qu'il soit beaucoup plus commun qu'on n'est fondé à le croire. Selon les rapports actuels c'est un des oiseaux les plus rares au Canada. Ses habitudes ne semblent pas différer beaucoup de celles des autres râles, sauf qu'il ne lui faut pas autant d'eau dans son habitat et qu'il se rencontre plus souvent sur les bords herbeux du marécage que dans les endroits mouillés et pleins de roseaux.

\section{Sous-famille-Gallnulinæ. Gallinules ou Poules d'eau.}

Description générale. Ce sont des oiseaux assez grands et ressemblant au canard, mais avec de longs doigts sans membrane ni partielle ni entière; bec conique qui s'étend sur le front et une plaque ou écu frontal (figure 26, p. 23).

Traits distinctifs. Se distingue des canards par l'absence des membranes et par la forme du bec; et de la foulque par l'absence des lobes digitaux; mais, par ailleurs, rossemblant énormément à celle-ci. 
Indications sur le terrain. Corps rond, de ntême forme que celui du canard; sa tenue quand il nage; coloration générale bleue ou gris bleu; bec et plaque frontale rouges; pattes et doigts longs, ou jaunes ou verts.

Si l'on surveille tranquillement les voies libres qui traversent un marais, il arrive parfois qu'on aperçoive un oiseau nageur, au profil de canard, se tenant droit dans l'eau, la queue en l'air, et avançant avec une succession de mouvements gracieux saccadés, la tête tantôt en avant tantôt en arrière. Cela suffit presque à faire reconnaitre les gallinules; mais le bec et la plaque frontale aux eouleurs brillantes, visibles à une grande distanee, et éclairés par un beau soleil, ne permettront plus aucun doute à cet égard. Quelquefois e'est l'observateur qui les fait partir lorsqu'il avironne tranquillement le long des détours d'un étroit chenal; tout à coup elles partent éelaboussant tout à la surface et eréant beaucoup d'agitation avant de prendre leur vol. Les gallinules nagent ordinairement et avec facilité, mais elles ne s'aventurent que rarement dans des eaux ouvertes comme les foulques, elles s'en tiennent à de petites mares dans les marécages, ou à des voies ou passages bien dégagés qui les dirigent.

\section{Genre-Ionornis. Gallinule de la Martinique.}

218. La Gallinule de la Martinique. (PURPLe Gallivule). Ionornis martinicus. $\mathrm{L}$, i3. Ressemble a la gallinule de la Floride, mais le cou et les parties de dessous sont irisés avec un bleu violacé très prononcé; la plaque frontale (figure 26, p. 23) est d'un bleu de plomb au lieu de rouge; les pattes sont jaunes au lieu de vertes. Les jeunes sont semblables, mais avec des tons réduits et seulement des traces d'irisation.

Traits distinctifs. On ne peut la confondre qu'avec la gallinule de la Floride, mais les caractères susdits et l'absence des raies blanches évidentes sur les flanes constituent des différences.

Indications sur le terrain. Un profil de gallinule ou de poule d'eau, une irisation bleue prononcée, pattes jaunes, et plumes sous la queuc toutes blanches.

Habitat. Amérique tropicale et sous-tropicale, toujours au nord des Carolines.

Ne se rencontre qu'occasionnellement au Canada. Ne doit se caeher que dans les parties les plus méridionales.

\section{Genre-Gallinula. Gallinule de la Floride.}

219. La Gallinule de la Floride. (FLonids galuinule). Gallinula galeata. L, 13.50. C'est un oiseau uniformément bleu d'ardoise plus foncé sur la tête; un peu plus clair plus bas, teinte d'un brun rougeâtre légèrement irisé; raies blanches très visibles sur les flanes et une petite bordure des mêmes raies sous la queue; le bec et la plaque frontale d'un rouge brillant (figure 26, p. 23); les pattes vertes avec jarretières rouges droit au-dessous des plumes.

Traits distinctifs. Cette gallinule se distingue de la gallinule de la Martinique par les caractères sus-mentionnés; de la foulque, à laquelle elle ressemble tout à fait, par le bec rouge au lieu de blanc et la plaque frontale également rouge, avec les lignes blanches des flancs, le dos brunatre et les doigts sans trace de membrane.

Indications sur le terrain. Bec et plaque frontale rouges; raies blanches aux flancs, dos brunâtre, et toutes les pointes des secondaires foncées quand l'oiseau vole.

Nid. Ordinairement sur un petit tertre tel qu'est un vieux nid de rat musqué dans les marais humides; le nid est fait de fragments de végétation de rebut.

Habitat. Plus au nord que celui de la gallinule de la Martinique; celle de la Floride est généralement commune au Canada seulement le long des grands lacs des régions basses.

Cette gallinule est la poule d'eau la mieux connue du Canada méridional. Sa taille assez grande et le bon goat de sa chair qu'il faut attribuer au riz dont elle est friande et à d'autres graines de marais, en font un gibier que les chasseurs recherchent. Elle demande plus que les râles des éten- 
dues d'eau bien libres mais elle ressemble aux râles en général par des habitudes communes. C'est un oiseau plutôt bruyant, surtout la nuit, et qui, pendant le jour, fait sa partie dans le concert de cris qu'une agitation inaccoutumée et inattendue a fait éclater. Parfois un oiseau fera entendre tout d'un coup une volée de caquets que répéteront successivement tous ses pareils et pendant quelques instants ce marécage désert en apparence deviendra le thếtre d'un véritable petit sabbat de bruits d'oiseaux.

Valeur économique. Sauf comme gibier, la gallinule n'a pas d'importance économique.

\section{Sous-famille-Fulicinæ. Foulques.}

Description générale Ce sont des oiseaux plutôt grands et semblables au canard, mais avec de longs doigts pourvus de lobes membraneux; le bec s'étend jusqu'au front en une plaque blanche frontale, espèce d'écu (figure 14, p. 21).

Traits distinctifs. Très pareils à ceux des gallinules; ces traits sont donnés à propos de la description des especes dans la section suivante.

$$
\text { Genre-Fulica. Foulques. }
$$

221. La Foulque d'Amérique. la podle d'end. (american coot.) Fulica americana. L, 15. C'est un oiseau dont la couleur est uniforme, gris d'ardoise, plus foncée sur la tête, plus claire au-dessous; le bec et la plaque frontale (figure 14, p. 21) sont blancs avec des taches isolées d'un brun rougeâtre au sommet de la plaque et au bout des mandibules. Les pattes sont d'un vert terne et les doigts bordés d'une dentelure de petits bouts de membrane (il y a de un à trois lobes a chaque doigt).

Traits distinctifs. Elle se distingue de la gallinule par un bec blanc et par les membranes digitales.

Indications sur le terrain. La taille, la couleur gris d'ardoise, le bec et la plaque frontale de couleur blanche et, quand l'oiseau vole, la bordure blanche aux extrémités des pennes secondaires sur les ailes.

Nid. Très semblable a celui de la gallinule de Floride.

Habitat. Plus au nord que celui des gallinules; on rencontre la foulque partout au Canada bien avant dans les terres cultivées; couve partout où elle se rencontre au Canada

A la différence des gallinules, qui quittent tranquillement nos marécages quand commence l'automne, les foulques s'y attardent jusqu'assez avant dans la saison, et leur nombre, augmenté de celui des oiseaux de passage venant du nord, se rencontre en larges troupes sur les petits laes et les étangs où ils sont souvent tués par les chasseurs qui, un peu plus tard, trouvent peu de goût à leur chair.

Valeur économique. La foulque se nourrit de matières végétales même plus que la gallinule; mais, à cause de son habitat, elle ne peut avoir d'autre importance économique que comme un but de chasse de deuxième ordre.

\section{Ordre-Limicolae. Oiseaux de rivage, Bécasses, Maubèches, Pluviers, etc.}

Description générale. Les oiseaux de rivage forment un ordre relativement facile à reconnaitre mais difficile à décrire succinctement. Tous les oiseaux qui ressemblent à la bécasse ou au pluvier sont compris dans cet ordre. Ils ont des pattes qui varient de longueur depuis les peu longues jusqu'aux très longues, et qui ont des formes tres fines, bien faites pour marcher dans des eaux peu profondes, et le long des bords des étangs; le cou et le bec son a l'avenant (figures 15, 21, 22, 24, p. 21-23). Les doigts peuvent être au nombre de trois ou de quatre, et ne sont guère faits pour percher. Ils peuvent n'avoir pas de membranes ou que des membranes partielles, soit situées à la base des doigts, soit formant des dentelures ou des bordures completes aux doigts (voir Phalaropes). Le doigt postérieur, quand il existe, est petit, faible et légèrement élevé au-dessus des autres. Les ailes sont longues et pointues et les secondaires tout près du corps sont allongées. 
Traits distinctifs. Certains oiseaux de rivage montrent une ressemblance superficielle avec les râles, tandis que d'autres par certains traits caractéristiques (courlis) peuvent être pris pour des ibis ou des hérons, mais peuvent s'en distinguer par le doigt postérieur ou petit et surélevé, ou absent, et par l'absence de peau dénudée entre l'œil et le bec.

Indications sur le terrain. Le profil général, le port, l'habitat et le vol sont des indications caractéristiques faciles à reconnaí tre.

Nid. Sur le sol; une espèce fait exception à la règle.

Habitat. Cet ordre, celui des oiseaux de rivage, est cosmopolite, et il y a peu de régions du monde que les membres de cet ordre n'occupent pas. Les formes particulières à l'hémisphère nord de l'ancien et du nouveau monde sont étroitement congénères; quelques-unes sont identiques, plusieurs sont apparentées comme sous-espèce, et d'autres, en petit nombre, comme le tournepierre, se rencontrent partout. La plupart de nos espèces boréales nichent dans l'extrême nord, quelques-unes même aussi loin que ces régions ont été explorées, quoiqu'il arrive à un petit nombre de nicher sur nos frontières méridionales et au deld.

Les oiseaux de rivage, dans les premiers temps où ils étaient abondants, constituaient un gibier très apprécié; aujourd'hui que le nombre en est si considérablement réduit on a perdu l'habitude de leur faire la chasse et on ne les tue que par occasion. Parmi les oiseaux de rivage de l'est du Canada, la bécasse et la grande bécassine sont les plus intéressants comme gibier. Les représentants de l'ordre dans le Canada oriental se divisent en six familles: Phalaropodida, phalaropes; les Recurvirostrida, les échasses et avocettes qui ne se rencontrent que par hasard dans l'est du Canada; Scolopacido, les bécassines et les bécasses d'Europe constituent le gros de nos espèces; Charadriid e, le pluvier; A phrizidœ, les tourne-pierres; Hœmatopodida, les huitriers, jadis rares sur notre côte de l'est, aujourd'hui probablement disparus de cet endroit.

Valeur économique. La plupart des oiseaux de cet ordre habitent les terrains déserts et n'ont que peu d'influence économique; d'autres, qui fréquentent les champs cultivés, sont plus importants et seront traités sous leurs noms d'espèces. De façon générale, cependant, cet ordre est ou inoffensif ou très utile à l'homme.

FAMILLE-PHALAROPODIDA. PHALAROPES. BECASSINES DE MER.

Description générale. Petits oiseaux, de $7 \cdot 75$ pouces à $8 \cdot 75$ de long, échassiers d'apparence mais au plumage serré et semblable à celui du canard. Ce fait, ajouté à celui de leurs doigts bordés de lobes membraneux, de bordures et de tarses aplatis, les rend relativement faciles à reconnaitre.

Traits distinctifs. Ce sont de petits échassiers caractérisés comme ci-dessus. Ne peuvent être confondus avec aucun autre oiseau.

Indications sur le terrain. La taille et la façon de nager et de se nourrir en eau profonde. Ce sont les seuls oiseaux de rivage qui nagent habituellement.

Nid. Sur le sol; nid garni d'un peu de mousse ou d'herbe.

Habitat. Dans le nord et dans l'ouest. Une espece couve dans les provinces des prairies les moins élevées, les deux autres le long des côtes arctiques et des lles avoisinantes. Ce sont des oiseaux migrateurs ordinaires le long des côtes atlantiques de l'est du Canada et plus bas, dans la vallée du Mississipi, mais de purs vagabonds dans la région des grands lacs.

Les phalaropes constituent une petite famille très originale d'oiseaux de rivage, dont les véritables rapports avec d'autres familles sont à peine bien compris ni bien établis. Ils nagent avec facilité et on les voit souvent en pleine eau, même en pleine mer, où ils sont autant dans leur élément qu'aucune espèce maritime.

Originaux quant à leur structure et à leurs affinités ordinaires, ils le sont tout autant dans leurs habitudes. C'est la femelle, et non le mâle, qui représente par ses belles couleurs le cercle familial, qui prend l'initiative des 
avances dans le temps des amours, qui fait toutes les démarches auprès $d u$ futur conjoint, personnage timide et au modeste plumage, auquel, après qu'clle a rempli ses devoirs de pondeuse, cette femelle laisse les soucis de la couvaison et de l'éducation des petits.

Valeur économique. Ces oiseaux fréquentent l'eau ou les rivages stériles et n'ont aucune importance économique.

\section{Genre-Phalaropus. Phalarope roux.}

222. Le Phalarope roux. (red phalarope, grey phalarope). Phaloropus fulicarius. $\mathrm{L}, 8 \cdot 12$. La femelle adulte est facilement reconnaissable par la couleur uniforme, brun rougeâtre terne de l'avant-cou et des parties de dessous. Le dos est de couleur ocre et noire en raies. Le mâle est tout semblable, mais les couleurs sont voilées, brisées et moins distinctes. En hiver cette espèce est d'un gris d'ardoise dessus et blanc dessous.

Traits distinctifs. Le phalarope roux peut se distinguer facilement de l'un ou de l'autre des deux membres de la famille par son bec et ses pieds, celui-là étant relativement large et plat. Les doigts sont palmés à la base et avec des dentelures des deux côtés. Voir l'espèce suivante.

Indications sur le terrain. Ses manières d'être en général et son habitat. Dans la saison de la couvaison la couleur est la meilleure indication pour le reconnaître. Sur mer cette espèce laisse voir, paraitt-il, plus de noir au sommet de la tête et aux ailes que les autres phalaropes. Dans le cas du plumage incomplet, le bec relativement court et aplati est peut-être le meilleur moyen d'identification.

Habitat. Cet oiseau niche tout le long de la côte aretique de l'Amérique; il voyage et descend sur les deux côtés de ce continent; il est rare à l'intérieur des terres dans la région des grands lacs.

\section{Genre-Lobipes. Phalarope hyperboréen.}

223. Le Phalarope hyperboréen. (Northern Phalarope). Lobipes lobatus. L, 7.75. Femelle adulte: les parties supérieures, celles de derrière le cou, et la tête, sont couleur d'ardoise foncée; la gorge et le dessous sont blanes; les côtés du cou, qui se rejoignent devant le bas du cou, sont d'un roux ardent, avec des lignes de la même couleur le long du dos sur les ailes refermées. Le mâle est tout semblable, mais voilé et de couleur atténuée. Les oiseaux d'hiver sont gris clair en dessus, blanc dessous, avec un simple soupçon de coloration en dessus.

Traits distinctifs. Bec très mince en forme d'alènc; ressemble plutôt au phalarope de Wilson mais plus court; les pieds ont de petites membranes et des bords dentelés, comme le phalarope roux.

Indications sur le terrain. Couleur générale et bec fin en forme d'aiguille. Le plus petit des phalaropes de l'est du Canada.

Habitat. Le même que le précédent.

\section{Genre-Steganopus. Phalarope de Wilson.}

224. Le Phalarope de Wilson. (wilson's phalarope). Steganopus tricolor. L, 8.75. Oiseau d'un plumage magnifique. La femelle adulte a des raies de couleur d'un contraste accusé sur la tête et le cou. Une couronne gris perle passant au blanc sur le derrière du cou, et au gris sur les épaules. Ligne noire passant par l'œil jusque sur le côté du cou et devenant d'un rouge châtain ardent et qui continue latéralement le long du dos. La gorge d'un blanc passant à une délicate couleur vineuse au cou et à la poitrine, et qui revient au blanc dans les parties de dessous. Le mâle est brun grisâtre en dessus, et blane en dessous, avec une apparence légère de la coloration plus brillante de la femelle.

Traits distinctifs. Bec très long, $1 \cdot 25$ pouce, mince, forme d'aiguille, c'est l'espèce précédente exagérée. Les pieds ne sont pas palmés, mais sont pourvus de rebords membraneux étroits, et uniformes.

Indications sur le terrain. Couleur, taille, bec très long et mince.

Habitat. C'est une espèce du milieu de l'Ouest, et plus méridionale que la précédente, couvant dans les provinces des prairies et qui n'apparait que par hasard dans l'Est sur la partie inférieure des grands lacs. 
FAMILLE-RECURVIROSTRID A. AVOCETTES ET ÉCHASSES.

Description générale. Ces oiseaux sont parmi les plus grands de ceux de rivage et sont reconnaissables a leurs couleurs, qui contrastent entre elles de façon frappante, et à la longueur singulière de leurs pattes et de leur bec. Ces caractères sont poussés à l'extrême chez les échasses qui, toutefois, ne rentrent pas dans nos limites. I'́avocette s'est rencontrée accidentellement dans nos contrées à l'est des prairies.

\section{Genre-Recurvirostra. Avocettes.}

225. L'Avocette d'Amérique. (AMERICAN AvoceT). Recurvitostra americana. L, 16.50. Oiseau de rivage grand et très frappant, avec une tête et un cou de couleur vineuse très chaude passant au blanc dans les parties de dessous; le noir et le blanc se rencontrent sur le dos en contraste violent.

Traits distinctifs. Bec très long, mince, en pointe, et qui se recourbe dans sa moitié extrême. Ce trait est toujours distinctif.

Nid. Dans un petit creux près de l'eau.

Habilat. C'est un oiseau qu'on rencontre à l'intérieur de l'Ouest central, qui niche dans les provinces des prairies et qu'on ne voit qu'accidentellement a l'est du Manitoba.

Cette espèce n'est comprise parmi nos oiseaux que parce qu'on en rencontre quelques spécimens à l'est des provinces des prairies.

\section{FAMILLE-SCOLOPACIDA. OISEAUX DU TYPE BECASSINE, ("T1P-UPS), MAUBEेCHES, ETC.}

Description générale. Ce sont des oiseaux de taille petite ou moyenne, le courlis étant l'espèce la plus grande. Les pieds ne sont jamais entièrement palmés ni les doigts pourvus de bordures ou de marges membraneuses. Quelques espèces ont de petites membranes entre la base des doigts, ce qui a fait appeler ces oiseaux semi-palmés ou à demi-membranes. Toutes les espèces, sauf une, le sanderling, ont quatre doigts. Les becs sont longs, minces, et pointus; ordinairement droits (fig. 21 et 22, p. 23); mais quelquefois recourbés en dedans; à l'occasion, comme chez les barges (p. 108) ils sont très légèrement recourbés en dehors; plutst flexibles et ordinairement un peu agrandis et très sensibles a la pointe.

Traits distinctifs. Ce sont manifestement des oiseaux de rivage, généralement connus sous les noms populaires ci-dessus. Le bec ne finit pas en une pointe finement acérée, comme celui des familles précédentes, et n'a pas la base tendre et la pointe cornée des tourne-pierres et des huitriers.

Nid. Tous, sauf une seule espèce, le chevalier solitaire, nichent sur le sol dans de petits creux garnis maigrement des matières végétales environnantes.

Habitat. Le plus grand nombre niche dans l'extrême nord, quoique certaines espèces se rencontrent au sud de la frontière des Etats-Unis. Ils émigrent le long de nos rivages ou à travers l'intérieur selon qu'ils appartiennent à telle espèce ou à tel habitat. Quelquesuns de ces oiseaux suivent des routes intéressantes dans leurs migrations.

Parmi ces oiseaux citons les bécasses et les bécassines des forêts humides et des marécages; les maubèches tachetées, à branle-queue et les autres maubèches que nous voyons le long de nos rivages et de nos rivières, et les courlis des hauts plateaux. Ces espèces formaient le gros des admirables compagnies d'oiseaux de rivage qui autrefois peuplaient nos rivages. Comme ils nichaient généralement bien loin par delà les limites des terres cultivées, l'occupation par les immigrants des terrains où ils avaient leurs nids n'a eu qu'une très minime influence sur leur nombre; mais c'est le fusil des chasseurs ignorants et stupides qui les a décimés. Comme ils volent en troupes serrées ils présentent une manière de cible facile à viser et on en a vu tomber quatre-vingts et davantage abattus par un seul coup de fusil; il n'y a donc rien d'étonnant à ce que ces oiseaux soient devenus relativement rares.

Valeur économique. Ils sont tout à fait inoffensifs ou même réellement utiles selon l'habitat. 
Genre-Philohela. Bécasse.

228. La Bécasse d'Amérique. La Bécassine. (AMErican wooncock). Philohela minor. L, 11. Planche VII B.

Traits distinctifs. Long bec (figure 21, p. 23) et les yeux situés très hauts dans la tête, couleur feuille morte des parties de dessous, les bruns très riches du dos sont distinctifs.

Indications sur le terrain. L'hahitat de cet oiseau, ajouté à son bec, à sa taille, à sa coloration ou d'un brun riche ou feuille morte la font aisément reconnaitre à l'état vivant.

Nid. Sur le sol parmi les feuilles mortes de l'année précédente avec lesquelles son plumage s'harmonise si bien.

Habitat. Toujours dans le sud de l'Ontario, dans la partie basse des grands lacs, quoique des individus vagahondent accidentellement sur un espace bien plus étendu.

Les bécasses fréquentent les plantations d'arbrisseaux simplement mouillés ou inondés, les bocages d'aunes ou de noisetiers, ou la lisière touffue des forêts humides. Si on les dérange, elles s'enlèvent soudain de terre, volent au hasard avec un singulier sifflement d'ailes et, passant par-dessus les broussailles, se laissent tout à coup tomber pour se cacher à quelques mètres de là. La génération présente se rappelle certainement que dans les clairières du sud de l'Ontario les bécassines pullulaient autrefois; tandis qu'aujourd'hui, les chasseurs pouvant donner carrière à leur passion, les terrains jadis délaissés ayant été déboisés et drainés, le chat domestique aidant peut-être aussi, la bécasse est devenue rare, presque introuvable. A l'inverse de la gelinotte huppée ou de la perdrix qui demandent des étendues considérables de terres vierges, il n'y a aucune raison essentielle qui empêcherait la bécasse d'être encore abondante et de procurer bien longtemps encore beaucoup d'exercice cynégétique aux amateurs. Il suffira aux besoins de cet oiseau de trouver le plus petit abri écarté parmi les arbrisseaux humides. La bécasse part pour le Sud peu après le commencement de la saison et ne reste pas longtemps exposée aux entreprises des chasseurs permissionnaires. Après l'homme, c'est le chat qui semble être le principal ennemi de la bécasse, et comme celle-ci vit et niche sur le sol, se fiant à la couleur de son plumage et à sa cachette pour la protéger, elle est singulièrement exposée aux attaques des félidés.

230. La Bécassine de Wilson. (wILsox's SNIPE). Gallinago delicata. L, 11.25. Planche VIII A.

Traits distinctifs. Il est très peu prohable qu'elle puisse être prise pour une autre espèce au Canada, mais la queue d'un brun rougeâtre, qui devient blanche sur les plumes extérieures et barrée de noir, la distinguera bien assez, au besoin.

Indications sur le terrain. L'habitat (prés herbeux bien découverts), long hec, vol curieux en spirale quand elle s'elève de terre, en sus de la taille, de la coloration générale de la queue d'un brun rougeâtre et blanchâtre. La bécasse et la bécassine rousse sont les seuls autres oiseaux semblables.

Habitat. Couve partout sur le continent, tout juste en dedans des limites des terres cultivées, et irrégulièrement sur les confins méridionaux. Elle est commune d'un bout à l'autre du Dominion.

\section{Sous-famille-Eroliinæ. Maubèches.}

\section{Genre-Limmodronus. Bécassines rousses.}

231. La Bécassine rousse. (DOWITCHER, RED-BREAsten-SNIPE). Limmodronus griseus. L, 10.50. Adulte au printemps: la gorge, le devant du coul, la poitrine et toutes les parties de dessous sont très rougeâtres. Le dos et les parties de dessus sont nuancés de teintes analogues et d'un brun foncé. Plumage d'automne: brun grisâtre terne sur la tête, le cou, le haut de la poitrine, les flancs et le dos, nuancé d'un brun plus clair sur ces dernières parties; hlanc en dessous. Le bas du dos est toujours blanc. Des teintes intermédiaires se présentent dans tout le plumage. 
Traits distinctifs. La même apparence générale que chez la bécassine de Wilson, mais le devant est rouge, ainsi que les dessous, au printemps; en automne, les tons bruns si riches de cette espèce disparaissent. Lẹ bec est plus long, comparé a la taille, que chez aucun autre oiseau, la bécassine de Wilson exceptée. Une poitrine rouge très semblable se voit chez la maubèche à poitrine rousse, mais le plus long bec de la bécassine rousse $(\mathbf{L}, 2 \cdot 10-2 \cdot 50$ contre $\mathbf{L}, 1.30$ ) est un moyen d'identification concluant.

Indications sur le terrain. C'est à peu près la taille et le profil général de la bécassine de Wilson mais avec du blanc très en vue sur le bas du dos, et plus blanc sur la queue. L'habitat est aussi différent.

Habitat. Il couve dans l'extrême Nord, dans l'Ouest, et probablement plus à l'est de la baie d'Hudson. Il est rare sur les grands lacs, plus commun sur la côte. Nos bécassines rousses de l'Est sont supposées être des oiseaux nicheurs du Nouveau-Québec, mais on n'a pas la-dessus des renseignements exacts.

SOUS-ESPECES. Une sous-espèce qui se différencie un peu: la bécassine rousse à long bec, L. g. scolopaceus, se rencontre dans l'Ouest. Elle se distingue par sa taille légèrement plus grande, son plus long bec, une poitrine plus fortement tachetee, et le rouge plus étendu en dessous. Il est difficile de différencier des plumages brillants; les oiseaux et les tout jeunes ne peuvent que rarement se distinguer les uns des autres. La becassine rousse à long bec ne peut être cherchée dans l'est du Canada que dans la région des grands lacs et sa présence ne devrait pas être signalée à moins que le spécimen n'ait été dûment comparé avec un exemplaire authentique.

C'est un oiseau qui recherche les terrains boueux plutôt que les prairies herbeuses.

\section{Genre-Micropalama. Maubèche à longs pieds.}

233. La Maubèche a longs pieds. (stiLT sANDPIPER). Micropalama himantopus. L, $8 \cdot 25$. Taches brunes sur un fond de blanc mat; parties de dessous plus claires, celles qui sont foncées sont disposées en barres uniformes qui se changent en raies obscures sur le devant du cou et en taches fines sur la gorge. Le plumage d'automne ne montre aucune trace de ces barres caractéristiques; le dos a des teintes variées de brun en un dessin accusé; les parties de dessous sont presque tout à fait blanches, légèrement voilées de teintes ocreuses sur la poitrine et le devant du cou, ou le brun présente des taches vaguement foncées.

Traits distinctifs. Les parties de dessous du plumage de printemps, uniformément barrées, sont telles qu'on ne peut pas s'y tromper. L'oiseau d'automne ressemble a plusieurs especes. La longueur du bec, 1.50 et celle du tarse, 1.60, sont plus grandes que celles d'aucun autre oiseau de rivage de taille d'ailleurs égale. La maubeche a dos roux a un bec d'à peu près égale longueur, mais il est plus massif et son bout extrême n'a pas le même élargissement abrupt en spatule.

Indications sur le terrain. Le contraste entre sa petite taille et son très long bec. Les couvertures sus-caudales, au lieu des sous-caudales comme chez la bécassine rousse, sont claires au printemps et blanches en automne.

Habitat. Elle couve sur la côte arctique au nord-ouest de la baie d'Hudson. Dans ses migrations elle est probablement plus commune dans l'intérieur que sur la côte. Elle est régulière, mais rare en automne sur le lac Ontario.

C'est un des oiseaux de rivage dans l'Est, et des plus rares, qui se trouve quelquefois en compagnie de la bécassine rousse et des grands chevaliers à pieds jaunes dans les marais boueux.

\section{Genre-Canutus. Maubèche à poitrine rousse.}

234. La Maubèche à poitrine rousse. (KNOT, RED-BREASTED PLOVER). Tringa

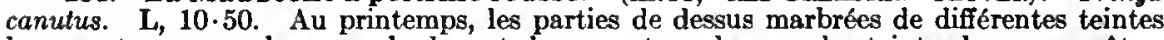
brunes et ocreuses; la gorge, le devant du cou, et au-dessous des teintes brun rougeatre, plus pales vers la queue. En automne, un gris clair fumeux, blanc pur en dessous, la poitrine et le devant du cou un peu plus foncés avec des taches fines, un peu obscurcies.

Traits distinctifs. Ces oiseaux au printemps peuvent, quant à la taille, être confondus seulement avec la bécassine rousse. Le bec court, $L, 1 \cdot 30$, en contraste avec $L, 2 \cdot 10-2 \cdot 50$, de la bécassine, constitue une différenciation concluante. Les oiseaux, en automne, sont 
très semblables à plusieurs espèces de même coloration. La coloration gris clair du dos est tout à fait pareille à celle de la maubèche à dos roux, mais chez la maubèche à poitrine rousse chaque plume a, en marge, une ligne à peine marquée de couleur plus claire qui donne l'impression d'une succession de demi-cercles, et qu'on ne voit chez aucun autre oiseau de rivage.

Indications sur le terrain. Au printemps, bec plus court que celui de la bécassine rousse à laquelle elle ressemble par ailleurs, avec une teinte grisâtre mais non très blanche sur la queue; c'est le meilleur signe indicateur.

Habitat. C'est une espèce circompolaire qui voyage dans une étendue d'un rayon considérable. Elle couve dans les îles du cercle arctique et dans ses voyages d'hiver va dans l'Afrique australe, en Patagonie, en Nouvelle-Zélande, etc. Elle parait être moins commune dans la région des grands lacs que sur la côte de l'Atlantique.

C'est un oiseau qu'on rencontre sur les plages sableuses aussi bien que dans les marécages boueux.

\section{Genre-Arquatella. Maubèche pourprée.}

235. La Maubèche pourprée. (Pưple sanpiper). Arquatella maritima. L, 9. D'un blanc grisâtre sur le dos, la tête comprise, et qui s'étend en travers du devant du cou, de la gorge, et le long des flancs. Plus pâle sur la gorge et très foncé sur le dos, blanc au-dessous. Sur les parties plus foncées on voit une couleur pourprée vaguement étendue et légèrement irisée, plus ou moins marbrée de teintes claires sur le bord des plumes. Le plumage d'automne est semblable, mais les bords clairs des plumes sont plus étendus. Les pattes et les pieds sont orange au printemps.

Traits distinctifs. C'est le seul oiseau de rivage avec un dos si uniformément noir.

Indications sur le terrain. Au printemps, couleur sombre générale et pattes courtes orange. En automne, la saison mếme de leur arrivée est en elle-même presque un signe indicateur, car elle arrive très tard, même presque en hiver alors que tous les autres oiseaux de rivage sont partis.

Habitat. La maubèche pourprée de l'Est couve probablement dans les îles arctiques. Quand elle émigre, elle est plus commune sur la côte maritime que sur les grands lacs où elle est très rare.

SOUS-ESPECES. La maubèche pourprée se rencontre dans les deux mondes, le nouveau et l'ancicn. La sous-espèce qui se rencontre sur la côte est du Canada c'est la maubèche pourprée de l'Est, le type de la race.

C'est un oiseau migrateur très tardif, qui arrive longtemps après que tous les autres oiseaux de rivage nous ont laissés. Les mois de novembre et de décembre sont les mois de leur arrivée. Leur apparente rareté peut être surtout attribuée à ce qu'elle arrive quand les rivages sont désertés par les chasseurs. Ils préfèrent les rivages rocheux au sable ou à la boue.

$$
\text { Genre-Pisobia. Maubèches. }
$$

239. La Maubèche à poitrine cendrée. L'AlOUETTE DEs PRés. (PECTORAL SANDpiper). Pisobia maculata. L, 9. Les parties de dessus sont bien foncées, chaque plume étant bordée d'une teinte d'ocre pâle; les dessous et la gorge sont blancs; le bas du cou et la poitrine sont étendus d'un brun chamois pâle avec des raies serrées d'un brun foncé.

Traits distinctifs. La taille et le devant qui est assez fortement rayé de brun chamois, sont bien distinctifs. La maubèche à croupion blanc et la maubèche de Baird peuvent lui ressembler à cet égard, mais le croupion de la première et la petite taille des deux sont en évidence.

Indications sur le terrain. Son habitat ordinaire dans les marécages herbeux fera de la bécassine de Wilson celle avec laquelle on pourra le plus facilement la confondre, mais la petitesse du bec de la maubeche à poitrine cendrée est manifeste. Celle-ci présente comme un oiseau d'un brun uniforme sans couleur claire au croupion ou ailleurs, assez semblable à une grosse maubèche de Wilson.

Habitat. Elle couve sur la côte arctique, au nord-ouest de la baie d'Hudson. C'est un voyageur ordinaire dans tout l'est du Canada. 
La maubèche à poitrine cendrée, comme la bécassine de Wilson, se rencontre dans les prés humides et herbeux, ou dans les terrains boueux, rarement sur les plages sablonneuse. Dans l'herbe un chien la tient facilement en arrêt, et la chasse en est parfois excitante. Sur le terrain de la couvaison le sac qu'elle a au cou peut s'enfler d'air de façon extraordinaire, et l'oiseau produit quand il vole, un chant qui est fort inaccoutumé chez les oiseaux de rivage qui sont généralement non chanteurs.

240. La Maubèche à croupion blanc. (WHITE-RUMPEd sandPIPER). Pisobia fuscicolitis. L, 7.50. Le dos et les parties de dessus sont brun foncé avec large bordure grisâtre et brun ocreux, le grisâtre prédominant. Le croupion et toutes les parties de dessous sont blancs, le devant du cou et le haut de la poitrine sont fortement et finement rayés de brun foncé. En automne la coloration est semblable, mais il y a plus de rouge ocreux au dos et les raies du devant sont plus fondues.

Traits distinctifs. La taille et le croupion blanc la distinguent d'espèces analogues. La barge de la baie d'Hudson et la maubèche à longs pieds ont les couvertures sus-caudales blanches, mais le premier de ces deux oiseaux est beaucoup trop grand pour se prêter à une erreur, et le bec plus long et plus mince et la poitrine rayée du second sont bien distinctifs. visible.

Indications sur le terrain. Taille et couleurs générales; gros croupion blanc bien

Habitat. Cette maubèche couve sur la côte arctique à l'ouest jusqu'a la frontière prochaine de l'Alaska. Pendant la migration elle est commune sur la côte de l'Atlantique, plutôt rare dans la région des grands lacs.

Cette espèce fréquente les terrains boueux et les rivages rocheux plutôt que les plages sablonneuses. Elle accompagne souvent les compagnies de maubèches de Wilson et de maubèches semi-palmées.

241. La Maubèche de Baird. (Baird's sandpiper). Pisobia bairdi. L, 7.40. Le dos jusqu'au sommet de la tête est brun foncé bordé d'une teinte ocreuse légère; audessous et à la gorge, du blanc; une bande d'un chamois pâle en travers de la poitrine; le bas du devant du cou est vaguement rayé de lignes brunes assez fines.

Traits distinctifs. Elle ressemble à la maubèche précédente mais avec le croupion plus foncé et plus de chamois étendu en travers du devant. Extrêmement semblable aussi à la maubèche de Wilson, mais plus grande.

Indications sur le terrain. Elle ressemble à une grande maubèche de Wilson avec du chamois étendu sur la poitrine.

Habitat. Couve sur la côte arctique d'un bout a l'autre du continent. Plus commune dans ses migrations vers les provinces des prairies que sur les cótes. N'est pas rare dans la région des grands lacs, mais l'est davantage plus à l'est.

Les parages sablonneux et boueux semblent attirer également cette espèce. On la trouve souvent en compagnie de maubèches de Wilson et de maubèches semi-palmées, quoiqu'elle soit moins dépendante de l'eau que plusieurs des membres de cette famille.

242. La Maubèche de Wilson. (LEAst sANDPIPER). Pisobia minutilla. L, 6. Les parties de dessus et la couronne sont brun foncé et bordées plus ou moins de diverses teintes de chamois ocreux et d'un rouge vermeil; blane en dessous. En travers de la poitrine et du devant du cou une teinte foncée étendue et quelquefois nuancée de chamois avec plus ou moins de raies et de taches d'un noir foncé.

Traits distinctifs. Cette maubèche se distingue de toutes les autres espèces, sauf de la maubèche semi-palmée, par sa taille minuscule, et de la maubèche semi-palmée par l'absence des membranes entre les doigts.

Indications sur le terrain. Elle se différencie de la maubèche semi-palmée par la couleur verdâtre foncé au lieu de noire des pattes.

Habitat. Elle couve sous les hautes latitudes, d'un rivage a l'autre du continent et, dans l'Est aussi loin, au sud, que les îles de la Madeleine dans le golfe du Saint-Laurent. Elle est commune, pendant ses voyages, à travers tout le Canada oriental. 
C'est un des oiseaux de rivage les plus répandus. Il est probable que sa toute petite taille l'a protégée contre les chasseurs, lors même que de ses troupes serrées on en peut tuer un grand nombre d'un seul coup de fusil. Elle fréquente les plages sablonneuses et les terrains boueux, c'est un oiseau apprivoisé et confiant. Elle se mêle volontiers à des troupes d'autres espèces que la sienne, bien que, si on l'effraie, elle s'en sépare pour les rejoindre plus tard. Elle arrive et repart plus tôt dans l'automne que les semipalmées.

\section{Genre-Pelidna. Pelidnes.}

243. La Maubèche à dos roux. (DUNLIN, ReD-BACK sandPIPER). Pelidna alpina. $L, 8$. Cet oiseau printanier est trop fortement caractéristique pour qu'on le confonde avec aucun autre. Le dos brun foncé a une si large bordure de rouge ocre que d'en être presque tout rouge. Une grande tache plus ou moins diffuse, presque noire, occupe la surface abdominale. Le bec est légèrement courbé en bas (figure 22, p. 23). L'oiseau d'automne manque de ces caractères frappants. La surface de dessus est presque uniforme, d'un gris brunâtre, pâle, étendu en travers de la poitrine et du bas du cou. Les dessous et la gorge sont blancs.

Traits distinctifs. Sa couleur d'automne est semblable à celle de la maubèche à poitrine rousse dans la même saison, mais sa plus petite taille et l'absence des demi-cercles pâles sur les bords des plumes la différencieront toujours, même en l'absence de traces accidentelles de plumage printannier. Cette coloration peut aussi faire penser au courlis ou bécasse de mer, mais les couvertures sus-caudales sont foncées au lieu de grisâtres.

Indications sur le terrain. Au printemps, le dos rouge et la tache noire en dessous sont très visibles. En automne, dos gris uniforme et, quand l'oiseau vole, une ligne de blanc sur l'aile. La recourbure du bec vers le bas quoique assez légère est cependant distincte et facilite l'identification.

Habitat. L'individu européen compris, cette espèce est circompolaire. Cette maubèche à dos roux d'A mérique couve par places sur la côte arctique, à travers le continent. C'est un migrateur ordinaire dans tout le Canada oriental.

SOUS-ESPECES. Le représentant de cette maubèche dans le nouveau monde est une sous-espèce. La maubèche à dos roux d'Amérique $P$. a. sakhalina, ne se distingue de celle de l'ancien monde que par sa taille légèrement grande.

Cet oiseau fréquente les bancs de sable, les terrains boueux ou les prés salés. Il est parmi les tout derniers des oiseaux de rivage à arriver à la fois au printemps et en automne.

\section{Genre-Erolia. Maubèches.}

244. La Maubèche courlis. (cuRLEw SANDPIPER). Erolia ferruginea. L, 8. C'est une petite maubèche à poitrine rousse. Oiseaux d'automne brun grisâtre en dessus et blanc en dessous.

Traits distinctifs. Elle ressemble à la maubèche à poitrine rousse ou à la bécassine rousse en ce qu'elle a la poitrine rousse, mais elle est beaucoup plus petite et de charpente plus légère.

Habitat. Elle couve dans la Sibérie arctique. Ne se rencontre que par hasard dans le Canada oriental. On l'a signalée plus souvent sur la côte de l'Átlantique que dans l'intérieur bien qu'elle l'ait été une fois près du lac Ontario.

C'est un oiseau de l'ancien monde vu quelquefois dans le nouveau. On dit qu'il ressemble à la maubèche à dos roux par le port, mais il est trop rare dans l'est du Canada pour en parler comme d'un visiteur régulier. Tout signalement de la présence d'un courlis doit être appuyé par des spécimens bien déterminés et soumis à un rigoureux examen. 


\section{Genus-Ereunetes. Maubèches semi-palmées.}

246. La Maubèche semi-palmée. (sEmipaLMated sandPiPer). Ereunetes pusillus. $\mathrm{L}, 6 \cdot 30$. Les parties de dessus, y compris la couronne, sont bordées d'un brun foncé avec un ocre ou un chamois pale, blanc en dessous avec une bande vague légèrement plus foncée en travers de la poitrine avec raies et taches obscures.

Traits distinctifs. Elle ressemble beaucoup par la taille et la coloration a la maubeche de Wilson dont on ne peut pas toujours la distinguer à moins de l'examiner de très près. Les pieds, toutefois, ont de petites membranes entre leurs doigts, d'où vient a cet oiseau le nom de semi-palmé.

Indications sur le terrain. Elle diffère de la maubeche de Wilson par sa taille légèrement plus grande, son dos d'un gris plus pur et la poitrine plus blanche et plus clairement délimitée. Les pattes et les pieds sont noirs au lieu d'être d'un vert olive foncé.

Habitat. Couve le long de la côte est du pôle, au sud du Labrador méridional. C'est un migrateur ordinaire dans tout le Canada oriental.

Cette maubèche est très semblable par son port et son apparence à la maubèche de Wilson (page 105). La maubèche de l'Ouest, Ereunetes mauri, est un individu très parent qui peut n'être qu'une sous-espèce de la maubèche semi-palmée. Elle se distingue par un bec légèrement plus long et par une plus forte quantité de roux sur le dos, spécialement au derrière de la tête. Il est fort douteux qu'on la rencontre dans la région des grands lacs.

\section{Genre-Crocethia. Sanderling.}

248. Le Sanderling. (sanderLing). Crocethia leucophaa. L, 8. Au printemps: les parties de dessus, $y$ compris la couronne, brun foncé, panaché de beaucoup d'ocre rouilleux pâle ou de blanc, ou de l'un et de l'autre. En dessous, du blanc. La gorge, le cou, et le haut de la poitrine sont étendus de plus ou moins d'ocre rougeftre et tachetés de brun. Les détails de ces colorations sont extrêmement variables. Lc dos peut montrer assez de ces différentes couleurs pour le rendre ou généralement grisâtre, ocreux, ou rouilleux, tandis que la gorge colorée et tachetée peut étre d'un blanc presque immaculé. L'oiseau d'automne est semblable sans avoir beaucoup de teintes chamois, ou de roux ou d'ocre; il est d'un blanc pur en dessous et sur le devant, et souvent, principalement gris ou cendré palle en dessus.

Traits distinctifs. A partir de simples traces jusqu'd de fortes teintes de rouille sur le cou et autour de la téte, au printemps, et à la blancheur générale en automne. Le sanderling peut se distinguer de toutes les autres maubeches par les trois doigts, au lieu de quatre, de son pied.

Indications sur le terrain. Teinte d'un brun rougeâtre répandue près de la tête chez quelques oiseaux de printemps; un contraste du blanc et du noir assez général sur l'aile; dans tous les plumages une ligne blanche le long de la base des plumes qui servent au vol. En automne, la poitrine d'un blanc très pur est également caractéristique.

Habitat. Couve dans les fles de l'Ouest du pôle arctique jusque dans l'Alaska. C'est un migrateur ordinaire sur les rivages sablonneux dans tout le Canada oriental.

Cet oiseau des plages sablonneuses fréquente rarement les terres boueuses. C'est un des petits oiseaux de rivage les plus beaux et intéressants. Il aime le bord de l'eau, suit volontiers la vague qui se retire, battant vite en retraite avant qu'elle revienne, menacé à tout instant d'être submergé par le ressac, mais y échappant tout juste. Quand ces oiseaux s'envolent audessus de l'eau bleue, que le soleil brille sur leur plumage étincelant, ils forment un tableau ravissant; tantôt tournant leur dos délicatement coloré de blanc et de noir, tantôt, comme obéissant à une seule et même impulsion, frôlant l'eau dans un vaste contour et montrant la blancheur éclatante de leurs dessous. 


\section{Genre-Limosa. Barges.}

249. La Barge marbrée. (Marbled godwit). Limosa fedoa. L, 18. C'est un gros oiseau de rivage; couleur générale: un chamois pâle vaguement teinté de rose sur tout le corps, sauf à la gorge, qui peut être blanche. Le dos, du derrière de son cou jusqu'à la couronne, nuancé de brun foncé et de teintes légères; la poitrine et les flanes plus ou moins rayés de lignes fines de la même couleur foncée.

Traits distinctifs. Pareils à ceux du courlis par son apparence générale, mais le bec est légèrement tourné en haut au lieu de l'être minifestement en bas. Se distingue de la barge de la baie d'Hudson par une marbrure fine, foncée sur le chamois rosé des primaires.

Indications sur le terrain. Les barges sont parmi les plus gros de nos oiseaux de rivage, d'une taille a peu près égale a celle des courlis. Leur bec, qui n'est pas recourbe en bas, les fera distinguer des courlis.

Habitat. Couve dans les provinces des prairies et aussi dans quelques régions cultivées; voyage vers l'un ct l'autre océan et se rencontre quelquefois dans l'est des provinces maritimes.

La barge n'abonde jamais dans le Canada oriental, cette espèce ayant été malheureusement décimée autant que d'autres gros oiseaux. Il n'est pas douteux que son habitude de nicher dans les régions cultivées ne soit la cause principale de sa disparition graduelle.

251. La Barge de la Bale d'Hudson. (hudsonian gonwit). Limosa homastica. L, 15. Au printemps: les parties de dessus brun foncé jusqu'a la couronne, maculées de plus ou moins de grisatre ou de chamois, avec des marques rouilleuses; les parties de dessous brun rougeâtre, plus ou moins rayées de teinte foncée étendue jusqu'au devant du cou. En automne: les parties de dessus sont uniformément gris brunâtre; celles de dessous, blanc chamois ou blanc sale; la poitrine plus grise.

Traits distinctifs. Elle se distingue soit des courlis par le bec légèrement tourné en haut au lieu de tourné en bas, soit de la barge marbrée par les dessous roux au printemps; en toute saison par les primaires toutes foncées, a tuyaux blancs sans marbrure. Cette espèce fait voir des variations presque interminables entre les susdits plumages, mais certaines données de la coloration printanière sont ordinairement reconnaissables chez tous ces oiseaux à l'exception des plus jeunes.

Indications sur le terrain. La grande taille, le bec droit ou recourbé légèrement, le croupion blanc a la base d'une queue noire feront distinguer cette barge soit des courlis de la barge marbrée, les seuls oiseaux qu'on serait tenté de confondre avec elle.

Habitat. Couve dans le nord-ouest, au dela des limites de la civilisation. Très commune à l'est des prairies jusqu'aux provinces maritimes en automne, et dans l'intérieur au printemps.

La barge de la baie d'Hudson est un bel oiseau bien près de disparaître complètement. Comme il arrive fréquemment, cet oiseau sembla disparaître tout à coup et avant qu'on se fût douté de sa rareté. On peut douter que les chasseurs soient absolument responsables de cet état de choses. Quand la quantité d'une espèce est déjà très réduite par une cause quelconque, toute autre influence adverse et qui sera relativement de peu d'importance, pourra suffire à éteindre cette espèce d'une façon inattendue. Toute protection est inefficace qui se fait si longtemps attendre que le nombre des individus reproducteurs est devenu trop réduit pour reconstituer l'espèce.

\section{Sous-famille-Numeniinæ. Maubèches et Courlis.}

$$
\text { Genre-Totanus. Chevaliers à pieds jaunes. }
$$

254. Le Grand Chevalier à pieds jaunes. LE CHeVALIER AUX PATtes JAUNES. (Greater yeLLow-LEGs). Totanus melanoleucus. L, 14. C'est un oiseau de rivage plutốt grand. Les parties de dessus jusqu'à la couronne vont d'un brun foncé au noir, avec de petites marques blanches ou grises avec plumes grises intrusives, faisant un effet gris, sans trace de chamois ou de brun rougeatre. Les parties de dessous sont blanches, rayées sur le devant du cou, la poitrine et les flancs avec des raies distinctes ou des ligues de la méme couleur foncée que sur le dos; pattes très longues et jaunes. 
Traits distinctifs. La taille, les pattes jaunes et l'absence de tout signe de chamois ou de rouille où que ce soit, sont les traits distinctifs des deux chevaliers a pattes jaunes. Dans les jeunes oiseaux d'automne les marques à la poitrine et au cou peuvent étre voilées et indistinctes. Cette espece et la suivante, prises à part, ne diffèrent que par leur taille.

Indications sur le terrain. Pattes longues, minces, absence complète de couleur ocreuse, la taille, et la grande quantité de blanc ou de couleur blanchâtre sur la queue et le croupion. Dès qu'on a appris à le connastre son vol est très reconnaissable.

Habilat. Il couve dans les hautes latitudes d'un bout a l'autre du continent. Dans l'Est, on le trouve au sud jusqu'a l'ile d'Anticosti et au rivage nord du golfe du SaintLaurent; il est commun dans les migrations par tout le Canada oriental.

Le grand chevalier à pattes jaunes est un des oiseaux de rivages les mieux connus. A cause de sa taille et du grand nombre relatif de ses congénères les chasseurs le recherchent beaucoup, et il paraît avoir tenu tête à leurs entreprises beaucoup mieux que d'autres espèces qui semblaient en être aussi capables que lui. Il aime les rives marécageuses et la boue plutôt que le sable, et on peut le voir au loin pataugeant dans ces marais, où il entre jusqu'aux cuisses, dans une eau trop profonde pour de plus petits échassiers. Son sifflement clair, comme flûté, en trémolo, et en gamme descendante est un son qui fait battre le cœur a tout vrai chasseur ou à l'ornithologue de profession. Cette espèce ne semble pas être aussi nombreuse, et montre à coup sar plus de circonspection que le petit chevalier. Avec cette réserve notre description s'applique à tous les deux.

255. Le Petit Chevaller à pleds jaunes. (LEBSER yellow-LEGs). Totanus flavipes. L, 10.75. Edition abrégée de la précédente espece.

Habilat. Il niche partout sur le continent dans les hautes latitudes. C'est un migrateur ordinaire dans tout le Canada oriental.

\section{Genre-Tringa. Chevalier solitaire.}

256. Le Chevalier Solitaire. L'AlOdETTE solitaire. (BOLTARY BANDPIPER). Tringa solitaria. L, 8.40. Les parties de dessus foncées, presque noires, avce un lustre légèrement verdâtre relevé par quelques petites taches blanches; les parties de dessous et la gorge, blanches; le bas du cou, la bande pectorale et les côtés des flancs, barrés et rayés avec des teintes plus claires de la couleur du dos; aucune trace de chamois ou d'autres nuances.

Traits distinctifs. Taille et coloration générale; les plumes axillaires blanches, rayées de noir, constituent un trait décisif.

Indications sur le terrain. Il ressemble a la fois a la maubeche tachetee et au petit chevalier à pieds jaunes. Il se distingue de la premiere par l'absence d'une ligne blanche sur l'aile déployée et par les barres blanches très visibles sur la queue et du second par la taille et par le croupion noir au lieu de blanc.

Nid. Pendant longtemps les habitudes du couvage de cette espèce demeurèrent inconnues et le problème de sa façon de nicher ne fut pas résolu jusqu'à ce qu'on eut découvert que cet oiseau se servait des nids abandonnés d'oiseaux percheurs dans les arbres et les buissons. La maubèche verte d'Europe, qui lui est très apparentée, a la même habitude.

Habitat. Il niche vers le nord à partir des limites intérieures des terres cultivées; il est assez commun dans tout le Canada oriental comme migrateur.

SOUS-ESPECES. Le chevalier solitaire est représenté au Canada par deux sousespeces, dont une seule, le solitaire de l'Est, l'individu type, se rencontre dans l'Est.

Ainsi que l'indique le nom, cette espèce est plutôt un oiseau solitaire qu'on rencontre soit tout seul soit avec un compagnon plutôt que par bandes, même au temps de la migration. Il fréquente les étangs fangeux et, comme la maubèche tachetée, il est le seul échassier qu'on voit ordinairement près de ces petits réservoirs d'eau tels que les fossés de drainage, ou le long de la lisière des bois submergés. 
Genre-Catoptrophorus. Chevalier semi-palmé.

258. "Le Chevalier semi-palme." (wILLET). Catoptrophorus semipalmatus. L, 15. Un gros oiseau de rivage; les parties de dessus gris chamois avec marques brunes plus foncées; les parties de dessous sont blanches étendues d'un pâle chamois grisâtre avec barres et raies gris foncé sur les flancs, la poitrine et le devant du cou; croupion blanc.

Traits distinctifs. Taille, coloration générale claire et grise; barre blanche de l'aile trè visible sur les primaires, croupion blanc, et plumes axillaires noires. Le bec caractéristique de la maubèche fera distinguer ce chevalier du pluvier à bec noir qui a aussi ces détails du croupion et des axillaires.

Indications sur le terrain. Par la taille il ressemble aux barges plus qu'à tout autre oiseau, mais le gris cendré et les ailes blanches et noires si visibles sont distinctives.

Habitat. I couve au sud de notre pays, d'habitude depuis la Virginie jusqu'd la Nouvelle-Ecosse dans l'Est, et vers l'Ouest Jusqu'aux parties centrales des provinces des prairies. Aujourd'hui il est très rare sur la côte, assez irrégulier dans la région des grands lacs, et assez commun dans l'Ouest dans certaines parties des provinces des prairies.

SOUS-ESPECES. L'espèce est divisée en deux sous-espèces: l'orientale et l'occidentale, celle-ci basée sur une taille un peu plus grande et sur une couleur plus grife. Le status précis de l'oiseau des grands lacs, en qualité de sous-espèce, n'est pas établi de façon satisfaisante. Très probablement le petit nombre de ces oiseaux qui reste dans les provinces maritimes sont des chevaliers de l'Est, tandis que ceux des grands lacs peuvent être le chevalier de l'Ouest, C. 8. inornatus, ou quelque oiseau intermédiaire. Nous avons trop peu d'échantillons sous la main pour nous prononcer à cet égard de façon définitive et, à moins que l'espèce ne recouvre quelques-uns de ses représentants originaux, nous ne serons probablement jamais capables d'établir de façon satisfaisante les limites du territoire spécial à ces deux formes.

Le ehevalier semi-palmé est une autre et importante espèce dont le nombre va disparaissant vite, disparition due peut-être surtout au vaste territoire où il niche et a l'imparfaite protection dont il est victime.

\section{Genre-Bartramia. Maubèche à longue queue.}

261. La Maubèche à longue queue. (UPLAND PLover). Bartramia longicauda. L, 11-50. Parties de dessus foncées; plumes à larges bords chamois, couleur qui s'étend fortement sur la tête, la poitrine et le cou. Marques foncées en forme de $V$ sur la poitrine qui se changent en barres sur les flancs et en raies sur le cou; parties de dessous, ternes, blanc crémeux.

Traits distinctifs. Si l'on se rappelle que cette espece est une maubèche et non un véritable pluvier, on trouvera que la taille et la teinte chamois sont caractéristiques. La barbe intérieure de la première primaire, fortement marquée de dents de scie pointues, foncée sur un fond blanc sur presque toute la longueur, est un caractère qui ne se présente chez aucun autre oiseau de rivage canadien qu'on puisse lui comparer. Le courlis de la baie d'Hudson présente un dessin semblable mais sur un fond chamois.

Indications sur le terrain. Le pluvier des hautes terres est a peine reconnaissable sur le sol, comme étant un échassier par ceux qui ne le connaissent pas, car il ressemble à une jeune gelinotte bien plutôt qu'à une maubèche. Quand il vole, toutefois, il fait voir sa véritable parenté. Sa taille, sa couleur générale, chamois, son habitat des plateaux sont des traits distinctifs. Son beau sifflement prolongé quand une fois on l'a entendu ne peut plus vous tromper.

Habitat. C'est probablement un oiseau de la région des prairies, mais qui, probablement, se propagea vers l'Est quand les forêts furent abattues. Il couve dans les parties plus méridionales du Canada oriental et dans les provinces des prairies, faisant d'occasionnelles apparitions sur la côte de l'Atlantique en qualité de migrateur.

Bien qu'on l'appelle pluvier, eette espèee est une véritable maubèche. Le terme de maubèche bartramienne est plus satisfaisant et devrait être généralement employé au lieu de pluvier des plateaux. Infiniment plus commun jadis, qu'il ne l'est aujourd'hui dans la région des grands laes, il est à présent fort rare. L'espèce niehait dans les contrées eultivées et y était exposée aux dangers que présentent ces localités: empêehement que 
les travaux agricoles opposent à la couvaison; le jeune homme toujours là et toujours armé de son fusil; le braconnier et le sportsman. Sa taille et sa facilité à s'apprivoiser ont été la cause de sa disparition alors que le plus petit et le plus prudent pluvier Kildir a su survivre dans des conditions analogues. Comme son nom l'indique cette espèce a déserté l'habitat ancestral trop humide de sa famille et s'est transporté vers les prairies des plateaux et leurs pâturages plus secs. Il est cependant rare de le trouver très éloigné de quelque petite nappe d'eau. Il se pose volontiers sur les barrières, sur les maisons ou quelquefois sur les arbres.

Valeur économique. Comme elle fréquente les terres cultivées, cette espèce se nourrit principalement d'insectes, de sauterelles, de chenilles et d'autres ennemis des cultures fourragères. On a vu de quelle importance elle pouvait être pour combattre le fléau des sauterelles; elle doit dono être parmi les espèces les plus utiles à l'agriculture et qui méritent d'être épargnées.

\section{Genre-Tryngites. Maubèches à poitrine jaunatre.}

262. La Maubèche à poltrine jaunatre. (BUFF-BREASTED SANDPIPER). Tryngites subruficollis. L, 8.50. Le dos et la couronne sont foncés, les plumes du bas du dos sont finement bordées de jaune crème, les parties de dessous sont blanches; la gorge, le cou, la poitrine et les flancs sont étendus de jaune chamois, couleur qui teinte presque toutes les parties de dessus.

Traits distinctifs. Petite taille et couleur généralement chamois; le dessous des barbes internes des primaires est finement moucheté de tons fonces aur du blanc. Ia surface de dessous de l'aile est admirablement marbrée d'une façon qui ne se retrouve dans aucune autre espece de l'Est.

Indications sur le terrain. Petite taille et couleur généralement jaune chamois. Sur les hautes terres elle peut ressembler a la maubeche a longue queue mais elle est beaucoup plus petite que cette dernière.

Habitat. Elle couve sur les rivages arctiques de l'extrême nord-ouest, voyageant au sud le long de la vallée du Mississipi; de la vient qu'elle est très rare dans les provinces maritimes, peu nombreuse dans la région des grands lacs, et plus commune vers Pouest.

Valeur économique. Ce qui a été dit de la maubèche à longue queue est probablement vrai aussi de cette espèce-ci.

\section{Genre-Actitis. Maubèche tachetée.}

263. La Maubèche tachetée. L'Alotetre BRANLE-QUEUE. (SPOTTED BANDPIPER). Actitis macularia. L, 7.50. Planche VIII B.

Traits distinctifs. Les adultes ont très manifestement des taches rondes a la poitrine et un éclat légèrement verdâtre sur le dos. Les jeunes oiseaux d'automne ressemblent au chevalier solitaire, mais se distinguent par des axillaires blanches au lieu de barrées.

Indications sur le terrain. La taille et les taches rondes bien distinctes sur la poitrine. Quand cet oiseau vole on peut le distinguer du chevalier solitaire, auquel il ressemble le plus, par la ligne blanche le long des bords des secondaires et par la beaucoup plus petite quantité de barres noires et blanches sur la queue. Le vol, quand l'observateur le connait bien, est aussi très caractéristique.

Nid. Un petit creux dans le sol non loin de l'eau, abrité par un bout de buisson ou d'herbe.

Habitat. Couve dans tout le Canada oriental jusqu'aux parties les plus septentrionales du Nouveau-Québec. Elle est commune dans toute l'étendue de ce territoire.

C'est la plus commune des maubèches d'été du Canada; quelquefois des couples sont vus le long des plus petits cours d'eau. Elle fréquente toute sorte de terrains, les plages sablonneuses, les bancs de gravier, les terrains fangeux ou les côtes rocheuses. Presque toute maubèche qu'on 
voit en été près de nos cours d'eau ou de nos étangs peut être regardée comme appartenant à cette espèce, à moins qu'il n'y ait de fortes raisons pour la croire d'une autre espèce. Son habitude de balancer parfois son corps de haut en bas, même quand on la croit au repos, ou très rapidement quand elle est agitée, lui a fait donner le nom de "Tip-up". Ses ailes à barres blanches, son vol bizarre où quelques battements d'ailes sont suivis d'une courte envolée avec les ailes rabattues, et son cri triomphant de "piou-it-piou-it-piou-it) quand elle se laisse choir sur le bord de la rivière, bien en avant de l'observateur, sont suffisamment connus de tous. On peut la chasser d'un lieu, à un autre, jusqu'à une certaine distance de son nid, quand, rattrapée par son camarade, elle viendra voler en cercle autour de l'intrus et puis retournera à la place d'où elle partit.

Valeur économique. Bien qu'elle fréquente d'habitude les bords de l'eau elle est souvent vue dans les champs voisins courant entre les sillons de la terre fraîchement retournée ou entre les rangs des plantes grandissantes. Sa nourriture se compose principalement, sinon entièrement, d'insectes, par où elle rend service à l'agriculture. L'espèce ne semble pas avoir beaucoup à souffrir du fait des chasseurs et paraît se maintenir dans les parages les plus cultivés.

\section{Genre-Numenius. Courlis.}

Description générale. Ce sont de gros oiseaux de rivage, entre 13 pouces 50 et 24 pouces de long. La coloration est de diverses teintes depuis le jaune crème au brun clair, pommelée en dessus, plus claire en dessous; le cou et la poitrine finement rayés, avec un jaune chamois, plus ou moins étendu ou seulement indiqué sur le tout. Les becs sont longs et recourbés vers l'intérieur.

Traits distinctifs. Taille grande; bec recourbé en dessous; couleur générale chamois. Se distingue des barges par le bec recourbé vers le bas au lieu de l'être légèrement vers le haut. corps.

Indications sur le terrain. Taille grande, bec recourbé, couleur chamois par tout le

Un grand corps constitue pour les oiseaux une menace certaine à leur existence. Les courlis en sont un bon exemple, et à moins que d'intelligentes mesures ne soient prises bientôt pour les protéger il n'en restera bientôt plus. Dans l'Est les courlis ont à peu près disparu, mais il en reste encore passablement dans l'Ouest. La partie végétale de leur nourriture est surtout le fruit sauvage, et dans les provinces maritimes ils fréquentent les marais déserts et ceux des plateaux pour y trouver les airelles et les myrtilles dites atocas. Dans les champs cultivés les insectes sont leur principal aliment, et comme beaucoup d'espèces nuisibles, les sauterelles incluses, sont mangées, la présence des courlis est certainement profitable au cultivateur.

264. Le Courlis à long bec. (LONG-BILLED cURLEw). Numenius americanus. L, 24. C'est le plus grand de l'espèce. Sa coloration de tous les courlis est tout a fait la même. Mais le courlis à long bec est visiblement chamois en dessous au lieu du blanc crémeux (voir titre précédent).

Traits distinctifs. Chez les spécimens de belle taille, la longueur extrême du bec (6 pouces) de cette espèce est un signe déterminant, mais, comme dans beaucoup d'autres espèces ou très spécialisées ou très grandes, la taille continue à croitre pendant quelque temps après la maturité, ce caractère ne suffit pas à lui seul à nous guider. La couronne, les axillaires et les caractères des primaires sont toutefois d'excellents critères pour les courlis. Chez cette espèce la couronne est foncée, uniformément tachée de points clairs non réunis en une ligne médiane, et les barbes intérieures des primaires sont marquées de dessins dentellés foncés sur un fond chamois pâle. Les axillaires ont une coloration totale sans raies. 
Indications sur le terrain. Pour reconnaltre le courlis voir la page précédente. On ne peut pas distinguer séparément les courlis a l'état vivant avec une certitude absolue.

Habitat. Il couve dans les provinces des prairies et tout à fait vers le sud. On le signalait à l'origine comme un migrateur plus ou moins commun sur la côte de l'Atlantique, au nord des provinces maritimes, mais on ne l'y rencontre pas actuellement; on le signale aussi dans la région des grands lacs mais sans preuve suffisante. La confusion qu'on fait de ce courlis et du courlis de la baie d'Hudson a été la cause de nombreuses fausses identifications dûment constatées et l'espèce ne devrait, à l'avenir, être signalée dans le Canada oriental que sur le témoignage irréfutable de spécimens probants.

265. Le Courlis de la Bale d'Hudson. (Hodsonian curLew). Numenius hudsonicus. $L, 17$. C'est un courlis plus petit que le précédent, mais plus grand que le suivant. Il est de la même coloration générale, mais le courlis de la baie d'Hudson est moins jaune chamois que celui à long bec, les dessous étant jaune crème mat; voir le titre du courlis, page précédente.

Traits distinctifs. Il a souvent été pris pour le courlis a long bec, mais il peut aisément se distinguer des autres courlis par la raie médiane très prononcée sur la couronne, plutôt que par les taches uniformément distribuées, ajoutée aux marques en dents de scie dans les barbes intérieures des primaires et les axillaires barrées.

Indications sur le terrain. Pour reconnaitre les courlis comme tels, voir Courlis, page précédente. Les courlis ne peuvent pas être facilement distingués à l'état vivant, avec une absolue certitude, mais la grosseur et la longueur du bec peuvent y aider. C'est la seule espèce de courlis qu'on ait quelque chance de rencontrer dans l'est du Canada.

Habitat. Il couve dans le nord-ouest du pôle. Il voyage le long de la côte du Pacifique et à travers le continent jusqu'à l'Atlantique où il est plus commun que dans l'intérieur. C'est un migrateur régulier quaique peu abondant dans la région des grands lacs.

C'est le seul courlis authentique dans la partie inférieure des grands lacs. L'expérience lui a appris à être un oiseau sauvage et prudent et comme il ne se rencontre actucllement en grand nombre que dans l'extrême est, on n'en prend que fort peu.

266. Le Courlls du Nord. le corbigea des esquimaux. (ebrimo curlew). Numenius borealis. L, 13.50. C'est le plus petit des courlis. De la même coloration générale que le courlis de la baie d'Hudson.

Traits distinctifs. Facilement distinguable de l'un quelconque des autres courlis par sa plus petite taille et ses primaires, sans marque, bien que les axillaires soient barrées comme chez le courlis de la baie d'Hudson et que la couronne soit tachetee uniformément, sans raie médiane, comme le courlis à long bec.

Indications sur le terrain. Voir l'espèce précédente. Cet oiseau est cependant trop rare de nos jours pour qu'il suffise de l'avoir vu pour en parler.

Habitat. Couve sur les terrains déserts de la région du Mackenzie. En automne il émigre vers le Labrador et puis il descend le long de la côte. Au printemps il remonte la vallée du Mississippi.

Cet oiseau est bien près de disparaitre. Qu'une chasse, ou permise ou illicite, soit ou non la cause principale de la grande diminution de cette espèce, car d'anciens récits parlent de ses bandes immenses, elle y a certainement contribué. C'est un nouveau cas de l'extinction d'une espèce avant qu'on ait aperçu la nécessité de la protéger, et de la menace de voir se perdre une espèce qui ne pourra plus se remplacer.

\section{FAMILLE-CHARADRIID E. PLUVIERS.}

Description générale. Les pluviers ont un corps plus gros et massif que les oiseaux du genre bécasse. Leur bec est plus court, mou à la base, mais terminé en pointe cornée et dure (fig. 24, p. 23). Le doigt postérieur manque dans toutes les espèces sauf dans celle à ventre noir ( $\left.{ }^{\circ} 270, p .114\right)$ dans laquelle il est très petit, presque rudimentaire.

Traits distinctifs. Avec la susdite description le pluvier ne court pas le risque d'être confondu avec aucun autre oiseau. 
Nid. Sur le sol dans un petit creux généralement garni d'un peu d'herbe, de mousse, d'autres végétaux, de rebut ou de petits cailloux.

Habitat. Représentée par des variétés très proches parentes ou presque identiques, la famille est circompolaire, couvant surtout au nord des régions habitées.

Les pluviers sont très connus des chasseurs. Leur taille moyenne est plus grande que celle des bécasses, et quelques-uns qui trouvent leur nourriture dans les champs des plateaux font passablement trimer les chasseurs.

Valeur économique. Considérés comme une famille, les pluviers recherchent les terres cultivées plus que ne font les autres oiseaux de rivage, ce qui les rend plus intéressants au point de vue économique. Ils prêtent à l'homme une assistance efficace.

\section{Genre-Squatarola. Pluvier à ventre noir.}

270. Le Pluvier à ventre noir. Lg VANNeAd onis. (BLACK-BELlied Plover). Squatarola squatarola. L, 11. Plumage de printemps: dos, presque noir avec beaucoup de pointes de pennes larges et blanches rassemblées sur les ailes, couronne, arrière-cou et croupion blancs ou presque blancs; joues, gorge, partie antérieure et poitrine jusqu'à l'abdomen sont noir pur, montrant souvent des plumes blanches qui survivent à la période d'imparfait développement. Plumage d'automne: dos, brun pâle avec bouts de pennes crême ou jaunâtres jusqu'au sommet de la tête; gorge, avant-cou et dessous blanchâtre terne avec bande indistincte de poitrine formée de raies voilées. Tous les plumages intermédiaires sont pris en dedans de nos frontières.

Traits distinctifs. A tous les caractères du pluvier; ne pourra être confondu qu'avec le pluvier doré auquel il ressemble tout à fait. La présence d'un doigt postérieur petit quoique bien formé est particulier à cet oiseau, car c'est notre seul pluvier qui ait un quatrième doigt.

Indications sur le terrain. Il ne doit être distingué que du pluvier doré: une bande assez peu distincte sur l'aile étendue; les axillaires noires, en violent contraste avec l'arrièreplan des dessous de l'aile quand l'oiseau vole, et croupion blanc qu'on pourra voir si les circonstances s'y prêtent.

Habitat. Espèce circompolaire couvant en Amérique le long des rivages polaires au nord-ouest de la baie d'Hudson. Plus ou moins commune pendant ses migrations vers des habitats propices dans tout l'est du Canada.

\section{Genre-Pluvialis. Pluviers dorés.}

272. Le Pluvier doré d'Amérlque. (AMEAican golnen Ploven). Plueralis dominicus. $\mathrm{L},-\mathbf{1 0 \cdot 5 0}$. Plumage de printemps: dos presque noir jusqu'au sommet de la tête, avec de nombreuses pointes de plumes jaunes, plus rares sur la couronne; le front et la ligne sur l'oil, qui descend vers les côtés de la poitrine, sont blancs; la gorge, le devant du cou et tous les dessous sont tout noirs. Plumage d'automne: dessus, brun terne avec beaucoup de taches d'un jaune crémeux sur le bord des plumes, se réunissant sur le croupion et la couronne; la gorge et la face légèrement tachetées de blanc; la poitrine et tous les dessous vaguement barrés d'un blanc mat et de légères teintes du même brun que sur le dos. On peut voir là tous les plumages intermédiaires.

Traits distinctifs. C'est un oiseau un peu plus petit que le précédent, mais avec lequel il est facile de le confondre. La forte quantité de jaune sur le dos du plumage de printemps et les barres indistinctes de la poitrine et des dessous en automne sont caractéristiqueş. L'absence de toute trace du doigt postérieur est une indication dans tout plumage.

Indications sur le terrain. Il n'est besoin que de le distinguer de l'cspèce précédente. L'aile déployée ne porte aucune indication de bande blanche; les axillaires sont gris fumée au lieu de noir très marqué; le croupion n'est pas blanc.

Habitat. Couve sur les terrains stériles depuis la baie d'Hudson vers l'Ouest. Il passe pour suivre des routes étranges dans ses migrations. En automne il se dirige a l'est vers le Labrador, puis au sud à travers le golfe du Saint-Laurent, jusque dans la NouvelleEcosse, d'où il traverse la mer jusqu'au Brésil sans toucher de terre intermédiaire à moins d'être entrainé par la tempête. Du Brésil il gagne les Pampas de l'Argentine. Au printemps il touche à l'ouest de l'Amérique du Sud, vers la république de l'Equateur, traverse 
l'isthme de Panama et le golfe du Mexique, puis remonte la vallée du Mississipi pour regagner les terrains où il a niché. Le fait que cet oiseau traverse au vol l'immense Atlantique sans se reposer, semble trop extraordinaire pour être vrai, mais comme il n'est pas nageur émérité, capable de se reposer sur l'eau, à volonté, il faut bien en croire des faits évidents.

SOUS-ESPECES. Le pluvier doré d'Amérique comprend deux sous-espèces; mais seul, le pluvier doré de l'Est comme forme typique, se rencontre dans le Canada oriental.

Il était jadis plus commun qu'aujourd'hui; on en voyait régulièrement des bandes considérables du vivant même des chasseurs de nos jours; aujourd'hui on n'en rencontre plus que par hasard. Ce pluvier est proche parent du pluvier doré d'Europe.

\section{Genre-Oxyechus. Pluvier Kildir.}

273. Le Pluvier klldir. (KILLdeEer Plover). Oxyechus vociferus. L, 10.50. Planche IX A.

Traits distinctifs. Comme le pluvier Kildir est le plus grand des pluviers semipalmés il ne peut guère être confondu avec ses congénères plus petits que lui. La bande double, au lieu de simple, de la poitrine l'en distingue aussi.

Indications sur le terrain. La taille, deux bandes de poitrine au lieu d'une, une queue et un croupion de couleur ocreuse, et un cri très particulier qui dit Kildi-kildi-kildi-kildi.

Nid. Un simple trou dans le sol maigrement garni de fragments de végétaux.

Habitat. C'est une espèce dont le territoire est remarquablement étendu, car elle couve depuis les limites nord de la culture agricole au Canada, jusqu'au golfe du Mexique. Elle est rare ou même absente des parages de l'une ou de l'autre cóte.

C'est un oiseau d'été commun par tout le Canada sauf dans les districts de la côte maritime. Son cri sonore est bien connu des habitants du pays et même des gens de passage. Quoique véritable oiseau de rivage, il fréquente les hauts plateaux desséchés, et les champs cultivés, faisant souvent son nid dans les sillons labourés au sein des récoltes, mais quand ses petits sont éclos il a coutume de les mener vers l'eau, toujours vers quelque étang prochain et paisible, et sitôt qu'ils sont capables de se conduire tout seuls ils se rassemblent en petites troupes séparées. Le fait que cette espèce a pu survivre et prospérer au sein même des régions cultivées et de la civilisation, alors que d'autres espèces tout aussi capables, en apparence, de se protéger ont cependant succombé, s'explique probablement par ses habitudes relativement solitaires et parce qu'elle n'a pas coutume de se rassembler en troupes serrées dont la chasse est avantageuse.

Valeur économique. Sa nourriture se compose largement d'insectes, et pendant le printemps et le commencement de l'été il fréquente des terres où il peut faire beaucoup de bien.

\section{Genre-Charadrius. Pluviers à collier.}

274. Le Pluvier semi-palmé. (semi-palmated plover). Charadrius semipalmatus. L, 6.75. C'est un petit pluvier qui ressemble de façon générale au Kildir, mais avec une seule au lieu de deux bandes foncées sur la poitrine, et sans le croupion et la queue couleur d'ocre de cette espèce. Les oiseaux d'automne sont semblables, mais leurs couleurs sont délavées et sans un noir pur où que ce soit.

Traits distinctifs. Avec sa taille et la description susmentionnée ce pluvier ne peut être confondu qu'avec le pluvier criard, mais la coloration du dos est à coup sâr brunâtre dans tous les plumages au lieu de gris fumée ou sable sec; la bande pectorale est toujours large, continue et bien marquée même en automne, et les couvertures d'oreilles derrière et au-dessous de l'œil, sont toujours parfaitement noires au lieu d'un blanc pur. Les pieds avec de petites membranes expliquent le surnom; c'est le seul pluvier au Canada qui ait cette membrane. 
Indications sur le terrain. Sa taille et la bande pectorale unique au lieu de double distinguent ce pluvier du kildir, et le dos très foncé comme du sable mouillé le distingue du pluvier criard qui est plus clair et gris comme du sable sec.

Nid. Dans un creux du sol ou dans des déchets de rivage.

Habitat. Il couve dans l'extrême nord à travers le continent; au sud, dans l'est, jusqu'au golfe du Saint-Laurent. Il est commun dans ses inigrations vers les localités qui lui agréent dans le Canada oriental.

C'est un joli petit pluvier qui fréquente indifféremment les lieux fangeux ou les plages sablonneuses. Très proche parent du pluvier rayé d'Europe et ne s'en distingue que par une plus petite taille et quelques menus détails.

277. Le Pluvier criard. (PIPING Plover). Charadrius melodus. L, 7-10. Plumage de printemps: mêmes marques que celles du semi-palmé précédemment décrit, mais le dos a des couleurs plus claires. Le dos et la couronne sont gris fumée clair, une barre noire en travers du devant de la couronne; le dessous jusqu'à la gorge, le col autour du cou et le front sont blancs, un anneau noir autour du cou, droit au-dessous du col blanc, quelquefois brisé au centre de la poitrine. Oiseaux d'automne: semblables mais sans lanneau noir et généralement de coloration plus faible.

Traits distinctifs. Ne peut êtré confondu qu'avec le semi-palmé, mais facilement distinguable par les caractères indiqués sous ce titre.

Indications sur le terrain. Distinguable du pluvier semi-palmé par sa coloration plus claire, comme le sable sec du sable mouillé. Son chant mélodieux ne s'oublie plus une fois entendu.

Nid. Dans un trou fait dans le sable par l'oiseau et ne contenant que quelques petits cailloux, des grains de sable ou des fragments de coquilles sur lesquels reposent les œufs.

Habitat. Il couve sur les rivages sablonneux dans des endroits situés entre les provinces des prairies, le golfe du Saint-Laurent et l'ile au Sable.

C'est un petit pluvier surnommé meloda, et un oiseau de plage sablonneuse qu'on ne voit jamais dans des places herbeuses ou marécageuses. Chez certains individus les bandes pectorales noires sont brisées, chez d'autres elles sont entières. Ces derniers ont été pendant quelque temps considérés comme une sous-espèce; tous, actuellement, ne constituent plus qu'une seule espèce. Le pluvier neigeux, œgialitis nivosa, a été pris sur le lac Ontario, mais on ne l'y reverra probablement plus. Il est un peu plus petit que le pluvier criard, de la même coloration mais avec une mouche noire sur l'oreille ou la joue, et seulement une tache noire sur les côtés de la poitrine au lieu d'une barre complète ou presque parfaite en travers. Un oiseau qu'on surprendrait plus au sud ou à l'ouest ne pourrait être qu'un vagabond.

\section{FAMILLE-APHRIZID AE. （SURF-BIRDS）TOURNE-PIERRES}

Description générale. Oiseau de rivage de taille moyenne avec un bec quelque peu court (figure 27, p. 24) corné en sa moitié terminale, le bout légèrement aplati (sur un plan horizontal) mais non distinctement agrandi comme chez le pluvier.

C'est unc petite famille dont l'habitat est le monde entier. Il n'y en a qu'une seule espèce dans l'est du Canada.

\section{Sous-famille-Arenariinz. Tourne-pierres.}

\section{Genre-Arenaria. Tourne-pierres.}

283a. Le Tourne-pierre à poitrine noire. LE TOURNE-PIERRE. (TURNSTONE). Arenaria interpres. L, $9 \cdot 50$.

Traits distinctifs. 'C'est un oiseau d'une coloration frappante. Le dos présente des amas assez étendus de rouge mat, de noir, et de blanc, couleurs plus ou moins entremêlées. Le croupion et la tête blancs, la couronne a des raies brunes ou noires. Les dessous d'un 
blanc pur, avec bande pectorale noire, allant des côtés du cou jusqu'à la face où elle fait un cercle en travers de l'oeil et autour d'une tache blanche locale. Les oiseaux d'automne ont leurs couleurs diffuses et la coloration du dos ou perdue ou vaguement indiquée, mais il reste toujours assez de marques sur la face de la poitrine pour faire admettre les traits distinctifs susdits.

Indications sur le terrain. La singulière mixte coloration de rouge, noir et blanc du plumage de printemps. En automne les couvertures blanches du bas du dos et celles du dessus de la queue sont séparées par une barre noire.

Nid. Dans un creux du sol garni de quelques feuilles mortes ou de fibres végétales.

Habitat. Le tourne-pierre considéré comme espece a un des habitats les plus vastes de la gent volatile; il y a, en effet, peu de pays ou il ne se rencontre pas. La sous-espèce américaine, le tourne-pierre à poitrine noire, couve depuis le rivage polaire, à l'ouest de la baie d'Hudson, vers le Nord; il est plus commun sur la cóte de l'Atlantigue que sur celle du Pacifique. Dans ses migrations, il est commun dans la région des grands lacs.

$S O U S-E S P E C E S$. Le tourne-pierre est représenté en Amérique par une sous-espèce A. $i$. morinella, le tourne-pierre à ventre noir, bien que la forme typique se rencontre, dit-on, dans l'ouest de l'Alaska.

C'est un oiseau des rivages sablonneux, boueux ou rocheux mais qui préfère les premiers. Son nom lui vient de son habitude de retourner les petites pierres et les cailloux de la plage pour chercher dessous ce dont il se nourrit; il est surprenant de voir quelles pierres, relativement grosses, il peut retourner. Il fait pénéter son bec sous le bord, donne une petite secousse et la pierre s'en va rouler ou glisser sur la plage à une grande distance. Cet oiseau est bon nageur. Il diffère du tourne-pierre de l'ancien monde par sa taille un peu plus petite, par moins de noir sur les dessus, et par une coloration plus forte des jambes.

\section{FAMILLE-H EMATOPODID E. HUITRIERS.}

Description générale. C'est un grand oiseau de rivage, de charpente plus lourde que ce n'est l'habitude dans cet ordre; gros bec corné, aplati sur les cótés vers la pointe. II n'y a qu'une seule espèce qui peut, peut-être, se rencontrer dans l'est du Canada.

\section{Genre-Homatopus. Huitriers.}

286. L'Huîtrier d'Amérique. (AMERican oYster-Catcher). Homatopus palliatus. L, 19. La tête, le cou et le haut de la poitrine sont noirs; le dos, brun olive, avec, en contraste, des plaques blanches aux ailes et au croupion. Tous les dessous, blanc pur; le bec, grand, rouge brillant.

Habitat. La côte de l'Atlantique du nord jusqu'en Virginie; autrefois jusqu'au Nouveau-Jersey et accidentellement au Nouveau-Brunswick. Il nichait à l'origine probablement dans toute l'étendue de son habitat.

L'habitat nord de cet oiseau remarquable était une fois sur nos côtes maritimes méridionales. Il y a longtemps qu'on l'a considéré comme disparu du Canada, et il y a peu de chance qu'il s'y représente de nouveau.

\section{Ordre-Gallinæ. Gallinacés.}

Ainsi que l'indique leur nom anglais "Scratching birds" ces oiseaux sont organisés pour obtenir leur nourriture en grattant la terre. Le type le plus populaire en est la volaille ordinaire de basse-cour, mais cet ordre se confond presque imperceptiblement avec les pigeons d'une part et les oiseaux de rivage d'autre part. Ils se trouvent dans presque tous les pays du globe. Au Canada nous n'avons qu'un seul sous-ordre de ce groupe, celui de Phasiani, les poules vraies. 

TES ET LAGOPEDES

Description générale. Ce sous-ordre se compose d'oiseaux pourvus de pieds forts, compacts, quatre doigts avec ongles obtus propres à gratter la terre (figure 28, p. 24). Bien qu'ils s'adaptent mieux à la vie terrestre ils se perchent facilement sur les arbres et bien souvent y mangent et s'y reposent. Bec court, corné avec culmen fortement arqué (figure 29, p. 24); narines enfoncées dans une matière molle à la naissance du bec; ailes courtes et arrondies. Ces oiseaux se servent rarement de leurs ailes, sauf pour franchir de courtes distances ou en cas de danger immédiat.

Nid. Ils nichent à terre, déposent leurs cufs sur l'herbe ou les feuilles mortes avec peu ou point de préparation.

Habitat. On trouve des especes de ce sous-ordre dans toutes les parties du Canada. La gelinotte huppée, le tétras et le dindon sauvage sont des oiseaux des bois; la caille, la poule des prairies et la gelinotte à queue aigué fréquentent la prairie; et le lagopède fréquente les terres stériles de l'extrême nord.

Trois familles de cet ordre sont représentées au Canada, savoir: Odontophoridœ, la caille d'Amérique, Tetraonidœ, la gelinotte, et Meleagridœ, le dindon.

Valeur économique.-Ils sont à la fois insectivores et végétariens, et mangent indifféremment grains, bourgeons, feuilles, fruits et insectes. Comme plusieurs espèces fréquentent les terres cultivées, leur régime économique intéresse particulièrement le cultivateur et a été l'objet de beaucoup de recherches dont les résultats démontrent qu'ils comptent parmi les oiseaux les plus utiles de la ferme. Le type d'insectes qui constituent une partie de la nourriture de certaines espèces est manifestement très malfaisant et l'on a très peu à se plaindre quant au reste de leur menu qui se compose principalement de déchets ou de matières sauvages de peu ou point d'utilité pour l'agriculteur.

De même que la plupart de nos grands oiseaux, leur nombre a été considérablement réduit et il faudrait qu'ils fussent protégés et que la chasse en fút limitée au surplus annuel de la reproduction afin de conserver en permanence un nombre plus que suffisant de reproducteurs.

\section{FAMILLE-ODONTOPHORTDE. CAILLES D'AMÉRIQUE.}

Description générale. Ce sont les plus petits oiseaux de ce sous-ordre au Canada. La narine est en partie recouverte d'une lame charnue qui n'est pas aussi bien dissimulée dans le plumage que celle de la gelinotte. Cette famille n'est représentée que par une seule espèce dans l'est du Canada.

Le nom de "caille» est improprement employé pour nos oiseaux canadiens. Ils n'ont rien de commun avec la caille d'Europe, mais ce sont de véritables perdrix. Par contre, nos "perdrix" sont des gelinottes. Voila des exemples de noms européens improprement appliqués à des formes du nouveau monde. Il y a beaucoup de cas semblables, ce qui donne lieu à la confusion au début, mais ils sont tellement implantés dans les usages nationaux qu'il est maintenant trop tard pour les corriger.

Cette famille habite plutôt les régions méridionales et c'est dans les états du Sud-Ouest et au Mexique qu'on trouve en plus grand nombre tant les espèces que les individus.

\section{Genre-Colinus. Cailles d'A mérique.}

289. La Caille d'Amérique. (BOB-WHITE, AMErican QUAIL). Colinus virginianus. L, 10. Planche IX B. 
Traits distinctifs. Ne peut être confondu avec aucun autre oiseau au Canada. Taille et couleur avec traits distinctifs des gallinacés.

Indications sur le terrain. Petites perdrix qui s'enlèvent soudainement et volent par battements rapides avee des coups d'ailes qui sentendent de loin.

Habitat. La caille d'Amérique et ses sous-especes alliées sont répandues sur toute la partie est de l'Amérique du Nord, dans l'Ontario-sud et plus au nord.

SOUS-ESPECE. La sous-espèce indigène à l'est du Canada est le spécinen type: la caille de la Virginie.

La caille d'Amérique n'apparaît, au Canada, que dans le sud de l'Ontario où elle est connue de tous les habitants de la campagne. A l'automne, le chasseur la poursuit avec des chiens et, au printemps, le laboureur et ses enfants trouvent son nid au cours des travaux de la ferme où chacun connait son sifflement perçant qui semble crier "Bob-white" ou ainsi que d'autres l'interprètent "More-wet". Ce n'est pas une espèce solitaire qui se retire dans les plus profonds sous-bois a l'époque de la culture; mais elle se tient dans les clairières, sur les lisières des bois, les lignes d'arbrisseaux, le long des clótures ou des taillis incultes au voisinage immédiat des champs. Quand elle manque de nourriture elle s'aventure dans la basse-cour pour manger avec les volailles. Elle mange dans la plaine et s'abrite dans les broussailles contre le danger. Avant que les terres fussent déboisées, la caille d'Amérique était peut-être rare au Canada, mais les progrès de la colonisation lui ont ouvert de nouveaux terrains. Même dans les parties les plus méridionales du pays aujourd'hui cet oiseau demeure de façon précaire et en nombre très variable; il est évident qu'il ne s'adapte pas très bien à cette limite septentrionale de son habitat. Il est très prolifique cependant, et à la suite de quelques hivers propices, si l'on en interdit la chasse, le nombre d'individus se doublera plusieurs fois; mais les abris sont presque invariablement mitraillés et les durs hivers réduisent périodiquement le nombre de ces oiseaux. Les conditions naturelles les plus dures qu'ils aient à combattre résultent de l'épais manteau de neige recouvrant la terre qui leur fournit à manger, comme de l'humidité glacée et du grésil qui non seulement les gèle mais les emprisonne sous une croute de glace lorsqu'ils se réfugient dans la neige la nuit. On dit, avec des preuves à l'appui, que le faisan à cou annelé qui est devenu un gibier très recherché est hostile à ces oiseaux. En sus de son importance comme gibier, la caille d'Amérique mérite d'être protégée par les cultivateurs au seul point de vue économique et, pour cette raison, on ferait très bien de le rayer de la liste des oiseaux-gibiers.

On a eu beaucoup l'habitude de repeupler les terrains de chasse épuisés avec des oiseaux importés des Etats du Sud. L'on ne s'est pas encore rendu compte si l'introduction de ces animaux non habitués au climat du nord a eu pour effet d'affaiblir la constitution des oiseaux indigènes. Plusieurs sous-espèces de la caille d'Amérique se présentent dans le Sud et, à cause des importations, il est devenu difficile de reconnaître les véritables caractères de nos oiseaux indigènes, lesquels ne peuvent maintenant être reconnus que sur des spécimens antérieurs a ces introductions.

Valeur économique. La caille d'Amérique se nourrit principalement de graine d'ivraie. Le grain qu'elle mange se compose de déchets glanés sur la terre. La partie insecte de sa nourriture, bien que plutôt faible, comprend certaines espèces que ne mangent pas ordinairement les autres oiseaux, c'est pourquoi elle est particulièrement importante. C'est l'un des rares oiseaux qui mange les doryphores ou insectes de la pomme de terre. 
FAMILLE-TETRAONIDAE. TETRAS.

Le tétras a les narines dissimulées sous un plumage qui occupe une plage de pénétration à la naissance du bec sur les côtés (figure 29, p. 24). Le tarse est soit complètement soit partiellement emplumé, et chez le lagopede, le plumage recouvre les doigts. Les doigts, s'ils ne sont pas emplumés, sont bordés de chaque côté d'une petite frange composée d'écailles cornées ou pectineuses (figure 28, p. 24), qui tombent au milieu de l'été. Le tétras du Canada, vulgairement appelé perdrix, est le gibier de savane le plus répandu de nos plaines et c'est l'oiseau favori de nos chasseurs. Les deux sexes sont semblables ou à peu près et, si l'on excepte le lagopède, subissent de légères variations saisonnières quant au plumage, et ces oiseaux n'émigrent généralement pas. Le lagopède à qui on peut attribuer l'inverse de ces remarques est d'autre part tellement bien caractérisé qu'il n'y a pas de confusion probable. Toutes les espèces font leur nid à ras de terre sans beaucoup de préparation pour les œufs. Il's pondent ordinairement beaucoup d'œufs, de six à dix-huit, et les petits, comme des poussins, suivent la mère dès qu'ils sont sortis de la coquille.

\section{Genre-Canachites. Tétras du Canada.}

298. Le Tétras du Canada. La Perdrix DE savane. (spruce Partridge, CANADA GROURE). Canachites canadensis. L, 15. Planche X A.

Traits distinctifs. Facile a distinguer par sa couleur, etc., de la gelinotte a fraise qui est à peu près la seule espèce dans l'est du Canada avec laquelle on peut le confondre. Il y a dans l'extrême Ouest certaines espèces qui lui ressemblent étroitement.

Indications sur le terrain. Couleur généralement bleuâtre et noire du mâle. Absence de huppe ou de collier de plumes et présence d'une crète remarquablement rouge au-dessus de l'œil, tant chez le mâle que chez la femelle, mais plus en évidence sur celui-là.

Habitat. Habite en permanence là où il se trouve dans les régions boisées de conifères du nord du Canada.

SOUS-ESPECES. Le tétras du Canada comprend plusieurs races géographiques dont deux, la gelinotte noire d'Amérique, Canachites canadensis canadensis, la forme type, et le tétras du Canada C.c. canace, fréquentent l'est du Canada. Le premier de ces oiseaux habite le Labrador et les collines plus au nord; le deuxième habite le Nouveau-Brunswick, le sud de l'Ontario, ete. Ils se ressemblent trop cependant pour qu'on les différencie dans un ouvrage de vulgarisation.

C'est un oiseau septentrional des épincttières. Son naturel confiant lui a fait donner le nom de "Fool Hen", car si l'on ne fait pas beaucoup de bruit, on peut souvent le tuer avec un bâton ou avec des pierres. Du fait qu'il se nourrit beaucoup de bourgeons de mélèze ou de conifères, sa chair a un gout trop fort pour un palais ordinaire.

Valeur économique. Comme il habite au nord et dans des forêts de conifères, il n'a aucune influence économique.

\section{Genre-Bonasa. Gelinotte à fraise.}

300. La Gelinotte à fraise. LA GELINOTTE hUPPEE. LA PERDRIX dE BOIS FrANC. (RUFFen Grouse. Pantridge). Bonasa umbellus. L, 17. Planche $\mathbf{X}$ B. (Pieds et bec, figures 28,29, p. 24 ).

Traits distinctifs. La gelinotte à fraise avec sa fraise soyeuse noire, sur les côtés du cou, sa grande queue en éventail et ses nombreuses petites taches semblables à des yeux sur le croupion et le bas du dos, ne saurait être confondue avec aucune autre espèce.

Indications sur le terrain. Grande taille et queue en éventail.

Habitat. Le gelinotte à fraise habite toutes les régions boisées du Canada vers le nord jusqu'aux limites des arbres. 
SOUS-ESPECES. On reconnait plusieurs races géographiques de la gelinotte à fraise. L'oiseau type habite le sud et est remplacé dans l'est du Canada par la gelinotte du Canada Bonasa umbellus togata qui est caractérisée par une coloration plus grise ou moins rouge.

C'est la "perdrix" de la plupart de nos chasseurs canadiens. Elle habite le fond des bois au milieu des brousailles, se cache à ras de terre, s'enlève comme une petite explosion et vole avec une grande rapidité à travers la forêt sombre de façon à éprouver l'habileté et l'adresse de tout bon tireur. La gelinotte du Canada ne se rencontre aujourd'hui que dans les coins de forêts où elle trouve suffisamment d'espace et d'abri pour se protéger et sur les lisières des terrains cultivés où elle n'existe encore qu'à titre précaire.

Les changements subits de température sont très mauvais pour cette gelinotte. Les printemps froids, humides, sont mortels pour les jeunes et un bon nombre d'oiseaux reproducteurs périssent en hiver par l'effet du grésil. Durant les hivers rigoureux, elle cherche un abri sous la neige ou se laisse recouvrir par ses tourbillons. Après un dégel suivi de temps froid, comme il arrive souvent, elle est gelée sous une croate qu'il lui est impossible de briser et par suite elle périt.

Le tambourinage de la perdrix est un bruit familier à tous ceux qui fréquentent les bois. C'est une série de palpitations sourdes et retentissantes produites par le battement rapide des ailes, et ce bruit a une intensité pénétrante qui fait qu'il est difficile de se rendre compte d'où il vient. Les battements commencent lentement et augmentent en vitesse jusqu'à ce qu'au bout de peut-être cinq secondes ils se confondent et se perdent dans un bruissement confus. L'oiseau se pavane ordinairement sur un tronc d'arbre renversé lorsqu'il s'arrête pour tambouriner. Durant ce tambourinage, il déploie tous ses ornements-queue, crête et collerette - et, bien que debout et immobile, ses ailes se perdent dans un brouillard de mouvement. On explique de deux manières cette espèce de tambourinage, soit pas le frottement des ailes l'une contre l'autre au-dessus du dos, soit par leur battement sur les côtés du corps. L'effet peut être produit de l'une ou de l'autre ou d'aucune de ces façons. Le bruit produit par le battement des ailes au moment où l'oiseau prend son vol est de même nature sauf en intensité et en mesure, et le seul battement de l'air semble suffire à produire cet effet. Le but de ce geste est évidemment d'attirer la femelle, comme fait la roue du paon ou du dindon. C'est au printemps surtout que l'on entend ce roulement, mais il se produit plus ou moins pendant tout l'été et encore plus fréquemment à l'automne. Chez la gelinotte huppée du Canada, Bonasa umbellus togata, il se présente deux phases de coloration bien définies qui ne dépendent ni du sexe ni de la saison ni de la localité. Il y a une certaine espèce qui est très rouge; un autre type rare a les huppes plutôt cuivrées que noires, la queue est remarquablement rouge et cette même couleur se retrouve plus ou moins sur les autres parties. Chez la gelinotte grise qui est peut-être la plus typique, la queue est manifestement grisée, il y a moins de rouge autre part et les huppes sont noires avec un faible éclat verdâtre. On rencontre toutes sortes de types intermédiaires.

Valeur économique. Comme cette espèce vit dans les bois on ne peut guère avoir à s'en plaindre et, à part son intérêt au point de vue sentimental et des plaisirs cynégétiques, elle n'a que peu d'importance économique. 
Genre-Lagopus. Lagopèdes.

Description générale. Le lagopède est un tétras plus septentrional et remarquable pour l'étonnante variation saisonnière de son plumage. En hiver il est tout blanc; en été il est rayé de diverses teintes de rouge, brun et ocre, principalement de rouge. Ses pieds sont emplumés jusqu'à l'extrémité des doigts et il fait régulièrement de longues migrations, allant à pied sur la majeure partie de leur parcours mais volant quelquefois d'un point à un autre ou pour traverser de grandes nappes d'eau comme par exemple le détroit d'Hudson. Comme il lui reste même en été des taches irrégulières de blanc à son plumage et que son pied est toujours emplumé jusqu'aux doigts, il est impossible de ne pas le reconnaftre. II babite les régions polaires et se trouve tant sur l'ancien que sur le nouveau continent. De même que bien d'autres oiseaux arctiques les lagopèdes sont très répandus au sud le long des chaines de montagnes où l'altitude transporte le climat boréal aux latitudes moins élevées. La gelinotte rouge d'Ecosse est un lagopède intéressant qui a perdu sa faculté de devenir blanc en hiver et, toute l'année, sa coloration estivale subsiste. Il y en a deux espèces dans l'est du Canada. En raison de la grande variabilité de cette espèce, les lagopèdes américains ont été divisés en un grand nombre de sousespèces qui ne peuvent être distinguées que par un spécialiste pourvu de nombreux spécimens de comparaison. Au point de vue économique le lagopède a peu d'importance sauf comme source d'alimentation pour les trappeurs, les chasscurs et les prospecteurs de l'extrểme nord.

301. Le Lagopède des saules. LA perprix Blanche. (Willow ptarmigan, WILLOW GROUSE). Lagopus lagopus. L, 15. Tout blanc en hiver, sauf la queue qui est tout à fait noire. En été il est presque uniformément rayé de noir sur tout le corps et de diverses teintes de brun, d'ocre et de rouille. Ce peut être soit l'ocre soit la rouille qui domine.

Traits distinctifs. En hiver, c'est la tête toute blanche et l'absence de ligne noire à travers les yeux qui le distingue du lagopède des rochers. En été sa taille supérieure qui se distingue surtout par la comparaison des becs le rend très facile à reconnaître.

Habitat. Régions polaires, à travers tout le continent, émigre au sud en hiver jusqu'aux frontières de la civilisation.

SOUS-ESPECES. La sous-espèce apparaissant dans la majeure partie du Canada est l'oiseau type. Une autre sous-espèce, le lagopède d'Allen $L$. $l$. alleni, se rencontre en Tcrreneuve et celle du lagopède $L$. $l$. ungavus se trouve dans l'extrême nord du Québec.

302. Le Lagopède des rochers. (Rоск pтARMigan). Lagopus rupestris. L. 13. Tout blanc en hiver sauf la queue qui est noire et une ligne à travers l'cil jusqu'd la naissance du bec. En été, il ressemble beaucoup à l'espèce précédente.

Traits distinctifs. En hiver, ligne noire des yeux. En été, on le reconnaft à sa petite taille, particulièrement celle du bec, comparée à celle du lagopède des saules.

Habitat. Régions polaires à travers tout le continent; en hiver habite un peu plus au nord que l'espèce précédente.

SOUS-ESPECES. Trois sous-espèces de lagopèdes de rochers sont reconnues aụ Canada: le lagopède de Reinhardt, $L$. $r$. reinhardti, dans l'extrémité nord du NouveauQuébec; le lagopède de Welsh, $L$.r. welchi, en Terreneuve; et la forme type, le, lagopede arctique, dans les autres régions.

\section{Genre-Tympanuchus. Gelinotte.}

305. La Poule des prairies. (PRAIRIE CHICKEN). Tympanuchus americanus. I, 18. Une gelinotte de la même taille que la gelinotte huppée, colorée avec des teintes de brun, d'ocre clair et de blanc, mais sans la longue queue en éventail, et dont la huppe souple est remplacée par quelques longues plumes droites et raides, dirigées de baut en bas au-dessus des épaules. Les rayures sur le ventre et la poitrine sont nettes et distinctes et se continuent à travers le dos, puis sur les parties supérieures et inférieures.

Traits distinctifs. Les indications précitées distinguent facilement cette espèce de la gelinotte huppée. On peut tout aussi facilement la distinguer de l'espèce suivante-la gelinotte à queue aiguë-par sa poitrine rayée au lieu d'étre recouverte de marques en forme de V. Les autres caractères distinctifs sont les longues plumes raides sur les côtés du cou et la queue courte et raide ne se terminant pas en pointe quand elle est fermée.

SOUS-ESPECES. Deux sous-espèces de poule des prairies sont reconnucs. Celle qui se rencontre au Canada est la race type-la poule des prairies du Nord. 
C'est la véritable poule des prairies qui fréquente les prairies de l'Ouest, bien que dans l'ouest du Canada, on donne vulgairement et improprement ce nom à l'espèce dont la description va suivre. Nous la faisons figurer ici seulement parce qu'on en a pris des spécimens dans le sud de l'Ontario qui semblent s'être répandus à partir du côté de Michigan de la rivière Détroit où elle était autrefois commune nais est devenue rare et restreinte à un petit nombre d'endroits. D'après les souvenirs de la présente génération, dans certaines parties de l'Ouest elle a considérablement empiété sur le territoire de l'oiseau suivant qu'elle a fini par cliasser.

Taleur économique.-Cet oiseau est trop rare dans l'est du Canada pour être étudié au long ici. Dans l'Ouest où il est très répandu, son importance économique est considérable, mais il n'est aucunement nuisible.

\section{Genre-Pediocetes. Gelinotte à queue aiguë.}

308. La Gelinotte à queue aiguë. (sharp-tailed Grouse). Pedioceles phasianellus. L, 17 -50. Décorée de vagues dessins de couleur rouge blanc ou crème sur les parties supérieures, qui se transforment en rayures seulement sur les épaules; parties inférieures d'un blanc pur. Sur la poitrine chaque plume est bordée d'un dessin foncé en forme de $V$ qui se change en rayure vers le cou et en fines taches sur la gorge qui est de couleur crème.

Traits distinctifs. Les marques en $\mathrm{V}$ sur la poitrine et la pointe molle et aigue de la queue lorsqu'elle est fermée constituent des marques d'identification concluantes et faciles a reconnaitre.

Habitat. L'espèce est plus septentrionale et moins nettement une poule de la plaine que la poule des prairies. Elle habite tout le continent au nord des terres actuellement cultivées dans l'Est mais ne se rencontre que par endroits isolés alors qu'il se trouve de vastes territoires où elle fait défaut ou ne sc rencontre que rarement. Elle vient irrégulièrement vers le sud à l'automne jusqu'aux confins des terres cultivées. Dans les provinces des prairies elle s'avance au sud jusqu'aux frontières des Etats-Unis et au dela.

SOUS-ESPECES. Cette espece est divisée en plusieurs races géographiques. Le long de la frontière manitobaine, on peut s'attendre à trouver la gelinotte des prairies a queue aiguê, Pedicecetes phasianellus campestris; dans les autres parties de l'est du Canada c'est la gelinotte à queue aiguē typique ou du Nord.

Dans l'Ouest cet oiseau est bien une poule des prairies mais, par endroits, elle est remplacée par l'espèce précédente.

\section{FAMILLE-MELEAGRID.E. DINDONS.}

\section{Genre-Meleagris. Dindons.}

C'est le plus grand de nos gallinacés, et il est si connu sous sa forme domestique qu'il est inutile d'en donner une description détaillée. Nous n'en avons eu qu'une seule espèce au Canada.

310. Le Dlndon sauvage. (WILD TURKEY). Meleagris gallopavo. L, 48.50. Ressemble tellement a notre dindon domestique qu'une description spéciale serait superflue.

Traits distinctifs. Le seul oiseau dont il a besoin d'être distingué c'est la variété apprivoisée ou domestique. Celle-ci est d'origine mexicaine et, par conséquent, a toujours un peu de blanc au bout de la qucue. La queue du dindon sauvage se termine toujours en une bande brun .cendré. Le sang d'une race apprivoisée est toujours indiqué par le bout blanc de la queue.

Habritat. Primitivement distribué sur toute la partie est de l'Amérique du Nord jusqu'au Maine et au sud de l'Ontario.

SOUS-ESPECES. On reconnalt plusieurs sous-espèces de dindon sauvage. L'oiseau type habite le Mexique. Le specimen canadien est le dindon sauvage du Nord, Meleagris galloparo silvestris. 
Le dindon sous sa forme sauvage n'a paru au Canada que dans le sud de l'Ontario et il a maintenant disparu depuis un nombre d'années. Actuellement le dindon sauvage n'habite plus que les régions boisées les plus reculées des Etats-Unis du Sud les plus déserts et même là, il tend à bientôt disparaître. Il y a probablement beaucoup de sang sauvage indigène chez les troupeaux de dindons apprivoisés, le long du lac Erié, et il y a un bon nombre de spécimens de soi-disant dindons sauvages qui sont certainement au moins à demi mêlés de sang domestique.

\section{Ordre-Columbæ. Pigeons et Colombes.}

Cet ordre, d'une distribution mondiale, est divisé différemment par les divers auteurs. D'après le système de classification de l'Union des Ornithologistes américains, toutes nos espèces américaines sont comprises dans une seule famille, les Columbida. Ils ont tout à fait la forme typique du pigeon, c'est pourquoi on peut les appeler les pigeons authentiques.

FAMILLF-COLUMBIDA. PIGEONS VRAIS ET COLOMBES.

On peut dire d'une façon générale que les pigeons et les colombes ressemblent, quant au profil et aux habitudes, à notre race domestique. Leurs caractères sont plus faciles à reconnaître qu'à décrire. C'est surtout à leur bec qu'on peut les reconnaître. Il est dur et corné à la pointe, laquelle s'élargit très légèrement. Il est pourvu, à la base, d'une membrane molle légèrement renflée dans laquelle s'ouvrent les narines (figure 35, p. 25). Les pattes et les pieds sont faibles, ne pouvant servir qu'a marcher sur une petite étendue plane ou pour simplement se percher. Nos pigeons domestiques ordinaires qui descendent du Biset d'Europe présentent tous les caractères les plus distinctifs de cette famille. Il n'existe aucune différence reconnaissable ou taxonomique entre ce qu'on appelle les pigeons et les colombes.

$$
\text { Genre-Ectopistes. Pigeons voyageur. }
$$

315. Le Pigeon voyageur. LA TOURTE. (PASSENGER PIGEaN). Ectopistes migratorius. L, 16.29. Planche XI A.

Traits distinctifs. La tourterelle de la Caroline est si souvent prise pour le pigeon voyageur que ces deux espèces devraient être distinguées l'une de l'autre avec soin. Le pigeon est un oiseau beaueoup plus grand; le mâle a la poitrine visiblement rouge avee la tête et les parties supérieures bleu ardoise; la femelle n'a pas le bleu foncé sur le dos mais la tête garde une teinte bleuâtre qui ne se rencontre jamais chez la tourterelle de la Caroline, laquelle a une couleur plus régulièrement fauve clair ainsi qu'une petite taehe noire sur le eôté du eou, droit au-dessous de l'oreille.

Indications sur le terrain. .Cette espèce étant maintenant tout à fait éteinte, toute indieation serait inutile.

Nid. Les pigeons voyageurs se eonstruisaient un nid grossier de branehettes dans les arbres et nichaient par bandes.

Habitat. Cet oiseau couvait dans les sections boisées de la majeure partie du Canada à l'est des montagnes et au sud jusqu'aux Etats-Unis du Centre, et hivernait dans les Etats du Sud et au dela.

Les immenses troupes de pigeons voyageurs qui autrefois obscurcissaient le ciel étaient l'une des merveilles de l'Amérique. Si les descriptions de leur nombre n'étaient accompagnées de témoignages détaillés, rapportés par des personnes absolument dignes de foi, elles sembleraient purement fantaisistes; des troupes, tellement compactes qu'un coup de fusil au hasard en aurait abattu des quantités, voyageaient avec rapidité, formant un front de plusieurs milles de largeur sur une longueur telle qu'il fallait des heures pour les voir passer. Audubon estime qu'une seule de ces troupes renfermait au delà d'un milliard d'oiseaux, en basant ce chiffre 
sur la densité et l'étendue du groupement et non sur une simple conjecture. Ils couvaient dans de denses refuges à corbeaux où leur poids brisait souvent les arbres de la forêt. On coupait les arbres qui renfermaient leurs nids et bien que chaque nid ne contînt qu'un seul pigeonneau, il y en avait tellement que l'on fit venir des cochons pour les leur donner en pâture. Plus tard les oiseleurs de profession les prenaient au filet et les expédiaient par pleins wagons aux divers centres de population. On comprendra que même ces immenses troupes de pigeons voyageurs ne purent pas résister à de pareilles attaques et graduellement ils ont diminué. Il a été question de mettre un terme à ce massacre à cette époque mais ces démarehes n'ont fait que provoquer des risées. L'on disait que leur nombre ćtait inépuisable, mais aujourd'hui l'espèce est éteinte et le dernier survivant, un oiseau captif, est mort il y a peu de temps à Cincinnati. Leur dernier grand ralliement fut près de Petoskey, Mich. A l'automne de 1878 les pigeons sont repartis pour le but accoutumé de leur voyage sans toutefois revenir en nombre exploitable le printemps suivant. Pendant quelques années, par la suite, on en a aperçu parfois de petits troupeaux et quelques ralliements isolés furent signalés, mais comme les oiseleurs ont suivi chaque cas de très près, il est devenu évident qu'il n'y avait plus rien à faire pour les chasseurs de pigeons. Ces oiseaux devinrent ensuite de plus en plus rares tous les ans jusqu'à ce que finalement on n'en ait plus aperçu du tout. Il y a même encore parfois des rumeurs que des bandes ont été vues en des endroits éloignés dans les montagnes occidentales du Mexique ou de l'Amérique du Sud et autre part, mais dans ehaque cas, les recherches ont démontré qu'il s'agissait d'autres espèces ou qu'on avait été mal informé. Pendant plusieurs années une forte récompense a été offerte à quiconque signalerait un couple reproducteur. Il va sans dire que la personne qui a offert la récompense a été inondée de lettres mais aucun des cas signalés n'a rien fait découvrir et la récompense n'a pas été gagnée; finalement cette offre a été retirée. Dans l'Est, e'est ordinairement la tourterelle de la Caroline que l'on prenait pour le pigeon voyageur et, dans l'Ouest, le pigeon à queue rayée. Même encore, de temps en temps, on entend certaines personnes raconter, avec force détails, qu'ils ont vu cet oiseau dans leur enfance, mais il n'est guère douteux que l'espèce est éteinte.

\section{Genus-Zenaidura. Tourterelle de la Caroline.}

316. La Tourterelle de la Caroline. (Iovnnivg pove). Zenaidura macraura. $\mathrm{L}, 11 \cdot 85$. Planche XI A.

Traits distinctifs. Plus petite et d'un fauve plus brunátre que le pigeon voyageur, sans le rouge caractéristique sur la poitrine ni le bleu sur le dos; porte une petite tache noire sur le côté du cou au-dessus de l'oreille. Ne saurait être confondue qu'avec l'espèce précédente.

Nid. La tourterelle de la Caroline se bâtit une simple plate-forme de bûchettes dans les branches inférieures des arbres ou les parties supérieures des buissons, rarement sur le sol. Elle niche solitairement et non par bandes.

Habitat. Elle couve sur les frontières méridionales de l'est du Canada et hiverne sur place dans les parties les plus méridionales et dans les Etats-Unis immédiatement au sud de la frontière jusqu'aux tropiques.

SOUS-ESPECE. La tourterelle plaintive (Mourning Dove) qui babite la majeure partie de l'Amérique du Nord est bien la tourterelle de la Caroline. $Z$. m. carolinensis, et c'est la seule sous-espèce généralement reconnue eomme apparaissant dans l'est du Canada. L'oiseau type dépasse habituellement les limites de son habitat.

Bien que le pigeon voyageur ait complètement disparu, la tourterelle de la Caroline existe encore et il est probable que le nombre en a considé$28588-9 \frac{1}{2}$ 
rablement augmenté depuis que l'on déboise le pays. Les régimes d'alimentation de ces deux oiseaux se ressemblaient beaucoup sauf quant à la proportion des divers éléments de nourriture. La tourterelle de la Caroline mange facilement des fruits d'arbres forestiers mais ceux-ci constituaient l'aliment principal du pigeon lequel était donc plutôt un oiseau forestier. La tourterelle de la Caroline a des habitudes plus solitaires et se rencontre rarement par bandes. Elle couve tout à fait seule. C'est probablement ce qui a le plus contribué à conserver l'espèce alors que son congénère de plus forte taille n'a pas survécu. La maladie ne pouvait pas se propager parmi ces oiseaux aussi complètement et toute autre calamité affectant les individus ou les petits groupements n'englobait pas toute l'espèce. Dans plusieurs sections, la tourterelle est considérée comme ou oiseau-gibier, mais le code de la chasse ne lui reconnait pas ordinairement cette qułalité. Il s'en tue cependant de grands nombres accessoirement aux autres chasses, malgré qu'elle soit protégée par la loi, et l'on ne peut pas dire que la vie de cet oiseau ne soit pas toujours exposée. C'est cependant une race forte et elle ne court en ce moment que peu de danger.

Son long cri plaintif "O-woe-woe-woe " est très connu et c'est ce qui lui a valu son nom anglais de (Mourning Dove". C'est un son très bizarre comme celui que l'on obtient en soufflant légèrement dans le goulot d'une bouteille vide.

Valeur économique. Bien que se nourrissant surtout des fruits d'arbres forestiers (glands, châtaignes et autres fruits semblables à l'écorce tendre), elle mange très bien le grain et aussi passablement d'insectes. Le grain qu'elle mange se compose en grande partie de déchets et la semence bien plantée et recouverte ne peut avoir à en souffrir, car elle ne gratte jamais la terre. On ne peut lui reprocher aucun dommage aux récoltes qui ne soit facile à prévenir et le bien qu'elle fait est manifeste.

\section{Ordres-Raptores. Oiseaux de proie.}

Description générale. Ce sont des oiseaux mangeurs de poissons avec quatre doigts bien developpés (figures 31 et 32, p. 25), armés d'une griffe puissante et crochue pour saisir et retenir leur proie. Le bec est recourbe (figures $30,33 a$ et b, et $34, p .24$ et 25) et la base en est revêtue d'une membrane gonflée et molle ou cire au sein de laquelle sont situées les narines. Les oiseaux de proie diffèrent des autres en ce que les femelles sont de beaucoup plus forte taille que les mâles, ce qu'il faut probablement attribuer aux plus grands efforts qu'elles doivent faire pour nourrir leurs petits; comme ce travail exige de la force, de la pesanteur et de l'endurance plutôt que de la finesse et de la dextérité, la femelle possède ces qualités à un plus haut degré que le mâle lequel, bien qu'il puisse porter secours à sa compagne, n'a pas la responsabilité finale d'élever la famille.

Habitat. Les rapaces sont distribués par tout le monde sauf dans le continent antarctique ou ils sont remplacés par les stercoraires, mouettes et autres rapaces de la mer.

Bien que la classification de cet ordre soit loin d'être satisfaisante et qu'elle doive être revisée, c'est l'habitude américaine de diviser notre espèce en trois sous-ordres: Sarcorhamphi, les vautours d'Amérique, qui sont différents de ceux de l'ancien monde; Falcones, les oiseaux de proie diurnes; et Striges, les oiseaux de proie nocturnes ou hiboux.

Valeur économique. Il n'est peut-être pas d'oiseau plus connu et en même temps plus mal compris d'une façon générale dans ses rapports économiques. Tout le monde connaît les éperviers, les hiboux et les 
aigles et leurs habitudes de carnivores, mais peu de gens se rendent compte que eertains d'entre eux comptent parmi les meilleurs amis de l'homme et que la manie de les tuer à première vue est désastreuse. Il y en a qui sont très malfaisants, mais e'est une très grave erreur au point de vue économique que de les condamner tous en bloc. Ceux de la première famille, les vautours, sont des oiseaux repoussants mais ils sont préeieux pour faire disparaître les déchets et il n'y a vraiment rien à redire à leur sujet. Quant aux deux autres divisions, les rapaces diurnes et nocturnes, la différence de leurs habitudes donne lieu à beaucoup de malentendus. Nous sommes heureux, en ce sens, de pouvoir donner une opinion basée sur des faits établis et non sur de simples théories. Le service biologique des Etats-Unis a fait une étude approfondie sur le régime d'alimentation des éperviers et hiboux d'Amérique et ses conclusions s'appuient sur l'examen de quelque 2,700 estomacs pris dans toutes les saisons de l'année dans diverses parties des Etats-Unis et du Canada. Toutes ces recherches accompagnées des témoignages qui en établissent l'authenticité sont publiees sous forme de rapport intitule: "The Hawks and Owls of the United States ), par le Dr A. K. Fisher'. Bien que ce travail soit fait au pays voisin pour son propre usage, toutes les espèces canadiennes sont étudiées et les résultats s'appliquent tout aussi bien au Canada qu'aux Etats-Unis. Comme certaines des espèces les moins malfaisantes n'apparaissent pas au Canada, les proportions ci-dessous devront être légèrement corrigées pour notre usage mais pas suffisamment pour modifier sensiblement les conclusions générales. Six seulement parmi les soixantetreize espèces étudiées sont malfaisantes, dont trois qui sont extrêmement rares au Canada et une ne mange que des poissons. Quant aux autres, 56 pour cent des estomacs examinés contenaient des souris et des petits mammifères, 27 pour cent des insectes, et seulement $3 \frac{1}{2}$ pour cent des volailles ou oiseaux-gibiers. En divisant les rapaces de l'est du Canada, en plusieurs groupes, il se trouve que trois espèces sont bienfaisantes et absolument inoffensives; seize sont prineipalement bienfaisantes et font véritablement plus de bien que de mal; quatre font à peu près autant de bien que de mal; et six sont manifestement malfaisantes. Trois seulement de ces dernières sont assez communes pour qu'on s'en occupe, et deux seulement, l'épervier brun et l'épervier de Cooper, qui sont assez répandus dans les localités à population dense, pour qu'on se prémunisse contre eux. L'autour à tête noire est une espèce plus septentrionale dont la distribution s'étend au delà des régions habitées dans le nord. Il est difficile de déterminer ce qui peut être considéré comme un juste milieu entre le bien et le mal que font ces oiseaux; la perte d'un poulet est une chose définie, facile à estimer, tandis que la disparition des souris et des insectes emportés par un oiseau de proie est un vague avantage difficile à vérifier ou à apprécier.

\section{SOUS-ORDRE-SARCORHAMPHI. VAUTOURS D'AMÉRIQUE.}

Ce sous-ordre se compose de vautours d'Amérique, lesquels sont de par la classification très distinets de ceux de l'ancien monde. Une seule famille est représentée au Canada, Cathartidœe, les vautours. Les vautours se nourrissent de chair corrompue, recherchent la chair morte et ne s'attaquent pas à une proie vivante à moins que celle-ci ne soit au dernier degré

"Voir aussi "The Hawks of the Canadian Prairie Provinces; their relation to agriculture". Commission gélogique du Canada, Bulletin du Musée, $\mathbf{n}^{\circ} 28,1818$. 
d'épuisement. Ils ne touchent ordinairement rien que de la viande en décomposition. L'on se figure ordinairement que c'est une question de choix, mais ce peut-être une nécessité, puisque leurs pieds ne sont pas constitués pour saisir la proie et leur bec est relativement faible. Ils sont donc peut-être incapables de déchirer de grosses carcasses encore saines et forcés d'attendre la décomposition qui rend la matière moins réfractaire.

FAMILLE-CATHARTID

Description générale. Ce sont de grands oiseaux, presque uniformément noirs de couleurs. Bec relativement long et moins fortement recourbé que chez les autres rapaces (figure 30, p. 24). La tête et le haut du cou sont dépourvus de plumage et ont une ressemblance superficielle avec ceux du dindon moins les caroncules ou excroissances verruqueuses. Pieds semblables à ceux du poulet plutôt qu'd̀ ceux de l'épervier. Les griffes sont sans pointes et le pied dans son ensemble n'est pas conformé pour saisir ou retenir une proie.

Habitat. Le vautour est essentiellement un oiseau des pays chauds. Il ne pénètre dans l'est du Canada que sur les frontières les plus méridionales.

On ne peut utilement étudier les vautours au Canada. Dans les Etats-Unis du Sud on peut les voir à chaque heure du jour planant haut dans les airs avec leurs ailes immobiles, fouillant la région de leurs yeux télescopiques pour y découvrir de la chair corrompue. Lorsqu'un animal meurt (ou même avant) il est tout de suite aperçu et une forme noire vient du ciel s'abattre auprès de lui; celle-ci est bientôt suivie d'une autre et, dans peu de temps, un endroit où l'on ne voyait pas un seul oiseau se trouve peuplé de ces rapaces s'arrachant cet infect repas. Bien que l'on constate à la dissection, de très puissantes narines, les vautours ne semblent pas être guidés d'une façon appréciable par l'odorat. On reconnaît par expérience qu'ils comptent sur la vue seule pour découvrir leur nourriture. Le vol du vautour constitue l'un des phénomènes qui émerveille le physicien. Cet oiseau reste suspendu dans l'air ou même s'élève à perte de vue sans aucun effort visible. Sur des ailes à demi étendues et immobiles il s'élève dans des spirales ascendantes, s'élevant toujours de plus en plus haut, et ensuite, toujours en tournant, maintient sa position dans l'air pendant des heures a la fois, sans apparemment donner un seul coup d'aile. Plusieurs explications de ce phénomène ont été apportées mais aucune jusqu'à présent ne saurait être concluante. Nous n'avons au Canada qu'une seule espèce dont l'habitat soit régulier bien que limité. Il y en a une autre qui n'apparaît qu'à de rares intervalles.

Valeur économique. Les vautours ne sont pas des oiseaux de proie dans l'acception ordinaire de ce terme, car ils ne tuent pas ce qu'ils mangent mais se nourrissent entièrement de charognes. On les a accusés, et peutêtre avec raison, de précipiter parfois la mort d'un animal, mais ils ne s'attaquent jamais à une proie qui n'est pas à la dernière phase de son existence. Cette espèce n'a que peu d'importance économique au Canada, mais dans le Sud, du fait qu'elle fait disparaitre les chairs corrompues c'est une bonne sauvegarde pour la. santé des familles sans précautions et dans bien des endroits ils sont vigoureusement protégés par la loi dans un but purement sanitaire.

\section{Genre-Cathartes.}

325. Le Vautour aura. (TURKeY vUlTure). Cathartes aura. L, 30 . Un oiseau tout noir; la tete et le cou sans plumage ou, chez les jeunes, recouverts d'un duvet brun grisâtre semblable À de la fourrure. 
Trails distinctifs. Cette espece ne saurait étre confondue qu'avec la suivante, mais comme l'habitat de chaque espèce au Canada est bien distinct il n'y a guère de danger que l'on se trompe. La base du bec est d'un rouge vif chez l'adulte, et la tête et le cou d'un rouge sombre. Ies surfaces sous les ailes sont dépourvues d'éclat argenté.

Indications sur le terrain. La tête et le cou sans plumes constituent la meilleure marque d'identification des vautours. On reconnalt cette espèce a la couleur rouge de ces parties.

Nid. Sur le sol, généralement dans un tronc d'arbre creux.

Habilat. Presque toute l'Amérique septentrionale, au nord de la frontière canadienne, qu'il ne traverse, dans l'Est, qu'au sud de l'Ontario. Il peut s'en introduire quelques-uns, pius ou moins régulièrement, qui viennent du Manitoba.

SOUS-ESPECES. L'espèce qui occupe la majeure partie des Amériques du Nord et du Sud est divisée en sous-espèces. Celle qui se présente dans le nord du Mexique est le vautour du Nord, Cathartes aura septentrionalis.

Valeur économique. Comme cette espèce se nourrit de chairs corrompues il n'y a rien à lui reprocher.

\section{Genre-Coragyps. Vautour noir.}

326. Le Vautour noir. (BLAck volture). Coragyps urubu. L, 24. Très semblable au vautour aura mais un peu plus petit.

Traits distinctifs. On le distingue du precédent par le cou, la tête et la base du bec qui sont blancs au lieu de rouges ou roses.

Indications sur le terrain. L'aspect généralement noir de la tête sans plumes et l'éclat argentê de la surface sous les ailes permettent de reconnaftre l'animal vivant. La queue cst plus courte et l'oisesu paraft plus noir que chez le vautour aura.

Habitat. Il est plus répandu dans l'Est que le vautour aura. Régulièrement il vient du sud de la Virginie et s'égare parfois a travers nos frontières dans les provinces maritimes.

Cet oiseau fréquente trop peu le Canada pour qu'on s'y arrête longuement. On ne peut s'attendre a le rencontrer que par occasion dans les provinces maritimes.

\section{SOUS-ORDRE-FALCONES. OISEAUX DE PROIE DIURNES.}

Description générale. Bec fortement recourbé a partir de la base (figure 33, p. 25 ) où il est recouvert d'une cire renflée ou d'une masse de tissus cireux jaunatre dans laquelle apparaissent les narines. Ce tissu est nettement plus mou que le bec proprement dit et ordinairement de couleur jaune. Les pieds sont forts et pourvus de puissantes serres pour capturer et retenir une proie vivante (figure 31, p. 25).

Traits distinctifs. Les membres de ce sous-ordre diffèrent des vautours en ce qu'ils ont le bec plus court et plus fortement recourbé, et la tête emplumée au lieu de sans plumes (a comparer les figures 30 et $33, \mathrm{p} .24$ et 25 ); et ils se distinguent du sous-ordre suivant, les hiboux, en ce qu'ils ne possedent pas les disques faciaux (figure 34, p. 25) autour des yeux qui obligent ces oiseaux à regarder droit en face au lieu de pouvoir regarder de côté comme la plupart des autres oiseaux.

Ce sont les oiseaux généralement connus sous les noms de faucons et aigles. Ils sont représentés au Canada par trois familles: Buteonida, les buses communes et les aigles: Falconida, les faucons vrais et caracaras; et Pandionida, les orfraies ou aigles pêcheurs.

\section{FAMILLE-BUTEONIDÆ. BUSES, MILANS, EPERVIERS, BUSARDS ET AIGLES.}

Description générale. La meilleure façon de décrire les éperviers, c'est de dire que ce sont ni des faucons ni des orfraies.

Traits distinctifs. Ailes courtes arrondies et concaves sauf chez les milans et les busards, et leur vol est relativement lourd. Le bec (figure 33b, p. 25) est dépourvu des dents aiguës de chaque côté de la pointe (figure 33a, p. 25) qui caractérisent celui des faucons. Les pieds (particulièrement sur la surface inférieure) n'ont pas de corrugations dures et aiguës pour retenir une proie glissante comme chez les orfraies ou aigles pêcheurs. 
Cette famille est composée d'un certain nombre de genres bien caractérisés dont chacun est relativement facile à reconnaître. Ces genres comprennent la majorité de nos oiseaux de proie. Bien que véritablement rapaces de nature, ils n'ont pas l'audace, l'adresse à l'attaque ou la robuste endurance des faucons vrais, et c'est pourquoi les anciens fauconniers les appelaient "éperviers méprisables".

\section{Genre-Elanoides. Milans.}

Les milans sont des oiseaux qui habitent le Sud et l'on n'en a jamais pris qu'une espèce au Canada.

327. Le Milan à queue d'aronde. (sWallow-TAILEd kITE). Elanoides forficatus. L, 24. (Les plumes extérieures de la queue avancent de 8 pouces sur celles du milieu). Un épervier plutôt petit, qui semble long en raison de la longueur démesurée des plumes extérieures de sa queue qui dépassent de 8 pouces celles du milieu. Ailes et queue d'un noir pur; tout le reste, y compris la tête, les épaules et la partie supérieure du dos, est blanc. Cet oiseau est remarquablement coloré en blanc et noir intenses; la queue profondément fourchue; il a les ailes longues et pointues d'une hirondelle des granges; il n'est guère possible de confondre cette espèce avec une autre.

Habitat. Régions tropicales et semi-tropicales de l'Amérique. Apparaít très rarement le long de notre frontière méridionale.

Cette espèce est de toute beauté mais trop rare au Canada pour mériter plus qu'une simple mention.

Valeur économique. Sa principale nourriture se compose d'insectes, de limaces et de reptiles; il ne touche jamais aux mammifères ou aux oiseaux.

\section{Genre-Circus. Busards.} XI B.

331. Le Busard des marais. (Marsh hawk). Circus hudsonius. L, 19. Planche

Traits distinctifs. L'espèce se distingue par un cercle incomplètement garni de plumes autour des yeux qui rappelle un peu ceux du hibou. L'adulte possède d'une façon générale les couleurs de la mouette et les jeunes sont caractérisés par des tons rouge chaud.

Indications sur le terrain. Les meilleures indications sont la coloration générale et le croupion blanc que l'on distingue très bien quand l'oiseau vole. . Les longues ailes pointues et sa longue et étroite queue lui donnent la silhouette d'un faucoṇ au vol, mais son mouvement est entièrement différent.

Nid. Il niche à terre sur un lieu sec des marais ou dans un champ de foin.

Habitat. Tous les Etats-Unis et tout le Canada jusque près des régions polaires. Couve dans tout le Canada.

L'un des plus communs d'entre nos éperviers, se rencontre presque partout dans l'est du Canada. Il fréquente les marais découverts, les prés et les champs; on peut le voir s'agitant de haut en bas, parcourant et fouillant le terrain comme un chien de chasse bien dressé. Pendant un moment il voltige au-dessus de sa proie et s'abat dessus pour s'élever un instant après et se poser sur un poteau de clôture ou autre élévation de cette nature afin de dévorer sa capture. Les jeunes ne sont pas sans méfiance, mais les adultes de couleur bleue comptent parmi les oiseaux les plus prudents et sont assez rarement atteints par le fusil du chasseur.

Valeur économique. Sur 116 estomacs qu'on a examinés, 7 contenaient des volailles ou oiseaux-gibiers; 34 , d'autres oiseaux; 57 , des souris; 22 , d'autres mammifères; 7, des reptiles; 2, des grenouilles; 14, des insectes, et 1 des matières indéterminées. Donc, sur 144 contenus alimentaires, 41 étaient nuisibles, 93 utiles et 10 indifférents. Sur les 41 aliments auxquels 
on peut trouver à redire, 3 seulement étaient des oiseaux de basse-cour et le reste des oiseaux sauvages, se composant de 46 individus de valeur beaucoup moindre que les variétés domestiques. Les 99 souris et 22 autres mammifères comprenaient environ 117 individus. Les insectes étaient surtout des locustes, des sauterelles et des coléoptères. La balance penche évidemment en faveur de cette espèce qui est incapable d'emporter des volailles, si ce n'est des jeunes, et seulement lorsque celles-ci s'aventurent jusque dans leur habitat. Si l'on élève des poules autour de la maison, elles sont pour ainsi dire parfaitement protégées contre cet oiseau. Comme il fréquente les marais, les plaines herbeuses et les haies embroussaillées, c'est l'ennemi naturel des souris des champs, et il contribue probablement plus que toute autre influence naturelle à réduire comme il convient le nombre de ees rongeurs.

\section{Genres-Accipiter et Astur. Accipitres, Eperviers aux courtes ailes.}

Description générale.-Eperviers à ailes courtes et arrondies et à longue queue.

Les accipitridés sont des oiseaux des bois qui voltigent autour du sommet des arbres ou sur les lisières des bois; ils n'ont pas l'habitude de s'élever très haut dans les airs. Ils prennent leur proie par surprise et attaquent vivement plutôt qu'après une longue poursuite. Leurs courtes ailes et leur longue queue leur permettent de prendre de rapides élans de vitesse et de faire de brusques évolutions, mais ne se prêtent pas à un effort soutenu.

Valeur économique. Ce sont les seules espèces communes d'éperviers canadiens qui peuvent être tant soit peu utiles. Ils sont pleins de vie et d'activité, et bien qu'ils n'aient pas la force et l'endurance des faucons vrais, ils font en réalité beaucoup plus de tort que leurs plus grands et plus puissants congénères. C'est d'après cet oiseau que l'on donne vulgairement le nom de "Chicken Hawk» à tous les petits éperviers. Ils ne mangent jamais de chair corrompue, ne cherchant toujours que la viande fraîche, et ne reviennent presque jamais à une proie déjà en partie dévorée. Heureusement les deux espèces les plus communes sont les plus petites, ee qui réduit d'autant le tort qu'ils peuvent faire. Le seul gros et puissant oiseau de ce groupe est l'Autour, mais il est moins répandu et, sauf au cours de certains hivers, il se rencontre rarement dans les parties les plus habitées du sud du Canada.

332. L'Epervier brun. L'Gmerillon. (Sharp-shinned ifawk). Accipiter velox. $\mathrm{L}, 11.25$. Planche XII A.

Trails distinctifs. En règle générale, e'est par la taille que l'on distingue l'épervier brun de tous les autres, sauf du faucon épervier, mais on ne le confondra pas avec celui-ci car il ne possède aucunement les caractéristiques du faucon. Une forte femelle est à peu près de la taille d'un petit épervier de Cooper mâle dont elle est une parfaite miniature comme coloration. Elle diffère de cette espèce par la queue qui est rectangulaire au lieu d'être arrondie; quand elle est fermée, les plumes extérieures sont tout aussi longues que les intérieures au lieu d'être manifestement plus courtes. Le tarse est relativement plus mince et plus élancé, une différence très perceptible quand on compare les spécimens.

Indications sur le terrain. Leurs ailes courtes et arrondies, leur longue queue et leur vol par coups rapides et uniformes, et leurs courtes envolées aideront à reconnaître cette espece comme un accipitridé. Sa queue rectangulaire au lieu d'arrondie facilitera son identification d'avec l'épervier de Cooper, mais c'est la taille qui constitue la principale différence.

Nid. Sur les arbres, ordinairement des conifères à une hauteur de 10 à 40 pieds.

Habilat. Presque toute l'Amérique du Nord, probablement jusqu'aux limites des régions boisées au nord. Dans l'est du Canada, il se reproduit partout, sauf dans la partie la plus inéridionale de la région inférieure des grands lacs. 
C'est l'un des plus petits éperviers que nous ayons. Il n'a pas la vigueur soutenue ou la persistance des "faucons nobles" mais il est actif et agile. Il attaque sa proie avec fureur mais revient rarement à la charge et n'attaque presque jamais sa proie au vol eomme le font les "oiseaux de proie nobles $"$.

Valeur économique. C'est cette espèce qui aurait dâ s'appeler le faucon épervier au licu du petit faucon auquel on a attribué ce nom. C'est un parent rapproché de celui-ci et c'est le représentant amérieain du fauconépervier d'Europe, lequel est aussi un accipitridé. Le nom de "Sparrow Hawk » lui conviendrait très bien puisqu'il se nourrit surtout de moineaux et autres petits oiseaux.

Sur 107 estomacs examinés, 6 contenaient des volailles ou des oiscaux gibiers; 99, d'autres oiseaux; 6, des souris, et 5 , des insectes. Cela nous donne 105 aliments qui condamnent l'oiseau contre 11 qui le font utile. Les souris ne se composaient pas de plus de 9 individus, mais les petits oiseaux se chiffraient à 115 , dont la taille variait entre celle du roitelet et celle de la tourterelle. C'est un fort argument contre cette espèce qui autrement est très intéressante. Un bon point qu'on peut relever en faveur de ce petit épervier c'est qu'il est friand des moineaux domestiques et qu'il en supprime des compagnies aux abords des petites villes. La pointe Pelée sur le lae Ontario, est un endroit favori de cet épervier et, tous les ans, il y a de grands nombres de grives, vireos, moincaux et autres petits oiseaux qui tombent victimes de ces entreprenants petits maraudcurs.

333. L'Epervier de Cooper. (COOPER's HAWK). Accipiter cooperi. L, 15.50. Semblable à l'épervier brun mais plus gros.

Traits distinctifs. Un petit épervier de Cooper mâle peut se rapprocher beaucoup comme taille d'une forte femelle de l'épervier brun, et une forte femelle de la même espèce peut ressembler à un petit autour mâle. L'épervier de Cooper peut être distingué de l'épervier brun par sa queue arrondie au lieu de rectangulaire et par son tarse relativement plus court et plus épais. Il ne possède à aueun âge la coloration de l'autour, mais chez les tout jeunes, les plumages se ressemblent beaucoup. La meilleure indication pour les distinguer c'est le plumage du tarse; il y en a environ un tiers de revêtu chez l'épervier de Cooper, et la moitié chez l'autour. Le plumage des jeunes peut aussi ressembler à celui de la busc à manteau roux (voir cet oiseau). Il peut aussi être pris faeilement pour le jeune de la buse de Pensylvanie, mais il a les quatre premières primaires au lieu des trois primaires extérieures brusquement rétrécies ou émargées.

Indications sur le terrain. On le reconnait d'abord à sa taille et sa silhouette d'accipitre. Sa queue arrondie au lieu de rectangulaire aidera à le distinguer d'avee l'épervier brun. La coloration des adultes est la seule indication sur le terrain, outre la taille, pour le différencier d'avec l'autour.

Nid. Il niche sur les arbres à peu près comme l'épervier brun, mais rarement sur des conifères.

Habitat. Depuis les frontières septentrionales des régions habitées en allant au sud à travers tous les Etats-Unis.

C'est une espèce plus malfaisante même que l'épervier brun, puisque sa plus forte taille lui donne plus de puissance et plus de moyens de nuire. Avec presque autant d'activité il poursuit une plus grosse proie et peut très bien emporter un poulet de taille ordinaire. Il s'attaque rarement à une poulc adulte, à moins que celle-ci ne soit faible.

Valeur économique. Sur 94 estomacs examinés, 34 contenaient des volailles ou des oiseaux-gibiers; 52 , d'autres oiseaux; 11 , des mammifères; 1, une grenouille; 3 , des lézards; et 2, des insectes. Les mammifères se composaient d'animaux rongeurs, la plupart malfaisants, mais il y avait cependant un écureuil gris. Cela fait un total de 87 aliments qui condam- 
nent l'espèce, 12 en faveur, et 4 indifférents. Comme c'est un épervier relativement commun dans un grand nombre des régions habitées du pays, e'est certainement une menace et il est, en bonne partie, cause de la mauvaise réputation de tout cet ordre d'oiseaux.

$$
\text { Genre-Astur. Autours. }
$$

334. L'Autour à tête noire. (AMerican Goshawk). Astur atricapillus. L, 22. Planche XII B.

Traits distinctifs. Les adultes, avec leur coloration caracteristique bleu ardoisé et finement vermiculaire, ne peuvent être confondus avec aucun autre oiseau d'Amérique. Les jeunes cependant sont très semblables aux autres jeunes accipitres et souvent un jeune mâle aura presque la même taille qu'une forte femelle de l'épervier de Cooper. Dans un pareil cas, on pourra les distinguer par le tarse qui est revêtu de plumes sur la moitié au lieu du tiers de sa longueur et par la légère teinte de chamois sur le blane des parties inférieures de cette espèce.

Indications sur le terrain. Les adultes se distinguent par leur coloration. Les jeunes se reconnaissent a leur taille et leurs silhouettes d'accipitres avec une longue queue et des ailes courtes et arrondies.

Nid. Sur les arbres.

Habitat. Il couve sur tout le continent dans les limites des régions habitées. Les mouvements migratoires sont ordinairement de peu d'étendue, bien qu'il visite parfois nos frontières méridionales en nombre considérable. De même que dans le cas du harfang, ces bandes se composent surtout d'individus adultes, bien que, normalement, les jeunes soient beaucoup plus communs au sud de la région où ils se reproduisent. Il est probable que ces envolées occasionnelles sont causées par une disette périodique de lapins dans leurs habitats usuels.

SOUS-ESPECES. L'autour à tête noire est divisé en deux sous-espèces, mais la forme typique seulement-l'autour de l'Est - se rencontre dans l'est du Canada.

C'est le représentant américain de l'autour d'Europe ou "Goose Hawk», désignation qui semble avoir été son nom primitif. C'était le seul épervier aux courtes ailes qui servît régulièrement dans l'ancienne fauconnerie. On lui faisait chasser les lièves, les perdrix, et ce genre de gibier de terre, que sa longue queue et ses courtes ailes lui permettait de suivre à travers les détours brusques et enchevêtrés, tandis que sa taille lui permettait de tuer du gros gibier. On ne le considérait pas cependant comme un oiseau chasseur de haut vol, parce qu'il n'avait pas la vigueur et l'énergie des faucons aux longues ailes et sa chasse n'excitait pas le même intérêt que les "oiseaux de proie nobles."

Valeur économique. Heureusement, eet oiseau n'est qu'un hôte irrégulier dans nos régions les moins habitées. Autrement il serait une menace sérieuse pour les basses-cours. Sur 20 estomacs examinées, 9 contenaient des volailles ou oiseaux-gibiers; 2 , d'autres oiseaux; 10, des mammifères; 3 , des insectes; et 1 , un mille pattes. Sur les 10 mammifères, 3 étaient des lapins et 1 un écureuil gris, deux espèces que l'on peut considérer comme utiles. Cela nous donne un total de 15 aliments qui condamnent l'oiseau, 15 en sa faveur et 1 qui est indifférent. Il n'y a aucun doute que cette espèce est plutôt malfaisante. Sa taille lui donne assez de puissance pour enlever un poulet et même une poule, et elle fait de la gelinotte du Canada sa nourriture favorite. Bien que le véritable habitat de l'épervier à tête noire soit dans les forêts les plus septentrionales sur les lisières des terres cultivées, lorsqu'une fois ils s'établissent au voisinage d'une ferme, on peut s'attendre à leur visite tous les jours. Ils fondent subitement, au-dessus ou autour des bâtiments, sur un troupeau de volailles, saisissent leur victime et l'emportent avant que le fermier ait le temps de la protéger. 


\section{Genres-Buteo et Archibuteo. Buses vraies.}

Description générale. Eperviers solidement bâtis, de taille moyenne ou forte, le bec dépourvu de dents (figure 33b, p. 25). Les ailes sont plutót courtes et arrondies et la queue longue et ample qui s'étale en un grand demi-cercle. Le genre Buteo a le tarse nu mais l'Archibuteo est emplumé jusqu'd la base des doigts.

Indications sur le terrain. L'arrondissement des ailes et la largeur, l'ampleur de la queue constituent les meilleurs signes pour reconnaitre les buses.

Ce sont les buses vraies. Le vautour d'Amérique (Turkey Vulture) est quelquefois improprement appelé buse dans le Sud. Les buses n'ont pas tout l'élan, la rapidité et la vigueur soit des faucons soit des accipitres; elles sont moins agiles, moins rusées et ne chassent que le petit gibier. Ce sont les éperviers de haut vol de la saison d'été, on peut les voir tourner en cercles dans les régions supérieures, mais c'est assez rare qu'ils se précipitent autour de fourrés comme les agiles accipitres. Si l'on excepte la buse de Pensylvanie, leur habitat caractéristique est le plein air, bien qu'ils fassent leur nid habituellement dans la forêt.

Valeur économique. D'une façon générale, le fait qu'ils mangent les souris et les insectes fait plus que compenser le tort incontestable que font certains individus de quelques espèces. Bien que les buses comprennent quelques-uns de nos plus grands éperviers, le tort qu'ils font n'est pas du tout comparable à celui qu'on peut attribuer aux plus petits accipitres. De fait, la plus grande espèce de ce groupe, la buse pattue, est la plus inoffensive. Ces oiseaux se nourrissent principalement de souris, d'autre petits mammifères, de reptiles et d'insectes; et la taille d'un bon nombre de ces bêtes nuisibles est un témoignage du bien qu'ils font sur une très grande échelle.

337. La Buse à queue rousse. (RED-TAILED HAWK). Buteo borealis. L, 20. Planche XIII A.

Traits distinctifs. C'est notre plus grand épervier commun; on le distingue ordinairement de tous les autres par sa taille. La buse pattue qui est de taille égale ou plus forte peut être distinguée facilement par son tarse emplumé. Une large queue rouge est un signe distinctif chez l'adulte. Les jcunes ne varient que très peu d'avec les buses à manteau rouge du même âge mais plus gros. A part cela, la différence la plus frappante entre les deux c'est l'absence de rouge chez cette espèce sur les épaules ou sur les couvertures supérieures des ailes et la présence d'une surface à peu près circulaire, sans taches, sur la poitrine.

Indications sur le terrain. On peut spécifiquement reconnaître les adultes sur le terrain par leur queue rouge, et tous les âges, génériquement par le profil, propre aux buses, des courtes ailes et de la large et ample queue arrondie. Les jeunes seulement peuvent être pris pour des buses a manteau roux, et c'est probablement par leur poitrine blanche et leur cri qu'on peut mieux les distinguer de celles-ci. Le chant de la buse à manteau roux est identique a certains cris du geai huppé. Les notes de celle-lì sont semblables mais il y a une assez forte différence dans le timbre et dans l'exécution pour les reconnaître quand une fois on les a entendues.

Nid. Sur les hautes branches des arbres dans les grandes régions boisées.

Habitat. L'est de l'Amérique du Nord jusque près de la limite des régions boisées. Couve au Canada partout où il se trouve.

SOUS-ESPECES. La buse à queue rousse est représentée au Canada par plusieurs sous-espèces mais on ne trouve guèrc dans l'Est, que la forme typique, la buse à manteau rouge de l'Est.

La buse à queuc rousse est un épervier timide et prudent. On la voit plutôt planer en grands cercles dans les régions élevées que près du sol. Elle exige pendant l'été des bois plus grands et plus sauvages que la buse à manteau rouge, bien que, comme cette autre espèce, elle chasse dans la contrée découverte du voisinage. 
Valeur économique. Ce grand et magnifique oiseau occupe une position discutable au point de vue de son utilité et cela dépend tellement des conditions locales et des caractéristiques personnelles de l'individu qu'on ne peut guère arriver, à son sujet, à une conclusion certaine et définitive. Sur 473 estomacs examinés, 54 contenaient des volailles ou oiseaux-gibiers; 51 , d'autres oiseaux; 278 , des souris; 131 , d'autres mammifères; 37 , des batraciens ou reptiles; 47, des insectes; 8 , des écrevisses; 1, une mille-pattes; et 13 , des restes. Parmi les autres mammifères, 16 étaient des lapins ou des écureuils gris. Cela nous donne environ 121 aliments qui condamnent l'oiseau et 464 en sa faveur et il y en a 48 qui peuvent être considérés comme indifférents à part les 13 qui se composent de restes qui ne peuvent être comptés que comme favorables. Il faut aussi se rappeler en étudiant ces aliments que ceux de ces oiseaux qui se rapprochent le plus des fermes sont les plus exposés à se faire tirer et se faire examiner l'estomac. Il y a ainsi une certaine sélection défavorable contre l'ensemble de l'espèce dans le choix des témoignages.

D'une façon générale cette espèce est extrêmement utile et fait beaucoup plus de bien que de mal. Certains individus, cependant, prennent souvent l'habitude de visiter régulièrement la basse-cour. Ce sont presque toujours de jeunes oiseaux de l'année à la recherche d'une proie facile. Les vieux oiseaux sont ordinairement trop prudents pour s'aventurer ainsi. Il est évident que ces individus devraient être éliminés le plus tôt possible, mais on ne peut certainement pas soutenir qu'une espèce généralement utile devrait être systématiquement persécutée en raison des méfaits de quelquesuns. En économie ornithologique c'est une bonne règle à suivre, quand les témoignages sont à peu près également divisés, que d'accorder à l'oiseau le bénéfice du doute.

339. La Buse à manteau roux. (RED-6HOCLDERED HAWK). Buteo lineatus. $\mathrm{L}, 18 \cdot 30$. Planche XIII B.

Traits distinctifs. Parties inférieures rougeâtres des adultes. Le plumage des jeunes peut être semblable à celui de la buse à queue rouge, mais leur plus petite taille et la présence d'au moins une indication de teinte rougeâtre sur les épaules ou les couvertures et les mouchetures au milieu de la poitrine faciliteront la distinction.

Indications sur le terrain. Avec son profil caractéristique de buse et ses habitudes, elle n'est guère susceptible d'être confondue avec aucune espèce autre que la buse d̀ queue rouge. L'absence de queue rouge et la teinte rougeâtre des parties inférieures feront reconnaitre les adultes. Pour ce qui est des jeunes, le chant et la taille sont probablement les indications les plus sûres. Le chant de cette espèce ressemble tellement à certains cris du geai huppé qu'on ne peut les différencier qu'avec difficulté. Les cris aigus de la buse à queue rouge sont de même nature mais très faciles à distinguer.

Nid. Sur les arbres, à une hauteur de 35 à 75 pieds de terre.

Habitat. Dans tout l'est de l'Amérique du Nord jusqu'aux limites des régious habitées. Couve partout où on la trouve au Canada.

SOUS-ESPECES. On reconnait plusieurs sous-espèces de la buse à manteau roux mais une seule, l'oiseau type, la buse à manteau roux de l'Est se rencontre dans l'est du Canada.

C'est probablement l'épervier le plus répandu dans l'est du Canada. Il a les mêmes habitudes que la buse à queue rouge, sauf qu'il ne recherche pas les bois profonds pour $y$ faire son nid, qu'il pourra construire dans n'importe quel petit bois, quelquefois a une surprenante proximité des habitations.

Valeur économique. Cette espèce est, dans le fond, très semblable, quant à son régime alimentaire, à la buse à queue rouge, mais comme elle est plus petite et plus légère elle peut faire proportionnellement moins de tort, 
tandis que les services qu'elle peut rendre sont à peu près sans mélange. Sur 206 estomacs examinés, 3 contenaient de la volaille; 12, d'autres oiseaux; 102, des sourís; 40, d'autres mammifères; 20, des reptiles; 30 , des batraciéns (grenouilles, etc.); 92, des insectes; 16, des araignées; 7 , des écrevisses; 1 , un ver de terre; 2 , des restes de table; et 3 , du poisson. On peut ainsi voir que sa nourriture est très variée. Il est difficile de décider si les musaraignes, grenouilles, reptiles et araignées doivent être comptés comme condamnant l'espèce, mais le grand nombre des souris et d'insectes contre 3 aliments se composant de volailles et 12 d'autres oiseaux, suffit évidemment à exonérer cette espèce de l'accusation de faire beaucoup de tort.

342. La Buse de Swainson. (SWAINson's HAWK). Butea swainsani. L, 20. $\AA$ peu près de la même taille que la buse a manteau rouge. Elle peut se présenter sous une phase soit presque noire, soit plus claire, soit entre les deux. Le plumage clair caractéristique laisse voir un dos, une tête et des parties supérieures uniformément bruns, des parties inférieures blanches avec une bande lie de vin sur la poitrine et de légères indications de bandes de même couleur sur les flancs.

Traits distinctifs. Beaucoup d'oiseaux ont l'extrémité de leurs primaires réduites en largeur comme si on avait taille le bord avec un canif. Chez cette espèce, les trois primaires extérieures sont ainsi atténuées ou émargées. Chez la buse à manteau roux avec laquelle on pourrait confondre certains plumages, quatre des primaires sont ainsi émargées.

Habitat. Les provinces des prairies et les régions à l'ouest. Au nord jusqu'aux régions polaires s'aventurant quelquefois jusqu'aux grands lacs des régions inférieures.

L'espèce n'apparaît dans l'Est que comme un traînard venant de l'Ouest; il faudra beaucoup de précaution pour l'identifier. Bien que ce soit un oiseau de proie, il ressemble trop aux espèces précédentes et se voit trop rarement pour mériter une description détaillée.

343. La Buse de Pensylvanie. (Broan-wingen hawk). Buteo platypterus. I, 15.89. La plus petite de nos buses. Adulte: brun aux parties supérieures, parties inférieures toutes rayées de brun rougeâtre et de blanc d'une façon plutôt irrégulière. Jeunes: parties supérieures brunes plus ou moins tachetées de blanc; aux parties inférieures, bandes blanches et brunes sur la poitrine jusqu'd la gorge, et rayures sur les flancs et les pattes.

Traits distinctifs. Les jeunes de cette espèce se distinguent des jeunes buses à manteau roux par leur taille. L'espèce se distinguera, tant d'avec celle-ci que d'avec l'épervier de Cooper avec lequel elle peut aussi être confondue, par ses trois primaires atténuées ou émargées au lieu de quatre. (Voir l'espèce suivante).

Indications sur le terrain. La taille, la coloration générale des adultes, et son cri allongé, aigu, ressemblant au grincement prolongé que fait la charnière rouillée d'une porte de grange, sont de bons signes pour la reconnaître.

Nid. Sur les arbres, à une hauteur de 25 à 70 pieds.

Habitat. L'est de l'Amérique du Nord jusqu'aux limites des régions habitées. Couve par endroits dans l'est du Canada, dans toutes les parties de l'Ontario sauf les plus méridionales et très loin au sud dans la vallée du Mississipi.

N'habite que certains endroits et préfère les régions les plus sauvages.

Valeur économique. Sur 57 estomacs examinés, 2 contenaient des petits oiseaux; 15 , des souris; 13 , d'autres mammifères; 11, des reptiles; 13, des batraciens (grenouilles, etc.); 30 , des insectes; 2, des vers de terre; 4, des écrevisses. Sans pousser plus loin l'examen, ces données sont suffisantes pour exonérer cette espèce de tout blâme.

\section{Genre-Archibuteo. Buse pattue d'Amérique.}

347a. La Buse pattue d'Amérlque. (ROvGH-LEGGEn HAWK). Archibutea lagopus sancti-johannis. $L, 22$. Le plus grand de nos vrais éperviers. Il se présente sous deux aspects: l'un tout foncé, presque noir, et l'autre clair, d'une variété presque infinie de nuances et de dessins; il y a aussi toutes les phases intermédiaires. La forme la plus 
commune a les parties supérieures brunes plus ou moins mêlées de jaune d'ocre, particulièrement autour de la tête, et les parties inférieures jaune d'ocre avec large bande foncée sur l'abdomen et des rayures éparses sur la poitrine et la gorge. C'est à cause du tarse emplumé jusqu'aux doigts que cet oiseau est ainsi nommé, et c'est là un trait caractéristique du genre.

Traits distinctifs. On la distingue par sa forte taille et son tarse emplumé jusqu'à la base des doigts.

Indications sur le terrain. Forte taille, larges amas noirs sur les parties inférieures, queue blanche a la base (le croupion n'est pas blane) et taches noires très visibles sur la surface inferieure de l'aile.

Nid. Dans l'extrême nord, elle niche à terre sur les bancs rocheux ou sur les arbres.

Habitat. Habite, en tant qu'espèce, les parties du nord de l'hémisphère boréal. Le représentant américain se rencontre depuis le Mexique jusqu'aux régions polaires. Couve dans les terrains incultes ou du voisinage.

SOUS-ESPECES. La buse pattue est représentée dans l'ancien et le nouveau continent par deux sous-espèces. Celle qui est propre à l'Amérique est la buse pattue d'Amérique $A . l$. sancti-johannis et se distingue de celle d'Europe et d'Asie par sa couleur légèrerement plus pâle ęt la plus grande rareté, chez cette espèce, de la phase noire.

Ce grand épervier n'est que passager dans les régions habitées du Canada et les terres incultes du nord. C'est un oiseau des grands marais et on peut le voir voleter au-dessus des prés jusqu'à une heure avancée de la nuit à peu près comme le busard des marais. On le voit ordinaircment, cependant, dans les hautes régions, faire de nombreuses évolutions en cercles interrompus par des pauses pour retrouver son chemin vers les lieux de nidification. En raison du dichromatisme de l'espèce il faut s'attendre à trouver chez clle une variété infinie de plumages.

Valeur économique. Bicn que ce soit notre plus grand épervier, e'est l'oiseau le moins malfaisant. Sur 47 estomaes examinés, 40 contenaient des souris; 5, d'autres mammifères; 1, un lézard; 1, était vide. Un pareil résultat suffit pour condamner la destruction générale des éperviers. Les pieds de la buse pattue sont faibles et incapables de retenir une grosse proie; c'est done un épervier chasseur des souris par excellence. Il se nourrit également de sauterelles et l'on sait qu'il a largement contribué à supprimer les fléaux occasionnés par ces insectes destructeurs.

\section{Genres-Aquila et Haliatus. Aigles.}

Les aigles sont nos plus grands oiseaux de proie. La taille seulement les distingue des éperviers. Tout oiseau de proie de plus de 30 pouces de long et de 6 pieds d'étendue est un aigle. Contrairement à l'idée répandue, l'aigle ne compte pas parmi ce qu'on appelle les oiseaux de proie nobles. C'est tout simplement une buse de taille démesurée. Il se nourrit en grande partie de grosses proies mais il est rarement funeste aux intérêts de l'homme. Dans certaines régions il est quelquefois une menace pour les jeunes agneaux mais, dans l'est du Canada, on peut les considérer plutôt comme nettoyeurs de déchets qu'autrcment, puisqu'ils n'enlèvent presque rien qui soit de valeur économique et qu'ils se nourrissent principalement de restes.

349. L'Ajgle doré. (GoLden Eaole). Aquila chrysaëtos. L, 30. Un grand aigle brun foncé, la tête saupoudrée de jaune d'ocre, ce qui fait qu'on l'appelle doré, et la première moitié de la queue oruée de barres grisâtres sur un blanc terne. Tarses emplumés jusqu'aux doigts.

Traits distinctifs. L'aigle doré est entièrement différent de l'aigle à tête blanehe adulte, mais très semblable au jeune de cette espèce. On peut toujours, cependant, quel que soit le plumage, le reconnaître à son tarse emplumé, l'aigle à tête dorée ayant toujours des pattes nues d'un jaune brillant.

Nid. Sur les rochers, les saillies rocheuses ou sur les arbres. 
Habitat. Dans la zone tempérée des deux hémisphères. En Amérique, il habite toute la partie nord, sauf l'extrême nord, et va au sud jusqu'au Mexique dans les plaines et les montagnes occidentales et sur les hautes terres de l'Est. Ne fréquente qu'accidentellement la région des grands lacs.

L'aigle doré est trop rare dans l'est du Canada pour qu'on s'y arrête longuement dans cet ourrage. C'est un oiseau magnifique et comme, de sa nature, il est moins porté à enlever des animaux ou des déchets, il s'adapte mieux dans la conception populaire que l'aigle à tête blanche, au titre de roi des oiseaux.

Valeur économique. Le point de vue économique de cet oiseau doit être considérablement influencé par les conditions locales. Il se nourrit surtout de mammifères et sa forte taille lui permet d'en enlever qui sont d'un poids considérable. Ce sont principalement des animaux sauvages, tels que marmottes, lapins et rongeurs malfaisants, mais il enlève de préférence les animaux nouveau-nés. Dans les régions de l'Ouest où l'on élève des moutons, les dégâts occasionnés par un certain nombre d'aigles dorés peuvent être sérieux, mais ils ne le sont jamais dans l'Est. Heureusement l'espèce est trop rare dans l'est du Canada, pour qu'on trouve beaucoup à redire; de fait, c'est à peine si l'on rencontre en moyenne un seul individu dans une année. On pourra done voir apparaître l'un de ces magnifiques oiseaux dans l'Est plutôt avec plaisir qu'avec crainte. Par occasion, cette espèce pourra se repaître de chair corrompue mais ne semble pas exiger cette nourriture comme son congénère l'aigle à tête blanche.

352. L'Aigle à tête blanche. (BALD EAGLE). Haliceteus leucocephalus. L, $32 \cdot 85$. L'adulte est d'un brun foncé avec la tête, le cou et la queue de couleur blanche. Les jeunes sont tout bruns avec plus ou moins d'indices, suivant l'âge, des parties qui deviendront blanches.

Traits distinctifs. Bien que le plumage du jeune oiseau soit semblable à celui de l'aigle doré on pourra toujours le reconnaftre, l'identifier à ses pattes jaunes et sans plumes.

Indications sur le terrain. Le profil de l'aigle et sa taille sont très caractéristiques quand on les connait bien. Le grand bec recourbé, le culmen qui s'avance en ligne droite avec la couronne et qui remplit toute la surface du front sont très apparents et tout de suite reconnaissables. Le blanc de la tête et de la queue sont des signes infaillibles pour reconnaí tre l'adulte et, en raison de la grosseur des détails, on distinguera les jeunes d'avec l'aigle doré plus facilement qu'on ne serait porté à croire. Le ton fauve doré sur le haut du cou chez l'aigle doré est très en évidence tandis qu'il ne se voit pas chez l'aigle à tête blanche. La queue blanchâtre ou mouchetée laisse voir par en dessous une pointe terminale qui forme un perceptible contraste avec sa base, variant de 2 a 4 pouces de profondeur, suivant l'âge; tandis que la queue du jeune aigle à tête blanche est entièrement d'un blanc sale avec peu ou point de bande terminale distincte, mais présentant à tout âge un aspect uniformément moucheté. Tout aigle remarqué dans l'est du Canada doit etre provisoirement reconnu comme un aigle de cette espèce, à moins qu'il n'y ait des raisons claires et concluantes pour que ce soit un aigle doré.

Habitat. Dans tous les Etats-Unis et le Canada, sauf sur les côtes extrêmes des régions polaires.

SOUS-ESPECES. Il y a deux sous-espèces dont une seule, l'aigle doré du nord, $H$. l. alascanus, a été rencontrée jusqu'à présent au Canada. La race typique habite plutôt le Sud.

Tandis que l'aigle doré est un hôte typique des montagnes et des plateaux, l'aigle à tête blanche est un oiseau de rivage et se rencontre rarement loin de l'eau. Bien qu'il fût autrefois une espèce typique des contrées de l'Est il est devenu tous les ans plus rare, et à l'heure qu'il est c'est tout un événement quand on en aperçoit un. L'aigle à tête blanche est une espèce à peu près inoffensive. Le dommage qu'il cause est insignifiant et à titre d'élément sauvage caractéristique de nos paysages lacustres cet oiseau pittoresque devrait être conservé. 
Valeur économique. Sur 15 estomacs examinés, 1 contenait du gibier; 5 , des mammifères; 9 , du poisson; et 2 , de la chair corrompue. En examinant ces données, il est a remarquer que les 6 estomacs contenant des mammifères et du gibier étaient des spécimens d'hiver et, à l'exception d'un seul, furent pris à une certaine distance de l'eau. Si les spécimens étudiés fussent provenus des lieux de nidification en été, les mammifères et oiseauxgibiers eussent probablement été remplacés par du poisson, car c'est surtout de cet aliment que se nourrit l'oiseau, Il s'en nourrit de diverses façons. Il plonge pour atteindre le poisson à la vraie manière de l'orfraie quand c'est nécessaire, mais d'habitude il le ramasse mort sur le rivage ou bien, là où les orfraies sont communes, il les lui enlève de force. Pour arriver à cette fin, il poursuit et tourmente l'oiseau pêcheur jusqu'à ce qu'il lâche sa proie que l'aigle, rapide comme l'éclair, attrappe dans l'air et emporte en triomphe. L'aigle à tête blanche n'est pas d'habitude aussi habile à ce manège que les oiseaux plus rapides, mais il est prompt à se saisir des canards ou autres oiseaux-gibiers blessés ou meurtris dans les marais. Quand l'occasion se présente, l'aigle à tête blanche mange volontiers des restes.

On reconnaitra qu'un nombre raisonnable de ces aigles ne peut êtrc une grande menace pour l'humanité. Ils s'attaquent rarement à la volaille. Le poisson qu'ils prennent n'est qu'une bagatelle, se composant surtout de déchets ou du surplus d'une grande abondance. Ils font beaucoup de bien comme enleveurs de déchets et dans l'Est surtout doivent être plutôt sncouragés que réprimés.

\section{FAMILLE-FALCONIDA. FAUCONS ET CARACARAS.}

La famille des falconidés comprend deux sous-familles: les faucons rrais, Falconido, et les caracaras, Polyborince. Cette dernière sous-famille rabite le Sud et il n'a été trouvé au Canada qu'un seul caracara.

Sous-famille.-Falconlnæ. Faucons vrais.

Les faucons étaient désignés par les anciens fauconniers sous le nom l'oiseaux de proie nobles ou aux grandes ailes et, en raison de leur vigueur, le leur force et de leur adresse, c'étaient les oiseaux préférés pour la chasse. seurs longues ailes leur donnent une grande rapidité, qu'une longue enduance leur permet de maintenir. Ils sont hardis et puissants et fondent sur eur proie pour la saisir quand c'est possible, mais, contrairement aux ccipitres, ils ne se découragent pas s'ils ont manqué leur coup. Leur preaière idée est de s'élever au-dessus de leur proie, ce qu'ils font au moyen l'une longue ascension en spirale. Une fois au-dessus de leur proie, ils ondent dessus et la frappent en même temps de leurs puissantes serres.

Le vol du faucon est très reconnaissable, il avance par coups rapides de cs ailes pointues, ne plane que très peu. Vue de près, la mandibule upérieure pourvue d'une dent, distingue toujours le faucon des autres éperiers. Par bonheur, il n'y a que les plus petits et les plus inoffensifs de cette jus-famille qui soient communs dans les régions cultivées, et ceux que leur zille rend redoutables sont ou très rares ou ne fréquentent que l'extrême ord où leurs dépréda'tions ne peuvent du tout nuire aux cultivateurs. Iême ceux qui apparaissent parfois dans les régions cultivées sont générament sauvages et assez prudents pour se tenir éloignés du voisinage imméiat des habitations. 


\title{
Genre-Falco. Faucons
}

\author{
Sous-genre-Hierofalco. Faucons.
}

\section{FAucons.}

Description générale. Grands rapaces de 20 à 22 pouces de longueur, ordinairement d'une coloration très blanche, quelquefois presque noire. Leur forte taille distingue ces oiseaux de tous les autres de la sous-famille.

Les faucons étaient très hautement prisés pour la chasse par les anciens fauconniers et, suivant les lois restrictives de l'époque, leur usage était réservé aux personnages du plus haut rang. Ils possèdent à la fois toute l'ardeur et l'endurance des petites espèces avec une plus forte taille et plus de vigueur; c'est pourquoi ils étaient utilisés pour le plus gros gibier qui pouvait se chasser avec des oiseaux. Les faucons habitent l'extrême nord du Canada et sont très rares dans les contrées habitées ou dans les terrains de chasse, sans quoi il faudrait probablement leur livrer une guerre d'extermination, car ce sont incontestablement de terribles destructeurs.

Il y a, au Canada, deux espèces. Elles habitent les régions polaires et se présentent dans les parties boréales de l'ancien et du nouveau continent.

353. Le Faucon blanc. (White grrfalcoN). Falco islandus. L, 22. Un très grand faucon, presque tout blanc avec quelques petites tacbes distinctes d'un brun clair.

Traits distinctifs. La blancheur prédominante et l'absence de taches sous les couvertures des ailes distingueront cette espèce de la suivante. Sa forte taille et ses caractéristiques visibles de faucon le feront tout de suite reconnaitre.

Nid. Sur des saillies rocheuses.

Habitat. Les régions polaires. Couve dans le Groenland et les contrées voisines en Amérique. Ne se montre que par occasion dans les parties habitées du Canada.

Aucun des faucons n'est commun dans les régions habitées du $\mathrm{Ca}$ nada, c'est pourquoi nous ne les étudierons pas en détail.

354a. Le Faucon noir. (Gyrfalcon). Falco rusticolus. L, 20. De méme taille que le faucon blanc mais d'une coloration beaucoup plus foncée, parties supérieures brun ardoisé, plus ou moins bordées ou rayées de crème ou de blanc. Téte et parties inférieures blanches ou blanc de crème rayé de noir.

Traits distinctifs. Chez le faucon blanc, c'est de beaucoup le blanc qui domine, tandis que chez le moins foncé de cette espèce le blanc et le brun ardoisé sont à peu près également répartis et, chez le plus foncé, c'est le brun qui l'emporte de beaucoup. Les couvertures inférieures de la queue sont toujours quelques peu rayées.

Nid. Sur les rochers ou sur les arbres.

Habitat. Les régions polaires de l'hémisphère boréal; ne fréquente qu'accidentellement les régions cultivées.

SOUS-ESPECES. Le représentant américain de cette espèce a été divisé en trois sous-espèces ou races géograpbiques, basées sur le degré de coloration foncée, le faucon gris, $F$.r. rusticolus; l'oiseau type, le faucon, F.r. gyrfalco; et le faucon noir, $F$. $r$. obsoletus. Certaines de ces subdivisions sont peut-être basées sur les plumages propres à l'âge ou sur la variation chez les individus et seront peut-être trouvées inutiles. Les divers noms se rattachent aux différences de couleur. Le premier a la tête largement ravée de noir sur un fond presque blanc, le deuxième a la tête presque complètement foncée et le foncé du dos est bordé d'une large bande plus claire, et le troisième a le dos presque noir et les parties inférieures si fortement rayées qu'elles sont presque noires.

356. Le Faucon pèlerin. (PERegrine Falcon). Falco peregrinus. L, 16.50. Planche XIV A.

Traits distinctifs. La coloration représentée planche XIV A, la taille comparative et. son véritable bec et son aile de faucon doivent servir à faire reconnaitre cet oiseau. 
Indications sur le terrain. Ses longues ailes de faucon, sa coloration et sa taille sont caractéristiques. Son vol est aussi facile à reconnaítre: il bat rapidement des ailes sans beaucoup planer.

Nid. Habituellement sur des bancs de saillies rocheuses, parfois dans des branches creuses des grands arbres.

Habilat. Il habite dans la plupart des régions polaires et tempérées de l'hémisphère boréal, n'étant commun nulle part mais vivant par couples isolés et retournant au même endroit tous les ans.

SOUS-ESPECE. Le faucon du nouveau monde qui porte le nom de faucon pèlerin est le seul représentant de l'espèce qui se rencontre dans l'Ámérique orientale.

Le représentant américain de cette espèce est une sous-espèce du fameux faucon pèlerin de l'ancien continent, qui, après le faucon proprement dit, était l'oiseau de chasse le plus reeherché par les fauconniers, et notre faucon pèlerin est presque impossible à distinguer de l'oiseau européen. Bien qu'il se rencontre dans toute la majeure partie de l'hémisphère boréal, il est partout rare et n'apparait que comme visiteur ou reproducteur casuel.

Valeur économique. On est étonné de la taille de la proie dont peut s'emparer cet oiseau. On l'a vu souvent abattre même un canard ordinaire pesant quelquefois trois fois autant que le faucon. L'auteur de cet ouvrage a trouvé autour d'un nid dans le district de Muskoka, les restes de plusieurs gelinottes de taille normale qui ont dú être transportées là de toute pièce. Un examen du contenu des estomacs de 16 spécimens ont donné les résultats suivants: 7 contenaient des volailles ou oiseaux-gibiers; 9 , d'autres oiseaux; 1, des souris; et 2, des insectes. Heureusement il est pour luimême aussi prudent que plein de vie. Les adultes se cantonnent ordinairement dans les champs et les marais où ils ne peuvent causer directement que peu de dommages.

\section{Sous-genre. Tinnunculus. Faucons des pigeons.}

357. Le Faucon des pigeons. L'EMERILlon. (PIGEON HAWK). Falcocolumbatius. L, 10. Un petit faucon. Adulte mâle: bleu ardoisé foncé en dessus, plus bleu sur le bas du dos et le croupion. Partie inférieure rayée de brun sur un fond jaune d'ocre, les rayures se reunissant légèrement sur le bas de la poitrine. Jeunes: semblables, sauf que le dos est brun au lieu de bleu ardoisé. Adultes femelles: entre les deux.

Traits disinctifs. Caractères du faucon vrai: la prédominance de coloration foncée et la petite taille de ce rapace sont caractéristiques.

Indications sur le terrain. Le vol et le profil du faucon, avec sa petite taille et sa couleur foncée, sont des signes distinctifs.

Nid. Dans les branches creuses des arbres ou sur des banes de rochers.

Habilat. Toute l'Amérique du Nord jusqu'au nord de l'Amérique du Sud. Couve au sud jusqu'aux confins des régions cultivées dans l'est du Canada.

SOUS-ESPECES. Il y a plusieurs sous-espèces du faucon des pigeons au Canada, mais on ne rencontre dans l'Est que l'oiseau type, le faucon des pigeons de l'Est.

$\mathrm{Au}$ point de vue de la vie et de l'activité c'est une miniature du faueon pèlerin, mais il est beaucoup plus commun qu'aueun des faucons précités. On dressait aussi cette espèce pour la fauconnerie royale.

Valeur économique. Ainsi que l'indique son nom, le faucon des pigeons s'attaquait probablement beaucoup aux pigeons sauvages. De nos jours, cependant, il poursuit ordinairement le petit gibier. Sur 51 estomacs examinés, 2 contenaient des jeunes poulets; 41, des petits oiseaux; 2, des souris; et 16 des insectes. On verra d'après ce résultat que le faucon des pigeons est funeste aux petits oiseaux sauvages, mais trop petit pour nuire sérieusement à l'éleveur de volailles. Il poursuit souvent les oiseaux de rivage et semble particulièrement friand du pluvier à ventre noir. Bien que plus répandu que le faucon pèlerin il n'est eneore nulle part un oiseau commun.

$$
28588-10 \frac{1}{2}
$$


Sous-genre-Cerchneis. Crécerelles.

360. Le Faucon-épervier.(american sparrow hawK). Falco sparverius. I, 10. Planche XIV B.

Traits distinctifs. La coloration de cet oiseau le rend impossible à confondre avec aucune autre espèce.

Indications sur le terrain. Avec son profil de faucon et sa petite taille on ne peut le prendre pour aucun oiseau sauf peut-être le faucon des pigeons. Sa forme plus svelte et sa plus longue queue le différencieront tout de suite quand ses vives couleurs ne sont pas perceptibles.

Nid. Dans des trous de pics abandonnés ou des cavités naturelles de bois mort.

Habitat. La majeure partio de l'Amérique du Nord, à partir des régions habitées jusqu'au golfe du Mexique. Couve partout od il se trouve au Canada.

SOUS-ESPECES. Deux sous-aspèces de faucon-tpervier sont reconnues au Canada, mais seulement l'oiseau type, le faucon-épervier de l'Est, se rencontre dans l'Est.

C'est le seul faucon qui soit commun en ce pays. C'est un magnifique oiseau, c'est lui qui de tous nos oiseaux de proie possède les couleurs les plus vives. Il chasse en plein champ et ses points d'observation préférés sont sur les plus hautes branches mortes d'arbres solitaires dans le's champs ou sur les lisières des prés; dès qu'il aperçoit sa proie il hésite un moment en battant rapidement des ailes, comme un martin-pêcheur, et alors il se précipite sur sa proie.

Le nom de faucon-épervier devrait plus proprement s'appliquer à l'épervier brun; c'est ce que l'on reconnaît dans l'ancien continent où le nom de faucon-épervier s'applique à un petit accipitre qui se rapproche beaucoup de cette espèce. Faucon des sauterelles serait un nom qui désignerait beaucoup mieux cet oiseau, puisqu'il se nourrit en grande partie de ces insertes. Quand on le prend jeune dans son nid, ce petit faucon s'apprivoise facilement.

Valeur économique. Bien que ce soit un épervier, cet oiseau est l'un des plus actifs et efficaces protecteurs de la ferme. Sur 291 estomacs examinés, 1 contenait un oiseau-gibier (caille); 53 , d'autres oiseaux; 89 , des souris; 13, d'autres mammifères; 12 , des reptiles ou batraciens (grenouilles, etc.); 215 , des insectes; et 29 , des araignées. Sur les oiseaux examinés, 43 furent pris pendant les mois d'hiver entre décembre et avril. Parmi les spécimens pris au cours de la saison pendant laquelle il y a des insectes, 10 estomacs seulement contenaient des oiseaux, ce qui prouve que ce faucon détruit les oiseaux plutôt par nécessité que par goût. Les «autres mammifères" sont surtout des rongeurs malfaisants avec seulement quelques écrevisses. Les insectes sont ordinairement des sauterelles qui font beaucoup de mal et dont il est difficile de se débarrasser. D'après ce témớgnage il eśt évident que le fahcon-épervier est bienfaisant et devrait être protégé.

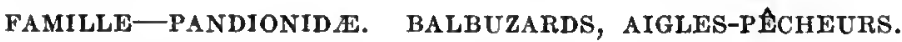

Les balbuzards ou aigles-pêcheurs forment une famille de rapaces se nourrissant entièrement de poissons, qu'ils capturent en plongeant en eau peu profonde. Il y a d'autres oiseaux qui mangent du poisson, mais ce sont ordinairement des poissons morts ou arrachés à des oiseaux pêcheurs. Comme il n'y a qu'une seule espèce d'aigle-pêcheur en Amérique il sera inutile d'en étudier la famille. 


\section{Genre-Pandion. Balbuzards.}

364. Le Balbuzard d'Amérique. L'ORFRAIE D'AMÉRIQUE, L'AIGLE-PECHEƯR. (osprey). Pandion haliaêtus carolinensis. I, 23-10. Planche XV A.

Traits distinctifs. La coloration de l'orfraie est tout à fait caractéristique. Ses pattes bleu pále et ses pieds recouverts d'écailles bérissées particulièrement sur la plante et les serres sont des caractères distinctifs.

Indications sur le terrain. L'envergure de cet oiseau est telle qu'on le prend souvent pour un aigle. Il est cependant beaucoup plus petit qu'aucun des aigles et il se distingue de ceux-ci par le blanc de ses parties inférieures.

Nid. Se compose d'un gros amas de buchettes sur les arbres ou à terre, et comme i] est augmenté d'année en année, il devient parfois de la taille d'une petite veillote.

Habitat. L'orfraie se rencontre dans la majeure partie de l'Europe, l'Asie, l'Afrique et dans le sud de l'Amérique jusqu'au nord de l'Amérique du Sud. Le balbuzard d'Amérique couve par endroits au Canada sauf dans les régions méridionales.

SOUS-ESPECES. L'orfraie qui habite et l'ancien et le nouveau monde est divisé en races sous-spécifiques dont le balbuzard d'Amérique est le représentant américain. Sa distinction d'avec le type européen est fondée sur des caractères trop subtiles pour être étudiée ici.

L'orfraie est un oiseau des plus pittoresques. Il plane en hauteur audessus de l'eau, s'arrête un moment, et puis fond soudainement, non pas comme une sonde, ainsi que fait le fou de Bassan, mais cn décrivant une longue spirale et 'vient s'abattre sur l'eau les pieds devant et les ailes redressées au-dessus du dos. On voit rejaillir en l'air une poussière blanche et l'oiseau remonte avcc un poisson enfermé dans ses fortes serres. Les orfraies fréquentent les bas-fonds larges, les platières de marées et les baies peu profondes, plutôt que les cndroits à eau profonde où il faudrait compter sur la chance de trouver des poissons à la surface. Dans la région des grands lacs, l'espèce devient plus rare. Son gros nid massif augmente d'une année à l'autre jusqu'à ce qu'il atteigne des proportions telles qu'il devient un point de mire dans tout le voisinage et s'attire ainsi les attaques des dénicheurs d'oiseaux, de sorte que ce n'est maintenant que dans les endroits les plus retirés que cet oiseau peut couver en toute tranquillité. Il est encore assez répandu le long des côtes de l'Atlantique et du golfe Saint-Laurent, mais, à l'intérieur, il est rare.

Valeur économique. Lors même que l'orfraie se nourrit entièrement de poisson, l'antipathie qu'éprouve le pêcheur à son égard est plutôt exagérée. Comme il ne pêche qu'en cau peu profonde il ne prend que peu de poissons d'importance économique. Sur la côte e'cst le carrelet, la petite morue ou tacaud et autres petites espèces qui constituent la majeure partie de sa nourriture. Sur les lacs d'eau douce c'est le crapet jaune, la perche et la carpe qui semblent composer son régime alimentaire. Le nombre de poissons gibiers ou de poissons marchands qu'il prend est incontestablement faible. Sur onze estomacs examinés, il n'y en avait pas un seul. Les truites de ruisseaux ne sont pas recherchées par cet oiseau et la plupart des poissons marchands tel que l'achigan et le brochet sont en eau trop profonde pour qu'il puisse les atteindre.

\section{SOUS-ORDRE-STRIGES. OISEAUX DE PROIE NOCTURNES. HIBOUX.}

Les hiboux sont facile à reconnâ̂tre. La cire dissimulée dans le plumage de la face et les cercles faciaux très en évidence autour des yeux (figure 34, p. 25) sont des signes distinctifs pour l'observateur le plus ordinaire. Ils sont principalement nocturnes, la chouette épervière et le harfang étant les seules espèces de l'est du Canada qui chassent habituelle- 
ment de jour. Même les oiseaux nocturnes cependant voient très bien le jour. Ils peuvent se trouver momentanément éblouis lorsqu'ils sont soudainement transportés de l'obscurité à la pleine lumière et certaines espèces ont tellement de confiance dans leur immobilité pour se dérober a la vue qu'on pourrait presque les prendre à la main. Le plumage est un des traits les plus intéressants chez les hiboux. Les plumes sont singulièrement molles et se tiennent ensemble de façon à empêcher l'air de passer a travers les petits interstices, ce qui leur assure un vol silencieux. Un hiboux pourrait passer assez près de vous pour vous éventer la figure de son aile sans qu'on ait rien entendu.

Deux familles sont représentées au Canada: Aluconidoe, effraies communes, représentées par une espèce qui est un visiteur aceidentel du sud, et Strigida, connues sous le nom de hiboux à aigrettes, lors même que beaucoup d'espèces canadiennes ne sont pas pourvues de ees ornements.

\section{FAMILLE-ALUCONIDA. FFFRAIES.}

Les effraies, appelées quelquefois chouettes à figure de singe, d'après la forme en cœur de leurs ccrcles faciaux réunis, sont représentées au Canada par une seule espèce laquelle est assez rare. La serre médiane est pectinée, étant munie de dentelures sur son bord intérieur comme le héron (figure 19, p. 22) ct le pouce est aussi long que le médian au lieu d'être un peu plus court comme chez les hiboux à aigrettes.

\section{Genre-Tyto. Effraies.}

365. L'Effraie d'Amérique. (AMERICAN BARN owL). Tyto pratincola. L, 18. Couleur générale de fond ocre rougeâtre, plus claire en dessous, cercles faciaux blanc sombre avec bordure extérieure d'un ocre plus foncé tirant sur le brun. Le dos jusqu'au sommet de la tête recouvert de gris cendré, œillé de nombreuses petites taches contournées de noir. Parties inféricures, gorge et tour de la face mouchetés de noir.

Traits distinctifs. Le disque facial en forme de cœur, clairement et nettement délimité, la coloration jaune claire avec nuance rosâtre et les tarses presque nus sont des traits distinctifs.

Nid. Dans des tours, clochers, greniers, terrasses ou arbres.

Habitat. Depuis le golfe du Mexique jusqu'aux Etats du milieu de l'Atlantique sur tout le continent. Il n'apparait au Canada qu'à titre de vagabond le long de la frontière sud.

C'est le représentant américain de la chouette européenne qui hante les ruines dans les vieilles légendes et chansons. Elle est d'une activité ćtonnante pour détruire les souris et, de ce fait, un oiseau précieux, mais elle est plutôt rare au Canada.

\section{FAMILLE-STRIGIDÆ. HIBOUX À AIGRETTES.}

Cette famille est caractérisée par un développement plus ou moins considérable de plumes formant aigrette au-dessus des oreilles qui ne se retrouve pas cependant dans toutes les espèces. Les pieds sont emplumés jusqu'à l'extrémité des doigts (figure 32, p. 25). Cette famille comprend tous les hiboux canadiens sauf l'effraie (Barn Owl) que nous venons de décrire.

$$
\text { Genre-Asio. Hiboux à oreilles. }
$$

366. Le Hibou à oreilles longues. (AMERICAN Long-Eared owL). Asio wilsonianus. L, 14.80. Hibou de taille moyenne ressemblant comme coloration au. duc de Virginie (p. 148) mais beaucoup plus petit et de charpente plus légère. 
Traits distinctifs. Bien que sa coloration se rapproche de celle du duc de Virginie, la différence de taille sert à les différencier. On peut le distinguer des hiboux à oreilles courtes par les touffes de plumes très apparentes au-dessus des oreilles qui se dressent sur le front à peu de distance l'une de l'autre, par l'absence générale de raies dans sa coloration et par la quantité de blanc et de noir étendue sur tout le corps.

Indications sur le terrain. Les aigrettes proéminentes se dressant presque verticalement au milieu du front, et le disque facial brun rouilleux différencient cette espèce de la suivante, la seule avec laquelle on pourrait la confondre.

Nid. Sur les arbres, s'empare ordinairement des nids abandonnés de corneilles ou d'éperviers, à une hauteur de 20 à 40 pieds du sol.

Habitat. Dans toute la zone tempérée de l'Amérique du Nord jusqu'aux limites des régions cultivées.

Cette espèce habite de préférence des fourrés d'aunes ou de conifères sur les bords des marais, pendant la migration, on les trouve quelquefois en compagnies, se reposant le jour dans les retraites sombres des forêts humides.

Valeur économique. Sur 92 estomacs examinés, 1 contenait un oiseau gibier (caille); 15, d'autres oiseaux; 84 , des souris; 5 , d'autres mammifères; et 1 , des insectes. D'après ces données, il est évident que l'espèce n'est pas très malfaisante. Sa préférence pour les souris suffit à en faire un oiseau utile et sa petite taille de même que ses habitudes nocturnes l'empêchent de s'attaquer aux jeunes volailles.

367. Le Hibou à orellles courtes. (american short-eared owl). Asio flammeus. L, 15.50. Hibou de taille moyenne à aigrettes courtes, a peines visibles. Couleur d'ensemble jaune d'ocre avec passablement de petites plumes blanches en dessous sur la face et des raies de brun nettement définies sur tout le corps, plus étroites en dessous et plus élargies au-dessus.

Traits distinctifs. La couleur générale chamois clair et les raies sont distinctives. Les aigrettes sont quelquefois indistinctes; lorsqu'elles sont perceptibles, elle se dressent verticalement comme celles de l'espèce précédente entre les yeux. L'absence de dessins noir et blanc dans la coloration sert à distinguer cette espèce d'avec le hibou à oreilles longues. terrain.

Indications sur le terrain. La couleur chamois constitue la meilleure marque sur le

Habitat. A peu près cosmopolite. Se rencontre partout au Canada et couve aux endroits où il se trouve.

C'est un véritable hibou des marais; il est un peu plus diurne dans ses habitudes que beaucoup de ses congénères. On l'aperçoit souvent sur la brunc survolant les marais avec un vol puissant comme celui des éperviers. Comme il vit dans les marais sur les lisières broussailleuses, il en est tous les ans un grand nombre de tués par les chasseurs.

Valeur économique. Sur 97 estomacs examinés, 11 contenaient de petits oiseaux; 77, des souris; 7, d'autres mammifères; et 7, des insectes. D'après ces données et par le fait que les lisières des marais, les lopins de terre incultes, les rangées de clôture que cet oiseau fréquente, sont précisément les endroits à partir desquels les petits rongeurs se répandent dans les terrains bien cultivés, il est évident qu'il s'agit ici d'une espèce très utile, qu'en l'exterminant on se priverait de l'un des plus efficaces destructeur's d'innombrables fléaux.

\section{Genre-Strix. Chouettes du Canada.}

368. La Chouette du Canada. (BARRED owl). Strix varia. L, 20. Planche $\mathrm{XV}$ B.

Traits distinctifs. Il est presque impossible de ne pas reconnaittre 'cet oiseau. La seule chouette sans aigrette qui lui ressemble est la chouette cendrée. La chouette du Canada peut etre reconnue par sa petite taille, ses yeux noirs au lieu de jaunes et ses raies et barres bien caractérisées en dessous. C'est le seul hibou avec l'effraie qui ait les yeux noirs. 
Indications. Taille, l'absence d'aigrettes et couleur générale brun grisâtre avec bandes sur la poitrine.

Nid. Niche dans des arbres creux ou dans des nids abandonnés ou corbeaux ou d'eperviers.

Habitat. L'est de l'Amérique du Nord depuis les limites de la colonisation jusqu'aux Etats de Kansas et Georgia.

SOUS-ESPECES. Il y a plusieurs sous-espèces de cette chouette dont deux se présentent au Canada. L'oiseau type qui se rencontre dans l'est du Canada est la chouette du Canada de l'Est.

Bien qu'apparemment d'assez forte taille cet oiseau, une fois dépouillé de ses plumes, est relativement petit. De plus, il est d'une nature plutôt douce et n'a pas le tempérament agressif de quelques-uns de ses congénères. Son cri retentissant et étrange s'cntend de loin au milieu d'une nuit tranquille.

Valeur économique. Lors même qu'on a vu souvent des volailles perchées impunément dans des arbres d'où l'on entendait tous les soirs le cri de cette chouette, elle est ordinairement considérée comme malfaisante et tuée sans merci. Sur 189 estomacs examinés, 5 contenaient des volailles ou oiseaux-gibiers; 13, d'autres oiseaux; 46, des souris; 18 , d'autres mammifères; 4, des grenouilles; 1, un lézard; 2, des poissons; 14, des insectes; 2 , des araignées; et 9 , des écrevisses. Les deux cas où l'on a trouvé des volailles peuvent être considérés comme accidentels, puisque celles-ci ont été prises en janvier alors qu'elles devaient être arrivées à toute leur taille et par conséquent trop robustes pour être abattues par cette faible chouette. La valeur économique de cet oiseau est très satisfaisante.

\section{Genre-Scotiaptex. Chouettes cendrées.}

370. La Ghouette cendrée. (cinereous owL). Scotiaptex nebulosa. L, 27. A peu près de la même couleur générale grisâtre que la chouette du Canada, mais de beaucoup plus forte taille.

Traits distinctifs. Le plus gros, tant en apparence qu'en taille, de tous nos hiboux, mais une fois dépouillé de son volumineux plumage, on est étonné de la petite taille de l'oiseau. Semblable à la précédente quant à la coloration et à l'absence d'aigrettes; mais les yeux sont jaunes au lieu de noirs et la coloration de la poitrine et du dessous est diffuse et dépourvue de raies et de bandes bien définies. trino.

Indications sur le terrain. Taille, coloration grisâtre et absence de bandes sur la poi-

Nid. Sur les arbres.

Habitat. Les forêts du Nord, à travers tout le continent; émigre parfois cn hiver dans les régions cultivées.

SOUS-ESPECES. La chouette cendrée se présente dans les parties nord de l'ancien et du nouveau monde, mais dans chacun sous une sous-espèce distincte. La forme européenne est la chouette de Laponie, $S$. $n$. lapponica, et l'américaine, la chouette cendrée qui est la race type. Canada.

Cet oiseau n'est qu'un visiteur occasionnel dans les régions habitées du

Valeur économique. La valeur économique, autant qu'il peut en avoir, est plutôt bienfaisante. On a peu de données sur son régime alimentaire. Sur 9 estomacs cxaminés, 1 contenait un petit oiseau; 7, des souris; et 4, d'autres mammifères. C'est évidemment un bon destructeur de souris.

\section{Genre-Cryptoglaux. Nyctales.}

371. La Nyctale de Richardson. (ARCTIC SAW-WHET OWL). Cryptoglaux funerea, L, 10. Petite chouette grise sans aigrettes. Brun cendré au-dessus, avec points blancs; blanchâtre au-dessous, avec raies indistinctes. Les taches de couleur sont légères et le dessin en est vague et diffus. 
Traits distinctifs. Cette espèce ressemble au hibou maculé quant à sa taille et à la coloration générale mais pas autrement; elle n'a pas d'aigrettes et les taches de couleur n'ont nulle part de contours définis. . La disposition des couleurs est trop indistincte pour être facilement différenciée d'avec celle qui caractérise la nyctale d'Acadie. On la reconnaitra cependant à sa taille manifestement plus forte.

Indications sur le terrain. Elle est trop rare pour être identifiée au moyen des vagues indications que nous pourrions donner.

Nid. Ordinairement dans des trous sous les arbres.

Habitat. Dans les forêts du Nord, jusqu'aux limites des régions boisées, depuis la vallée du Mackenzie en allant vers l'Est.

SOUS-ESPECE. C'est une espèce européenne en mêmc temps qu'américaine, mais le type habitant le nouveau monde est une sous-espèce distincte de celle de l'ancien et porte le nom de chouette de Richardson, C.f. richardsoni.

C'est peut-être l'espèce la plus rare de tous les hiboux canadiens, et qui ne peut être considérée, dans l'est du Canada, que comme un oiseau passager d'hiver très irrégulier.

Valeur économique. Bien que trop rare pour avoir une valeur économique notoire on peut considérer cette espèce comme plutôt bienfaisante. Sur 9 estomacs examinés, 1 contenait un petit oiseau; 7, des souris; et 4, d'autres mammifères.

372. La Nyctale d'Acadle. (sAw-Whet owl, acadian owL). Cryptoglaux acadia. $L, 8$. Une très petite chouette. Le dessous est d'une couleur brun cendré chaud, vaguement marqué de taches blanches peu apparentes, particulièrement sur le derrière du cou, se transformant sur la tête en de fines raies distinctes qui se réunissent en une seule ligne au-dessus du disque facial. Ie dessous est blanc avec petites raies brun clair et une vague tendance a former une bande massive, sur la poitrine. Certains individus ont un rare plumage qui leur a fait donner le nom de chouette de Kirtland; l'on a reconnu, cependant, que ce n'était qu'une phase passagère du plumage des jeunes nyctales d'Acadie, bien que l'on ne s'explique pas la raison de sa rareté. Le dessus est completement brun, un peu plus rougeat tre que chez l'adulte et cette couleur se prolonge à travers la poitrine en une bande presque rouge; le dessous est d'un jaune d'ocre uniforme. Le disque facial autour des yeux est presque noir formant un contraste saillant avec le blanc qui surmonte et sépare les yeux.

Traits distinctifs. Son plumage ordinaire est tellement semblable à celui de la chouette de Richardson qu'on ne peut les distinguer que par la taille. L'absence d'aigrettes et de taches de couleurs nettement dessinées la rend facile a reconnaitre d'avec le hibou maculé.

Indications sur le terrain. Très petite taille et absence d'aigrettes.

Nid. Dans des trous sur les arbres, quelquefois des trous naturels, d'autres fois dans ceux faits par les pics et les écureuils.

Habitat. Principalement la zone tempérée de l'Amérique du Nord. Elle couve d'une façon irrégulière et ordinairement dans les parties nord de son habitation à de hautes altitudes plus au sud.

SOUS-ESPECES. Il y a plusieurs sous-espèces de aSaw-whet Owl » au Canada, mais une seule, l'oiseau type, la nyctale d'Acadie, se rencontre dans l'Est.

Cette miniature de chouette est le plus petit des oiseaux de proie de l'est du Canada. Elle fréquente les sombres fourrés des marais de cèdre et de tamarac, passant la journée accolée à un tronc d'arbre de façon que son plumage se fond imperceptiblement avec la couleur de l'écorce. Elle a tellement confiance en sa coloration pour se protéger qu'on peut l'approcher de très près et même parfois la prendre avec les mains. Son nom indigène de "Saw-whet" vient de son cri d'appel que l'on dit ressembler au son produit par une scie que l'on affûte.

Valeur économique. Sur 19 estomacs examinés, 1 contenait un moineau; 17, des sourís; et 1 , une mite. Ce résultat devrait suffire pour que l'on protège l'espèce. 


\section{Genre-Otus. Hiboux maculés.}

373. Le Hibou maculé. (screech owL). Otus asio. L, 9.40. Planche XVI A.

Traits distinctifs. Cette espèce est dichromatique, c'est-à-dire, se présente sous deux couleurs très distinctes sans égard au sexe, à l'âge ou à la saison. L'un des types est d'un beau brun avec nuances de gris et de blanc, et chez l'autre le gris est remplacé par un rouge vif. Le dessin du plumage est le mêtme chez les deux types. Il se rencontre beaucoup de phases intermédiaires. Sa taille apparente varie entre celles des chouettes de Richardson et d'Acadie, mais la présence d'aigrettes bien caractérisées sur les côtés de la couronne et le dessin nettement défini de ses panachures sont caractéristiques.

Indications sur le terrain. La taille et les aigrettes bien en vue sont les meilleures indications.

Nid. Dans les arbres creux.

Habitat. Toute la zone tempéré de l'Amérique du Nord. Couve au Canada partout où il se trouve.

SOUS-ESPECES. Le hibou maculé comprend un grand nombre de sous-espèces: le catalogue de l'American Ornithologists' Union en reconnaît neuf races géographiques dans l'Amérique du Nord. La seule trouvée dans l'est du Canada, cependant, est l'oiseau type, le hibou maculé de l'Est.

Lors même qu'on l'appelle "Screech owl), le chant de cet oiseau est mélodieux et agréable avec une note mélancolique et n'a rien de dur ni d'irritant. Son cri le plus ordinaire est un long sifflement, doux et chevrotant sur un ton uniforme ou une gamme descendante. Parfois ce sont des fredonnements ou glouglous ou autres notes tranquilles et familières. Ce petit oiseau quoique inoffensif fait preuve toutefois d'un courage étonnant pour défendre ses petits. Le soir, il s'abat sur la tête de tout intrus en émettant un sinistre "hou-hou" au son creux et faisant claquer son bec. Juste au moment de frapper, cependant, il s'écarte un peu pour fondre avec plus d'élan. Ces attaques, bien que déconcertantes, sont loin d'être dangereuses. L'habitude qu'il a de faire son nid dans les vieux pommiers creux de la ferme où la chasse qu'il fait aux souris est de toute première importance, en fait pour le cultivateur un oiseau très précieux.

Valeur économique. Les recherches sur le régime alimentaire du hibou maculé indiquent qu'il doit être protégé. Sur 212 estomacs examinés, 1 contenait de la volaille; 38 , d'autres oiseaux; 91 , des souris; 11 , d'autres mammifères; 2 , des lézards; 4, des grenouilles et crapauds; 1, du poisson; 100, des insectes; 5, des araigríées; 9, des écrevisses; 7, des aliments divers; 2, des scorpions; et 2 , des vers de terre. L'élément volaille doit être considéré ici comme accidentel, car l'oiseau en question, qui était un pigeon, est une proie de trop forte taille pour ce hibou.

\section{Genre-Bubo. Grands Ducs.}

375. Le Duc de Virglnie. (GReat hoRned owL). Bubo virginianus. L, 22. Planche XVI B.

Traits distinctifs. C'est notre seul hibou de plus de 15 pouces de longueur qui porte des aigrettes. Sa coloration ocreuse, et noire et blanche, est le signe distinctif.

Indications sur le terrain. Forte taille, couleur ocreuse et aigrettes proéminentes. creux.

Nid. Niche ordinairement dans des nids abandonnés d'éperviers ou dans des arbres

Habitat. Plusieurs sous-espèces du duc de Virginie fréquentent toute l'Amérique du Nord. Pour celles qui habitent le Canada, leur nom indique suffisamment leur habitat.

SOUS-ESPECES. Cette espèce comprend dans l'Amérique du Nord un grand nornbre de races géographiques ou de sous-espèces dont quelques-unes apparaissent comme migratrices dans l'est du Canada. Le spécimen arctique $B$. v. subarcticus est presque blanc de couleur et manque presque totalement des parties ocreuses et rousses de la variété commune, et les taches noires sont très réduites. Le duc de Virginie de l'Ouest, $B . v$. pallescens, est à peu près de taille intermédiaire entre celui de la région polaire et celui de 
l'Est-l'oiseau type représenté par l'illustration. Celui du Labrador est un oiseau très foncé. Attendu qu'il y a encore, entre ces sous-espèces, des variétés intermédiaires difficiles à distinguer, et qui dépassent leur habitat dans les migrations, on ne saurait les désigner qu'avec beaucoup de circonspection, sauf dans les cas de plumages distinctifs, et seulement après comparaison avec des spécimens authentiques.

Le duc de Virginie est le mauvais génie des bois. Il s'infiltre silencieusement à travers l'ombrageux feuillage et règne en maître, sauf sur les plus grands animaux. L'animosité qu'éprouvent les corbeaux à l'endroit de cette espèce doit résulter d'une cruelle expérience.

Valeur économique. La valeur économique de cet oiseau dépend de la contrée qu'il habite. Dans les bois profonds, loin des habitations, il est évidemment inoffensif à l'homme, et il n'y a que les animaux sauvages lui servant de proie qui ont à en souffrir. Mais, dans les régions habitées, il faut de toutes les façons possibles se mettre en garde contre lui. Sur 110 estomacs examinés, 31 contenaient de la volaille ou des oiseaux-gibiers; 8, d'autres oiseaux; 13 , des souris; 65 , d'autres mammifères; 1 , un scorpion; 1 , du poisson; et 10, des insectes. Ce relevé est donc manifestement défavorable à l'oiseau. On peut lui faire la guerre sans craindre de l'exterminer puisqu'il est parfaitement capable de se défendre, et il y a de vastes régions sauvages où l'espèce peut régner en souveraine sans être molestée.

\section{Genre-Nyctea. Harfangs.}

376. Le Harfang. LE RIBOU BLANC. (8NowY OWL). Nyctea nyclea. L, 25. Un gros hibou blanc avec des bandes courtes et distinctes d'un brun foncé sur presque tout le corps, sans aigrettes visibles. L'adulte arrivé à son plein développement est souvent presque tout blanc.

Traits distinctifs. Le seul hibou qui soit distinctement blanc, sauf le duc de Virginie de la région polaire qui parfois s'en rapproche. Toutefois l'absence d'aigrettes ou d'oreilles le distingue.

Indications sur le terrain. Presque tout blanc, sans aigrettes.

Nid. Sur le sol.

Habitat. Régions polaires de l'Amérique du Nord, émigre au sud en hiver jusqu'd la latitude des grands lacs, sur tout le continent.

Il apparaít à titre de visiteur en hiver dans les régions habitées du Canada. Il fréquente les marais gelés et les rives des lacs et c'est typiquement un oiseau de plein air. Habituellement les spécimens qui viennent du Nord sont des jeunes passablement blancs, mais il s'en présente parfois des vols parmi lesquels les adultes très blancs et presque sans taches sont en majorité. Il est probable que les jeunes sont naturellement plus vagabonds que les adultes, lesquels n'émigrent au loin que lorsqu'ils y sont forcés par le manque de nourriture ou attirés vers le Sud par une grande abondance de lapins.

Valeur économique. La nourriture du hibou blanc se compose surtout de la gent à plumes. Sur 26 cas examinés, 2 estomacs contenaient du gibier; 9, d'autres oiseaux, et 20 des souris, des rats et des lièvres. N'étaitce que ce hibou se mêle à la civilisation quand la plupart des autres oiseaux l'ont quittée, il pourrait faire beaucoup de mal. Dans l'Est il en cause probablement assez peu même en hiver alors qu'ils sont plus abondants; mais dans l'ouest, vers ce temps-là, dans les plaines à ciel ouvert, où se trouve son habitat preféré, il peut exercer une influence sérieuse sur la poule des prairies et d'autre gibier des terres déboisées. 


\section{Genre-Surnia. Chouettes épervières.}

377. La Chouette épervière. (HAwk owL) Surnia ulula. L, 15. Une chouette de taille moyenne ayant a peu près la charpente et les habitudes de l'épervier. Les parties de dessus sont d'un beau brun chaud diversement maillé de blanc. Toutes les parties inférieures et la poitrine sont nettement et régulièrement barrées de brun et de blanc. Une ligne d'un beau brun de phoque contourne extérieurement les disques faciaux dont les deux côtés se rencontrent sous la gorge. Le disque facial n'est pas aussi parfait.

Traits distinctifs. Figure moins aplatie et moins typique de la chouette, queue longue et parties inférieures distinctement et régulièrement rayées.

Indications sur le terrain. Les habitudes diurnes, sa coloration générale et sa longue queue sont de bonnes indications. Tout hibou chassant durant le jour ou perché ouvertement en pleine lumière du jour appartient probablement a cette espece, bien que les chouettes à oreilles longues et à oreilles courtes en fassent parfois autant.

Nid. Sur les arbres conifères ou dans des trous d'arbres morts.

Habitat. Régions septentrionales boisées du continent; ne se présente que rarement. en hiver dans les régions habitces.

SOUS-ESPECE. La chouette epervière habite tout le nouveau ainsi que l'ancien monde à titre de sous-espèce, mais le type est européen. La chouette épervière d'Amérique, $S$. $u$. caporoch, est la seule qui se trouve au Canada.

C'est la plus strictement diurne parmi les chouettes du Canada. Elle possède bien la forme et le mouvement de l'épervier étant plus svelte et d'une charpente plus légère que les autres membres de cette famille. On la voit souvent perchée à l'extrémité d'un tronc d'arbre sec, et elle rappelle beaucoup le faucon-épervier, tant par son profil que par sa façon distinetive d'agiter sa queue.

Valeur économique. C'est un oiseau septentrional qui n'apparaît que dans les régions cultivées, seulement en hiver et peu souvent. Nous n'avons que peu de renseignements sur son régime alimentaire, mais il doit sans doute se nourrir de souris car il est trop petit pour s'attaquer à de plus gros animaux.

\section{Ordre-Coccyges. Coucous et Martins-pêcheurs.}

Les zoologistes à système ne s'accordent pas dans la classification de ces oiseaux. Le catalogue de l'Union américaine des Ornithologistes (édition de 1910) les reconnaît comme constituant un ordre complet, et divise ceux du Canada en deux sous-espèces: Cuculi, comprenant les coucous américains et les familles qui vivent au delà de leur habitat, et Alcyones, les martins-pêcheurs. Les traits distinctifs sont donnés sous les titres de la sous-famille et des diverses espèces.

\section{SOUS-ORDRE-CUCULI. COUCOUS, ETC.}

Ce sous-ordre est représenté en Amérique du Nord par une seule famille, les Cuculidés, comprenant les Anis et deux groupes de coucous.

FAMILLE-CUCULIDE. COUCOUS D'AMÉrique.

Cette famille est représentée dans l'Amérique du Nord par trois familles dont une seule est représentée au Canada, les Coceyzinés, coucous d'Amérique. 
Sous-famille-Coccyzinæ. Coucous d'Amérique.

Description générale. Cet oiseau a le pied faible et les doigts conjugués; deux doigts sont dirigés en avant et deux en arrière (figure 37, p. 26). Le bec est plutôt long, légèrement recourbé et pointu. Le plumage est doux et lâche, manque de cohésion et est très soyeux an toucher comme celui de nombreuses espèces des tropiques. La queue est longue, molle et graduée.

Traits distinctifs. On le distingue du pic, qui a également les doigts conjugués, disposés par paires, par la différence entre les bees et les queues. Chez le pic, les becs sont droits, gros, avec le bout en ciseau, et la queuc est courte, très rigide, et hérissée à la pointe.

Les coucous habitent principalement les tropiques. Les deux coucous canadiens descendent de la souche principale de l'espèce dans les plus chaudes latitudes. Ils possèdent une grâce sensuelle et une élégance tropicale incompatible avec les climats du nord. C'est ce que l'on constate dans leur tenue souple et sinucuse, leur belle gorge profonde et arrondie, leur queue longue et élégante, et leur plumage mince mais doux et soyeux. Ils fréquentent les jungles d'arbrisseaux chaudes et humides et planent rapidement à travers les espaces déboisés avec un vol silencieux et onduleux qui semble bien en rapport avec leur nature exotique. On trouve dans la littérature anglaise beaucoup d'allusions au coucou, mais qui n'ont que peu de rapport avec le coucou canadien. Ce coucou n'est pas une espèce qui apparait de bỏne hcure: elle arrive doucement et silencieusement au printemps, de façon que pendant un certain temps après son arrivée on ignore sa présence. Son cri est aussi tout différent de celui que nous ont maintes fois décrit les auteurs européens. Ils est moins musical mais il possède un charme particulier et un caractère exotique estraordinaire qui s'harmonise avec sa nature. Les deux espèces canadiennes ont à peu près les mêmes cris; son cri saisissant de "caou-caou-acou» est l'un des plus caractéristiques et par un temps calme en été, on peut l'entendre d'un quart de mille ou de plus loin encore. Ils ont un autre cri de "couc-conc-couc" rappelant le bruit d'une grosse horloge qui marque les secondes; celui-ci n'a pas autant de portée que l'autre mais il s'entend aussi à une distance considérable. En ce qui concerne les soins maternels, nos oiseaux semblent beaucoup mieux se rendre compte de leurs responsabilités que ceux d'Europe. Ils sont légèrement parasites dans leurs habitudes, c'est-à-dire qu'ils déposent parfois leurs cufs dans les nids d'autres oiseaux auxquels ils confient le soin d'élever leurs petits, mais ils n'en font pas une pratique commune. L'ancien nom anglais de "Cuckold" se rapporte à cette habitude de l'oiseau européen. Quant à l'américain, ce n'est pas chez lui une pratique habituelle comme chez son congénère d'Europe, mais plutôt accidentelle.

Valeur économique. Les coucous se nourrissent presque exclusivement d'insectes mais mangent aussi parfois des fruits sauvages. Ils sont particulièrement précieux en raison de leur préférence pour certains insectes auxquels les autres espèces ne touchent presque jamais. Les chenilles velues, qui, en raison de leur manteau hérissé, sont à l'épreu've des oiseaux les plus dédaigneux sont une proie favorite des coucous. On trouve l'intérieur de l'estomac du coucou tapissé de poils épineux de chenilles fixés aux parois et formant comme une fourrure.

\section{Genre-Coccyzus. Coucous.}

387. Le Coucou a bec jaune. (yellow-BILled cuckoo). Coccyzus americanus. L, $12 \cdot 20$. Planche XVII A. 
Traits distinctifs. Le coucou à bec jaune ne peut être confondu qu'avec son congénère le coucou à bec noir. Le jaune sur le bec d'où lui vient son nom est le meilleur signe d'identification. Les autres marques distinctives sont, une grande surface de couleur cannelle répandue sur les, ailes, que l'on aperçoit quand l'oiseau vole et beaucoup plus de blanc à l'extrémité des plumes de la queue.

Indications sur le terrain. Le long et flexible profil quand il vole et la coloration générale de cet oiseau le font reconnaítre, comme un coucou. L'espèce se distingue par sa mandibule inférieure jaunâtre, ses marques couleur cannelle sur l'aile, et la forte quantité de blanc sur sa queue.

Nid. Construit avec des branchettes non loin du sol.

Habitat. Cette espèce habite plus au sud que la suivante, mais les relevés semblent indiquer une augmentation du nombre et une extension de l'habitat durant les trente dernières années. Son habitat dépasse tout juste les frontières méridionales d'Ontario, de Québec et du Nouveau-Brunswick.

SOUS-ESPECES. On reconnait deux races de cette espèce au Canada. Le coucou de l'Est-l'oiseau type-est répandu dans l'Ouest jusqu'aux plaines.

388. Le Coucou à bec noir. (BLACK-BILled cuckoo). Coccyzus erythrophthalmus. L, 11.85. Planche XVII A.

Traits distinclifs. Se distingue du coucou à bec jaune par son bec tout noir, l'absence de teinte caunelle sur les ailes et la quantité beaucoup moindre de blanc à l'extrémité de la queue.

Indications sur le terrain. Son habitat est plus répandu et plus septentrional que celui du coucou à bec jaune; il fréquente irrégulièrement les parties habitées de la région au sud du golfe Saint-Laurent et à l'ouest de la frontière manitobaine.

Nid. Structure lâche de branchettes non loin du sol.

Habitat. Son habitat est plus vaste et plus septentrional que celui du coucou a bec jaune; fréquente irrégulièrement les parties plus peuplées de la région au sud du golfe Saint-Laurent et à l'uuest de la frontière du Manitoba.

\section{SOUS-ORDRE-ALCYONES. MARTINS-PÊCHEURS.}

Les martins-pêcheurs représentent un groupe bien caractérisé dans presque toutes les parties du monde. Bien qu'évidemment ils s'adaptent bien à, la pêche, quelques-uns ont abandonné leurs habitudes ancestrales et le bord des eaux pour aller dans les bois se nourrir d'insectes et de vers de terre. Cependant tous ces types exceptionnels vivent en dehors de leur habitat et principalement aux tropiques; le "Laughing Jackass" d'Australie est un grand martin-pêcheur anormal. Les martins-pêcheurs de l'Amérique du Nord sont tous compris sous une seule famille, celle des Alcédinidés.

\section{FAMILLE-ALCEDINIDE. MARTINS-PECHEURS.}

Attendu qu'il n'y a qu'une seule espèce de cette famille au Canada la description de cette espèce servira pour la famille entière.

$$
\text { Genre-Ceryle. Martins-pêcheurs. }
$$

390. Le Martin-pêcheur. (Belted Kingfisher). Ceryle alcyon. L, 13.02. Planche XVII B.

Traits distinctifs. Avec sa grosse touffe hérissée et son dos ardoisé, le martin-pêcheur ne peut guère être confondu avec aucun autre oiseau d'Amérique. Les pieds faibles, les trois doigts en avant avec les deux extérieurs réunis (figure 36, p. 26) sur la moitié de leur longueur, et les surfaces singulièrement grossières des doigts sont des signes distinctifs des martins-pêcheurs.

Indications sur le terrain. Sa huppe hérissée et sa grosse tête, sa coloration générale et son habitude de rester perché sans mouvement au bout d'une perche surplombant la rivière, puis de plonger avec fracas, permettent de reconnaître facilement le martin-pêcheur a l'etat vivant.

Nid. Ordinairement sur le sol, à l'extrémité d'un tunnel percé dans la paroi d'un talus.

Habilal. Toute l'Anćrique du Nord; ccuve partout où il se trouve au Canada. 
Tous les habitués des eaux canadiennes connaissent le martin-pêcheur. Il s'installe immobile sur une perche qui domine la rivière, afin de guetter le poisson qui peut se trouver au-dessous. Tout-à-coup il s'enlève, reste suspendu un moment dans l'air et se précipite dans l'eau avec grand fracas pour remonter un instant après avec un malheureux poisson dans son large bec et disparaît au tournant du cours d'eau. Dans la région qu'il fréquente journellement le martin-pêcheur connaît toutes les perches ou les branches du haut desquelles il peut avoir une bonne vue sur ses lieux de pêche. Il ne fréquente pas seulement les cours d'eau, on le remarque également au bord des étangs, des lacs et même de la mer. Ces oiseaux pêchent quelquefois à des distances considérables de leurs nids et souvent on les aperçoit dans des endroits où les talus dont ils ont besoin pour faire leurs nids sont rares. Cependant ils s'adaptent aux conditions qui se présentent, si bizarres soient-elles; ils utiliseront, par exemple, la terre qui s'attache aux racines d'un arbre renversé ou les parois d'un fossé d'égouttement.

Valeur économique. Le martin-pêcheur se nourrit de menu poisson et l'on ne saurait dire sans hésitation si oui ou non cela constitue un grave clanger au point de vue économique. Les tout petits poissons que capture cet oiseau, dans nos grands cours d'eau, étangs ou lacs, sont certainement sans importance mais lorsqu'il fréquente les petits ruisseaux à truite réservés, il peut parfois commettre des dégâts sérieux. Leur influence sur les grandes rivières où l'on pêche le saumon est moins évidente. Ordinairement le poisson qu'ils prennent se compose de petites perches, ablettes barbeaux et autre frétin qui fréquente les eaux chaudes de surface ou peu profondes. Le nombre de poissons-gibiers qu'ils prennent ne saurait être bien grand. Quant aux eaux réservées à la culture de la truite, c'est une autre question. Les poissons dont ils se nourrissent en ces endroits sont relativement assez gros et, même s'ils ne sont pas très nombreux, le pêcheur à la ligne ne peut pas voir cet oiseau d'un bon œil.

Sur les rivières à saumon les martins-pêcheurs sont très mal vus et les gardes-pêche s'emploient ordinairement à en réduire le nombre par le fusil et au piège, chaque fois que l'occasion s'en présente; ils offrent même des primes pour leurs têtes et leurs nids. On peut se demander jusqu'à quel point ils ont raison. Dans beaucoup de ces cours d'eau les poissons ne trouvent guère d'autre nourriture que les plus petits individus de leur propre espèce. Tous les gros poissons, sauf les reproducteurs du printemps, se nourrissent des petits. Le fretin se nourrit évidemment de micro-organismes et de planctons, de saumoneanx, et ainsi de suite. Ce sont les saumoneaux que prend le martin-pêcheur. Or, si le nombre final de saumons adultes dépend des saumoneaux, si le saumoneau constitue la phase critique de la vie du saumon, au delà de laquelle ses chances de survivre sont considérablement augmentées, le martin-pêcheur est susceptible de commettre des dégâts appréciables; mais si, d'autre part, cette phase critique se produit plus tard, pendant la vie marine du poisson, par exemple, le résultat de l'enlèvement de même un nombre considérable de digitales sera sans importance. De toute façon il faudra plusieurs martins-pêcheurs pour faire autant de tort qu'un seul relativement petit poisson dans les eaux fréquentées par le saumon. Il semble donc que l'on peut facilement exagérer le mal fait par le martin-pêcheur. 


\section{Ordre-Pici. Pics.}

Ce groupe répandu par tout le monde est une division plutôt hétérogène comprenant de nombreuses subdivisions et les opinions sont très partagées quant a leurs exactes relations. Il n'existe au Canada qu'une seule famille de cet ordre-Picide, les Pics.

FAMILLE-PICIDE. PICS.

Description générale. Les pics sont des oiseaux faciles à reconnaître. Ils ont soit trois, soit quatre doigts armés d'ongles recourbés leur permettant de bien se cramponner après l'écorce rugueuse des arbres. Ils sont comme ceux du coucou, mais deux des doigts sont situés en avant. Chez l'un des groupes celui des pics à trois doigts, l'un des doigts postérieurs fait défaut. Le bec est droit, fort et se termine en forme de ciseau (figure 41, p. 27). La queue est ben développée, pas remarquablement longue mais forte et hérissée, usée par le frottement contre les rugosités de l'écorce.

Traits distinctifs. Les particularites des pieds, du bec, et de la queue constituent de bons signes distinctifs.

Indications sur le terrain. Cet oiseau grimpe sur les arbres; vole par une série de coups d'ailes rapides interrompus par de courtes pauses, ce qui donne à son parcours une direction onduleuse formant comme une succession de festons.

Nid. Dans des trous creusés dans les arbres ou dans les souches.

Le pic est très connu par sa faculté de se maintenir avec ses doigts sur des surfaces verticales ou surplombantes. Son fort bec en forme de coin se prête admirablement à la perforation du bois d'où il peut extraire des larves de xylophages ou autres insectes. La langue est modifiée en une longuc lance extensible munie d'une pointe aigue et armée de barbes minuscules lui permettant de retenir sa proie transpercée et l'extraire du bois. Les os hyoïdes sont tellement longs qu'à son état normal de repos, ils s'enroulent par dessus la base de la tête, le long de la couronne, et, chez certaines espèces, pénètrent les narines au-dessous de l'étui du bec et enfin appuient leurs extrémités près de la pointe du bec. Pour leur faciliter le travail la langue est enduite d'une matière visqueuse à laquelle se prennent les petits insectes. Quelques-uns de ces oiseaux, par exemple les "pics maculés», ont le bout de la langue élimé de façon à ressembler à une sorte de brosse qui doit servir évidemment à recueillir la sève.

Valeur économique. Il ne peut guère y avoir de doute quant à l'utilité des pics, si l'on excepte le pic maculé. Ils sont presque entièrement insectivores. Ils poursuivent les vers percebois en formant des trous même dans des arbres d'apparence saine mais réellement infestés, et sont ainsi plutôt utiles que nuisibles.

\section{Genre-Dryobates. Pics chevelus et Pics minules.}

393. Le Pic chevelu. (hairy woonpecker). Dryobates villosus. L, 9.40. Presque exactement semblable à l'espece suivante mais plus gros. Planche XVIII A.

Traits distinctifs. Le pic minule est la seule espèce avec laquelle on peut confondre cet oiseau. C'est surtout par la taille qu'on le reconnastra, mais il y a aussi le blanc des plumes extérieures de la queue qui est intact au lieu d'être rayé de noir.

Indications sur le terrain. La coloration blanche maillée de noir caractérise le genre, et c'est par la taille qu'on le distingue du pic minule.

Nid. Dans des trous approfondis dans des arbres sains ou des souches.

Habitat. Le pic chevelu avec ses diverses sous-espèces se trouvc dans toutes les régions boiś́es du Canada et couve partout sauf peut-être dans les parties méridionales de l'Ontario. 
SOUS-ESPECES. Cette espèce, dans l'est du Canada, comprend deux races géographiques: le pic chevelu de l'Est qui est l'oiseau type, et le pic chevelu du Nord, $D$. $v$. leucomelas. Ce dermier est ordinairement mais improprement représenté comme l'oiseau du Canada méridional. Il est légèrement plus gros et habite plutôt le nord; il s'avance seulement par occasion dans les régions habitées, en hiver, mais ne se montre peut-être jamais dans la région des grands lacs inférieurs.

Le pic chevelu est nominé d'après ses plumes blanches sur le dos, lesquelles retombent sur les bordures noires d'une manière éparse et lâche de façon à représenter vaguement une touffe de cheveux. C'est l'un des pics communs et il représente bien le type de la famille par ses habitudes. On ne le rencontre pas aussi familièrement autour des habitations et des vergers que le pic minule, car il préfère les bois aux vergers et aux arbres feuillus.

Valeur économique. Le 77 pour cent de la nourriture de cette espèce se compose d'insectes; ce sont en partie des escarbots mais il y a aussi des fourmis, des silphes et des mouches à scie; le 22 pour cent se compose de matière végétale, presque entièrement de fruits sauvages.

394. Le Pic minule. (Downy woonpecker). Dryobates pubescens. I, 6.83. Planche XVIII A.

Traits distinctifs. Le pic minule se distingue du précédent par sa taille plus petite, ct les rayures noires sur les plumes extérieures de la queue.

Indications sur le terrain. La taille est la meilleure indication.

Nid. Dans des trous creusés dans des arbres morts et dans des souches.

Habitat. Le pic minule avec ses diverses sous-espèces occupe toute la zone tempérée de l'Amérique et couve au Canada partout où il se trouve.

SOUS-ESPECES. Cette espèce comprend plusieurs races géographiques; celle de l'est du Canada--le pic minule du Nord, $D$. p. medianus-ne se distingue de l'oiseau type que par une légère différence de taille.

Le pic minule est notre pic le plus commun. Il vient tout près des maisons et se sent tout à fait à l'aise dans les vergers et au milieu des arbres ombreux des villes et des parcs. Comme il habite le Canada toute l'année, c'est un oiseau particulièrement utile au cultivateur.

Valeur économique. Ce pic étant le moins sauvage de toutes nos espèces et se rapprochant des fermes et des habitations où il rend les plus grands services, est un oiseau extrêmement précieux. En fouillant dans toutes les fentes et crevasses des arbres feuillus et des arbres fruitiers, et en les perforant pour extraire les insectes qui sont plus au fond, cet oiseau complète très bien le travail des mésanges et des sittelles. De fait, ces trois espèces voyagent souvent en compagnies pendant l'hiver et très peu d'insectes sont oubliés quand toutes les trois travaillent ensemble. L'alimentation du pic minule cst la même que celle du pic chevelu, mais, comme l'on pourrait s'y attendre en raison de sa plus petite taille et de sa présence plus commune en été, elle comprend un plus grand nombre des plus petits insectes. Ce sont les divers coléoptères qui composent la majeure partie de sa nourriture et il capture plus de larves de teignes, y compris la teigne des tentes et la pyrale des pommes.

\section{Genre-Picoides. Pics.}

400. Le Pic arctique. (ARCTIC THREE-TOED poodPECKER). Picoides arcticus. L, 9.50. Planche XVIII B.

Trails distinctifs. Ce pic n'a que trois au lieu de quatre doigts et le dos est d'un noir uniforme. 
Indications sur le terrain. Si l'on excepte le pic à tête rouge, c'est le seul pic à dos noir de l'est du Canada. On reconnait l'espèce à son dos noir et le genre à la tache jaune sur la couronne du mâle. (Planche XVIII B). prairies.

Habitat. Les forêts de conifères du Nord, et vers l'ouest jusqu'aux provinces des

C'est un oiseau des forêts de conifères du Nord, qui se présente rarement à la vue d'un observateur ordinaire sauf sur les confins des régions habitées où il apparaît régulièrement.

Valeur économique. Ce pic devient plus important pour la protection des forêts de conifères contre les fléaux d'insectes importés ou autres qui ont considérablement augmenté en ces dernières années.

401. Le Pic d'Amérique. (american three-Toen wooppecker). Picoides americanus. L, $8 \cdot 75$. Presque absolument semblable au pic arctique, sauf que le milieu du dos est rayé de blanc.

Traits distinctifs. La couronne jaune chez le mâle est un signe distinctif du pic d'Amérique. On peut distinguer cette espèce de la précédente par son dos rayé de blanc au lieu d'être tout noir. Si l'on excepte le pic à ventre rouge, c'est le seul de l'espèce dans l'est du Canada qui ait le dos rayé. du dos.

Indications sur le terrain. La couronne jaune du mâle et les raies blanches au milieu

SOUS-ESPECES. C'est le pic d'Amérique de l'Est-la race type de l'espèceque l'on rencontre dans l'est du Canada. Dans l'Ouest, il présente d'autres formes.

C'est un oiseau beaucoup plus rare que le précédent mais il lui est identique au point de vue des habitudes et de l'habitat; il y en a cependant des sous-espèces répandues dans les montagnes de l'Ouest.

\section{Genre-Sphyrapicus. Pics maculés.}

402. Le Pic maculé. (yellow-BlLled sapsucker). Sphyrapicus varius. L, 8.56. Planche XIX A.

Traits distinctifs. La calotte rouge bien caractérisée sur le sommet de la tête permet de distinguer cette espèce. Certaines femelles ont une couronne noire, mais la coloration générale est toujours reconnaissable même chez les jeunes, bien que chez ceux-ci, elle soit voilée et plutôt indiquée que affirmée.

Indications sur le terrain. La calotte rouge chez les deux sexes et la gorge rouge chez le mâle sont les signes les plus apparents. Dans d'autres plumages la large bande blanche qui s'aperçoit au bord de l'aile et la tache noire au-dessous de la gorge sont des signes distinctifs.

Nid. Dans des trous d'arbres morts.

Habitat. L'est de l'Amérique du Nord depuis les régions cultivées du Nord en allant au sud. Couve partout dans l'est du Canada sauf dans les parties les plus méridionales.

SOUS-ESPECES. Le type de l'Est du pic maculé, représente la race type-le pic maculé de l'Est. Une autre sous-espèce se rencontre dans l'extrême Ouest.

Cette espèce semble avoir abandonné ses anciennes habitudes de piquer le boís. Bien qu'elle soit très répandue durant la migration à travers le sud du Canada, elle couve rarement dans la région des grands lacs inférieurs tout en étant commune dans les autres endroits.

Valeur économique. C'est le seul oiseau de cette famille qui semble être nuisible. Il cause des dommages aux arbres fruitiers et autres arbres à écorce lisse en en extrayant le suc dont il se nourrit; pour cela il pratique autour des troncs et des branches des trous carrés régulièrement espacés, en lignes horizontales, pénétrant à la fois l'écorce externe et l'écorce interne jusqu'à l'aubier. Il entreprend ainsi quelquefois plusieurs arbres à la fois et les visite à tour de rôle à mesure que le suc se dégage. Lors même que 
c'est principalement le suc qu'il recherche il mange également les insectes qu'il rencontre, car bien que la sève des arbres soit son principal aliment, il lui faut aussi des insectes comme nourriture.

Ie dommage causé aux arbres qui sont ainsi perforés est loin d'être aussi considerable qu'on pourrait le croire, mais ceux-ci sont parfois endormmagés pour toujours et peuvent même mourrir. Tous sont affaiblis et deviennent un repaire de végétation fongoĩde et d'insectes. Si, cependant, ils ne sont pas sérieusement et à plusieurs reprises attaqués, la plupart des arbres survivent. Même la végétation forestière est sujette à des dégâts considérables de la part de cet oiseau; de bons arbres à bois de service sont attaqués et les nœuds et les taches qui en résultent dans la fabrication qui sont des marques des anciennes blessures maintenant refermées, faites par le pic maculé, réduisent la valeur marchande des bois de construction. Toute cette question des dégâts occasionnés par le pic maculé a été traitée à fond dans un Bulletin du Service biologique des Etats-Unis, le numéro 39, intitule: "Woodpeckers in relation to trees and wood products" par W.-L. McAtee. Sous le titre de mesures défensives contre les pies maculés, l'auteur conseille un usage modéré du fusil là où l'espèce cause des dommages appréciables, ou bien l'usage du poisson. Si l'on emploie le fusil, il faut prendre bien garde de ne tuer que les pics maculés et, quant au poison, il ne faut pas oublier que les oiseaux-mouches et autres petites espèces, particulièrement les fauvettes, peuvent être également atteints.

\section{Genre-Phlootomus. Pic à huppe écarlate.}

405. Le Pic à huppe écarlate. (PILEated woodpecker). Phlootomus pileatus L, 17. Planche XIX B.

Traits distinctifs. Grâce à sa taille, ce pic est facile à reconnaitre. Il ne peut être confondu avec aucun autre pic canadien.

Indications sur le terrain. Forte taille, huppe rouge remarquable et taches blanches sur les ailes déployées.

Habitat. Autrefois répandu sur tout l'est du Canada, il se cantonne maintenant dans les régions les plus sauvages et incultes du Nord.

SOUS-ESPECES. Le pic à huppe écarlate comprend deux sous-espèces, dont le pic à huppe écarlate du Nord, $P$. p. abieticola est la seule qui se rencontre au Canada.

Par suite de la destruction immodérée de ce magnifique oiseau qui était autrefois très répandu, on ne le trouve plus maintenant que dans les parties retirées des forêts du Nord. Le nom vulgaire de "Cock-of-thewood" qui lui est assigné est devenu par corruption dans le Nord, "Woodcock" et l'emploi de ce nom pour cette espèce est une source de confusion et de malentendus. Le pic a huppe écarlate n'est pas proprement dit un oiseau gibier; il n'est pas "tenu en arrêt par le chien», ne peut pas être chassé par les méthodes habituelles des chasseurs et il est trop petit pour être servi sur la table, mais il est très précieux pour la conservation des forêts. Il faudrait donc une loi sévère pour le protéger au point de vue tant économique que sentimental.

Valeur économique. Le pic à huppe écarlate se cantonne maintenant dans les régions les plus sauvages. Il est principalement utile pour la protection des arbres forestiers et, par conséquent, c'est un oiseau précieux pour le marchand de bois. Son régime d'alimentation est à peu près le même que celui des autres pics mais il est plus robuste, ce qui lui permet. de creuser plus profondément et d'extraire des larves et des insectes qui sont à l'épreuve d'un oiseau moins puissant. 
Genre-Melanerpes. Pics.

406. Le Pic à tête rouge. (RED-HEADED wOODPECKER). Melanerpes erythrocephalus. $\mathrm{L}, \mathbf{9} \cdot \mathbf{7 5}$. Planche XX A.

Traits distinctifs. La tête d'un rouge vif et les contrastes de masses blanches et noires dans le plumage du corps sont faciles à reconnaître.

Indications sur le terrain. La coloration remarquable de cette espèce la rend facilement reconnaissable.

Nid. Généralement dans des trous d'arbres morts et parfois dans des poteaux télégraphiques ou autres.

Habitat. Cet oiseau habite plutôt le Sud, ne pénétrant régulièrement et fréquemment au Canada que par la frontière sud le long des grands lacs inférieurs.

Le pic à tête rouge est une des espèces communes autour des vergers ei des terres à bois. Il est par conséquent bien connu partout où il se trouve. Il possède un peu moins les habitudes des pics que les espèces déja étudiées, ou bien il s'est développé chez lui des aptitudes de gobemouches en sus de celles qu'il possédait déjà.

Valeur économique. Ce pic détruit moins de larves et de vers que les autres espèces, et des coléoptères plus utiles, les carabes. Toutefois, il est encore plutôt bienfaisant que nuisible, car alors même qu'il mange des fruits, sa nourriture se compose en grande partie de hannetons ou de scarabées de et charançons. On l'a accusé de manger des fruits et, sans doute, l'accusation n'est pas sans fondement, mais une étude approfondie a démontré que ce sont des cas spéciaux se bornant à des régions restreintes et peut-être à de certains individus.

\section{Genre-Centurus. Pics de la Caroline.}

409. Le Pic de la Caroline. (RED-BeLled wOODPECKer). Centurus carolinus. $L, 9 \cdot 50$. Dos entièrement noir et parties supérieures nettement et régulièrement rayées de noir et de blanc. Tout le dessus du cou jusqu'à l'épaule et, chez le mâle, le sommet de la tête, d'un rouge vif. Tout le reste et les parties inférieures d'un blanc cendré avec légère teinte olivâtre. Abdomen légèrement teinté de rouge.

Traits distinctifs. Les rayures uniformes sur tout le dos et le rouge de la couronne et de la nuque sont faciles à distinguer.

Habitat. Depuis le golfe du Mexique jusqu'au nord des Etats-Unis; apparaissant à titre de vagabond jusqu'au nord de la frontière dans le sud de l'Ontario.

Ce pic n'apparaît que rarement et par endroits, seulement, au Canada. Sa valeur économique est satisfaisante bien qu'il soit friand de fruits sauvages et que parfois il se nourrisse des variétés cultivées.

$$
\text { Genre-Colaptes. }
$$

412. Le Pic doré. Le "PIVert. Le "PIvant". (Flicker). Colaptes auratus. L, 12. Planche XX B.

Traits distinctifs. Un pic de forte taille avec les surfaces inférieures des ailes et la queue d'un jaune brillant.

Indications sur le terrain. Habitudes générales des pics; taille; le dessous des ailes jaunes et le croupion blanc se voient bien quand l'oiseau vole.

Nid. Dans des trous creusés dans les trones d'arbres morts, ordinairement en plein air et rarement sinon jamais dans les épaisses forêts. Les singulières relations entre des espèces distinctes sont bien mises en évidence par cet oiseau. Les trous, qu'il a approfondis pour lui servir de nid, une fois abandonnés, sont utilisés par beaucoup d'autres espèces incapables de creuser les leurs propres. Par exemple les faucons éperviers, les hirondelles bicolores, les moucherolles huppés et autres espèces utiles sont ainsi directement tributaires du pic, particulièrement du pic doré pour l'établissement de leurs nids. Il existe dans la nature bien d'autres cas semblables de dépendance mutuelle; il y en a qui sont 
bien en évidence et très connus, d'autres que nous supposons, et il peut s'en trouver d'autres encore que nous ne soupçonnons pas et ne pouvons même pas actuellement soupçonner. Voilà précisément une bonne raison pour agir avec circonspection avant de rien déranger à l'ordre établi de la nature.

Habitat. Les diverses sous-espèces du pic doré sont répandues sur toute la partie est de l'Amérique du Nord en allant au nord jusqu'aux limites des régions boisées. Il couve partout où il se trouve au Canada.

$S O U S-E S P E C E$. Le pic doré comprend plusieurs sous-espèces; la variété propre à l'est du Canada est désignée sous le nom de pie doré du Nord, C. a. luteus.

C'est celui des pies qui est le plus connu du public en général, ainsi qu'on peut le voir par le grand nombre de désignations populaires qui lui ont été assignées; on l'appelle en anglais: "Golden-winged Woodpecker, Highhole, Yellow-hammer»; et, en français: "Pivart" ou "Poule des bois». Le cri aigu de "Fliqueu, fliqueu, fliqueu que fait entendre le mâle et le strident " $P i$-iu-iou " piopre aux deux sexes sont des sons très connus et faciles à reconnaître. Bien que ce soit un vrai pic, le pic doré se nourrit essentiellement sur terre et est particulièrement friand de fourmis, lesquelles composent bien souvent une bonne partie de son alimentation. Il prend plaisir à se cramponner à un tronc d'arbre, creux, sonore, et à le frapper à coups de becs rapides et retentissants que l'on entend de très loin. Il semble faire ce bruit pour appeler sa compagne ou alors c'est quelquefois pour donner livre cours à sa bonne humeur. Les facultés de reproduction de cet oiseau sont phénoménales. En enlevant les œuf́s à mesure qu'ils sont pondus, on a pu en recueillir trente ou plus en une seule saison. Il semble pouvoir en déposer tant que la série n'est pas complète.

Valeur économique. Près de la moitié de sa nourriture se compose de fourmis. Les autres insectes dont il se nourrit sont des espèces soit utiles soit nuisibles, mais ce sont surtout ces dernières qui l'emportent. Il mange aussi des fruits, du grain et des fruits d'arbres forestiers; mais, dans l'ensemble, on peut le considérer comme utile plutôt que nuisible. Pcut-être bien que la plus grave accusation qu'on puisse porter contre cette espèce est de disséminer les graines du chêne et du sumac vénéneux et contribıer ainsi à répandre ces plantes nuisibles.

\section{Ordre-Macrochires. Engoulevents, Martinets et Oiseaux- mouches.}

Dans cet ordre sont groupés un certain nombre d'oiseaux qui, après des recherches plus complètes pourront être classés différemment. La classification actuelle de l'Union des Ornithologistes américains, est censée être provisoire et n'est adoptée que jusqu'à ce que l'on puisse s'entendre pour établir un système permanent. Les divers sous-ordres de la division sont plus faciles à reconnaître par leurs différences que par leurs concordances et nous appuierons plutôt, dans les descriptions qui vont suivre, sur les traits qui les différencient.

\section{SOUS-ORDRE-CAPRIMULGI. ENGOULEVENTS.}

Ce sous-ordre est très répandu et divisé en plusieurs familles. Une description de l'unique famille représentée dans l'Amérique du Nord servira à faire reconnaître l'espèce indigène. 
FAMILLE-CAPRIMULGID A. ENGOULEVENTS.

Description générale. Les cngoulevents ont la tête aplatie, le bec très petit avec une large ouverture qui se prolonge jusqu'au-dessus des yeux (figure 38, p. 26). Les pieds sont petits et très faibles et l'ongle médian est pectiné ou pourvu de dentelures en forme de peigne comme chez les hérons (figure 19, p. 22). Le plumage est d'une texture très molle et coloré de brun cendré de chamois et de gris.

Traits distinctifs. Les traits précités devraient suffire à distinguer cette famille puisqu'ils ne se rapportent à aucun autre oiseau canadien.

Le nom de "Goatsuckers" ou "Tète-chèvre" leur a été attribué parce que l'on croyait autrefois, évidemment à tort, qu'ils suçaient le lait des chèvres dans les prairies au-dessus desquelles on les voyait tournoyer, et leurs immenses bouches de même que leurs gorges roses semblaient appuyer cette croyance populaire. De fait, les oiseaux que l'on voyait ainsi survoler les pâturages étaient en train de chasser au vol les insectes attirés par ces animaux. Les oiseaux de cette famille sont nocturnes ou crépusculaires. Ils se nourrissent exclusivement d'insectes attrapés au vol et n'atterrissent que rarement sauf pour nicher ou se reposer. Leurs pieds sont trop petits et trop faibles pour se fixer fermement à une branche et quand ils perchent ils s'assoient normalement sur de grosses branches, mais en longueur et non pas en largeur comme font la plupart des oiseaux.

\section{Genre-Antrostomus. Engoulevents criards.}

416. L'Engoulevent de la Caroline. (CHUCK-WILL's wibow). Antrostomus carolinensis. L, 12. Un gros engoulevent criard. Voir l'espèce suivante.

Traits distinctifs. Taille; il est de beaucoup plus forte taille que l'engoulevent criard; il a de longs poils autour de la bouche recouverts de petits cheveux à leur base au lieu d'être dénudés sur toute leur longueur.

Habitat. Etats-Unis du Sud. Ne se présente qu'accidentellement à l'intérieur de nos frontieres.

Nous faisons figurer cet oiseau dans notre ouvrage parce qu'il en a été trouvé un spécimen à la pointe Pelée sur le lac Erié, et un autre à Pictou (Nouvelle-Ecosse). Il est plutôt rare et ressemble à l'engoulevent criard quant à son apparence, à ses habitudes et à son chant.

417. L'Engoulevent criard. LE BOIS POURR. (WHIP-POOR-WILL). Antrostomus vociferus. L, 9.75. Marbré de brun cendré et de gris avec légères teintes de roux et d'ocre jaune. Il n'y a pas de tache dominante dans la coloration, mais beaucoup de petits détails. Les parties inférieures ne laissent voir qu'une faible indication de rayures et la coloration de l'ensemble est semblable à celle d'une grosse teigne brune.

Traits distinctifs. Ne saurait être confondu qu'avec l'engoulevent d'Amérique et l'engoulevent de la Caroline. On le distingue du premier de ces oiseaux par les trois signes suivants: la gorge est foncée au lieu de blanche; il y a une étroite bande blanche au bas de la gorge; la moitié extérieure des pennes de la queue, sauf les deux du centre, est blanche chez le mâle et se termine en blane nuancé de chamois chez la femelle. L'aile déployée ne laisse voir aucune tache blanche.

Nid. Dépose ses œufs à terre ou sur les feuilles mortes.

Habilat. Très répandu sur toute la majeure partie des régions habitées de l'est du Canada; plus rare dans l'extrême Est, et plus commun dans les sections moins fréquentées que dans la où il se fait beaucoup de culture.

SOUS-ESPECES. On reconnait deux espèces d'engoulevent criard dans l'Amérique du Nord dont l'oiseau type-l'engoulevent criard de l'Est-est la seule qui se présente au Canada.

Il n'y a pas dans nos bois canadiens de cri à la fois plus poétique et lugubre que celui si fréquemment entendu de l'engoulevent criard. Il faut ordinairement assez d'imagination pour exprimer par des paroles le chant des 
oiseaux mais celui-ci semble dire bien dictinctement: "Whip-poor-will, whippoor-will" ou encore "Bois pourri." Pour faire entendre ce cri il s'installe sur une perche se détachant d'un tronc d'arbre mort, sur une branche défeuillée, sur un toit de bâtiment ou même un mât de tente. Il fréquente régulièrement ses diverses stations chaque soir et semble y revenir à tour de rôle. Entre ses périodes d'appel, l'oiseau voltige et tournoie au sommet des arbres en formant de grands cercles entrelacés et vient s'abattre parfois vers la terre en un long mouvement de balancier. Dans le jour il cherche un coin de terre tranquille sous la brousse où il passe sa journée au repos. S'il est dérangé par un intrus, il s'enlève d'un vol lâche et maladroit qui ne donne aucune idée de sa merveilleuse maîtrise de l'air; en d'autres temps, il voltige à peu de distance au-dessus des broussailles et retombe de nouveau à terre.

L'engoulevent criard est souvent regardé comme identique à l'engoulevent d'Amérique, ce qui est une erreur très naturelle quand on les voit côte à côte, car ils sont assez semblables pour être confondus.

Valeur économique. Cet oiseau se nourrit principalement de coléoptères nocturnes particulièrement de hannetons et de scarabées.

\section{Genrc-Chordeiles. Engoulevents d'A mérique.}

420. L'Engoulevent d'Amérique. LE MANGeor DE MARINGoUing. (NIGHTHAWK) Chordeiles virginianus. L, 10. Planche XXI A.

Traits distinctifs. L'engoulevent d'Amérique et l'engoulevent criard sont souvent pris l'un pour l'autre. Cette espèce peut être distinguée d'avec l'autre cependant par les traits suivants: la gorge est blanche au lieu de foncée; il n'y a pas de bande blanche audessous de la gorge, et les longs poils de la bouche font défaut. La queue est légèrement fourchue au lieu d'être arrondie et laisse voir peu ou point de blane sauf une étroite bande semi-terminale. Les parties inférieures sont distinctement rayées et les ailes ont une tache blanche à la base des primaires au lieu d'être tout à fait noires.

Indications sur le terrain. L'engoulcvent d'Amérique voltige en plein jour et à la tombée de la nuit, tandis que l'engoulevent criard ne vole jamais en plein air avant le soir. On aperçoit très distinctement, au vol, une tache blanche à l'aile, qui, vue d'une certaine distance, ressemble à un trou de balle bien tranché. Sa façon de se précipiter comme une flèche vers la terre avec accompagnement de bruit sourd est aussi caractéristique de l'espèce.

Nid. Niche sur le sol en un endroit découvert, ordinairement sur les sommets dénudés de rochers aplatis, en plein air. Il dépose ses oufs par terre avec peu ou point de préparation; il les dépose aussi souvent sur les toits plats de constructions recouverts de graviers.

Habitat. I'Amérique du Nord et du Sud en allant au nord jusqu'aux limites des régions boisées; couve au Canada partout où il se trouve.

SOUS-ESPECES. On reconnait au Canada plusieurs sous-espèces de cet oiseau, mais la seule qui apparaisse dans l'est du Canada est l'oiseau type-l'engoulevent du Canada de l'Est.

Bien qu'on l'appelle "Nighthawk » cette espèce n'a aucun rapport avec les rapaces quant à la tenue, à sa structure ou à son apparence extérieure. Les grands yeux dirigés légèrement de face le font quelquefois prendre pour un hibou par un observateur d'occasion. Niche assez souvent sur les toits plats des constructions, recouverts de graviers. On peut voir ces gracieux aéronautes évoluer à la tombée de la nuit, au-dessus des villes et exécuter avec leurs ailes très longues et résistantes des mouvements bizarres, longs, puissants, mais légèrement irréguliers. A un moment donné, on en voit un monter de plus en plus haut dans l'espace en rapides spirales, puis alors se retourner vers la terre et se précipiter verticalement comme une pierre qui tombe. Au moment où il tombe en entend un bourdonnement 
sourd en trémolo. Juste à l'instant où l'observateur s'attend à le voir atteindre la terre, l'oiseau se ressaisit et remonte en l'air pour recommencer les mêmes évolutions. Son chant n'est pas mélodieux et, cependant, du haut des régions supérieures son cri aigre et perçant nous arrive adouci et harmonisé par la distance.

Valeur éconómique. Il est peu d'oiseaux dont on peut dire autant de bien et si peu de mal. Il se nourrit exclusivement d'insectes et les prend presque tous au vol dans les hautes régions où ils s'accouplent et à une époque où leur destruction rend le plus de services. On est étonné de voir comme l'oiseau est petit une fois dépouillé de son épais manteau de plumes molles, mais il exige une forte quantité de nourriture. La liste des espèces qu'il capture comprend un grand nombre de fourmis, de scarabées, de punaises de courges, de punaises des bois, de jasses, et autres espèces nuisibles. L'habitude assez commune, par endroits, de se servir de cet oiseau comme point de mire pour s'exercer au tir est inexcusable, et ceux qui s'en rendent coupables devraient être sévèrement punis par la loi. L'on devrait se rendre compte que chaque infraction aux lois qui protègent les insectivores est plus grave qu'une contravention en principe contre l'Etat; c'est une attaque directe contre le bien-être de la communauté.

\section{SOUS-ORDRE-CYPSELI. MARTINETS.}

Un sous-ordre très répandu composé d'une famille dont nous n'avons dans l'est du Canada qu'une seule espèce.

FAMILLE-MICROPODIDE. MARTINETS.

Les martinets de l'Amérique du Nord sont divisés en deux sousfamilles dont une seule, Chœturinœ, est représentée dans l'est du Canada.

\section{Sous-famllle-Chæturinæ. Chœtures, Martinets à queue éplneuse.}

Les martinets forment un groupe d'oiseaux ressemblant superficielleinent aux hirondelles, mais ils sont d'une structure très différente, la similitude étant occasionnée par des besoins communs et non par des rapports mutuels. Nous donnons, sous le titre spécifique qui suit, une description qui s'applique à la famille entière.

\section{Genre-Chotura. Martinet des cheminées.}

423. Le Martinet des cheminées. L'HIRONDELle DEs CHEMINÉEs, LE RAMoneur. (CHIMNeY swifT). Chœtura pelagica. L, 5.43. Planche XXI B.

Traits distinctifs. La couleur brun de suie uniforme devenant plus elaire à la gorge et plus foncée vers l'anus suffit presque pour faire reconnaitre l'oiseau. On le reconnaît aussi tout de suite a ce que les tuyaux des pennes de la queue (figure 39, p. 26) dépassent les barbes sous forme d'épines hérissées et aiguës.

Indications sur le terrain. Leurs ailes bizarres, longues, etroites, en forme de massue, que l'on remarquera très bien dans l'illustration; le battement rapide et presque continu des ailes; la coloration très foncée; et l'habitude de pénétrer dans les cheminées sont des signes distinctifs et caractéristiques.

Nid. Autrefois dans le tronc des arbres ou dans une fissure de rochers et, maintenant, sur la majeure partie de son habitat, dans des cheminécs abandonnées. Le nid est une solide structure de buchettes cimentées ensemble au moyen d'une glu naturelle sécrétée par les glandes salivaires. Les nids comestibles qui sont si recherchés par les épicuriens chinois se composent d'un ciment produit par un proche parent de ce martinet.

Habitat. L'est de l'Amérique septentrionale en allant au nord jusqu'aux limites des terres cultivées. Couve au Canada partout ou il se trouve. 
C'est une espèce intéressante, ressemblant à l'hirondelle quant à son apparence extérieure et à sa manière de poursuivre les insectes, mais elle est d'une structure bien distincte de celle de l'hirondelle. C'est un curieux exemple d'un développement parallèle de caractères absolument différents déterminé par des besoins de même nature. Les habitudes de cet oiseau ont complètement changé depuis l'apparition de l'homme blanc, et, après avoir abandonné les arbres creux, il ne compte plus guère maintenant que sur les cheminées pour y établir son nid. On ignore ou ce martinet va passer l'hiver. Il ne faut pas cependant exagérer ce mystère, car il y a un grand nombre de martinets dans l'hémisphère occidental qui ressemblent beaucoup à celui-ci et on l'a probablement perdu de vue dans ses refuges d'hiver ou confondu avec des congénères étroitement alliés. Le martinet est la plupart du temps au vol et s'arrête rarement pour se reposer sauf dans une cheminéc ou un arbre creux. A l'automne, avant la migration, de grands nombres se rassemblent et, vers le soir, viennent s'abriter dans une vaste cheminéc pour y passer la nuit. On peut les voir, sur la brune, sillonner l'air en tous sens auprès de la cheminée de leur choix, et, lorsque le soleil se couche, tournoyer jusqu'à ce que, ayant redressé leurs ailes verticalement au-dessus du dos ils se laissent tomber en battant des ailes dans ce refuge de nuit, les uns à la suite des autres comme dans un semblant de gouffre en miniature. Ils se cramponnent en masses, par centaines, aux parois verticales de la cheminée comme autant d'amas de suie. Parfois il en est un, mal affermi, qui sc détache et tombe de quelques pieds en faisant lâcher prise à d'autres au-dessous de lui. On cntend un moment battre des ailes, puis un résonnement de fines brisures qui tombent jusqu'à ce que les oiseaux se cramponnent de nouveau et s'installent pour la nuit.

\section{SOUS-ORDRE-TROCHILI. OISEAUX-MOUCHES.}

Ces tout petits oiseaux ressemblent à des insectes, avec leur brillante coloration pareille à celle des fleurs, leur vol semblable à celui des insectes et leur remarquable diversité de formes, constituent un ordre typiquement américain. D'une certaine façon, ils occupent beaucoup la même position dans le nouveau monde que les Souïmangas dans l'ancien, mais la ressemblance entre les deux n'est que superficielle et il n'y a aucun lien de parenté. Beaucoup d'espèces sont éminemment spécialisées et laissent voir les formes les plus étranges dans le monde ailé, comprenant aigrettes, fraises, volants, et manchons, queues fantastiques, pennes démesurées et becs énormes en formes de sabres ou effilés en forme d'alènes; mais le trait le plus remarquable chez ces oiseaux est la colorátion d'un brillant métallique qui recouvre les diverses parties du corps et reluit au soleil. Ils se nourrissent principalement du suc des fleurs. La langue est très longue et extensible comme chez les pics, avec ses rebords tournés vers le milieu de façon à former un double tube usé à son extrémité comme la pointe d'un pinceau, constituant un excellent organe pour sucer les liquides. Ils absorbent cependant un grand nombre de petits insectes avec le suc des fleurs, lesquels d'après des expériences d'alimentation pratiquées sur des sujets en captivité, semblent être nécessaire à la santé de l'oiseau. Ce sont ordinairement des insectes minuscules qu'ils trouvent sur les fleurs dont ils extraient le suc.

En tant que groupe ces oiseaux fréquentent les régions tropicales et sous-tropicales et sont beaucoup plus nombreux en allant vers le sud; il y a cependant une espèce dans l'Est qui visite le climat du Nord. 
FAMILLE-TROCHILIDE. OISEAUX-MOUCHES.

Comme il n'y a qu'une seule famille d'oiseaux-mouches représentée dans l'est du Canada, on pourra consulter les rubriques précédentes et. suivantes pour en trouver la description générale.

\section{Genre-Archilochus. Colibri àgorge rubis.}

428. Le Colibri à gorge rubis. L'OISEAU-MOUChe. (RUBY-THROATED hUMMingBIRD), Archilochus colubris. L, 3.74. Planche XXII A.

Traits distinctifs. La taille, le ber ténu (figure 40, p. 26) et la coloration métallique sont des traits distinctifs.

Nid. Une petite merveille de construction recouverte avec des parcelles de lichens et des toiles d'araignées, fixée au sommet d'une branche.

Habitat. L'est de l'Amérique du Nord jusqu'aux limites des terres à culture.

Les oiseaux-mouches volent en avant, en arrière, à droite, à gauche ou restent complètement suspendus dans les airs sans le moindre effort-un autre exemple de développement parallele-un oiseau qui vole comme un insecte et qui possede cependant tout à fait la structure de l'oiseau. La vibration des ailes est tellement rapide qu'on ne peut la mesurer qu'au moyen du diapason employé pour l'étude des insectes. Ce système de vol est fondamentalement différent de celui des autres oiseaux et, par conséquent, les ailes diffèrent du type habituel. Elles sont longues, étroites, non flexibles, et le bréchet du sternum est très considérablement approfondi afin de servir d'appui aux grands muscles qui les mettent en mouvement. En proportion de son envergure l'oiseau-mouche a un bréchet trois fois plus grand que celui du pigeon qui est un oiseau de vol normal, ou quarante fois plus grand que celui de l'albatros.

Valeur économique. Si l'on se rappelle que certains petits insectes nuisibles sont les plus destructeurs, on pourra se rendre compte que l'importance économique de l'oiseau-mouche peut être beaucoup plus considérable qu'on ne le soupçonne. A part le suc des fleurs, son régime alimentaire comprend des moucherons, des cousins, de minuscules abeilles, des guêpes et autres insectes qui se posent sur les fleurs et se nourrissent de pollen. II ne semble pas qu'on puisse accuser cette espèce d'aucun dégât et, par contre, elle peut rendre des services tout à fait hors de proportion avec sa taille.

\section{Ordre-Passeres. Oiseaux percheurs.}

L'ordre des passereaux ou oiseaux percheurs constitue la division la plus considérable et la plus importante des espèces modernes. Les types inférieurs et plus généralisés d'oiseaux étaient autrefois en majorité; mais aujourd'hui ce sont les passereaux aux innombrables espèces qui dominent; ils constituent à peu près, sinon tout à fait, la moitié de tous les oiseaux vivants et sont placés en tête de la classification par des savants méthodiques. Ils possèdent en plus grand nombre qu'aucun autre ordre, de traits qui leur sont propres à chacun, mais qui sont spéciaux relativement aux autres ordres, comme par exemple un appareil vocal muni de muscles spéciaux très compliqués, et bien d'autres caractères particuliers. En règle générale on peut assigner un tel oiseau à cet ordre par voie d'élimination, en constatant qu'il ne relève d'aucun des ordres précédents. Les pieds (figure $42, \mathrm{p}$. 27) ne sont pas palmés, le doigt de derrière est aussi long que celui du 
milieu, et tout le pied est bien constitué pour que l'oiseau puisse percher. Le bec est dur ou corné sans cire ou membrane de base, ct les narines ne communiquent pas entre elles comme dans certains autres ordres. Deux sous-ordres sont représentés au Canada: Clamatores, les oiseaux criards, et Oscines, les oiseaux chanteurs.

\section{SOUS-ORDRE-CLAMATORES. OISEAUX CRIARDS.}

Ce sous-ordre est constitué sur une base de structure anatomique. Le nom anglais "Songless"- on les appelle en français "Criards"-n'implique pas qu'ils sont silencieux, mais que lcur chant est moins harmonieux que celui des chanteurs et leur larynx moins spécialement développé. Une seule famille de ce sous-ordre apparait au Canada, les Tyrannidés ou Moucherolles.

\section{FAMILLE-TYRANNIDE. MOUCHEROLLES.}

Description générale. Il est très facile, parmi les oiseaux canadiens, de reconnaitre les moucherolles à leur bec (figure 42, p. 27) qui est relativement long, un peu aplati et élargi à la base, plus large que haut et légèrement recourbé à son extrémité.

Indications sur le terrain. Facile à reconnaitre \& l'état vivant par ses habitudes et ses attitudes caractéristiques avec lesquelles un observateur attentif arrive à se familiariser. Quand ils perchent ils se posent ordinairement dans une attitude verticale et restent immobiles sauf à s'ébranler parfois par un coup de queue. S'ils voient passer un insecte ils s'élancent et l'attrappent au vol d'un mouvement et d'un coup de bec rapide.

C'est l'une des familles les plus difficiles à identifier au point de vue de l'espèce. Certains moucherolles sont nettement caractérisés, mais, parmi les plus communs, il y a plusieurs espèces qui se ressemblent au point d'intriguer un ornithologiste expérimenté quand ils sont silencieux ou hors de leur habitat normal. Pour les identifier à l'état vivant il faut bien tenir compte de leur chant. Le chant de l'oiseau et le type d'habitat où on le trouve sont de bonnes indications pour le différencier quand il s'agit des espèces les plus embarrassantes.

Valeur économique. Ils se nourrissent presque entièrement d'insectes attrapés au vol, leur large bec se prêtant très bien à ce genre de capture. Comme ils prennent la majeure partie de leur nourriture dans l'air non loin du sol, ils attrapent des variétés d'insectes que ne prennent pas les autres oiseaux. Les espèces trouvés dans leurs estomacs se composent de coléoptères, mouches, guêpes, tipules, fourmis, sauterelles, teignes des tentes et mites. De fait, ils s'attaquent à presque toutes les espèces d'insectes nuisibles ot doivent être classés parmi les oiseaux très utiles.

\section{Genre-Muscivora. Moucherolle à queue en ciseaux.}

443. Le Moucherolle à queue en ciseaux. (scisson-TARen FLycATCHER). Muscivora forficata. L, 13 (à peu près). (Queue, 9). Ce moucherolle est un visiteur occasionnel à l'intérieur de nos frontières, venant de l'Amérique sous-tropicale. Il est impossible de ne pas reconnaitre cet oiseau: il est à peu près de la taille du moucherolle de lo Caroline, de couleur cendré clair, avec les ailes plus foncées, la calotte écarlate ou orangée, même couleur sous le sailes, et avec une queue longue de 8 à 9 pouces, fendue à une profondeur de 6 pouces ou davantage, laquelle lorsque l'oiseau est au repos, $s^{2}$ ouvre et se referme comme une paire de ciseaux. Chose assez curieuse, l'un des meilleurs spécimens reconnus authentiques que nous ayons à notre disposition provient de York Factory, sur la baie d'Hudson. En présence d'un pareil spécimen de cette provenance, il est difficile d'affirmer positivement quelle espèce peut ou ne peut pas se trouver au Canada. 


\section{Genre-Tyrannus. Moucherolles de la Caroline.}

444. Le Moucherolle de la Caroline. LE TRITRI. (KINGBIRD, BEE MARTIN). Tyrannus tyrannus. L, 8.51. Planche XXII B.

Traits distinctifs. Coloration noire et blanche; la tache orangée de la couronne apparaít dans des moments d'excitation, et la queue noire visiblement terminée de blanc comme si on l'eut trempée dans de la peinture blanche.

Indications sur le terrain. Facile à reconnastre à l'état vivant au moyen des marques précitées. La couronne orangée ccpendant se voit rarement. A l'état vivant, la tête et la queue semblent être d'un noir mat en opposition vive avec le blanc des parties inférieures.

Nid. Sur les arbres, de 5 à 40 pieds au-dessus du sol; le nid est une jolie structure composce de tiges, d'berbes, et de débris végétaux, tapissée de duvet végétal, de radicelles et de fines herbes. Le fait que cet oiseau vient souvent nicher dans les vergers et non loin des terres à culture est beaucoup en sa faveur.

Habitat. L'Amérique du Nord vers le nord jusqu'aux limites des bois. Couve au Canada partout où il se trouve.

Le moucherolle de la Caroline est une espèce familière que l'on voit autour de nos maisons et de nos vergers, et la présence d'un couple venant nicher dans le voisinage est l'une des meilleures garanties contre les dégâts des éperviers ou des corbeaux. Ces oiseaux ne se montrent pas aux abords de son nid sans être vigoureusement poursuivis. Ce moucherolle se met à la poursuite des intrus avec une énergie qui étonne chez un oiseau si petit et si faible. Il ne peut pas leur faire grand mal, mais il sait les repousser et ses hauts cris annoncent de loin qu'il faut se méfier. Grâce à sa petite taille et à ses mouvements agiles dans l'air il peut attaquer un gros oiseau de tous côtés et se mettre en garde contre tout oiseau plus fort et moins agile.

Valeur économique. Le nom de "Kingbird" s'explique par lui-même, mais celui de "Bee Martin » qu'on lui assigne également donne naturellement licu à des soupçons sur son régime alimentaire. On l'accuse de capturer les mouches à miel et l'on a des preuves irréfutables qu'il n'est pas sans reproche à cet égard. Le relevé que nous donnons ci-après montre que l'accusation est en partie fondée, mais il fait voir aussi que les abeilles capturées sont en majeure partie des bourdons dont on peut très bien se passer. L'ancienne croyance que le moucherolle étale sa brillante huppe pour amorcer l'abeille qui est à sa portée, en lui faisant croire que c'est une fleur, peut être mise de côté, comme étant de la pure légende, malgré que beaucoup de personnes y aient ajouté foi. Sur 264 estomacs de moucherolles de la Caroline, examinés en 1911, par le ministère de l'Agriculture des EtatsUnis, 22 contenaient un total de 61 abeilles, dont 51 étaient des bourdons, 8 des ouvrières et 2 indéterminées. Le reste des aliments se composaient d'autres insectes, comprenant de nombreuses espèces nuisibles, puis quelques fruits sauvages et des baies.

\section{Genre-Myiarchus. Moucherolles à huppe.}

452. Le Moucherolle à huppe. (CRESTEd Flycatcher). Myiarchus crinitus. L, 9.01. Parties supérieures d'un brun olivatre tournant au roux sur les barbes internes de la queue. Gorge et haut de la poitrine d'un gris cendré. Parties inférieures d'un jaune soufre.

Traits distinctifs. La coloration précitée est distinctive. Aucun autre moucherolle dans l'est du Canada n'est ainsi coloré.

Indications sur le terrain. La couleur jaune vif des parties inférieures et la longue queue rousse sont les marques les plus frappantes. Son cri aigu, strident et prolongé de a ou $i-i-i-p$ et celui plus faible de aouip-ouip-ouip sont très caractéristiques, bien qu'on les confon de quelquefois avec ceux du moucherolle aux côtés olive. 
Nid. Dans des trous abandonnés par les pics. Cet oiseau fait voir une prédilection pour les vieilles peaux de serpents dans la confection de son nid. C'est a peine si l'on trouve un nid appartenant à cette espece qui n'en contienne une ou plusieurs.

Habitat. L'est de l'Amérique du Nord au Canada, tout le long de la frontière méridionale; couve partout ou il se trouve.

C'est un moucherolle du sommet des arbres dans les bois touffus. On entend son cri constamment pendant l'été, mais rarement à une distance quelconque d'une épaisse forêt.

Valeur économique. Son régime alimentaire se compose surtout d'escarbots, sauterelles, fourmis, grillons, mouches et teignes. Il capture plus de guêpes parasites et d'escarbots que la plupart des oiseaux mais pas suffisamment pour contrebalancer ce qu'il détruit d'insectes malfaisants.

\section{Genre-Sayornis. Moucherolles bruns.}

456. Le Moucherolle brun. (Рнсвв). Sayornis phobe. L, 6.99. Planche XXIII A.

Hatitat. Les petits moucherolles verdâtres sont peut-être les plus difficiles des oiscaux américains à discerner. Heureusement chacun a son habitat typique et son chant caractéristique qui permettent de les différencier. Le moucherolle brun est le plus gros de ces petits oiseaux de nature intrigante, et le plus facile à identifier. On est très exposé à le confondre avec le moucherolle verdâtre, mais quand on le tient dans la main, on reconnaft facilement les pattes et les pieds plus gros et plus robustes du moucherolle.

Indications sur le terrain. La tête du moucherolle brun semble un peu plus foncée et présente un contraste plus frappant avec le eorps que chez les autres moucherolles. L'extension latérale de la queue est caractéristique et, chez les adultes, le manque de barres sur les ailes. Le cri de "Phébé" ccpendant, qu'il fait entendre rapidement en accentuant longuement la première syllable, constitue l'indication la plus facile à reconnaitre. Le cri du moucherolle verdâtre est plutôt allongé tandis que celui du petit moucherolle est court et détonnant.

Nid. Une simple construction faite de boue mélangée de mousse et d'herbes, et installée sous les ponts, les parties saillantes des édifices ou des rochers.

Habitat. L'est de l'Amérique en allant vers le nord jusqu'aux limit:s des régions boisées. Couve au Canada partout où il se trouve.

Ce moucherolle niche de préférence sur les solives de vieux ponts au-dessus de quelque petit ruisseau où il trouve dans l'atmosphère surmontant le cours d'eau une ample provision d'insectes. Dans bien des parties de ce pays il n'y a guère un pont où l'on ne rencontre pas un couple de moucherolles bruns en été. Toutefois, ces nids de boue ne s'aperçoivent pas seulement sous les ponts, car l'on en trouve collés aux moindres saillies sous les gouttières des édifices ou même sous les porches des habitations. C'est un oiseau doux et familier qui s'approche facilement de l'homme quand on l'attire. Malheureusement les grands nids de cette espèce sont souvent des repaires de milliers d'insectes parasites. On s'en débarrasse ordinairement en renversant les nids avec des bâtons et en y jetant de l'eau bouillante. Il vaut mieux encore se servir de poudre insecticide, ce qui fera disparaître les insectes malfaisants tout en conservant auprès des habitations cet oiseau si attrayant et si facile à apprivoiser.

\section{Genre-Nuttallornis. Moucherolle aux côtés olive.}

459. Le Moucherolle aux côtés olive. (OLIVE-8Ided Flycatcher). Nuttallornis borealis. L, $7 \cdot 39$. Ressemble beaucoup à un gros moucherolle brun, mais on y remarque moins de vert olive et il présente de grandes taches foncées de chaque côté de la poitrine.

Traits distinctifs. Dans la main, les grandes taches foncées de chaque côté de la poitrine et les couvertures sous-caudales avec pointes de couleur claire serviront à différencier cette espèce d'avec le moucherolle brun auquel il ressemble. Sur les côtés du dos, on 
remarque ordinairement cachées sous les ailes fermées, mais parfois étalées au-dessus, des surfaces de plumage fin et soyeux de couleur blanche ou crème. C'est ce qui permettra de distinguer cette espèce d'avec toutes les autres.

Indications sur le terrain. A l'état vivant, ce moucherolle ressemble au moucherolle de la Caroline à poitrine foncée plutôt qu'au moucherolle brun ou à tout autre moucherolle. Les deux taches foncées de la poitrine séparées par une ligne blanche permettent assez facilement de faire la distinction. Lorsque les plumes blanches et soyeuses s'aperçoivent au-dessus des ailes sur les côtés du dos, ainsi qu'il arrive parfois, il est impossible de ne pas bien identifier cette espèce. Son cri d'appel ressemble un peu, quant au timbre et ì l'exécution, à ceux du moucherolle à huppe, mais un peu d'attention et d'expérience suffiront pour faire apprécier la différence entre les deux.

Habitat. L'Amérique du Nord. Couve au Canada depuis la limite des forêts jusqu'aux frontières des régions régulièrement cultivées.

C'est un oiseau ty pique des crêtes brûlées du nord. Il perche de préférence sur le sommet d'un grand arbre mort et solitaire d'ou l'on peut entendre de très loin sa voix pénétrante. Dans ses migrations il se tient à certaines localités; et bien qu'il en passe de grands nombres d'une manière inaperçue à travers les régions populeuses du sud, il est ordinairement considéré comme un oiseau mlgrateur peu commun.

Valeur économique. Il est trop rare et passe trop rapidement dans les régions habitées pour avoir une grande valeur économique, mais il est certainement un oiseau bienfaisant.

\section{Genre-Myiochanes. Moucherolles verdâtres.}

461. Le Moucherolle verdâtre. (WOOD PEWEE). Myiochanes virens. L, 6.53. Très semblable comme coloration au moucherolle brun, mais plus petit.

Traits distinctifs. Le moucherolle verdâtre peut être différencié d'avec le brun et les autres moucherolles de taille semblable, par son petit tarse et ses longues ailes, lesquelles sont manifestement plus longues que la queue.

Indications sur le terrain. Le moucherolle verdâtre ne joue pas de la queue comme le moucherolle brun. Les côtés de la poitrine sont légèrement plus foncés ce qui donne une ligne médiane blanche plus distincte et plus étroite. On le reconnaîtra cependant plus facilement à son cri d'appel qui ressemble beaucoup à celui du moucherolle brun mais est plus prolongé et peut se traduire par ces notes: pi-e-oui, qu'il prononce sans accent appréciable mais avec une inflection croissante à la fin. Le cri de la femelle varie en ce qu'elle élimine la dernière note, ce qui fait pi-i-i.

Nid. Une construction bien façonnée mais légère, faite de plantes fibreuses et de radicelles recouvertes de lichens et fixée sur une branche à une hauteur de 20 à 40 pieds.

Habitat. Il est répandu sur à peu près toute la partie est de l'Amérique du Nord en allant vers l'ouest jusqu'aux prairies. Il couve au Canada partout où il se trouve.

Son cri plaintif et prolongé de pi-e-oui est une note caractéristique des régions boisées au printemps, et après que les autres oiseaux sont retombés dans le silence au cœur de l'été on entend encore parfois cette note mélancolique.

Valeur économique. Le régime alimentaire de cet oiseau est tout à fait semblable à celui des autres moucherolles mais modifié, bien entendu, en raison de son séjour dans les bois. Comme il n'est pas casanier et se plaît à fréquenter librement les bosquets et les vergers, c'est un oiseau précieux pour l'agriculteur.

\section{Genre-Empidonax. Moucherolles d̀ ventre jaune.}

463. Le Moucherolle à ventre jaune. (Yellow-BELlied FlyCatcher). Empidonax faviventris. L, 5.63. Semblable au moucherolle verdâtre et au brun comme couleur, mais de plus petite taille, et avec les taches brunes et olive remplacées par un vert olive distinct et le blane par un jaune soufre terne. 
Traits distinctifs. Grâce à la couleur distinctement jaune de tout l'oiseau, c'est le plus facile à reconnaitre parmi les petits moucherolles.

Indications sur le terrain. La couleur jaune particulièrement sur la gorge, est la meilleure indication. Son cri de Ti-pi-a est distinctif-trois syllabes, celle du milieu étant la plus accentuée. Son Bi-wic ressemble un peu au Phébé du mouch rolle brun, mais la première syllabe n'est pas accentúé.

$\mathrm{Nid}$. Niche sur le sol; son nid est construit avec de la mousse garnie, à l'intérieur, d'herbes fines.

Habitat: Depuis les grandes plaines en allant a l'est et au nord jusqu'au bout des régions cultivées. Couve au Canada sur la frontière nord de son habitat, et non dans la région inférieure des grands lacs.

C'est encore un moucherolle des bois mais il fréquente moins les grands arbres; il préfère les fourrés qui bordent les forêts.

Valeur économique. Régime alimentaire très semblable à celui des autres petits moucherolles. Son habitude de se tenir sur les bords des forêts qui longent les terres à culture en fait un oiseau précieux pour le cultivateur.

465. Le Moucherolle d'Acadie. (ACADIAN FLYCATCHER), Empidonax virescens. $\mathrm{L}, 5 \cdot 63$. Un petit moucherolle à peu près de la même taille que le moucherolle à ventre jaune, le moucherolle des aulnes, et le petit moucherolle; plus petit que le moucherolle verdâtre et beaucoup plus petit que le moucherolle brun. Comme couleur, se rapproche de ce dernier mais est distinctement vert olive dans les parties supérieures et plus jaunâtre dans les parties inférieures. Les bandes des ailes sont plutôt prononcées.

Traits distinctifs. Cette espèce se rapproche tellement, comme taille et coloration, du moucherolle des aulnes et du petit moucherolle qu'il est extrêmement difficile de les différencier. Il n'est pas aussi jaune que le moucherolle à ventre jaune, n'ayant de jaune pur sur aucune de ses parties, mais c'est le plus jaunâtre et le plus verdâtre des autres petits moucherolles. Il est très rare au Canada et on ne peut actuellement accepter son signalement que de la part d'ornithologistes compétents.

Habitat. L'est de l'Amérique du Nord en allant au nord jusqu'aux frontières du Canada le long de l'extrémité ouest du lac Erié.

466. Le Moucherolle des aulnes. (TRAILL's FLyCATCHER). Empidonax trailli. $\mathrm{L}, 6 \cdot 09$. Un petit moucherolle légèrement plus gros que le moucherolle à ventre jaune ou le petit moucherolle et presque de la même coloration que ce dernier et que le moucherolle d'Acadie.

Traits distinctifs. Le dos est plus brun que chez le moucherolle d'Acadie et dépourvu de la teinte verdâtre de ce dernier. Quelquefois on ne peut guère le différencier du petit moucherolle que par sa taille.

Indications sur le terrain. Les détails de couleur ou de taille sont peu utiles sur le terrain pour l'identification de cette espèce ou de la suivante. On peut se fier cependant à l'habitat et au cri d'appel. Il fréquente les bosquets d'aulnes, de saules, ou de framboisiers et les épaisses lisières de marais. Le petit moucherolle est un oiseau des bois; et bien que l'on puisse parfois rencontrer l'un ou l'autre dans d'autres habitats, le voisinage est ordinairement très utile à considérer au point de vue de l'identification. Il y a aussi le cri d'appel que l'on reconnaft facilement; il peut se rendre par: $i-z i-i-o p p$, avec l'accent sur la deuxième syllabe, tandis que celui du petit moucherolle est un ichébec détonant qu'il répète plusieurs fois avec vivacité.

Nid. Dans une fourche d'arbuste près du sol; il le construit avec de grosses herbes, du duvet végétal et des plantes fibreuses garnies d'herbes fines.

Habitat. Le moucherolle des aulnes en tant qu'espèce, fréquente presque toute l'Amérique du Nord. La sous-espèce typique de l'est, le moucherolle des aulnes proprement dit, se rencontre depuis la côte orientale de l'est du Canada en allant vers l'Ouest à travers les montagnes.

SOUS-ESPECES. Il y a deux sous-espèces de moucherolles des aulnes au Canada. Le type oriental, moucherolle des aulnes, $E$. t. alnorum, est le seul qui se rencontre dans le territoire que nous étudions. Le moucherolle des aulnes de l'Ouest, $E$. $t$. trailli, est un type plus méridional et plus occidental. 
Valeur économique. Semblable à celle des autres moucherolles. Il se nourrit presque entièrement d'insectes, il détruit surtout les espèces qui fréquentent les terres incultes.

467. Le Petit Moucherolle. (Least FlyCatcher, Chebec). Empidonax minimus. L, 5:41. C'est le plus petit de nos moucherolles. Sa coloration est celle du type moucherolle brun et, à cet égard, on ne peut que très difficilement le différencier d'avec les moucherolles d'Acadie et des aulnes.

Traits distinctifs. Plus brun sur le dos et dépourvu de la nuance verdâtre qui distingue le moucherolle $d$ 'Acadie, mais presque identique au moucherolle des aulnes comme couleur; on ne peut parfois le différencier d'avec celui-ci que par sa taille. Son habitude de fréquenter les régions boisées plutôt que les broussailles des contrées désertes pourra aider à l'identifier et son cri d'appel est différent de celui de toute autre espèce: c'est un rapide et détonant tché-bec qu'il répète souvent plusieurs fois et qui rappelle assez bien une toux intermittente.

Nid. Ordinairement sur une fourche d'arbre à une bauteur de 15 à 30 pieds; construit avec duvet végétal, plantes fibreuses, radicelles et longs poils.

Habitat. Dans tout le Canada, vers l'Ouest jusqu'aux montagnes et vers le Nord jusqu'au dela des régions cultivées. Couve au Canada partout où il se trouve.

Cet oiseau fréquente les vergers et les arbres au voisinage des habitations, les haies très développées et autres endroits familiers où la proximité des terres cultivées rend ses services remarquablement avantageux.

Valeur économique. Son régime alimentaire ne diffère pas beaucoup de celui de ses congénères, mais sa familiarité et sa confiance le mettent en contact journalier avec l'homme auprès de qui ses qualités utiles ont un maximum d'influence.

\section{SOUS-ORDRE-OSCINES. OISEAUX CHANTEURS.}

Le sous-ordre Oscines est une vaste division des oiseaux placés en tête de la liste de ceux qui présentent le plus haut degré de développement dans la classe Aves. Il comprend un grand nombre de familles et d'espèces dont les relations exactes d'un très grand nombre n'ont jamais été définitivement établies. Suivant la coutume américaine actuellement, ce sous-ordre commence avec les alouettes et se termine avec les grives qui sont considérées comme constituant la famille la plus développée. Les caractères qui distinguent les Oscines d'avec les Clamatores sont techniques et anatomiques et n'entrent pas dans le cadre d'un ouvrage de vulgarisation. Comme l'indique leur nom, ces oiseaux possèdent des organes et muscles vocaux très particulièrement développés dont on a tenu compte dans leur classement. Les divers membres de ce sous-ordre se reconnaissent plus facilement d'après les descriptions des familles que d'après ce titre général.

\section{FAMILLE-ALAUDID A. ALOUETTES.}

Les alouettes composent une grande famille répandue au loin. Les marques distinctives qui caractérisent cette famille sont d'un ordre plutôt technique et comme nous n'avons qu'une espèce à étudier au Canada, elles seront décrites sous le titre de l'espèce qui va suivre.

\section{Genre-Otocoris. Alouettes ordinaires.}

474. L'Alouette ordinaire. L'ORTOLAN. (HORNED LARK). Otocoris alpesiris. L, 7.75. Planche XXIII B.

Traits distinctifs. L'ongle du doigt postérieur très allongé (figure $44 \mathrm{~b}, \mathrm{p} .27$ ) est une des marques distinctives des alouettes. On retrouve cependant cette particularité chez la farlouse (figure 60, p. 29) et le plectrophane; ce dernier cependant est un moineau ayant le bec conique qui caractérise les moineaux et qui est très différent de celui des 
alouettes. L'alouette ordinaire se distingue par sa gorge jaune ou crème avec gorgette noire au-dessous, et deux petites touffes allongées en forme de cornes au-dessus des oreilles (figure $44 \mathrm{a}$, p. 27).

Indications sur le terrain. Les taches de couleur sur les parties supérieures et les cornes constituent des indications faciles à reconnaltre.

Nid. Sur le sol. Il est fait avec des herbes sèches; on le trouve souvent dans la neige au début du printemps. Nord.

Habitat. On trouve cet oiseau sous ses diverses formes dans toute l'Amérique du

SOUS-ESPECES. L'alouette ordinaire comprend un grand nombre de races ou sous-espèces géographiques. Elle est très répandue dans l'Amérique du Nord et comme elle s'adapte à de nombreuses et diverses conditions, elle s'est développée dans différentes parties du pays sous divers aspects. Ainsi dans les contrées désertes elle est petite, grêle et pâle, tandis que, au nord-est où c'est l'oiseau type, élle est forte et vivement colorée. La forme plus commune dans l'est du Canada est l'alouette ordinaire des prairies: $O$. $a$. praticola. Autrefois lorsque la contrée était boiscé c'était probablement l'alouette des prairies et elle ne devait pas se rencontrer dans l'est du Canada. C'est le déboisage, la création de prairies artificielles qui lui a permis de venir s'y établir, et aujourd'hui elle y couve régulièrement. Dans le Nord, à l'est de la baie d'Hudson, on rencontre le type original que nous avons décrit: $O$. a. alpestris lequel n'a pas maintenant de nom vulgaire reconnu que nous appelons l'Alouette ordinaire de l'Est. C'est un grand oiseau avec une forte teinte de jaune sur la face et la ligne des sourcils. Comme la différence moyenne dans la longueur totale est d'environ un demi-pouce et comme toutes les tailles intermédiaires peuvent se présenter, on comprendra qu'il peut être assez difficile de différencier les divers types de cette espèce. On a essayé dans l'illustration, de représenter la différence dans la coloration. L'alouette ordinaire de l'Est n'apparaft dans les districts colonisés de l'est du Canada qu'à titre de migrateur hivernal et rarement, sauf dans les provinces maritimes ou c'est le type hivernal ordinaire. Une autre race, l'alouette ordinaire de Hoyt, $O$. a. hoyti, est propre au Nord; elle reproduit à l'ouest et au nord-ouest de la baie d'Hudson. Les couvertures des oreilles brunâtres au lieu de grisâtres permettent de l'identifier, mais la différence n'est pas grande. Ce dernier type se rencontre parfois dans la région iuferieure des grands laes à titre de migrateur hivernal.

La distribution des diverses races et leurs migrations au Canada n'ont pas été complètement déterminées et ce travail comporte de telles difficultés qu'il est inutile d'essayer à déterminer les sous-espèces sans le concours d'un ornithologiste et d'une bonne collection de spécimens authentiques comme moyen de comparaison.

L'alouette ordinaire est un oiseau de plein air qui fréquente les champs incultes, les grèves et les routes. En hiver elle se nourrit principalement des graines de mauvaises herbes qui percent la neige et il s'en trouve beaucoup sur les routes fréquentées, tant pour manger les herbes qui croissent sur les bords que pour recueillir le grain partiellement digéré dans le crottin laissé par les chevaux. Il en passe quelquefois en hiver de grandes compagnies. C'est dans ces occasions que l'on devrait rechercher les plus rares types migrateurs.

\section{FAAILLE-CORVIDE. GEAIS ET CORNEILLES.}

La famille des Corneilles est très grande et très variée; elle comprend beaucoup d'oiseaux magnifiques et aux couleurs très vives; de fait le fameux oiseau de paradis lui est étroitement allié. Le bec (figure 45, p. 27) en est la partie la plus facile à reconnaitre. Il est passablement long et fort avec un culmen bien arqué. A la base, sont d'épaisses touffes de soies longues et raides qui recouvrent les narines. Les pics et les mésanges ont quelquechose de semblable mais celles-ci sont toutes petites et ceux-là possèdent d'autres caractères distinctifs. Ce ne sont pas, à proprement parler, des oiseaux chanteurs; leur voix est enrouée et rauque, mais leurs organes vocaux sont extrêmement compliqués et il en est quelques-uns à qui l'on peut faire articuler certains mots. Ils comptent parmi nos espèces les plus intelligentes et certains auteurs les ont classés en tête de la liste de tous les oiseaux.

$$
28588-12
$$




\section{Sous-famille-Garrulinæ. Ples et Geais.}

Oiseaux de taille moyenne; un bon nombre sont vivement colorés et sont garnis de houppes et de queues flottantes. On peut très facilement les reconnaître d'après la description de chaque espèce.

$$
\text { Genre-Pica. Pies. }
$$

475. La Pie d'Amérique. (Magpie). Pica pica. L, 15·20. (Queue, 10). Le corps légèrement plus gros que celui d'un geai huppé, mais beaucoup plus long en raison de sa queue qui atteint environ 10 pouces. Coloration frappante à cause des contrastes de blanc et de noir. La tête, le cou jusqu'au haut de la poitrine sont noirs; le dos, la queue et une bonne partie des ailes sont noirs et à reflets verdatres sur les ailes et la queue. Une barre blanche très apparente sur le dos au-dessus des ailes. Barbes internes des primaires, bas de la poitrine et parties inférieures toutes blanches.

Traits distinctifs. Facile à reconnaître à sa longue queue et aux contrastes de blanc et de noir.

Habitat. Comme espèce, la pie fréquente les régions tempérées de l'ancien et du nouveau monde. Le type américain est plutôt un oiseau de l'Ouest; il fréquente régulièrement les provinces centrales des prairies et apparait parfois plus a l'Est.

SOUS-ESPECES. La pie d'Amérique est une sous-espèce connue sous le nom $P$. p. hudsonia d'une espèce commune tant à l'ancien qu'au nouveau monde, et ne diffère de la pie d'Europe que par des détails sans importance. C'est une question à discuter pour les experts.

Ce n'est passans quelque hésitation que nous comprenonsla pie parmi les oiseaux indigènes du Canada. L'espèce est commune dans le Nord-Ouest et plusieurs de ces oiseaux nous ont été signalés dans les provinces maritimes et la région des grands lacs inférieurs, qui nous semblent aussi authentiques que possible sans les spécimens. En 1879, un certain nombre furent importés d'Angleterre et mis en liberté à Lévis en face de Québec. Quelques-uns des signalements se rapportent peut-être à la progéniture de ces oiseaux introduits dans le pays et non à des indigènes. Nous serions très désireux de posséder des spécimens de ces oiseaux de l'Est. L'espèce est trop rare pour l'étudier au point de vue économique.

\section{Genre-Cyanocitta. Geais bleus.}

477. Le Geai bleu huppé. (BLUE JAY). Cyanocitta cristata. L, 11.74. Planche XXIV A.

Traits distinctifs. La huppe et la coloration sont très caractéristiques.

Indications sur le terrain. La prédominance du bleu dans la coloration d'ensemble, les bordures bleues de la queue, et la huppe. Le vol du geai huppé est facile à reconnaśtre. Ses nombreux cris sont distinctifs et varient entre le cri rauque de $d j e-d j e$, son grincement comme celui d'une porte de grange et divers gloussements doux et familiers.

Nid. Composé de branchettes et de racines, de 10 à 20 pieds du sol.

Habitat. L'est de l'Amérique depuis les confins des régions habitées jusqu'au golfe du Mexique. Couve partout où il se trouve au Canada.

Le geai huppé est alerte, curieux et malfaisant. Il est facilement attiré par un bruit étranger dans les bois ou par un être en mouvement et il s'en approche sournoisement; s'il reconnait un ennemi, il se sauve plein de terreur en poussant des cris perçants. C'est ainsi que des geais ont bien gêné des expéditions de chasse et causé toute sorte d'ennuis aux chásseurs. A l'automne, il est prévoyant et amasse des glands qu'il a la précaution de mettre de côté dans des crevasses d'arbres et autres cachettes. On ne sait pas très bien si jamais il revient chercher ses provisions. 
Valeur économique. Au point de vue économique le geai occupe plutôt une place douteuse. Quant à sa nourriture il est carnivore et mange tour à tour insectes, fruits, glands, grain, œufs ou oisillons. Il n'y a pas de doute que, pendant la saison des glands, c'est de ce fruit qu'il se nourrit régulièrement. C'est un oiseau dont il ne faut pas encourager la présence autour des vergers quand les autres oiseaux plus utiles font leurs nids.

\section{Genre-Perisoreus. Geais du Canada.}

484. Le Geai du Canada. la pie. (Canada Jay). Petisoreus canadensis. L, 13. Planche XXIV B.

Traits distinctifs. Taille: coloration gris neutre avec calotte noire et front blanc. Les jeunes que l'on aperçoit rarement avec leur plumage primitif ont la tête et le cou uniformément noirs. Tous ont un plumage lache, duveteux et inégal (rsgged).

Indications sur le terrain. Taille, coloration d'un gris uniforme, face et front blancs.

Nid. Construit de petites branchettes, de plantes fibreuses, soigneusement garni de fourrure et de plumes de façon à former une construction compacte et profonde servant à protéger les coufs qui sont quelquefois éclos à une température de 30 degrés au-dessous de zéro Fahrenheit.

Habilat. Les bois de conifères du Nord à travers tout le continent.

SOUS-ESPECES. Il y a plusieurs sous-espèces de cet oiseau, lesquelles présentent de très légères différences. Dans l'est du Canada, le geai de l'Est du Canada qui est l'oiseau type, est le plus répandu avec le geai du Labrador, $P$. c. nigricapillus, qui habite Terreneuve et le Nouveau-Québec.

Le geai du Canada, connu sous le nom vulgaire de pie, possède d'une façon exagérée à peu près toutes les caractéristiques du geai huppé. Tous les villégiateurs dans les forêts du nord connaissent le "Whisky Jack". Dès que l'on allume un feu de campement, on est sûr de le voir apparaitre en quête de déchets de table. Il est très friand des restes de gibier et, à peine s'il a entendu le coup de carabine qu'il est déjà sur les lieux pour attraper quelque lambeau de chair. . Son chant présente toute sorte de surprises pour ceux qui ne le connaissent pas bien. De fait, dans son propre habitat, tout chant d'oiseau que l'on ne saurait attribuer à une autre espècc peut 3tre provisoirement assigné au geai du Canada.

Valeur économique. C'est essentiellement un oiseau des forêts sans imites et il ne se rencontre que rarement dans les régions peuplées. Sa valeur économique est très semblable à celle du geai huppé mais il se tient rop à l'écart pour être nuisible au cultivateur.

\section{Sous-famille-Corvinæ. Corneilles.}

Les corneilles forment une famille bien caractérisée, d'apparence remarjuablement semblable. Elles sont répandues à peu près partout et pour eur description il n'y a qu'à se reporter à la planche XXV A. Le freux !'Europe appartient à cette division.

\section{Genre-Corvus. Corbeaux et Corneilles.}

486. Le Corbeau. (RAven). Corvus corax. L, 22. Semblable à la corneille lanche XXV A) mais plus gros.

Traits distinctifs. Le corbeau dans son apparence extérieure est une très grosse rneille. Le caractère le plus saillant est la forme allongée et pointue des plumes de la rge; elles sont posées distinctement l'une sur l'autre sans être fondues ensemble comme icz la corneille. 
Indications sur le terrain. Outre la taille, qui constitue toujours un signe incertain dans une vaste étendue de plein air, la marque la plus certaine est le cri de l'oiseau. Son aroassement est rauque et strident et non pas clair et distinct comme le cri de la corneille. Il y a cependant de jeunes corneilles qui croassent presque absolument comme le corbeau et, quand on est en présence des deux espèces, il faut quelquefois avoir recours à d'autres indications. Quand on aperçoit un corbeau on peut facilement s'imaginer ses caractères saillants quant aे la forme et au vol, mais nous devons avouer qu'ils sont rarement perceptibles \& l'observateur ordinaire avant que l'on ait identifie l'oiseau.

Nid. Ordinairement sur des saillies de rochers, quelquefois sur de grands arbres; le nid se compose de buchettes.

Habilat. Cette espèce se rencontre tant dans l'ancien que dans le nouveau continent jusque dans les régions polaires où sa livrée noire contrastant avec la blancheur de co milieu semble combattre la tendance trop facile à étendre la théorie de la coloration protectrice à tous les anirnaux de la création. Cette espèce fréquentait autrefois tout le Canada mais il se cantonne maintenant dans les parties les moins babitées au nord et au sud, le long des chaines de montagnes sur les deux littoraux et pénètre assez loin jusque dans les Etats-Unis.

SOUS-ESPECES. Cette espèce se divise en plusieurs races géographiques. Lo corbeau européen est l'oiseau type. Nous avons au Canada le corbeau du Nord, $C$. $c$. principalis. Les divers types présentent en somme peu de différences.

La tradition veut que le corbeau soit un oiseau de mauvais augure. A vec sa couleur sombre, son cri lugubre, ses habitudes sauvages et solitaires, il remplit le même rôle dans le Nord que le vautour dans le Sud. II se tient à l'écart des endroits fréquentés par l'homme. Au fur et à mesure que la civilisation a progressé dans les vastes contrées primitives, le corbeau, contrairement à son congénère la corneille, s'est éloigné, et il est devenu aujourd'hui ce qu'il était au début, un oiseau des lieux déserts. Si l'on en juge seulement par les nécessités physiques et le régime d'alimentation des deux espèces, on est naturellement porté à croire que le corbeau peut vivre dans les régions habitées aussi bien que la corneille. Il est omnivore et peut s'adapter à presque toute sorte de nourriture. Il est robuste et peut vivre sous des climats et dans des conditions qui seraient fatals à son plus faible congénère, et eependant, pour certaines raisons inexpliquées, les corneilles augmentent et les corbeaux diminuent cn nombre à mesure quc le pays se colonise.

Valeur économique. Le corbeau prend une nourriture tant végétale qu'animale, mais préfère de beaueoup eette dernière. Il convoite les restes de gibier laissés par le chasseur ou le gibier lui-même, quand il peut se l'approprier. Il rôde autour des nids des autres oiseaux et s'empare des œufs et des oisillons. Au bord de la mer, il fouille les rivages a la marée basse pour y trouver des erabes et autres crustacés ou tout ce que la mer peut lui apporter de mangeable. Comme il évite plutôt les régions cultivées, il n'a que peu d'influence directe sur les récoltes.

488. La Corneille d'Amérique. (AMErican Crow). Corvus brachyrhynchos. L, 19.30. Planche XXV A.

Traits distinctifs. Peut être confondue avec le corbeau mais l'oiseau est plus petit et n'a pas sur la gorge les plumes lancéolées, longues et pointues de celui-ci.

Indications sur le terrain. Son aspect général que l'on apprend vite à connaître.

Nid. Sur les arbres; construits avec de petites branches.

Habitat. Toute la zone tempéréc de l'Amérique du Nord.

SOUS-ESPECES. Deux sous-espèces de la corneille de l'Amérique se présentent au Canada; mais nous n'avons dans l'Est que l'oiseau type, la corneille de l'Est.

Alors que le corbeau disparaît en présence des progrès de la civilisation, la corneille augmente en nombre. Elle est omnivore, se nourrissant volontiers de n'importe quoi depuis la chair corrompue jusqu'au maîs germant 
Il n'y a pas de doute que la corneille s'est énormément répandue dans le pays depuis la disparition des forêts et il est probable que son avènement dans l'est du Canada date de l'arrivée de l'homme blanc. C'est un oiseau partiellement migrateur. La plupart des corneilles s'en vont dans le Sud en hiver, mais un bon nombre, attirées par les abattoirs et les tas d'ordures ménagères au voisinage des villes, demeurent en permanence au pays.

Valeur économique. La valeur économique de la corneille ne peut pas se résumer en quelques mots. Elle est certainement très bienfaisante mais elle fait aussi beaucoup de dégâts. "Seulement, le mal qu'elle fait est eoneentré et facile à a pprécier, tandis que le bien est réparti çà et là, et plutôt difficile à établir. Les résultats détaillés d'environ un millier d'examens d'estomacs et des témoignages provenant de toutes les parties de l'Amérique du Nord ont été publiés dans le Bulletin $n^{\circ} 6$ du département de l'Agriculture des Etats-Unis, par W.-A. Barrows et E. -A. Schwarz. Le plus grand reproche qu'on lui fait dans l'est est de manger les jeunes pousses de céréales, surtout du maĩs. On a constaté que le maiss constitue le 29 pour cent de sa nourriture annuelle mais, chose assez curieuse, depuis le mois de mai jusqu'au mois d'août, il n'en composait que du 8.4 au 17.7 pour cent et augmentait en décembre jusqu'au $\mathbf{4 8 . 2}$ pour cent, alors que ces grains ne peuvent être que des glanures des champs moissonnés. La corneille mange aussi beaucoup d'insectes et si l'on compare les especes nuisibles avee les utiles qu'elle détruit, la balance penche évidemment en faveur de l'oiseau. Elle se nourrit aussi considérablement de souris et autres rongeurs it de reptiles; c'est même la nourriture qu'elle préfère à toute autre. Elle recherehe avidement à l'automne les fruits d'arbres forestiers (glands, faînes, ete.). Si alors on en juge seulement par son régime slimentaire, la corneille est un oiseau précieux. Il faut cependant envisager la question autrement, car on ne peut pas ignorer qu'elle fait tout de même des dégâts; il importe peu au cultivateur qu'elle détruise toutes les chenilles dans son champ si elle emporte également tout le grain. D'une façon générale, il ne paraît guère $y$ avoir de bonne raison pour prendre à son égard de nouvelles mesures de précautions; il n'y a d'ailleurs aueun danger qu'elle disparaisse et elle a probablement besoin d'être surveillée.

\section{FAMILLE-STURNIDE. VRAIS EtTOURNEAUX.}

Les vrais étourneaux forment une famille de l'ancien monde, distincte des étourneaux américains en ce qu'ils ont, entre autres signes distinetifs, dix primaires au lieu de neuf; les premières peuvent n'être que rudimentaires comme dans l'espèce ci-dessous, oiseau importé, non indigène, mais l'unique représentant de la famille, en Amérique.

L'Étoumeau vulgaire. (starlivg). Sturnus vulgaris. L, 8.50. Taille à peu près celle de l'étourneau à ailes rouges, mais avec la queue plus courte. En automne tout le corps est d'un noir métallique, couvert de taches foncées de la forme d'un $V$ et de couleur chamois-crème. Au printemps, les taches les plus claires ont disparu, mais le bec est jaune. Dans le jeune áge, brun grisátre avec une gorge blanchâtre.

Traits distinctifs. Au printemps c'est un mainate à bec jaune; a l'automne un mainate à tachetures fines et claires. Au jeune âge c'est un mainate brunâtre avec une gorge blanchâtre. En toutes saisons c'est un mainate à courte queue.

Indications sur le terrain. Voir les traits distinctifs. A un vol énergique, bien caractérisé, nullement celui d'un mainate. 
L'Étourneau vuigaire (staRLING). Sturnus vulgaris. L, 8.50. Taille à peu près celle du loriot de Baltimore, mais de charpente plus grande. Tout entier d'un noir métallique, couvert de taches foncées de la forme d'un $V$ et de couleur chamois-crème.

Traits distinctifs. Petit mainate à tachetures fines et claires.

Indications sur le terrain. Voir les traits distinctifs.

Nid. Dans de grands tas d'berbes en désordre, avec des ramilles et des déchets dans les fentes de murs et d'arbres creux. S'installera volontiers dans des boites-nids.

Habitat. Originairement dans l'ouest et le centre de l'Europe. Importé à NewYork en 1890 il s'est répandu sur une étendue de pays de plus en plus grande. Après quelques apparitions dans le sud de l'Ontario on l'a pris, enfin, à Kingston. Ce que sera son dernier établissement dans le Dominion on ne peut pas le prédire.

Valeur économique. Ies recherches entreprises par le Service biologique des Etats-Unis pour se rendre compte des résultats qu'un facteur si troublant pourra avoir sur l'état de choses en Amérique, ont abouti à ce qui suit. En tant que destructeur des insectes du sol, l'étourneau a peu d'égaux parmi les oiseaux du nord-est de l'Amérique du Nord. L'objection la plus sérieuse qu'on puisse avoir à sa présence vient de son goût prononcé pour les cerises. Il s'attaque bien à d'autres fruits mais pas à un degré sérieux. Il se niche de façon disgracieuse; il en veut à d'autres oiseaux; son penchant à former d'immenses compagnies qui perchent ensemble dans les quartiers les plus recherchés contrebalance le bien que peuvent faire ses gouts insectivores.

Comme si les sociétés d'acclimatation, et d'autres sociétés du même genre, n'avaient pas fait dejà assez de mal en introduisant le moineau domestique en Amérique, elles ont ajouté un autre agent de conséquences possibles encore inconnues sous la forme de l'étourneau qui va disputer le terrain aux espèces indigènes, développer des aptitudes inattendues dans ses nouveaux alentours et, en l'absence d'une continence naturelle, se multiplier à l'infini. Déjà l'étourneau existe par milliers dans le Massachusetts et il envahit les Etats voisins en nombres toujours plus grands et en cercles toujours plus étendus. Tout espoir de l'extirper aujourd'hui est probablement parfaitement vain.

Par ses mœurs, l'étourneau promet de compléter le moineau domestique; il s'empare des vergers, des parcs, des faubourgs comme fait le moineau dans les villes. A cause de sa taille plus grande, ses mœurs de couvaison sont peut-êtrc encore plus criticables que celles du moineau domestique, et le fait qu'il occupe des trous en fait un concurrent sérieux des oiseaux indigènes tels que les rouges-gorges bleus qui ont déjà bien du mal à trouver des places pour couver, eu égard aux méthodes en usage dans nos vergers. L'espoir va s'évanouissant que nos hivers canadiens, si rigoureux soient-ils, pourront l'empêcher de s'implanter fermement dans le Dominion, puisqu'il a su adapter ses mours migratrices aux habitudes américaines, et par ce moyen achever la conquête du territoire canadien. Pendant les années qui viennent l'étourneau devra être surveillé, son champ d'activité devra être connu, ses mœurs et leurs conséquences devront être miscs au jour.

\section{FAMILLE- ICTERIDA. ÉTOURNEAUX D'AMÉRIQUE.}

Cette famille comprend les étourneaux, orioles et étourneaux des prés qui représentent sur ce continent les sansonnets ou étourneaux d'Europe. Ils sont étroitement alliés aux moineaux et l'on ne peut pas nettement les différencier dans une description ordinaire. La plupart de ces oiseaux ont un long bec conique et pointu (figures $46,47,48,49, \mathrm{p} .28$ ) et quelques-uns 
ont le milieu du culmen qui remonte en forme de quille sur le front. Le goglu et l'étourneau ordinaire ont tout à fait des becs de moineaux mais ce sont des becs raccourcis d'ictérinés plutôt que de passereaux. On peut également différencier ces espèces par leurs taches de couleur.

\section{Genre-Dolichonyx. Goglu.}

494. Le Goglu. (вовоLINK). Dolichonyx oryzivorus. L, 7·25. Planche XXV B.

Traits distinctifs. Le mâle printanier avec son plumage pie se reconnait immédiatement. La femelle et les oiseaux d'automne de l'un ou l'autre sexe n'ont aucun rapport comme couleur avec le mâle du printemps et sont facilement confondus avec certains moineaux (bec, figure 46, p. 28). A l'automne avec leur nouveau plumage ils sont de couleur chamois, non rayés sur les dessus et fortement rayés en dessous. La femelle printanière, plus fatiguée et fanée et légèrement olivâtre, ressemble beaucoup à la femelle du moineau domestique, mais on la reconnait a son dos et sa couronne fortement rayés et a sa ligne couleur chamois au-dessus de l'oeil.

Indications sur le terrain. Le mâle printanier n'exige aucune indication spéciale pour être reconnu; les raies et le jaune des autres plumages et la ligne chamois de l'cil avec les barres sur la couronne sont de bonnes marques d'identification qui devraient avec l'habitat et l'ensemble des caractères empêchcr qu'il y ait confusion.

Nid. Sur le sol; construit avec des herbes secbes.

Habilat. L'Amérique du Nord; il hiverne dans l'Amérique du Sud; dans l'est du Canada, le long de la frontière méridionale, couvant partout où il se trouve.

Le goglu, au printemps et en été, est un oiseau qui fréquente les champs de foin et de trèfle. On peut le voir tous les jours d'été, perché sur une clôture du voisinage ou s'élançant dans les airs en frissonnant des ailes et faisant entendre son chant extatique. Plus tard dans la saison, le mâle aux gestes folâtres se dépouille de ses couleurs gaies pour endosser le jaune d'ocre plus terne avec les raies brunâtres de la femelle. Son chant est remplacé par des tintements métalliques et, en compagnies de plusieurs centaines d'autres oiseaux de cette espèce réunis en troupes, il recherche les marais jusqu'à l'automne. Au moment de quitter le Canada pour ses quartiers d'hiver en Amérique du Sud, il s'arrête quelque temps dans les champs de riz des Carolines où il est reconnu non pas comme le goglu, gai chanteur, aimé de tous tant pour des raisons pratiques que sentimentales, mais comme l'ortolan de riz qui s'abat par milliers sur les récoltes et devient le fléau de l'agriculteur. Dans le Sud, on les tue au fusil et on les vend en grand nombre comme nourriture.

Valeur économique. Le goglu, au Canada, est un oiseau irréprochable qui nous charme de son chant harmonieux et auquel nous n'avons pas encore découvert de mauvaises habitudes. En mai et en juin, le 90 pour cent de son alimentation consiste en insectes nuisibles et le reste en graines de mauvaises herbes et quelques insectes utiles. En juillet et aout, il mange un peu de grain. Toutefois on considère cet oiseau comme un fléau dans les Etats du Sud.

\section{Genre-Molothrus. Etourneaux ordinaires.}

495. L'Étourneau ordinaire. (CowBird). Molothrus ater. L, 7.92. Planche XXVI A.

Traits distinctifs. Un petit étourneau avec la tête et le cou noir de phoque. La femelle est d'un brun cendré, plus pâle sur la gorge et peut étre distinguée d'avec les moineaux auxquels elle ressemble a cause de son bec conique, par sa coloration uniforme et peu prononcée. 
Indications sur le terrain. Petit étourneau avec bec court et conique; accents rauques et criards et cri écorchant.

Nid. Dépose ses cufs dans des nids d'autres espèces, ordinairement plus petites, sur le sol ou en des endroits plutôt bas.

Habitat. Dans la majeure partie de l'Amérique du Nord. Au Canada, dans la partie nord jusqu'aux limites de la culture. L'espèce est commune, sauf dans les provinces côtières de l'extrême Est.

L'étourneau ordinaire est notre seul oiseau habituellement parasite. Jamais il ne construit de nids, ni ne couve ni ne s'occupe de ses petits. En l'absence d'un autre oiseau qui couve il profite d'une occasion pour déposer l'un de ses propres œufs dans le nid non gardé. D'habitude, les propriétaires du nid acceptent l'œuf sans protester, mais parfois ils s'y opposent énergiquement puis finalement se résignent. Dans certains cas, le nid est déserté ou un nouveau nid est construit par dessus l'œuf étranger; c'est ainsi que fait quelquefois la fauvette d'Amérique. L'incubation présente un cas intéressant d'adaptation. On remarque que l'œuf de l'étourneau est éclos ordinairement quelques heures avant ceux de l'oiseau nourricier et, par conséquent, l'intrus est déjà fort et développé alors que les premiers occupants du nid ne font que briser leur coquille. Cela lui permet de s'emparer de la nourriture et d'augmenter ainsi son surcroît de vigueur de telle façon qu'il peut finalement rejeter ses concurrents hors du nid et recevoir pour lui seul les soins qui devaient aller à toute la nichée. C'est ainsi que, de fait, la naissance de chaque étourneau signifie la destruction d'une nichée d'une autre espèce, et l'on doit probablement considérer l'étourneau comme l'un des ennemis les plus terribles des espèces qui sont ainsi dupées. Une fois que le père et la mère nourriciers ont accepté l'œuf étranger ils ne font aucune différence entre celui-ci et les leurs propres. Cet oiseau a été appelé "Cowbird" par suite de son habitude de suivre les bêtes à cornes, sans doute dans le but de capturer les mouches et insectes qui s'assemblent autour de ces animaux.

Valeur économique. Si l'on en juge d'après son alimentation, l'étourneau semblerait être simplement un oiseau utile. Il consomme de nombreuses graines de mauvaises herbes et beaucoup d'insectes nuisibles mais une petite quantité seulement de grain ou de fruits; le grain se compose principalement des glanures et les fruits sont des fruits sauvages. Le mal qu'ils font cependant vis-à-vis d'autres oiseaux également utiles donne un tout autre aspect à leur influence économique. On peut dire que chaque étourneau élevé jusqu'à l'état d'oisillon signifie la destruction de toute une nichée d'une autre espèce. Il est possible que l'influence économique des oiseaux substitués compense celle des oiseaux disparus mais cette substitution ne peut tout de même pas être envisagée froiclement.

\section{Genre-Xanthocephalus. Étourneau à tête jaune.}

497. L'Étourneau à tête jaune. (YeLLow-Headed BLACKBjRD). Xanthocephalus xanthocephalus. L, 10. Un étourneau avec des taches blanches sur les rémiges primaires, et avec la tête, le cou et la poitrine jaunes. La femelle est semblable mais de couleur brunâtre plutôt que noire; le jaune est moins brillant et le blanc fait défaut sur les ailes.

Traits distinctifs. Les indications précédentes suffisent a le faire reconnaittre.

Habitat. L'ouest de l'Amérique du Nord jusqu'au nord des provinces des prairies. N'apparait qu'accidentellement dans l'est du Canada.

C'est un oiseau des inarais qui apparaît rarement dalis l'est du Canada. 


\section{Genre-Agelaius. Etourneaux à ailes rouges.}

498. L'Étourneau à ailes rouges. (RED-Winged BLACKBIRD). Agelaius phoniceus. L, 9.51. Planche XXVI B.

Traits distinctifs. Le plumage du mâle a toujours les épaules au moins tachetées de rouge, bien que parfois, on ne remarque que ça et la des mouchetures d'orange. La femelle se distingue toujours des autres étourneaux par sa coloration nettement rayée.

Indications sur le terrain. Le mâle est distinctement caractérisé par ses épaules rouges et on le reconnait à toutes les phases de son plumage par ses accents caractéristiques; son cri le plus ordinaire qui n'est propre qu'au mâle, peut se traduire par "O-ki-li» ou "O-ki-ri" avec une inflexion de plus en plus forte à la fin.

Nid. Une construction bien faite avec des joncs et des herbes, attachée à 2 ou 3 pieds au-dessus de l'eau après des roseaux, des massettes ou des arbrisseaux dans des endroits marécageux.

Habitat. Comme espèce, cet étourneau est répandu sur toute l'Amérique du Nord jusqu'aux limites des régions boisées.

SOUS-ESPECES. Il y a plusieurs sous-espèces de cet oiseau. La plus commune dans l'est du Canada est l'oiseau type, l'étourneau à ailes rouges de l'Est. Dans l'extréinité ouest de l'Ontario, nous avons probablement celui du Nord, A. p. arctolegus qui vient des régions centrales du Nord. Celui-ci est caractérisé par sa taille un peu plus forte mais il n'y a qu'un expert qui puisse faire la distinction exacte.

Il n'y a pas de marais typique au Canada où l'on ne trouve un ou plusieurs couples d'étourneaux aux ailes rouges se poursuivant les uns les autres ou se eramponnant aux roseaux, les mâles étalant leurs ailes et leurs queues et se tortillant en des attitudes forcées pendant qu'ils font entendre leur " $O-k i-r i »$ avec un trille sur la dernière syllabe, en présence des femelles. Au printemps, les étourneaux arrivent ordinairement par grandes compagnies d'espèces mélangées qui demeurent ensemble pendant quelques jours et ensuite se séparent. Les étourneaux à ailes rouges se dirigent vers les marais et, avant que les roseaux commencent à pousser, ils s'occupent de leur installation domestique. Lorsqu'ils ont rempli leurs devoirs de famille pour la saison, toutes les espèces d'étourneaux se rassemblent de nouveau en troupes qui obseureissent le firmament, se perchant ensemble dans les marais lorsque cela est possible et s'éparpillant durant le jour en groupes plus ou moins nombreux pour parcourir les champs de récoltes.

Valeur économique. En raison de son régime alimentaire, cet oiseau est réellement utile. Le 80 pour cent de sa nourriture se compose de graines de mauvaises herbes et d'insectes malfaisants et le grain qu'il mange se monte à environ 15 pour cent. C'est en juillet et août qu'il consomme le plus de grain et, au début de la colonisation, lorsque la superfieie des terres à culture était restreinte et que les étourneaux étaient en grand nombre, c'était une sérieuse menace pour la moisson.

\section{Genre-Sturnella. Étourneaux des prés.}

501. L'Étourneau des prés. (MEADowlank). Sturnelia magna. L, 10.75. Planche XXVII A.

Traits distinctifs. Impossible à confondre avec une autre espèce dans l'est du Canada. (Bec, figure 47, p. 28).

Indications sur le terrain. La poitrine jaune très apparente et un collier noir bien tranché sont des marques sûres. Au vol, les plumes extérieures blanches de la queue et la façon singulière de voler sont de bonnes indications. Le sifflement clair et prolongé de l'étourneau des prés est caractéristique. de four.

Nid. Sur le sol; construit avec des herbes sèches; le nid est ordinairement en forme

Habitat. L'est de l'Amérique du Nord jusqu'aux confins du territoire cultivé. 
La voix claire de l'étourneau des prés est souvent la première indication de la venue du printemps. Il arrive en même temps, quelquefois même avant le merle et le rouge-gorge, et fréquente les pâturages élevés; on entend alors de très loin, du sommet d'un arbre isolé ou d'un poteau de clôture, ses accents doux et sonores.

Valeur économique. L'étourneau des prés est l'un des oiseaux les plus utiles au cultivateur. Comme il se tient près du sol, il s'attaque à la plupart des pires ennemis de la moisson. Son alimentation est répartie comme suit: 75 pour cent d'insectes, 12 pour cent de graines de mauvaises herbes, et 13 pour cent de céréales, presque tout recueilli à la fin de l'automne et au début du printemps, évidemment. en raison de la pénurie d'insectes à ces époques. Cet oiseau mérite tout à fait d'être protégé.

\section{Genre-Icterus. Loriots des vergers.}

506. Le Loriot (ou l'Oriole) des vergers. (ORCHARD ORIOLE). Icterus spurius. L, 7.32. Un petit étourneau ressemblant au loriot de Baltimore (planche XXVII B), l'orangé de cet oiseau étant remplacé par un brun de phoque; sa queue est noire. La femelle est d'un vert terne et uniforme. Le jeune mâle est comme la femelle mais il a la gorge noire.

Traits distinctifs. La coloration brun de phoque et noire du mâle se reconnait immédiatement. La femelle a une certaine ressemblance avec la femelle du tangara, mais elle est plus petite et d'une forme plus délicate, et son bec est fin, pointu, et non denté. (Comparer les figures 48 et 53, p. 28).

Indications sur le terrain. Couleur, taille et chant à peu près semblables à ceux du loriot de Baltimore, mais la voix est plus riche, et il possède certains traits qui lui sont propres.

Nid. Composé d'herbes vertes et suspendu à une enfourchure d'arbre. C'est une jolie construction mais pas aussi bien finie ni aussi profonde que le nid du loriot de Baltimore.

Habitat. C'est une espèce plus méridionale que le loriot de Baltimore; elle apparait régulièrement au Canada sur les rives du lac Erié et parfois au nord jusqu'à l'extrémité sud du lac Huron.

Le loriot des vergers ne se rencontre communément que le long des frontières sud de l'Ontario et ses mœurs sont absolument les mêmes que celles du loriot de Baltimore.

\section{Sous-genre-Yphantes. Loriot de Baltimore.}

507. Le Lorlot (ou l'Oriole) de Baltimore. (BALTmone oniole). Icterus galbula. L, 7.53. Plancbe XXVII B.

Traits distinctifs. Coloration.

Indications sur le terrain. Ses taches éclatantes d'orange doré et sa riche voix de contralto sont des marques d'identification certaines. Cette espèce ne peut être confondue qu'avec des individus d'un autre habitat.

Nid. Son nid est l'une des curiosités de la gent ailée. Il a la forme d'un sac tissé avec des plantes fibreuses, du duvet végétal, des crins et des ficelles, le tout artistement enlacé et suspendu à l'extrémité d'une longue branche inclinée vers la terre. Avec son bec pointu comme une alène la femelle enfonce un filament dans la paroi du nid, puis, l'ayant saisi par en dedans, le tire et l'enlace solidement pour faire un travail serré, et recommence ainsi avec un autre filament jusqu'a ce que le nid une fois terminé se trouve tellement bien tricoté, tissé et feutré que malgré qu'il soit balancé à l'extrémité d'une longue branche flexible au milieu des tempêtes des quatre saisons, il demeure intact pendant plusieurs années.

Habitat. L'est de l'Amérique du Nord en allant au nord jusqu'aux limites des régions bien habitées.

Le loriot de Baltimore fréquente de préférence les régions découvertes, parsemées de bosquets avec ça et là, de grands ormes isolés. Le nom de cet 
oiseau lui vient de son magnifique plumage orangé et noir, couleurs officielles de lord Baltimore sous le patronage duquel l'Etat de Maryland fut d'abord colonisé et en l'honneur duquel les premiers colons ont nommé cet oiseau.

Valeur économique. La nourriture de ce loriot se compose surtout d'insectes qui sont par ordre de quantité: chenilles, taupins, dont on connait les larves pestilentielles, hannetons et sauterelles. Il capture très peu de coléoptères rapineurs. Il consomme peu de matière végétale Cet oiseau occupe donc une place très importante comme destructeur d'insectes. On l'a quelquefois accusé de gâter les fruits et aussi de piquer le raisin pour en extraire le jus. Ce n'est pas de ce qu'il en extrait que l'on se plaint mais c'est de la quantité de vigne qu'il gâte car il va d'une grappe à l'autre piquant partout le raisin tout en en consommant très peu. Cette habitude semble cependant être purement locale et peut n'être qu'individuelle; elle n'a d'ailleurs que peu d'importance sauf dans un pays de vigne. Au Canada, le loriot de Baltimore s'en va peu après la mi-aout et avant que la saison des fruits à l'automne soit avancée, de sorte que les raisins sont ordinairement trop verts pour leur faire envie. Par conséquent, bien qu'on ne puisse pas entièrement l'exonérer des accusations portées contre lui, les dégâts causés par le loriot au Canada ont été certainement très exagérés. Les services rendus par cet oiseau sont constants et importants; quant au mal qu'il peut faire, ce n'est que par moments et c'est peu de chose.

\section{Genre-Euphagus. Mainates couleur de rouille.}

509. Le Mainate couleur de rouille. (RUSTY BLACKBIRD, RUSTY GRACKLE). Euphagus carolinus. L, 9.55. A peu prds de la taille d'un étourneau a ailes rouges, mais tout noir avec reflets verdâtres et des yeux couleur de paille. A l'automne, les plumes sont largement bordées d'un ton rouilleux, plus pale sur la couronne et sur la tête. La femelle est un oiscau presque uniformément gris foncé avec des traces de marques rouilleuses au printemps, beaucoup plus étendues chez les deux sexes à l'automne, alors qu'elles forment une calotte rougeâtre et une ligne sourcilière bien caractérisée.

Traits distinctifs. Taille petite relativement a celle du mainate bronze, la seule autre espece aux yeux clairs; couleur noire ou teinte rouilleuse uniforme passant a une ligne pâle au-dessus de l'œil; les yeux sont d'un jaune paille.

Indications sur le terrain. La taille, la coloration et les yeux jaune paille.

Nid. Sur des conifères ou sur le sol; se compose d'herbes ou de mousse.

Habitat. L'est et le nord de l'Amérique du Nord; couve ordinairement juste au nord des régions cultivées au Canada.

Le mainate couleur de rouille se présente chez nous en grand nombre au printemps et à l'automne; il est mêlé pour une large part aux grandes troupes d'étourneaux que l'on aperçoit aux abords des champs et des marais. Le nom anglais de "Grackle" qui est ordinairement appliqué aux deux étourneaux aux yeux jaunes (presque blancs) provient sans doute de leur cri aigre et pétillant.

\section{Genre-Quiscalus. Quiscales.}

511. Le Mainate bronzé.(CROW BLACKBIRd, BRonzed Grackle). Quiscalus quiscula. L, 12. Planche XXVIII A.

Traits distinctifs. Forte taille, partout d'un noir iridescent; les yeux jaune paille.

Indications sur le terrain. Le corps tout noir, les yeux jaune paille et la taille sont de bonnes indications. Quand on l'aperçoit au vol, la longue queue du mâle est tout étalée avec les cótés tournés en haut de façon a former la lettre aU. C'est un trait bien caracteristique qui se dessine très nettement sur le ciel au printemps, mais est moins visible ou même absent à l'automne. 
Nid. Ordinairement par groupes sur des conifères; le nid est de structure grossière et composé d'herbe et de boue.

Habitat. Toute la zone tempérée de l'Amérique du Nord à l'est des Montagnes Rocheuses. Le mainate bronzé occupe la section canadienne de l'habitat et empiète sur celui du mainate pourpre, au sud de nos frontières.

SOUS-ESPECES. Il y a trois sous-espèces. Le mainate bronzé, Q. q. oneus, est l'oiseau canadien. L'oiseau type, le mainate pourpre, est un oiseau plus méridional qui n'a pas encore été capturé au Canada, bien qu'il devrait se trouver sur nos frontières méridionales; on le reconnaît aux plumes du dos qui présentent une iridescence pourprée en demi-cercle, au lieu d'un ton uniforme de cuivre métallique. La troisième sous-espèce apparaît en Floride.

Le mainate bronzé est un oiseau grégaire et se plaît à nicher en compagnie de ses congénères. Il niche de préférence dans les conifères et s'installe souvent dans les haies décoratives des jardins. Avec ses couleurs métalliques et ses yeux jaunes, c'est un oiseau magnifique et d'un éclat frappant. Il se pavane sur le gazon avec une affection comique et des gestes bizarres pendant qu'il laisse entendre ses accents plutôt discordants.

Valeur économique. Pendant qu'il habite le Canada, les insectes constituent le 29.7 pour cent de sa nourriture et les matières végétales, le 70.3 pour cent. Les insectes comprennent certaines espèces qui vivent de proie mais pas en grand nombre. La matière végétale comprend environ 48 pour cent de grain et de fruits d'arbres forestiers et graines de mauvaises herbes. Une bonne partie du grain consiste en déehets et les fruits cultivés ne comptent que pour le 2.9 pour cent. D'une façon générale, l'influence du mainate bronzé est bienfaisante mais il ne faudrait pas trop en laisser le nombre s'accroître. C'est un implacable destructeur de nids et comme tel il ne fait pas bon l'avoir autour de la maison si l'on désire avoir des espèces plus attrayantes.

\section{FAMILLE-FRINGILLIDAA. MOINEAUX, LINOTTES, PINSONS, OU BRUANTS.}

Description générale. Telle que représentée au Canada, cette famille est généralement facile à reconnaître. Elle se compose de petits oiseaux -il n'y a guère d'espèce canadienne de plus de $8 \frac{1}{2}$ pouces de longueur - avec bec conique non échancré, se prêtant bien au cassage des graines et l'ouverture de la bouche ordinairement tournée en dessous (figures 50, 51, 52 , p. 28). Ce dernier trait n'est pas développé au méme degré chez toutes les espèces et certains étourneaux d'Amérique, ainsi que nous l'avons déjầ dit (p. 176) le possèdent d'une façon prononcée; mais ils ont d'autres marques dictinctives qui permettent de les différencier d'avec les moineaux.

Traits distinctifs. C'est au bec qu'on les reconnait le mieux; celui du serin domestique est bien nettement du type passereau. Les oiseaux qu'on est le plus portế à confondre avec ceux de cette famille sont le goglu (figure 46, p. 28) et l'étourneau ordinaire (p. 177) de la famille précédente, et les tangaras, de la famille suivante. On peut facilement différencier ceux-ci par leurs couleurs éclatantes (voir à chacun des titres). Les tangaras ont des échancrures aux bords tranchants et, à la pointe, des mandibules ce qui les rend faciles à reconnastre (figure 53, p. 28). Dans un groupe de fringillidés, les becs-croisés, les pointes du bec se croisent (figure 52, p. 28); dans un autre, les gros-becs, le bec est très gros et très robuste (figure 50, p. 28).

Les passereaux (sparrows) constituent la plus grande et la plus importante famille des percheurs et c'est probablement la plus importante famille d'oiseaux du monde entier. On les reneontre partout sauf en Australie; ils sont représentés dans tous les habitats, dans les marais humides, plateaux herbeux et épaisses forêts comme dans les plaines sèches et les régions sablonneuses. Les termes: Moineau, Linotte, Pinson, et Bruant sont à peu près synonymes et s'appliquent à diverses espèces sans égard à leurs rapports mutuels. Le nom de Passereau (sparrow) a par conséquent une 
acception très vaste et peut s'appliquer a beaucoup d'espèces différentes en importance ou en valeur. Il est fâcheux que certaine espèce introduite au pays ait été cause de la mauvaise réputation attribuce à toute une vaste famille qui comprend beaucoup de magnifiques et de précieux oiseaux, dont quelques-uns nous charment par leur chant délicieux. Le caractère le plus typique de cette famille dans l'esprit populaire est une coloration gris terre uniforme, mais elle comprend aussi des oiseaux au plus éclatant plumage et au lieu du simple pépiement du moineau auquel on s'attend d'ordinaire, on entend parfois le chant le plus agréable. On peut diviser cette famille en espèce terrestre, espèce des arbres, migrateurs d'hiver et grosbecs. Pour l'observateur superficiel, les oiseaux de la première espèce sont d'apparence terne, mais si on les examine de près, ils présentent souvent de magnifiques harmonies de couleur. L'espèce des arbres est souvent très brillamment colorée. Les migrateurs d'hiver présentent ordinairement beaucoup de rouge terne. Les gros-becs que l'on reconnait tout de suite a leur gros bec robuste (figure $50, \mathrm{p}$. 28) sont vivement colorés. Il ne s'agit pas ici d'une subdivision scientifique, mais ces oiseaux sont tellement difficiles à distinguer par l'amateur que toute classification pouvant lui servir ne sera pas sans utilité.

\section{Gener-Hesperiphona.}

514. Le Gros-bec a couronne noire. (EVEnisg grosbeak). Hespetiphona vespertina. L, 8. C'est un des plus gros passereaux avec un tres grand et puissant bec qui est typique chez les gros-becs (figure 50, p. 28). Sa coloration est par larges masses d'un jaune intense avec les ailes, la queue et la couronne noires et une bande blanche en travers de l'aile. La femelle est toute semblable, mais de couleur plus terne avec des tons cendrés sur tout le corps.

Traits distinctifs. La taille, le bec, et la forte quantité de jaune. La femelle garde assez de jaune mêlé à la couleur cendrée pour être facilement reconnue. Ie jaune ou vertjaunâtre du bec fait reconnaitre tous les plumages. Pour la comparaison de la femelle avec le gros-bec des pins, voir cette derniere espece.

Indications sur le terrain. La taille et la coloration jaune. Comme c'est un hôte de nos hivers seulement, on ne peut pas le confondre avec d'autres oiseaux.

$N^{\top} i d$. Sur les arbres; nid fait de petites ramilles, garni d'écorce, de poils et de radicelles. Le nid a rarement été vu.

Habitat. Le centre et l'ouest de l'Amérique du Nord; au sud le long des montagnes. Il est migrateur vers l'Est, mais de façon irrégulière.

SOUS-ESPECES. Le gros-bec à couronne noire comprend une sous-espèce de l'Est, et une autre de l'Ouest; c'est la première, la forme type, qui seule se rencontre dans l'est du Canada. Les localités où ce gros-bec de l'Est niche et couve n'ont pas encore été déterminées avec exactitude.

Cet ciseau est seulement un migrateur irrégulier en hiver, dans l'est du Canada. Il s'écoule quelquefois des années sans qu'on voie cet oiseau et puis, tout à coup, il se montre partout. Les causes de ses apparitions irrégulières n'ont pas été expliquées de façon définitive. Est-ce l'absence de nourriture qui a tenu ces oiseaux éloignés de leurs stations d'hiver, ou bien sont-ils attirés vers d'autres régions par une abondance inaccoutumée d'aliments? C'est ce que l'on ne peut dire. La nourriture est probablement la cause déterminante.

Valeur économique. Le gros-bec à couronne noire, qui ne vient qu'en hiver, ne peut faire que très peu de dommage. Sa nourriture de prédilection, c'est la graine de l'érable du Manitoba, qui demeure pendue aux branches de cet arbre, ou encore le fruit du frêne sauvage. Le fait que l'érable du Manitoba a, dans ces dernières années, été planté en quantité dans 
toutes les parties du Canada, peut avoir influé sur les habitudes migratrices de cet oiseau. Presque tout fruit sec lui est bon et il aime à retirer les pépins des vieilles pommes pourries qui pendent aux arbres en hiver. On l'accuse d'endommager les arbres en arrachant les bourgeons; c'est un peu vrai, mais cela ne saurait être la source de dégâts appréciables.

\section{Genie-Pinicola. Gros-becs.}

515. Le Gros-bec des pins. (PINe grosbenk). Pinicola enucleator. L, 9.08. Planche XXVIII B.

Traits distinctifs. A s'en rapporter aux descriptions, on pourrait confondre les femelles des gros-becs des pins avec celles des gros-becs a couronne noire. car elles sont toutes d'un gris étendu de jaune; mais le jaune chez le gros-bec des pins est plus foncé, surtout sur la tête et le croupion, d'un jaune citron plutôt que clair et qui recouvre la coloration du corps au lieu d'avoir l'air de se laisser deviner en dessous. Le bec foncé de l'espèce est également distinctif.

Indications sur le terrain. La taille, le bec foncé du gros-bec et la coloration rouge des mâles adultes. La plupart des oiseaux qui visitent le Canada ont la livrée terne des femclles, de là vient que l'effet général est celui d'une bande de gros oiseaux, couleur ardoise mate, passant à un jaune chaud sur la tête et le croupion, vue sous une lumière favorable et accompagnée à l'occasion de quelques individus rougeâtres. Les notes ordinaires de cet oiseau sont d'une faiblesse et d'une finesse ridicules pour un gros oiseau, bien qu'on lui connaisse aussi un sifflement clair et retentissant.

Nid. Sur les arbres coniferes; fait de ramilles et de radicelles.

Habitat. En tant qu'espèce, son territoire se borne aux régions du nord de l'hémisphère boréal. Le gros-bec des pins du Canada couve dans les forêts de conifères du Nord, à l'ouest du fleuve Mackenzie; ses migrations vers les régions colonisées n'ont lieu qu'en hiver.

SOUS-ESPECES. Il y en a plusieurs au Canada, l'individu de l'Est, le gros-bec des pins du Canada-Pinicola enucleator lencura-étant le seul qui puisse figurer dans le cadre du présent ouvrage.

Ces oiseaux sont des hôtes irréguliers venant du Nord en hiver. Leur présence chez nous ne peut que rarement être prévue, bien qu'ils puissent apparaître pendant l'hiver dans n'importe quelle localité de l'est du Canada. Ils fréquentent les arbres conifères, mais ont aussi beaucoup de gout pour les petits fruits du frêne sauvage, du sorbier et aussi du sumac.

Valeur économique. Comme le gros-bec des pins passe l'été dans les forêts du Nord et ne visite qu'en hiver les régions colonisées, les dégâts qu'il peut causer se réduisent à fort peu de chose. Il mange du fruit sauvage qu'on laisse perdre et qui pend aux arbres, si bien que son influence économique est absolument négligeable. On l'a accusé de détruire les graines des fruits, mais le mal qu'il fait ainsi est minime. Il y a toutes les meilleures raisons du monde pour protéger cette espèce qu'on ne peut accuser de rien de sérieux.

\section{Genre-Carpodacus. Pinsons pourprés.}

517. Le Pinson pourpré. L'orsead Rodge. (PURPle Finch). Carpodacus purpureus. L, 6.22. Planche XXIX A.

Traits distinctifs. La taille et la coloration générale du mâle sont distinctives. La femelle a des raies d'un brun olive, mais l'uniformité générale de la teinte olive dans cette coloration et l'abondance des raies en dessous sont tout à fait caractéristiques. Le bec est plutôt plus gros pour la taille de l'oiseau que chez les autres espèces, le gros-bec excepté.

Indications sur le terrain. La taille, la coloration générale; parfois une ressemblance entre ce pinson et le petit gros-bec des pins, et un chant très frappant; telles sont les meilleures indications sur le terrain.

Nid. Dans les arbres conifères; nid fait de brindilles, d'herbes et de radicelles. 
Habitat. En tant qu'espèce le pinson pourpré occupe toute l'Amérique, du Mexique au Canada. Dans l'est du Canada, il va au nord jusqu'a l'extrême limite de la colonisation établic. Le long des confins méridionaux c'est un hôte régulier d'hiver qui couve ha moins souvent que plus au nord.

SOUS-ESPECES. Le pinson pourpré est représenté par deux sous-espèces; l'individu de l'est du Canada, le pinson pourpré de l'est, est le type de l'espèce.

Le pinson pourpré, malgré son nom, n'est pas du tout pourpré; la couleur "magenta " (rose violacé) décrirait mieux sa coloration. C'est un de nos meilleurs chanteurs et on le met parfois en cage pour cette raison. Comme ses congénères, le gros-bec des pins et les becs-croisés d'Amérique, une fois en captivité il perd le beau rouge de son plumage et revêt un jaune vermeil si particulier et caractéristique que des oiseaux qui ont réussi à s'envoler de la cage se reconnaissent à première vue. Le chant est une suite continue et claire de sons mélodieux comme ceux que module le fameux viro gris-olive, mais d'une exécution plus rapide. Le jeune mâle, en automne, chante presque aussi bien que l'adulte.

Valeur économique. Le pinson pourpré se nourrit surtout de bourgeons et de fruits. Le nombre de fruits qu'il mange dans l'est du Canada est insignifiant vu que cette espèce d'oiseaux n'est pas nombreuse en été dans les localités où l'on cultive les arbres fruitiers. Cet oiseau quitte les confins méridionaux et se rend dans les parages moins eultivés dans la saison de la couvaison. Les fruits qu'il prend sont principalement ceux que l'homme a dédaignés et laissé perdre, des fruits sauvages dont il est friand comme ceux du frêne sauvage et du sorbier. Le procès qu'on lui fait à cause des bourgeons qu'il mange est plus sérieux, mais n'a pas été jusqu'ici appuyé par autre chose que des affirmations générales et non par le résultat d'un examen de l'estomac des oiseaux. Il se peut que, parfois, le pinson pourpré fasse des dégâts considérables en certaines localités, en picotant les bourgeons à fruits de l'année suivante; mais il reste à prouver qu'il fait assez de tort de cette façon pour nuire à la récolte des fruits.

\section{Genre-Passer. Moineau domestique d'Europe.}

520. Le Moineau domestique. (notse sparrow). Passer hostilis. L, 6.50. Planche XXIX B.

Traits distinctifs. La bavette noire du mâle est distinctive. Les femelles ont une légère indication de la couleur olive et pourraient se prendre pour la femelle du pinson pourpré, n'était-ce qu'elles n'ont pas de raies en dessous. La femelle, ou les plumages d'automne du goglu, font un peu penser à ce plumage, mais les raies très prononcées en dessus et la couleur jaune générale sont tout à fait caractéristiques du goglu. Un peu d'attention donnée à l'espèce dans nos rues et dans nos cours de fermes suffira à empêcher qui que ce soit de prendre cette espèce pour aucune autre.

Indications sur le terrain. Les notes caractéristiques et le gazouillement du moineau domestique seront toujours la meilleure indication sur le terrain pour le reconnastre.

Habitat. A l'origine son territoire comprenait toute l'Europe et la plus grande partie de l'Asie. Aujourd'hui il est dans toute l'Amérique du Nord jusqu'aux confins des parties colonisées.

Cet oiseau n'est pas originaire d'Amérique, mais e'est un des produits les moins désirables qu'on ait importés d'Europe. Malgré son habitude bien connue de manger du grain il fut introduit en Amérique en qualité de destructeur de chenilles. En effet, à l'instar de la plupart des oiseaux qui quelquefois mangent des chenilles, le moineau en mange, mais à cet égard il n'approche pas de la capacité des espèces qu'il a évincées. Comme e'est un oiseau des villes et des cours de fermes, son activité se donne carrière sur- 
tout dans les localités où il y a abondance de nourriture pour les non-insectivores, c'est-à-dire de déchets de cuisines, de grains qu'on laisse perdre, etc. En automne il se livre à des excursions à la campagne et visite les champs en grandes bandes, surtout après les moissons quand il reste beaucoup de grain perdu par terre; quelquefois il arrive avant la moisson et alors il cause de grands dégâts. Son régime d'alimentation est donc nuisible, ou ne l'est pas suivant les circonstances, si bien que dans un règlement de compte, c'est en faveur de l'oiseau que le bilan s'établirait. Les trois autres principaux griefs qu'on fait valoir contre le moineau domestique sont les suivants: il évince des espèces plus utiles que la sienne; il salit les alentours des maisons; on le suspecte de propager les maladies des oiseaux de basse-cour.

Les moineaux domestiques chassent les autres oiseaux de trois façons: en monopolisant les provisions alimentaires; en s'emparant des places pour leurs nids; par leurs mœurs querelleuses et batailleuses. Pendant le temps de la couvaison, quand les petits reçoivent leur becquée, les moineaux et d'autres espèces se font concurrence pour la nourriture à donner à leurs petits, c'est-à-dire pour les mêmes insectes (les petits de tous les passereaux demandent des insectes, bien que ceux de l'espèce présente ne soient pas longtemps purement insectivores). Jusqu'ici peut-être les moineaux pourraient passer pour être aussi utiles que les individus qu'ils évincent, mais la plupart des oiseaux évincés sont de perpétucls chasseurs d'insectes, tandis que le moineau ne l'est que temporairement. Aussitôt que les devoirs de la couvaison sont finis ils retournent aux déchets et aux matières qu'on laisse perdre et leur importance baisse d'autant, tandis que les oiseaux évincés continuent à rendre des services pendant toute la saison. Les moineaux domestiques restent avec nous pendant tout l'hiver, ne montrant aucune disposition à émigrer, aussi sont-ils sur le terrain dès les premiers jours du printemps et, lorsque arrivent nos hôtes d'été, oiseaux originaires du pays (presque tous plus ou moins migrateurs, soit comme espèces soit comme individus) ils trouvent déjà prises toutes les meilleures places pour y mettre leurs nids. La difficulté qu'on trouve à écarter les moineaux des nichoirs est la meilleure preuve de l'état de choses en question. Ils sont querelleurs, en outre, et quoique, une fois établies, la plupart des espèces natives soient très capables de résister aux agressions, ils n'aiment pas le perpétuel état de guerre où les fait vivre le voisinage du moineau anglais. Il est bien plus facile de l'éviter que de le combattre. De là vient que peu d'oiseaux d'autre espèce se soucient de vivre dans leur voisinage. Les nids sont grands, massifs, des amas malpropres de paille et d'herbe, et l'habitude qu'ont ces oiseaux de boucher les tuyaux de gouttières, les rend très désagréables. Pour ajouter aux inconvénients provenant des nids des oiseaux domestiques, la réunion de ces oiseaux en nombres considérables, pendant toute l'année, dans des coins abrités sous des corniches et des porches, entraîne des accumulations d'ordures qui irritent les habitants des maisons. De nos jours un des importants problèmes de l'architecture consiste à imaginer des détails satisfaisants et qui ne fourniront aucun abri aux moineaux dont les saletés défigurent les moulures les plus soignées et finissent par désagréger les matériaux dont l'édifice est composé. Le dernier grief, celui de colporter les maladies, n'est pas le moindre de ceux qu'on fait à cette espèce. Le fait de picoter familièrement avec les poules et celui de voleter en liberté d'une basse-cour à l'autre donnent à ces moineaux toute facilité pour être des colporteurs de maladies. I Ia chose, il est vrai, n'a pas 
encore été absolument démontrée, mais qu'elle soit possible et même probable, cela est assez évident. C'est une circonstance bien instructive que les dindes sont de nos jours sujettes aux ravages de certains boutons qui se sont propagés rapidement dans notre pays, alors que dans certaines íles eôtières du Massachusetts, d'où le moineau anglais est absent, le dindon s'élève aussi facilement qu'autrefois.

Il n'est pas douteux que l'introduction en Amérique du moineau domestique a été une erreur. Il était déjà eonnu dans son pays d'origine comme une espèce peu recommandable, et nullement propre à l'usage auquel on le destinait en l'important en Amérique. Dans ce pays, éloigné de tout ee qui pouvait enrayer ses défauts et le tenir en dépendance, il a multiplié au delà de toute raison et, si ses mœurs les plus critiquables ont empiré, ses moins mauvais côtés ne se sont guère perfectionnés. Quoi qu'il en soit, le moineau domestique est ici pour y rester. On a légiféré contre lui, de grosses sommes ont été dépensées pour le tenir en échec, rien n'y a fait Des mesures locàles ont été prises pour en réduite le nombre, de temps à autre, mais elles n'ont servi qu'à attirer du dehors de nouvelles hordes quand l'effet de ces mesures s'est épuisé. De constants efforts maintiendront le nombre de ces oiseaux en de certaines limites, mais ces efforts devront s'étendre à tout le continent si l'on veut en finir avee cette malfaisante engeance. Des pièges, du poison, une destruction systématique des nids seront les meilleurs moyens d'en venir à bout. L'effet du poison est certain, mais il faut faire attention de ne s'en servir que dans les saisons et les lieux où aucune autre espèce ne puisse avoir accès. Les pièges fait de fils de fer et toujours en place, prendront un grand nombre de ces oiseaux à la fois et donneront pleine satisfaction. Un bon modèle de ces pièges a été décrit par la Commission biologique des Etats-Unis, dans le Farmers' Bulletin $n^{\circ} 493$.

\section{Genre-Loxia. Becs-croisés.}

521. Le Bec-crolsé d'Amérique. (crossbill). Loxia cuririostra. L, 6.19. C'est un passereau de taille moyenne avec le prolongement des deux bouts du bec qui s'entrecroisent quand le bec se ferme (figure 52, p. 28). Le mâle d'un rouge terne, plus brillant au croupion; les femelles et les jeunes sont semblables avec le rouge remplacé par du verdâtre ou du jaune. Pas de barres sur les ailes.

Traits distinctifs. Ce bec entre-croisé est distinctif de cet oiseau, l'absence de la barre blanche sur l'aile indique l'espèce.

Indications sur le terrain. C'est un petit oiseau d'hiver qui arrive souvent en grandes bandes; quelques individus font voir une coloration rouge. Les notes de sa voix sont à peu près semblables à celles des chardonnerets. Habitudes de grimper à la façon des perroquets.

Nid. Ordinairement dans les arbres conifères; nid fait de brindilles et d'herbes, garni de mousse et de radicelles.

Habitat. Les forêts de conifères dans les deux hémisphères. Le bec croisé d'Amérique couve au nord des lieux colonisés et très populeux, au sud des montagnes, à l'Est et à l'Ouest. Sa présence est irrégulière en hiver dans le sud du Canada.

SOUS-ESPECES. Le bec-croisé d'Amérique est une sous-espèce du bec-croisé, une espèce de l'ancien comme du nouveau monde, et porte le nom en trois mots de Loxia curvirostra minor.

Les becs-croisés sont également des oiseaux qui descendent irrégulièrement du nord vers le sud du Canada, en hiver, reviennent d'habitude pendant plusieurs saisons successives, et puis ne reparaissent plus de bien des années. 
Valeur économique. Cette espèce se nourrit presque entièrement de graines de conifères: pins, sapins du Canada, et cèdres; des baies de la douce-amère grimpante et aussi des pépins de pommes pourries qui pendent aux arbres. La vitesse et l'adresse avec lesquelles ces graines sont retirées des cônes de pins nous feraient croire que le bec-croisé est nécessaire pour ce genre d'opération jusqu'au jour où l'on découvre que l'espèce au bec normal peut faire la même chose avec la même facilité.

522. Le Bec-croisé à ailes blanches. (wHITe-WINGed crossBILL). Loxia leucoptera. L, 6.05. Semblable à l'espèce précédente, mais avec des barres blanches sur les ailes.

Traits distinctifs. D'une coloration peut-être un peu plus brillante que le bec-croisé d'Amérique, mais avec des modifications considérables de teinte et de nuance. Les barres blanches de l'aile et le bec croisé sont toujours distinctifs.

Cet oiseau est si semblable au précédent par ses mœurs et par ses visites qu'aucune discussion du sujet n'est nécessaire.

\section{Genre-Acanthis. Sizerins.}

Description générale. Ce sont de petits passereaux au bec court et aiguisé; une couronne avec calotte d'un cramoisi mat, une tache d'un noir diffus sur le menton; le dos et les flancs ont des raies brunes, cendrées et blanches. Les mâles adultes ont la poitrine d'un rose pâle, le croupion plus ou moins teinté de rose; une idée de cette teinte se montre en d'autres plumages.

Traits distinctifs. La petite calotte cramoisie est toujours distinctive.

Nid. Dans des buissons plutôt bas: nid fait d'herbes garni de poils, de peau de lapin blanc ou de renard, de plumes ou de duvet végétal.

Habitat. Régions circompolaires et arctiques pour la couvaison, émigrant vers le Sud assez irrégulièrement en hiver.

Il y a dains l'est du Canada deux espèces de sizerins lesquelles se divisent en cinq sous-espèces, toutes si pareilles les unes aux autres qu'il faut une expérience toute spéciale pour les différencier avec exactitude. La ligne de démarcation des espèces est très subtile, les sous-espèces se mélangent et, comme il y a des modifications considérables quant aux individus et quant à l'âge, c'est seulement par une attention donnée aux petits détails qu'on peut les distinguor.

Les traits distinctifs sont donnés plutôt comme des indications que comme des différenciations définitives.

\section{Valeur économique. Voir le sizerin à tête rouge.}

527. Le Sizerin de Hornemann. (horNemann's ReDPoll). Acanthis hornemanni. L, 5. Ce sont des sizerins de couleur claire, avec un croupion blanc ou rose et sans tache. Les adultes ont des plumes dont les bords sont clairs si bien qu'un oiseau typique ressemble à un sizerin ordinaire vu à travers un voile blanc.

Traits distinctifs. Le croupion sans tache est le caractère le plus constant et le plus facile à reconnaître.

Habitat. Les régions arctiques et sous-arctiques de l'hémisphère boréal. Le sizerin du Groenland couve dans l'Europe aretique, le Groenland et peut-être dans l'Amérique voisine. Le sizerin blanc couve dans l'Amérique arctique, de l'Ungava aux parties adjacentes de l'Asie.

SOUS-ESPECES. Le sizerin de Hornemann comprend deux sous-espèces en Amérique: un individu de bonne taille, le sizerin du Groenland, l'individu type; et le sizerin blanc, $A$. $h$. exilipes. Ils se distinguent très facilement l'un de l'autre par la taille, le premier ayant une aile de 3 pouces $\cdot 37$ et le second de 3 seulement. 
Par leurs mours générales ces oiseaux se rapprochent tant de l'espèce suivante, qui est d'ailleurs beaucoup plus commune, qu'une discussion spéciale n'est pas nécessaire. Cette espèce est en général si rare dans les districts colonisés que toute identification des individus doit se faire avec des grandes précautions. Pendant certains hivers cette espèce arrive en nombres plus ou moins grands, avec de grandes bandes de sizerins ordinaires, mais il n'y a aucune régularité dans leurs visites.

528. Le Sizerin à tête rouge. (REDPoll Linner). Acanthis linaria. L, 5-5.5. C'est un sizerin plutôt foncé, avec un croupion plus ou moins fortement rayé et imperceptiblement lustré de blanc.

Traits distinctifs. Le croupion rayé est le trait le plus facilement reconnaissable.

Habilat. Le nord de l'hémisphère boréal. Le sizerin commun couve partout dans les régions du nord du continent américain; dans les régions du sud, il couve dans l'est jusqu'au golfe du Saint-Laurent. Le sizerin de Holboll couve en Amérique dans les iles arctiques de l'Ouest, et le grand sizerin couve dans le Groenland.

SOUS-ESPECES. L'espèce comprend trois sous-espèces: le sizerin commun, Acanthis linaria linaria, qui est le plus commun de fait comme de nom; le sizerin de Holboell, $\boldsymbol{A}$. $\boldsymbol{l}$. holboelli, individu un peu plus grand; et le grand sizerin, $\boldsymbol{A}$. $\boldsymbol{l}$. rostrata, le plus grand de l'espèce. Il y a de petites différences, visibles à l'cil du connaisseur, dans les becs et les détails de la coloration, mais à tout prendre, la taille est le meillcur critérium, bien qu'il ne faille pas trop s'y fier, car il se fait parfois des mélanges entre les sous-spèces. L'aile du sizerin commun devrait être de 2 pouces .9 , celui du Holboll, de 3 pouces, avee un bec légèrement plus long, et celui du grand sizern de 3 pouces $\cdot 5$, avec un bec plus court et plus gros. Cependant, quand on identifie des sizerins, il conviendrait de se rappeler que le sizerin commun est le seul qu'on ait quelque chance de rencontrer; aucune autre caractérisation ne devrait se faire qui ne fut pas appuyée de l'opinion d'une autorité.

Avec plus ou moins de régularité nos champs d'hiver et nos terrains abandonnés sont envahis par d'immenses bandes de petits moineaux qui se nourrissent des bouts de mauvaise herbe qui émergent de la neige, ou qui perchent sur les arbres un peu bas et dans les buissons d'alentours. De cette foule s'élève un gazouillement continu sortant de ces petits gosiers, non pas qu'un seul de ces oiseaux produise un chant, au sens propre du mot, mais tous ensemble ils font une musique en sourdine qui est certainement agréable à entendre. Les corps arrondis duvetés et couverts de plumes, la petite calotte richement cramoisie, et parfois quelque rayon rose parti de ces poitrines et du croupion, disent assez que ce sont des sizerins. Ces petits êtres sont apprivoisés et sans méfiance; si celui qui les observe se fait discret, ils pourront d'aventure se poser autour de lui, même sur lui, avec autant de sans-gêne que s'il était une bâche ou quelque partie inanimée du paysage. Ils demeurent dans la contrée jusqu'a l'heurc printanière du départ; alors ils disparaissent jusqu'au retour de l'hiver. Leurs migrations hivernales sont irrégulières et très changeantes.

Valeur économique. Comme ils arrivent en grand nombre, qu'ils cherchent avidement les bouts des mauvaises herbes qui percent la neige, les sizerins doivent être accueillis avec joie par les cultivateurs, non seulement à cause de leurs gentilles manières mais surtout pour le bien qu'ils font en détruisant les semences d'ivraie. Il est impossible de parcourir le terrain où ces oiseaux ont pâturé et de l'examiner de près sans être frappé de la quantité de bon travail qu'ils y ont fait. Leurs traces se voient partout sur la neige et tous les bouts d'herbes semble avoir été examinés au microscope. Vu leur nombre, et qu'ils viennent dans les plus grands froids, il est évident que leur présence doit avoir une influence destructive des mauvaises herbes de la saison suivante. 


\section{Genre-Astragalinus. Chardonnerets.}

529. Le Chardonneret jaune. (Americangoldfinch). Astragalinustristis. L, 5. 10. Planche XXX A.

Traits distinctifs. En été, le mâle avec son corps jaune qui contraste fortement avec le noir des ailes, de la queue et de la calotte est extrêmement caractéristique. En hiver les couleurs sont moins marquées et distinctives, mais il y a toujours un soupçon de jaune vers le cou, la tête et le dos, sinon ailleurs; les ailes et la queue restent absolument noires quoique avec plus ou moins de bordures blanches ou chanois sur les barres de l'aile.

Indications sur le terrain. Par leurs mœurs, leur manière d'être et la qualité générale de leurs notes les chardonnerets d'hiver ressemblent aux chardonnerets des pins ou aux becs-croisés. On peut les distinguer des premiers par l'absence de raies, et des autres par l'absence de coloration rouge, plus claire et du croupion blanc.

Nid. Fait d'herbes, de duvet végétal, et garni de ce même duvet.

Habitat. En tant qu'espèce, tous les Etats-Unis et le sud du Canada, jusqu'aux limites de la colonisation. Le chardonneret de l'Est va à l'Ouest jusqu'au Manitoba inclusivement.

SOUS-ESPECES. Le chardonneret jaune comprend trois sous-espèces reconnues, dont une seule, le chardonneret de l'Est, l'individu type, se rencontre dans l'est du Canada.

C'est un des oiseaux d'été les plus gais et qui, parfois, reste en hiver dans les parties les plus méridionales du Canada. C'est un grand amateur du chardon blanc duveté et des graines de pissenlit; on peut souvent le voir picotant le duvet, coupant la partie qui mûrit et laissant la partie la plus légère s'envoler avec le vent. Son chant est aussi agréable que son apparence, quand il est posé sur quelque élévation isolée et chante: "suit-swittchewit-tchewit-tchewit') ou qu'il s'envole dans l'air avec une bande joyeuse qui répète son "pertchic-o-pie». Le chardonneret jaune, quoique apparenté à l'oiseau du même nom dans l'ancien monde, est une espèce bien différente qui fut dénommée comme les premiers colons avaient coutume de le faire pour beaucoup d'oiseaux, e'est-à-dire d'après des ressemblances réelles ou imaginaires avec les individus qu'on avait connus dans la mèrepatrie.

Valeur économique. C'est un oiseau qui n'a aucune mauvaise habitude et qui en a plusieurs de bonnes. Les semences des mauvaises herbes sont sa nourriture principale, mais jl touche rarement au grain. Si les moineaux domestiques ou anglais n'en ont pas épuisé prématurément le stock, les graines du tournesol sont le meilleur moyen d'attirer les chardonnerets, et si l'on met de ces graines le long des palissades de derrière la maison, ces oiseaux y seront en permanence pendant l'automne et l'hiver. Ils prennent aussi plus ou moins d'insectes et un peu de fruit, des espèces plutôt sauvages car on ne s'est jamais plaint qu'ils touchassent aux variétés cultivées.

\section{Gener-Spinus. Tarins.}

533. Le Chardonneret des p̈ins. (PINE siskin). Spinus pinus. L, 5. Ce sont de petits oiseaux, pareils aux chardonnerets, à raies d'un brun olive parfois sur un fond blanc mat, quelquefois légèrement teinté de jaune; plus clair en dessous et avec une tache jaune citron et les ailes légèrement nuancées do cette couleur.

Traits distinctifs. Rayures générales et tache jaune étendue sur l'aile. donneret.

Indications sur le terrain. Mœurs et rayures générales, pareilles à celles du charvidítal.

Nid. Dans les arbres conifères; nid fait de ramilles et de radicelles, garni de duvet

Habitat. Les forêts de conifères du nord, a travers tout le continent; émigre vors les régions colonisées en hiver, et y couve par endroits. 
C'est un hôte d'hiver des parages méridionaux du Canada et niche ordinairement dans Québee et les provinces maritimes. Il affectionne les arbres conifères et leur perpétuelle verdure.

Valeur économique. Comme il ne visite qu'en hiver le Canada colonisé et qu'il a un goât prononeé pour les fruits des conifères, c'est plutôt une espèce neutre ne faisant peut-être aucun bien, mais à coup sâr aucun dommage.

\section{Genre-Plectrophenax. Plectrophanes des neiges.}

534. Le Plectrophane des neiges. L'orsead blanc. (snow benting). Plectrophenax nivalis. $\mathrm{L}, \mathbf{6} \cdot 88$. Planche XXX B.

Traits distinctifs. Le violent contraste du blanc et du noir avec la bordure rouilleuse des plumes, surtout sur la tête, le dos et la bande pectorale. Pendant l'hiver les bordures rouilleuses disparaissent peu à peu, et le plumage noir et blanc de la couvaison succède sans mue. Le motif général de la coloration du plectrophane ne se trouve chez aucun autre oiseau du Canada.

Indications sur le terrain. Ce sont des moineaux vivant en troupe sur le sol et montrant de grandes parties de blanc sur leurs ailes noires quand ils rolent.

$\mathrm{Nid}$. Sur le sol dans la mousse, nid fait d'herbe, de radicelles, garni de mousse, de plumes et de poils.

Habitat. Les régions circompolaires, arctiques. Au Canada, ils courent depuis la limite des terres stériles dans la direction du nord, d'un bout a l'autre du continent.

SOUS-ESPECES. Bien que le plectrophane comprenne plusieurs sous-especces, on ne rencontre dans l'est du Canada que le type de l'espece, le plectrophane commun.

Ces oiseaux sont les hôtes d'hiver du sud du Canada, se nourrissant de têtes de mauvaises herbes émergeant de la neige dans les champs de commune propriété et perehant rarement sur les arbres. Une troupe d'oiseaux s'abat sur une neige tachetée de ces têtes d'herbes et la traverse en faisant son petit travail, l'arric̀re-garde s'élevant de temps à autre comme une bourrasque de neige pour prendre les devants; la chose se répète jusqu'à ce que le champ entier soit pareouru.

\section{Genre-Calcarius. Plectrophanes.}

536. Le Plectrophane de Laponie. (LAPLANn longspor). Calcarius lapponicus. L, 6.25. C'est un moineau d'hiver, vivant en troupe et fréquentant les champs de commune propriêté. Ies mâles, brun foncé en dessus fortement rayé de chamois jusqu'à la couronne de la téte, avec un col brun rougeâtre en travers de l'arrière-cou; les joues, la gorge et la bavette sont noires. Blanc, en dessous. Les femelles et les jeunes sont semblables, mais le noir de la face et de la gorge est remplacé par un nuage de brun et de chamois, ou bien il ne se montre que vaguement sur les plumes irrégulièrement dispersées. L'ongle du doigt postérieur est très allongé comme dans l'alouette ordinaire (fig. 44, p. 27).

Traits distinctifs: Par ses mœurs ce plectrophane pourrait etre pris pour le plectrophane des neiges mais le dos fortement rayé, l'absence de blanc sur l'aile et la griffe de derrière allongée sont des traits distinctifs. L'absence de touffe aux oreilles, de jaune à la gorge, le bec de moineau feront distinguer ce plectrophane de l'alouette ordinaire. Une forte taille, l'habitude de se tenir au sol, et sa venue en hiver en troupe feront distinguer le plectrophane de Laponie de tout autre moineau de même coloration.

Indications sur le terrain. L'absence d'amas blanc sur les ailes fera distinguer le plectrophane de Laponie de celui des neiges, et l'absence de touffes aux oreilles et de jaune à la gorge le distinguera de l'alouette ordinaire, oiseaux avec lesquels on est exposé à l'état vivant.

Habitat. C'est une espèce circompolaire qui ne descend vers les régions colonisées qu'en hiver. En Amérique il couve sous les hautes latitudes partout dans ce continent.

SOUS-ESPECES. Dans l'Est on ne rencontre jamais qu'une sous-espece de ce plectrophane de Laponie, celui de l'Est, l'individu type.

Semblable par ses mœurs au plectrophane des neiges il accompagne souvent des bandes de ces oiseaux et d'alouettes ordinaires. 


\section{Genre-Poccetes. Pinson à ailes baies.}

540. Le Pinson à ailes baies. (vesper sparrow). Poccetes gramineus. L, 5.75. Planche XXXI A. Moineau du sol, de couleur terne, délicatement rayé de teintes brunes, en dessus; en dessous, du blanc, avec des raies vaguement indiquées sur les flancs, en travers de la poitrine, et sur les côtés de la gorge. Les épaules sont d'un brun rougeâtre.

Traits distinctifs. Ce pinson peut se distinguer des autres pinsons de couleur terreuse par le brun-rouge des couvertures supérieures de l'aile ou des épaules, et par le blanc sur les plumes extérieures de la queue.

Indications sur le terrain. Le pinson a ailes baies peut facilement être confondu avec le pinson chanteur, a l'état vivant, mais l'absence de la tache pectorale causée par la réunion des raies, et la présence des plumes blanches extérieures de la queue, qui se voient quand l'oiseau vole, sont des marques distinctives. Le pinson niverolle, oiseau d'un gris uniformément foncé, est la seule autre espèce ayant une marque caudale de ce genre, qu'on puisse comparer au pinson à ailes baies.

Nid. Dans l'herbe sur le sol; nid fait d'herbages, de radicelles, de poils, et d'herbes plus délicates à l'intérieur.

Habitat. En tant qu'espèce cet oiseau habite toute la partie tempérée de l'Amérique du Nord. Il couve partout où on le rencontre au Canada.

SOUS-ESPECES. Bien qu'on reconnaisse des sous-espèces de l'Est et de l'Ouest de ce pinson, celui de l'Est, l'individu type, se rencontre seul dans l'est du Canada.

Le pinson a ailes baies peut se comparer au pinson chanteur par ses mœurs, son chant et son apparence générale. Il est moins un oiseau des buissons, toutefois, et d'habitude il fréquente la lisière des champs ou les endroits qui offrent un abri, comme le voisinage des fossés ou des routes. Son chant ressemble a celui du pinson chanteur, mais une oreille cxercée ne s'y trompera pas et saura l'en distinguer.

Valeur économique. C'est un des plus utiles d'entre tous les passereaux. Il cherche sa nourriture au loin dans les champs, plus loin que la plupart des moineaux ordinaires d'été et prend une plus grande quantité d'insectes et de grandes quantités de semences de mauvaises herbes. On trouve du grain dans son estomac, mais la chose s'explique par le fait que c'est du grain perdu avec l'éteule ou le long du chemin. Le pinson a ailes baies mérite donc d'être protégé de toutes les façons.

\section{Genre-Passerculus. Pinsons des prés.}

541. Le Pinson d'Ipswich. (IPswich sparrow). Passerculus princeps. L, 6.25. Ressemble à un gros et très pale pinson des prés (voir l'espc̀ce suivante).

Traits distinctifs. Il se dintingue du pinson des prés par une plus grande taille, une coloration claire et l'absence de la tache jaune en avant de l'œil. En automne, le jaune à la courbure de l'aile est complètement absent. Il se distingue du pinson a ailes baies par une coloration claire, l'absence do rouge sur les épaules, et, au printemps, par du jaune à la courbure de l'aile et en avant de l'cil. Il se distingue du pinson chanteur par sa couleur claire et, au printemps, par du jaune, comme ci-dessus. baies.

Indications sur le terrain. Un pinson très pâle, à peu près de la taille du pinson à ailes

Ce pinson a un habitat restreint et isolé. La seule place où il couve c'est l'île du Sable, à environ 60 milles au sud de Cap Breton. En hiver il émigre et descend la côte de l'Atlantique sans jamais aller loin dans l'intérieur. Il a peu de valeur économique.

542. Le Pinson des prés. (savannah sparrow). Passerculus sandwichensis" $\mathrm{L}, 5 \cdot 68$. C'est un assez petit pinson rayé, en dessus, de brun, de gris cendré et de teintes intermédiaires. En dessous, blanc avec des raies d'un brun tranché sur la poitrine, sur les flancs et, en certains cas, sur la gorge. Du jaune sur la courbure de l'aile et une tache en avant de l'ail. Les oiseaux de l'automne sont lavés d'une teinte chamois, et les marques sont aussi plus légères et plus étendues. 
Traits distinctifs. Le pinson des prés peut se distinguer du pinson chanteur par les taches jaunes en avant de l'œil et sur la courbure de l'aile, et par l'absence de ces raies jointes ensemble qui forment une tache au milieu de la poitrine du pinson chanteur. Il peut se distinguer d'autres pinsons apparentés, des groupes du pinson des sauterelles et du Passerherbulus, par les raies bien tranchées sur un fond blanc en dessous.

Indications sur le terrain. Le pinson des prés peut se reconnaitre sur le terrain par la ligne jaune au-dessus de l'œil et par les notes de sa voix. Son chant est une fine modulation, \& la façon des insectes et dit: atrip-tsip-ion-ri-i-i-i-i-i-iou», les premières notes à peine assez claires pour être entendues, et le tout lancé avec une vigueur qui porte les sons très loin et si fortement qu'on se demande si ces sons viennent de tres pres ou de très loin. Cela ressemble au chant tout pareil du pinson des sauterelles, mais celui-ci omet la note finale.

Nid. Sur le sol; nid fait d'herbes et garni de matières plus délicates.

Habitat. L'Amérique, du nord des rivages arctiques. Le pinson des prés de l'Est, P. s. savanna, occupe l'est de l'Amérique du Nord à l'ouest jusqu'aux prairies, ou il est remplacé par le pinson des prés de l'Ouest.

SOUS-ESPECES. Notre individu, le pinson des prés de l'Est, $P$. s. savanna, est une sous-espèce dont la forme type se rencontre dans les fles Aléoutiennes. Il y a plusieurs autres races géographiques mais les différences entre elles sont trop légères pour étre familières au public.

C'est un oiseau soit des prés humides, des terrains vagues et délaissés, où l'herbe croit en touffes fortes et grossières, et où l'eau est presque à la surface, soit encore des terrains sablonneux et stériles où l'herbe et les mauvaises herbes croissent en touffes éparses, Ce pinson court là comme ferait une souris, et puis s'enlève d'un rapide coup d'aile, souvent avant même qu'on ait pu le bien observer. C'est un oiseau petit, intéressant mais de si minime apparence que le plus souvent l'observateur d'occasion ne le remarque pas.

Valeur économique. En sus de grandes quantités de graines de mauvaises herbes, le pinson des prés consomme plus d'insectes que la plupart des passereaux et plus d'escarbots qu'aucun autre de son espèce. Les insectes saisis comprennent un grand nombre de charançons et d'autres espèces nuisibles. Bien que ce pinson habite des endroits incultes il fréquente aussi des champs cultivés, et cela assez souvent pour faire de lui un aide actif du cultivateur.

\section{Genre-Ammodramus. Pinson des sauterelles.}

546. Le Plnson des sauterelles. (GRAs8hopper sparrow). Ammodramus savannarum. L, 5.38. C'est un petit pinson qui vit beaucoup dans l'herbe. Le dos est marqué de fines et courtes raies d'un chamois brun, cendré et clair, d'un dessin indéterminé; blanc mat en dessous, lavé en travers de la poitrine, d'un chamois clair qui se fane sur les cótés de la gorge. Tache jaune en avant de l'œil; les couvertures supérieures de l'aile et la courbure de l'aile sont jaunes ou jaunâtres.

Traits distinctifs. Les couvertures supérieures de l'aile sont jaunatres et distinctives de l'espèce. La poitrine sans raie et sans tache le fera distinguer de la plupart des autres petits pinsons herbicoles.

Indications sur le terrain. Cette espèce peut être distinguée de la plupart des autres petits pinsons par sa poitrine sans raje et vaguement colorée de chamois. Son chant rappelle la dernière partie de celui du pinson des prés, moins la syllabe finale, et finit peu à peu en $a z-b z-b z-b z-z-z-z)$.

Nid. Sur le sol; nid fait d'herbes et avec un toit arqué.

Habitat. Depuis les Etats-Unis jusque dans l'Amérique du Sud; il traverse régulièrement la frontière canadienne dans le voisinage du lac Erié, et seulement en cet endroit.

SOUS-ESPECES. Le pinson des sauterelles de l'Est est une sous-espèce, A. s. custralis. La forme type se rencontre en dehors des limites du présent oiseau. Une autre espèce se trouve dans l'Ouest. 
Ce pinson doit se chercher dans les prairies herbeuses en même tcmps qu'on y cherche le goglu et l'étoưrneau des prés; mais son habitat se borne décidément à certains lieux.

Valeur économique. C'est un pinson rare, de minime valeur économique et en tout cas ne causant aucun dommage.

\section{Genre-Passerherbulus. Pinson à queue aiguë.}

547. Le Pinson de Henslow. (henslow's sparrow). Passerherbulus henslowi. L, 5. C'est un très petit pinson herbicole. La nuque et le bas du cou sont d'un olive 'jaunâtre; le dos est vinacé, chacune de ces deux couleurs est marquée de courtes lignes de brun. En dessous du blane finement rayé de brun foncé en travers de la poitrine et sur les flancs. Bec gros pour la taille de l'oiseau, les plumes et la queue sont pointues.

Traits distinctifs. La couleur olive et la vinacée sont les couleurs de fond des parties supérieures; la poitrine a de fines raies.

Indications sur le terrain. C'est un petit oiseau qui court dans l'herbe et qu'il est très difficile de faire s'envoler. Il s'élève d'un vol rapide, bas, en zig-zag et se laisse rechoir dans l'herbe a vec une soudaineté inattendue. Le meilleur moyen de l'identifier à l'état vivant c'est d'écouter ses notes, les deux sons si-slik très pénétrants d'un si petit volume que d'en être presque difficile à entendre même de très près, et cependant, assez prononcés pour avoir une force considérable de portée.

Nid. Sur le sol; nid fait d'herbes et extraordinairement bien caché.

Habitat. Le pinson de Henslow de l'Est se rencontre dans l'est du Canada seulement dans le sud de l'Ontario. Une autre sous-espèce se trouve dans l'Ouest.

SOUS-ESPECE. La forme de ce pinson de l'Est c'est le pinson de Henslow de l'Est qui est la sous-espèce type de la race.

Il peut arriver qu'on se trouve au milieu même d'une colonie de pinsons de Henslow sans qu'on s'en doute, pour la raison qu'on les voit rarement, à moins que leurs notes n'attirent l'attention. Les prés herbeux dont on laisse perdre l'herbe sont leurs séjours de prédilection.

548. Le Pinson de Leconte. (Leconte's sparrow). Passerherbulus lecontei. I, 5. C'est un très petit pinson herbicole, habile à se jouer de vous. En dessus, couronne brun foncé avec raie médiane d'un chamois clair, la nuque est vinacée avec raies gris chamois, le dos brun foncé avec raies d'un chamois clair bien tranchées. En dessous, blanc lavé d'ocre sur la poitrine, la gorge et les joues.

Traits distinctifs. Lre contraste de la raie médiane d'un chamois clair avec la nuque vinacée et le dos brun foncé.

$N i d$. Sur le sol; nid fait d'herbes fines.

Habitat. Le centre de l'Amérique du Nord. C'est un oiseau des prairies qui ne sc rencontre qu'accidentellement en dedans du territoire dont s'occupe le présent ouvrage.

Cette espèce n'a été signalée qu'une seule fois dans l'est du Canada, à Toronto. A cause de ses habitudes toutes pareilles à celles des souris, ce pinson est difficile à trouver ou à reconnaitre, si on le trouve; il peut donc être plus commun qu'on ne croit.

549. 1. Le Pinson à queue aiguë. (NElson's sharp-TaIled sparrow). Passerherbulus nelsoni. L, 5.9. Un petit pinson herbicole. En dessus, la ligne médiane de la couronne, d'un bleu ardoisé avec bordure brun foncé, et ligne d'ocre au-dessus de l'œil. Une bande vaguement ardoisée et olive en travers de la nuque et des épaules. Dos à raies bien tranchées d'un brun ardent et chamois pâle lavé d'un ton ardoisé clair. En dessous du blanc avec poitrine de couleur ocre qui s'étend plus ou moins aux joues, aux flancs, et pâle sur la gorge. La puitrine est quelquefois marquée de raies indistinctes et plus foncées; les flancs le sont toujours.

Traits distinctifs. La raie médiane de la couronne est de couleur ardoisée; de longues raies sur le dos de même couleur.

Indications sur le terrain. La coloration générale de couleur chamois ou jaune foncé en dessous; la ligne jaune au-dessus de l'oil et les raies un peu vagues sur la poitrine.

Nid. Sur le sol; nid fait d'herbes délicates. 
Habitat. L'est de l'Amérique, surtout au nord des Etats-Unis et au sud du Canada. La forme type est limitée aux prairies et l'autre à la côte de l'Atlantique.

SOUS-ESPECES. Le pinson à queue aiguē comprend deux sous-espèces: la forme type, le pinson a queue aiguê, race de la Prairie; et le pinson à queue aiguë d'Acadie, $P . n$. subvirgatus, qui est une race de l'extrême Est. Le pinson d'Acadie se cantonne dans les marécages salins de la côte orientale de l'île du Prince-Edouard et au-dessous du golfe Saint-Laurent. Sa poitrine et sa gorge sont plus claires, couleur cbamois plutôt que d'ocre, il est distinctement quoique faiblement rayé sur la poitrine. La coloration du das est plus effacée et plus étendue; la ligne médiane de teinte ardoisée est plus large et fait moins contraste avec la brune. On ne court guère le risque de les confondre car les races peuvent être réparties géographiquement avec beaucoup de certitude. Le pinson à queue aiguẻ ne se rencontre que par occasion et par accident dans la région des grands lacs, tandis que le pinson d'Acadie est en réalité confiné dans le voisinage de l'eau salée.

Les pinsons à queue aiguë sont des oiseaux de marécages, courant dans les herbes, s'y cachant et refusant de s'envoler jusqu'à ce qu'ils y soient forcés.

\section{Genre-Chondestes. Pinsons ordinaires.}

552. Le Pinson ordinaire. (LARE SPARRow). Chondestes grammacus. L, 6.25. C'est un pinson d'apparence frappante et portant des marques bien visibles. En dessus, rayé de brun et de brun chamois, en dessous blanc avec flancs chamois. Couronne brun châtain avec ligne médiane visible et pâle. Les couvertures des oreilles sont brun châtain en violent contraste avec la face blanche; puis, trois lignes noires bien tranchées, l'une en travers de l'autre, une autre depuis la mandibule inférieure jusqu'aux couvertures de l'oreille; une dernière le long des côtés de la gorge. Les plumes extérieures de la queue, les extrémites de toutes les autres, sauf de celles du milieu, sont blanches.

Traits distinctifs. Les couvertures des oreilles d'un brun rougeâtre brillant, contrastant avec la face noire et blanche sont des caractères très distinctifs.

Indications sur le terrain. La tête et la face fortement marquées et la forte quantité de blanc à la queue sont des indications faciles à reconnaitre.

Nid. Sur des arbres plutôt bas et dans les buissons; nid fait d'herbes et garni d'herbes plus fines, de radicelles et de poils.

Habitat. De la vallée du Mississippi vers l'Ouest. Le pinson ordinaire de l'Est, la forme type, s'étend depuis les grandes plaines a l'Est et au Nord jusque dans le sud de l'Ontario. Une autre sous-espece se rencontre dans l'Ouest.

SOUS-ESPECE. La sous-espèce de l'Est de cet oiseau est le pinson ordinaire de l'Est, la forme type de l'espèce.

Le pinson ordinaire est rare dans l'est du Canada. Il faut l'aller chercher dans les terrains à broussailles incultes et le long des lisières boisées des champs.

\section{Genre-Zonotrichia. Gros Pinsons des bois.}

553. Le Pinson de Harris. (harris' sparrow). Zonotrichia querula. L, 7.50. Gros pinson rayé de brun et de brun cendré en dessous; blanc, en dessus. La couronne, la face, la gorge et le front entièrement noirs, et passant à l'état de raies sur les flancs.

Traits distinctifs. Chez les adultes, la face est noire comme si elle avait été trempée dans de l'encre qui se serait répandue sur la couronne et la gorge.

Nid. Sur le sol; au pied d'un bouleau nain; nid fait d'berbes et rarement découvert.

Habritat. Les régions des prairies, en Amérique; au nord jusqu'aux confins des terres stériles.

Cet oiseau peut être plus commun qu'on ne croit dans l'ouest de l'Ontario, près de la frontière du Manitoba, mais c'est à coup sûr la seule région où l'on puisse rechercher cette espèce. On a signalé un de ces pinsons à London, Ontario, mais ce n'a été, naturellement, qu'accidentellement. 
554. Le Pinson à couronne blanche. (WHITE-Crowned SPARrow). Zonotrichia leucophrys. L, 6.88. Planche XXXI B.

Traits distinctifs. Le seul qu'on puisse probablement confondre avec le pinson a gorge blanche, l'espèce suivante, et il est possible de l'en distinguer, sous quelque plumage que ce soit, par l'absence de la tache jaune en avant de l'œil et, chez l'oiseau adulte, par la gorge d'un gris presque uniforme au lieu de la gorge très blanche de cette espèce.

Indications sur le terrain. La couronne blanche et l'absence de blanc a la gorge ou de la tache jaune en avant de l'œil. A l'état vivant le dos a une couleur plutốt grise que rougeatre.

Nid. Sur le sol ou dans les buissons un peu bas; nid fait d'herbe et de petites fibres végétales, de radicelles, etc.

Habitat. L'Amérique du Nord à partir de la limite des arbres dans la direction du Sud. Notre pinson à couronne blanche de l'Est, le type de l'espèce, s'étend vers les provinces des prairies de l'Ouest, où sa place est prise par une sous-espèce, le pinson de Gambel, Z. i. gambeli.

SOUS-ESPECES. Quoique trois sous-espèces de cette espèce soient reconnues au Canada, une seule, le pinson à couronne blanche de l'Est, la forme type, se rencontre dans l'Est.

C'est l'un des plus beaux pinsons. Lors même qu'il manque de couleurs très brillantes, sa couronne noire et blanche, en contraste frappant avec le gris de la gorge et du cou, lui donne un air de distinction. Son chant aussi est mélodieux, vu qu'il a cette limpidité qui distingue le chant de son proche parent le pinson a gorge blanche; malheureusement on ne l'entend surtout à son avantage que dans les parages du nord où a lieu sa couvaison.

Valeur économique. Bien qu'il ne séjourne que pendant deux semaines, au printemps et en automne, dans les régions cultivées, il y fait cependant un utile travail. Les semences de mauvaises herbes forment une partie considérable de ses aliments, la renouée et le chiendent y jouant le principal rôle. Les insectes ne constituent qu'une faible partie de sa nourriture.

558. Le Pinson à gorge blanche. Le giFfledr. (WHite-THRoated SPARrow). Zonotrichia albicollis. $\mathrm{L}, 6 \cdot 74$. Planche XXXII A.

Traits distinctifs. Quand il revêt sa livrée de l'age adulte il est facile de le confondre avec le pinson à couronne blanche, précédemment mentionné, mais sa gorge absolument blanche, et la tache jaune en avant de l'œil sont distinctives. Les jeunes oiseaux peuvent être pris pour des pinsons de marais, mais la tache jaune et la coloration plus rouge les feront distinguer toujours l'un de l'autre.

Indications sur le terrain. La gorge blanche, les lores jaunes, et le dos rougeâtre au lieu de grisatre, feront facilement distinguer les adultes d'avec les pinsons à couronne blanche. Les jeunes sont un peu plus difficiles à identifier et à distinguer de plusieurs autres espèces, mais au cas où ni la tache lorale jaune, ni la gorge vaguement blanche que suggere parfois le pinson des marais, ne seraient reconnaissables, le dos beaucoup plus rouge suffira ordinairement a l'identification des pinsons a gorge blanche.

Nid. Sur le sol ou dans des buissons peu élevés; nid fait d'herbes grossières, de radicelles et de mousse, garni d'herbes plus fines.

Habitat. L'est de l'Amérique du Nord jusqu'à la limite des arbres au nord. Il couve partout où on le trouve au Canada sauf dans les régions les plus méridionales.

Nous avons ici le plus fameux chanteur des forêts du Nord. Quand il chante de son mieux, sa voix est limpide, flatée, lentement mesurée pour siffler ce qu'on a cru pouvoir rendre par les mots: "Hard-times-can-a-da-can-a$d a-c a n-a-d a)$, ou bien "Poor-Bill-Pea-body-Pea-bo-dy-Pea-body). Le pinson à gorge blanche est un oiseau des broussailles; les fourrés embroussaillés, les buissons de ronces, en tas tout près des champs, constituent sa demeure favorite. Dans la plupart des régions cultivées du Canada, cet oiseau n'est qu'un oiseau de passage, et son chant se fait rarement entendre. En automne quand les jeunes oiseaux s'envolent vers le sud, leurs notes se font entendre en des sons adoucis et abrégés. 
Valeur économique. Le pinson à gorge blanche est un oiseau de beaucoup de valeur. Il a de l'importance comme destructeur de semences de mauvaises herbes, surtout de renouée; il consomme force insectes et un peu de fruits sauvages. Comme cette espèce arrive en nombre considérable dans les régions fortement cultivées, au commencement de l'automne, son influence sur la masse des semences de la saison suivante ne peut être que très prononcée.

\section{Genre-Spizella. Petits Pinsons des bois.}

559. Le Pinson de montagne. (TREe sparnow). Spizella monticola. I, $6 \cdot 36$. Planche XXXII B.

Traits distinctifs. Ressemble beaucoup au petit pinson a couronne rousse et au pinson des champs mais est plus gros, le bec jaune, a pointe noire, au lieu d'etre noir comme chez le précédent ou couleur cannelle comme chez le dernier, et avec une tache foncée à moitié cachée au milieu de la poitrine.

Indications sur le terrain. Calotte brun rouge, barres blanches des ailes en évidence; gorge gris cendré, et tache foncée au milieu de la poitrine sans tache et uniformément colorée.

Nid. Sur le sol ou tout près; nid fait d'herbes, de radicelles et de poils.

Habitat. L'est de l'Amérique du Nord. Couve dans l'extrême nord par delà des limites de la civilisation; hiverne dans le nord des Etats-Unis.

SOUS-ESPECES. Les sous-espèces de l'Est et de l'Ouest du pinson de montagne se rencontrent au Canada. La premiere est la forme type et la seule qui se trouve dans la région dont s'occupe le présent ouvrage.

Dans la foule de pinsons qui se donnent rendez-vous au sein des bouquets d'arbrisseaux, en automne, ou qui y reviennent de bonne heure au printemps, on est sûr de trouver le pinson de montagne. Dans les parties méridionales du Dominion, il y reste quelquefois tout l'hiver mais ailleurs il passe en migrateur. C'est un petit oiseau très propre, dont le modeste chant, au début du printemps, est fort bien accucilli après le long silence de l'hiver.

Valeur économique. Ce pinson est précieux parce qu'il détruit beaucoup de semences de mauvaises herbes et qu'il parait n'avoir aucune mauvaise habitude.

560. Le Petit Pinson à couronne rousae. (Chipping sparrow). Spizella passerina. I, 5.37. Planche XXXIII A.

Traits distinctifs. C'est un oiseau bien connu, à distinguer par la taille du pinson des marais et du pinson de montagne, qui eux aussi, comme lui, ont une calotte rouge; on le distinguera aussi d'eux et du pinson des champs par son bec noir et par la raie noire en travers de l'oil. Les jeunes ont la téte rayée et ressemblent extrémement au pinson coulcur d'argile, oiseau de l'Ouest qui, à l'occasion peut être confondu avec eux dans l'extréme ouest de l'Ontario. Ie pinson à couronne rousse, cependant, a un croupion couleur d'ardoise au lieu d'être d'un olive chamois.

Indications sur le terrain. C'est un petit oiseau, mince avec une calotte rouge, son chant prolongé, traśrant, n'est qu'une suite de gazouillements sans accent, qui se mélent pour ne former qu'une trille unique, soutenue, fort caractéristique.

Nid. Dans les arbres ou dans les buissons; nid fait d'herbes, de radicelles, et de fibres nombreuses entrelacées avec de longs poils. La quantité de crín de cheval employé dans ce nid eat l'origine d'un des noms populaires anglais de cet oiseau (Hair-Bird).

Habitat. L'est de l'Amérique du Nord jusqu'au nord de la civilisation. Il couve au Canada partout où il se trouve.

SOUS-ESPECES. Le type du pinson a couronne rousse qui se rencontre dans l'est du Canada, c'est le pinson a couronne rousse de l'Est, l'individu type. Une autre sousespèce se rencontre dans l'Ouest. 
Le petit pinson à couronne rousse est rarement éloigné du voisinage des habitations de la banlieue ou des villages, car il s'approche volontiers des maisons et fréquente les vergers, les arbres feuillus, la cour devant la maison et même le pas de la porte d'entrée. Il n'a pas peur de l'homme, mais, quoique ne l'évitant pas, il ne se fait pas remarquer de lui, par suite de ses mœurs paisibles et modestes.

Valeur économique. Le petit pinson à couronne rousse est un plus grand insectivore qu'aucun autre oiseau de la même famille. En réalité, pendant tout lo mois de juin, le 93 pour cent de sa nourriture se compose d'insectes, dont une fraction minime, le 1 pour cent seulement, renferme des espèces utiles telles que les coléoptères carnassiers et les guêpes parasites. La moyenne de l'année est de 38 pour cent d'inscetes, ct pour les mois que le pinson passe au Canada la moyenne doit être beaucoup plus élevée. La matière végétable qu'il consomme se compose de petites semences de mauvaises herbes parmi lesquelles le panic sanguin, le bon-henri et la renouée prédominent. On sc convaincra sans peine qu'un oiseau qui a des goûts si désirables et qui survient dans le voisinage immédiat d'un jardin est infiniment utile et mérite d'être protégé de toute façon.

561. Le Pinson couleur d'argile. (Chay-Colodren sparrow). Spizella pollida. L, 5.20. C'est un petit pinson dont les parties de dessus sont rayées d'un chamois pâle et d'un brun foncé jusqu'a la couronne, où se remarque une bande médiane blanchâtre. Un col un peu vague d'une couleur ardoisée étendue vers l'arrière-cou. Les dessous sont blancs, légèrement teintés de chamois sur les flancs.

Traits distinctifs. En raison de sa taille et de ses mœurs, cet oiseau ne saurait être confondu avec aucun autre pinson qu'avec celui à couronne rousse; mais le dos est plus pâle que chez ce dernier, plus couleur d'argile, et le croupion au lieu d'être vaguement ardoisé est quelque peu d'un olive chamois.

Nid. Sur le sol ou dans les buissons; nid fait d'herbes et garni de poils.

Habitat. L'intérieur de l'Amérique. C'est un résident des prairies qui va au nord jusqu'aux limites des provinces des prairies et qui ne se rencontre que par accident dans l'est du Canada sauf peut-être dans le pays qui touche à la frontière du Manitoba.

C'est un oiseau de l'Ouest, qui se montre rarement dans les confins de l'ouest de la région dont s'occupe le présent ouvrage.

563. Le Pinson des champs. (FIELd sparrow). Spizella pusilla. L, 6.68. C'est un petit pinson de la même couleur, en général, que le pinson à couronne rousse, mais dont les couleurs sont atténuées, étendues et fondues ensemble. La couronne rouge est plus foncée et peu manifeste, la ligne noire en travers de l'œil est absente. Le bec est couleur cannelle au lieu de noir.

Traits distinctifs. Les traits qui précèdent suffisent à distinguer l'oiseau.

Indications sur le terrain. Couronne rouge mat; absence de marques faciales autres qu'une pointe rouge aux extrémités des couvertures des oreilles; bec couleur cannelle. Son chant est le trait caractéristique le plus facile à reconnaitre, et une fois connu il constitue le meilleur moyen d'identification.

Nid. Sur le sol ou dans des buissons peu élevés; nid fait d'herbes plutôt grossières, tiges d'herbe et radicelles, garni d'herbes plus délicates et de poils.

Habitat. L'est de l'Amérique; le Canada dans les parties les plus colonisées; un habitat cependant plutốt local que général mais un oiseau étrangement absent de certaines localités comprises dans son territoire.

SOUS-ESPECES. Le pinson des champs comprend deux sous-espèces: celle de l'Est et celle de l'Ouest; la première, la forme type, est la seule qui se rencontre dans l'est du Canada.

Le pinson des champs est un oiseau qui n'attire pas les regards et qui, quoique très commun, ressemble tant au pinson à couronne rousse avec son plumage habituel qu'on peut le confondre avec lui. C'est un oiseau des champs et des rangs de clôture, quoiqu'il ne soit ni timide ni extrêmement solitaire, il faut le chercher et l'écouter pour le trouver. 
Valeur économique. Très semblable au pinson à couronne rousse par ses habitudes alimentaires; prenant quelques insectes utiles mais cependant pas en si grand nombre qu'il en devienne nuisible.

\section{Genre-Junco. Pinsons niverolles.}

567. Le Pinson niverolle. (Jonco). Junco heimalis. L, 6.27. Planche XXXIII B.

Traits distinctifs. Gris ardoisé foncé sur tout le dessus du corps et la poitrine, contrastant violemment avec les dessous blancs.

Indications sur le terrain. Ligne tranchée de la poitrine foncée contre les dessous blancs, et contre les plumes extérieures de la queue qui se montrent pendant lc vol.

Nid. Sur le sol ou tout près; nid fait d'herbes, de mousse, de radicelles et garn d'herbes plus délicates et de longs poils.

Habitat. En tant qu'espèce, toute l'Amérique jusqu'à la limite des arbres. Le pinson niverolle couleur d'ardoise s'étend à l'ouest jusqu'à l'Alberta.

SOUS-ESPECES. Les pinsons niverolles comprennent plusieurs sous-especes, dont une seule, le pinson niverolle ardoisé, la forme type, se rencontre dans l'est du Canada.

Le pinson niverolle avec sa poitrine noire, son bec de couleur claire, sa queue à bordure blanche est remarquable parmi les grandes bandes de pinsons qui sont de passage ou qui séjournent pendant le printemps et l'automne.

Valeur économique. L'influence du pinson niverolle en agriculture est presque entièrement bienfaisante. Pendant son séjour dans les parties les plus populeuses du pays il consomme de grandes quantités de semences de mauvaises herbes. Les insectes qu'il mange sont presque tous nuisibles. On ne peut pas lui en vouloir puisqu'il ne fait de mal ni aux récoltes ni aux fruits.

\section{Genre-Melospiza. Pinsons chanteurs.}

581. Le Pinson chanteur. le rossignol. (song sparrow). Melospiza melodia. L, 6.30. Planche XXXIV A.

Traits distinctifs. Il ressemble plutôt au pinson à ailes baies par la taille et la coloration générale, mais les tons sont plus foncés et plus accusés; il lui manque les plumes blanches extérieures de la queuc. Les raies de la poitrine sont aussi plus tranchées, d'un brun plus foncé, et réunies vers le milieu en une tache bien définie. L'absence de la bande jaune au-dessus de l'cil distingue le pinson chanteur du pinson des prés, et la poitrine aux raies tranchées le distingue de l'un quelconque des autres pinsons de taille et de mours analogues.

Indications sur le terrain. Poitrine à bandes tranchées et tache centrale. L'absence des plumes blanches extérieures de la queue empêchera qu'on confonde ce pinson avec son confrère à ailes haies; la qucue plus longue, l'absence des lora jaunes, la voix et la tenue générale feront distinguer le pinson chanteur de celui des prés.

Nid. Sur le sol, plus rarement dans les buissons; nid fait d'herbes grossières, de radicelles, de feuilles mortes, de bandes d'écorce, etc., garni d'herbes délicates et quelquefois de longs poils.

Habitat. En tant qu'espèce, le pinson chanteur habite partout en Amérique jusqu'à Ia limite des arbres. Notre individu de l'Est s'étend à l'Ouest jusqu'aux provinces centrales des prairies.

SOUS-ESPECES. Le pinson chanteur est une espèce qui habite un vaste territoire et qui comprend plusieurs sous-espèces, dont une vingtaine sont reconnues dans l'Amérique du Nord et plusieurs autres sont proposées. La plupart de ces sous-espèces sont des formes de l'Ouest, originaires des terres accidentées de la Côte du Pacifique ou des colonies isolées et des conditions variées ont favorisé de nombreuses déviations du type. Dans l'est du Canada, la forme reconnue est celle du pinson chanteur de l'Est, M. $m$. melodia, la race type. 
Il est difficile de se faire une idée juste et sans parti pris de la place qu'occupe le pinson chanteur dans l'orchestre ailé. Ce pot-pourri de gazouillements et de trilles donne une certaine durée à un chant qui offre à ceux qui l'écoutent avec plaisir une jovialité, une limpidité et une douceur qu'il est difficile de surpasser. Cet oiseau semble être partout à la fois. Il vit parmi les arbrisseaux qui entourent la maison, et il est l'un des hôtes habituels du jardin. Il fréquente les bosquets à la lisière des bois ou sur le bord des ruisselets. Ies forêts profondes et les champs découverts sont les seules places d'où il est en général absent, mais quelquefois, là même, il nous surprend par une fanfare de notes limpides.

Valeur économique. Le nombre considérable de ces pinsons chanteurs leur donne une importance très grande aux yeux du cultivateur. Une analyse des aliments de cet oiseau fait voir que le 2 pour cent seulement est composé d'insectes utiles et que le 18 pour cent est formé par des insectes nuisibles. Le grain qu'on laisse perdre constitue le 4 pour cent et les semences de mauvaises herbes le 50 pour cent. Le reste est composé de fruits sauvages et d'autres matières sans importance. On peut voir par ce qui précède que le pinson chanteur a une valeur économique considérable. Des recherches à ce sujet ont montré qu'un quart d'once de scmences de mauvaises herbes par jour est une évaluation assez exacte de la quantité consommée par un pinson mangeur de ces semences. Pendant les neuf mois que le pinson chanteur est parmi nous dans une localité moyenne de l'est du Canada, la consommation se monte à quatre livres et quart par individu, et par année. Si nous estimons qu'il peut y avoir 75 pinsons chanteurs par mille carré-chiffre qui est très loin d'être exagéré-nous arrivons à un total-pour les parties cultivées de l'Ontario-de plus de onze mille tonnes de ces semences détruites chaque année par cette espèce, à elle seule.

583. Le Pinson de Lincoln. (Lincoln's sparrow). Melospiza lincolni. L, 5.75. Ressemble au pinson chanteur mais avec une ceinture chamois en travers de la poitrine, laquelle est aussi parsemée de petites taches très fines.

Traits distinctifs. Les traits précédents distingueront le pinson de Lincoln de tous les autres avec lesquels il pourrait être confondu.

Indications sur le terrain. De bons yeux feront voir la poitrine vaguement chamois et les taches fines de l'oiseau. A cela près, on le distinguera avec peine, a l'état vivant, du pinson chanteur. Le dos est plutôt plus gris que le pinson chanteur, et de là résulte le soupçon qu'il pourrait s'agir du pinson de Lincoln, soupşon que viendraient confirmer d'autres caracteres.

Nid. Semblable à celui du pinson chanteur, sur le sol.

Habilat. L'espèce est répandue par toute l'Amérique, couvant comme elle fait, dans les bois conifères du Nord.

SOUS-ESPECES. Les pinsons de Lincoln, soit ceux de l'Est soit ceux de l'extrême Ouest, peuvent être différenciés en deux espèces. Le type qui occupe le territoire ici mentionné est le pinson de Lincoln de l'Est, M. l. lincolni, la race type.

Bien qu'il soit rare, ce pinson est fort intéressant. Autant qu'aucun oiseau, il a élevé à la hauteur d'un art le talent de se cacher dans les broussailles. Dès qu'il est dérangé il sautille sur une branche, d'où il peut bien voir ce qui se passe; il regarde un instant l'intrus, et puis plonge dans l'enchevêtrement des broussailles, et puis.... ni vu ni connu! La recherche la plus active ne donnera ensuite pas autre chose qu'une vue rapide d'une ombre brune qui disparaitra dans le plus prochain tas de broussailles. Cette espèce se réduit à un hôte passager, migrateur à travers les parties colonisées du Canada, et se fait rare. 
584. Le Pinson des marals. (swamp sparrow). Melospiza georgiana. L., $5 \cdot 89$ Tres semblable au pinson chanteur, mais d'une coloration plus forte et moins fondue, et sans aucune raie ni marque distincte.

Traits distinctifs. Le pinson des marais est difficile a distinguer de plusieurs autres formes de coloration et de taille analogues. On peut le distinguer du pinson chanteur, très facile à confondre avec lui, par la poitrine sans raie et, chez les oiseaux adultes, par la couronne rouge. Les jeunes oiseaux d'automne ressemblent fort aux jeunes pinsons a gorge blanche, mais ils n'ont pas la tache lorale vaguement jaune; ils ne sont pas non plus aussi uniformément rouges sur le dos, et ordinairement ils ont comme l'esquisse d'une barre cendrée en travcrs des épaules à la base du cou, unc teinte cendrée a la couronne, et des lignes sourcilières qui manquent à cette espèce. Il peut se distinguer du pinson de montagne par l'absence de la tache foncée au milieu de la poitrine ou des barres blanches de l'aile.

Indications sur le terrain. Sa ressemblance avec le pinson chanteur mais sans les raies de la poitrine, l'absence de la tache jaune lorale du pinson à gorge blanche, ou des barres de l'aile du pinson de montagne. En été, quand le pinson de montagne n'est pas sur les lieux, la calotte rouge est distinctive.

Nid. Semblable a celui du pinson chanteur, sur le sol, quelquefois dans l'herbe.

Habitat. L'Amérique du Nord à l'est des prairies; il couve dans la plupart des parties inhabitées du Canada.

Comme l'indique son nom, ce pinson fréquente les terres basses et humides et les marais. Les autres herbes et les bords des marécages sont les parages qu'il affectionne. Vers la fin de l'automne il rejoint les grandes bandes de passereaux de toute sorte dans les buissons et sur les barrières entrecroisées où il se trouve alors dans un plus proche voisinage de l'homme.

Valeur économique. Le genre d'aliments que recherche le pinson des marais ne diffère pas grandement de celui des pinsons qui lui ressemblent. Comme il passe sa vie dans les terrains incultes, son alimentation est sans importance.

\section{Genre-Passerella. Pinsons fauves.}

585. Le Pinson fauve. (Fox sparrow). Passerella iliaca. L, 7.26. C'est un assez gros pinson. En dessus il est d'un brun rougeâtre brillant, entièrement brun d la queue et au croupion, mais avec une teinte ardoisée mate visible a travers le rouge aur l'arrière-cou et la couronne. En dessous, blanc fortement taché et avec des raies rouges comme le dos, sur les côtés de la gorge, en travers de la poitrine et sur les flanes. La gorge est presque dépourvue de marques et les taches tendent à se grouper sur la poitrine en une tacbe centrale.

Trails distinctifs. La taille plutôt grande et la coloration générale d'un rouge fauve brillant sont distinctives.

Indications sur le terrain. Coloration d'un rouge intense surtout sur le croupion et la queue. La grive solitaire lui ressemble beaucoup quand elle vole, mais le haut du dos est plus olivacé.

Nid. Sur le sol ou sur des arbres peu élevés et dans les buissons; nid fait d'herbes grossières, garni d'herbes délicates, de poils, de mousse et de plumes.

Habitat. En tant qu'espèce le pinson fauve a pour territoire toute l'Amérique du Nord, au nord jusqu'à la limite des arbres. Le type canadien, le pinson fauve de l'Est, s'étend à l'ouest jusqu'au pied des Montagnes Rocheuses, où sa place est prise par plusieurs autres sous-espèces.

SOUS-ESPECES. Le pinson fauve est une espèce qui présente beaucoup de variétés et qui se préte a des formes nouvelles, aussi distingue-t-on plusieurs sous-espèces bien caractérisées. Celle qui se rencontre dans l'est du Canada, c'est le pinson fauve de l'Est, $P$. i. iliaca, la race type.

Ce pinson ne demeure dans les parages eivilisés que pendant quelques jours au printemps et en automne. Il lui arrive quelquefois, au printemps, de nous saluer d'une voix pleine et limpide que peu d'autres oiseaux sauraient égaler et qu'aucun, peut-être, ne serait capable de surpasser. 
Valeur économique. Ce pinson ne séjourne que trop peu de temps au milieu de nous, et en trop petit nombre, pour $y$ avoir beaucoup d'importance aux yeux du cultivateur. Il mange un peu plus de fruits que la majorité des pinsons, mais dans le temps de ses visites, il n'y a que peu de fruits cultivés à sa disposition. Quand à la partie de son alimentation faite d'inscctes et de semences de mauvaises herbes, elle n'est pas de nature à alarmer qui que ce soit.

\section{Genre-Pipilo. Pinsons aux yeux rouges.}

587. Le Pinson aux yeux rouges. (Towhes). Pipilo erythrophthalmus. L, $8 \cdot 35$. Chez le mâle tous les dessus sont d'un noir pur, y compris la queue, la tête, la gorge et la poitrine, où ce noir se détache en une ligne tranchée sur le blanc clair des dessous. Les flancs larges sont rougeâtres ou bais. Quelques bordures blanches des plumes qui servent au vol, et beaucoup de blanc sur les bords de la queue. La femelle est toute pareille mais chez elle le noir est remplacé par du brun rougeâtre.

Traits distinctifs. Par suite de sa grande taille, de sa longue queue et de sa remarquable coloration, ce pinson ne peut guère être pris pour aucun autre oiseau. Les jeunes oiseaux ne donnent pas beaucoup l'idée de ces couleurs distinctivcs, car cette coloration est vague, généralement rouilleuse, à peine rayée, et cela encore de façon interrompue; mais la longue queue, la taille et les contours généraux sont d'habitude suffisants à faire reconnaitre l'oiseau.

Indications sur le terrain. Si l'on voit bien la tête et le dos ou noirs ou bruns, les flancs rouges, cette espèce ne peut guère être prise pour une autre. Iorsque l'oiseau s'élánce dans les dessous des buissons, le noir et le blanc si fortement accusés des ailes et de la queue du mâle, ou le brun rougeâtre et le blanc sur la queue de la femclle sont facilement reconnaissables.

Nid. Sur le sol ou tout près, nid fait de feuilles mortes, de bandes d'écorce et garni d'herbes délicates.

Habilat. L'est de l'Amérique du Nord, depuis le Canada méridional jusqu'au golfe du Mexique. Il ne se rencontre au Canada que le long de la frontière méridionale et n'est commun que dans la région inférieure des grands lacs.

SOUS-ESPECES. Le pinson aux yeux rouges de l'est du Canada, P. e. erythrophthalmus, est le pinson aux yeux rouges de l'Est, le représentant typique de la race.

Le pinson aux yeux rouges est un oiseau des broussailles et des lisières de bois, où sa note " $C h i-w i)$ ou " $T i-w i)$ est un son bien connu. Il aime à se percher au bout d'une branche isolée dans la broussaille et à faire entendre son petit cri limpide de "dik-iou-tchidoll, tchiddoll, tchiddoll». S’il est dérangé il se laisse choir dans les broussailles du pied de l'arbre, sa livrée noirc et blanche y brillant un instant, puis il disparaît dans l'enchevêtrement des plantes d'où il se laisse apercevoir et fait entendre son "Tchi-wi " habituel et inquisitif. Pour chercher sa nourriture il gratte la surface comme une poule, faisant voler les feuilles mortes tout autour de lui.

\section{Genre-Cardinalis. Cardinaux.}

593. Le Cardinal gros-bec. (CARninaL). Cardinalis cardinalis. L, 8.25. C'est un grand pinson et un gros-bec typique. Le mâle est d'un rouge cardinalice brillant avec une pointe de noir autour de la base du bec et de la gorge. Les deux sexes ont une buppe très visible et aussi protubérante que celle du geai buppé. La femelle est d'un chamois ardent, comme coloration, presque blanche en dessous et d'un olive-chamois sur le dos; les ailes, la queue, et la buppe ayant presque la couleur rose du mâle. La face et la gorge noires du mâle sont vaguement indiquées.

Traits distinctifs. Impossible de le prendre pour autre chose que ce qu'il est. Le grosbec des pins peut faire penser au cardinal, mais le rouge n'est jamais si général ni si brillant, puis il n'a pas la huppe ni la marque noire si frappante de la face. Le tangara écarlate est d'un rouge aussi brillant, mais il n'a ni la huppe ni la marque faciale, puis les ailes et la queue sont noires. 
Indications sur le terrain. La coloration rouge de tout l'oiseau, le jet de rouge ardent sur les ailes et la queue de la femelle; la huppe en saillie et le gros bec rouge dans les deux sexes.

Nid. Dans les buissons, nid fait de ramilles, de radicelles et bandes d'écorce, et garni d'herbes et de radicelles.

Habitat. Le cardinal, dans ses diverses sous-espèces, occupe un vaste territoire aux Etats-Unis; la forme type traverse la frontière canadienne, en général le long de l'extrémité ouest du lac Erié. Il se présente ou comme individus séparés ou en groupes isolés soit la soit dans des localités voisines. Le cardinal de l'Est, celui dont il est ici question, est l'individu type de l'espece.

Le cardinal gros-bec n'habite pas, en général, partout au Canada, mais il est toujours à demeure là où on le rencontre. Sa coloration somptueuse et son sifflet retentissant lui donnent un charme de plus. Il peut paraître étrange que cet oiseau du Sud se rencontre parfois au Canada, mais dans quelques parages le long du lac Erié on le voit non seulement de façon régulière mais il y est même commun. Il conviendrait de le protéger de façon étroitc et à cause de sa beauté et aussi pour d'autres raisons pratiques.

Valeur économique. Le cardinal se nourrit surtout de sauterelles, de cigales, de doryphores des pommes de terre, des hannetons de rosiers, des insectes de prunes ct de ccrises, des chenilles, des charançons, et d'autres fléaux destructeurs. En outre il mange les semences des mauvaises herbes en grand nombre et un peu de fruit sauvage. Il n'y a aucune preuve qu'il nuise aux variétés cultivées.

\section{Genre-Zamelodia. Gros-becs.}

595. Le Gros-bec à poitrine rose. (ROsE-Breasted Grosbeak). Zamelodia ludoviciana. L, $8 \cdot 12$. Planche XXXIV B.

Traits distinctifg. Le mâle avec son dos noir et sa bavette rose ne peut se confondre avec aucun autre oiseau. Ia femelle est dans l'est du Canada le seul gros-bec à raies bien tranchées. Les jeunes malles d'automne ressemblent beaucoup à la femelle, mais il y a une légère teinte rose qui apparaft au travers du chamois de la poitrine. Ils varient beaucoup, mais on peut ordinairement apercevoir les indices des plumages plus accusés du printemps.

Indications sur le terrain. Une vue complète de l'un ou de l'autre sexe avec la coloration caractéristique de chacun et du gros bec, est suffisamment distinctive. Le mále au dos noir avec son croupion blanc et les barres blanches des ailes peut être reconnu au simple coup d'oeil, même quand il disparait dans les broussailles. La femelle, si elle n'est pas nettement aperçue, peut être prise pour la femelle du pinson pourpré, qui est beaucoup plus petite, mais les dessous qui sont sans raie, la tête plus fortement marquée avec la ligne bien manifeste au-dessus de l'oil, et les barres blanches des ailes qui ressortent plus distinctement, servent d'habitude a l'identifier.

Nid. Dans les buissons ou les arbres, entre 5 et 20 pieds au-dessus du sol; nid fait de fines ramilles, de tiges d'herbes et de radicelles.

Habitat. L'est de l'Amérique, au nord bien au delà de la colonisation. L'oiseau couve au Canada, partout oú il se rencontre.

Le gros-bec à poitrine rose est un des plus beaux oiseaux et des meilleurs chanteurs. Il préfère les fourrés très touffus, séparés par des espaces dégagés et de grands arbres. Il fréquente les fourrés le long des rivières, les lisières de bois qui confinent à des terres défrichées, des palissades recouvertes de végétation et quelquefois des vergers.

Valeur économique. Si le nombre des gros-becs à poitrine rose pouvait se multiplier beaucoup dans les fermes, le fléau des doryphores des pommes de terre ne tarderait pas à disparaître. Cet oiseau est un des rares spécimens de son espèce qui mange les vers blanc et qui les prend soit $28588-14$ 
quand ils sont adultes, soit à l'état de larves. Un dixième du contenu des estomacs examinés se composait de ces vers blancs de pommes de terre, et la dite espèce est tout aussi active contre d'autres insectes non moins pernicieux. Il pourrait être difficile d'augmenter le nombre des gros-becs à poitrine rose, mais il pourrait l'être moins de conserver ceux que nous avons, de les protéger contre une destruction qui peut s'éviter, et de voir à ce que de bons coins à nid soient ménagés dans ces endroits de la ferme ou du bois dont on ne tire aucun parti. Quand on se met en train d'exécuter des projets de bonnes cultures et d'élimination de places perdues, il faudrait avoir soin de laisser subsister de petites plantations d'arbrisseaux où pourraient s'aller réfugier ces oiseaux qui, privés de ces lieux de refuge, seraient condamnés à disparaître. La conservation de ces oiseaux ferait plus que compenser les pertes minimes qu'ils pourraient occasionner.

\section{Genre-Guiraca. Gros-becs bleus.}

597. Le Gros-bec bleu. (BLUE orosbeak). Guiraca corulea. L, 7. C'est un petit gros-bec de la même coloration que le gros pinson indigo, mais pas tout a fait aussi brillant, et avec des barres d'un brun chatain sur l'aile et sur l'épaule.

Traits distinctifs. Les plumages, dans les changements quí dépendent des saisons et du sexe, se conforment tout a fait a ceux du gros pinson indigo dont on peut distinguer notre gros-bec bleu par la taille et le bec. Dans un plumage mêlé il peut ressembler au rouge-gorge bleu, méme quant à la poitrine rougêtre, mais le bec est absolument différent de celui de ce dernier (voir figure 50, p. 28).

Indications sur le terrain. Les traits qui précedent pourront tenir lieu de ces indications mais le gros-bec bleu est trop rare au Canada pour qu'on puisse se fier seulement à la vue pour l'identifier.

Habitat. L'est de l'Amérique du Nord, au nord il s'arréte d'habitude bien arant la frontière canadienne.

SOUS-ESPECE. La sous-espèce du gros-bec bleu qu'on peut espérer voir au Canada c'est le type de l'est, le gros-bec blcu de l'Est, la race type de l'espece.

Les cas que l'on a cités de la présence de cet oiseau au Canada sont trop isolés pour qu'on puisse parler de lui autrement que comme un vagabond accidentel et rare. Il ne faut espérer le rencontrer que dans les régions méridionales.

\section{Genre-Passerina. Passerines.}

598. Le Pinson indigo. ua PAsserine BLeUe. (Indigo BUNtino). Passerina cyanea. L, 5.59. C'est un pinson plutôt petit; le mâle d'un bleu brillant par tout le corps, la femelle presque uniformément d'un chamois rouilleux avec, par occasion, de vagues soupçons du bleu propre au mâle, légèrement blanchâtre en dessous avec de vagues raies assez indistinctes. Les oiseaux d'automne font voir des phases intermédiaires entre la coloration susdite, ou un rouge rouilleux plus accentué.

Traits distinctifs. Le pinson indigo est notre seul oiseau tout bleu. Le rouge-gorge bleu, qui est le seul qui puisse lui étre comparé, a une poitrine rougeâtre. La couleur uniforme, presque invariablement d'une couleur rouille mate, de la femelle et des jeunes oiseaux les distingue d'autres espèces.

Indications sur le terrain. La couleur absolument bleue du mâle, et celle uniformément rougeâtre des femelles et des jeunes oiseaux.

Nid. Généralement à la fourchure des branches d'un buisson, à 2 ou 3 pieds au-dessus du sol, nid fait d'herbes, de feuilles mortes, de bandes d'écorce, et garni d'herbes délicates, de radicelles et de longs poils.

Habitat. L'Amérique du Nord, à l'est des plaines et au nord assez loin a l'intérieur du Canada.

Le pinson indigo fréquente habituellement des terrains de broussailles recouverts d'une végétation qu'on laisse.perdre, ou que le feu a consumée, ou que des abatis ont réduits en clairières. Son chant est agréable. 
Valeur économique. Nos renseignements au sujet des aliments du pinson indigo sont incomplets. Il est hors de doute que ses mœurs alimentaires sont ceux de sa famille, et on lui fait honneur des grands services qu'il rend en détruisant les larves de lépidoptères à queue brune sur les arbres. Cet oiseau a autant de droit que d'autres de son espèce à être protégé.

\section{Genre-Spiza. "Dickcissel".}

604. Le "Dickcissel". (DICkcissel). Spiza Americana. L, 6. Le dos a des raies brun foncé et chamois rougeAtre qui se changent en un rouge mat sur les couvertures des ailes; l'arrière cou est ardoisé jusqu'a la couronne où il est fortement teinté de jaunc; les joues sont grises avec la ligne sourcilière d'un jaune pur; blanc en dessous; la poitrine d'un jaune pur et la gorge avec une tache noire ou bavette de contour très tranchée.

Traits distinctif8. La poitrine jaune, la gorge noire, qui fait songer à l'étourneau des prés, sont distinctives.

Habitat. L'est de l'Amérique, surtout dans les parties de l'intérieur et du centre. Dans l'est du Canada, assez irrégulièrement au delà de nos frontières dans le sud-ouest de l'Ontario.

Cette superbe espèce d'oiseau des champs découverts est rare au Canada. Quelques-uns y sont parus pendant un petit nombre d'annees consécutives à l'extrémité ouest du lac Erié, puis ont disparu pour revenir quelques années plus tard.

FAMILLE-TANGARIDA. TANGARAS.

Description générale. Ce sont des oiseaux aux couleurs brillantes avec un bec qui ressemble a celui des pinsons mais légèrement allongé et avec un culmen uniformément recourbé. Le bord tranchant de la mandibule supérieure est dentelé et échancró quoique chez quelques espèces ces marques sont trop petites pour être vues à moins d'une cxtrême attention. (figure 53, p. 28).

Traits distinctifs. En sus des caractères précités du bec, l'espèce de l'est du Canada peut être facilement reconnue à sa couleur. Les males du printemps sont des oiseaux d'une brillante coloration rouge, les femelles et les oiseaux de l'automne sont d'un jaune ou d'un vert ardent sans marques ni détails bien tranchés et avec peu de variété dans la coloratíon sauf dans les amas uniformes des ailes et de la queue. Les males du printempe ne peuvent se comparer qu'avec le cardinal au point do vue de la couleur; les oiseaux d'automno et les femelles qu'avec les orioles femelles, mais l'absence de huppe chez les tangaras les distinguera facilement d'avec le cardinal, et les caractères du bec les distingueront et du cardinal ou des orioles.

Les tangaras constituent une famille essenticllement américaine qui attcint son plus haut développement sous les tropiques et n'est parfaitement représentée que par une seule espèce dans l'est du Canada.

En tant que famille les tangaras sont si étroitement alliés aux pinsons que l'on ne connaît pas encore le status de certaines espèces qui dépassent cet habitat.

\section{Genre-Piranga. Tangaras.}

608. Le Tangara écarlate. (scarlet tanager). Piranga erythromelas. L, 7·25. Planche XXXV A.

Traits distinctifs. A part l'espèce suivante, le tangara vermillon, le tangara écarlate ne peut se comparer qu'au cardinal, mais il est facilement reconnaissable a son manque de huppe, son bec plus léger et plus long, au noir de ses ailes et de sa queue. Le vert uniforme de la femelle est distinctif, et il n'est à peu près égalé que chez un petit nombre de lauvettes et d'orioles. Le mâle d'automne est pareil à la femelle, mais avec des ailes et la queue noires.

Indications sur le terrain. Brillante couleur écarlate, avec les ailes et la queue noires chez le mâle, la coloration verte uniforme et la taille chez d'autres plumages.

Nid. D'habitude près de l'extrémité d'une branche, a environ 20 pieda au-dessus du sol: nid fait de feuilles, de bandes d'écorce, etc.

Habitat. L'est de l'Amérique, au nord jusque vers la limite des endroits colonisés.

$28588-14 \frac{3}{2}$ 
Le tangara écarlate fait voir de remarquables changements de plumage selon la saison et le sexe. Au printemps les sexes sont si complètement différents l'un de l'autre qu'on s'étonne qu'ils soient de la même espèce, et en été l'écarlate étincelant du mâle prend peu à peu le vert mat de sa compagne.

Le tangara écarlate est un oiseau des terrains plantés de bois clairsemés où des arbres puissants croissent avec des broussailles légères à l'entour, mais au printemps l'oiseau visite à l'occasion les vergers. Quand il árrive, au printemps, le tangara écarlate attire facilement les regards, mais à mesure que les arbres se revêtent de leurs feuilles l'oiseau ne se laisse apercevoir qu'avec bien des précautions, et si ce n'était sa note si distinctive "tchip-tchur», qui attire les regards de son côté, il serait très difficile de le découvrir. Son chant est gai, rythmique et assez bien soutenu, un peu comme celui du rouge-gorge, mais plus lié et pas tout à fait aussi limpide.

Valeur économique. La nourriture du tangara écarlate se compose surtout d'insectes et de fruits. Les insectes sont partout des espèces forestières et il importe aux forestiers et aux cultivateurs d'arbres fruitiers de détruire ces insectes. Le fruit qu'ils attaquent est surtout sauvage, en réalité la plupart des oiseaux préfèrent les espèces sauvages aux cultivées, et quand les premières sont abondantes les autres sont rarement attaquées. Le tangara écarlate ne leur cause aucun dommage sérieux.

610. Le Tangara vermillon. (sUMmer TANager). Piranga rubra. L, 7.50. Très pareil au tangara écarlate, mais avec la queue rouge au lieu de noire, avec les ailes d'un brun mat bordées et teintées de rouge; les femelles se comportent comme coloration au regard du mâle, comme font celles du tangara écarlate.

Traits distinctifs. Les ailes et la queue diffèrent de celles du tangara écarlate, le rouge est.plus rosé, moins brillant et plus clair en dessous que sur le dos. La femelle a des tons vert d'orange très chaud, et d'une bien différente nuance que les verts froids de la femelle apparentée du tangara écarlate. Elle ressemble d'assez près à la femelle du loriot de Baltimore, mais la coloration uniforme du dos et des ailes sans aucune marque et le bec du tangara rendront la distinction facile.

Habitat. Le sud-est des Etats-Ünis et le nord jusqu'à la latitude du Ohio méridional. On l'a signalé au Canada près de la frontière du sud le long des rives inférieures des grands lacs, ainsi qu'au Nouveau-Brunswick et dans la Nouvelle-Ecosse.

Le tangara vermillon est un traînard d'occasion dans le Canada, à partir du sud, le long des rives inférieures des grands lacs, ainsi que dans la Nouvelle-Ecosse et le Nouveau-Brunswick.

\section{FAMILLE-HIRUNDINID E. HIRONDELLES.}

Description générale. Ce sont pour la plupart de petits oiseaux, à ailes longues et pointues; pieds petits et fajbles, impropres à la marche; tête aplatie et bec très court et très large (figure 54, p. 28).

Traits distinctifs. Ressemblent superficiellement aux engoulevents, mais beaucoup plus petits et d'un different type de coloration. Risquent plutôt d'être confondues avec le martinet des cheminées qu'avec aucune autre espèce, mais sans les épines de l'extrémité des pennes de la queue.

C'est une famille répandue dans le monde entier, de mours aériennes, descendant rarement sur le sol sauf pour y chercher de quoi bâtir leur nid. Leurs pieds sont faibles et ne sont bons qu'à se poser sur de petites branches, des fils télégraphiques ou de semblables perchoirs. Elles saisissent leur nourriture au vol et on les voit souvent glisser au-dessus des étangs et en sillonner la surface tout en s'abreuvant. Ce sont d'habiles bâtisseurs de 
nids; elles construisent une remarquable variété de formes depuis des structures en cou de bouteille faites de boue pétrie jusqu'a des trous en tunnel sous des bancs de terre.

Valeur économique. Les insectes ailés constituent presque toute l'alimentation des hirondelles. Volant haut ou bas dans l'air, selon que leur gibier les y invite, les hirondelles attaquent maint insecte ailé qui autrement ne serait pas inquiété. Au-dessus des champs de céréales, autour des granges, où il se trouve peu d'oiseaux insectivores, les hirondelles s'assemblent et prêtent un secours efficace au cultivateur. Leur malpropreté est quelquefois désagréable quand elles nichent en grand nombre sous les gouttières des maisons. Il est bien, en pareil cas, de leur procurer des endroits favorables où elles puissent nicher dans les bâtiments de la ferme où elles se rassembleront sans causer de désagréments, car une compagnie d'hirondelles autour de la cour d'une ferme est d'un grand avantage pour le fermier.

\section{Genre-Progne. Hirondelles.}

611. L'Hirondelle pourprée. (PURPLe vartin). Progne subis. L, 8. Planche XXXV B.

Traits distinctifs. C'est la plus grande de nos hirondelles. La taille et la coloration noir bleu irisce du mâle devrait suffire pour distinguer les espèces.

Indications sur le terrain. La taille, la couleur, le vol, presque pareil à celui du faucon, et la voix sont des indications parfaites.

Nid. A l'origine, dans le creux d'un arbre. Dans l'état civilisé, des nichoirs artificiels pour oiseaux, des cavités sous les corniches des maisons, etc. Ce sont des nicheuses sociables et qui préfèrent bâtir parmi des compagnies de leur espèce.

Habitat. L'Amérique du Nord et l'Amérique du Sud. Une sous-espéce occidentale occupe la côte du Pacifique. L'espèce orientale- qui est la race type-fréquente la plupart des établissements agricoles de l'est du Canada.

SOUS-ESPECE. La sous-espèce de l'hirondelle pourprée qui se rencontre dang l'est du Canada est celle de l'hirondelle pourprée de l'Est $P$. 8. subris, la forme type.

Pendant le jour ces oiseaux se répandent dans la contrée, revenant à de fréquents intervalles, avec de la nourriture pour leurs petits. Le soir ils reviennent tous à la maison qu'ils occupent et s'y mettent à l'abri pour la nuit. Les petits restent très longtemps dans le nid et même après la première sortie en l'air les parents et les jeunes reviennent ensemble au nid. Les hirondelles pourprées sont domestiques et sociables et se saluent mutuellement par des gazouillis et un babillage de bienvenue. Chaque hirondelle s'intéresse aux affaires des autres et il se fait un continuel échange de visites entre voisins. Elles se disputent bien rarement entre elles mais se liguent contre l'ennemi commun qui n'est autre que le moineau anglais. Une colonie d'hirondelles peut fort bien maintenir ses positions contre cette engeance, lorsqu'elles se sont bien installées. Une maisonnette pour cette hirondelle devrait renfermer plusieurs chambres de 8 pouces sur 8 , à l'abri du mauvais temps et des courants d'air tout autour sauf a la porte qui devrait avoir 2 pouces de diamètre et être à 1 pouce et demi au-dessus du plancher. La dite maisonnette devrait être à 15 ou 20 pieds au-dessus du sol, placée en plein air et de façon à pouvoir se descendre pour être nettoyée.

Il y a des faits qui prouvent que l'hirondelle pourprée se fait plus rare. D'anciennes colonies se démembrent de temps en temps et il ne s'en crée que peu de nouvelles pour les remplacer. Les hirondelles pourprées reviennent à leurs quartiers d'été de très bonne heure au printemps et parfois 
elles souffrent beaucoup des gels tardifs et des pluies froides qui arrotent le vol des insectes et privent l'oiseau de la grande et régulière quantité de nourriture qui lui est si nécessaire.

Valeur Economique. L'hirondelle pourprée, comme les autres hirondelles, est un oiseau qui n'a pas de mauvaises habitudes et qui en a tant de bonnes qu'on devrait tout tenter pour en multiplier le nombre.

\section{Genre-Petrochelidon. Hirondelle à front blanc.}

612. L'Hirondelle à front blanc. (CLIFF swallow). Petrochelidon lunifrons. L, 6.01. Semblable par la coloration générale à l'hirondelle des granges (planche XXXVI A) mais différente dans les détails et sans la queue longue et grandement fourchue.

Traits distinctifs. La gorge de couleur plus riche et d'un brun plus rougeatre que celle de l'hirondelle des granges; la couleur brune, qui s'étend en une bande étroite en travers de la partie de derrière du cou et avec une tache noire au milieu de la gorge inférieure au lieu d'une barre noire brisée en travers de la poitrine. Le front qui est d'un blanc crémeux au lieu de rougeâtre fait un contraste frappant. Les dessous sont d'un blanc terne. La queue est presque carrée.

Indications sur le terrain. Le front blanc et le croupion rougeâtre sont les meilleures indications. La queue carrée la distinguera de toutes les hirondelles des granges, bauf des toutes jeunes qui n'ont pas encore la fourche.

Nid. Le nid typique est fait entièrement de terre gâchée portée en petites boules dans le bec et sur les pieds. La terre est mélangée avec de la salive et appliquée boule à boule contre le mur sous les chéneaux de quelque batiment. Le nid est d'abord une tablette construite sur le mur, puis façonné en soucoupe, puis en tasse, et sous l'une ou l'autre de ces formes il est regardé comme achevé. Dans les meilleurs exemples de ces nids les côtés sont continués jusqu'a ce que le nid prenne la forme d'une bouteille avec son cou recourbé et dirigé vers l'extérieur.

Habitat. L'Amérique. Dans l'est du Canada jusqu'au nord des parages civilisés.

SOUS-ESPECE. La sous-espèce de l'hirondelle au front blanc qui occupe la plus grande partie de l'Amérique du Nord et tout le Canada, c'est l'hirondelle au front blanc de l'Est, $P$. $l$. linufrons, la forme typique de l'espece.

Cette hirondelle habitait à l'origine les falaises, comme l'indique son nom anglais, mais au sein de la civilisation elle fait maintenant son nid presque toujours dans des granges et autres bâtiments. Les groupements sont plutôt distribués par localités vu que les différentes colonies d'hirondelles nichent bien souvent dans des fermes fort éloignées les unes des autres.

\section{Genre-Hirundo. Hirondelle des granges.}

613. L'Hirondelle des granges. (BARN sWaLLow). Hirundo erythrogastra. L, 6.95. Planche XXXVI A.

Traits distinctifs. Une barre noire brisée en travers de la poitrine, au-dessous de la gorge rougeatre; croupion noir et, chez l'adulte, queue longue et fourchue, tels cont les traits distinctifs de l'espèce.

Indications sur le terrain. Teintes brun rougeatre en dessous et croupion noir sont des indications sâres; en outre, chez l'adulte la queue longue et fourchue, et chez les tout jeunes le blane dans la queue.

Nid. Le nid est loin de ressembler à la belle architecture de celui que batit l'hirondelle à front blanc. Il est surtout fait de boue mélangée avec des herbes, garni d'herbes et de plumes, et planté sur un support comme une poutre ou une traverse; mais souvent la plus petite projection sera utilisée comme un point d'appui pour y bâtir. Il arrive que des cultivateurs, pour attirer ces oiseaux et les engager a faire leurs nids ld ou on les verra volontiers, leur fournissent de petits supports pour leurs nids tout a côté et sous les chéneaux de leurs granges, où en dedans là où ils seront protégés sans causer aucun dommage. dentes.

Habitat. L'Amérique. Probablement ne va pas aussi au nord que les espèces précé- 
C'est l'hirondelle qui d'habitude niche dans les granges et les annexes de la maison de ferme. Non seulement elle battit son nid sous les chéneaux mais elle pénètre dans le bâtiment et se loge à l'intérieur. Sa longue queue d'hirondelle» en fait peut-être la plus gracieuse de toutes les hirondelles canadiennes.

\section{Genre-Iridoprocne. Hirondelle bicolore.}

614. L'Hirondelle blcolore.(TREE swallow). Iridoprocne bicolor. L, 5.90. Planche XXXVI B.

Traits distinctifs. Le noir uniforme avec des reflets d'acier très prononcés dans les dessus, la blancheur immaculée des dessous sont bien distinctifs.

Indications sur le terrain. La blancheur parfaite, sans marque aucune des parties de dessous, constitue une indication facilement reconnaissable sur le terrain. Chez les jeunes oiseaux le noir des dessus est remplacé par du brun mat et un soupçon de brun étendu sur les côtés de la poitrine, mais tout cela ne forme jamais une bande continue en travers de la poitrine comme chez l'hirondelle de rivage.

Nid. Se fait dans les trous laissés par les pics, dans des couches mortes, dans des arbres creux ou dans des nichoirs. Le nid est garni d'herbes et de plumes.

Habitat. L'Amérique, dans le nord jusque vers la limite des bois.

Bien qu'ordinairement elle profite des trous de pics, dans les souches mortes au-dessus de l'eau, l'hirondelle bicolore se laisse facilement aller à nicher dans des boîtes dans un jardin. La beauté de sa brillante irisation et la grâce de son vol vaut largement la peine qu'on prendra de leur préparer un abri, même si sa présence n'était pas à elle seule une importante protection contre les insectes dans le jardin. La permanence de l'espèce est menacée par la rareté toujours plus grande des places favorables à sa nidification, à moins qu'un effort ne soit tenté pour créer des nids artificiels.

\section{Genre-Riparia. Hirondelles de rivage.}

616. L'Hlrondelle de rivage. (BanK swallow). Riparia riparia. L, 5.20. Planche XXXVII A.

Traits distinctifs. Un brun mat au lieu d'une coloration irisee du dos; les dessous blancs avec une bande pectorale distincte et noire sont des traits de ce genre. La bande est toujours pleine et complète et le blanc très pur. La bande pectorale complète fera distinguer l'hirondelle de rivage de la jeune hirondelle bicolore, et aussi de l'hirondelle à ailes bérissées chez laquelle le blanc n'est pas pur et chez qui la poitrine et la gorge sont uniformément étendues d'un brun cendré.

Indications sur le terrain. Les dessous blanes sont traversés par une barre foncée très visible. C'est la meilleure indication sur le terrain.

Nid. Presque tout le monde a vu combien vite les côtés en vue d'une excavation de sable ou de gravier sont creusés de petits trous à nids par les hirondelles. Trop souvent les petits gamins fouillent ces trous et en font sortir les oiseaux. C'est là une action à la fois dangereuse pour l'enfant, parce que l'excavation peut s'effondrer, et méchante en ellemême parce que c'est un coup droit porté a l'existence d'un des meilleurs amis du fermier.

\section{Genre-Stelgidopteryx. Hirondelle à ailes hérissées.}

617. L'Hirondelle à alles hérissées. (ROđGH-WINGEd swallow). Stelgidopteryx serripennis. $L, 5 \cdot 75$. Très semblable à l'hirondelle de rivage par la coloration générale mais avec la poitrine et la gorge teintées d'un brun cendré pâle au lieu d'étre traversées par une bande brune bien tranchée.

Traits distinctifs. La susdite différence, jointe a l'absence de beaucoup de blane pur en dessous et d'un blanc quelconque sur la gorge, est tres indicative. Les oiseaux de croissance achevée ont la petite membrane externe des primaires extérieures convertie en une série de petits crochets presque imperceptibles a l'oel nu mais que le doigt peut sentir quand on le passe le long du bord jusqu'à la pointe. Sur les jeunes oiseaux cela ne se voit pas bien, quelquefois pas du tout, et les différentes étapes de ce développement se font remarquer. On remarque la chose en quelque degré chez tous les spécimens du printemps. 
Indications sur le terrain. La meilleure indication de ce genre pour distinguer l'hirondelle à ailes hérissées de l'hirondelle de rivage c'est la poitrine uniformément étendue de la même teinte au lieu de la poitrine blanche avec une large barre noire. Quand on porte son attention sur une compagnie un peu mêlée d'oiseaux, l'hirondelle à ailes hérissées peut être ordinairement distingúe grâce à son dos un peu plus rouge ou de couleur rouille qui ressort beaucoup plus dans la vie ordinaire que dans la main.

Nid. A cet égard cette hirondelle ressemble à celle de rivage mais elle est plus solitaire et peut être plus disposée à nicher dans les crevasses de roches superposées, de falaises ou de batisses.

Habitat. L'Amérique. A partir du nord à travers la frontière canadienne jusqu'a la région inférieure des grands lacs.

Les hirondelles à ailes hérissées appartiennent à une espèce très dispersée dans le monde; elles montrent toute la forme spéciale de l'aile qu'on ne peut expliquer dans l'état actuel de nos connaissances.

FAMILLE-BOMBYCILLID A. IASEURS.

Les jaseurs sont des oiseaux extraordinaires, répandues à travers les parties septentrionales des deux mondes. Ils sont représentés en Amérique par deux espèces si bien caractérisées pour la forme et la couleur et si pareilles l'une de l'autre que toute description ici est inutile. Ils offrent très peu de variations par rapport soit aux saisons soit aux sexes, et la planche XXXVII B comme la figure 55 , page 29 , les désigne très distinctement. Les tuyaux des secondaires et quelquefois ceux de la queue s'élargissent vers les bouts en des appendices de brillantes couleurs qui ressemblent à des morceaux de cire à cacheter.

\section{Genre-Bombycilla. Jaseurs.}

618. Le Jaseur de Bohème. (вонемian waxwing). Bombycilla garrula. L, 8. Il est presque tout à fait semblable par la forme et la couleur au jaseur du cèdre, mais plus grand; les secondaires ont à leurs extrémités un peu de blanc; la plupart des primaires ont du blanc ou du jaune, ou l'un et l'autre; il y a aussi une petite barre à l'aile. Les couvertures sous-caudales sont marron et l'abdomen est grisâtre sans être étendu de jaune.

Traits distinctifs. Aucun autre de ces traits n'est nécessaire; le jaseur du cèdre est la seule espèce avec laquelle on pourrait le confondre.

Indications sur le terrain. Leurs figures proprettes et leurs crêtes si frappantes font reconnaitre les jaseurs. Le blanc ou le jaune sur les ailes, le marron des couvertures souscaudales sont les indications les plus déterminantes sur le terrain.

Nid. Sur les arbres, composé de ramilles, de racines, de mousse, etc.

Habitat. Les sections boréales de l'hémisphère nord. En Amérique, couve dans l'extrême nord, au nord-ouest de la baie d'Hudson, et visite les districts colonisés en hiver, mais irrégulièrement.

Leurs mœurs irrégulières et migratoires en hiver ont fait donner à ces oiseaux le nom de "bohémiens》) lequel, en ce sens, est synonyme de "errants". Ce sont des oiseaux du nord-ouest, mais qui descendent à l'occasion dans les régions cultivées du Canada oriental, en hiver, comme fait le gros-bec à couronnc noire. Ils sont trop rares pour avoir aucune valeur économique. Leur nourriture favoritc se compose des fruits desséchés qui restent pendus aux arbres pendant l'hiver.

619. Le Jaseur du cèdre. (Cenar Waxwing). Bombycilla cedrorum. I, 7·19. Planche XXXVII B.

Traits distinctifs. Avec l'aide de la gravure le jaseur du cèdre ne peut guère être confondu avec aucune autre espèce sauf avec le jaseur de Bohème. Pour les traits distinctifs voir cette espèce. Ce jaseur-là ést le seul qu'on rencontre dans l'est du Canada, en été, et qu'on peut probablement rencontrer en hiver dans les districts méridionaux. 
Indications sur le terrain. La forme et le port gracieux ainsi que la huppe droite si apparente sont des indications faciles à reconnaitre. La voix, un sifflement aigu, est caractéristique et vite reconnue. Voir la précédente espèce.

Nid. Souvent placé gur des arbres fruitiers ou ombreux, c'est un assemblage massif de morceaux d'écorce, de feuilles, d'herbes, de ramilles, de petites racines, de mousse etc., garni de matières plus fines de même nature.

Habritat. L'Amérique, au nord dans le Canada jusqu'aux limites des régions cultivées et même par delà.

Le jaseur du cèdre est un des oiseaux qui fréquentent nos vergers. Il niche en été dans les arbres fruitiers et n'est que trop connu dans le voisinage des cerises à maturité précoce. En hiver il recherche les diverses espèces de vicux fruits desséchés qui pendent aux branches. La coloration est tendre, harmonieuse avec tout juste assez de contraste de couleurs pour lui donner du caractère. Le tissu bizarrement lisse et soyeux du plumage semble être cause que les plumes sont pour ainsi dire collées, l'une à l'autre et jamais en désordre. La façon dont les tuyaux des secondaires et quelquefois les plumes de la queue se terminent comme des bouts de cire à cacheter rouge, façon commune à ce jaseur et à celui de Bohème, est unique en son genre, parmi les oiseaux d'Amérique et donne à ces deux individus un cachet de plus d'individualité.

Valeur économique. Environ le treize pour cent de leurs aliments est formé d'insectes nuisibles, le reste se compose en majeure partie de fruits. Ces fruits sont surtout d'espèce sauvage et sans valeur économique; c'est même un fait que la plupart des oiseaux préfèrent de beaucoup les fruits sauvages aux fruits cultivés. Néanmoins, quand les cerises précoces mûrissent avant les cerises sauvages le dommage que les jaseurs peuvent faire est considérable. La même quantité de ce fruit répartie sur un grand nombre d'arbres plus tardifs pourrait passer inaperçue, mais lorsque le dommage se concentre sur la partie la plus précoce et la plus précieuse de la récolte, la perte se fait vivement sentir. La protection du fruit précoce contre les dégâts causés par cette espèce d'oiseau et quelques autres de même mœurs est une affaire qui a demandé une attention considérable. Quant à tuer à coups de fusil tous les oiseaux qui visitent le verger, ce serait évidemment une solution du problème, mais qui ne laisserait pas d'être piteuse. La protection du fruit ne serait qu'incomplète et devrait se répéter à chaque saison; car tant qu'il reste de ces oiseaux dans le voisinage, leur multiplication annuelle viendrait annuler les résultats des entreprises précédentes. En outre, le village entier serait privé de l'utile secours d'un certain nombre d'espèces à la seule fin de protéger une quantité donnée de fruits. Un filet qu'on étendrait sur le fruit reviendrait d'ordinaire à meilleur marehé que la fusillade en question. Comme les oiseaux préfèrent le fruit sauvage au fruit cultivé mûr de bonne heure, des arbres à fruits sauvages plantés dans des coins inutilisés et le long des palissades donneraient une protection à bon marché. Le mûrier russe et le sorbier, plus tard le cassis, l'alisier, le framboisier, le sumac, l'aune, la vigne sauvage, la douce-amère, la morelle noire, la symphorine, le sureau, sont tous des fruits sauvages qui rempliront l'office voulu.

FAMILLE-LANIIDE. GRIÈCHES, PIES-GRIÈCHES.

Description générale. Les pies-grièches sont des oiseaux du genre passereau, de taille moyenne et de caractère rapace. On les reconnait facilement a leur bec qui est distinctement crochu a l'extrémité et pourvu d'une entaille et d'une dent près du bout de la mandibule supérieure (figure 56, p. 29). Les deux espèces qui se rencontrent au Canada sont très semblables par la coloration et ne diffèrent que par de menus détails seulement (planche XXXVIII A). 
Les pies-grièches sont d'intéressants exemples d'oiseaux du genre passereau vivant de graines et d'insectes et faits pour une vie de rapaces. Les vrais rapaces, les éperviers, etc., qui font leur proie d'être plus élevés qu'eux dans l'échelle animale, ont des pieds puissants avec lesquels ils tiennent leur proie tandis qu'ils la déchiquettent. Les pies-grièches n'ont pas ces membres faits pour saisir et tenir, car leurs pieds ne sont pas plus forts que ceux d'un moineau ou d'un merle de même taille. De là vient qu'elles saisissent leur proie avec leur bec et que pour la tenir tout en la mangeant, elles ont pris l'habitude de l'empaler sur de fortes épines; c'est cette habitude qui leur a fait donner le nom d'oiseaux-bouchers. Les piesgrièches sont hardies, courageuses et montrent autant d'audace et de savoirfaire, toute proportion gardée, qu'aucun des véritables oiseaux de proie. C'est une famille très nombreuse et fort répandue. Un seul genre est représenté en Amérique et il y en a deux espèces dans l'Amérique du Nord.

\section{Genre-Lanius. Pies-grièches.}

621. La Pie-grièche boréale. (NORTHERn sHRIKE), Lanius borealis. L, 10.32. Elle est semblable à la pie-grieche migratrice (planche XXXVIII A) mais est plus grande et avec une série de lignes fines, ondulées ou vermiculées vaguement marquées en travers des dessous.

Traits distinctifs. La coloration générale et le bec encoché feront reconnaltre la pie-grièche. La taille et les vermiculures bien marquées en dessous la caractériseront comme étant la pie-grieche boréale.

Indications sur le terrain. Le contraste très accusé du noir et du blanc sur les ailes et la queue, les dessus gris et la bande noire en travers de l'œil. Toute pie-grièche aperçue dans l'est du Canada en hiver, d'octobre à mars, appartiendra à cette espèce.

Nid. Dans les arbres un peu bas ou dans les buissons; nids faits de branchettes, d'herbes, etc.

Habitat. L'Amérique du Nord; l'oiseau couvant au delà de la colonisation et à travers le continent, au sud en hiver.

La pie-grièche boréale est la plus hardie et la plus vigoureuse des deux espèces. Elle couve dans le nord et ne se rencontre que dans les régions cultivées, en hiver, où elle suit les bandes de sizerins à tête rouge, etc. Elle a montré quelque disposition à pénétrer dans les villes et les villages à la poursuite du moineau domestique ou anglais, intention qu'il conviendrait d'encourager de toute façon. Des souris et des oiseaux desséchés, petits cadavres, sont quelquefois trouvés cloués à des épines, à des clôtures de fils de fer barbelés, ou pendus à la fourche étroite de deux branchettes; ce sont ordinairement des victimes de cette espèce.

Valeur économique. Bien que de sa nature elle soit rapace à tous crins, la pie-grièche boréale ne peut cependant point être regardée comme faisant beaucoup de dommage. Elle n'est pas suffisamment commune dans ces petits centres nouveaux pour être un facteur important dans la vie des oiseaux des champs. Elle attrape beaucoup de souris et il est probable que la guerre qu'elle leur fait ainsi qu'au moineau anglais fera pardonner ses entreprises sur les oiseaux mangeurs de grains.

622. La Pie-grièche migratrice à croupion blanc. (LOGaerhead BHrike). Lanius ludovicianus. L, 9. Planche XXXVIII A.

Traits distinctifs. Cette espèce ne peut guère être confondue qu'avec la pie-grièche boréale, et elle est beaucoup plus petite que cette dernière. L'oiseau adulte n'a pas les fines vermiculures de la poitrine et, chez les toutes jeunes, elles ne sont qu'à peine indiquées. C'est, au Canada, un oiseau d'été et non d'hiver. 
Indications sur le terrain. Le blanc clair et le gris pâle du plumage de leur corpe, les ailes noires et la queue fortement marquée de blanc, enfin, la bande noire sur la face, sont particuliers aux pies-grièches. Toute pie-grièche d'été au sein des régions cultivées sera de cette espece.

Nid. Fait de morceaux d'écorce, de ramilles, de fibres végétales, et garni de laine et de plumes bien ajustées.

Habitat. En tant qu'espèce, l'Amérique du Nord, jusqu'd la limite des terres cultivées, au nord. La pie-grieche migratrice occupe l'est de l'Amérique du Nord, au nord des Etats du golfe du Mexique et à l'ouest Jusqu'aux provinces des prairies.

SOUS-ESPECES. La pie-grièche migratrice, comme bien d'autres especes dont le territoire cst très vaste, dévcloppe différents caractères selon les localités des parties du territoire où ellc vit, chaque partie donnant lieu à une sous-espece reconnue. La piegrièche de l'est du Canada, est la pie-grièche migratrice: Lanius ludovicianus migrans qu'on distinguera de la sous-espèce type des Etats-Unis du Sud, appelée pie-grièche au croupion blanc de l'Ouest, par de légères différences de couleur et de proportions seulement.

La pie-grièche migratrice est un oiseau des pâturages et des coteaux découverts et broussailleux. Les arbres appelés stramoines, émondés et tondus par le bétail jusqu'à en devenir ramassés et repoussants sont les endroits favoris que l'oiseau recherche pour y nicher, et dans ce voisinage on peut ordinairement l'apercevoir sur quelque observatoire haut placé, comme la pointe d'un plançon mort ou un fil de télégraphe, qui scrute d'un œil perçant la contrée environnante. L'empalement de sa proie n'est pas une habitude aussi fortement développée dans cette espèce que dans la précédente, probablement parce qu'elle est plus insectivore et qu'elle peut manier ses plus petites victimes sans les empaler. En tout cas on ne voit que très rarement des restes plantés sur des épines dans les retraites où cette espèce est commune et où l'on s'attendrait à trouver bcaucoup de traces de cette coutume. Le chant de la pie-grièche migratrice est mélodieux et agréable, mais son cri d'appel est dur et discordant.

Valeur économique. Les habitudes de cette pie-grièche par rapport aux aliments sont semblables à celles de la pie-grièche boréale avec la scule différence qu'on peut attendre d'un oiseau plus petit et plus faible, d'un oiseau d'été plutôt que d'hiver. C'est ainsi que nous trouvons qu'elle prend moins d'oiseaux et de mammifères et plus d'insectes; quand la saison des insectes bat son plein on voit même qu'ils constituent presque toute la nourriture de la pie-grièche. Au commencement de l'été, des quantités d'esearbots sont mangés, qu'ils soient utiles ou nuisibles, il s'en mange autant des uns que des autres. Plus tard ce sont les sauterelles et les criquets qui forment une grande proportion de la nourriture, mais une forte quantité de chenilles, dont plusieurs sont velues, des larves, des guêpes, des araignées et d'autres formes d'insectes servent de nourriture à la piegrièche. L'alimentation de cet oiseau, pendant l'année, rend service, selon la Commission biologique des Etats-Unis, dans la proportion de 4 a 1 .

\section{FAMILLE-VIREONIDE. VIREOS.}

Description générale. Des oiseaux petits, pareils aux fauvettes, avec du vert et du blanc comme coloration, et plus ou moins de jaune en masses délicatement étendues, et sans marques bien distinctes. Le bec est visiblement encoche et recourbe a son bout, comme celui de la pie-grieche (figure 56, p. 29) mais de proportions beaucoup plus petites et plus légères.

Traits distinctifs. Les vireos peuvent facilement être pris pour des fauvettes auxquelles ils ressemblent par le port, la taille et la coloration générale. Toutefois, leur bec est plus gros, plus fortement arqué au sommet, plus élevé eu égard à sa largeur et plus 
visiblement recourbé et encoché au bout. La fauvette à poitrine jaune a un bec qui pourrait répondre à cette description dans ses grandes lignes, mais il n'est pas recourbé et n'a rien qui ressemble à une encoche à son extrémité.

Indications sur le terrain. En sus des marques spécifiques qui constituent le meilleur guide pour reconnaître l'espèce, les vireos peuvent se reconnaître à leurs habitudes d'oiseaux chanteurs et à leurs mouvements plus lents et plus paresseux, quand ils vous regardent fixement de dessous les feuilles et grappollent nonchalamment sur les branches et les ramilles.

Les vireos forment une petite famille spécialè à l'Amérique. Il y en a trois genres au Canada, représentés par six espèces.

Valeur économique. Au point de vue économique, les vireos peuvent être traités dans le même paragraphe car ils ont les mêmes habitudes quant à leur alimentation. Celle-ci se compose pour le 91 pour cent d'insectes et de fruits qui restent sur les arbres et qui appartiennent presque tous aux variétés sauvages. Les insectes poursuivis sont parmi les plus nuisibles, et comprennent les cicindèles et autres espèces apparentées qu'aucun autre oiseau que les vigilants vireos, à la vue perçante, ne recherche généralement. Les vireos sont parmi nos oiseaux les plus utiles.

\section{Genres-Vireosylva, Lanivireo et Vireo. Vireos.}

624. Le Vireo aux yeux rouges. (RED-EYED viREo). Vireosylva olivacea. L, 6.23. Planche XXXVIII B.

Traits distinctifs. Ce vireo peut être distingué des autres vireos grâce à sa taille plus grande, à l'absence de jaune, au gris restreint à la couronne, et à la ligne blanche du sourcil bordée de noir soit au-dessus ou au-dessous. L'iris est rouge, mais ceci ne peut se remarquer que vu de très près.

Indications sur le terrain. Les marques sur la face de ce vireo constituent la meilleure indication sur le terrain. Le sourcil blanc bordé de noir et la ligne inférieure en travers de l'œeil peuvent ordinairement se voir quand l'oiseau braque ses yeux à travers le feuillage sur l'importun visiteur.

$N i d$. Suspendu après la fourche d'une petite branche de 5 à 15 pieds au dessus du sol, ce nid ou cette coupe suspendue, et tressée avec des morceaux d'écorce, des fibres de bois mort, du papier, du duvet végétal ou de l'écorce de bouleau garni de matières fines.

Habitat. L'Amérique du Nord jusqu'à la limite des arbres.

Le vireo aux yeux rouges est un des hôtes les plus familliers de nos bocages et de nos bois. Son chant, répétition agrémentée de petites variations de la même phrase, avec des pauses entre-deux, et continuées ad libitum, peut être entendu au sommet des arbres à peu près partout au Canada, et a valu à cette espèce le nom d'oiseau prêcheur.

626. Le Vireo de Philadelphie. (PHILAdelphia vireo). Vireosylva philadelphica. $\mathrm{L}, 4.75$. Presque tout à fait semblable comme coloration au vireo gris-olive (planche XXXIX A) mais d'un jaune plus complètement diffus sur la poitrine, les flancs et en dessous.

Traits distinctifs. Le vireo de Philadelphie peut toujours être distingué du vireo gris olive par la longueur de la première des plumes primaires. Dans cette espèce-ci elle est presque aussi longue que la seconde, tandis que chez le vireo gris olive elle est réduite à un état rudimentaire et n'a qu'à peine trois quarts de pouce de long.

Indications sur le terrain. A l'état vivant ce vireo ressemble au vireo gris olive avec une poitrine et les dessous exceptionnellement jaunes.

Nid. Suspendu à une branche qui est à environ 8 pieds au-dessus du sol; ce nid est fait de fibres et d'écorce de bouleau.

Habitat. L'Amérique orientale. Ce vireo couve au sein d'un territoire qui est plus boréal que celui du vireo gris olive; il n'est que migrateur dans la plupart des régions colonisées du Canada.

Ce vireo est généralement assez rare et trop peu remarquable pour être souvent aperçu et reconnu par l'observateur ordinaire. 
627. Le Vireo gris ollve. (Warblivo vireo). Vireosylva gilva. L, 5.80. Planche XXXIX A.

Traits distinctifs. Sa coloration uniforme sans marque spéciale et a petite taille distingueront ce vireo de tout autre sauf du vireo de Philadelphie. L'absence de couleur jaune répandue sur le devant et les dessous servira ordinairement de marque indicatrice. Mais la preuve déterminante de l'espèce sera l'état rudimentaire de la première qui est à peine longue de trois quarts de pouce au lieu d'être d'un pouce trois quarts.

Indications sur le terrain. Les dessous d'un blanc presque pur au lieu de la diffusion jaune du vireo de Philadelphie constituent la meilleure façon de les distinguer l'un de l'autre, de même que la petite taille et la coloration terne uniforme font distinguer le présent vireo de tous les autres.

$N i d$. Suspendu dans la fourche d'une petite branche à une hauteur de 15 à 50 pieds au-dessus du sol; le nid est fait de bandes d'écorce très fines et de fibres végétales.

Habitat. En tant qu'espèce ce vireo occupe toute l'Amérique tempérée; le vireo oriental s'etend à l'ouest jusqu'aux provinces des prairies.

SOUS-ESPECES. Le vireo gris olive forme deux sous-especes: celle de l'Est et celle de l'Ouest. Le vireo gris olive de l'Est, $V . g$. gilea, le type de l'espèce, est le seul qu'on rencontre dans l'Est du Canada.

Le vireo gris olive, caché dans les sommets feuillus d'un arbre est plus souvent entendu que vu. Son chant est très différent de celui du vireo aux yeux rouges, puisqu'il est continu et non composé de phrases entrecoupées.

628. Le Vlreo à front jaune. (YELLOW-THROATED VIREO). Lanitireo flavifrons. L, 5.95. La tête, les joues et le dos sont verdâtres; le croupion et la queue couleur d'ardoise; la poitrine et la gorge jaune clair; les dessous blancs; les ailes ont deux barres blanches distinctes.

Traits distinctifs. La poitrine jaune claire et la gorge de cette espece sont distinctives. Le vireo à tête bleue et le vireo aux yeux blancs sont les seuls autres vireos avec des barres blanches. Dans le premier de ces deux les barres sont blanches comme sur la poitrine; dans le second les barres sont jaunatres. Comme coloration, le vireo à front jaune est très semblable à la fauvette des pins, mais il y a beaucoup moins de blanc dans les dessous de cette dernière espèce, le jaune se répandant pour en couvrir la plus grande partie au lieu de s'arrêter presque brusquement à la ligne de la poitrine.

Indications sur le terrain. La gorge et la poitrine d'un jaune brillant sont parfaitement reconnaissables et empéchent qu'on prenne ce vireo pour aucun autre vireo indigène. La voix, semblable à celle du vireo aux yeux rouges, mais avec des phrases qui se suivent moins rapidement, à 2 ou 3 secondes d'intervalle au lieu d'une par seconde, le fera distinguer d'autres oiseaux de sa famille ou de celles des fauvettes.

Nid. Dans un nid fait de bandes d'écorce, de fibres végétales, l'oiseau est suspendu à une branche fourchue, à une hauteur de 10 à 80 pieds au-dessus du sol. Le nid est garni d'herbes délicates et couvert à l'intérieur de lichens, de toiles d'araignées, etc.

Habitat. L'Est de l'Amérique du Nord. Ce vireo n'est commun dans l'est du Canada que dans les parties méridionales de la région inférieure des grands lacs.

C'est un oiseau des pays boisés et des vergers. A part son chant caractéristique cet oiseau fait entendre bien des sons bizarres, et il a une gamme assez étendue.

629. Le Vireo à tête bleue.(solitary viREo, BLUE-HEAdEd viReo). Lanivireo solitarius. L, 5.61. Dos verdâtre tirant sur un bleu cendré sur la tête et sur les portions contiguës des joues; une tache lorale blanche et un anneau blanc autour de l'cil; tous les dessous sont blancs avec du jaune disséminé sur les flancs; les ailes avec deux barres blanchâtres.

Traits distinctifs. La tête bleu cendré avec des lores blancs très marqués comme l'anneau autour de l'œil sont distinctifs.

Indications sur le terrain. Ia tête bleuâtre avec l'anneau blanc autour de l'œil, les lores bien visibles, et la gorge d'un blanc pur sont des indications très en évidence. 
Nid. Dans un nid fait de fibres ligneuses, de bandes d'écorce, d'aiguilles de pin, de duvet végétal, l'oiseau est, suspendu entre les fourches d'une petite branche à une bauteur de 5 a 10 pieds au-dessus du sol.

Habitat. En tant qu'espèce le vireo a tête bleue se trouve dans toute l'étendue des Etats-Unis et du Canada boréal jusqu'aux limites extrêmes de la colonisation. Le vireo à tête bleue, représentant de l'espèce à l'Est, va jusqu'au centre de l'Alberta a l'Ouest.

SOUS-ESPECE. Le vireo solitaire forme plusieurs sous-espèces, dont une seule, le vireo à tête bleue, $L$. s. solitarius, la forme type, se rencontre dans l'est du Canada.

C'est un joli petit vireo et un habitant ordinaire des terres boisées ct des vergers pendant les migrations. Le chant est semblable à celui du vireo aux yeux rouges, mais une oreille exercée saura saisir la différence.

631. Le Vireo aux yeux blancs. (WHITE-EYED vireo). Vireo griseus. L, 5.27. C'est un petit vireo comme le vireo gris olive, mais d'un vert beaucoup plus riche sur le dos, et avec une marque lorale et un anneau oculaire jaunes; l'iris est blanc. La gorge et les dessous presque d'un blanc pur; jaune foncé sur les flancs; les ailes ont deux barres jaunâtres.

Traits distinctifs. Iris blanc, anneau oculaire et marque lorale jaunes, jaune foncé sur les flancs, poitrine et abdomen blancs.

Indications sur le terrain. Cette espèce est trop rare dans le Canada et trop semblable à d'autres espèces pour qu'on puisse en parler après ne l'avoir vue que de loin.

Nid. Généralement semblable a celui du vireo aux yeux rouges.

Habitat. Tous les individus de cette espèce sont du midi. Le vireo aux yeux blanes qui est de l'Est se rencontre dans les Etats-Unis de l'Est, au nord des Etats de Now-York et de Massachusetts, et n'est qu'accidentellement rencontré au Canada.

SOUS-ESPECES. Le vireo aux yeux blancs est représente par plusieurs sousespèces, l'individu type, le vireo aux yeux blancs du Nord $V . g$. griseus étant le seul qui se rencontre au Canada.

C'est un oiscau du Sud noté pour sa voix remarquable. Il n'est question de cette espèce dans notre travail que sur la foi de quelques signalements rassemblés le long des frontières méridionales du Canada.

\section{FAMILLE-MNIOTILTIDAS. FAUVETTES DES BOIS.}

Description générale. Ce sont de petits oiseaux, une seule espèce canadienne ayant plus de 6 pouces $\cdot 28$, et quelques-unes seulement dépassent 5 pouces $\cdot 75$. Ce sont généralement des oiseaux aux couleurs voyantes des régions boisées et on les voit perchés au sommet des arbres, lors même qu'il en est quelques-uns qui habitent à terre et dans l'herbe. Ils sont, en tant que famille, difficiles à définir, et les genres peuvent être plus facilement reconnus a leurs becs (figures 57,58 , et 59, p. 29) bien qu'il faille une connaissance très grande de l'espèce pour en reconnǻtre les caractères génériques.

Indications sur le terrain. On ne peut donner aucune de ces indications-là s'appliquant à toute la famille. Toutefois, avec quelque expérience de la chose, leur petite taille, leurs couleurs vives, leurs mouvements animés sont vite reconnaissables. On pourra très probablement les confondre avec les vireos.

Nid. Les habitudes de cette famille par rapport au nid qu'elle se fait sont assez variées: quelquefois c'est sur les arbres, depuis le bas près du sol jusqu'au sommet des arbres les plus élevés; d'autres fois c'est sur le sol ou dans l'herbe ou encore dans les trous de trones morts, etc.

Habitat. La plupart des fauvettes couvent dans les forêts de sapin du Nord, au delà des limites de la colonisation générale. Quelques-unes font leur nid dans les régions plus cultivés, d'autres n'bésitent pas à franchir la frontière internationale. Tous ces oiseaux sont migrateurs et passent l'hiver, selon l'espèce, depuis le nord des Etats-Unis jusqu'au sud le long du fleuve des Amazones, dans l'Amérique du Sud.

Les fauvettes des bois forment une nombreuse famille particulière aux deux Amériques. De fait elles partagent cet honneur-celui du nom- 
bre-avec les moineaux. Les fauvettes font les délices de l'amateur ornithologiste. Elles sont si petites que peu de gens, si ce n'est les plus passionnés pour cette étude, les aperçoivent jamais; mais elles sont en même temps si nombreuses et de couleurs si brillantes, que, rien qu'à les apercevoir, le commençant voit s'ouvrir devant lui tout un monde nouveau, d'un grand intérêt. Les sexes sont d'habitude dissemblables et le plumage change beaucoup avec les saisons. Ce fait, compliqué encore par le grand nombre des espèces, rend presque sans espoir pour le commençant la tâche de les identifier toutes. Cela n'est cependant pas aussi difficile qu'il y parait d'abord. Les mâles du printemps portent ordinairement des marques distinctes, et comme beaucoup d'entre eux portent des noms descriptifs, il devient relativement aisé de les différencier. Comme, d'autre part, les femelles et les oiseaux d'automne gardent des traces des marques caractéristiques des mâles du printemps, la difficulté est réellement moindre qu'on ne s'y attendait d'ordinaire. Sans doute, des spécimens embarrassants se rencontrent pour intriguer un peu les connaisseurs, mais il s'agit d'ordinaire d'une alternative entre deux espèces qu'on finit par éclaircir en donnant plus d'attention à un ou à plusieurs petits détails. Quand on s'applique à l'étude des fauvettes, l'observateur est averti qu'il devra d'abord se familiariser avec les mâles printaniers. Dès que les mâles de l'espèce commune sont connus, chose relativement aisée, grâce à des formes très caractérisées, la plupart des femelles sont reconnues sans trop de peine, vu qu'elles portent des vestiges atténués du dessin vivement coloré de leur conjoint. En automne la plupart des jeunes oiseaux ressemblent assez parfaitement aux femelles pour rendre la reconnaissance assez peu difficile. Il n'y a donc, relativement que peu de plumages, ì part les mâles printaniers, qu'il faille apprendre à connaître individuellement. Les fauvettes canadiennes représentent douze genres dont sept sont représentés par une seule espèce. Le genre Dendroica a seize espèces, le Vermivora cinq, et trois autres sont représentés chacun par trois espèces. Les détails génériques des plus importants de ces genres seront élucidés sous des titres particuliers.

Bien qu'on les dénomme Modulateurs" (Warblers) leur chant ne devrait guère être honoré de ce titre. A peu d'exceptions près ces chants ne sont que d'insignifiantes petites notes sans grande continuité, mais comme ils servent souvent à distinguer les espèces, quiconque s'applique à cette étude est invité à y apporter une attention soutenue car, lorsque commencent les grandes migrations des fauvettes, la présence d'une espèce nouvelle ou rare est souvent révélée par une seule modulation peu connue qui détourne l'attention qui se portait sur la masse vers la fauvette qui risquait de passer inaperçue.

Valeur économique. Les fauvettes sont éminemment insectivores. Quelques-unes prennent plus ou moins de grain et un peu de fruit qui est presque toujours sauvage, et aucune plainte ne s'est jamais élevée contre aucun membre de cette famille. Leur action est donc, en somme, assez bienfaisante. Elles atteignent toutes les sortes d'habitat des insectes, depuis l'aisselle des feuilles les plus élevées jusqu'aux brins d'herbe sur le sol; et comme ces oiseaux sont petits, ils se contentent des insectes et des œufs de ces insectes qui sont de trop peu d'importance ou trop bien cachés pour attirer l'attention d'oiseaux bien plus gros. 


\section{Genre-Mniotilta. Fauvette grimpeuse.}

636. La Fauvette noire et blanche. (BLACK AND WhItE WARBLER). Mniotilta varia. L. 5.30. Planche XXXIX B. Le plumage varie peu.

Trails distinctifs. C'est une fauvette noire et blanche qui se glisse autour des trous et des branches comme un pivert. En automne les couleurs du jeune oiseau sont semblables à celles de l'adulte mais légèrement voilées avec une petite dissémination de chamois et moins de noir sur la gorge. Au printemps on ne peut la confondre qu'avec la fauvette rayée, mais la raie blanche médiane sur la couronne, au lieu d'être toute noire, peut la distinguer de cette espèce.

Indications sur le terrain. Son habitude de ramper, sa forte coloration noir et blanc avec la raie médiane sur la couronne. En automne c'est la seule fauvette toute noire et blancbe qu'on rencontre dans le Canada oriental.

Nid. Sur le sol au pied d'un tronc, d'une buche, ou d'une grosse pierre dans un nid fait de bandes d'écorce, d'herbes, etc., garni de radicelles et de longs poils.

Habitat. L'est de l'Amérique du Nord; couve dans presque tout le Canada au nord bien au delà de la partie colonisée.

C'est une des fauvettes qui arrivent le plus tôt au printemps et une de celles qu'il est le plus facile d'identifier en tout temps, vu qu'elle a toujours des marques bien reconnaissables et qu'il y a peu de différence dans la coloration soit saisonnière soit sexuelle.

\section{Genre-Protonotaria. Fauvette dorée.}

637. La Fauvette protonotaire. (PRothoyotary WARBLer). Protonotaria citrea. L, 5.50. C'est une fauvette jaune d'or, un chrome riche et brillant ou doré sur la tête et sur presque tous les dessous, avec ailes, croupion et queue grisâtres. Il y a peu de variation de plumage.

Traits distinctifs. C'est la seule fauvette avec une intense couleur d'or sur la tête, le cou et la poitrine, sans barres sur les ailes. La fauvette des pins et la fauvette de Wilson ont une couronne verte ou noire; la fauvette jaune n'a ni les ailes ni la queue grises, elle est jaune citron plutôt qu'orange ou or.

Indications sur le terrain. La fauvette protonotaire est une espèce trop rare au Canada pour la signaler d'après des spécimens vivants.

Nid. Dans un trou d'une souche ou d'un tronc d'arbre; son nid est fait de radicelles de fines ramilles, de mousse, de duvet végétal ou de plumes.

Habitat. Le vallee du Mississipi; au nord à peine jusqu'a la frontière canadienne, qu'elle passe accidentellement dans la région inférieure des grands lacs.

Cet oiseau n'est compris dans la liste canadienne que par suite de quelques rencontres qu'on en a faites dans la région inférieure des grands lacs. C'est un oiseau des terrains submergés et des buissons qu'entourent des eaux stagnantes.

\section{Genres-Helmitheros, Vermivora et Compsothlypis.}

\section{Fauvettes vermivores.}

Le genre Vermivora est un groupe de petites fauvettes de charpente délicate et chez lesquelles les caractères suivants sont très facilement discernés. Le bec est petit, très pointu, presque pareil à une épine, et la ligne du culmen est droite ou presque concave plutôt que convexe ou légèrement arquée (figure $57, p .29$ ). Les queues sont fortement colorées et sans taches blanches.

639. La Fauvette vermivore. (WORM-EATING WARBLER). Helmitheros vermivorus. L, 5.51. Olive mat en dessus; la tête chamois avec des lignes brun foncé, qui forment un contraste marqué, en travers de l'oil et de la couronne en bordure. Peu de variété dans le plumage. 
Traits distinctif8. C'est la seule fauvette avec cette marque distinctive foncée et cbamois à la tête. Le bec est plutôt massif pour une fauvette de cette espèce.

Indications sur le terrain. Oiseau trop rare au Canada, pour qu'on puisse se fier a un simple coup d'cil pour l'identifier.

Nid. Sur le sol; nid de radicelles, de feuilles et d'écorce.

Habitat. L'est des Etats-Unis; un seul signalement mentionné au Canada, dans le sud de l'Ontario.

C'est un oiseau des plateaux boisés ou des taillis marécageux; se nourrit près du sol.

641. La Fauvette aux ailes bleues. (BLOE-WINGED WARBLER). Vermivora pinus. L, 4.80. C'est une fauvette verte avec un front jaune, la gorge, la poitrine et les dessous de la même coulcur; une ligne noire fine en travers de l'oil, les ailes et la queue gris bleu. Deux barres blanches aux ailes, la femelle semblable mais plus terne. Peu de variété dans le plumage.

Traits distinctifs. La face et les dessous jaune clair, avec une raie noire a l'oil, les ailes gris bleu avec barres blanches, tout cela est distinctif.

Indications sur le terrain. Cet oiseau est trop rare au Canada pour qu'on puisse se fier a des indications de ce genre pour l'identifier.

Nid. D'ordinaire sur le sol au milieu ou tout près des secondes récoltes dans un nid d'écorce et de feuilles garni de fines bandes d'écorce et de vrilles, solidement entourées de feuilles.

Habitat. L'est de l'Amérique du Nord; s'arrête régulièrement au sud de la frontière canadienne et ne la traversant qu'accidentellement dans le sud dc l'Ontario.

Bien qu'elle n'ait été prise qu'une seule fois au Canada, c'est une très intéressante espèce car elle s'accouple avec l'espèce suivante, la fauvette aux ailes dorées, procréant des sujets hybrides déconcertants qui furent longtemps regardés comme des espèces séparées sous les noms de fauvettes de Lawrence et de Brewster.

642. La Fauvette aux ailes dorées. (GOLDEN-wingen warbler). Vermitora chrysoptera. L, 5.10. C'est unc fauvette gris bleu; le mâle a du blanc ou du gris très clair en dessous, qui devient foncé sur les flanes, avec bonnet jaune et plaque de même couleur sur l'aile; joues et gorge noires. La femelle est semblable au mále, mais un peu moins brillante et les surfaces noires sont représentées par du gris foncé. Il y a peu de variation dans le plumage selon l'âge et la saison.

Traits distinctifs. Son corps gris bleu, la tache jaune de l'aile, la gorge et les taches noires autour de l'oeil sont distinctives.

Indications sur le terrain. Les indications susdites sont facilement reconnaissables chez l'oiseau en vie. La gorge noire rappelle un peu la mésange à tête noire mais les autres marques suffisent tout aे fait à l'en distinguer.

Nid. Sur le sol ou dans les champs a buissons ou de seconde récoltc; c'est un nid asscz pareil à celui de la fauvette aux ailes bleues.

Habitat. L'est des Etats-Unis; elle traverse nos frontières régulièrement dans le sud de l'Ontario le long du lac Erié et dans l'angle inférieur du lac Huron.

Cet oiseau est ordinairement trouvé dans les terres incultes chargées d'arbrisseaux ou le long des lisières buissonneuses des forêts. On ne peut s'attendre à le voir que dans l'Ontario méridional.

645. La Fauvette de Nashville. (Nashville warbler). Vermivora rubricapilla. L, 4.77. C'est une fauvette jaune et verte avec une tête grisâtre et une tache marron plus ou moins dissimulée sur la couronne. Le sexe, la saison et l'âge font varier les plumages seulement quant à l'intensité du jaune et à la quantité de couleur marron sur le bonnet. Chez les femelles le bonnet peut étre entièrement dissimulé par les bords gris des plumes, et même il peut étre tout a fait absent.

Traits distinctifs. C'est le vert indistinct au-dessus, et le jaune de tous les dessous jusqu'a la queue, mais surtout brillant sur la gorge et la poitrine; la tête et les joues ou grises ou grisâtres sont encore les traits distinctifs. Cette tête grisâtre et le derrière du cou peuvent n'être pas bien marqués, mais on les voit toujours comme formant une légère 
différenciation d'avec le vert du dos. Quand elle est présente la couronne marron (non point orange-rougeâtre comme chez la fauvette à couronne orangée) est un caractère spécifique qui ne peut pas tromper. grises.

Indications sur le terrain. Les dessous d'un jaune brillant, sans raie, tête et joues

Nid. Sur le sol dans des terrains en partie défrichés ou des pâturages boisés; dans des nids faits d'herbes et de mousse, garnis d'herbes plus fines et de fines radicelles.

Habritat. L'est de l'Amérique du Nord. Au Canada, dans le nord au delà des régions colonisées. La fauvette de Nashville, de l'Est, s'étend à l'ouest jusque vers les montagnes.

SOUS-ESPECES. La fauvette de Nashville comprend celle de l'Est, qui est la forme typique, V.r. rubricapilla, et celle de l'extreme Ouest qui s'appelle la fauvette de Calaveras.

Cette fauvette se rencontrera surtout dans les plantations d'arbustes et dans les haies qui bordent les chemins de campagne.

646. La Fauvette à couronne orangée. (ORANGE-CRowned WARBLER). Vermivora celata. L, 5 . C'est une fauvette d'une jaune mat, gris vert, plutôt jaune en dessous, avec une tache orange-rougeatre dissimulée sur la couronne. Très peu de différence sexuelle. Les toutes jeunes n'ont pas la tache sur la couronne et l'éclat du jaune des dessous est ramené presque a la coulcur du dos.

Traits distinctifs. Cette fauvette est semblable à celle de Nashville, mais sans le gris ou le grisâtre sur la tête. La tache sur la couronne, quand elle existe, est encore plus dissimulée que celle de la fauvette de Nashville; elle est même tout à fait cachée jusqu'd ce que les plumes soient séparées pour laisser voir la couleur de leurs bases. La gorge jaune est plus terne que dans la fauvette de Nashville. L'oiseau très jeune est presque d'un vert grisâtre uniforme avec de vagues soupçons cendrés et assez semblable à la très jeune fauvette du Tennessee, mais sans la ligne claire et vague du sourcil; elle est plus uniformément colorée et sans indication quelconque de blanc dans les dessous.

Indications sur le terrain. Pareille à une fauvette de Nashville de couleurs très ternes, ou a une jeune fauvctte du Tennessee sans la ligne vague du sourcil.

Nid. Sur le sol ou tout près, nid fait de feuilles et d'herbes fines.

Habitat. Le centre et l'ouest de l'Amérique. Celle à couronne orangée de l'intérieur couve depuis l'Alaska jusqu'au Manitoba et n'est que migratrice a travers le Canada oriental.

SOUS-ESPECES. L'espèce comprend trois sous-espèces. La fauvette à couronne orangée de l'Intérieur $V$. c. celata-la forme type-avance \& l'ouest jusqu'aux montagnes.

C'est une des plus rares parmi les fauvettes particulières au Canada oriental. Il ne suffit pas de l'avoir simplement aperçue pour en garantir la présence dans le Canada oriental.

647. La Fauvette du Tennessee. (TENNESseE WARBLER). Vermivora peregrina. L, 5. Le dos du mâle est vert; les dessous sont presque d'un blanc pur. La tête et l'arrière du cou d'un gris cendré, disséminé sur les joues. La ligne du sourcil est blanche avec une apparence de ligne noire en travers de l'œil. Chez les femelles et les toutes jeunes fauvettes le gris de.la tête et de l'arrière-cou est remplacé par le vcrt du dos qni s'étend plus ou moins en un jaune mat ou un jaune verdâtre sur la poitrine et les desssous. La ligne sourcilière est toujours visible comme une coloration plus claire du vert.

Traits distinctifs. La coloration générale verte et blanche, et la ligne claire du sourcil sont les meilleurs de ces traits dans tout plumage.

Indications sur le terrain. La coloration générale comme ci-dessus, avec la ligne claire du sourcil et sans les barres blanches de l'aile, les taches à la queue ou la tache blanchatre à la base des primaires de la fauvette bleue à gorge noire.

Nid. Dans la mousse sur le sol parmi la végétation petite et compacte des conifères; le nid fait d'herbes ténues, de radicelles et de longs poils.

Habitat. L'est de l'Ámérique, probablement jusque vers la limite nord des forêts. D'habitude cet oiseau est rarement migrateur à travers nos régions colonisées, mais il est commun dans nombre de localités.

Cette fauvette est plutôt rare dans presque toutes les parties du Canada oriental. Dans le temps de la migration on la trouve vers le sommet des arbres. 
648. La Fauvette d'Amérique. (PARUla WARBLER). Compsothlypis americana. I, 4.73. Les dessus et les cótés de la face et du cou sont bleus, un bleu presque brillant, avec du jaune étendu sur le milieu du dos. Les dessous sont blancs, la gorge et la poitrine jaune clair avec une bande vaguement noire (ou noir bleuâtre) et rougeâtre en travers de la poitrine. Les femelles et les tout jeunes oiseaux sont plus ternes, sans la bande pectorale, et avec le jaune plus ou moins disséminé sur tous les dessus, mais très foncé au milieu du dos. Des barres blanches sur les ailes dans tous les plumages.

Traits distinctifs. Le dos bleu, ou pur ou mélangé de jaune; les barres des ailes, la poitrine et la gorge jaunes sont toujours distinctifs.

Indications sur le terrain. Voir le numéro précédent.

Nid. Dans des touffes pendantes d'usnée (barbe de vieillard) ou d'autres lichens pendants.

Habitat. Principalement l'est de l'Amérique. La fauvette d'Amérique du Nord occupe le nord de ce territoire particulier, jusqu'aux limites de la colonisation actuelle; elle ne couve pas dans la région des grands lacs inférieurs.

SOUS-ESPECES. La fauvette d'Amérique comprend deux sous-espèces: celle du Nord et celle du Sud. L'individu canadien, la fauvette du Nord, (Northern Parula) C. a. usnea, est ainsi nommé d'aprés le lichen dans lequel il bâtit son nid.

\section{Genre-Dendroica. Fauvettes des régions boisées.}

Le genre Dendroica est composé de fauvettes d'une charpente plus robuste que celle du genre Vermivora. Le bec est plus long et le culmen sensiblement plus arqué (figure 58, p. 29). La queue, sauf celle de la fauvette jaune, a beaucoup de blanc.

650. La Fauvette du cap May. (Cape may warbler). Dendroica igrina. L, 5. Le mâle: la gorge, la poitrine et presque tous les dessous jaune brillant rayé de noir au bas de la gorge, de la poitrine et des flancs. Une tache marron au milieu de la joue se détache fortement sur le jaune. Le sommet de la tête d'un noir qui continue sous forme de taches allongées sur le vert jaune du dos. Le croupion est jaune et l'aile a une grande tache blanche. La femelle: les dessus gris olive; les dessous d'un blanc sale réchauffé par du jaune répandu en travers de la poitrine, laquelle est vagucment rayée de jaune foncé. Le croupion est jaunâtre et la tache blanche de l'aile est remplacke par des barres vaguement distinctes. Les jeunes: semblables aux oiseaux de printemps, mais moins brillants; le mâle n'a pas les joues marron. Les femelles: gris olive uniforme et terne, légèrement jaune au croupion; un blanc terne légèrement olive en dessous, vaguement rayé de lignes tendres et foncées, et quelques vagues touches d'olive jaunÁ tre sur la poitrine et les flancs.

Traits distinctifs. Les mâles sont reconnaissables à leurs couleurs tigrées d'un jaune rayé de noir. Les femelles adultes laissent voir assez du dessin propre au male pour qu'on puisse les reconnaitre. Les jeunes femelles sont plus difficiles \& reconnaftre. Toutefois, toutes font voir au moins assez de jaune dans les parties internes des plumes sur les côtés du cou juste derrière les oreilles. Cette légère teinte se montre quelquefois, chez l'oiseau vivant, quand il tourne la tête, mais si l'oiseau est dans la main, il faut séparer les plumes pour apercevoir cette teinte.

Indications sur le terrain. Les adultes et les tout jeunes máles sont faciles a distinguer. Les toutes jeunes femelles peuvent se reconnaître à la raie très fine et obscure de la poitrine qui ressort sur un fond légèrement chamois, gris olive clair, ou encore à la tache jaune, dissimulée sur les côtés du cou, comme c'est décrit plus haut.

$\mathrm{Nid}$. Sur les branches basses des petits arbres dans les pâturages ou les terrains boisés; le nid est en partie suspendu et fait de ramilles et d'berbes réunies ensemble par des fils d'araignée et garni avec du crin de cheval.

Habitat. L'est de l'Amérique, à l'ouest jusqu'aux prairies, au nord jusqu'au delà des: régions colonisées.

Cette fauvette est une des plus belles; elle est considérée ordinairement comme rare, mais devenue plus commune selon les localités. C'est une espèce qui aime le sommet des arbres dans les régions boisées, mais qu'on voit souvent dans les vergers et dans les arbres feuillus.

$$
28588-15 \frac{1}{2}
$$


652. La Fauvette jaune. (Yellow Warbler). Dendroica æastiva. L, 5.10. Planche XL A.

Traits distinctifs. La fauvette jaune peut facilement être confondue avec quelques autres espèces. Sa queue, avec du jaune sur les barbes intérieures des plumes, la fera distinguer de toutes les autres espèces uniformément jaunes ou vertes.

Indications sur le terrain. Le jaune brillant égal et uniforme des oiseaux de printemps ne peut pas tromper. Quelques femelles ternes sont plus vertes que jaunes, mais les ailes à bords verts, la queue jaune, les contrastes dus à l'absence de couleur, la taille et les mouvements de l'oiseau sont facilement reconnaissables.

Nid. Il est placé dans une fourche droite de branches, dans les buissons et les petits arbres; il est fait de fibres délicates et d'une bonne quantitế de duvet végétal; garni avec ce même duvet et quelquefois de longs poils.

La fauvette jaune est une des rares espèces qui parfois refusent de couver des oufs d'étourneau. Au lieu de jeter dehors l'œuf intrus, elle bâtit un nouveau nid au-dcssus de l'ancien qu'elle ensevelit avec tout son contenu, y compris, souvent, quelques-uns de ses propres oufs dans les fondements de la nouvelle construction, au sein de laquelle une nouvelle série d'œufs est déposée.

Habitat. Presque toute l'Amérique du Nord. La fauvette jaune de l'Est habite tout le Canada, a l'exception du versant du Pacifique.

SOUS-ESPECES. La fauvette jaune comprend quatre sous-espèces; la fauvette jaune de l'Est, $D$. astiva, qui occupe la plus grande partie du Canada, est le type de l'espèce.

Cette fauvette est la couveuse la plus commune dans le sud du Canada. Elle partage avec le chardonneret le nom populaire de "Canari sauvage", mais l'absence de noir la fera reconnaître au premier coup d'œil. On la rencontre dans les lieux buissonneux en pleine campagne ou le long des cours d'eau et sur le bord des marais. C'est une visiteuse habituelle des jardins où son petit chant jovial fait plaisir à entendre. En automne la fauvette jaune est une des premières espèces à partir. Un peu après le mois de juillet elle disparaît, et vers la mi-août il ne reste plus que quelques traînards. Elle part avant que maint observateur ait commencé à penser aux migrations d'automne, c'est ainsi que le détail de ses mouvements d'automne est difficile à suivre.

654. La Fauvette bleue à gorge noire. (BLACK-THroated blue warbler). Dendroica carulescens. L, $5 \cdot 28$. Planche XL B.

Traits distinctifs. Le male, dont le nom est si descriptif et qui est fortement caractérisé, est très facile à reconnaître. La femelle, cependant, l'est beaucoup moins, surtout en automne; elle peut ressembler soit au jeune oiseau du Tennessee, soit à la fauvette rayée de l'automne, soit à la fauvette à poitrine baie. Le dos rayé et les barres de l'aile, cependant, des deux dernières sont absentes. Elle est plus foncée et plus vert-grisâtre que l'oiseau du 'Tennessee, en dessus, et plus chamois en dessous. Une tache blanche ou claire, indistincte et en partie dissimulée à la base des primaires fait toujours reconnaître les femelles de cette espèce.

Indications sur le terrain. Le mâle est facile à distinguer. La femelle sous quelque plumage que ce soit peut ordinairement être distinguée de toutes les fauvettes de couleur uniforme vert mat, grâce à une tache parfois très vague et claire à la base des primaires, tache qui se voit bien plus à l'état vivant qu'on ne s'y attendrait.

Habitat. L'est de l'Amérique du Nord, couvant dans le nord, mais au sud des régions colonisées.

SOUS-ESPECES. La fauvette bleue à gorge noire comprend deux sous-espèces dont une seule se trouve au Canada, la forme typique $D$.c. corrulescens, la fauvette à gorge noire du Nord, quoique parfois, des spécimens se rapprochent beaucoup de la variété méridionale, la fauvette de Cairn $D$. c. cairnsi, par le fait qu'ils ont comme un soupçon de taches noires sur le dos.

655. La Fauvette à croupion jaune. (MYRTLE WARBLER). Dendroica coronata. L, 5.65. Planche XLI A.

Traits distinctifs. C'est une espèce très facilement reconnaissable. Tous les plumages ont au moins des soupçons du jaune de la couronne, du croupion et des marques latêrrales. Les tout jeunes sont principalement brun rouilleux en dessus, et ont plus ou moins d'une nuance de cette même couleur en travers de la poitrine avec des rajes très faiblement indiquées. 
Indications sur le terrain. Le croupion jaune est toujours distinct et voyant, formant l'indication la plus visible sur le terrain. Sa présence et les marques de méme couleur du flanc et de la couronne toujours présentes quoique parfois indistinctes, en automne, constituent des guides sûrs pour l'identification a l'état vivant.

Nid. Dans les arbres coniferes entre 5 et 10 pieds au-dessus du sol; le nid est fait de fibres végétales et garni d'herbages.

Habitat. Presque toute l'Amérique du Nord jusqu'a la limite des arbres. Elle couve juste au dela de la colonisation populeuse.

C'est une fauvette qui arrive de bonne heure au printemps et qui est la dernière à repartir en automne. Parfois, en cette dernière saison, les lieux buissonneux, les bords des routes, et les clôtures que la végétation recouvre, sont pleins de fauvettes à croupion jaune, chacune montrant, comme elle s'envole, son croupion bien reconnaissable en preuve de son identité.

657. La Fauvette a tête cendrée. (Magnolu warbler). Dendroica magnolia. L, 5.12. Planche XLI B.

Traits distinctifs. La poitrine d'un jaune voyant et les dessous fortement rayés de noir, les joues noires, les dessus grisatres sont parfaitement reconnaissables au printemps. Les oiseaux d'automne ont des restes bien reconnaissables du plumage adulte, mais les marques de la poitrine sont absentes et celles des flancs sont tres amoindries. La tête et les joues sont uniformément grisatres et le dos est grisâtre et ombré de vert jusqu'au croupion qui est étendu de jaune.

Indications sur le terrain. Le jaune en dessous et au croupion fera distinguer la fauvette à tête cendrée de toutes les autres sauf de la fauvette du Cap May. La poitrine jaune de cette dermière est nettement et plus uniformément raýe de noir et elle a la tache marron à l'oreille. Un examen très attentif de la fauvette a tête cendrée, dans son plumage d'automne, fait toujours apercevoir une barre vague, cendrée en travers du haut de la poitrine. La queue est aussi un bon moyen d'identification. Les marques blanches si caractéristiques de la queue se trouvent en arrière de la pointe et sont assez étendues, donnant quand on les regarde de dessous l'impression d'une queue blanche avec une large bande de noir.

Nid. Est placé sur les arbres conifères a une hauteur de 3 à 6 pieds au-dessus du sol; il est fait de ramilles fines, de tiges de feuilles et garni de radicelles ressemblant à des poils.

Habilat. L'est de l'Amérique du Nord, dans le nord jusqu'à la limite des arbres; elle couve surtout au nord des régions cultivés.

Cette fauvette est une des plus belles de son espèce; quand la migration a lieu, elle se loge dans les arbres feuillus et dans les vergers où ses belles couleurs se marient de façon charmante aux arbres en fleurs.

658. La Fauvette bleue. (CERULean warbler). Dendroica cetulea. L, 4.50. Le mâle: bleu de ciel et blanc. En dessus, tout bleu avec de fines marques noires sur le dos et sur les côtés de la couronne. Au-dessous, blanc pur avec une bande pectorale bleue et des raies bleues aux flancs; les barres des ailes sont blanches. La femelle: en dessus, un bleu verdâtre uniformément terne; blanc en dessous, plus ou moins taché de quelques soupçons de vert et de jaune. Les tout jeunes sont semblables, mais plus jaunes partout.

Traits distinctifs. Le mâle ne peut donner lieu a aucune erreur. D'autres plumages ont une teinte bleuâtre singulière au lieu d'olive ou de jaunatre; le vert des dessus est tout a fait caractéristique. Le tout jeune avec ses dessous verdâtres et jaunâtres est assez semblable à la jeune fauvette du Tennessee, puisqu'il a la même raie au sourcil, mais ses barres blanches a l'aile et les taches a la queue l'en distingueront assez.

Indications sur le terrain. Le bleu de l'adulte et l'éclat bleuâtre d'autres plumages. Autrement il ressemble a la jeune fauvettc du Tennessee, mais avec les barres de l'aile et le blanc sur la queue.

Nid. Dans un arbre, entre 20 et 60 pieds au-dessus du sol; un nid fait de fibres fines liées avec des toiles d'araignées, garni de bandes de fibres d'écorce et de quelques lichens à la surface extérieure.

Habitat. L'est des Etats-Unis, sauf la côte, au nord le long du lac Erié jusqu'd un peu au delà de la frontière canadienne.

Cette fauvette est trop rare au Canada pour qu'on puisse s'attendre à la rencontrer sauf dans une région limitée du sud de l'Ontario. 
659. La Fauvette de Pensylvanie. (chestnut-siden warbler). Dendroica pensylvanica. L, 5.14. Le mâle: couronne jaune; dos noir et gris, en raies, étendu d'un vert jaunatre; en dessous du blanc avec des bandes marron le long des flancs, deux barres aux ailes, ou blanches ou jaunatres. Les jeunes: en dessus un vert jaunâtre presque uniforme; en dessous du blanc, les joues sont grises; ordinairement avec des soupçons de cette couleur marron que le mâle a aux flancs.

Traits distinctifs. Une attention superficielle apportée à la description qui précède pourrait faire confondre cette espece avec la fauvette a poitrine baie, mais la gorge blanche est distinctive. Les oiseaux de printemps avec leur bonnet jaune, leurs flancs marron et leurs dessous blancs; et les oiseaux d'automne blancs en dessous, vert-jaunatre en dessus, et avec les barres jaunâtres aux ailes sont facilement reconnaissables.

Indications sur le terrain. Les parties de dessous blanches et le vert singulièrement citron des dessus sont des indications excellentes même dans les plumages où les marques caractéristiques ne se laissent pas voir.

Nid. Dans les buissons à quelques trois pieds au-dessus du sol; nid fait de bandes d'écorce, de tiges de feuilles, etc., garni de vrilles et de radicelles.

-- Habitat. L'est de l'Amérique du Nord, a l'ouest jusqu'aux provinces des prairies, au nord au delà des limites de la colonisation. Les nids sont dans les localités où on la trouve dans l'est du Canada, sauf dans la partie d'extrême sud.

La fauvette de Pensylvanie est ordinairement trouvée dans les lieux buissonneux et secs, dans les secondes récoltes et dans les arbustes à framboises.

660. La Fauvette a poltrine bale. (BAY-BREAsted warbler). Dendroica casta* nea. L, 5.63. Le mâle: finement rayé d'ocre-olive mat et de noir en dessus; les dessous sont blancs; le haut de la tête, la gorge, l'avant-cou et les flancs sont de couleur baie (marron rougeâtre); le front et les joues sont noirs; il y a une tache d'ocre pâle sur le côté du cou. La femelle a toutes ces marques caractéristiques très propres à la faire facilement reconnaître, quoique voilées et vaguement indiquées. Les oiseaux d'automne, toutefois, sont absolument différents; en dessus un vert-jaunâtre vaguement rayé de noir, en dessous un blanc plus ou moins teinté d'un vert-jaunâtre ou chamois; la couleur baie des côtés est souvent indiquée par un rouge un peu plus chaud ou par des plumes individuelles entièrement colorés.

Traits distinctifs. Les oiseaux de printemps sont bien faciles a distinguer. Les individus d'automne ressemblent à la fauvette rayée de façon si étroite que souvent on ne peut les en distinguer qu'avec peine, même quand on en tient dans la main. La fauvette à poitrine baie a presque toujours une certaine quantité d'ocre de tons très cbauds sur les flancs, ce que n'a pas la fauvette rayée, et les couvertures sous-caudales sont crème au lieu d'un blanc pur. La présence de barres aux ailes fera distinguer ces deux espèces de toute autre fauvette simplement verte.

Indications sur le terrain. Le mâle adulte est reconnaissable à la couleur. La femelle de printemps fait toujours voir assez de sa poitrine baie pour qu'on la reconnaisse. Les oiseaux d'automne adultes ont aussi d'ordinaire une trace de bai sur les flancs et les chauds tons ocreux de ces parties peuvent d'ordinaire se voir sur les jeunes. Mais lorsque ces caractères manquent pour distinguer l'espèce, une attention marquée fera voir que la couleur de la poitrine est parfaitement uniforme, et avec des yeux perçants ou des verres grossissants on apercevra d'ordinaire des raies vaguement foncées qui se montrent sur les cótés de la poitrine de la fauvette rayée. Aucune de ces indications, cependant, ne se verra sinon dans les circonstances les plus favorables, mais dans les troupes mélangées on peut habituellement dire la proportion de chaque espèce avec passablement d'exactitude.

Nid. Dans des arbres conifères, de 5 a 20 pieds au-dessus du sol, dans un nid fait d'herbes et de plantes fibreuses, garni avec du duvet de ces plantes et de longs poils.

Habitat. L'est de l'Amérique du Nord, a l'ouest jusqu'à l'autre côté des prairies; au nord jusqu'à la limite des forêts. Elle couve au dela des régions babituellement cultivées.

La fauvette à poitrine baie pendant la migration du printemps préfère les broussailles dans les localités sablonneuses, et le bord des chemins, etc. cependant elle se risque près des maisons dans les arbres feuillus et les vergers. La ressemblance entre la fauvette à poitrine baie et la fauvette rayée d'automne, oiseau d'un plumage complet tout à fait différent, est un des phénomènes très intéressants de la coloration des oiseaux. Les plumages d'automne de ces deux oiseaux n'étaient jadis nullement distingués l'un de l'autre, mais confondus sous le nom de fauvette d'automne. 
661. La Fauvette rayée. (BLAck-PoLl WARBLER). Dendroica striata. L, 5.56. Le mâle: raies noires et blanches avec une couronne entièrement noire; des lignes fines de noir et de blanc sur le dos; les dessous tout blancs avec du noir, depuis la base du bec jusqu'aux côtés du cou, qui se divise en raies sur les flancs; joues blanches. La femelle, les dessus verdâtres; le blanc des dessous est étendu de vert sur la gorge et la poitrine mais avec suffisamment de raies noires, plus ou moins visibles, pour qu'on reconnaisse l'oiseau comme étant la femelle du précédent. Les oiseaux d'automne ne peuvent presque pas se distinguer de la fauvette à poitrine baie (voir ci-dessus).

Traits distinctifs. Les mâles de printemps avec leurs marques blanches et noires très distinctes ne peuvent étre confondus qu'avec le grimpereau blanc et noir, mais leurs habitudes n'étant pas grimpantes, et leur couronne qui est entièrement noire, au lieu d'avoir une large raie médiane blanche, les feront aisément distinguer. La rayure de la femelle est bien distinctive. Les oisealux d'sutomne peuvent se distinguer de la fauvette a poitrine baje par l'absence de couleur ocreuse, baie ou chamois sur les flancs et par les couvertures sous-caudales absolument blanches.

Indications sur le terrain. La fauvette rayce se montrc sous trois différents plumages: le mâle est noir ct blanc; la femelle est verdâtre; les dessous gont blanes et plus ou moins rayés de noir; les oiseaux d'automne, comme les fauvettes a poitrine baie avec de ragues raies, visibles seulement pour des yeux perçants ou avec de bonnes loupes, se montrent dans les flancs jaunftres.

Nid. Généralement dans les gapins à 6 pieds environ au-dessus du sol; nid fait de branchettes, de mousses, de radicelles etc., garni d'herbes fines et de vrilles.

Habitat. Presque le même que celui des espèces précédentes.

La fauvette rayéc est une des dernières de cette famille à arriver au printemps, d'habitude après que la plupart des autres bandes migratrices sont parties au nord.

662. La Fauvette de Blackburn. (BLACKBURNAN WARBLER). Dendroica fusca. L, 5.25. Planche XLII A. Les parties de dessus qui sont noires, et l'orangé éclatant de la gorge, sont des marques distinctes dans le cas du male adulte. Chez la femelle la couleur orange de la gorge, quoique indigtincte, est cependant reconnaissable. Les jeunes en automne sont comme la femelle adulte, lors même que les jeunes femelles aient des couleurs plus ternes, la gorge et la poitrine ayant retenu, seules, une coloration janne assez vague.

Traits distinctifs. Les males adultes et de l'automne sont absolument reconnaissables, sans erreur possible. Les femelles de l'automne peuvent être confondues avec les fauvettes rayées et les fauvettes a poitrine baie de l'automne, mais le jaune plus clair de la gorge, l'absence de teinte grisatre en dessous, et les couvertures sombres de l'oreille avec la ligne chamois des sourcils sont des traits distinctifs caractéristiques.

Indications sur le terrain. Le brillant orangé, ou le jaune a tons chauds bornés à la gorge et à la poitrine et la raie jaune-orangé ou chamois des aourcils, en contraste avec les joues foncées, constituent les meilleures indications sur le terrain.

Nid. Dans les sapins, de 10 a 14 pieds au-dessus du sol. Nid fait de fines branchettes et d'herbes, garni d'herbes et de vrilles.

Habitat. L'est de l'Amérique du Nord, à l'ouest des provinces des prairies; clle couve aurtout au nord des localités populeuses.

Le mâle de la fauvette de Blackburn possède ce plumage brillant qu'on attribue communément au climat des tropiques plutôt qu'à celui de nos contrées plus froides. L'espèce s'associe avec beaucoup d'autres fauvettes au sommet des arbres de forêts ou de vergers.

667. La Fauvette a poitrine noire. (BLACK-THRoated oreen warbLer). Dendroica virens. $\mathrm{L}, 5 \cdot 10$. Planche XLII $B$.

Traits distinctifs. C'est une fauvette verdâtre avec des joues d'un jaune brillant et en contraste marqué avec le noir de la gorge et de la poitrine. Chez les femelles et les jeunes le noir est presque absent ou seulement indiqué par de vagues diffusions de noir, parfois interrompues, mais surtout plus fortes sur la poitrine. Chez les jeunes femelles le noir se réduit à de petits nuages ternes sur les côtés de la poitrine et des flancs. Dans tous les plumages les dessous sont blanes, le dos et la couronne sont clairs, même verts. Le jaune brillant prédomine sur les joues. 
Indications sur le terrain. Pour les tout jeunes, le dos vert, les joues jaunes, et les dessous blancs. Pour les adultes, la gorge et la poitrine noires contrastant avec la face jaune et les dessous blancs.

Nid. Sur les sapins entre 5 et 30 pieds au-dessus du sol, un nid fait de petites branchettes et de mousse, garni de radicelles, d'herbes ténues et de vrilles.

Habitat. L'Amérique du Nord, à l'ouest jusqu'aux montagnes. La couvaison a lieu dans l'est du Canada, partout oư le cèdre ou les halliers toujours verts se rencontrent sauf dans les régions très méridionales.

C'est un oiseau très frappant, ami des arbustes toujours verts et chétifs, mais aussi des vergers et de la sommité des arbres a feuilles décidues.

670. La Fauvette de Kirtland. (KIRTLand's warbler). Dendroica kirtlandi. L, 5.75. Gris bleu en dessus fortement rayé de noir sur le dos, et finement rayé sur la couronne. Tous les dessous, sauf sous la queue, sont jaune pâle avec des raies noires sur les côtés de la poitrine et sur les flancs. Les femelles et les tout jeunes sont semblables mais plus ternes, les raies de la poitrine sont brisées et forment des taches.

Traits distinctifs. La fauvette de Kirtland ressemble à la fauvette du Canada, mais elle est plus grande; le jaune est plus pâle et les raies noires sont sur les flancs et ne servent pas a simuler un collier suspendu aux oreilles, comme dans cette espece; en outre, le dos est marqué de noir au lieu d'être gris clair. La seule autre fauvette qui pourrait être confondue avec celle-là, c'est la fauvette à tête cendrée, mais le noir ne traverse pas la poitrine comme c'est le cas pour les fauvettes à tête cendrée adultes, et il n'y a ni jaune ni vert sur le dos ou sur le croupion.

Indications sur le terrain. Cette espèce est trop rare pour qu'on accepte l'identification sur une simple vue.

Nid. 'Sur le sol au pied d'un chêne; nid fait d'écorce tendre, de bandes fibreuses végétales, d'herbe, et garni d'herbes ténues, d'aiguilles de pins et de poils.

Habitat. Pendant l'hiver, aux fles Bahama. La seule station connue pour la couvaison se trouve dans une région limitée des plaines de pin gris (jack-pine) dans la partie nord de la péninsule inférieure de l'Etat de Michigan. Cette espèce n'a encore été prise que deux fois au Canada, et chaque fois ce fut dans la région des grands lacs inférieurs. Son nid doit être cherché dans la péninsule Bruce de l'Ontario et autour des grands lacs à l'ouest de la région du lac Supérieur.

En hiver cette espèce est confinée en dedans d'un habitat insulaire de l'océan et constitue un exemple des difficultés que rencontre une multiplication anormale d'une espèce indigène. Il est évident que nous n'aurons jamais, dans le nord, une plus grande quantité de fauvettes de Kirtland que ne peuvent en nourrir en hiver les îles Bahama.

671. La Fauvette des pins. (PINE warbler). Dendroica vigorsi. L, 5.52. C'est une fauvette d'un vert mat, le vert se changeant en jaune mat sur la gorge et la poitrine avec des ailes et la queue grises ou brunes, et d'indistinctes barres aux ailes. Quand le plumage est le plus beau, le jaune de la gorge et de la poitrine devient rarement assez brillant. Peu de changement soit en raison du sexe ou de la saison.

Traits distinctifs. Très semblable au vireo à front jaune, mais de couleur plus verte et avec le jaune des dessous plus foncé et plus étendu. L'abdomen blanc n'attire pas les regards. Le bec est léger, pareil à celui de la fauvette au lieu d'être fort, crochu et du type vireo. Cette fauvette peut se distinguer de la fauvette jaune par sa couleur plus terne et par le contraste entre ses ailes, la queue et le corps. Son goút pour les pins fait à tout le moins deviner son identité.

Indications sur le terrain. C'est une fauvette d'un vert mat, jaune sur la poitrine avec des ailes d'un brun grisâtre, des barres blanches aux ailes. Les spécimens canadiens ont d'habitude une apparence sale et crottée. On la trouve presque toujours dans les pins.

Nid. Dans les pins, de 10 à 80 pieds au-dessus du sol; nid fait de bandes d'écorce, de feuilles, de fibres végétales.

Habitat. L'est de l'Amérique du Nord, a l'ouest jusqu'aux prairies et au nord jusqu'aux limites des bois de pins; la couvaison, au Canada, se fait surtout au dela de la. colonisation. 
La préférence marquée pour les pins que manifeste cette fauvette est frappante. On la trouve rarement, peut-être même jamais, loin de ces arbres; elle est par conséquent exposée à avoir son plumage plus ou moins sali par la poix, ce qui lui donne un air terne et usé. Il est rare de trouver chez nous des oiseaux de cette espèce qui soient propres et brillants.

672. La Fauvette d couronne rousse. (PALM warbLer). Dendroica palmarum. L, 5.25. C'est une fauvette de couleur mate ou verdatre. Le mâle: tous les dessous sont brillants ou étendus de jaune; la calotte est marron rougeâtre. La poitrine et les flanes sont rayés plus ou moins de brun rougeâtre, et une ligne jaune aux sourcils contraste avec les joues foncées et la calotte rouge. Les femelles sont pareilles mais leur couleur est plus pâle. Les tout jeunes et les oiseaux d'automne sont brun grisâtre en dessus, chamois en dessous avec des raies pâles, presque blanches sur la gorge et étendues d'un jaune assez distinct qui devient très pur sur les couvertures caudales. Dans tous les plumages il y a une tache au croupion d'un jaune fondu dans la couleur générale.

Traits distinctifs. Les dessous jaunes et la calotte rouge ne peuvent prêter à aucune erreur chez les oiseaux du printemps. En automne les dessous vaguement rayés, le dos brun et les couvertures sous-caudales sont bien distinctifs.

Indications sur le terrain. Les mouvements habituels et saccadés de la queue, pareils à ceux de la maubeche, feront distinguer cette fauvette de toutes les autres avec des dessous jaunes, sauf de celle dite ades près". Le jaune brillant restreint aux couvertures souscaudales, la gorge clair et la poitrine vaguement rayée de chamois des jeunes oiseaux permettront de distinguer cette espece d'autres formes qui peuvent lui être comparées.

$N$ id. Sur le sol, ou tout près, dans un terrain marécageux, ou dans des mousses stériles; un nid fait d'herbes grossières, garni de plumes approprićes.

Habitat. L'est de l'Amérique du Nord, vers l'ouest, jusqu'aux prairies et au nord jusqu'à la limite des forêts; couvant la plupart du temps au dela des limites de la civilisation. La fauvette jaune à couronne rousse a un habitat de l'est, et va vers l'ouest jusqu'd la frontière est de l'Ontario. La fauvette à couronne rousse de l'intérieur occupe le reste du Canada oriental.

SOUS-ESPECES. La fauvette à couronne rousse comprend deux sous-espèces, la forme typique: la fauvette à couronne rousse de l'intérieur, Dendroica palmarum palmarum, qui se distingue de la suivante par le plumage printanier de l'oiseau adulte; et la fauvette jaune à couronne rousse, Dendroica palmarum hypochrysea qui se distingue par la moindre quantité de jaune et le moindre éclat brillant des dessous, qui sont légèrement teintés de grisâtre. Toutefois les deux formes sont trop semblables pour qu'on puisse les distinguer sans un spécimen de chaque sous-espèce pour faire la comparaison.

C'est un oiseau qui vit habituellement à terre. Au temps de la migration il faut le chercher dans des terrains bas, à végétation chétive, sablonneux et d'aucun usage; au temps de la couvaison, dans les marécages moussus.

673. La Fauvette des prés. (PRAIRIE WARBLER). Dendroica discolor. L, 4.75. C'egt une fauvette verte et jaune. La gorge, les joues et tous les dessous du mâle sont d'un jaune brillant; une ligne noire en travers de l'cil, un coin noir au-dessous des couvertures des oreilles, et une succession de lignes noires le long des cótés de la poitrine et des flancs. Le milieu du dos a une selle de taches rougeâtres. La femelle est semblable, mais quelquefois elle n'a pas du tout ou presque pas cette couleur rougeâtre sur le dos. Les tout jeunes sont pareils, mais les couleurs sont atténuées et voilées, les dessous sont jaunes, brillantes, sur la poitrine et les lignes noires de la face, des cótés de la poitrine et des flancs ne sont qu'indiquées.

Traits distinctifs. Les marques faciales fines et noires tout à côté d'un jaune brillant, leur prolongement très accentué le long des flanes, et les taches rougeâtres du dos sont les traits qui distinguent le mieux les adultes. Chez les jeunes oiseaux, les dessous sont surtout brillants sur la poitrine plutôt que sur la gorge ou ailleurs et les barres simplement indiquées sur les côtés au lieu de l'être au milieu de la poitrine constituent les meilleurs moyens de reconnaitre cette fauvette.

Indications sur le terrain. Elle est trop rare au Canada pour être distinguée à la simple vue, a moins que l'observateur n'ait acquis une grande expérience du sujet.

$N i d$. Dans les buissons de ronces, le nid est fait de fibres végétales et de duvet végétal, garni de radicelles et de longs poils. 
Habitat. L'est des Etatg-Unis, rarement au nord. L'oiseau est peut-être régulièrement en certaines places au delà de la frontière canadienne dans la région inférieure des grands lacs.

La fauvette des prés préfère les terrains abandonnés, secs, et sablonneux, où de seconde végétation. D'après les données qu'on a touchant sa présence au Canada, il faudrait la chercher quand elle niche dans le voisinage de l'extrémité inférieure du lac Huron.

\section{Genre Seiurus-Fauvettes hochequeues.}

Les oiseaux du genre Seiurus ressemblent plus à des grives qu'à des fauvettes, comme on l'indique par les noms populaires de "grive couronnée" et de "grive des ruisseaux" qu'on leur donne quelquefois. Mais ce sont, en réalité, de véritables fauvettes de mœurs forestières; oiseaux du sol qui marchent au lieu de sautiller; de taille plutôt grande pour des fauvettes; de couleur brune ou d'un olive foncé en dessus, les dessous blancs avec la poitrine fortement rayée. Toute proportion gardée, les becs sont semblables à ceux des fauvettes (figure 59, p. 29). On peut les prendre pour des grives, soit qu'on les voie en liberté ou dans la main; mais en faisant attention aux caractères spécifiques, on pourra les distinguer facilement.

674. La Grlve couronnée. (ovenBrnd). Seiurus aurocapillus. L, 6.17. Planche XLIII A.

Traits distinctifs. La tache qui simule une couronne d'un or mat en partie cachée, bordée de brun, servira toujours à déterminer l'espèce.

Indications sur le terrain. Bien que très semblable à la grive, il n'est pas du tout probable que cette fauvette puisse être prise pour une grive. La gorge d'un blanc pur, comme son avant-cou, les dessous où ne se voit que peu ou point du tout d'autre couleur, enfin des raies plutôt que des taches d'un si frappant contraste sur la poitrine rendent la distinction très facile.

Nid. Sur le sol dans une construction massive d'herbes grossières, de pétioles, de feuilles et de radicelles; recouvert de feuilles avec une entrée sur un côté, comme un four, d'ou lui vient son nom anglais.

Habitat. Les parties boisées de l'Amérique du Nord, à l'est des Montagnes Rocheuses, au nord par dela les localités colonisées. La couvaison se fait au Canada partout où se rencontre cet oiseau.

C'est un oiseau des régions boisées, ordinairement commun partout où des arbres d'accès facile se trouvent mêlés à ceux plus scrrés d'une forêt. Son chant habituel Titche-titche-titche, d'abord un peu sourd et finissant très fort est un son bien connu dans les forêts et qu'on n'oublie pas.

675. La Grive des rulsseaux. (NORTHERN WATER-THRUSH). Seiurus noveboracensis. L, 6.04. D'un brun olive foncé en dessus, d'un blanc jaunâtre finement strié de raies foncées en dessous; des lignes séparées sur la gorge, la poitrine et les flancs. Une ligne fondue, couleur chamois, au-dessus de l'œil, et une autre fine, accentuée et foncée en travers de l'oil.

Traits distinctifs. Facile à distinguer de la grive couronnée grâce aux rayures plus fines, plus uniformes, plus foncées, et aux dessous jaunâtres, grâce aussi à l'absence de la tache qui forme la couronne. ' Cette grive peut se distinguer de la grive des ruisseaux de la Louisiane par la ligne chamois au lieu de blanche au-dessus de l'ceil, par les dessous jaunâtres au lieu de chamois ou crème et par la présence de taches sur la gorge.

Indications sur le terrain. Les grives des ruisseaux peuvent être reconnues dans la vie ordinaire par les coups saccadés, habituels de la queue quand elles marchent ou se tiennent debout, mouvements très semblables à ceux de la maubèche tachetée commune. Ces deux grives des ruisseaux peuvent être distinguées par la différence des détails de coloration donnés plus haut.

Nid. Sur un banc de mousse ou dans les racines d'un arbre déterré, habituellement près ou au-dessus de quelque eau; dans un nid garni de mousse de vrilles et de fines radicelles. 
Habitat. Dans le nord de l'Amérique du Nord jusqu'aux limites de la colonisation. Couve au Canada partout où on la rencontre dans les régions les plus méridionales.

SOUS-ESPECES. La grive comprend deux sous-especes: une de l'Est et l'autre de l'Ouest. La grive de l'Est, $C$. n. noveboracensis, forme typique, occupe un territoire vers l'ouest jusqu'd l'Ontario méridional, ou elle ae mele avec la grive des ruisseaux de Grinnell, $S$. n. notabilis qui occupe la contrée vers l'ouest. cèdre.

Le domaine favori de la grive des ruisseaux est dans les marécages du

676. La Grive de la Loulslane. (Loulsiana water-THRush). Seiurus motacilla. $\mathbf{L}, 6 \cdot 28$. D'un brun olive foncé en dessus, d'un blanc, en dessous, fnement rayé de lignes brisées noires bien tranchées, allant du haut du cou à la poitrine et aux flanc8. Une ligne blanche, très tranchée au-dessus de l'ceil et une ligne assez fine d'un noir fondu en travers.

Traits distinctifs. Facile à distinguer de la grive couronnée par l'absence de la raie colorée formant couronne; très semblable a la grive des ruisseaux, mais s'en distinguant par sa taille un peu plus grande, par des dessous chamois plutót que jaunâtres, et par la blancheur des marques de la face.

W. Indications sur le terrain. La grive des ruisseaux de la Louisiane a l'habitude des sarcades de la queue dont la grive des ruisseaux est coutumière, et ne peut se distinguer de celle-ci, dans la vie ordinaire qu'en faisant attention aux différences notées ci-dessus.

Nid. Semblable a celui de la grive des ruisseaux.

Habitat. L'est des Etats-Unis et ne ae montrant au Canada que dans le sud-ouest de l'Ontario.

Cette grive est très semblable à la grive des ruisseaux par ses habitudes aussi bien que par la couleur et la forme. C'est cependant un oiseau d'un habitat plus méridional que l'autre et qui n'est que rarement aperçu au Canada.

Genres-Oporornis, Geothlypis et Icteria. Fauvettes terrestres.

Les fauvettes dites du sol sont plutôt plus grandes que les fauvettes des bois mais beaucoup plus petites que les fauvettes hochequeues et avec de grosses pattes et de courtes ailes. Leur coloration est généralement verte et d'un jaune brillant. Elles habitent des buissons plutốt bas et sont rarement vues fort au-dessus du sol.

677. La Fauvette du Kentucky. (KENTUCKY WARBLER). Oporornis formosus. L, 5.40. Verdatre en dessus; tous les dessous sont jaune clair; le front est noir et devient plus clair au milieu de la couronne: ligne sourcilière jaune brillant encerclant l'œil. Une tache noire bien tranchée a'étend de la base du bec entourant les lores et la plupart des couvcrtures auriculaires, jusqu'au côté du cou. La femelle et les oiseaux de l'automne sont semblables mais plus ternes, la marque noire de la face étant plus ou moins voilée quoique toujours visible.

Traits distinctifs. De coloration très semblable a celle de la fauvette des prés, mais le noir ne laissant qu'une étroite ligne sourcilière en avant de l'cil au lieu d'une joue presque toute jaune et sans les raies latérales. Assez semblable a la fauvette canadienne mais avec le dos verdâtre au lieu de gris et sans marque quelconque sur la poitrine.

Indications sur le terrain. Trop rare au Canada pour qu'on puisse la reconnaitre a première vue dans la vie ordinaire.

Nid. Sur le sol ou tout près, dans un nid massif fait de branchettes et de radicelles bien enveloppées de plusieurs épaisseurs de feuilles et garni de fines radicelles.

Habitat. L'est des Etats-Unis et n'atteignant pas la frontière canadienne a moins que l'oiseau ne soit un trainard par accident.

Cette espèce n'a eté prise au Canada que dans très peu d'occasions. On ne peut raisonnablement s'attendre à la trouver que dans les sections les plus méridionales de la région des grands laes inférieurs.

678. La Fauvette du Connecticut. (CONNEcticut WARBLER). Oporornis agilis. L, 5.40. C'est une fauvette verdatre. Le male est, en dessous, d'un jaune claire de citron, la face et la gorge jusqu'au haut de la poitrine, d'un gris bleuatre uniforme avec un joli cercle blanc autour des yeux. La femelle est semblable mais d'un gris plus pale. Chez les tout jeunes le gris est remplacé par une teinte chamois plus claire de la coloration du dos. 
Traits distinctifs. Cette espèce ressemble tellement à la fauvette de Philadelphie que parfois il est difficile de les distinguer l'une de l'autre. Les mâles adultes qui ont un cercle très visible autour des yeux, la gorge et la poitrine parfaitement uniformes, sont asse $z$ faciles a reconnaitre. Les femelles se reconnaissent par le cercle autour des yeux et par le fait qu'au sommet de la tête l'olive du dos est fortement étendu, et qu'il n'y a pas de gris clair. Les tout jeunes, quand ils ont le cercle autour des yeux, sont d'habitude bien reconnaissables, bien que les fauvettes de Philadelphie du même âge en ont comme une apparence. Quand le cercle autour des yeux ne fournit pas de preuve concluante, la différence de couleur de la gorge et de la poitrine, un olive chamois au lieu d'un jaune pur uniformément clair et gris dans les dessous, est un bon guide.

Indications sur le terrain. La gorge uniformément grise et le cercle blanc autour de l'œil chez les adultes et, chez les tout jeunes, la gorge olive chamois et le cercle chamois autour de l'œil. Cette fauvette et celle de Philadelphie marchent au lieu de sautiller.

Nid. Sur le sol et formé d'herbe sèche.

Habitat. L'est de l'Amérique du Nord à l'ouest des Alléghanies. Elle paraît couver sur la limite de la colonisation, au Canada, mais nous manquons de renseignements positifs. Ses migrations ne sont régulières que dans des localités le long des rives du lac Erié et du lac Ontario.

La fauvette du Connecticut est une des plus rares parmi les fauvettes d'habitudes régulières. Elle arrive tard au printemps et vit tellement isolée qu'on la voit rarement en automne. Nous n'avons pas assez de faits précis à cet égard pour dire si le champ de sa migration est purement local, ou si on n'y a pas assez fait attention. Il convient d'aller chercher cet oiseau tout près du sol dans des broussailles perdues.

679. La Fauvette de Philadelphie. (MOdRning Warbler). Oporornis philadelphia. L, 5.63. C'est une fauvette verdâtre. Le mâle a les dessous jaune citron; toute la tête, le cou et la poitrine et la gorge gris bleuâtre avec taches à demi cachées sur la poitrine et la gorge, où l'imagination voit quelque ressemblance avec du crêpe, d'où son nom anglais de fauvette en deuil. La femelle est pareille, mais le gris est plus clair et sans les marques de crêpe sur la poitrine, ressemblent ainsi au mâle de la fauvette du Connecticut. Les tout jeunes ont le gris de la couronne remplacé par la couleur verte du corps et le jaune des dessous s'étend du cou à la poitrine légèrement modifié par des teintes plus claires et grisâtres.

Traits distinctifs. La fauvette de Philadelphie peut, d'ordinaire, se distinguer par le crêpe sur la poitrine ou par un soupçon de la chose, par la couleur grise de la couronne et l'absence du cercle autour des yeux, quoique les jeunes aient quelquefois et très vaguement les dits cercles. Voir les espèces précédentes.

Indications sur le terrain. Le crêpe noir de la poitrine, l'absence du cercle autour des yeux chez les adultes, et chez les tout jeunes, l'absence de distinction tranchée entre la poitrine, la gorge et les dessous jaunes.

Nid. Sur le sol ou tout près, nid fait de bandes d'écorce et d'autres matières fibreuses et garni de poils.

Habitat. L'est de l'Amérique du Nord surtout à l'ouest des Alléghanies. Elle couve le long des frontières nord de la colonisation à l'ouest à travers les parties boisées des provinces des prairies.

Cette fauvette est une des plus tardives à arriver au printemps et une des plus tôt reparties, en automne, traversant le pays avant que des observateurs aient guetté les fauvettes migratrices, et par conséquent qui filent sans être observées. C'est dans la seconde moitié d'août qu'il faut guetter ces fauvettes de Philadelphie. Dès le premier jour de septembre elles sont presque toutes parties.

681. La Fauvette trichas. (MARYLAND yellow-throat). Geothlypis trichas. L, 5.33. Planche XLIII B.

Traits distinctifs. Le mâle adulte avec son masque noir est facilement reconnaissable et $\mathbf{n}^{\prime}$ exige pas d'être autrement caractérisé. Les jeunes mâles montrent suffisamment d'indices du masque en question pour être vite reconnus. Les femelles adultes et les toutes jeunes sont très semblables. On peut les reconnaître à leur coloration uniforme en dessus, leur gorge d'un jaune ardent, leurs dessous blanc chamois lavés d'un jaune plus foncé sur les flancs, et les couvertures sous-caudales jaunâtres. On les confondra 
très probablement avec les fauvettes de Philadelphie ou du Connecticut, mais la séparation tranchée entre les couleurs de la gorge et celles des joues, le brillant de la gorge et la teinte jaune généralement plus chaude servira à les distinguer. On les a confondues avec la fauvette de Nashville et la fauvette du Tennessee, mais on pourrait facilement arriver à les distinguer grâce à la tête et à la couronne gris clair plutốt que chamois ou d'un olive vermeil chez l'oiseau de Nashville et a la poitrine presque blanche au lieu de jaune tres distinct chez la fauvette du Tennessee.

Indications sur le terrain. En sus de la coloration, l'habitat marécageux, les habitudes clandestines et les mouvements caractéristiques de la fauvette trichas deviennent vite familiers à l'observateur. Sa note grondeuse, qui rappelle celle du roitelet, est aisément reconnaissable.

Nid. Sur le sol ou tout près, il est massif, fait de bandes d'écorce d'herbage grossier, de feuilles mortes, garni d'herbes fines, de vrilles et de radicelles.

Habitat. Dans l'Amérique du Nord, au nord, jusqu'aux limites de la colonisation. La fauvette trichas du Nord, notre sous-espece canadienne de l'Est, qui est la forme type, couve dars l'ouest jusqu'aux prairies, et au sud jusqu'en Virginie, étant remplacée dans les deux directions par d'autres sous-espèces.

SOUS-ESPECES. La fauvette trichas comprend plusieurs sous-especes dont une seule, la fauvette trichas du Nord, $G$. $t$. trichas, la forme type, se rencontre dans l'est du Canada.

Les lieux spécialement fréquentés par la fauvette trichas sont les marécages humides où le pâturin est long et épais. Elle considère le voisinage immédiat de son nid comme sa propriété particulière et s'indigne avec colère qu'on ose mettre le pied chez elle. Le chant ordinaire de cette fauvette trichas est caractéristique des prairies humides. On l'a traduit poétiquement dans les sons "Witcheri-witcheri-witcheri", qui se rapprochent tout à fait de la réalité phonétique.

683. La Fauvette à poitrine jaune. (YELLow-Breasted chat). Icteria virens. L, 7.44. C'est la plus grande de cette famille et la moins semblable aux fauvettes. Tous les dessus et les joues sont verts, les lores sont noirs, bordés en dessus et en dessous de blanc; de la gorge à la poitrine, jaune clair; les dessous sont blanes; le bec relativement plus court et plus gros que celui des autres fauvettes.

Traits distinctifs. Sa taille suffit a faire distinguer cette fauvette en tout temps, mais ses couleurs ne sont pas moins caractéristiques.

Indications sur le terrain. Sa grande taille, l'avant-corps jaune clair, lores noirs bordés en dessus et dessous de blanc, sont des indications frappantes.

Nid. Dans une fourche de branches près du sol, nid plutót massif d'herbes grossières, de feuilles et de bandes d'écorce, garni d'herbes plus fines.

Habitat. L'est des Etats-Ünis et de l'autre cóté de la frontière canadienne dans l'Ontario méridional le long du lac Erié.

Cette fauvette fréquente les fourrés épais et les terrains perdus et embroussaillés; elle arrive et s'en va sans être vue mais on l'entend fort bien. C'est un oiseau rare au Canada et qu'on trouve ordinairement dans le comté d'Essex (Ontario) seulement le long des rives du lac Erié.

\section{Genres-Wilsonia et Setophaga. Fauvettes muscivores.}

Ce sont de petites fauvettes, presque toujours de couleur jaune. Le bec est légèrement aplati ou élargi à la base, et fait penser à celui des gobe-mouches; il a des poils bien développés autour de la bouche. A cet égard ces fauvettes ressemblent à celle dite à queue rousse, mais pas de façon si excessive. La fauvette à queue rousse, cependant, n'a que peu ou point de tout de jaune clair et peut être facilement reconnue.

684. La Fauvette mitrée. (hooded warbler). Wilsonia citrina. L, 5.67. Le mâle: vert en dessus et jaune brillant en dessous; toute la tête et le cou sont noirs avec un masque jaune brillant de forme semblable à celui tout noir de la fauvette trichas. La femelle n'a pas de noir, sauf une tache un peu vague derrière la tête et le masque jaune passe délicatement à la teinte jaune de la gorge et des parties de dessous. 
Traits distinctifs. Le mâle adulte de cette espèce est trop bien précisé pour prêter à aucune confusion. La face jaune de la femelle avec la couronne foncée de derrière est aussi facilement reconnaissable.

La fauvette mitrée est comprise dans cette liste sur la foi de quelques relations canadiennes dans la région du lac Erié du sud de l'Ontario.

685. La Fauvette de Wilson. (BLACK-CAPPED WARBLER). Wilsonia pusilla. I, 5 . C'est une petite fauvette verte. Le malle est d'un jaune brillant partout en dessous, avec un capuchon d'un noir bien tranché sur la couronne. Les sexes se ressemblent mais quelques femelles manquent tout a fait du capuchon; chez d'autres il existe mais moins parfait que chez les adultes.

Traits distinctifs. Le capuchon noir et la coloration entièrement verte et jaune sont distinctifs des adultes et des jeunes males. Quand le capuchon est absent la ligne sourcilière vaguement jaune est caractéristique.

Indications sur le terrain. La petite taille, le corps entier d'un jaune et vert brillants, avec le capuchon noir, ou seulement des traces de ce capuchon, ou la ligne sourcilière jaune si le capuchon manque.

Nid. Sur le sol; nid fait presque en entier d'herbages fins et garni de quelques poils; nid très concave et solide pour une fauvette.

Habitat. L'Amérique du Nord. Le sous-espèce dite fauvette de Wilson se rencontre au Canada, s'étend a l'ouest vers la région centrale des prairies, et couve d partir de la colonisation dans le nord jusqu'd la limite des forêts.

SOUS-ESPECES. Les fauvettes de Wilson se répartissent en trois sous-espèces qui toutes se rencontrent au Canada, bien qu'une seule, la fauvette de Wilson, Wilsonia pusilla pusilla, la forme type, se rencontre dans l'Est.

C'est une très jolie petite fauvette qui ordinairement se rencontre près des saules ou d'autres arbres semblables et dans les buissons près de l'eau.

686. La Fauvette du Canada. (CANada warbler). Wilsonia canadensis. I, 5.61. Le mâle: en dessus d'un gris uniforme légèrement marqué de noir sur la couronne; tous les dessous sont d'un Jaune brillant, sauf les couvertures sous-caudales qui sont blanches, la ligne prélorale et le cercle autour des yeux qui sont jaunes; les lores noirs se prolongent le long des côtés du cou et forment un collier d'étroites raies en travers du haut de la poitrine laissant la gorge d'un jaune clair. La femelle est pareille, mais le collier et le cou sont atténues, quoique assez fortement marqués pour maintenir le caractère tres reconnaissable de l'espece. Les tout jeunes ressemblent a la femelle, mais le collier est presque effacé et ne se montre que sous forme de nuages vagues, disséminés et fragmentaires.

Traits distinctifs. Le gris uniforme en dessus sans marques, et le jaune dessous avec le collier noir et ses marques ou bien tranchées ou vagues ou seulement suggérées.

Indications sur le terrain. Voir les traits distinctifs.

Nid. Sur les bancs de mousse, ou sous des racines; nid fait de bandes d'écorce et de menus morceaux de bois mort enveloppés dans des feuilles, et garni de fines radicelles.

Habitat. L'est de l'Amérique du Nord, vers l'ouest pres du pied des collines et vers le nord, jusqu'à la limite des gros arbres; couvant par occasion et seulement dans certaines localités du sud du Canada, sauf dans les parties les plus méridionales, et toufours vers le nord à partir de la lisière des régions colonisées.

C'est une fauvette gaie et vive avec quelque penchant à attraper les mouches. Son habitat typique est semblable à celui de la fauvette de Wilson, mais on la trouve plus souvent en haut des arbres.

687. La Fauvette a queue rousse. (AMERICAN REDstart). Setophaga ruticilla. L, 5.41. Planche XLIV A.

Traits distinctifs. C'est un oiseau tout à fait caractéristique sous tous ses plumages. Aucune autre fauvette n'a quoi que ce soit qui ressemble à cette combinaison de rougeorange et de noir, la premiere de ces couleurs faisant place chez la femelle à des plaques analogues jaunes ou blanches sur la queue et les ailes. Les tout jeunes ont un peu de jaune répandu sur la poitrine et n'ont pas la tacheture de l'aile, mais celle de la queue y est toujours. Quelques jeunes mâles, au printemps, ressemblent à des femelles, mais avec les taches irrégulières du parfait plumage mâle paraissent sur la gorge. 
Indications sur le terrain. La couleur est la marque distinctive la plus facile à remarquer, mais même une silhouette noire permet de voir distinctement et de rendre familiere a l'observateur la façon dont la longue queue est jetée de droite et de gauche.

Nid. Dans la fourche de deux branches d'un jeune arbre; nid fait de fines bandes d'écorce, de fibres, de duvet végétal avec vrilles et radicelles, et presque toujours recouvert extérieurement de bandes d'écorce argentée.

Habitat. Le nord de l'Amérique du Nord jusqu'a la limite des grands arbres. Sa couvaison a lieu au Canada où l'on trouve la fauvette en question.

L'éclat brillant d'une fauvette à queue rousse dont le riche plumage se détache sur le vert sombre des arbres est une perpétuelle source de plaisir pour les ycux de l'observateur même le plus blasé, un spectacle qui ne perd jamais de son charme. Les mouvements si vifs de cet oiseau, son voltigement incessant, le déploiement de ses ailes et de sa queue témoignent d'une vivacité qui n'appartient qu'à peu d'autres espèces. Ainsi que cela est arrivé à d'autres oiseaux d'Amérique, le terme de Redstart fut donné à cette fauvette par les premiers colons en souvenir d'un oiseau de l'ancien monde qui ne lui ressemblait cependant que de loin.

\section{FAMILLE-MOTACILLIDE. HOCHEQUEUES ET PIPITS.}

\section{Genre-Anthus. Pipits.}

Il n'y a qu'une seule espèce de cette famille dans l'est du Canada, et pour les caractères de cette famille, le lecteur doit s'en rapporter à la description suivante de la dite espèce.

697. La Farlouse d'Amérique. (AMERican P1PIT). Anthus rubescens. L, 6.38. C'est un oiseau couleur de terre et qui affectionne le sol; le bec est tout a fait celui des fauvettes; la griffe de derrière est allongée comme celle du plectrophane et de l'alouette ordinaire (figure 60, p. 29 , comparez avec figure 44, p. 27 ). Le male adulte du printemps est grisâtre en dessus, d'un gris très pur sur la tête, qui devient légèrement olive au croupion; le dos est vaguement bigarré de plumes noiratres au centre; rase chamois en dessous avec de fines raies d'un gris brunâtre éparses sur la poitrine et qui tendent a former un collier en travers de la poitrine et à se prolonger le long des côtés; les ailes sont brunes avec des plumes d'un brun fané sur les bords. Les oiseaux de l'automne et les femelles du printemps sont d'un olive mat légèrement pommelé en dessus, d'un blanc chamois en dessous avec des taches répandues plus ou moins rassemblées et qui descendant sur les côtés de la gorge se prolongent en travers de la poitrine et le long des flancs.

Traits distinctifs. Le joli bec de la fauvette ajoute à la longue griffe de derrière le pied sont des traits distinctifs. Les seuls autres oiseaux ayant pareille griffe sont l'alouette ordinaire et le plectrophane de Laponie, mais la griffe de l'un et le bec de moineau de l'autre (figure 51, p. 28 ) rendent la différenciation facile.

Indication sur le terrain. C'est un oiseau de couleur terreuse qu'on voit en plein air dans les parties colonisées du Canada au printemps et en automne, souvent par grandes bandes dispersees, comme le plectrophane des neiges et l'alouette ordinaire. La coloration uniforme, son habitude constante de baisser sa queue, et la blancheur frappante des plumes exterieures de la queue sont de bonnes indications.

Nid. Sur le sol, nid composé d'herbages.

Habitat. L'Amérique du Nord; elle couve dans les hautes latitudes au delà de la limite des foréts.

C'est un oiseau migrateur au printemps et vers la fin de l'automne, qui se rencontre quelquefois en grandes bandes, qui trouve sa nourriture dans les prés, dans les champs cultivés, ou sur des hauteurs sèches et sablonneuses, aussi sur les rivages. Dans les terres où il couve il a la coutume de l'alouette de monter et de chanter à des grandes hauteurs dans l'air, puis de redescendre par une chute perpendiculaire, comme celle d'une pierre. 
Valeur économique. Arrivant, comme elle fait, alors que les champs sont dénudés et s'en retournant après les moissons, la farlouse doit se contenter des graines d'herbages et des insectes soit du printemps soit de l'automne. Son rôle est done utile.

\section{FAMILLE-MIMDA. MOQUEURS ET MERLES.}

La faculté d'imitation de l'oiseau moqueur, d'où est venu le nom même de la famille, a beaucoup de représentants dans le Canada. Cette famille d'oiseaux est particulièrement propre à l'Amérique et, comme c'est le cas de bien des subdivisions de l'ordre des passereaux, très difficile à distinguer en un language autre que purement technique. Ce sont des oiseaux plutôt grands. Le merle-chat ou grive de la Caroline et la grive polyglotte ont des teintes uniformes gris de pierre et la grive rousse est d'un brun rougeâtre brillant en dessus avec des dessous fortement tachés de blanc ou de jaune crème et une queue extraordinairement longue et abondante. Ces oiseaux sont tous d'excellents moqueurs et savent varier leur chant par des imitations de tous les sons qu'ils entendent autour d'eux, y compris les chants ou les cris d'autres oiseaux; ils sont même capables de produire de tels effets en ce genre qu'on a pu dire qu'ils n'étaient égalés que rarement par les plus célèbres chanteurs de l'ancien ou du nouveau monde. L'une quelconque de ces espèces suffit à contredire de façon complète et écrasante l'assertion qu'il n'y a pas d'oiseau chanteur en Amérique.

\section{Genre-Mimus. Oiseau moqueur.}

703. La Grive polyglotte. L'OISEAU MOQUEUR, LE MERLE MOQUEUR. (MOCKINGBIRD). Mimus polyglottos. L, 10.50. Elle ressemble à un merle-chat mais sans la calotte noire ni le rouge sous la queue; les dessous sont presque blancs et il y a considérablement de blanc dans l'aile et dans la queue.

Traits distinctifs. I Les caractères susdits serviront à distinguer la grive polyglotte du merle-chat ou grive de la Caroline. Elle ressemble de très près aux pies-grièches par la coloration, mais elle n'a pas la tache noire si visible en travers des yeux.

Indications sur le terrain. La couleur grise assez générale, et les taches blanches sur l'aile et la queue avec l'absence du masque noir de la face.

Nid. Dans les fourrés de branches grossières et de tiges herbacées, nid garni de radicelles et de bouts de coton.

Habitat. Les Etats-Unis du sud et au nord jusqu'au Canada, à l'extrémité ouest du lac Erié. C'est la seule localité où cette espèce ait pris pied de façon permanente au Canada. On a vu quelques couples y passer l'été en ces dix dernières années.

SOUS-ESPECES. La grive polyglotte forme deux groupes, celui du sud-est et celui du sud-ouest; le premier, c'est la grive polyglotte commune, la race type de l'espèce la seule sous-espèce qu'on puisse s'attendre à trouver au Canada.

Cette espèce est rare au Canada. Elle ressemble beaucoup à la grive de la Caroline et presque tout ce qui est dit de cette dernière s'applique avec encore plus de force à la grive polyglotte, qui n'est, à bien des égards, qu'une grive de la Caroline plus célébrée et qui est probablement le plus fameux chanteur natif de l'Amérique.

\section{Genre-Dumetella. Grive de la Caroline.}

704. La Grive de la Caroline. Le merla-chat. (CATBIRd). Dumetella carolinensis. L, 8.94. Planche XLIV B. (Figure 62, p. 30.)

Traits distinctifs. Cet oiseau d'un gris uniforme ne peut être confondu qu'avec la grive polyglotte; la calotte noire, les couvertures rouges sous-caudales, l'absence de blanc sur l'aile, sur la queue, ou en dessous, sont autant de traits distinctifs. 
Indications sur le terrain. Couleur grise uniforme; calotte noire et notes d'appel, tout spécialement le "miaou du chat qui a fait donner à l'oiseau son nom anglais.

Nid. Dans les fourrés ou dans les buissons a feuillage épais; nid fait de branchettes, d'herbes, de feuilles et garni de radicelles.

Habritat. L'est de l'Amérique du Nord; au Canada en y comprenant la plupart des régions plus peuplées.

Quoique inférieure à la grive polyglotte, la grive de la Caroline, par ses meilleurs représentants, occupe un rang élevé comme oiseau chanteur, lors même que l'excellence de ses talents varie beaucoup selon l'individu. Sa note d'appcl, qui ressemble au miaulement du chat, et que l'oiseau émet dans le buisson tandis qu'il examine avec curiosité l'intrus qui cnvahit son domaine, est bien connue de la plupart de ceux qui fréquentent la campagne et, fait bizarre, a provoqué la critique d'une psychologie dévoyée.

Cet oiseau hante les fourrés et, comme pour bien d'autres espèces qui fréquentent des habitats analogues, où l'observation très serrée peut se faire d'objets dangereux, sans trop de risque pour l'observateur caché, la curiosité de cette grive est bien aiguisée. Perchée sur quelque haute ramille qui émerge du fourré, on la voit en plein soleil, la queue basse et le corps penché sur la branche, qui lance tous les sons variés d'un véritable pot pourri. Les phrases mélodieuses se succèdent rapidement et des fragments de tous les chants d'oiseaux du voisinage sont entre-mêlés de sons plus durs et mécaniques que la dite grive exécute avec autant de brio que les sons les plus mélodieux. La grive de la Caroline est un voisin tout à fait désirable.

Valeur économique. La grive de la Caroline se nourrit surtout des fruits de la saison dont un tier's peut-être peuvent passer pour fruits cultivés; mais elle prend aussi beaucoup d'insectes. Les fruits sont petits, des variétés molles, et il est très rare qu'un dommage sensible en résulte.

\section{Genre-Toxostoma. Grives rousses.}

705. La Grive rousse. (Brown thrasher). Toxostoma rufum. L, 11.42. Planche XLV A. (Figure 62, p. 30.)

Traits distinctifs. La grive rousse, avec son dos brun rouge, sa poitrine à raies bien tranchées, a toute l'apparence extérieure d'une grive, mais sa forte taille, l'incarnat de 89 couleur brune et sa longue queue sont fort distinctifs.

Indications sur le terrain. Son dos brun rouge brillant, sa poitrine à raies tranchées, sa longue queue, son maintien et ses mœurs en général.

$N i d$. Dans des fourrés ou sur le sol; nids faits de ramilles, de radicelles grossières et de feuilles, garnis de fines radicelles.

Habitat. L'est des Etats-Unis, le sud du Canada, sauf la côte de l'Atlantique, au nord les régions de la colonisation la plus dense.

La grive rousse est probablement le meilleur oiseau chanteur ordinaire du Canada, de l'Ontario à l'ouest.

Son chant, très semblable à celui de la grive chanteuse d'Europe, est une succession de phrases comme celles de la grive de la Caroline, mais sans les fausses notes accidentelles, un timbre plus doux et plus moelleux. Les notes sont très rapprochées et se prolongent pendant plusieurs minutes, parfois avec une grande variété. Thoreau les a représentées comme disant: "drop-it-drop-it-cover-it-up-cover-it-up-pull-it-up-pull$i t-u p$ ». La répétition de chaque variation est une des singularités du chant de la grive rousse, au moyen de laquelle on peut la distinguer de la grive de la Caroline.

$$
\text { 28588-16 }
$$


C'est aussi un oiseau des fourrés, qui habite les bosquets, les buissons dans les prés, les lisières de forêts et des clôtures. La grive rousse aime plus l'isolement que celle de la Caroline, elle se laisse aussi moins facilement attirer près des habitations.

Valeur économique. C'est tout à fait un oiseau utile, car plus de la moitié de sa nourriture se compose d'insectes nuisibles, de scarabées, de chenilles, de sauterelles, etc. Le reste est composé surtout de fruits dont une petite partie est formée de fruits cultivés, surtout de framboises. En somme il fait peu de mal et beaucoup de bien.

\section{FAMILLE-TROGLODYTIDA. TROGLODYTES, ROITELETS.}

Les roitelets sont de petits oiseaux bruns qui vivent tous près du sol. Quoique de taille minime ils sont très actifs et, à moins qu'ils ne couvent ou ne dorment, ils se tiennent rarement tranquilles. On peut les reconnaître à leur petite taille, à leur brune couleur, à leur petite queue ramassée, souvent rejetée sur le dos, leurs manières agitées, entrant dans les plus épais buissons ou en ressortant plutôt à la façon des souris qu'à celle des oiseaux. Les roitelets constituent une nombreuse famille bien distribuée dans le monde mais mieux représentée dans le nouveau monde que dans l'ancien. Leur habitat varie entre des marécages inondés et des plateaux très secs ou entre des fourrés en pays ouvert et des forêts épaisses et profondes. Ce nom de Troglodytes, qui veut dire habitants des cavernes, vient de leur habitude de loger leurs nids dans des trous.

\section{Genre-Thryothorus. Roitelet de la Caroline et autres.}

718. Roltelet de la Caroline. (CAROLINA WREN). Thryothorusludovicianus. L, 5.50. Le plus gros de nos roitelets et le plus rouge; le dos se rapprochant du rouge de la grive rousse, d'une teinte plus claire mais visiblement chaude dans les dessous.

Traits distinctifs. La taille, l'incarnat relatif du dos, et la ligne sourcilière parfaitement claire, sont distinctifs de cette espèce.

Indications sur le terrain. Les traits susdits constituent les plus sures indications sur le terrain. Le roitelet des marais à long bec a une ligne sourcilière toute semblable, mais la taille, la couleur générale et l'habitat empêcheront qu'on ne les confonde l'un avec l'autre.

Nid. Dans des trous d'arbre, de tronc d'arbre, dans des creux et des lézardes de mur; nid fait d'herbages, de plumes, de feuilles, etc., garni d'herbes délicates, de longs poils, etc.

Habitat. L'est des Etats-Unis, au nord de façon intermittente au Canada dans la section de l'ouest du lac Erié. Pendant nombre d'années l'espèce était fort commune à la pointe Pelée et dans l'sle Pelée, mais depuis 1913 elle semble être devenue plus rare.

SOUS-ESPECES. Des trois sous-espèces du roitelet de la Caroline, une seule, le roitelet du nord de la Caroline, la race type, peut se rencontrer au Canada.

Le roitelet de la Caroline est le plus beau chanteur de son espèce, son chant n'est ni prolongé ni long, mais il a une qualité flùtée de douceur et de frappante beauté. L'espèce est rare au Canada, et son chant n'est entendu de façon régulière ou par occasion que dans peu de localités.

Valeur économique. Trop rare au Canada pour y avoir aucune influence économique. 


\section{Genre-Thryomanes. Roitelet de Bewick et autres.}

719. Le Roitelet de Bewick. (BEwTCK's WREN). Thryomanes bewicki. L, 5. Semblable au troglodyte adon, mais plus blanc en dessous, avec une queue plus longue, plua grande et évidemment grisátre. Il a une légère ligne sourcilière. l'espèce.

Traits distinctifs. Les signes caractéristiques données plus haut serviront a identifier

Indications sur le terrain. C'est un troglodyte acdon avec une longue queue et un chant fort et pareil a celui du moineau.

Nid. Tout aemblable à celui du troglodyte aédon.

Habitat. L'est des Etats-Unis et sans aller jusqu'à la frontière du Canada, sauf comme vagabond dans le sud de l'Ontario.

SOUS-ESPECE. L'individu de l'est du Canada n'est que le roitelet de Bewick de l'Est, la race type de l'espèce.

Ce roitelet n'est qu'un rare visitcur venant du sud, et n'a été que bien rarement signale dans l'est du Canada. Il conviendrait de n'en faire mention que sur des témoignages absolument dignes de confiance.

Valeur économique. Espèce trop rare au Canada pour y être d'aucun intérêt économique.

\section{Genre-Troglodytes. Troglodyte aédon.}

721. Le Troglodyte aédon. (Hoose wren). Troglodyte ædon. L, 5. Planche XLV B.

Traits distinctifs. C'est le roitelet le plus commun dans le sud du Canada. Le dos d'un brun ligneux uniforme, la gorge et la poitrine teintées d'un brun clair; les dessous presque blancs, et les raies restreintes aux flancs, le distingueront des autres roitelets indigènes.

Indications sur le terrain. Les dessous clairs et la queue plus longue le distingueront du troglodyte d'hiver, et la couleur brune uniforme du dos, tout autant que l'babitat, les distinguera tous deux du troglodyte des marais.

Nid. Dans un trou d'arbre, ou une boite à oiseau, ou dans des places analogues; nid fait de branchettes, garni d'herbes et de plumes, ete. Le troglodyte aédon se mettra volontiers dans toute espece de boite à moineau avantageusement plackée. Le moineau anglais peut être tenu à distance en faisant très petit le trou de l'entrée de la bolte; un trou fait avec une tarière d'un pouce suffit à laisser passer le troglodyte et à évincer tout à fait les moineaux.

Habitat. Comme espece, le troglodyte est partout aux Etats-Unis et au Canada, au nord jusqu'aux limites des parties colonisées. L'individu de l'Est, qui est l'individu type, le troglodyte aédon de l'Est, habite les contrées à l'est des grands lacs.

SOUS-ESPECES. Le troglodyte ádon comprend deux sous-espèces: celle do l'Est et celle de l'Ouest; celle de l'Est constitue la forme type.

Le troglodyte aédon est un oiseau tout a fait attrayant dans un jardin; il se faufile partout, sur ou sous tout ce qui se rencontre; pas de fissure, pas de lézarde dans une palissade qui n'attire son petit bec investigateur; c'est à peine si une tige de feuille n'a pas été soigneusement inspectée une fois pour les insectes qui s'y trouvent. On l'a accusé de percer et de détruire les aufs d'autres espèces qui nichent dans son voisinage immédiat; il n'est pas douteux que cela n'arrive parfois, mais le dommage ainsi causé vient probablement d'une habitude particulière à tel troglodyte et n'est peut-être pas suffisant pour justifier des mesures préventives contre l'espèce entière.

Valeur économique. Comme la nourriture du troglodyte aédon se compose presque entièrement d'insectes, il n'y a rien à lui reprocher à cet égard. Sa taille minime l'oblige à ne s'occuper que d'insectes minuscules qui échappent à l'attention de plus gros oiseaux, et c'est ainsi que le troglodyte se rend maître d'animalcules nuisibles avant qu'ils soient de taille à causer du dommage ou a attirer d'autres oiseaux. 


\section{Genre-Nannus. Troglodyte d'hiver.}

722. Le Troglodyte d'hiver. (WINTER WREN). Nannus hiemalis. L, 4.06. De structure et de coloration absolument typique du troglodyte. Il ressemble beaucoup au troglodyte aédon, mais est plus petit et plus foncé en dessous, aussi plus ou moins finement rayé en travers de l'abdomen.

Traits distinctifs. Les raies qui couvrent les dessous sont distinctives de cette espèce.

Indications sur le terrain. C'est un petit troglodyte très foncé, avec une queue courte; on le trouve dans les foréts épaisses.

Nid. Dans les racines d'un arbre, ou dans un tas de broussailles, ou encore dans le flanc d'une buche moussue: nid fait de petites branchettes et de mousse, garni de plumes.

Habitat. En qualité d'espèce il est partout dans l'Amérique du Nord jusqu'à la limite des arbres; il couve partout au Canada dans les bois de conifères, sauf dans les parties plus méridionales.

SOUS-ESPECES. Le troglodyte d'hiver, distribué a travers presque tout le continent, comprend un bon nombre de races que distingue la géographie, ou de sous-espèces dont l'individu type, le troglodyte d'hiver de l'Est est le seul qui rentre dans nos limites géographiques.

Cet oiseau qui n'est que migrateur dans le plupart des régions plus méridionales du Canada, couve ordinairement dans les forêts profondes et froides. Son chant, qui ressemble assez par sa verve et son caractère à celui du troglodyte aédon pour qu'on reconnaisse leur affinité, est cependant d'une qualité plus sonore et plus riche, d'un ton plus pur. Entendu dans le silence de la paisible forêt, ce chant a la beauté sauvage des bois qu'aucune espèce indigène ne possède.

Valeur économique. Cet oiseau étant un hôte habituel des contrées boisées ne s'approche de l'homme que de loin en loin, mais son action, autant qu'on en peut parler, est tout à fait utile.

\section{Genres-Cistothorus et Telmatodytes.}

724. Le Roitelet de marais au bec court. (SHORT-BILLen MARSH WREN). Cis tothorus stellaris. L, 4. Très semblable au roitelet des marais à long bec, mais plus petit et plus finement rayé.

Traits distinctifs. Les deux roitelets de marais sont les seuls roitelets indigènes dans le Canada qui aient des raies très tranchées en dessus. Le roitelet à bec court est le seul qui ait une couronne rayée.

Indications sur le terrain. C'est un petit roitelet de marais avec une faible teinte incarnate, peut-étre même point du tout, dans sa coloration brune et avec une tête rayée. Les sons qu'il lance sont très différents de ceux du roitelet al long bec, avec lequel on risquera probablement de le confondre.

Nid. Sur le sol humide, un nid qui est une boule d'herbe verte tressée près du sommet de bouquets d'herbes, avec un trou circulaire sur le cốté comme entrée du nid.

Habilat. L'est des Etats-Unis jusque dans le Canada, à l'est tout le long du lac Erié, et dans la contrée environnante, mais l'oiseau est plus commun dans les provinces des prairies.

Le roitelet des marais à bec court occupe un territoire plutôt local et peu régulier; une année il se trouvera dans telle localité et n'y sera plus l'an d'après; on sait peu de chose de son habitat. Il fréquente les marécages humides, herbeux plutôt que des marécages submergés, et se réunit d'habitude à d'autres pour former de petites colonies.

725. Le Troglodyte de marais. LE ROITELET de Marais a LONG BEc. (LongBILLED MARSH WREN). Telmatodytes palustris. I, 5.2. C'est un roitelet d'une riche coloration avec un manteau presque noir qui tombe de l'arrière-cou sur les épaules où il est rayé de blanc; tout le reste est brun rougeâtre en dessus, d'un blanc crème en dessous avec les flancs lavés de la méme couleur que le dos. 
Traits distinctifs. La variété et les couleurs du dos distingueront les deux roitelets de marais de tous les autres; la couleur informe de la couronne ou n'ayant seulement qu'une raie médiane d'un brun étendu, au lieu de plusieurs courtes et fines raies, le distingueront du roitelet a bec court.

Indications sur le terrain. Ia localité qu'il fréquente-marécages couverts d'eau, de roseaux et de massettes-suffit d'habitude a l'identifier, mais la couronne foncée en contraste avec la légère ligne sourcilière est toujours une preuve spécifique.

Nid. Est placé près du sommet des roseaux ou des joncs dans de vastes terrains marécageux et humides; c'est une boule de feuilles mortes de massettes, d'herbages ou de roseaux. Très différente de bien d'autres hôtes des marécages cette espèce n'est pas attirée par des marais de petite dimension. Un étang marécageux de quelques yards de large attire l'étourneau rouge et peut-être un ou deux râles, mais le roitelet de marais a long bec exige une vaste étenduc. Un trait intéressant du roitelet de marais c'est son habitude de construire plusieurs faux nids a côté de celui qu'il occupe. L'usage auquel servent ces nids n'est pas connu, mais on en a vu parfois huit ou neuf qu'on pouvait avec raison attribuer au travail d'un seul couple.

Habitat. En tant qu'espèce c'est les Etats-Unis et le Canada méridional. Notre roitelet des marais de l'Est, le type de la sous-espece, se rencontre à l'ouest de la région des grands lacs.

SOUS-ESPECES. Le roitelet de marais a long bec comprend plusieurs sous-especes; le roitelet de marais de l'Est, le seul individu auquel nous nous intéressions directement dans l'est du Canada, constitue la forme typique de l'espèce.

Les vastes étangs marécageux et les marais pleins de massettes agitées par le vent ou de roseaux sont les lieux fréquentés par ce roitelet.

\section{FAMILLE-CERTHIID E. GRIMPEREA UX.}

Le nom du seul grimpereau de l'est du Canada, le grimpereau brun, décrit parfaitement cet oiseau. C'est un petit oiseau brun qui rampe ou grimpe à la façon des pics sur les troncs et les plus grosses branches des arbres de forêts. Il est plus petit qu'aucun pic du Canada et son bec relativement long, léger, délicatement appointé et en forme de faucille (fig. 64, p. 30) est très propre à extraire les menus insectes et leurs œufs des étroites cavités mais non à découper le bois, même le plus mou, ou l'écorce pour les atteindre. La queue est plutôt longue et raide, et les griffes sont très longues et bien recourbées.

\section{Genre-Certhia. Grimpereaux.}

726. Le Grimpereau d'Amérique. (BRown creeper). Certhia familiaris. L, 5.66. XLVI A.

Traits distinctifs. Les raies brunes et blanches, où ne ressort aucun dessin; le bec finement délicat, en forme de faucille et les longues plumes raides de la queue, usées au bout, sont des marques distinctives de l'espece et facilement reconnaissables.

Indications sur le terrain. C'est notre seul petit oiseau brun avec les habitudes prononcées d'un grimpereau.

Nid. Derrière l'écorce à demi-détachée des arbres, nid fait de branchettes, de bandes d'écorce, de menus morceaux de bois mort, de mousse, etc.

Habitat. En tant qu'espèce qui occupe la plus grande partie de l'bémisphère nord, on le trouve dans l'est de l'hémisphère boréal. Dans l'est de l'Amérique du Nord, lé grimpereau de l'Est est la sous-espece indigène; au Canada, il va vers l'ouest jusqu'aux provinces des prairies et vers le Nord au dela des limites de la colonisation.

SOUS-ESPECES. Le grimpereau brun se rencontre dans l'ancien monde aussi: bien que dans le nouveau. L'espèce se divise en plusieurs sous-especes, en Amérique, dont une seule, le grimpereau brun de l'Est, Certhia familiaris americana, se rencontre dans l'est du Canada.

Pressé étroitement contre le tronc d'un arbre de la forêt, le grimpereau brun peut être aperçu grimpant en spirale le long du tronc perpendiculaire 
et retirant avec ardeur quelque insecte de chaque fissure et de chaque lézarde. Arrivé à cette partie de l'arbre où les branches commencent à s'amincir et l'écorce à devenir lisse il se laisse choir à la base d'un arbre voisin et recommence son travail ascensionnel, toujours se dépêchant, jamais ne s'arrêtant, emplissant son estomac de petits escarbots, de larves et d'œufs d'insectes. L'adresse avec laquelle cet oiseau peut s'accrocher à des surfaces lisses est remarquable. L'auteur a vu un jour un grimpereau brun grimper le côté poli d'une bibliothèque en noyer noir, et le faire sans plus d'inquiétude que si cela eât été l'écorce la plus rugueuse d'un chêne des bois.

Valeur économique. Le grimpereau brun est purement insectivore et l'attention continuelle et microscopique qu'il donne au moindre tout petit trou dans une écorce rugueuse ne s'explique que par la présence du fléau d'innombrables insectes. La majeure partie de son activité se dépense dans les bois, mais comme ce petit oiseau se montre souvent dans les vergers et sur des arbres feuillus ou servant à embellir les alentours des villes et des maisons, l'espèce a certainement une très grande et très heureuse influence.

FAMILLE-SITTID \&. SITTELLES.

Les sittelles sont de petits oiseaux semblables aux pics quant aux mœurs générales, mais leurs doigts sont du type ordinaire de ceux des passereaux, les trois doigts de devant et un doigt de derrière au lieu des deux doigts antérieurs et postérieurs caractéristiques des pics. Le bec est assez semblable à celui du pic commo contour, mais sans la pointe en forme de ciseau; il forme un léger angle avec la tête, ce qui lui donne l'air retroussé (fig. 65, p. 30, comparez avec fig. 41, p. 27). Les couleurs de cette cspèce sont caractéristiques. Le nom anglais de "Nuthatch" vient de leur habitude de faire entrer de force des noisettes, ou quelque aliment dur, dans des fentes, et de les ébrécher jusqu'à ce qu'elles soient dedans. Bien que ces oiseaux soient capables de creuser le bois ou l'écorce ils n'emploient pas cette force à faire des trous profonds dans les arbres, mais ils se contentent d'éliminer les parcelles écailleuses qui se détachent de l'écorce et de fouiller les cavités et les jointures.

\section{Genre-Sitta. Sittelles.}

727. La Sittelle de la Caroline. (carolina nothatch). Sitta carolinensis. L, 6.07. Planche XLVI B.

Traits distinctifs. Au moyen de la gravure et de la description de la famille cette espèce ne peut être confondue qu'avec la sittelle du Canada. C'est, toutefois, un plus gros oiseau, et bien qu'il y ait du marron sur le bas-ventre et sur les couvertures souscaudales, et même quelquefois cette couleur légèrement lavée sur les flancs, la poitrine et presque tous les dessous sont d'un blanc pur au lieu d'être uniformément étendus d'une couleur brun rouge ou rouilleuse. Les côtés de la face sont tout a fait blancs au lieu d'avoir les couvertures de l'oreille noires et les raies sourcilières parfaitement noires.

Indications sur le terrain. Le dos d'un gris bleu uniforme et la couronne noire caractérisent les sittelles; la pureté du blanc en dessus et l'absence de la ligne blanche sourcilière distinguent la sittelle de la Caroline de celle du Canada.

Nid. Dans un trou d'arbre ou dans quelque cavité naturelle; nid fait de plumes, de feuilles, etc.

Habritat. En tant qu'espèce, la sittelle de la Caroline habite toutes les parties tempérées de l'Amérique du Nord. 'Notre sous-espèce de l'Est, la sittelle de la Caroline, le type de l'espèce, avance à l'ouest jusqu'aux provinces des prairies au Canada, et au nord jusqu'aux limites des grandes forêts.

SOUS-ESPECES. La sittelle de la Caroline comprend géographiquement plusieurs races dont une seule, la sittelle a poitrinc blanche, la forme typique, se rencontre dans l'est du Canada. 
Le talent qu'ont les sittelles de monter et de grimper aux arbres est merveilleux. Elles voyagent en haut, en bas, en avant, en arrière, perpendiculairement ou horizontalement, se cramponnant en dessous des branches comme des mouches au plafond, et avec une aise toute semblable. Leur cri d'appel est un rauque "coin coin"); elles voyagent souvent en couples et en petits groupes de compagnie avec les mésanges et les grimpereaux.

Valeur économique. C'est un des oiseaux les plus utiles. Bien qu'il soit très attaché aux arbres forestiers il fréquente souvent les vergers et les arbres au riche feuillage, et en qualité de membre de la susdite compagnie qui inspecte d'un œil microscopique toutes les parties des troncs d'arbres, à la rccherche des insectes, il consomme de grandes quantités de ces animalcules pernicieux, soit à l'état adulte ou d'œufs ou de larves.

728. La Sittelle du Canada. (RED-BREAsted NUTHatch). Sitla canadensis. L, 4.62. Semblable a la sittelle de la Caroline (planche XLVI B), mais plus petite et avec les dessous lavés de brun rougeâtre; les couvertures des oneilles sont noires avee une raie blanche sourcilière.

Traits distinctifs. La taille et les différences de couleur en dessus dintingueront cette espece de tout autre oiseau.

Indications sur le terrain. C'est une sittelle evidemment plus petite que l'espèce précédente et avec une ligne blanche sourcilière très marquée; tous les dessous sont visiblement rougeâtres.

Nid. Dans un trou d'arbre ou de souche; nid fait arec des herbes.

Habitat. La couvaison a lieu plus au nord que celle de la sittelle précédente. Elle est migratrice dans la plupart des parties cultivées du Canada. Son territoire comprend presque toute l'Amérique du Nord et au nord, jusqu'à la limite des arbres.

C'est un oiseau un peu plus actif que le précédent et plus pressé de. fourrager à l'extrémité des branches. A cela près ses habitudes sont tout à fait les mêmes.

FAMILLE-PARIDE. MÉSANGES.

Les mésanges sont des oiseaux répandus sur un vaste territoire dans l'hémisphère nord, et aussi connus des habitants de l'Europe que de nousmêmes. Ce sont de petits oiseaux avec un bec plutôt court, mais relativement très arqué (fig. 66, p. 30 ). Leur plumage a certains caractères facilement reconnaissables.

\section{Genre-Boolophus. Mésanges huppées.}

731. La Mésange huppée. (TUFTED TITMOdge). Boeolophus bicolor. L, 6. La plus grande de nos mésanges et qui n'a pas la coloration caractéristique des mésanges à tête noire. En dessus, une couleur uniforme gris-pierre; en dessous, blanc légèrement la vé de brun rougeâtre sur les flancs. Une huppe bien distincte d'un bleu de geai.

Traits distinctifs. Is gris uni et la coloration sans marque quelconque, avec une huppe assez frappante, ce sont id des traits distinctifs et des indications sur le terrain auxquels on ne peut pas se tromper. Son cri ordinaire, fort et distinct: "Pittle-pittle pittle" est très caractéristique mais ressemble beaucoup a l'une des phrases du loriot des vergers.

Nid. Dans de vieux trous de pies, dans les trous d'arbres, etc.; nid fait de feuilles, de mousse, de bandes d'écorce, de plumes, etc.

Habitat. Le sud et l'est des Etats-Unis, au nord, jusqu'aux frontières du Canada dans la région inférieure des grands lacs.

Les seules mentions canadiennes faites de cette mésange huppée sont les deux de Pointe Pelée au sud de l'Ontario, dans la contrée à l'ouest du lac Erié. Cet oiseau est assez commun du côté Michigan de la rivière Détroit et même dans la grosse île au milieu du courant; on le trouverait probablement aussi du côté canadien de la rivière quoique, jusqu'ici, il n'y ait été fait aucune mention de sa présence. 


\section{Genre-Penthestes. Mésanges à tête noire.}

735. La Mésange à tête noire. (BLACK-CAPPED CHICKAdEe). Penthestes atricapileus. L, 5.27. Planche XLVII A.

Traits distinctifs. Cette espèce ne peut guère être confondue, dans l'est du Canada qu'avec la mésange du Canada, mais c'est une espèce bien plus commune et plus largement distribuée.

Indications sur le terrain. Tout dans cette mésange â tête noire peut servir d'indication sur le terrain. Sa forme: un paquet tout rond de plumes avec une queue et presque pas de cou, la vivacité de ses allures, son penchant à se renverser la tête en bas tandis qu'elle inspecte le bout des branches, ses couleurs, une calotte noire et la gorge de même, des joues blanches, un dos d'un gris tendre, enfin son cri: Tchick-a-di-die, qui prononce si distinctement son nom; tout en un mot, annonce et proclame immédiatement son espèce.

Nid. Dans de vieux trones d'arbre, ou dans des trous d'arbre, etc.; nid fait de mousse, d'herbes, de plumes et de duvet végétal.

Habitat. En tant qu'espèce elle s'étend du centre, environ, des Etats-Unis jusqu'a la limite des bois, au nord. La mésange à tête noire se rencontre à partir du sud de la frontière canadienne vers le nord, s'étendant à l'ouest vers les provinces des prairies où sa place est occupée par des sous-espèces apparentées.

SOUS-ESPECES. A l'instar d'autres espèces très nombreuses et répandues au loin, la mésange à tête noire, sous l'influence de conditions variées dans ce contiment est représentée dans l'Amérique du Nord, au point de vue géographique, par diverses races ou sous-espèces faciles à reconnaitre. Dans l'est du Canada il n'y en a qu'une seule forme, le type de l'espèce, la mésange de l'Est. Au sud on trouve la mésange de la Caroline, $P$. carolinensis, espèce étroitement apparentée quoique distincte et qu'on peut considérer comme accidentelle dans la région inférieure des grands lacs et qui a passé dans le Michigan par dessus la frontière toute voisine. Les distinctions spécifiques cependant, sont trop subtiles pour pouvoir être exactement définies ici, et on ne peut en parler que sur la vue de spécimens.

De tous les oiseaux des champs ou des bois la mésange est le plus gai et le plus joyeux. La mésange est souvent le centre d'une petite société d'espèces mélangées de fauvettes, de vireos, de roitelets, de sittelles, et parfois d'un pic duveté et d'un grimpereau d'Amérique. Quand les migrateurs sont partis pour l'hiver, les plus robustes restent en arrière ensemble jusqu'à ce que la couvaison du printemps ait dispersé cette aimable petite société. La mésange a un autre cri composé de seulement deux notes d'un registre naturel assez élevé, clair et qui ressemble à un coup de sifflet. La première note est prolongée, la seconde plus courte est de deux tons plus bas et a été traduite en anglais comme "Spring's here."

Valeur économique. Peu d'oiscaux sont plus utiles aux hommes que la mésange. Bien que petite elle est constamment au travail et, comme elle passe tout l'hiver parmi nous, son travail continue toute l'année. Les insectes sont tous très minuscules dans les tout premiers temps, aussi le petit oiseau qui dévore d'un seul coup tout un paquet d'œufs peut être aussi utile a l'agriculture qu'un plus gros oiseau qui fait son repas d'une ou de deux grosses chenilles ou d'insectes adultes, mais qui fait fi des plus minimes. Les habitudes fouilleuses de la mésange et de ses compagnons: la sittelle et le grimpereau, et leur inspection diligente des plus petites fentes, ou beaucoup d'insectes se cachent ou passent l'hiver, rendent leurs services inappréciables au cultivateur, surtout en hiver quand les ennemis des insectes sont rares. La masse d'insectes pris de la sorte pendant l'année par ces oiseaux doit être très grande. Ces petits oiseaux si actifs exigent des quantités relativement considérables de nourriture pour résister à un froid intense, et la petitesse de leur gibier nécessite l'absorption d'innombrables individus. 
La nourriture de la mésange est de 68 pour cent animale et de 32 pour cent végétale. La première sorte comprend des œufs, des larves, des chrysalides, de petits insectes, beaucoup de charançons et quelques-uns des pires fléaux de nos vergers et de nos récoltes. La nourriture végétale est surtout composée de petites semences et de fruits sauvages. On ne s'est jamais plaint que ces oiseaux fissent du tort aux fruits cultivés. Les mésanges peuvent être facilement attirées autour de nos fermes en hiver, et en les cajolant un peu elles deviendront assez apprivoisées pour se poser sur la personne et manger dans sa main. Un morceau de graisse attaché à un tronc d'arbre ne manque jamais de les attirer et nous assure de leurs visites répétées.

740. La Mésange du Canada. (Brown-hended chickadee). Penthestes hudsonicus. L, 5.12. Semblable à la mésange à tête noire mais de couleur plus terne et foncée; calotte brun grisâtre d'à peu près la même couleur que le dos; tache à la gorge visible mais voilée; les flancs teintés de brun rougeâtre.

Traits distinctifs. La calotte et le dos brunâtres, la coloration générale plus terne et avec moins de contraste.

Indications sur le terrain. Une mésange très foncée avec une coloration étendue et d'un dessin manquant de précision. Le cri caractéristique de atchik-a-di" est rauque, mais, a cela près, semblable à celui de la mésange ordinaire.

Nid. Dans des trous d'arbres ou de souche; nid fait de mousse et feutré de poils. forêts.

Habitat. L'Amérique du Nord a partir des jégions colonisées jusqu'à la limite des

SOUS-ESPECES. La mésange du Canada est représentée par deux sous-espèces dans l'est du Canada. La forme type, la mésange de la baie d'Hudson, s'étend jusqu'au centre de l'Ontario; a l'est de cette région elle est remplacée par la mésange acadienne $P$. h. littoralis qui n'en diffère que légèrement par la taille et la couleur.

La mésange du Canada est si semblable de mours à la mésange à tête noire qu'une description plus longue ne serait guère qu'une répétition.

\section{FAMILLE-SYLVIID F. FAUVETTES, ROITELETS ET GOBE-MOUCHES DE L'ANCIEN MONDE.}

C'est une famille de l'ancien monde représentéc en Amérique par un petit nombre d'espèces. De celles-la, les fauvettes de l'ancien monde, qu'il ne faut pas confondre avec nos fauvettes des bois, ne se montrent pas dans l'est du Canada; les roitelets sont représentés par deux sous-espèces, et les gobe-mouches par une seule.

\section{Sous-famille-Regulinæ. Roitelets.}

Description génkrale. Les roitelets sont les plus petits des oiseaux canadiens, le colibri excepté. Ils ressemblent au troglodyte par leur petit corps tout rond, mais encore plus aux mésanges par leurs mours et leurs actions. Leurs couleurs sont d'un olive verdâtre mat, plus clair en dessous, et ils ont a la couronne de petites taches brillantes de rouge, d'orange ou de jaune. Le bec est petit et droit semblable, quoique moins gros, à celui de la mésange (voir fig. 67, p. 31 ).

Traits distinctifs. Les roitelets pourraient être pris pour quelques-unes des fauvettes de couleur mate et uniforme, mais comme tous les plumages des roitelets, sauf ceux de la femelle et du tout jeune roitelet a couronne rubis, ont des taches brillantes à la couronne, cette circonstance previendra généralement une confusion de ce genre que la taille seule, d'ailleurs, suffirait à prévenir.

Indications sur le terrain. Coloration verdâtre et mate, agitation pareille à celle des mésanges, habitude de se suspendre, la tête en bas, à des branchettes pendantes, telles sont les indications caractéristiques. Leurs petits cris: "tsi-tsi-tsi» deviennent bientôt familiers et sont facilement reconnaissables. 


\section{Genre-Regulus. Roitelets.}

748. Le Roitelet huppé. (OOLDEN-CROWNED KINOLET). Regulus satrapa. L, 4.07. Planche XIVII B.

Traits distinctifs. Les roitelets, si semblables les uns aux autres par la coloration générale, peuvent être facilement distingués par leurs couronnes. Le roitelet huppé a une ligne noire au-dessus de l'œil laquelle n'existe pas chez le roitelet a couronne rubis, et la tache à la couronne est orange et jaune chez le mâle, ou simplement jaune chez la femelle au lieu de rouge rubis ou olive uniforme comme chez le roitelet a couronne rubis.

Indications sur le terrain. La couleur de la couronne et les sourcils de couleur claire sont les meilleures indications sur le terrain, mais, vu la petitesse de l'oiseau, il faut parfois une très grande somme de patience pour distinguer ces indications quand l'oiseau est sans cesse en mouvement à une hauteur considérable dans les grands sapins.

Nid. En générale parmi les conifères. Nid suspendu, fait de mousse verte avec de fines bandes intérieures d'écorce tendre, de minces radicelles noires et de plumes.

Habitat. En tant qu'espèce, c'est l'Amérique du Nord. Le roitelet huppé de l'Est habite un territoire qui s'étend à l'Ouest jusque près des montagnes; l'oiseau couve dans la zone des conifères.

SOUS-ESPECES. Le roitelet huppé comprend deux sous-espèces: une de l'Est et une de l'Ouest. Celle de l'Est, qui est la forme type, est la seule qui se rencontre dans l'Est du Canada.

On se trouve souvent environné d'une grande multitude de ces petits oiseaux qui voltigent autour d'un épais feuillage pour y entrer ou s'en échapper, s'élancer çà et là, sans s'inquiéter de la présence d'un intrus, et de mouvements si rapides qu'il est difficile de remarquer les caractères spécifiques de ces oiseaux. Les uns se suspendent la tête en bas, à une touffe de branchettes, les autres sont tout à leur travail sur les branches et les ramilles, échangeant avec d'autres leur cri aigu de tsi-tsi-tsi.

Le roitelet huppé reste au Canada presque tout l'hiver, quelquefois même l'hiver entier. Il aime les arbres toujours verts et souvent hante les conifères qui ornent les alentours des villes et des maisons. Il n'a peur de rien, est très confiant, mais ne s'apprivoise pas comme fait la mésange.

Valeur économique. Les roitelets sont tellement insectivores qu'on peut à bon droit les considérer comme très utiles. Ils sont petits, il est vrai, mais leur quantité, quand ils se trouvent là, supplée à ce qui manque à leur taille; ce qui a été dit des mésanges, à cet égard, s'applique également aux roitelets.

749. Le Roitelet à couronne rubis. (RUBY-CROWNED KINGLET). Regulus calendula. L, 4.41. Planche XLVII B.

Traits distinctifs. Le roitelet à couronne rubis peut probablement être pris pour les espèces précédentes seulement, mais l'absence de raies noires sur la tête est toujours distinctive.

Indications sur le terrain, Eu égard à la rapidité des mouvements de troupes d'oiseaux si vifs et à l'ombre épaisse que projettent des conifères très rapprochés, il est parfois difficile de saisir les marques distinctives de la tête des roitelets. On remarquera, cependant, que le roitelet à couronne rubis a l'habitude d'agiter ses ailes, à l'occasion, par quelques courts et rapides mouvements vibratoires, pendant certains repos momentanés et sautillant de droite et de gauche mais sans voler. Tout cela évoquera souvent chez l'observateur l'idée de la susdite espèce, bien que cela ne puisse cependant pas être regardé comme une preuve d'identité.

Nid. Dans les conifères; nid fait de mousse et de fines bandes d'écorce proprement entrelacées, et garni de plumes; le nid est ordinairement à moitié suspendu.

Habitat. Le nord de l'Amérique du Nord. Dans l'est du Canada la couvaison se fait dans le nord au dela de la région colonisée, et plus au nord que le roitelet huppé.

SOUS-ESPECES. Le roitelet à couronne rubis comprend trois sous-espèces dont une seule, celle de l'Est, la forme type, se rencontre dans l'est du Canada. 
Cette espèce ressemble tant au roitelet huppé qu'il n'y a pas lieu d'en étudier davantage ni les mœurs ni la valeur économique. Le chant du roitelet à couronne rubis est, toutefois, une des surprises de la nature. Il est fort, clair, de plein gosier et peut s'entendre à une distance surprenante; il portc aussi loin que celui du pinson pourpré. Quand on l'entend pour la première fois on l'attribue presque toujours à l'un des pinsons qu'on sait être les meilleurs chanteurs plutôt qu'à ce minuscule oiselet.

\section{Sous-famille-Polioptiling. Gobe-mouches.}

C'est une petite famille composée d'un seul genre et particulière à l'Amérique. La coulcur est un bleu-cendré tendre avec du blanc. Le bec est d'une fauvette, à la surface, mais la première primaire de l'aile est petite et presque abortive, étant de plus de la moitié moins longue que sa voisine, comme c'est le cas chez le vireo gris-olive. Il n'y a qu'une seule espèce de cet oiscau au Canada.

\section{Genre-Polioptila. Gobe-mouches.}

751. Le Gobe-mouches gris-bleu. (BLUE-GRey anatcatchen). Polioptila carulea. L, 4.05. Les dessus sont d'un gris bleu uniforme; les ailes foncées, la queue noire, les plumes blanches du dehors de la queue; les dessous et la face tout blancs. Le malc a une ligne noire en travers du front et au-dessus de l'œil.

Traits distinctifs. La petite taille, la coloration uniforme bleu et blanc et la très courte plume primaire de l'aile, tout cela est distinctif.

Indications sur le terrain. Les couleurs d'un petit oiseau qui habite les plus hautes branches d'arbres très élevés, sont habituellement malaisćes à distinguer, cependant les contours, la longue et étroite queue balancée comme celle de la fauvette à queue rousse, et ses notes caractéristiques, plutôt rauques, sont d'excellentes indications.

Nid. Fait de vrilles, de fines bandes d'écorce, d'herbes délicates, solidement entrelacées et recouvertes à l'extérieur de fils d'araignée et de lichens. Il a la forme d'une cheminée, haut avec côtés tout droits. C'est un des plus beaux nids d'oiseau américain.

Habitat. Le sud-est des Etats-Unis allant au nord jusqu'd la frontière canadienne le long du lac Erié ct la rivière Détroit.

SOUS-ESPECES. La sous-espece de l'Est, le gobe-mouches de l'Est, est la forme type. Une sous-espèce de l'ouest se rencontre dans les Etats-Unis du sud-ouest.

C'est un oiseau des forêts de grands arbres, où il habite à l'ordinaire et fait son nid parmi les cimes des arbres. Comme on ne le trouve de façon régulière que dans le sud de l'Ontario, le long des rives de l'ouest du lac Erié, et qu'on ne l'a rencontré ailleurs que par occasion au Canada, il faut le considérer comme un oiseau rare dans ce pays.

Valeur économique. Trop rare au Canada pour pouvoir être envisagé à ce point de vue.

\section{FAMILLE-TURDIDE. GRIVES ET OISEAUX APPARENTES.}

C'est iei un groupe presque cosmopolite systématiquement séparé, non sans difficulté, de la dernière famille mentionnée, lors même que les individus décrits dans les pages qui suivent sont assez fortement marqués pour etre facilement reconnaissables. Dans l'est du Canada il n'y a qu'une seule sous-famille qui soit représentée.

\section{Sous-famille-Turdinæ. Les Grives vraies.}

La plupart des grives canadiennes sont faciles à reconnaître comme telles. Au point de vue systématique elles sont clairement indiquées par 
le nombre des primaires et par leurs pieds squameux. Comme ces détails sont plutôt difficiles à distinguer pour un amateur, il sera peut-être plus facile de différencier ces oiseaux au moyen d'autres caractères plus frappants et qui s'appliquent aux représentants de ce groupe dans l'est du Canada. Dans ce but, on peut distinguer d'un côté les grives proprement dites, et de l'autre le merle d'Amérique, le rouge-gorge bleu et le traquet motteux, ce dernier très rare et les dcux autres si bien caractérisés que d'être aisément reconnus. Les grives proprement dites sont des oiseaux de taille moyenne, bruns en dessus et blanes en dessous, avec la poitrine plus ou moins tachée, sauf une espèce, la grive de Wilson, où elle est très visiblement tachée. Tout oiseau canadien de cette description, entre 6.25 ct 8 pouces $\cdot 30 \mathrm{dc}$ long, avec la première primaire très petite et amoindrie, appartient à ce groupe. Les grives sont toutes des oiseaux qui se tiennent au sol et habituellement plus ou moins solitaires. Leur principale nourriture se compose d'insectes et de fruits tendres. Les espèces des contrées boisées sont de minime importance immédiate, bien que leur influence soit bienfaisante. Toutes les grives de ce groupe sont d'aimables chanteurs.

\section{Genre-Hylocichla. Grives des bois.}

755. La Grive des bois. (woon thrush). Hylocichla mustelina. L, 8.29. Planche XIVIII A.

Traits distinctifs. Cette grive est facilement reconnue pour telle, bien qu'elle ait un peu la couleur générale de la grive rousse. La courte queue, le bec tout droit, l'œil foncé au lieu de clair, sont de bons traits distinctifs. Elle peut se distinguer des autres membres de l'espèce par sa plus grande taille, la blancheur absolue de la poitrine, la délimitation plus tranchée des taches rondes et par la teinte jaunâtre plutôt que rougeâtre ou d'olive du dos, teinte très brillante sur la tête.

Indications sur le terrain. Les taches bien tranchées sur une poitrine toute blanche, la teinte basanée du dos, sont les meilleures indications sur le terrain.

Nid. Sur de jeunes arbres a environ 8 pieds au-dessus du sol; nid fait de feuilles, de radicelles, de fines ramilles, de tiges d'herbes solidement entrelacées, et garni de boue et de fines radicelles.

Habitat. L'est de l'Amérique du Nord; du nord au sud de l'Ontario; se trouve par occasion ou de passage dans les régions voisines. La grive des bois est un oiseau des contrées boiśées plutôt méridionales; elle est commune au Canada seulement dans les parties plus méridionales.

La grive des bois est un oiseau des contrées boisées plutôt méridionales; elle est commune au Canada seulement dans les parties plus méridionales.

756. La Grive de Wllson. (WILson's THRUsH, VEERY). Hylocichla fuscescens. L, 7.52. Planche XLVIII B.

Traits distinctifs. Chez cette grive le brun est presque aussi basané que chez les grives des bois mais le dos est de couleur uniforme et nullement plus brillant sur la tête et la queue qu'il ne l'est ailleurs. Les taches de la poitrine se réduisent à une vague série de taches plus foncées sur les côtés du cou et sur une région teintée en travers de la poitrine supérieure.

Indications sur le terrain. La coloration uniforme, claire du dos et la couleur claire pour ainsi dire saupoudrée, des taches de la poitrine.

$\mathrm{Nid}$. Sur le sol ou tout auprès; nid fait de bandes d'écorce, de radicelles, de feuilles enveloppées d'autres feuilles, et garni de radicelles.

Habitat. Le nord de l'Amérique du Nord jusqu'aux limites de la colonisation.

SOUS-ESPECES. Au Canada, la grive de Wilson est représentée par deux sousespeces, la présente qui est la forme type et la grive des saules qui habite l'ouest, avançant vers l'est jusqu'au Manitoba et ne se rencontrant que par occasion dans l'est du Canada. 
La grive de Wilson occupe un vaste territoire dans les régions colonisées. Au delà du territoire occupé par la grive des bois et où les autres grives ne sont pas nombreuses, cette espèce est commune, et les notes qu'elle lance, semblables à celles des cloches, tombant en cassades, au coucher du soleil, dans les sombres buissons, sont très agréables à entendre.

757. La Grive d'Alice. (ALICE's THROSH OU GREX-CHEEKED THROSH). Hylocichla alicia. $\mathrm{L}, \mathbf{7} \cdot 58$. Planche XLIX A.

Traits distinctifs. Tout en étant une espèce distincte, cette grive ressemble tellement a la suivante-la grive de Swainson-qu'il est assez difficile de l'en distinguer. Toutes deux peuvent être distinguées d'avec d'autres grives par leur dos d'un olive foncé uniforme et par leur poitrine fortement mouchetee de taches fondues ensemble par places et n'en faisant qu'une. Chez la grive d'Alice les cotés de la face et les taches de la poitrine sont parsemés d'un gris terne et froid au lieu de l'être d'un chamois de tons chauds.

Indications sur le terrain. La poitrine fortement tachetce, le dos et la queue uniformement colorés feront distinguer cette grive d'avec toutes les autres, sauf de la grive de Swainson. L'absence de tons chamois sur le cóté de la face, la différenciera de cette espèce, bien que, sur le terrain, ce soit seulement dans les plus favorables conditions que ce caractère pourra être discerné avec certitude; aussi bien, des spécimens aperçus dans l'obscurité des bois ou a la faveur d'instants très passagers, consacrés a ces observations, resteront non identifiés.

Habitat. Le nord de l'Amérique du Nord; la couvaison se fait surtout au Canada à partir des régions colonisées dans le direction du nord.

SOUS-ESPECES. La grive d'Alice comprend deux races au point de vue géographique: celle d'Alice, le type, et la grive de Bicknell H.A. bicknelli. Cette dernière, au Canada, se trouve reléguée dans les provinces maritimes au-dessous du golfe du SaintLaurent, mais l'habitat des deux formes, dans l'est du Canada, n'est pas bien précisé.

758a. La Grive de Swainson. (oLIve-BAcked THRUsh). Hylocichla ustulata. L, 7.i7. Planche XLIX A.

Traits distinctifs. Le dos uniformément coloré sans différence de couleur soit sur la tête soit sur la queue, et la poitrine fortement mouchetée de taches distingueront cette grive de toutes les autres sauf de la grive d'Alice. La nuance chamois au lieu d'un gris froid dans les joues, la différenciera de cette dernière espèce.

Indications sur le terrain. Le dos et la poitrine caractérisés comme ci-dessus. La coloration faciale qui la distingue d'avec celle d'Alice ne peut être aperçue que dans les circonstances les plus favorables et il y a bien des individus qu'on n'a jamais pu identifier.

Nid. Dans les buissons ou sur de petits arbres à environ 4 pieds au-dessus du sol; nid fait d'herbes grossières, de mousse, de radicelles, de feuilles et d'écorce; garni dé radicelles et d'herbe.

Habitat. En tant qu'espèce, toute l'Amérique du Nord; la couvaison a lieu principalement au Canada, depuis les limites des régions cultivées dans la direction du Nord. La grive de Swainson existe dans l'Ouest jusqu'aux Montagnes Rocheuses.

SOUS-ESPECES. La grive à dos olive, dite de Swainson, comprend deux sousespèces. La race type, sous le nom de grive au dos brun rougeâtre est un oisesu de la côte du Pacifique. La grive de l'Est, dite à dos olive ou de Swainson, $H . u$. swainsoni, est la seule de son espèce dans l'est du Canada.

Au printemps et a l'automne, les bois peu épais sont envahis par d'innombrables grives de couleur uniforme, à poitrine tachetée, qui vont a leur couvée ou en reviennent. Elles sont plutôt circonspectes et la proportion numérique de chaque espèce peut en général se supputer.

759. La Grive solitaire. (hermt throsh). Hylacichla guttata. L, 7.17. Planche XLIX B.

Traits distinctifs. Très semblables aux deux dernières, mais la queue brun rougeâtre fait contraste avec le brun olive du dos.

Indications sur le terrain. Le dos brun, la poitrine d'un blanc tacheté la fait reconnaftre comme une grive. La coloration rougeatre de la queue, qui contraste avec le dos olive, sert à identifier l'espèce.

Nid. Sur le sol, nid fait de mousse, d'herbes grossières et de feuilles, garni de radicelles et d'aiguilles de pin. 
Habitat. En tant qu'espèce, c'est tout le nord de l'Amérique du Nord. La grive solitaire de l'Est va vers l'Ouest jusque près des montagnes et vers le Nord jusqu'à la limite des arbres; elle couve d'habitude tout juste au delà les régions cultivées, mais assez irrégulièrement près de nos limites méridionales.

SOUS-ESPECES. La grive solitaire, représentée en Amérique par six sous-espèces authentiques, est un oiseau qui se plie facilement aux circonstances. La forme typique est une race de l'Alaska. Le Canada de l'Est n'a qu'une seule sous-espèce, la grive solitaire de l'Est, H.g. pallasi.

La grive solitaire est un de nos plus fameux chanteurs. Malheureusement il est ordinairement muet quand il traverse le sud du Canada; il ne se fait entendre à son avantage que dans les bois de conifères du Nord.

\section{Genre-Planesticus. Merles d'A mérique.}

761. Le Merle d'Amérlque.(american robin). Planesticus migratorius. L, 10. Planche L A.

Traits distinctifs. Le merle est un oiseau trop parfaitement caractérisé et connu pour demander une description spéciale.

Indications sur le terrain. Le merle est aisément reconnu, par ceux qui connaissent l'espèce, même à de grandes distances quand aucune couleur n'est visible, et cela grâce à sa silhouette, à sa façon de se tenir et aussi de voler.

Nid. Fréquemment sur des arbres fruitiers ou très feuillus; près des maisons, nid fait d'herbes grassières, de feuilles, de radicelles, etc., avec une séparation intérieure faite de boue; garni d'herbes fines.

Habital. C'est dans toute l'Amérique du Nord que se rencontre cette espèce et vers le nord jusqu'a la limite des forêts. Le merle de l'Est habite partout au Canada coincidemment avec celui de l'Ouest sur la côte du Pacifique.

SOUS-ESPECE. Le merle comprend trois sous-espèces desquelles celui de l'Est, l'individu type, est le seul représentant dans l'est du Canada.

Le merle a plus que tout autre oiseau des relations étroites avec l'homme. Sa voix joviale annonce le printemps. Son chant est le premier a se faire entendre le matin, et le dernier, vers le soir; en automne quand il a dépouillé le sorbier de ses dernières baies et qu'il a disparu, on sait que l'hiver est aux portes. Bien qu'il soit appelé d'après le fameux oiseau de l'ancien monde, il ne ressemble que de très loin au rouge-gorge d'Angleterre. Les seuls points de ressemblance entre eux sont la poitrine rouge et les mœurs familières.

Le pire ennemi des merles c'est probablement le chat domestique. Comme ils nichent à des endroits très accessibles, les jeunes merles sont exposés à bien des dangers; ils quittent souvent le nid avant d'être capables de se défendre tout seuls, et sont ainsi la proie du chat. La poitrine tachetée du jeune merle montre qu'il descend d'un ancêtre tacheté et qu'il est apparenté aux grives de l'espèce précédente. De fait, les jeunes de la plupart des oiseaux de cette famille ont la poitrine tachetée.

Valeur économique. Bien que le merle soit un aide très utile au cultivateur, son goût pour les fruits lui occasionne parfois des désagréments de la part du cultivateur qui élève des arbres fruitiers. Le 42 pour cent de sa nourriture fait de cet oiseau un insectivore, le reste se compose de baies et d'autres petits fruits tendres dont un peu plus du 4 pour cent consiste en du fruit cultivé.

\section{Genre-Enanthe. Traquet motteux.}

765. Le Traquet motteux. (WHEATEAR). Enanthe onanthe. L, 7.01. Oiseau qui ressemble à la farlouse, gris clair en dessus, blanc dessous, un chamois aux tons chauds a la gorge; un ruban noir en travers de l'œil et un croupion blanc. La femelle et le tout jeune sont semblables, mais plus ternes et d'un chamois plus uniforme. 
Traits distinctifs. Le croupion blanc très large et visible avec la coloration générale indiquée plus haut sont caractéristiques.

Indications sur le lerrain. Avec son croupion blanc si évident l'oiseau ressemble à une farlouse partiellement albinos.

SOUS-ESPECES. La sous-espèce du traquet motteux qui se rencontre dana l'est, du Canada, c'est le traquet du Groenland, $O$. o. leucorhoa.

Nous avons ici un oiseau européen qui se rencontre assez régulièrement dans le Groenland et peut-être aussi dans les régions voisines du NouveauQuébec, mais seulement par occasion ou accidentellement ailleurs au Canada.

\section{Genre-Sialia. Rouges-gorges bleus.}

766. Le Rouge-gorge bleu. (BLderird). Sialia sialis. L, 7·01. Planche L B.

Traits distinctifs. C'est lc seul oiseau parmi les espècea canadiennes qui soit complètement bleu avec une poitrine rougeâtre. L'oiseau indigo est bleu mais il lui manque la poitrine rouge. Les femelles et les tout jeunes sont plus ternes de couleur et les poitrines des jeunes oiseaux sont tachetées de brun, mais tous ont le dos d'un bleu très caractéristique.

Indications sur le terrain. La brillante coloration bleue du dos et le rouge terreux de la poitrine.

Nid. Dans des arbres creux, ou sur des poteaux ou des souches, aussi dans les maisons à oiseaux: nid fait d'herbes.

Habitat. L'est de l'Amérique du Nord, a l'ouest jusqu'aux provinces des prairies, et au nord, presque jusqu'aux limites de la colonisation.

SOUS-ESPECES. La sous-espèce de rouge-gorge bleu qui se rencontre au Canada c'est la race type, le rouge-gorge bleu de l'Est.

L'oiseau bleu arrive aux premiers jours du printemps en même temps que le merle et l'étourneau des prés. C'est un oiseau plein de confiance, construisant son nid dans les creux de vieux pommiers, dans les trous des poteaux de clôtures, dans les nichoirs, ou dans les nids abandonnés des pics. Son caractère c'est d'être essentiellement un oiseau de verger et, pour peu qu'il y soit encouragé, il construira son nid dans les maisonnettes à oiseaux d'un jardin où ses jolies manières, ses susurrements aimables et sa brillante coloration le feront regarder comme une précieuse acquisition.

Valeur économique. Le rouge-gorge bleu se nourrit surtout d'insectes et par conséquent se trouve être infiniment utile. Les semences d'herbes forment une partie importante de sa nourriture; il mange aussi du fruit tendre, mais, en fait, d'aucun fruit cultivé. De tout cela il résulte que l'oiseau bleu peut être considéré a juste titre comme éminemment utile.

\section{GLOSSAIRE.}

Albinisme La présence ou fortuite ou purement capricieuse de spécimens blancs, ou tout à fait ou à peu près blancs, zoit gur tout le corps soit par taches irrégulières, chez des espèces qui ne sont pas d'habitude blanches. Ce n'est là qu'une fantaisie de la nature cauaée par un manque de matière colorante dans le plumage (voir p. 7).

Axillaires. Un groupe de plumes en forme d'éventail sous l'aile et qui bouchent l'espace entre les plumes les plus internes, qui gervent au vol, et le corps quand l'aile est étendue pour voler.

Barres. Dans les descriptions de la coloration des oiseaux, les barres désignent les lignes tirées en travers du corps et qui ne sont pas parallèles aux tuyaux des plumes (voir raies).

Cire. Membrane qui a l'apparence de la cire et qui recouvre la base de la mandibule supérieure de certains oiseaux, surtout des éperviers (voir figure 33a et $b$, page 25). 
Cou. Espace compris devant entre la gorge et la poitrine et derrière, entre l'occiput et les épaules. Il se compose d'un avant-cou et d'un arrière-cou, dont la signification est bien claire (fig. 1, p. 19). crâne.

Couronne. Le sommet de la tête depuis le front jusqu'à tout près de la base du

Couvertures. Ce sont des plumes qui recouvrent les bases des plumes plus grandes qui servent au vol et des plumes de la queue. Il y a les couvertures en dessus et en dessous de l'aile, comme il y a les couvertures sus-caudales et sous-caudales. Les couvertures en dessus de l'aile se divisent en grandes couvertures et en petites couvertures, les premières forment la plus grande ligne succédant tout a fait aux tuyaux qui servent au vol et leur ressemblent en quelque mesure au point de vue de la texture (figure 1, p. 19).

Crépusculaire. Qui se rapporte au demi-jour, qui précède la nuit.

Croupion. Le bas du dos et qui précède la racine de la queue (fig. 1, p. 19).

Culmen. Le culmen peut s'appeler la ligne de faíte du bec. Vue de cóté cette ligne dessine le tracé du haut du bec à partir de la naissance des premières plumes du front jusqu'à la pointe du bec. On le mesure en ligne droite comme avec le compas, sans suivre les courbes comme avec un yard à ruban (fig. 1, p. 19).

Dichromatisme. C'est la présence normale de deux colorations différentes dans la même espèce, et qui ne sont dues ni au sexe, ni à la saison ni à l'âge, et ne sont que partiellement héréditaires. Les deux colorations peuvent se présenter dans la même couvée bien que la tendance soit que la même espèce n'engendre que l'espèce semblable, et un individu peut prédominer dans une localité donnée quelconque (voir p. 7).

Emargine. Quand ce mot est employé à propos de la forme des plumes, il indique qu'une membrane a été plus ou moins coupée comme si on l'avait ébarbée avec un couteau de poche.

Espèce. En classification zoologique c'est le plus petit groupe permanent. C'est le synonyme du mot "sorte" dont on se sert dans le language ordinaire. Ainsi le chat domestique est une espèce, que ce chat soit maltais, moucheté ou angora; de même pour le chien, qu'il soit lévrier ou épagneul, et pour le chevàl, qu'il soit shetlandais ou arabe (voir p. 5).

Famille. Dans la classification zoologique, la famille constitue l'un des grands groupes d'animaux qui sont assez semblables l'un à l'autre pour être classés ensemble à l'exclusion de tout autre individu. C'est le groupe que, par la grandeur, précède le agenre et qui par ses limitations vient de suite après l'ordre ou le sous-ordre. Par exemple, tous les canards, oies et cygnes appartiennent à la même famille: Anatidæ (voir p. 5).

Flancs. Les côtés du corps, plus bas que l'aile, sous l'aile. Ils sont souvent recouverts par un faisceau de plumes détachées qui peuvent être placées à volonté ou sur ou sous les tuyaux de l'aile refermée (fig. 1, p. 19).

Genre. Dans la classification zoologique le genre est un des plus petits groupes d'animaux qui ont assez de ressemblance entre eux pour être classés ensemble et à part de tous les autres groupes du même rang. C'est une sous-division d'une famille ou sousfamille et qui précède l'espèce. Un genre est donc un groupe d'espèces, et un groupe de genres est une famille.

Hors des limites. Quand on décrit l'habitat des oiseaux on dit d'un individu qui se rencontre au dela des confins de la région en question qu'il est hors des limites.

Hybride. Signifie qui est né de deux espèces différentes; soit une race croisée.

Iris. Partie colorée de l'œil. La pupille, sauf dans le cas d'albinisme, est toujours noire, et le cercle de couleur qui l'entoure s'appelle l'iris.

Lancéolé. Signifie: qui a la forme d'un fer de lance, c'est-â-dire longue, étroite, avec des bords parallèles ou se terminant graduellement en pointe.

Longueur. On abrège ce mot par l'initiale $\mathrm{L}$ et on la donne en pouces et dixièmes de pouce. La longueur est prise en ligne droite, comme avec le compas, depuis la pointe du bec jusqu'au bout de la plus longue plume de la queue, l'oiseau étant mis à plat sur son dos et suffisamment étiré pour rendre droites les courbes du cou.

Lores. Petite tache entre l'œil ct la base du bec (fig. 1, p. 19). 
Mandibules. Les deux pièces qui forment le bec; il y a la mandibule supérieure et la mandibule inférieure.

Manteau. Terme qui comprend la région dorsale, les épaules, les couvertures de dessus de l'aile et les secondaires. Ce terme s'applique plus spécialement aux mouettes chez lesquelles la couleur uniforme de ces parties fait penser à un manteau qui recouvre la portion supérieure du corps et les ailes refermées.

Mélanisme. C'est le contraire de l'albinisme. C'est le phénomène plus ou moina irrégulier, d'individus très foncés ou même noirs quoique appartenant à une espèce qui normalement est de couleur claire. D'habitude ce phénomène est moins fréquent que l'albinisme, lors méme que certaines espèces y sont plus exposées; dans quelques cas le mélanisme devient peu à peu et imperceptiblement du dichromatisme. L'albinisme est d'habitude le signe d'un manque de virilité. Le mélanisme ne aemble pas étre une preuve de faiblesse vitale, de la vient qu'il a plus de chance de se perpétuer. Un animal mélanistique s'appelle, dit-on, un mélano (voir p. 7).

Miroir. Partie brillante, rectangulaire formant par sa couleur une tache au centre de la surface supérieure de l'aile. Cette tache offre souvent une irisation métallique et constitue un caractère commun de coloration dans certaines familles, comme celle des canards. p. 19).

Nuque. Petite place derrière le cou, juste au-dessous de la base du crâne (fig. 1,

Ordre. Dans la classification zoologique on appelle ainsi un groupe de familles qui se ressemblent assez l'une de l'autre pour qu'on puisse les distinguer de tous les autres groupes. C'est le groupe le plus grand avant la famille et la plus grande subdivision d'niseau que nous ayons à décrire au Canada (voir page 5 ).

Pectiné. Pourvu de dents ayant la forme des dents d'un peigne. Fn ornitloologie cc terme a'applique surtout aux griffes de certaines espèces qui sont pourvues de dents ile cette forme (voir fig. 19, p. 22).

Pélagique. Se dit des oiseaux qui fréquentent la haute mer.

Poche oesophagienne. Poche membraneuse qui dépend de la mandibule inférieure, cntre ses bras en forme de $Y$ et qui la relie au cou. Certaines especes n'en ont qu'une simple trace; d'autres l'ont extrêmement développée, bien que dans la plupart des espèces elle soit entièrement absente.

Primaires. Les grandes plumes ou pennes insérées à la première partie de l'aile, c'est-à-dire à la main, depuis le poignet jusqu'à l'extrémité (voir aecondaires) (voir fig. 1, p. 19).

Race. Dans l'acception ordinaire, ce mot est synonyme de sous-espèce. En général, c'est tout groupe dans une espèce qui fait voir des caractères communs, reconnaissables, ct qui le différencient d'autres groupes de la méme espèce.

Raies. Dans les descriptions ornithologiques les raies suivent toujours le sens de la longueur de l'oiseau; ai ces lignes sont en travers de leur corps, on les appelle des barres.

Secondaires. Les grandes plumes ou pennes insérées à la seconde partie de l'aile c'est-à-dire à l'avant-bras depuis le poignet jusqu'au coude (voir primaires) (fig. 1, p. 19).

Sous-espèce. Dans la classification ornithologique ce mot eat synonyme de race ou de variété géographique; il indique une division de l'espèce ordinairement en rapport avec des conditions géographiques. La sous-espèce differe essentiellement de l'espèce en ce qu'elle offre des états intermédiaires qui la relient à des races alliées du même rang. Si nous prenons le cheval comme représentant une espèce, les différentes races comme l'arabe, le Clydesdale ou Shetland, etc., sont des sous-espèces (voir p. 7, pour la discussion du sujet).

Sternum. C'est l'os pectoral. Chez l'oiseau c'est un creux profond en forme de quille, auquel viennent s'attacher les muscles de l'aile.

Suspendu. Se dit des nids quant ils pendent comme un sac entre les deux ramifications formant fourcbette d'une branche, ou d'un autre point d'appui sans rien dessous pour supporter le nid.

$28588-17$ 
Tarse. Les os métatarsiens du pied se fusionnent en un seul os. C'est la ce que nous appelons communément la patte de l'oiseau, mais qui n'en est que le pied, s'étendant depuis la jointure des doigts jusqu'au bout de la cuisse. Une comparaison avec les articulations de la jambe de l'homme fera vite voir que le genou se trouve entre la cuisse et la "seconde articulation" de la volaille, et que la première articulation externe chez l'oisean correspond à notre talon, les "pieds n n'étant que les doigts proprement dits.

Type. Dans la nomenclature zoologique la wforme type est la forme parfaitement décrite et dénommée, et le spécimen d'après lequel la description a été faite est le spécimen type. Cela ne veut cependant pas dire que la forme soit typique dans le sens ordinaire du mot, bien que, pour la commodité du sujet on admette que les choses sont ainsi (voir p. 8).

Vermiculures. Dans les descriptions du plumage, le mot vermiculures se rapporte a des raies sinueuses imitant celles que produisent d'innombrables petits vers dans le bois.

Vinacé. Qui a la couleur du vin. C'est une teinte rose pourprée qu'indique la coloration de quelques oiseaux. 


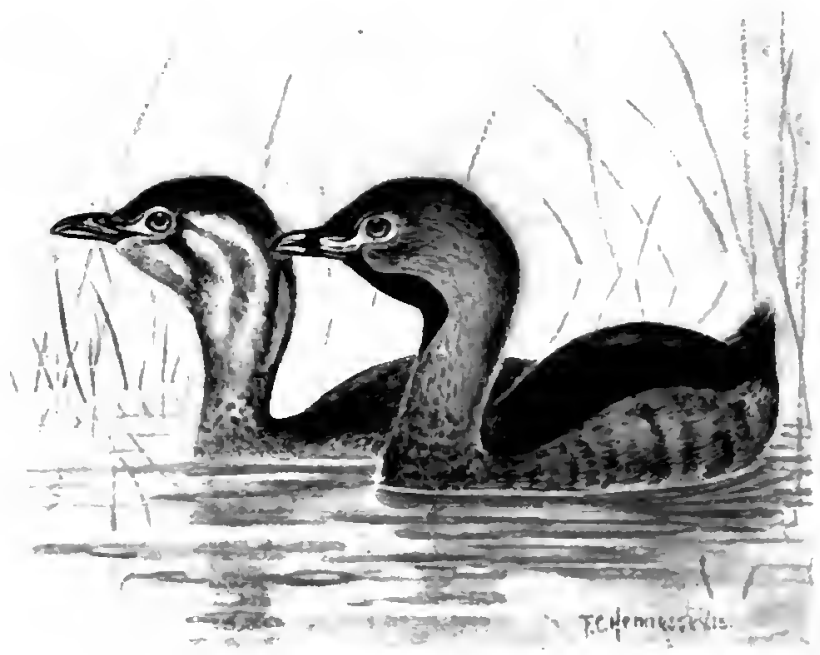

A. Le Grèbe au bee bigarré (p. 45).
Jeune
Alulte

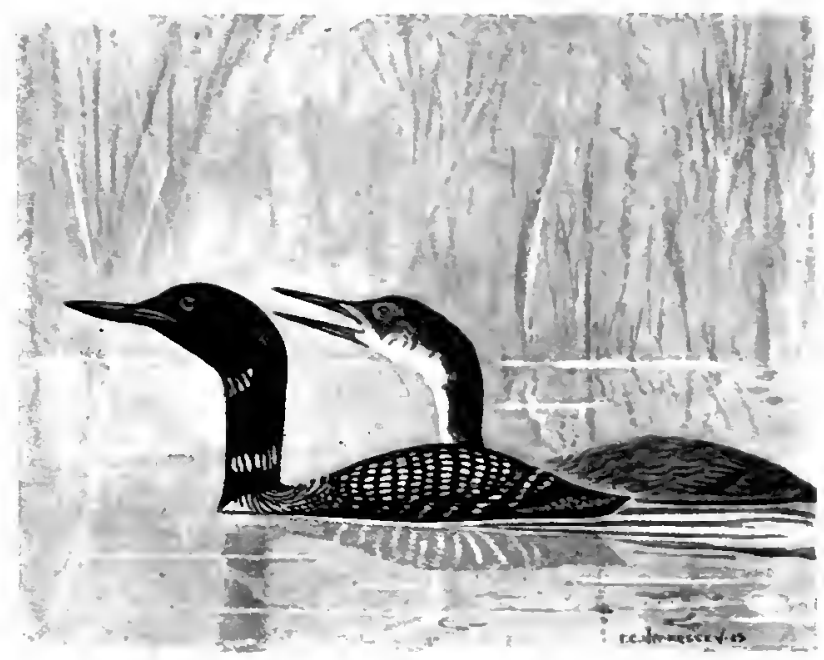

B. Le I'longeon à collier, le Huard (p. 46).

Adulte Jeune" 


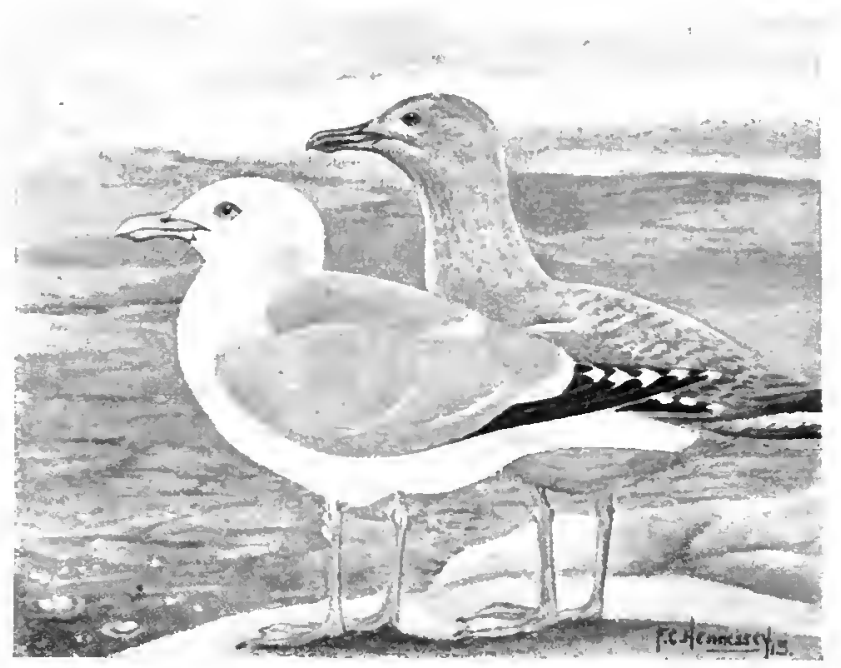

A. Le Goéland argenté (p. 5̃̃). Adulte Jeune

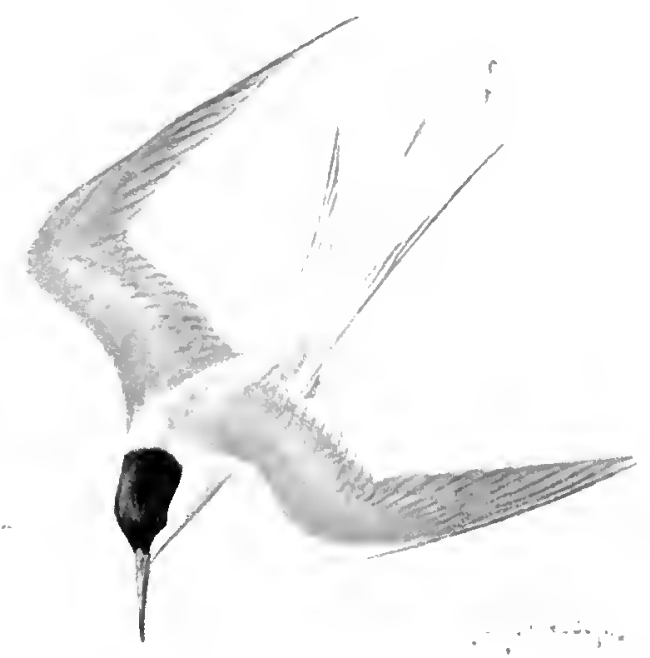

B. La Sterne commune (p. 59). 
Platehe III.

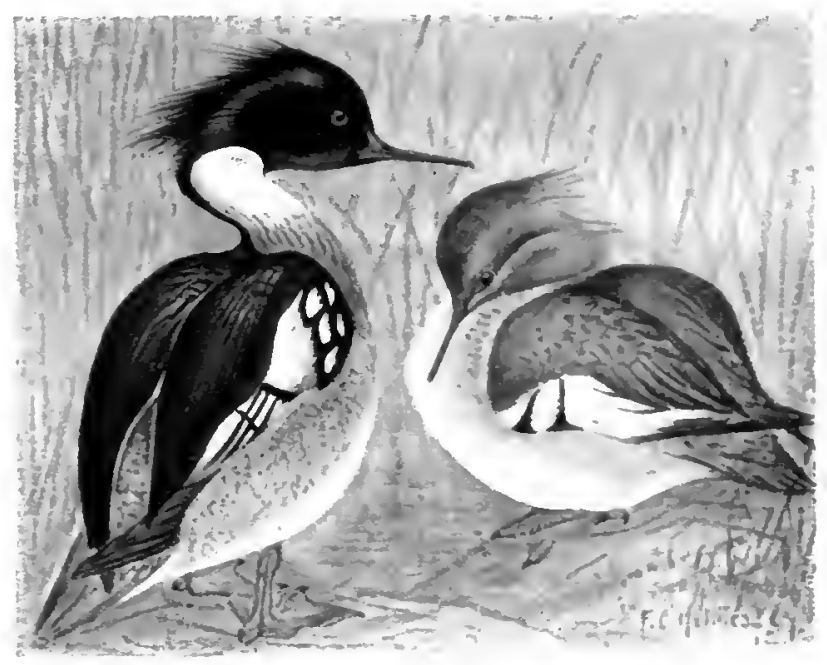

A. Le Harle à poit rine rousse (p, 69).

Mâle

Femelle

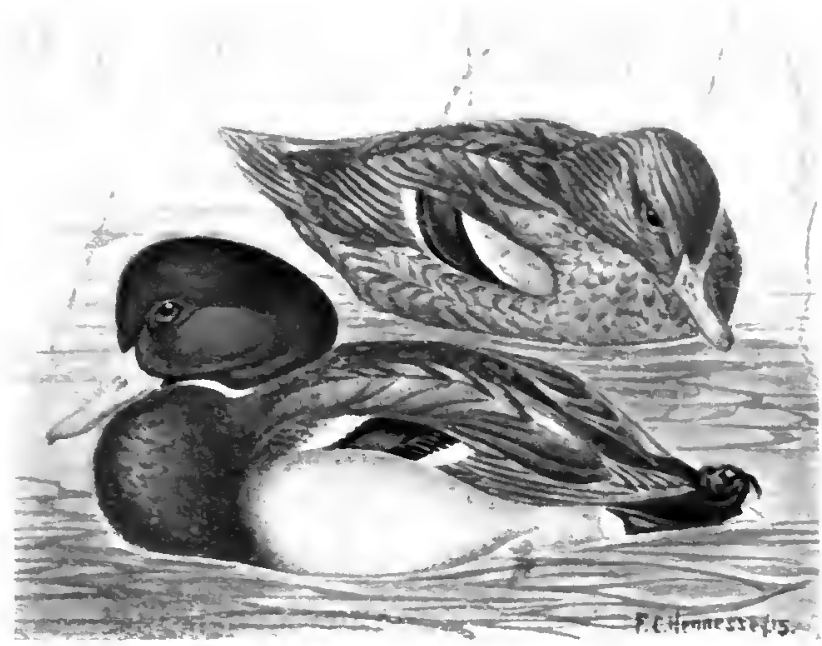

B. Le Canard ordinaire (p. 70).

Mâle

Femelle 
Planche IV.

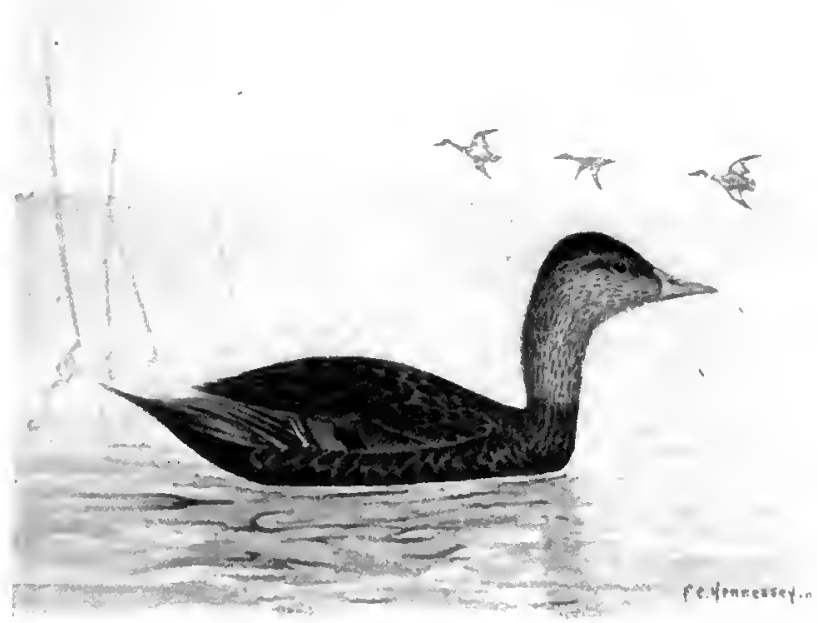

A. Le Canard noir (p. 70).

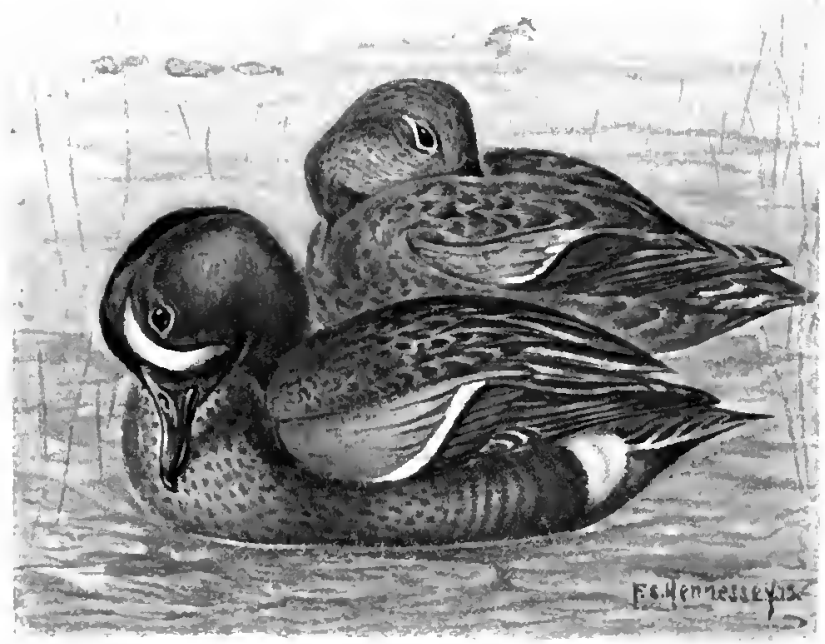

B. La Sarcelle à ailes blenes (p, 72) .

Mâle

Femelle 


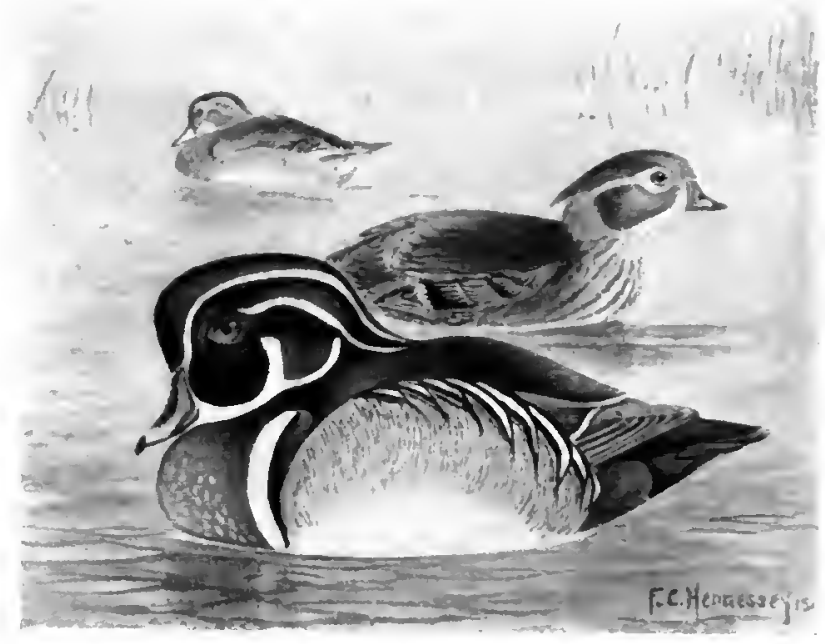

A. Le Canard huppé (p. 73). Mâle Femelle

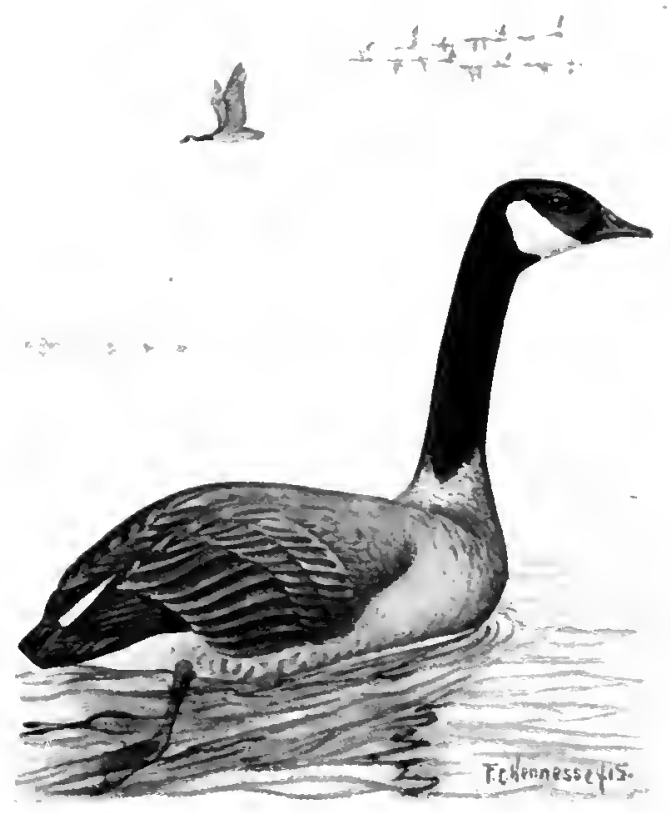

B. La Bernache du Canada (p. 83). 
Planche VI.

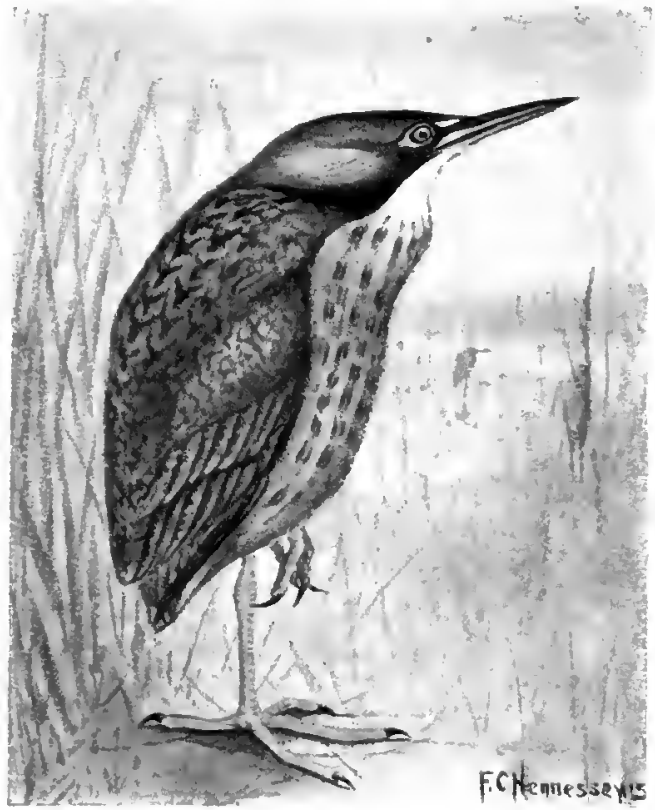

A. Le Butor d'Amérique (p. S7).

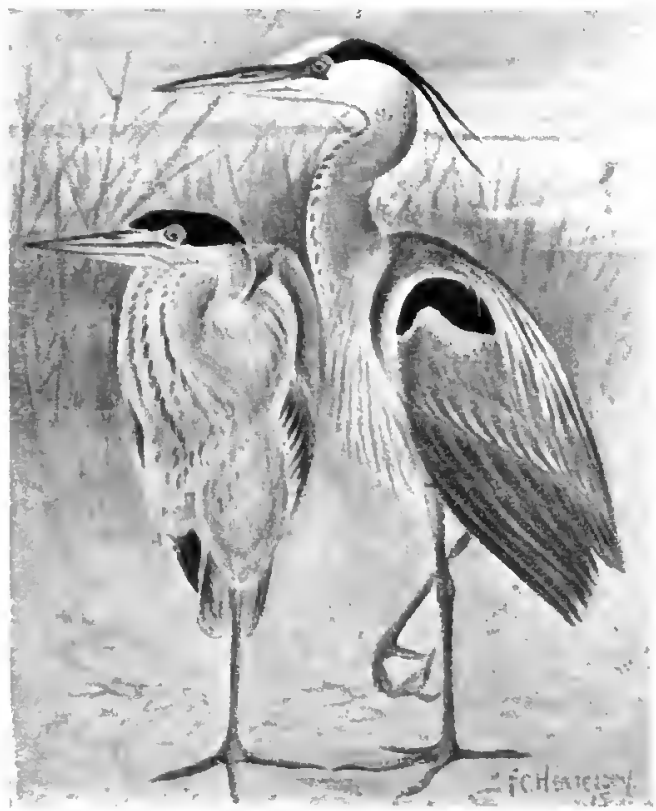

B. Le Grand Héron bleu (p. 89).

Jeune Adulte 
Plaxche VII.

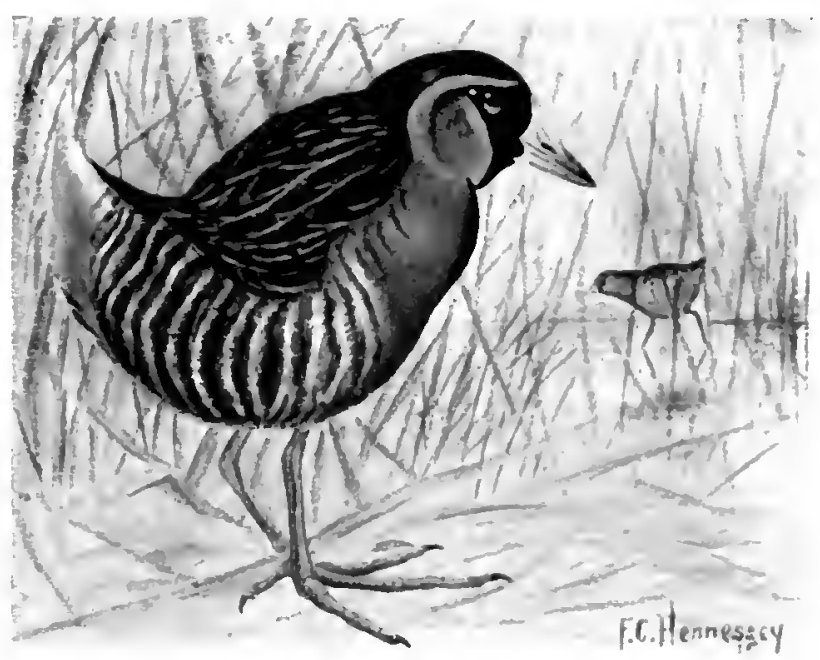

A. Le Râle de la Caroline (p, 96).

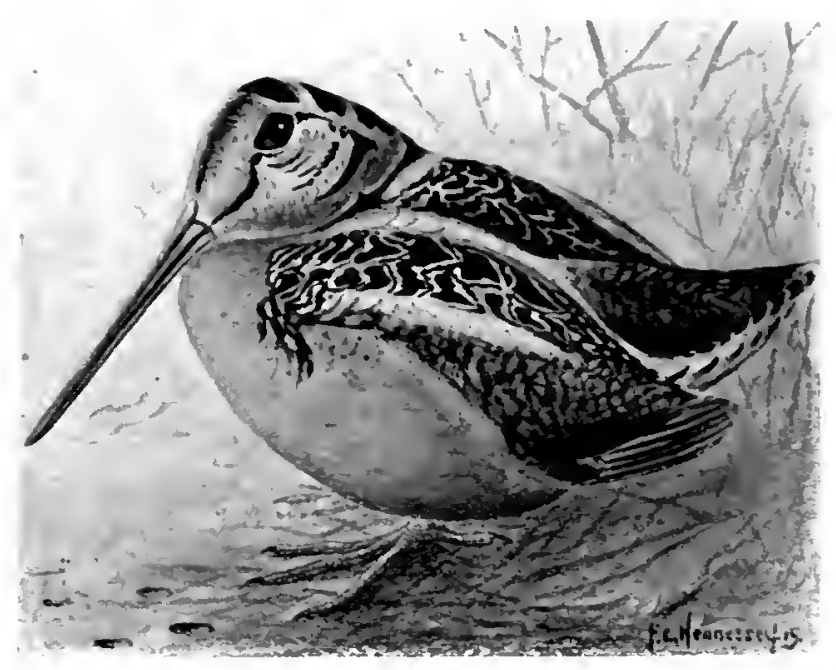

B. La Bécasse d'Amérique (p. 102). 
Planche Vili.

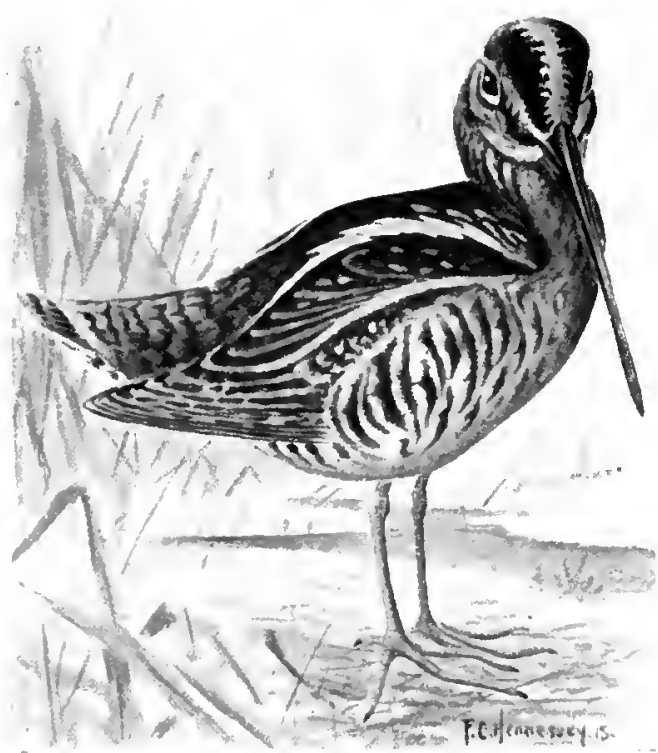

A. La Bécassine de Wilson (p. 102).

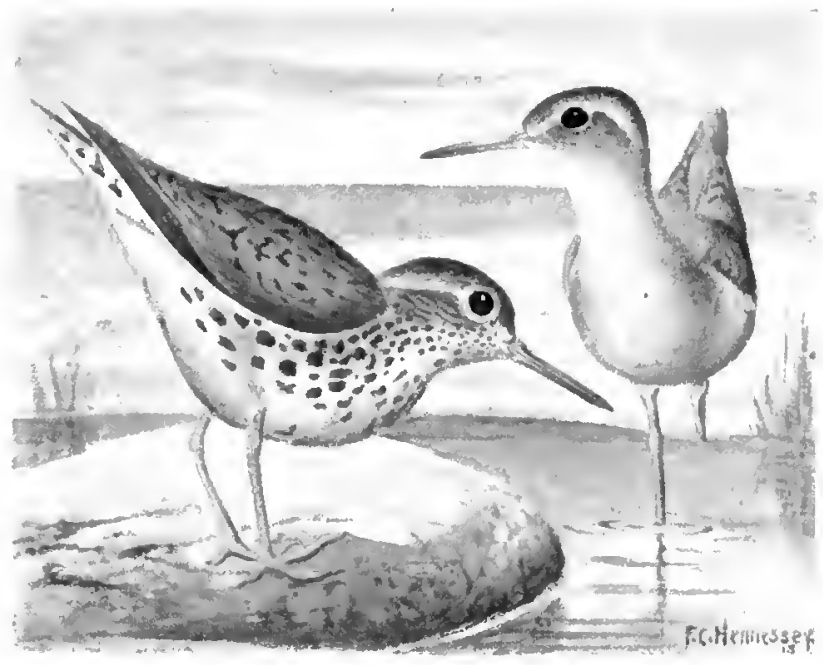

B. La Maubèche tachetée (p. 111). Adulte Jeune 
Puaxche IX.

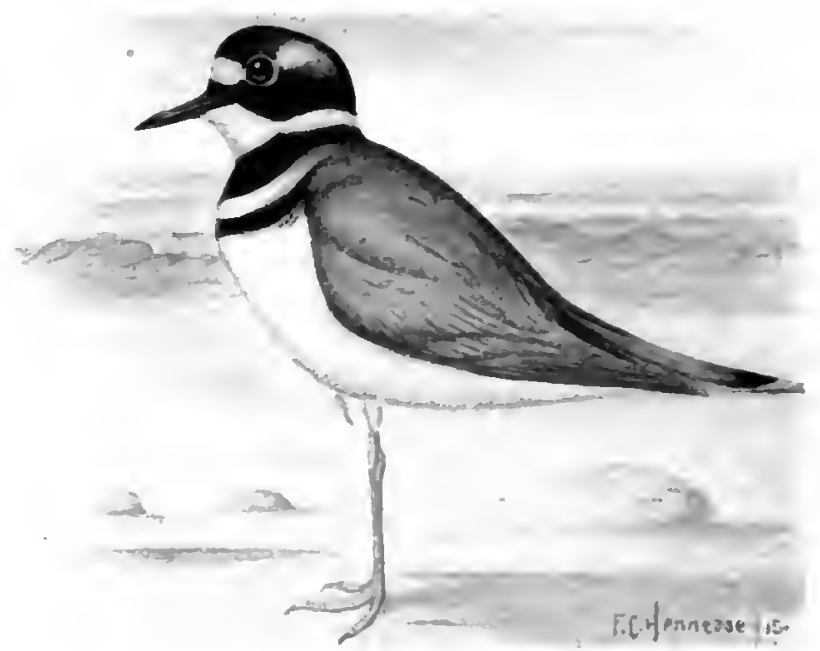

․ Le Pluvier kildir (p. 115).

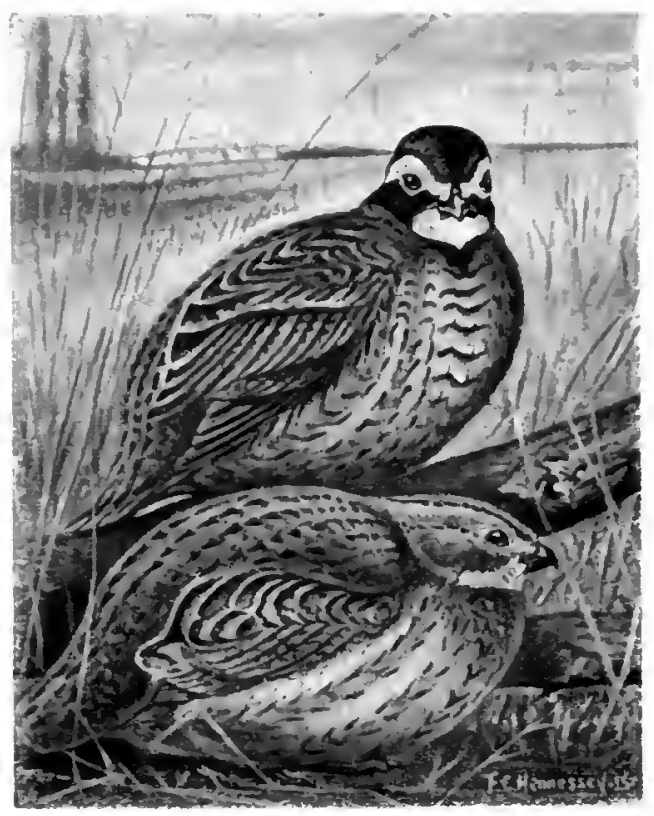

B. La Caille d'Amérique (p. 11s).

Mâle

Femelle 


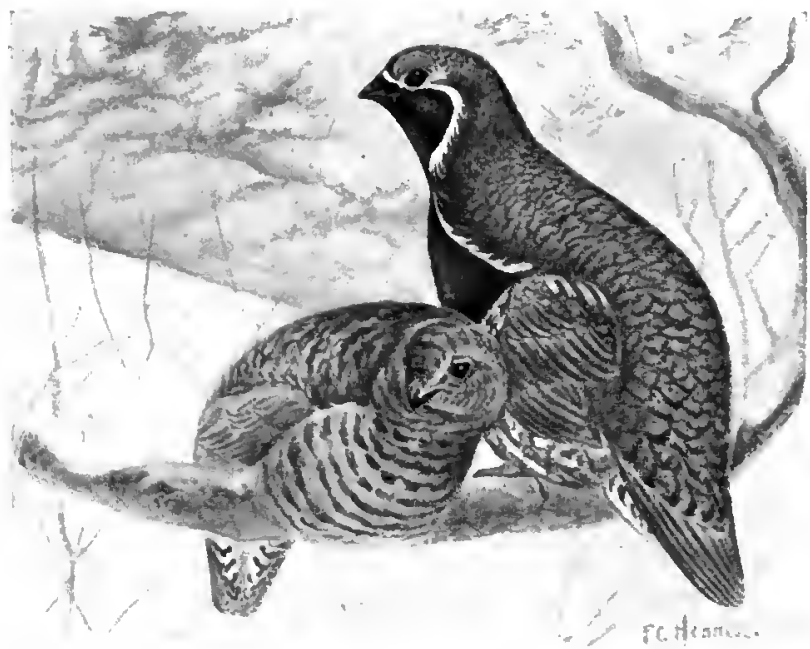

A. Le Tétras du Canada (p. 120).

Femelle Mâle

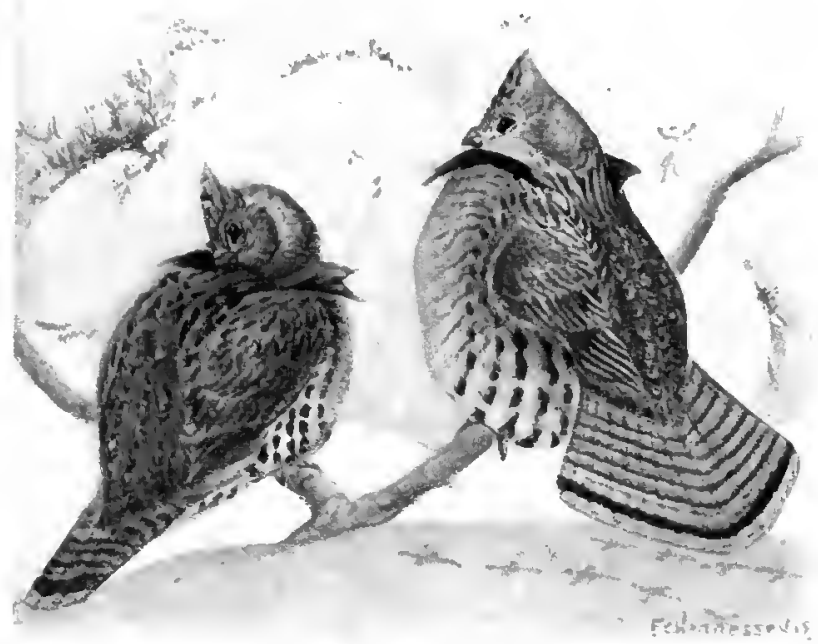

B. La Gelinotte à fraise (p. 120).

Phase rouge Phase grise 
P'LANCHE XI.

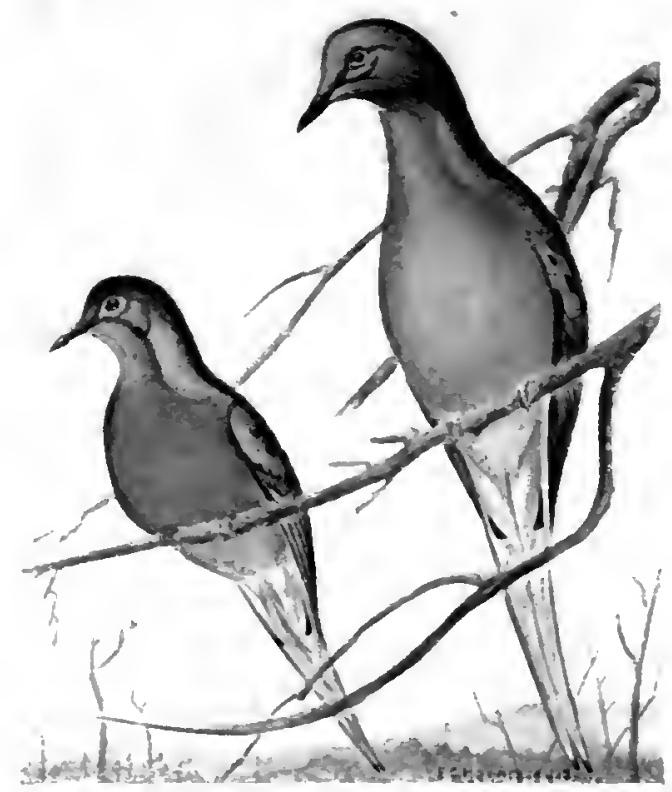

A. La Tourterelle de la Caroline et le Pigeon voyageur (p. 124 et 125).

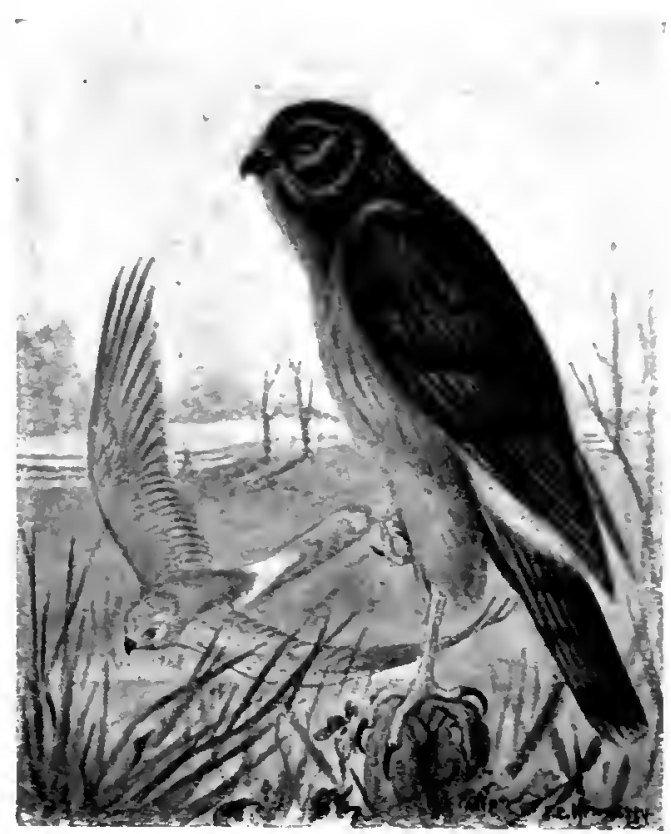

B. Le Busard des marais (p. 130). Adulte Jeune 


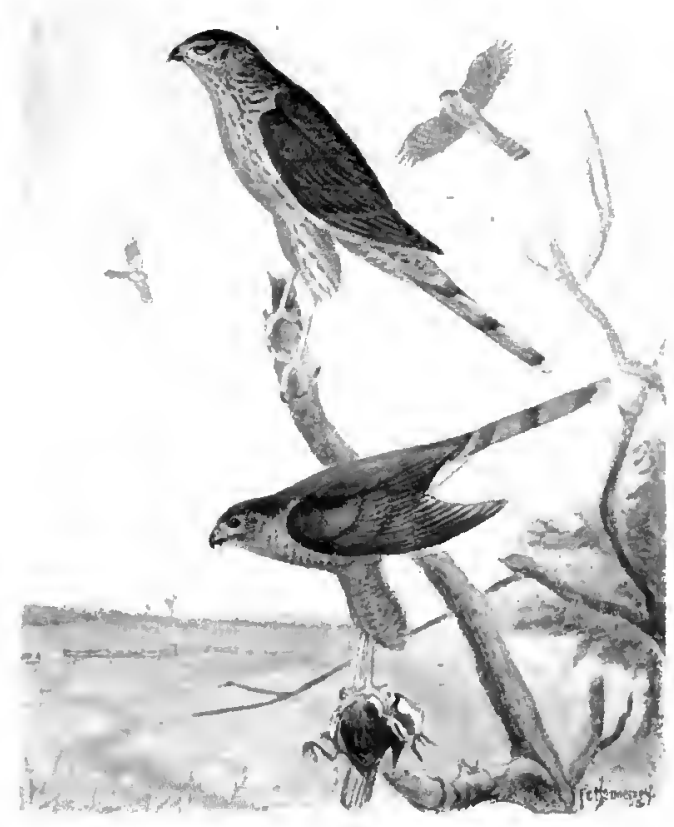

A. L' 'Épervier brun (p. 131).

Jeune femelle Mâle adulte

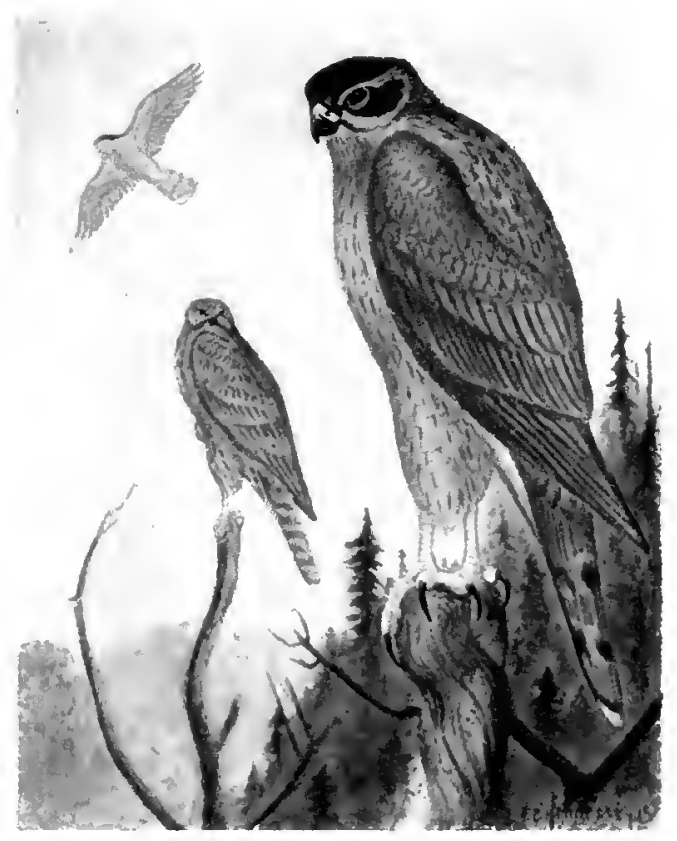

13. L'Autour à tête noire (p. 133).
Jeune
Adulte 
Platche XiII.
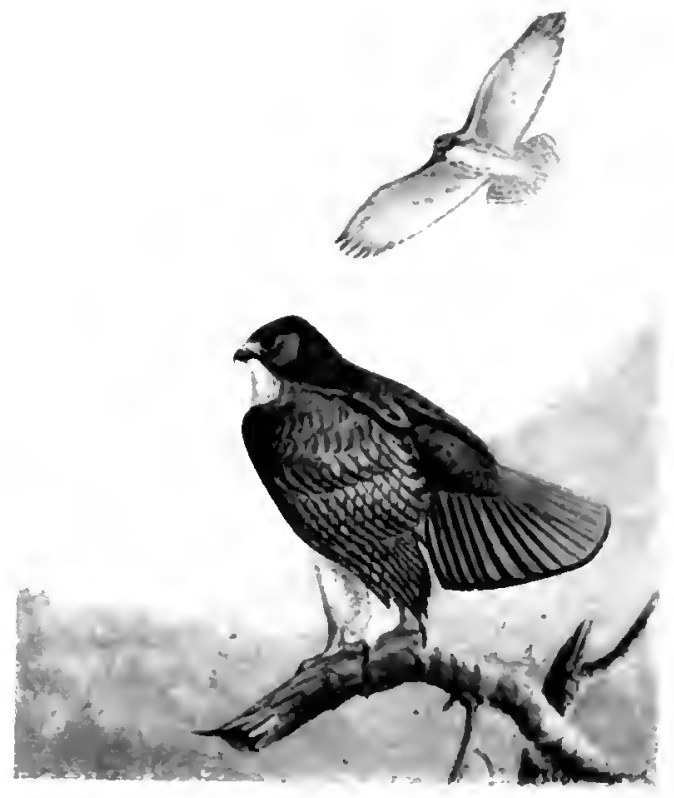

A. La Buse à queue rousse (p. 134).

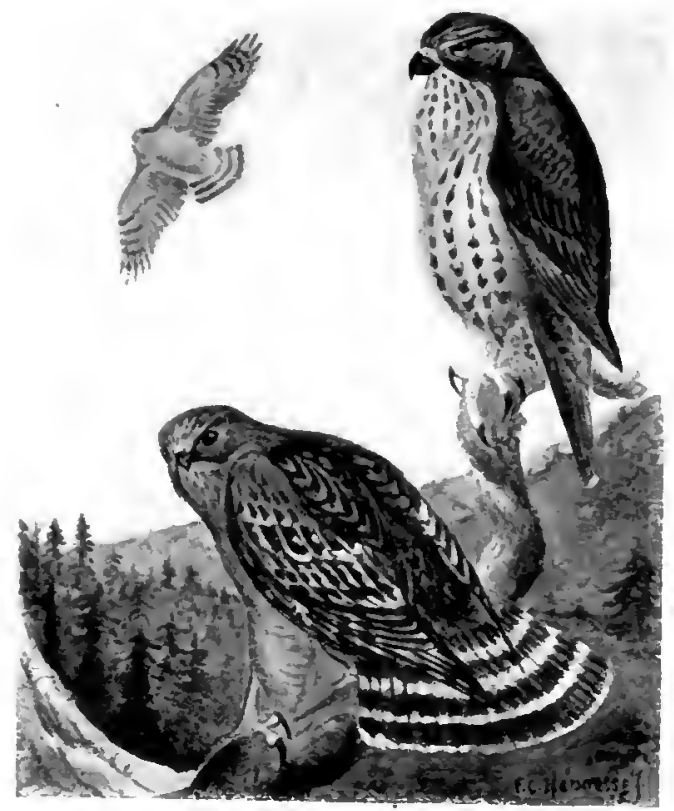

B. La Buse à manteau roux (p. 135̃).
Adulte
Jeune 
Plancie NiV.

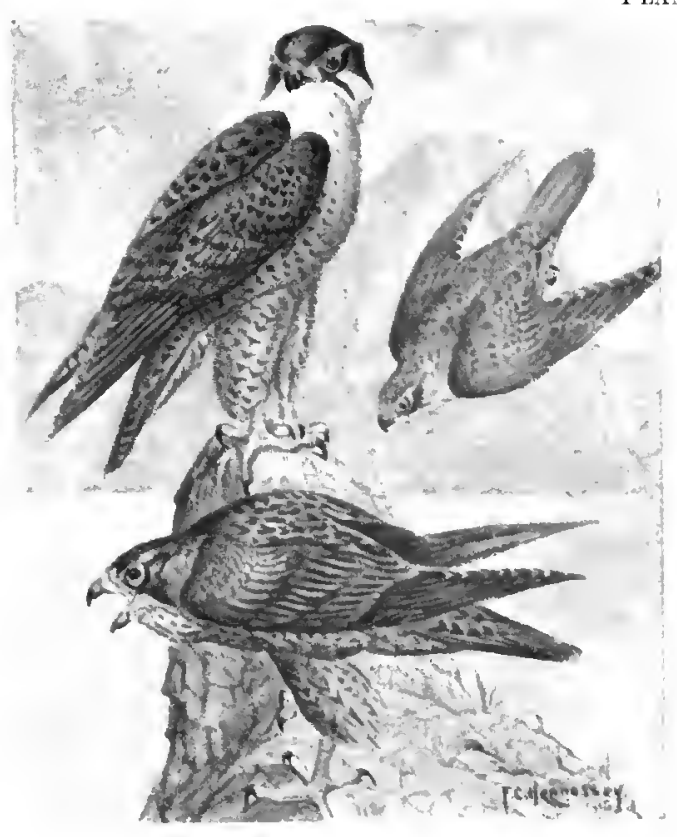

A. Le Faucon pèlerin (p. 140). Adulte Jeune

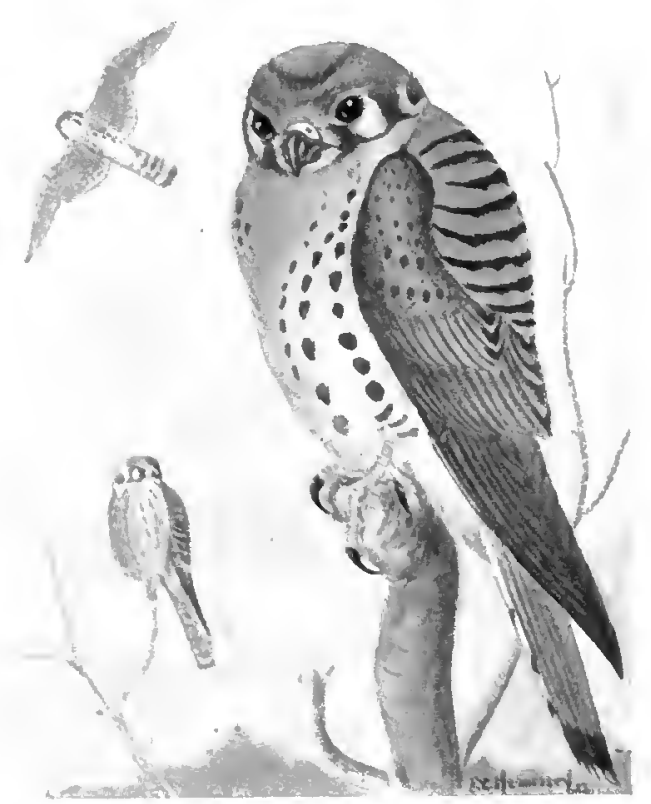

B. Le Faueon épervier (p. 142).

Femelle

Mâle 
Plaxche XV.

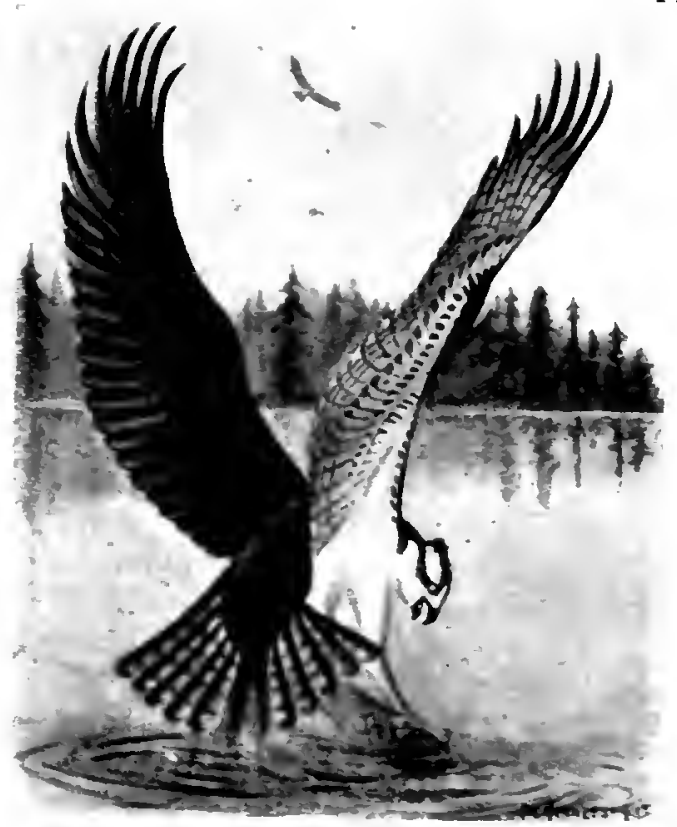

A. Le Balbuzard d'Amérique (p. 143).

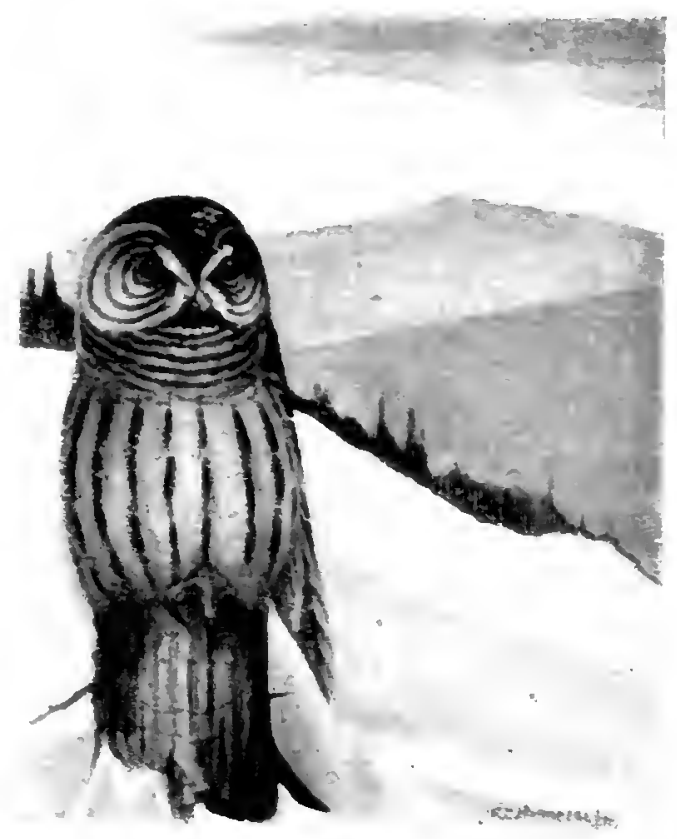

B. La Chouette du Canada (p. 145). 
PlaAche XVI.

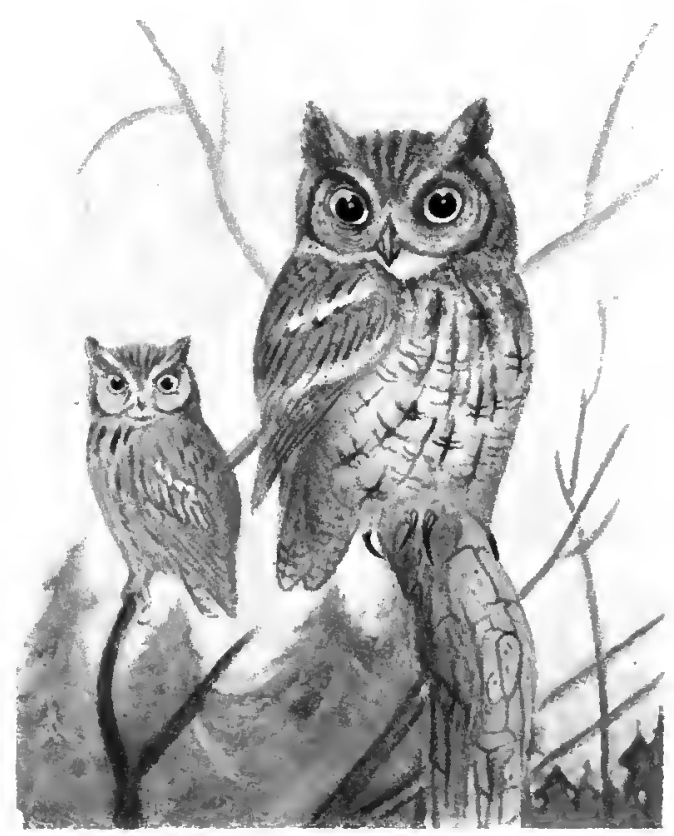

A. Le Hibou maculé (p. 148).

Pliase rouge Phase grise

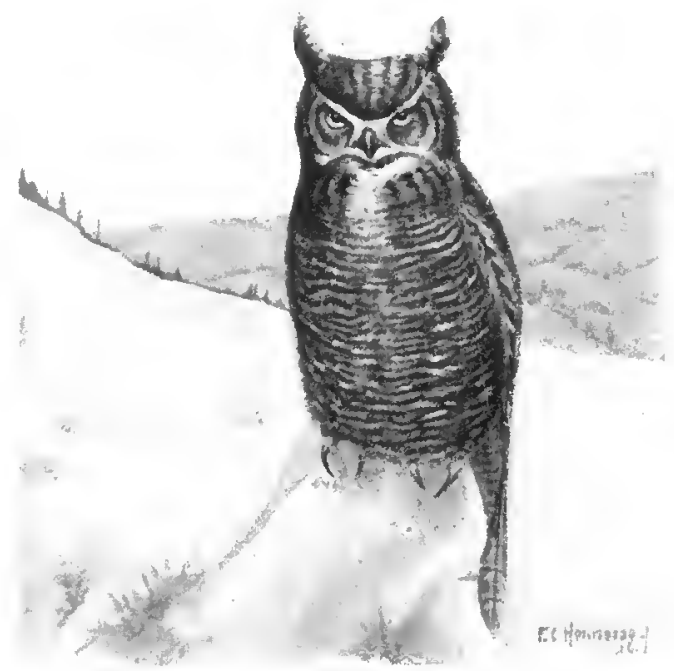

B. Le Duc de Virginie (p. 148). 


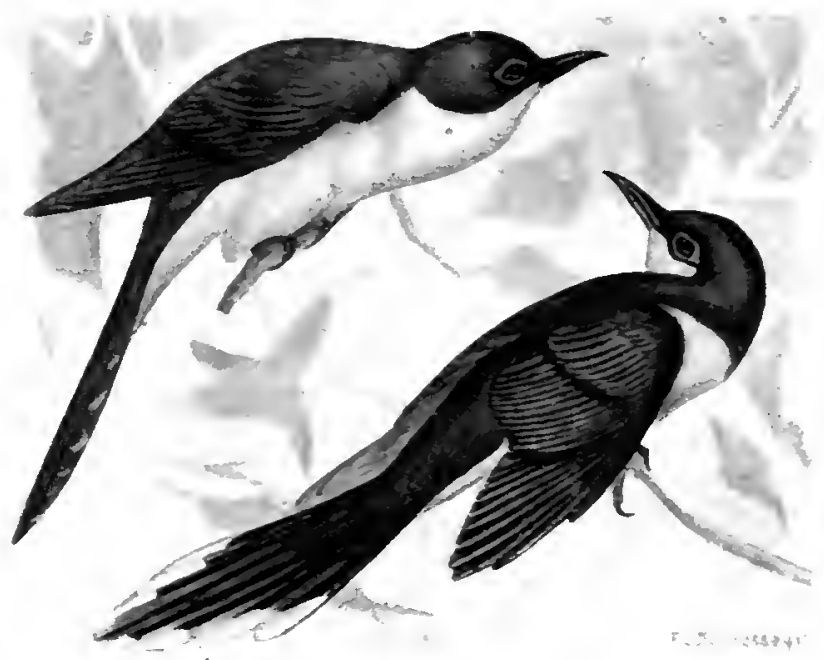

1. Le Concou à bee noir (p, 152).

Le Coucou à bec jaune (p. 151).

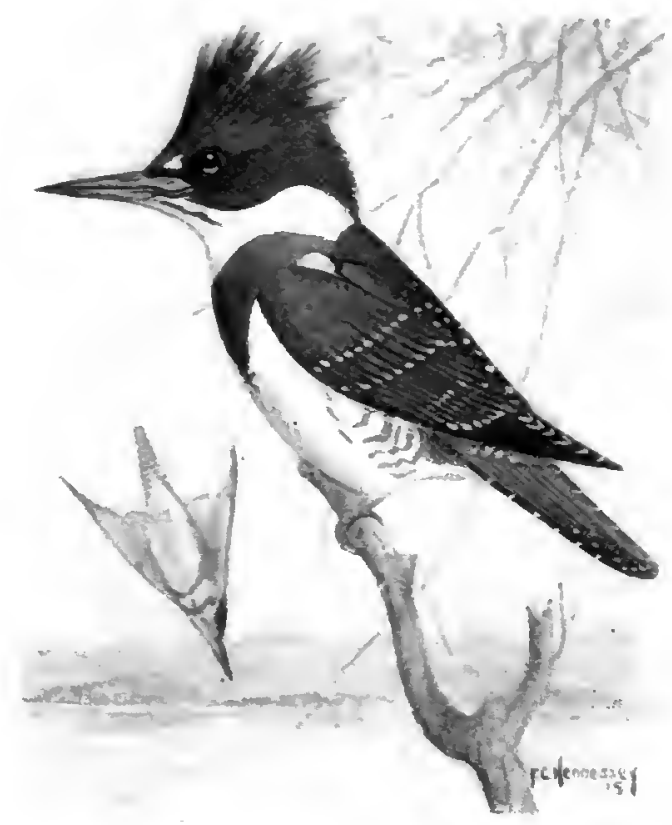

B. Le Mlartin-pêcheur (p. 152),

Femellc

Mâle 
Planche xitil.

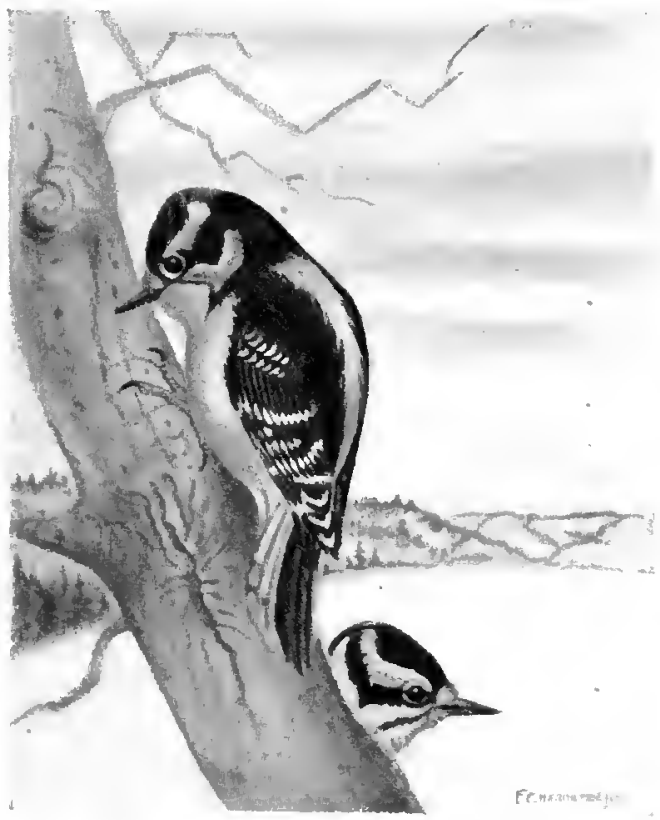

A. Le Pic minule (p. 155).

Mâle Femelle

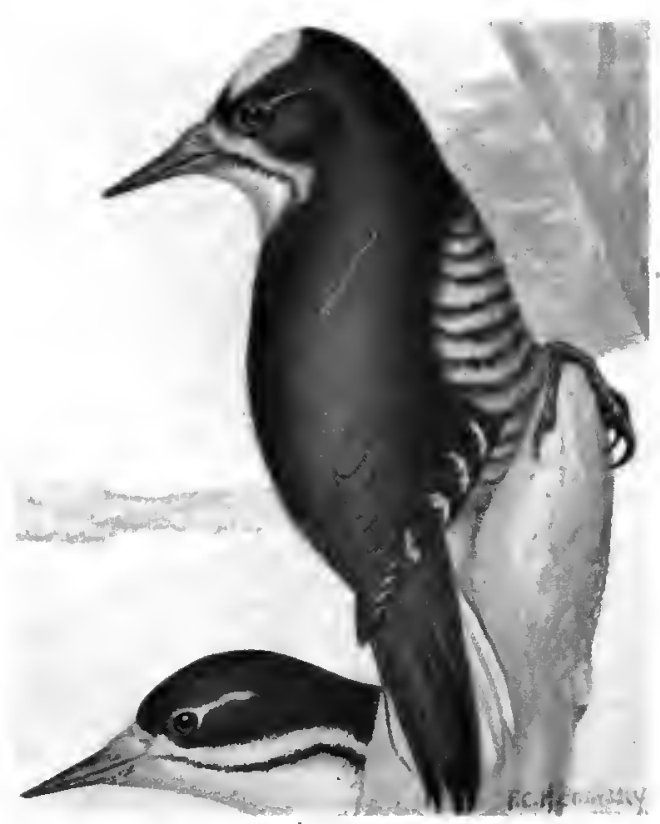

B. Le Pic arctique (p. 155).

Femelle

Mâle 
Pulache Xix.

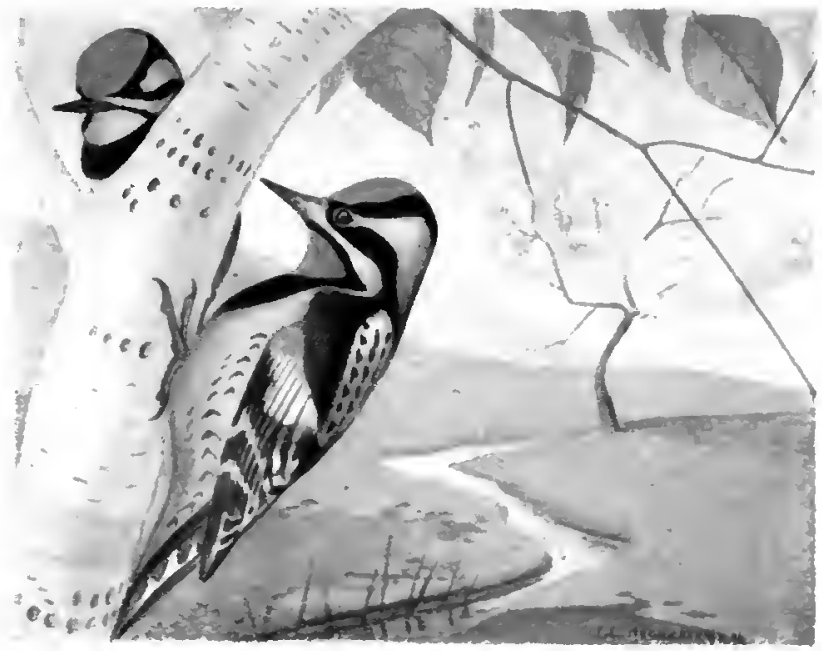

A. Le Pie maculé (p. 156).

Femelle Mâle

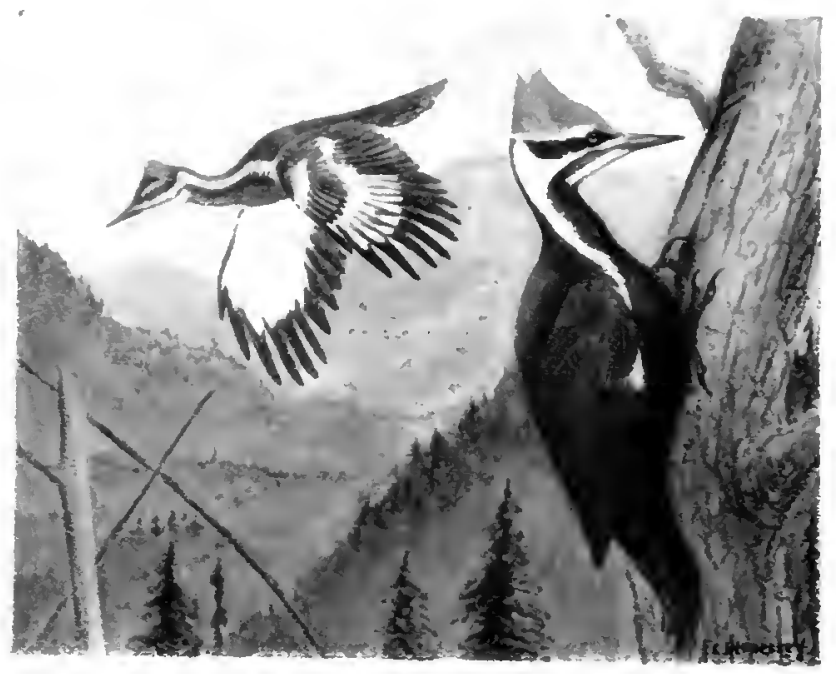

B. Le Pic à huppe écarlate (p. 15̃j). 
Planche XX.

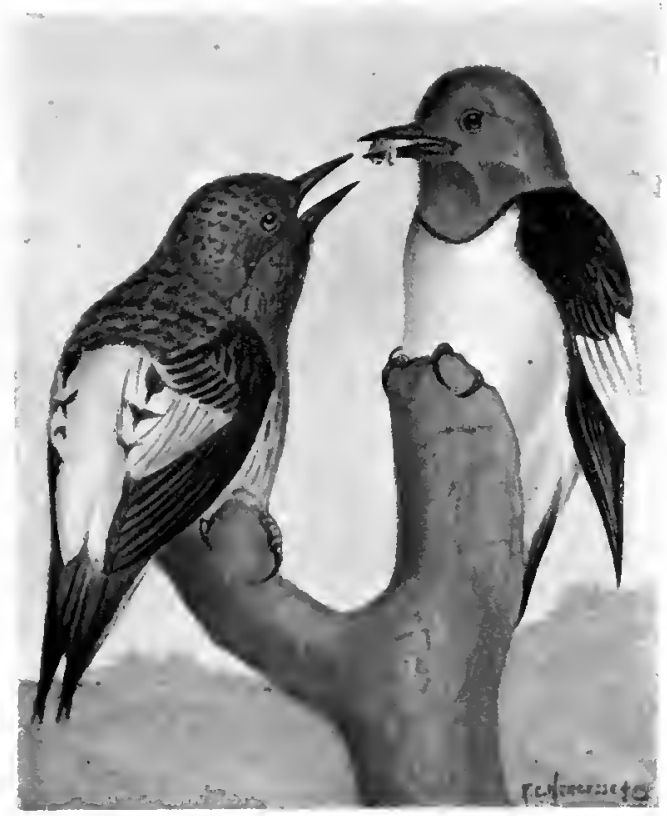

A. Le Pic à tête rouge (p. 158). Jeune Arlulte

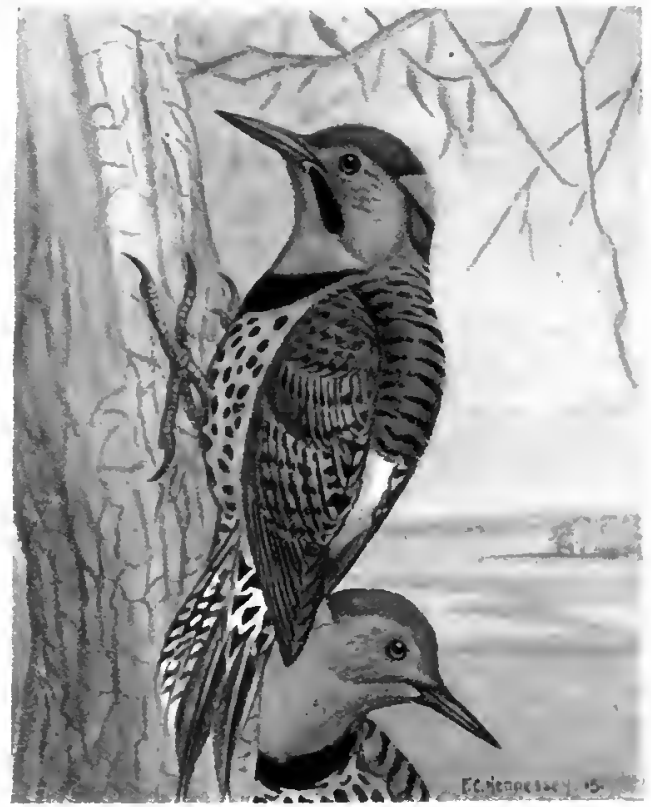

B. Le Pie doré (p. 158). Mâle Femelle 
Plaxche XXI.

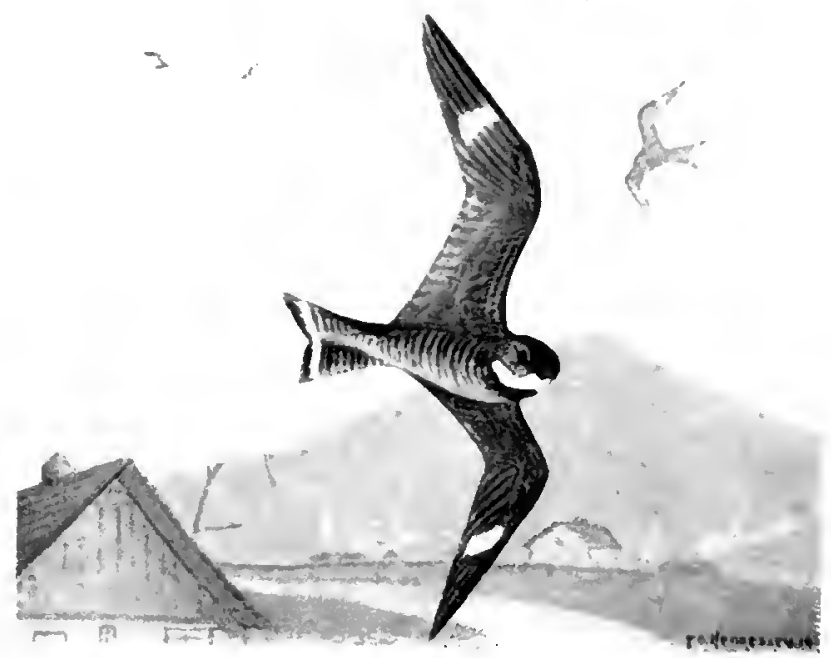

A. L'Engoulevent d'Amérique (p. 16I).

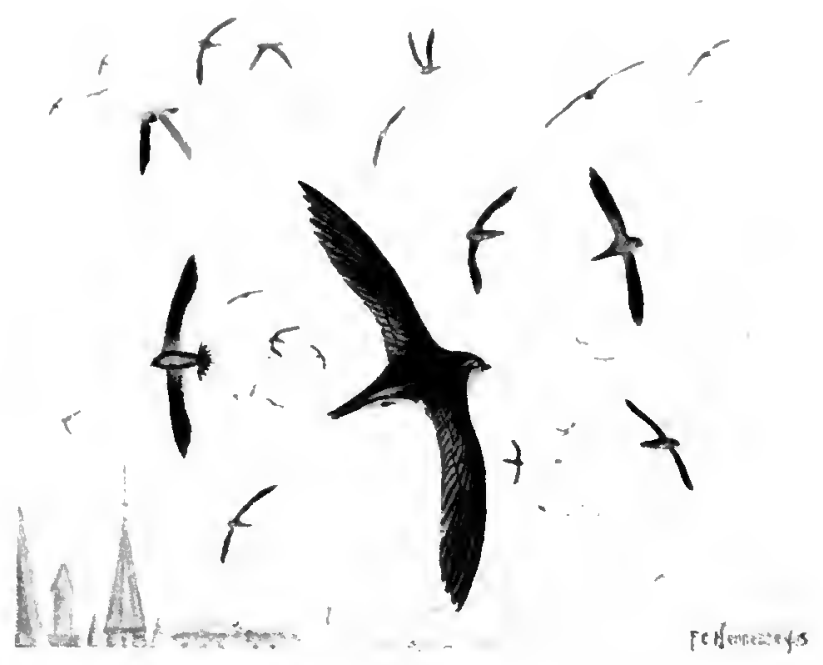

B. Le Martinet des cheminées (p. 162). 


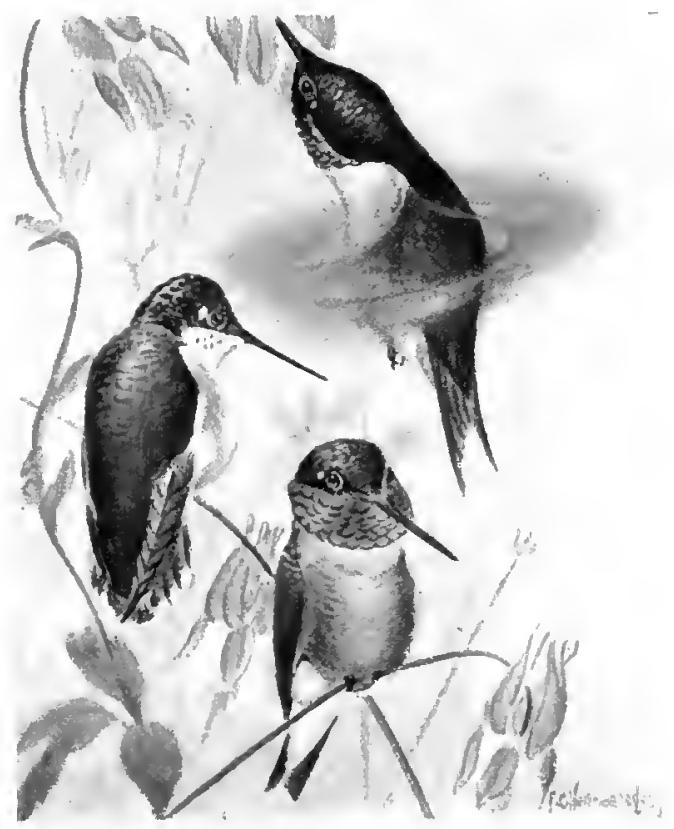

A. Le Colibri à gorge rubis (p. 164). Femelle Mâles

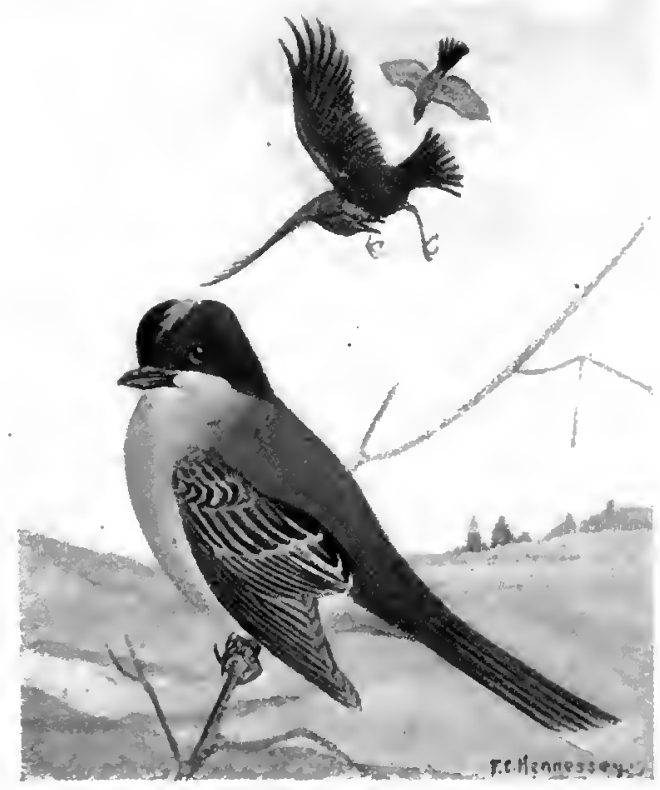

B. Ise Moucherolle de la Caroline (p. 166). 
Plaxche XXIII.

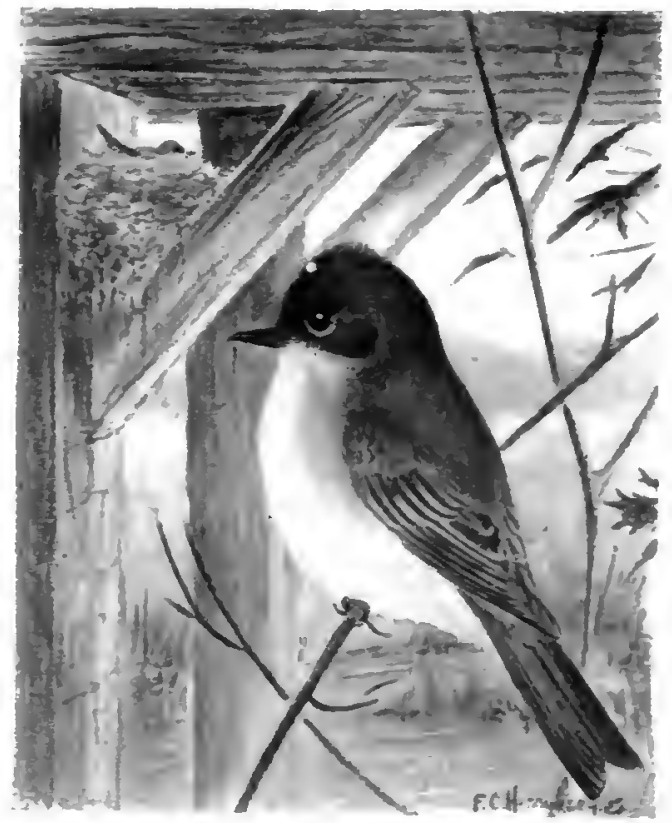

1. Le Moueherolle brun (p. 167).

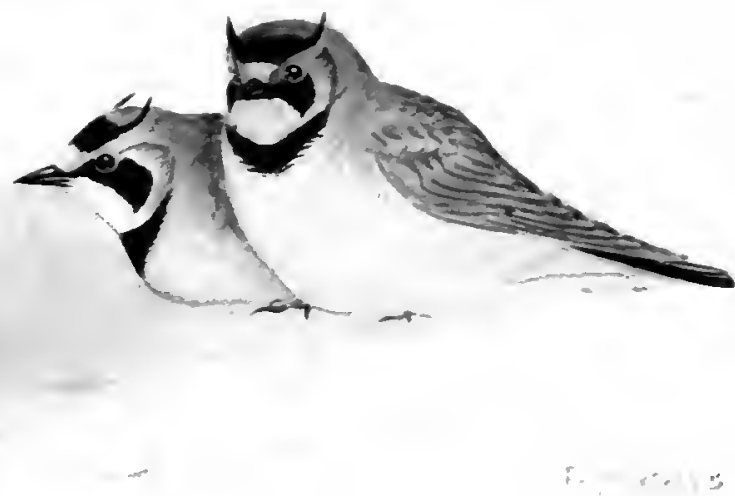

B. L'Alouette ordinaire (p. 170).

LiAlonette ordinaire de l'Est. L'Alouette ordinaire des Prairies. 


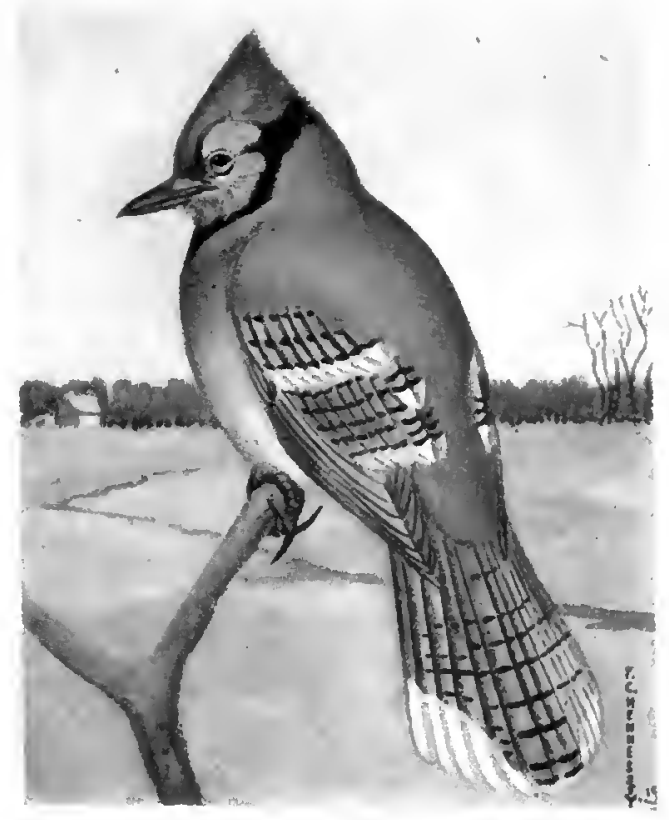

A. Le Geai bleu huppé (p. 172).

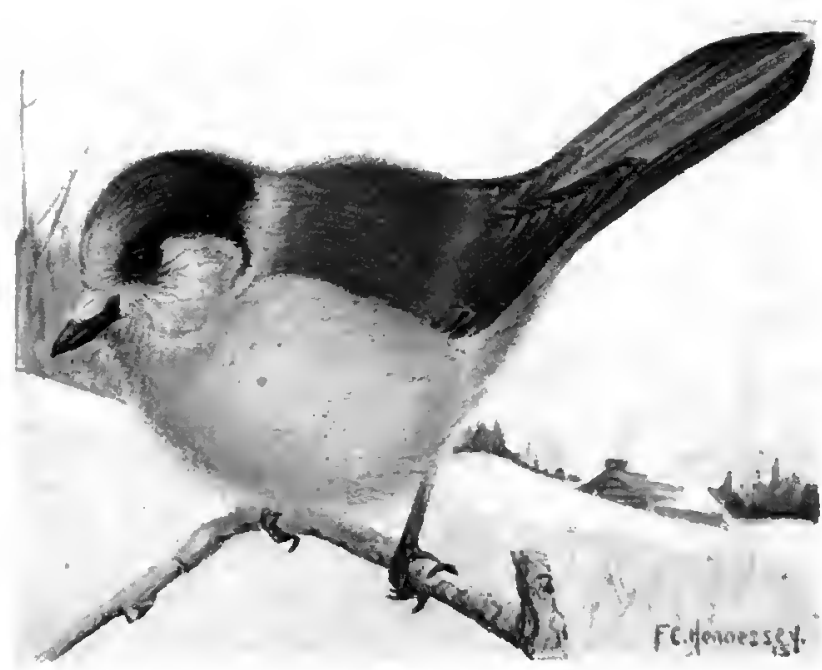

B. Le Geai du Canada (p. 173). 


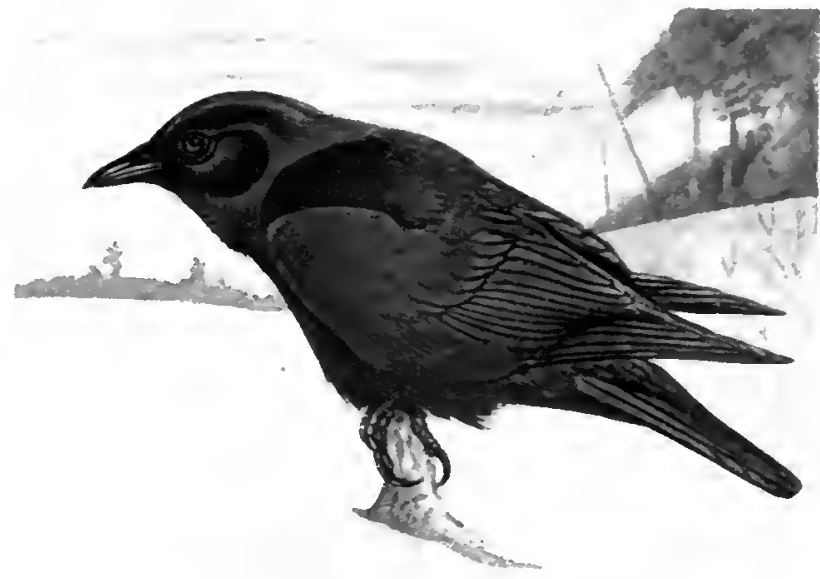

A. La Corneille d'Amérique (p. 174).

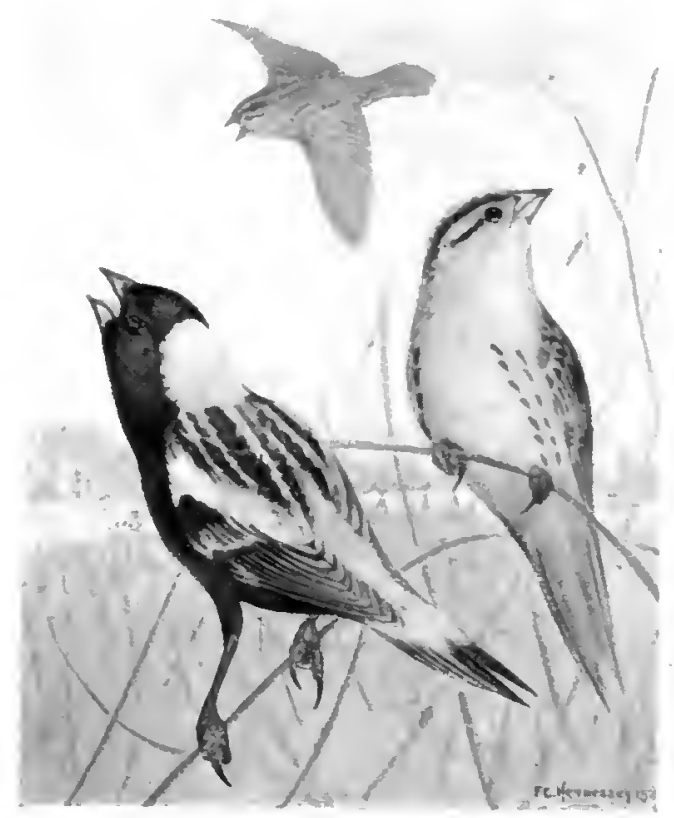

B. Le Goglu (p. 177).
Mâle
Femelle 


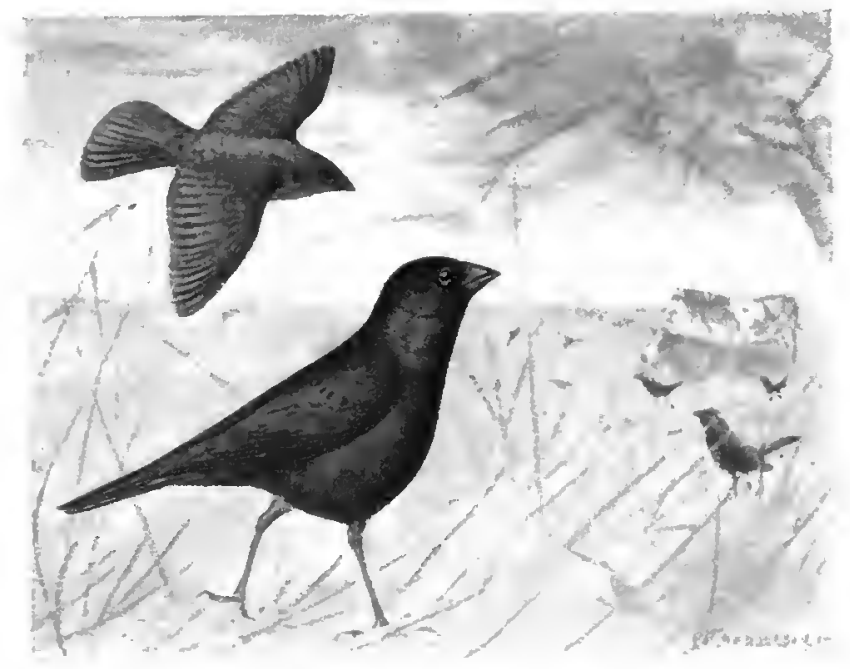

A. L'Thtoumeau ordinaire (p. 175).

Femelle Mî̀le

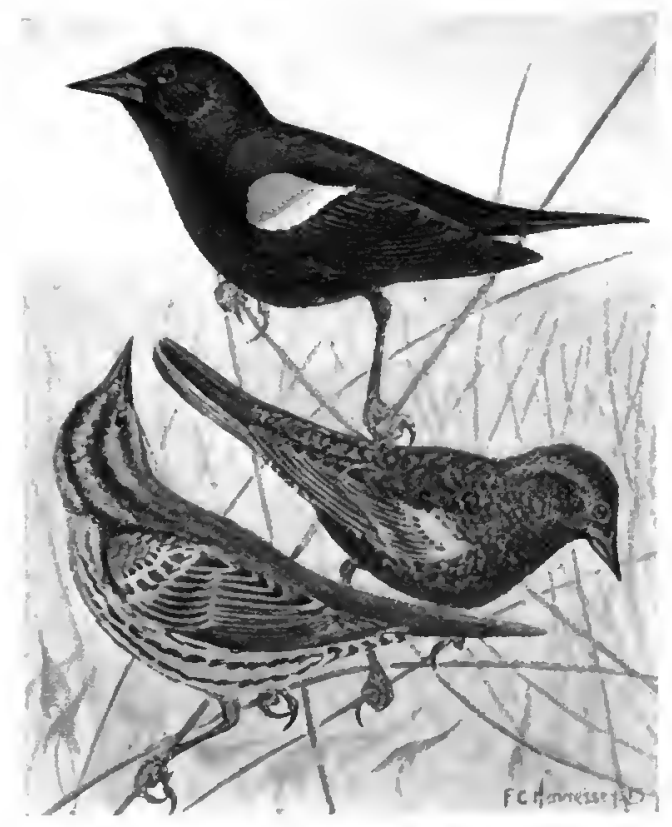

B. L'Étourneau à ailes rouges (p. 179).
Jeune
Mâle
Femelle 


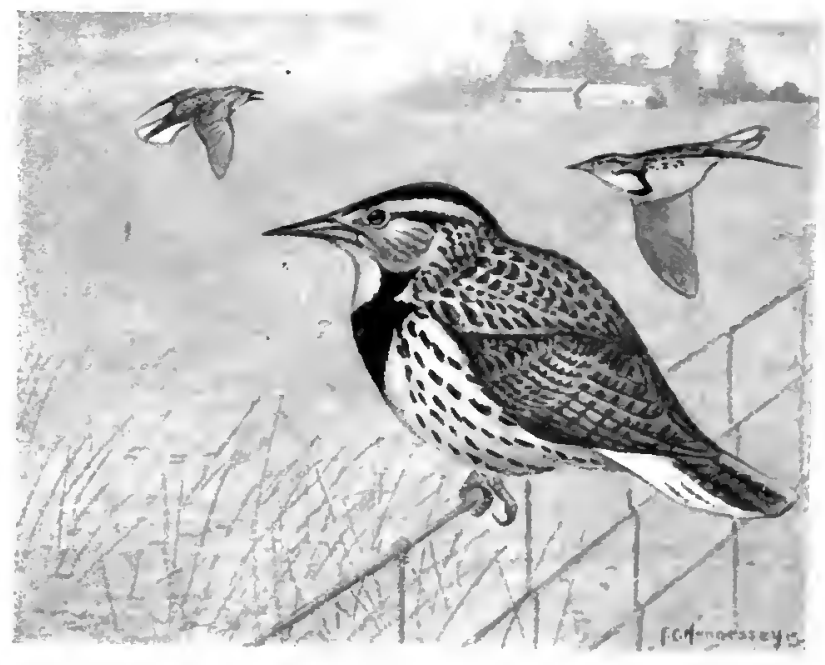

A. L'Étourneau des prés (p. 179).

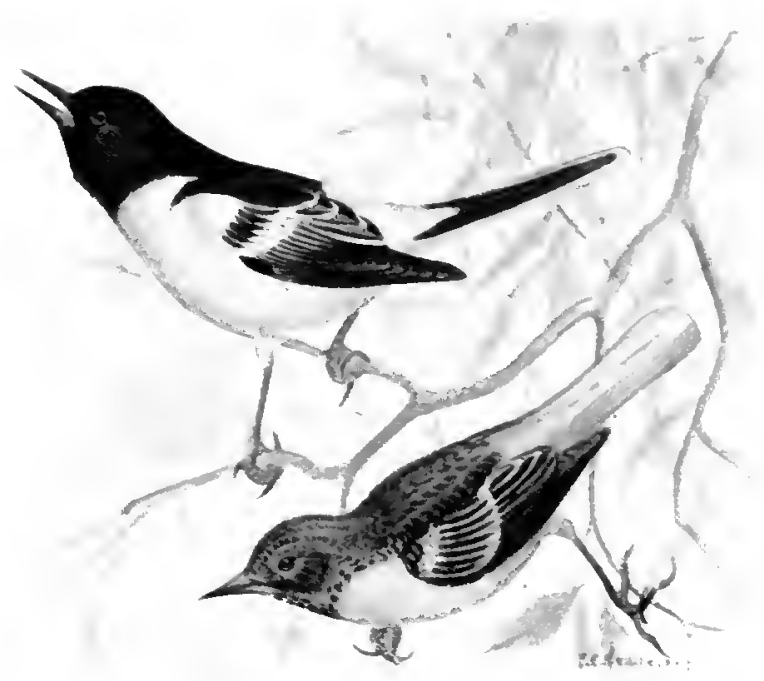

B. Le Loriot de Baltimore (p. 180).
Mâle
Femelle 


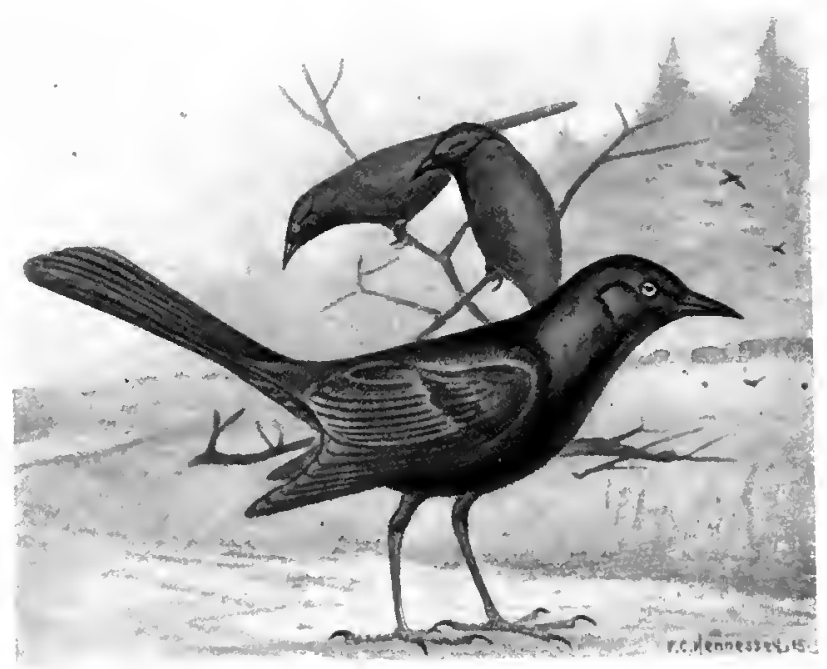

A. Le Mainate bronzé (p. IS1).

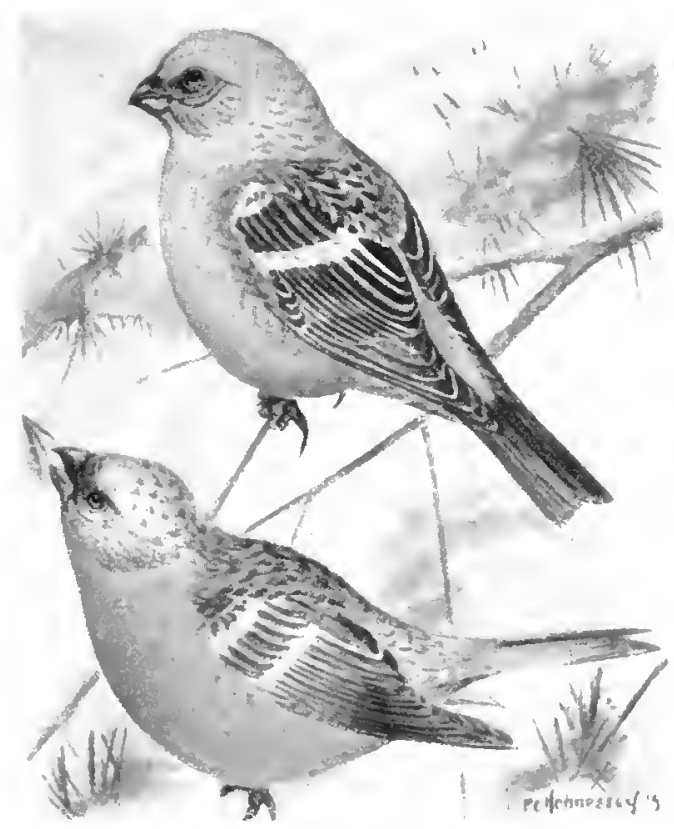

13. Le Gros-bee des pins (p. 184). Femelle Mâle 
Plaxche XXIX.

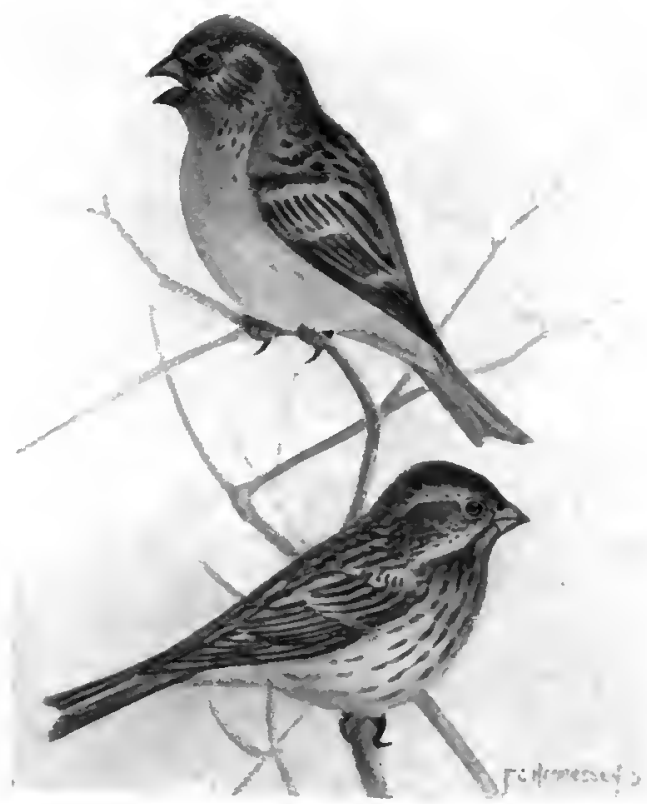

A. I.e Pinson pourpré (p. 184).

Mâle

Femelle

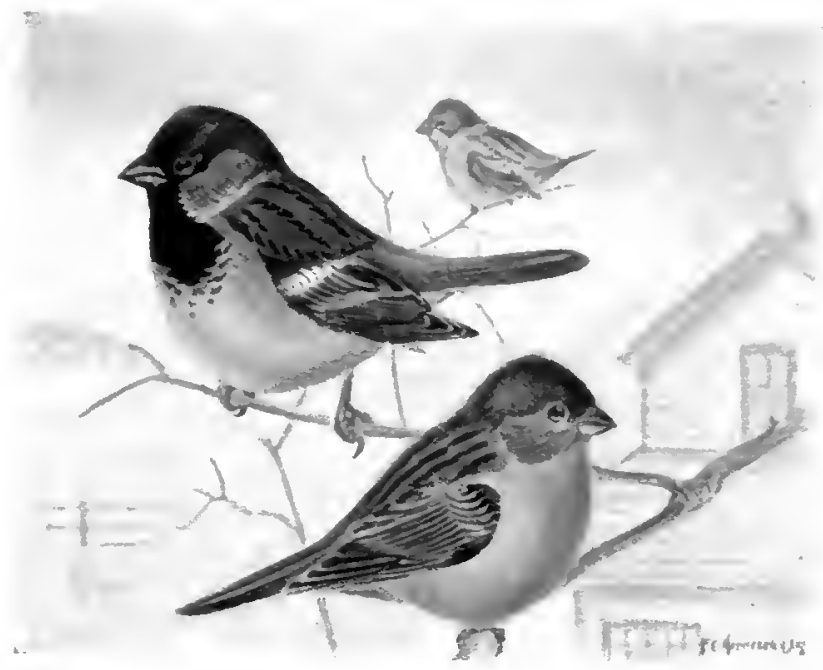

B. Le Moineau domestique (p. 185).

IIâle

Femelle 


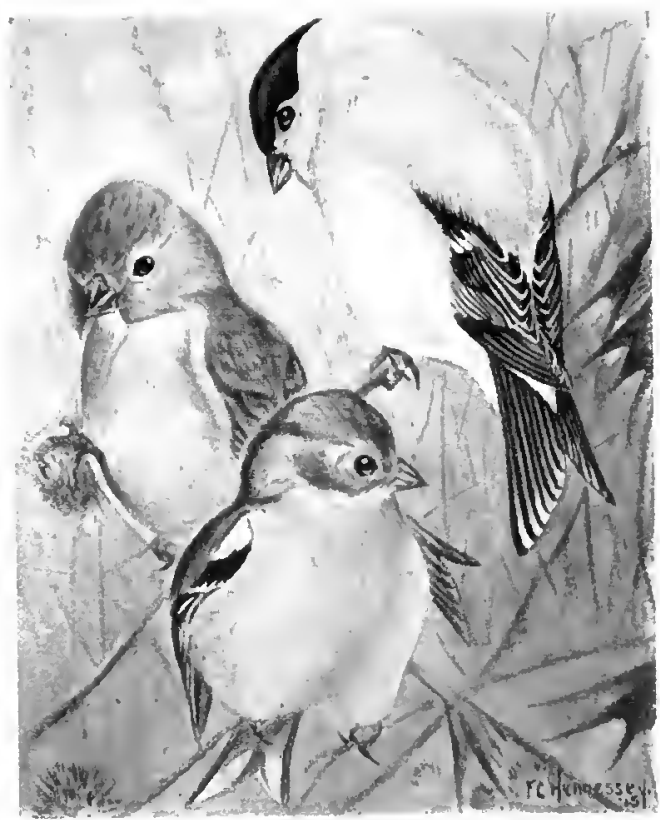

A. Le Chardonneret jaune (p, 190).
Jeune
Femelle
Iâle

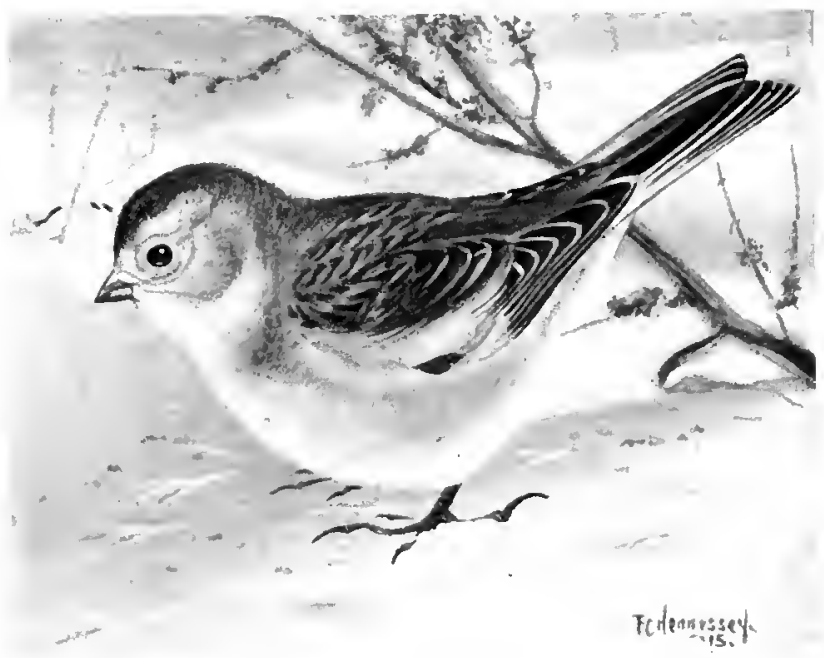

B. Le Plectrophane des neiges (p, 19I). 
Plaxche XXXI.

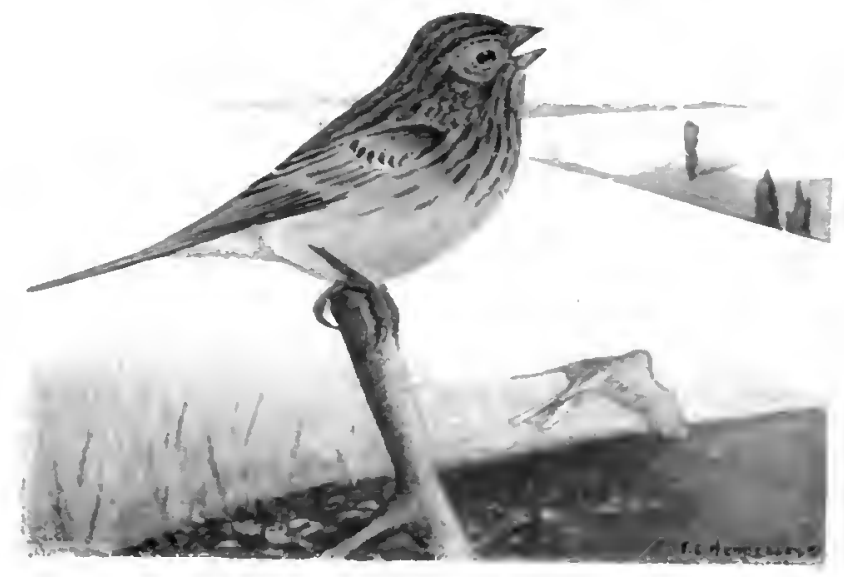

A. Le P'inson à ailes baies (p. 1921.

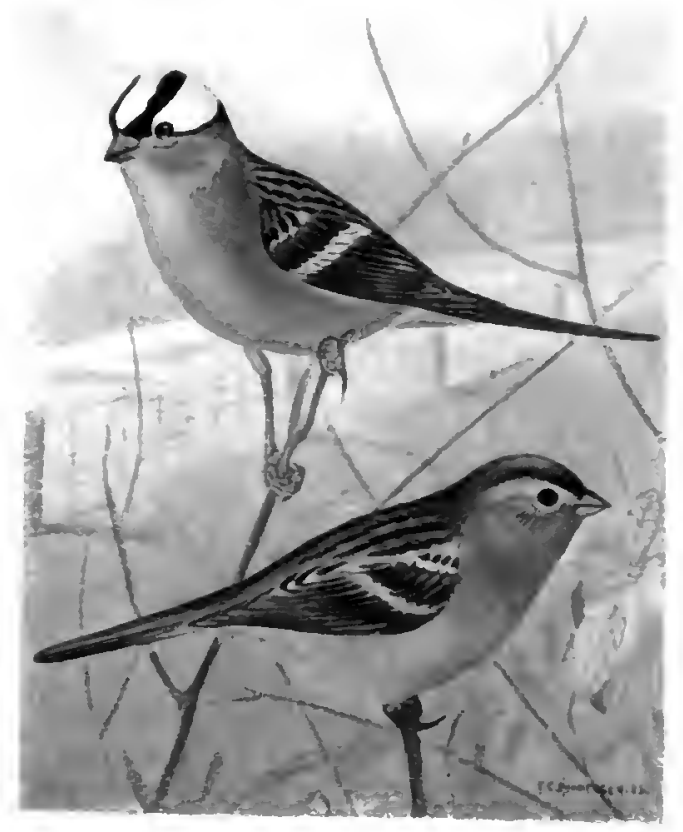

B. Le Pinson à courome blanche (p. 196).

Aclulte Jeune 


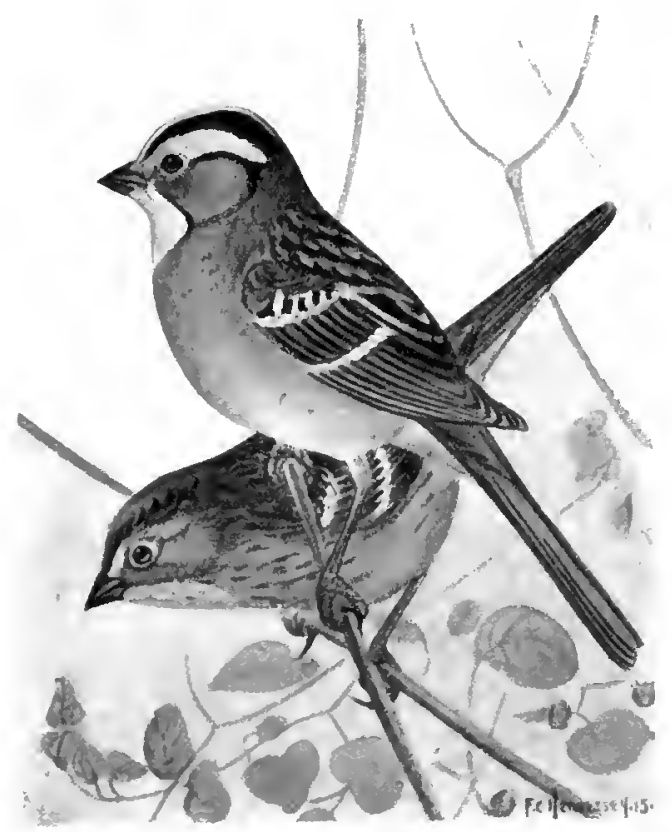

A. Le Pinson à gorge blanehe (p, 196).

Adulte

Jeune

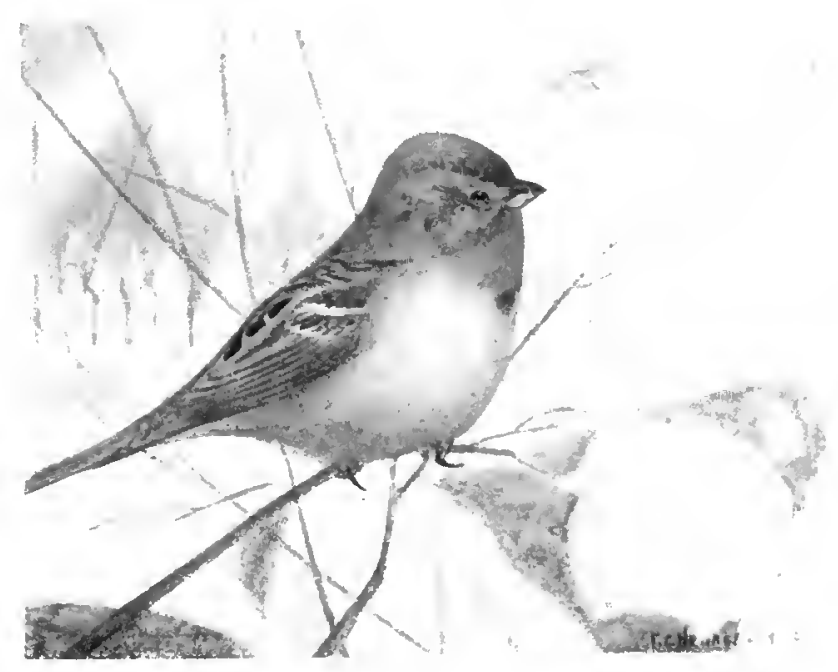

B. Le Pinson de montagne (p. 197). 


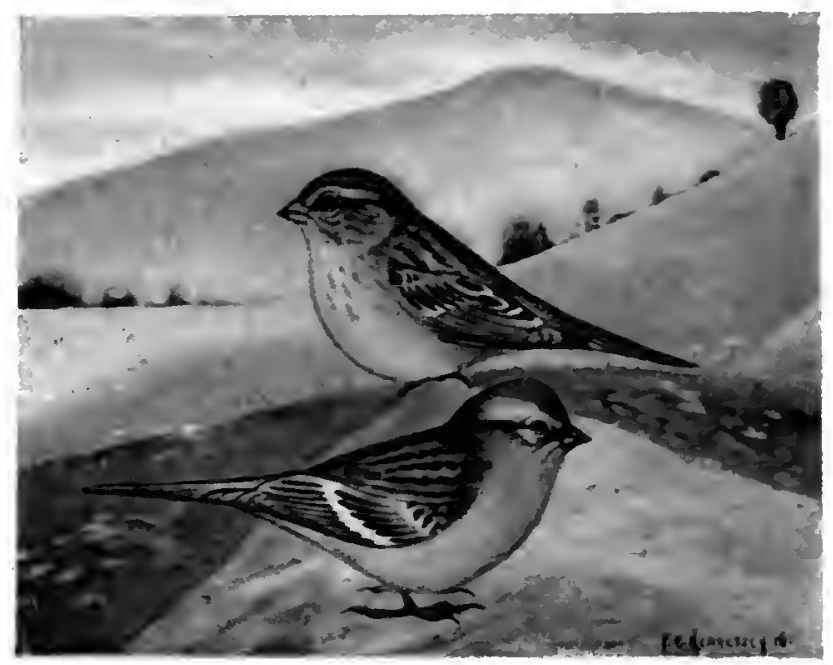

A. Le Petit Pinson à couronne rousse (p. 197).

Jeune

Adulte

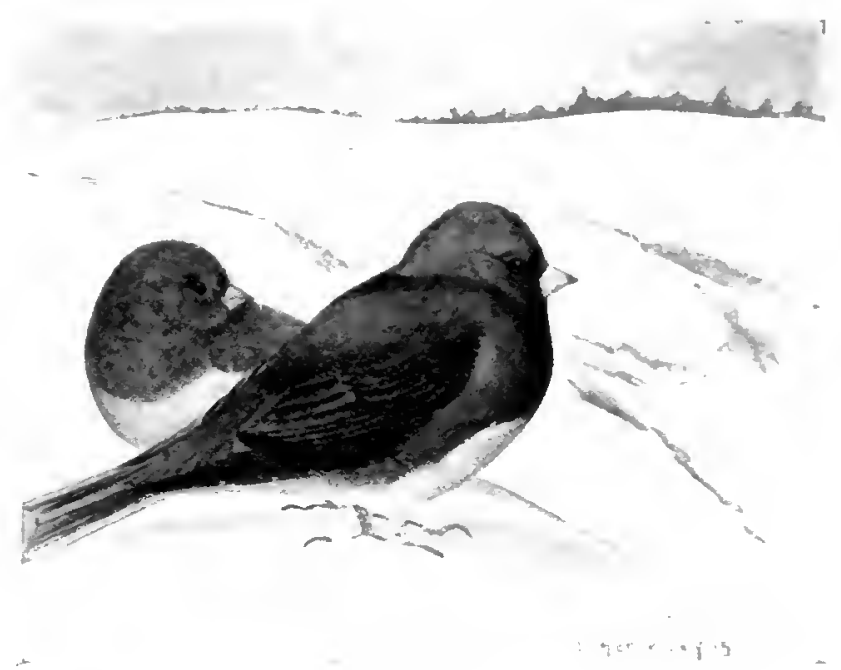

B. Le Pinson niverolle (p. 199).

Femelle Mâle 


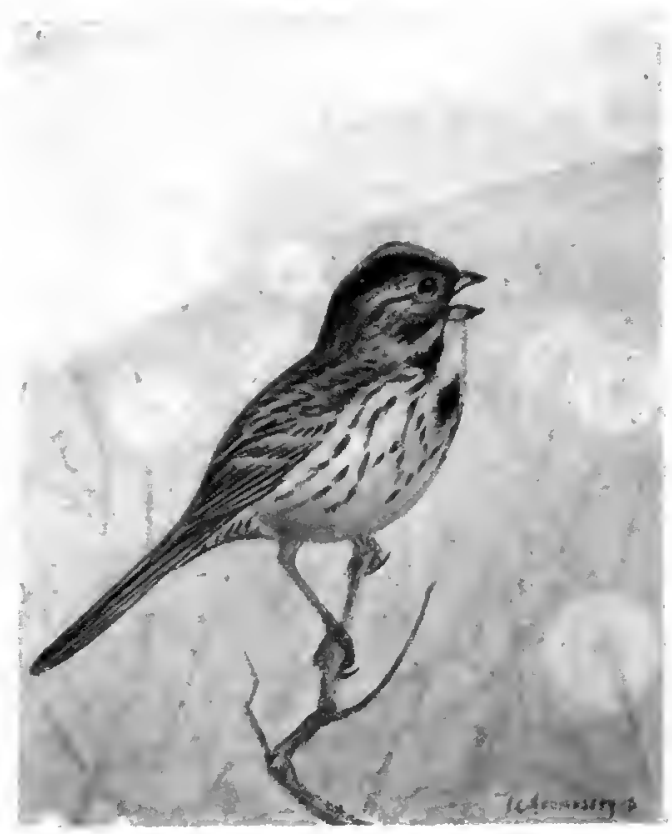

A. Le Pinson chanteur (p. 199).

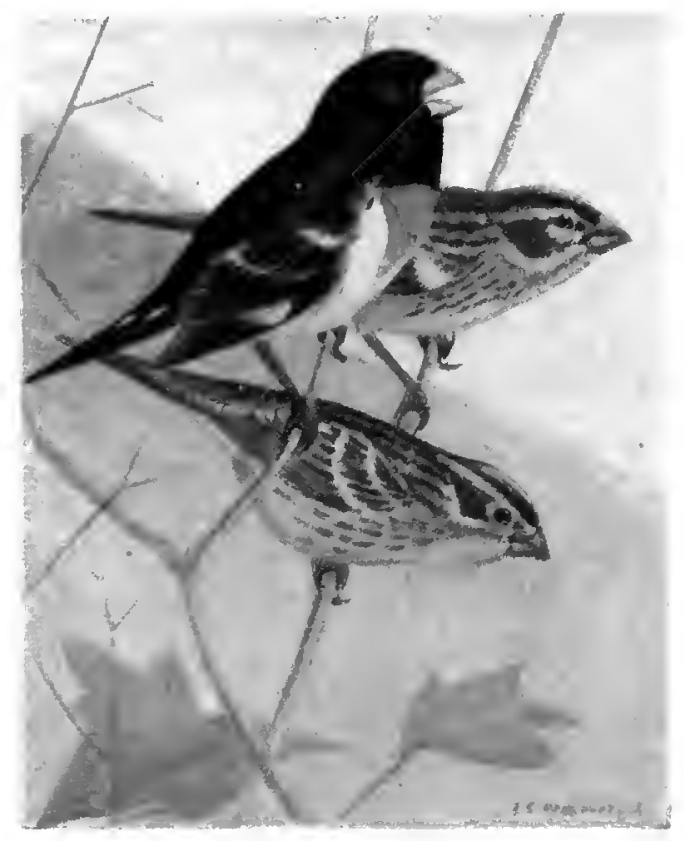

13. Le Gros-bec à poitrine rose $(p, 203)$. Mâle Femelles 


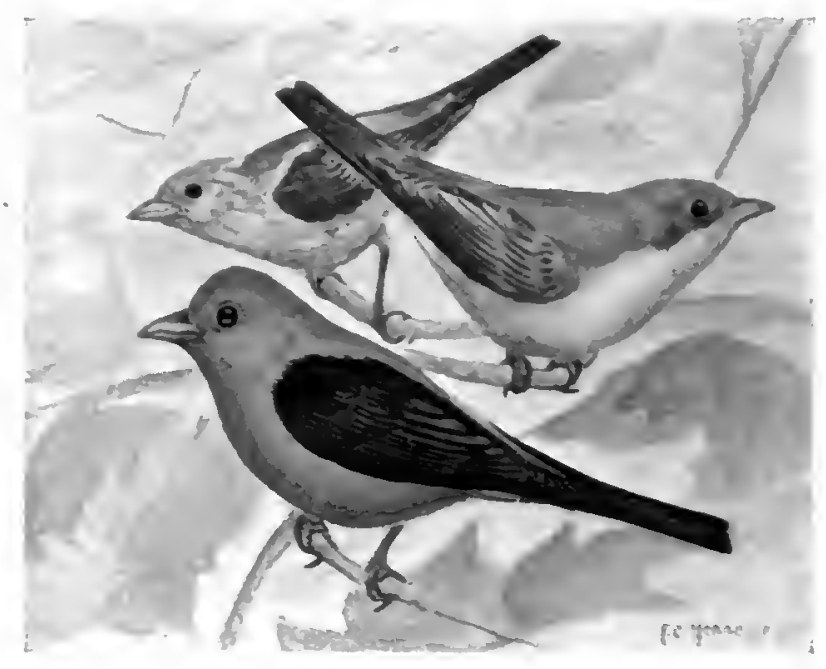

A. Le Tangara éearlate (p. 205).

Le mâle change son plumage à l'automne Mâle Femelle

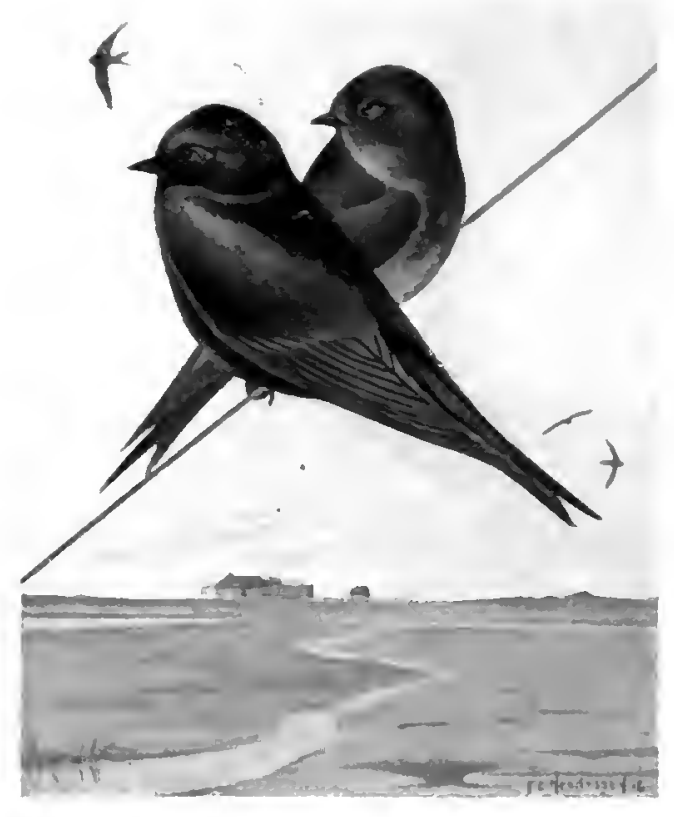

“B. L'Hirondelle pourprée (p. 207).

Mâle Jeune 
Plaxche AXXVi.

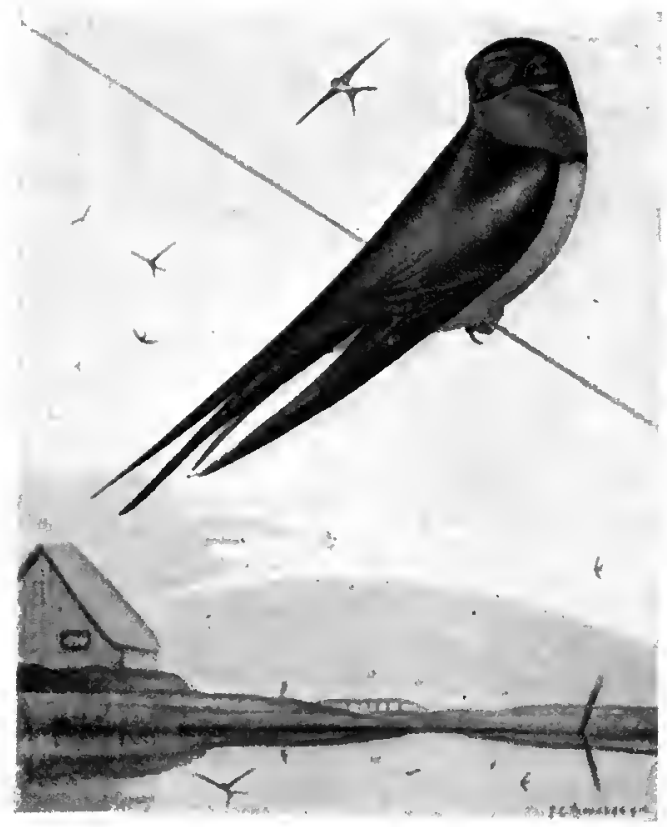

A. I.Hirondelle des granges (p. 208).

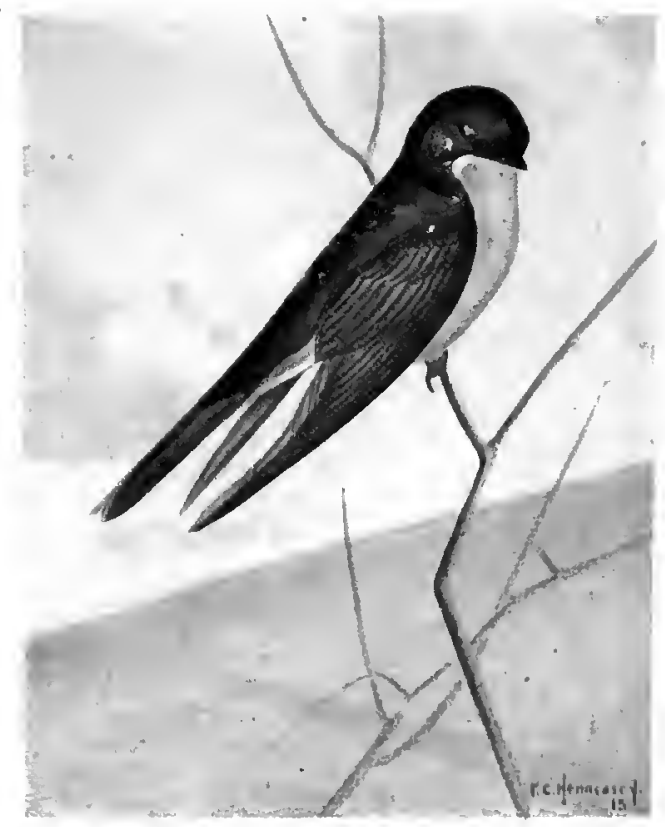

B. L'Ilirondelle bicolore (p. 209), 
Planche Mxyil.

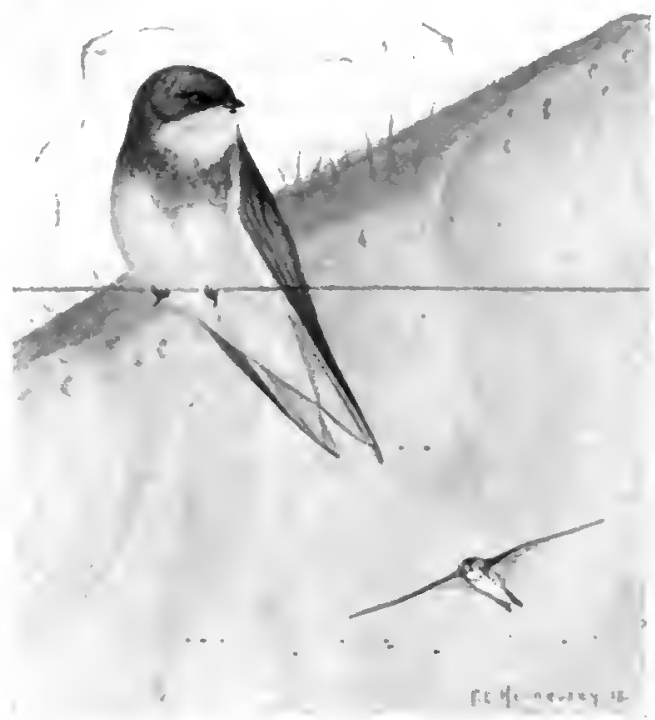

A. L'Hirondclle de rivage (p. 209).

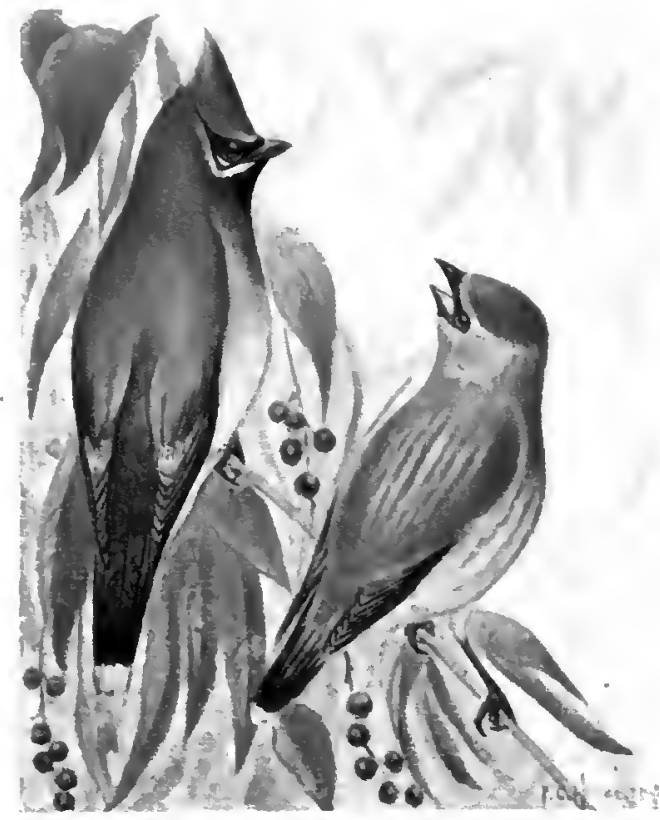

B. Le Jaseur du cèdre (p. 210).
Adulte
Jeune 


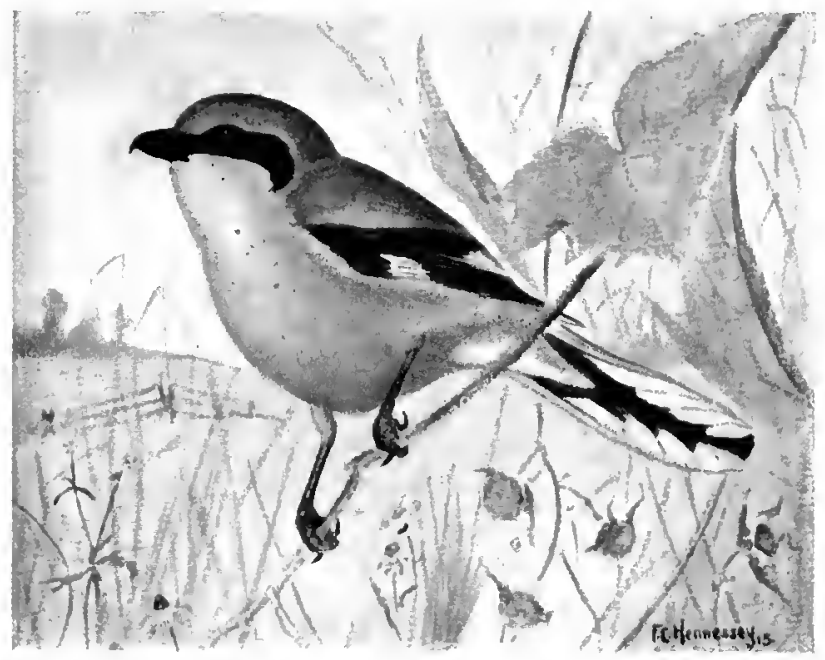

A. La Pie-grièche à eroupion blane (p. 212).

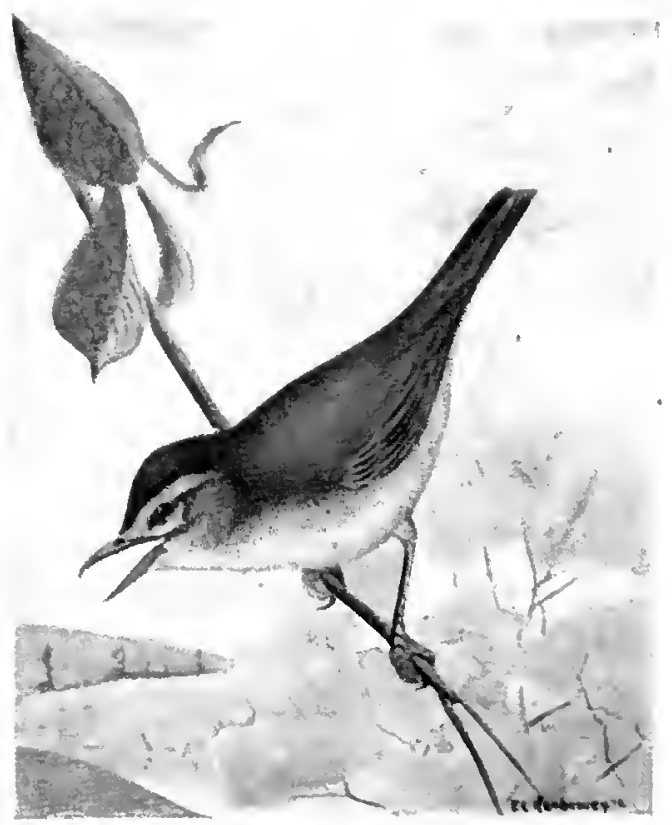

B. Le Vireo aux yeux rouges (p. 214). 
Plaxche xxxix.

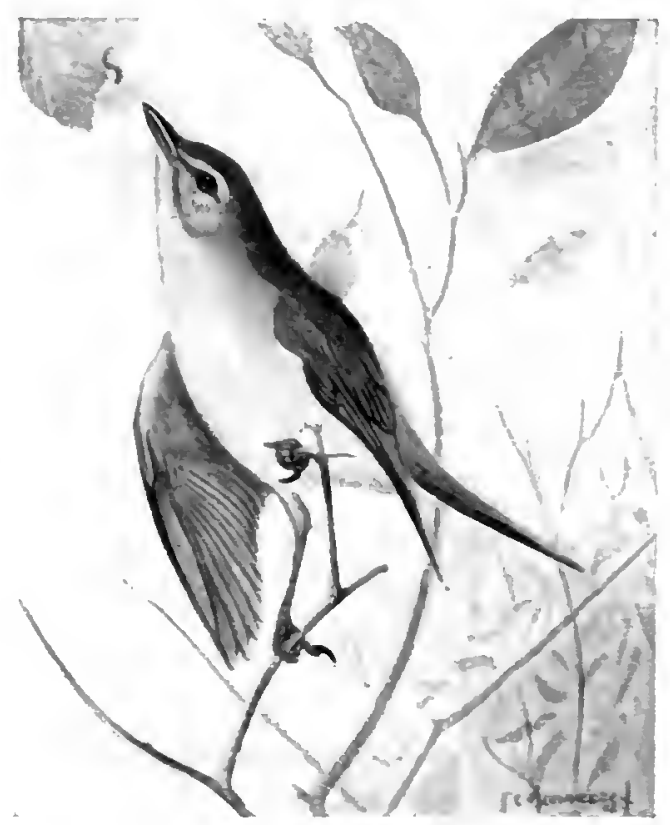

A. Le Vireo gris olive (p. 215).

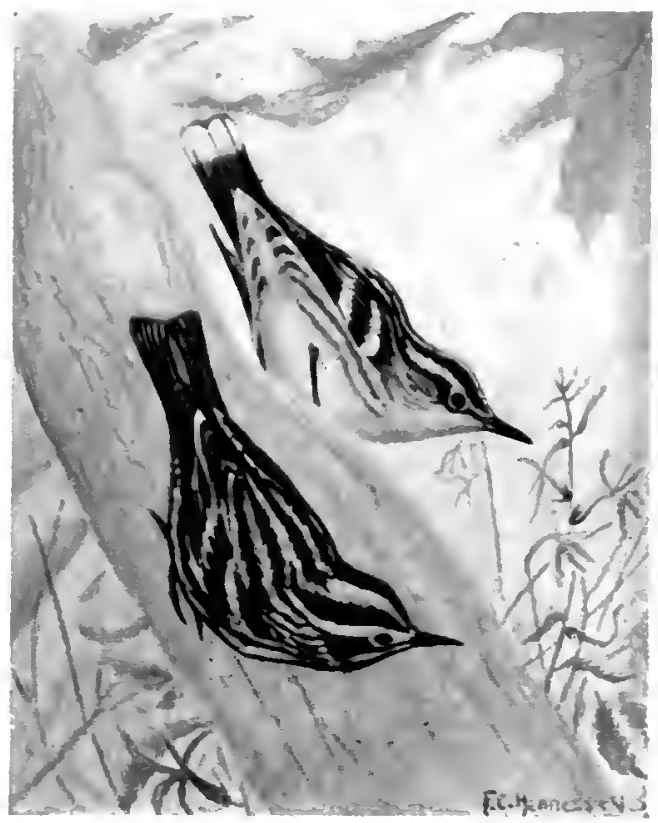

B. La Faurette noire et blanehe (p. 21s).

Plumage du printemps Plumage d'automne 


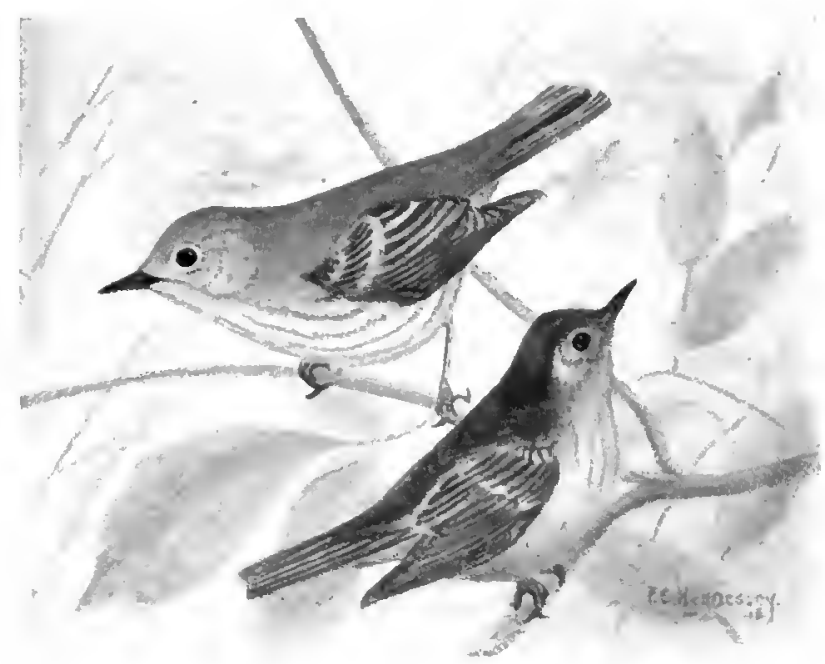

A. 1a Faurette jaune (p. 2222).

Mâle remelle

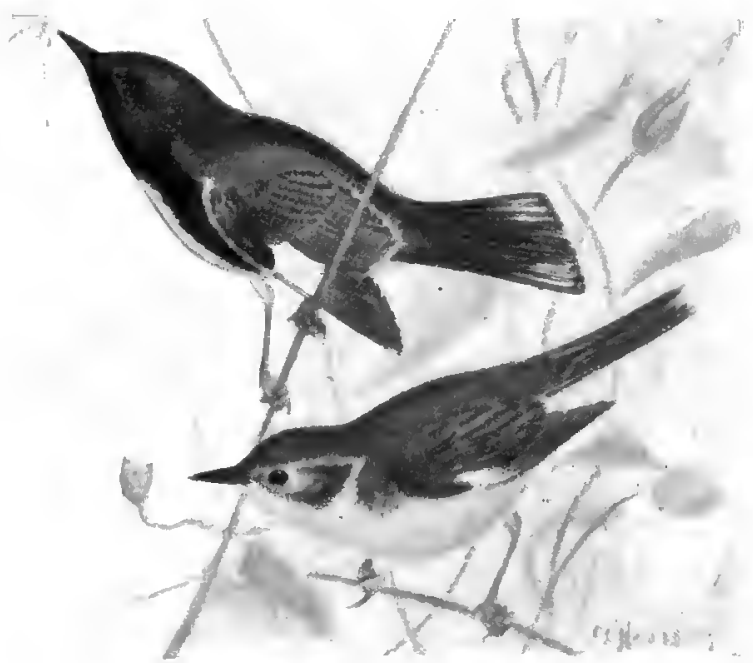

B. La frauvette bleue à gorge noire (p, 222),

Mâle

Femelle 
Plaxche Xli.

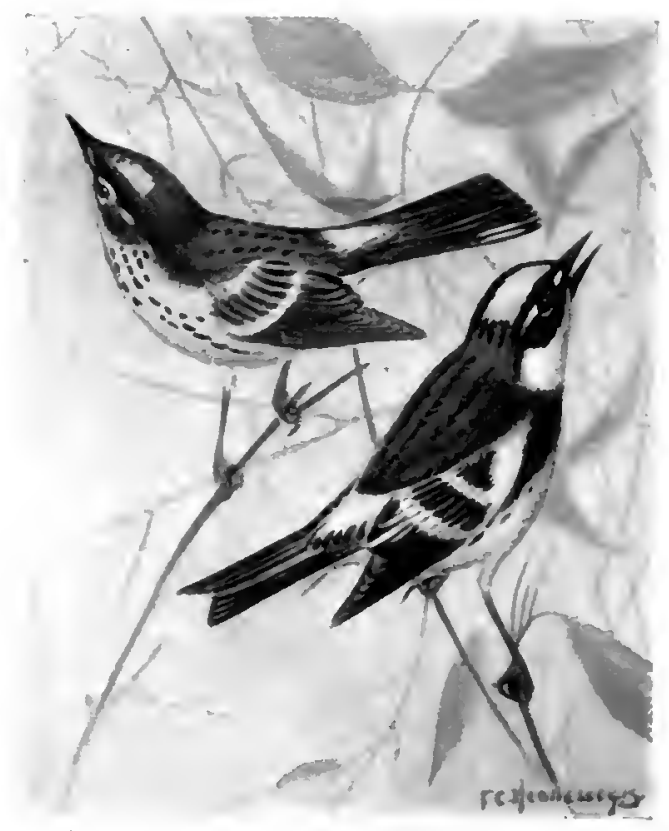

A. La Fauvette ì croupion jaune (p. 222).

Femelle Iâle

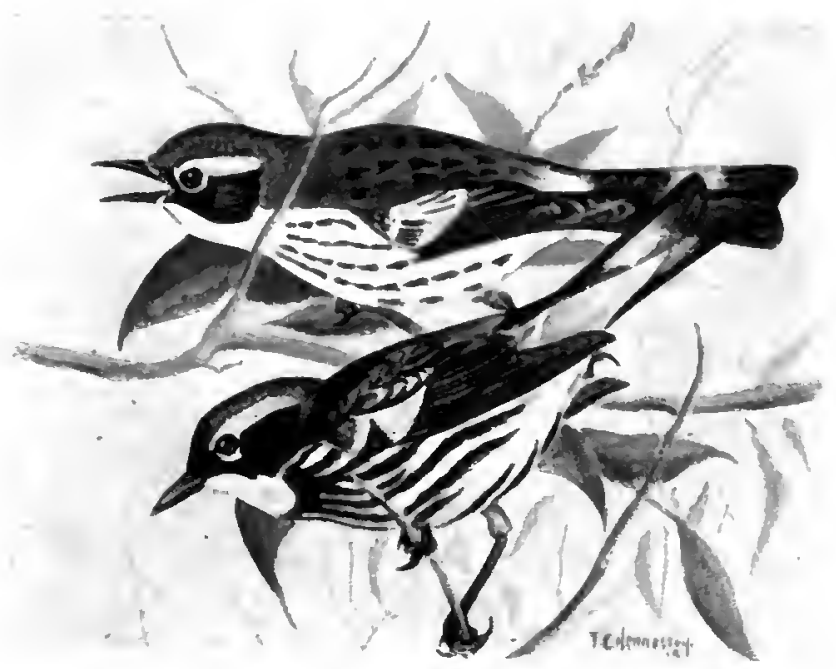

B. La Fauvette à tête cendrée (p. 223).
Femelle
Mâle 。 


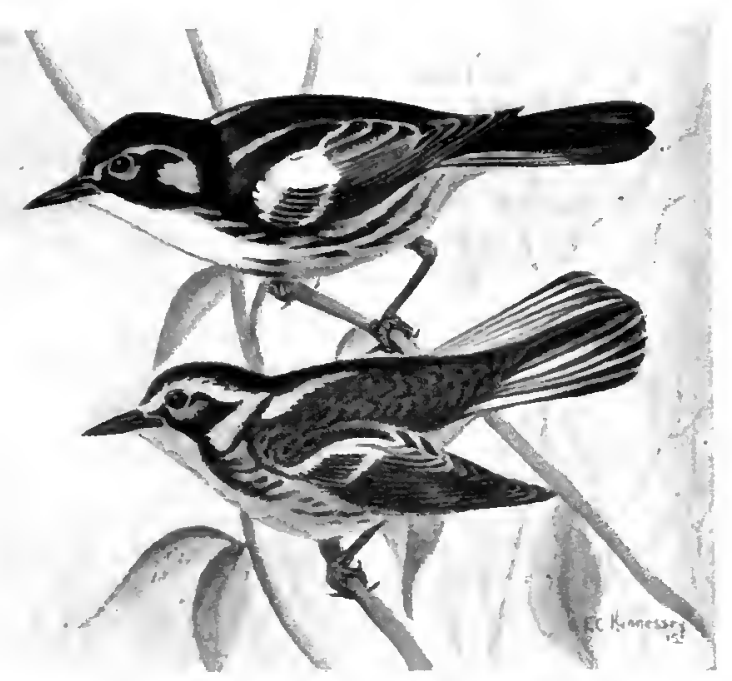

A. La Fauvette de Blackburn (p. 225).

Mâle Femelle

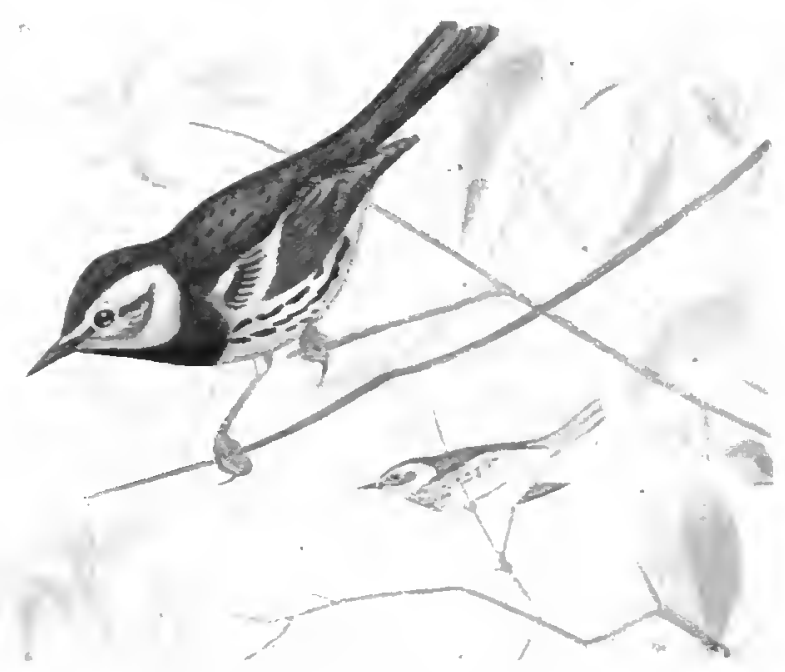

B. La Fauvette à poitrine noire (p. 225).

- Mâle Femelle 


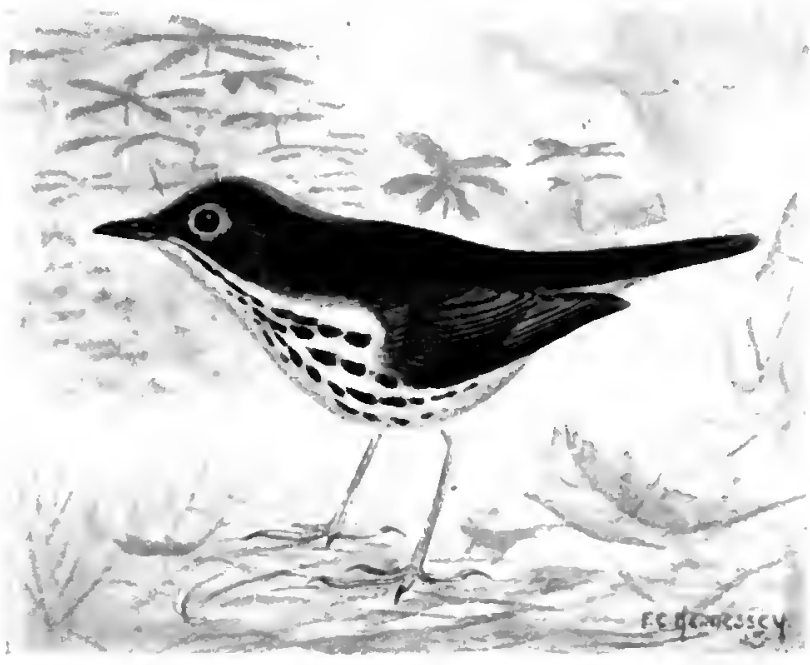

A. Lil Grive couronné (p. 22s).

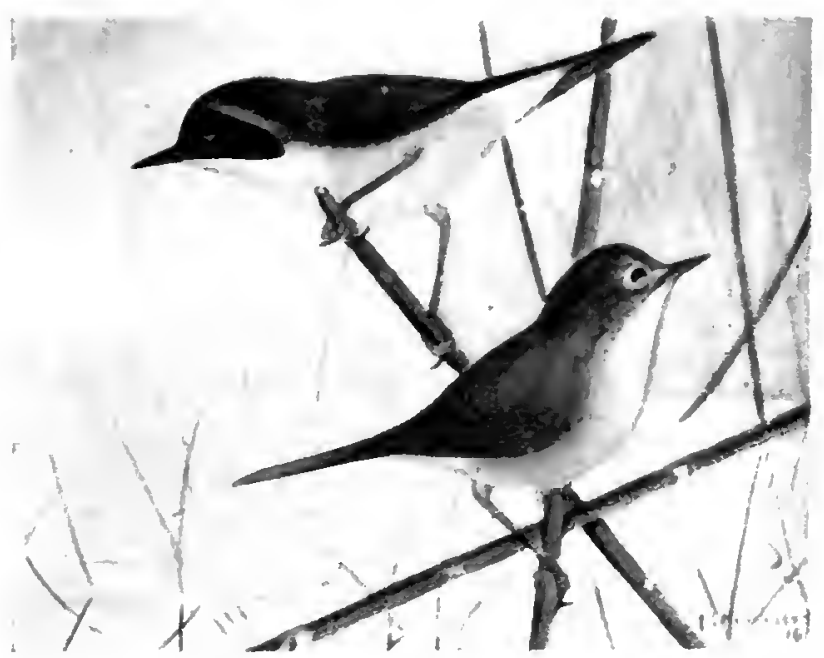

B. La Fauvette trichas du Nord (p. 231).

Mâle

Femelle 
Plaxche Xlit.

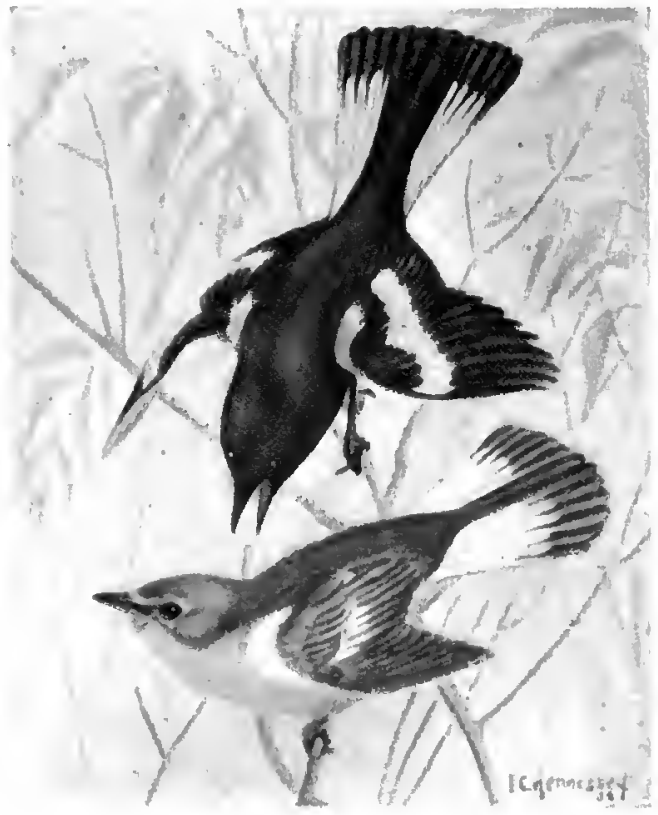

1. I Fanvette à queue ronsse (p. 232).

Mâle

Femelle

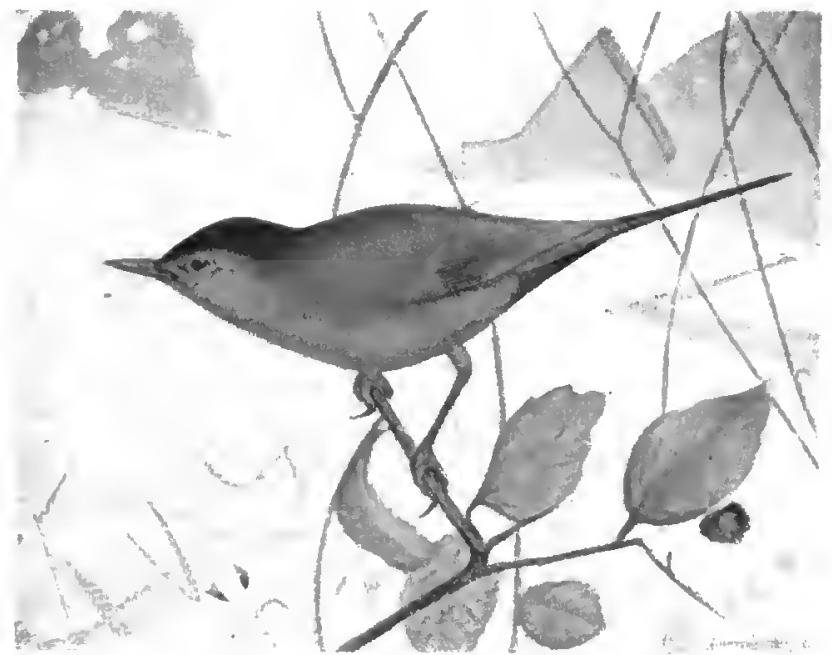

B. La Grive de la Caroline (p. 234) 
Plaxche Xlu.

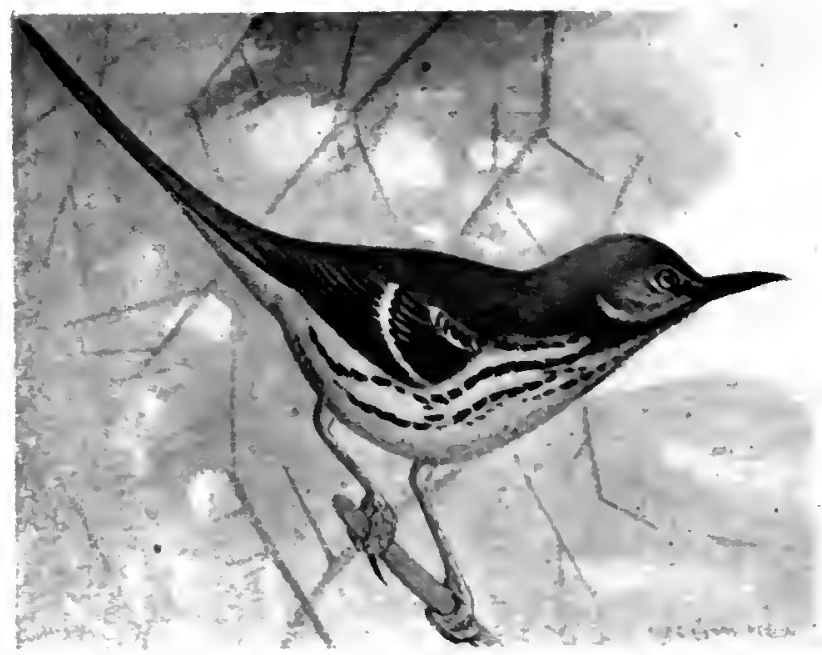

A. La Grive rousse (p. 235).

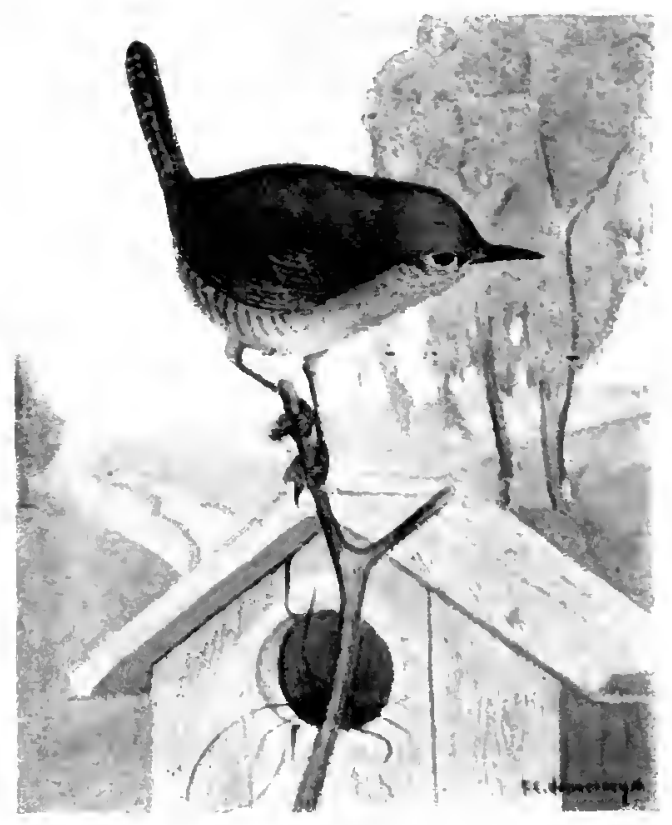

B. Le Troglodyte aédon (p. 237). 


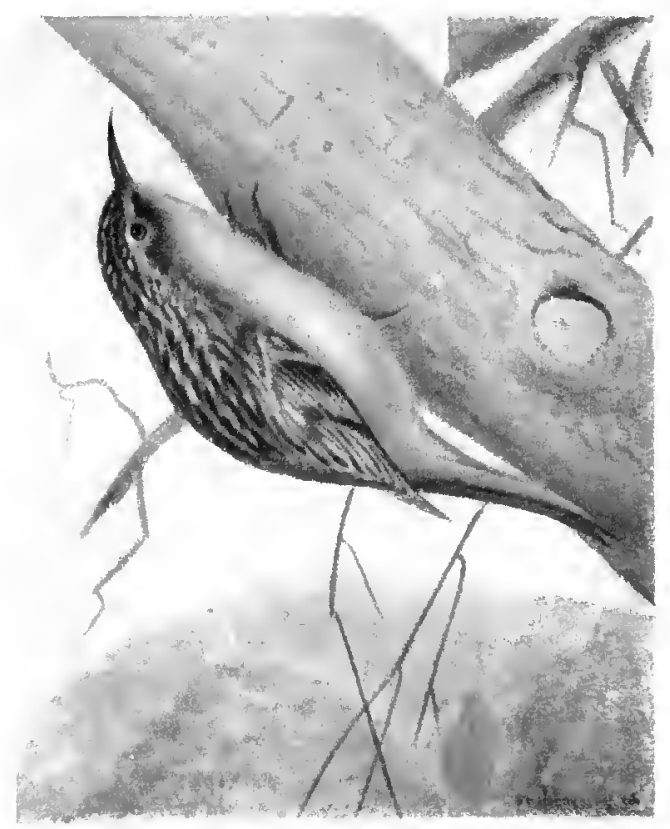

A. Le Grimpereau d'Amérique (p. 239).

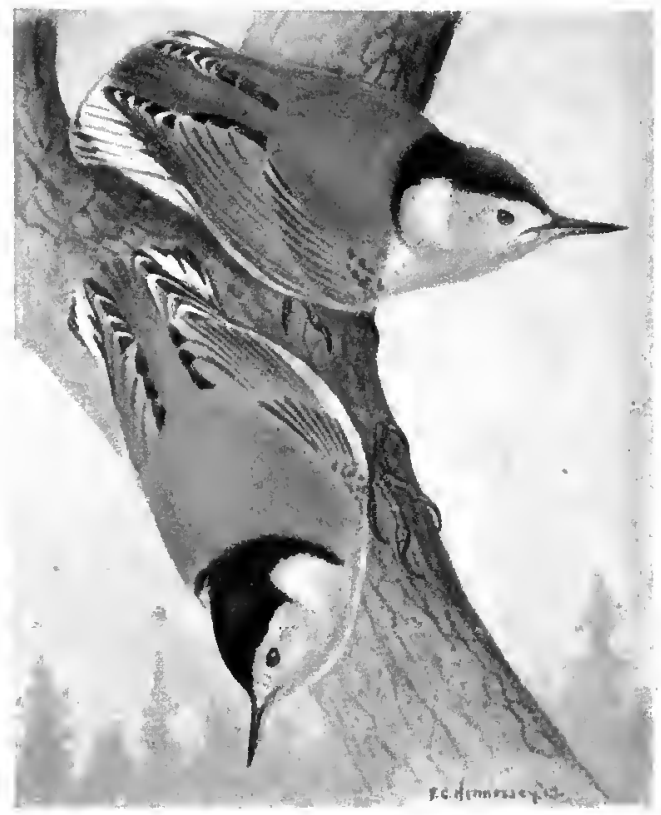

13. La Sittelle de la Carvline (p. 240). 


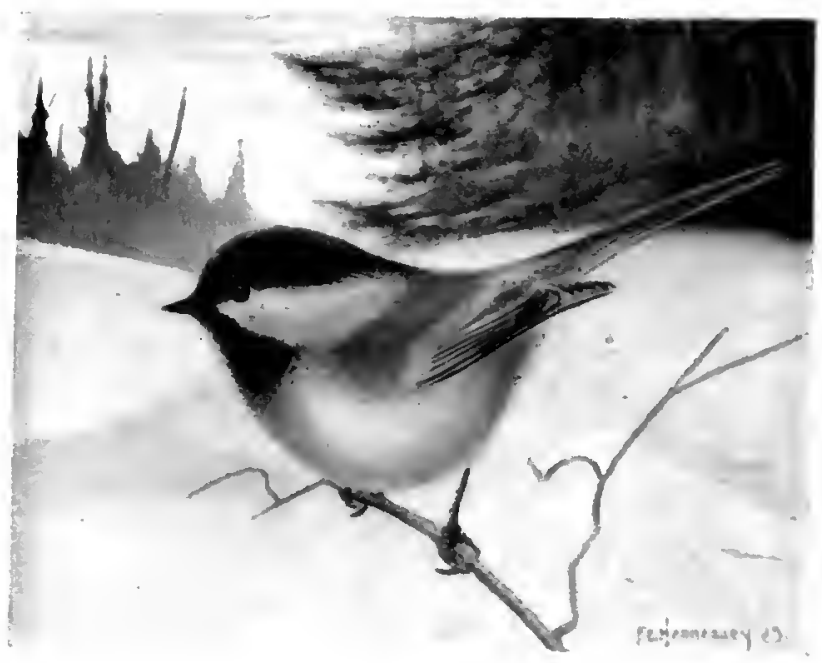

A. La Mésange à tête noire. (p. 212).

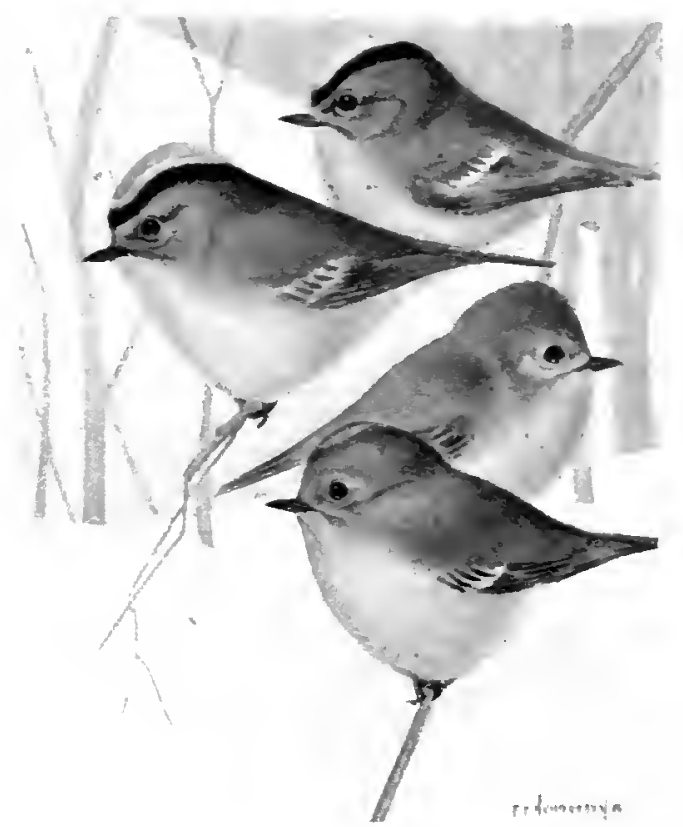

B. Le Roitelet huppé.

Mâle Femelle
Le Roitelet ì couronne rubis (p. 244). Mâle Femelle 


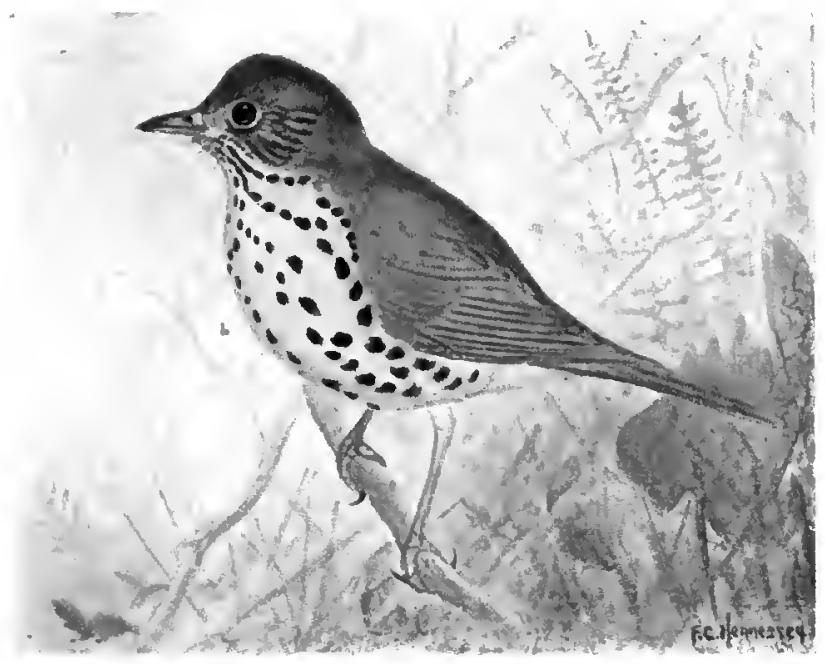

A. La Grive des bois (p. 246).

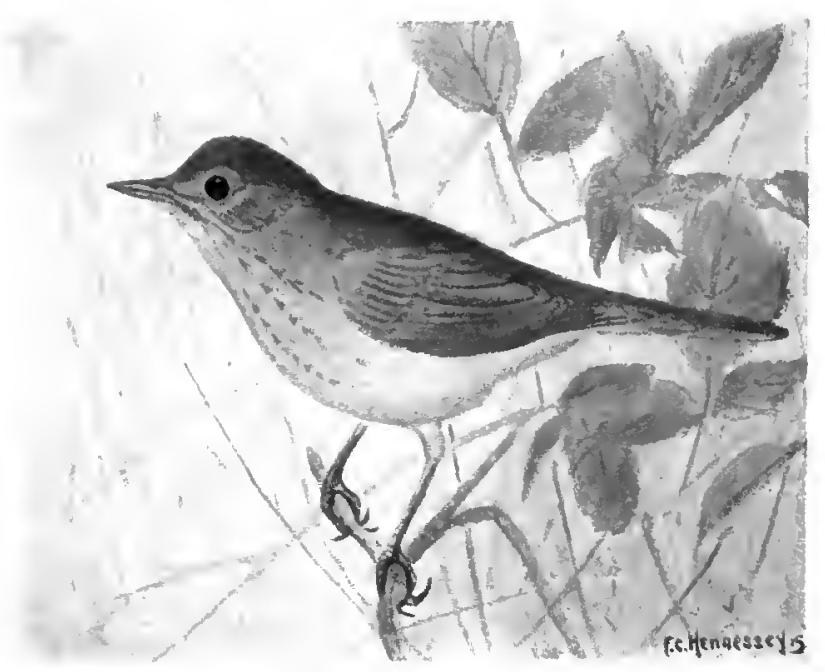

13. La Grive de Wilson (p. 246). 
Planche NLIX.

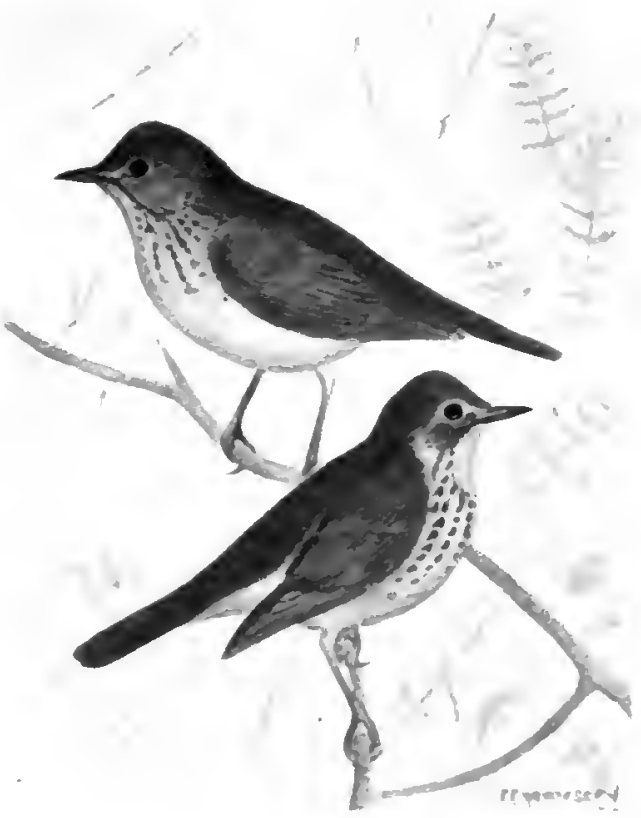

1. La Cirise d'Alice ( $\beta$. 2) 24 .

La (irive de swatison p. pata)

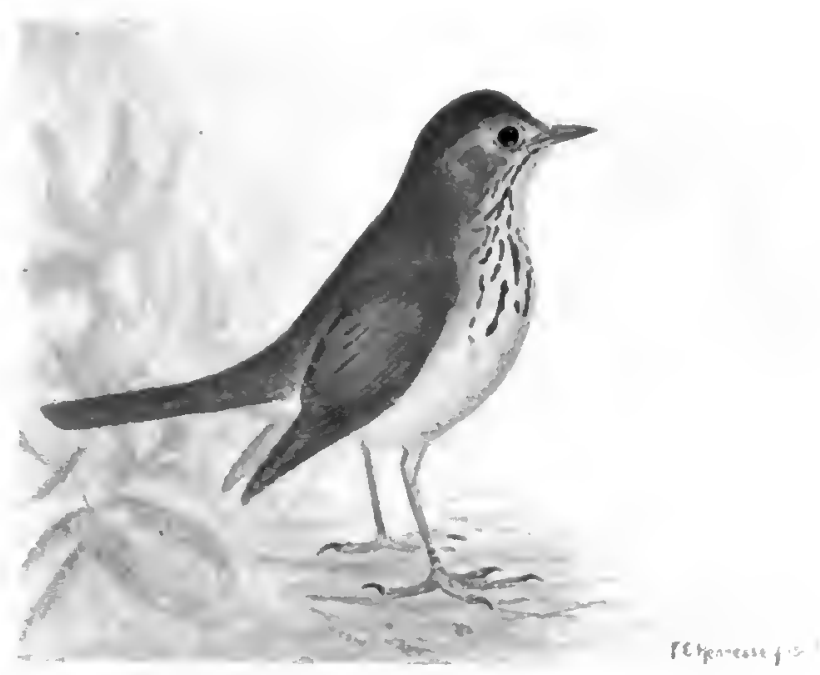

B. La Cirive solitaire (p, 247 , 


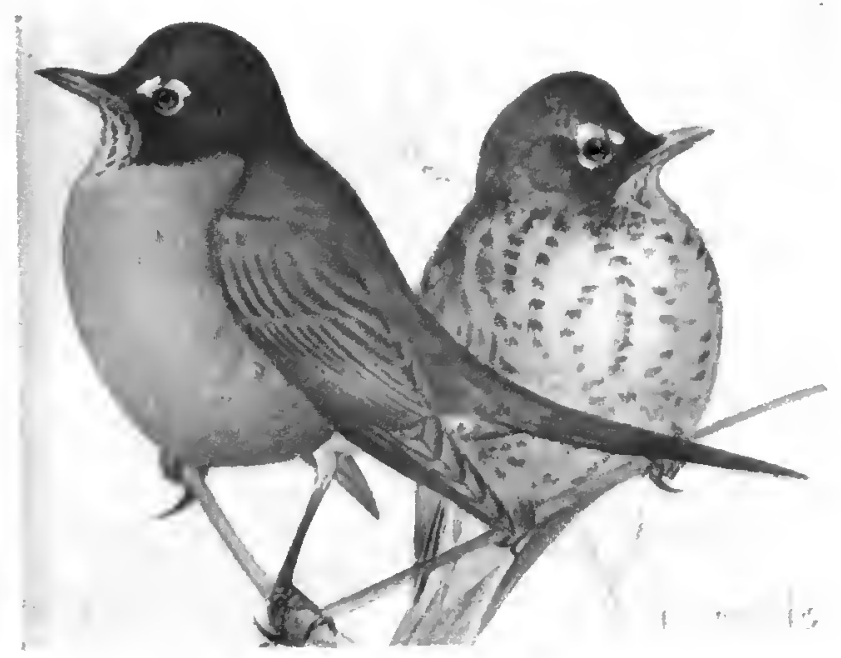

A. Le Nerle d'Amérique (p. 2ts).

Adulte Jeune

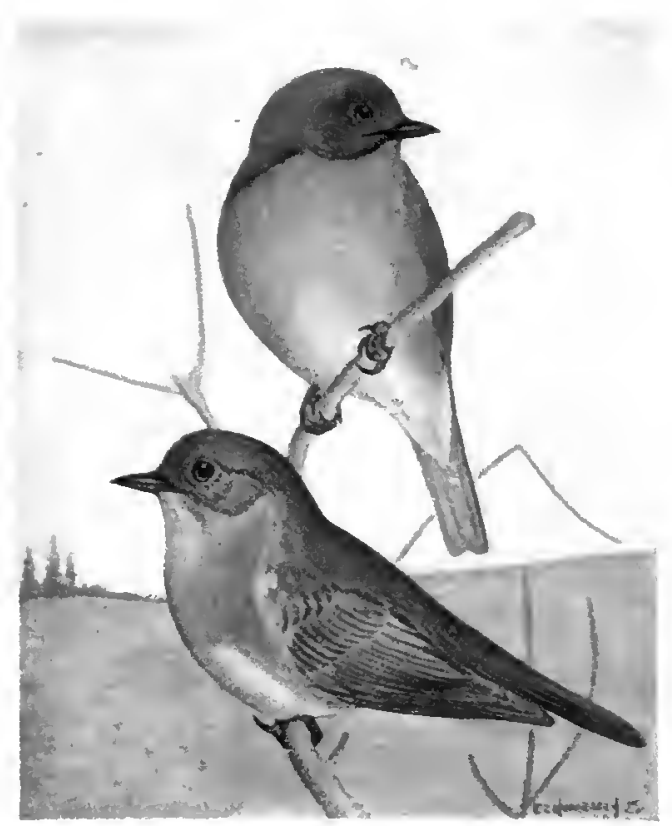

B. Le Rouge-gorge bleu (p. 249).

Ilâle

Femelle 


\section{INDEX}

$\boldsymbol{\Lambda}$.

PAGE

PAGE

Acanthis................. 188, 189

Accipitres.................131, 132

Actitis.................... 111

Athiins................. 48

Agelaius................... 179

Aigles............... 25, $129,137,138$

Aigles-pêcheurs .............142, 143

Aigrettes...................... 89,90

Aix...................... 73

Alaudidæ.................... 170

Alca........................ 49

Alcedinidx.................. 152

Alcidæ..................... $\quad 47$

Alcyones.................. 152

Allinæ....................... 50

Alouettes......27, 104, 109, 111, 170, 275

Aluconid $x . . . \ldots \ldots \ldots \ldots \ldots \ldots \ldots, 144$

Ammodramus................ 193

Anas......................... 70

Anatid $æ . . \ldots \ldots \ldots \ldots \ldots \ldots \ldots 6,67,68$

Anatina................... 69

Anser.................... 82

Anseres.................... 67

Anserinæ.................... 81

Anthus.................... 233

Antrostomus................. 160

Aphrizidæ................... 116

Aquila...................... 137

Aramidæ...................... 93

Archibuteo...................134, 136

Archilochus................... 164

Ardea...................... 89

Ardeidæ.................... 87

Arenarinæ.................... 116

Arquatella...................... 104

Asio....................... 144, 145

Astragalinus...................... 190

Astur....................... 131, 133

Auks............................ 49,50

Autour...................... 133, 264

Avocettes...................... 101

B.

Becs-scies................68, 69

Bernaches..............20,83, 257

Bibliographie................... 14

Bitterns.............. 87, 88, 258

Blackbirds.............178, 179, 181

Bluebills. Voir Morillons.

Bluebird..................... $\quad 249$

Bob-white..................... 118

Bobolink........................ 177

Bois pourri................. 160

Boftes a oiseaux.............. 14

Bombycillidæ................... 210

Bonasa.................... 120

Botaurinæ................ 87

Brant (Branta)............. 83

Broad-bills. Voir Morillons.

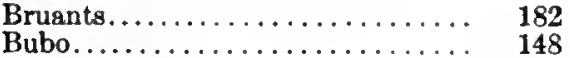

Bucéphales..................... 76,77

Buffle-head....................... 77

Buntings....................191, 204

Busards.................... 129, 130, 263

Buses...........10, 129, 134-136, 265

Buteo......................134-136

Buteonidæ...................... 129

Butorides..................... 91

Butors.................. $87,88,258$

C.

Cailles................24, 118, 261

Calcarius................. 191

Canachites.................. 120

Canards.........20, 68-82, 255-257

Canne de roche............... 78

Canvas-back.................. 74

Canutus...................... 103

Caprimulgi................... 159

Caracaras...................... 139

Cardinal........................ 202

Carinatæ.................. 43

Carpe allemande................. 75

Carpodacus................. 184

Catbird................... 234

Bæolophus................. 241 Catbartidæ.................... 128

Balbuzards.................142, 143, 267 Catoptrophorus................... 110

Baldpate.................... 71 Centurus........................ 158

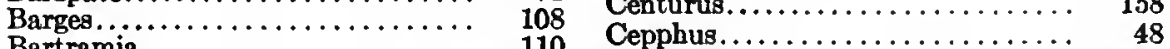

Bartramia................... 110 Cerchneis........................... 142

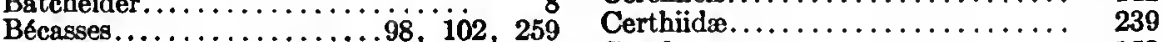

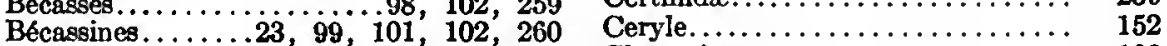

Becs-croises.............28, 187, 188 Chæturinæ................ 162

Becs d'oiseaux................. 19-31 Chapman, Frank............... 5 
Charadriidæ................. 113

Charadrius.

Chardonnerets.

Charitonetta.

190,282

Chat.................... 231

Chaulelasmus................. 71

Chebec................ 170

Chen ................... 82

Chevaliers...................108-110

Chickeu, Prairie................ 122

Chikadees.................242,243

Chondestes.................... 195

Chordeiles.................... 161

Chouettes.........145, 146, 150, 267

Chuck-will's Widow............ 160

Cigognes...................86, 87

Circus................... 130

Cistothorus................. 238

Clamatores................... 165

Clangula.................... 76

Classification............... 5

Coccyges..................... 150

Colaptes................... 158

Colibri.................. 164, 274

Colinus................. 118

Colombes (Columbx)............ 124

Colymbidæ.................. 44

Compsothlypis................ 218

Coots.................... 98

Coragyps..................... 129

Corbeau.................... 173

Corbigeau................... 113

Cormorans................. 65

Corneilles. . . . . . . 27, 171, 173, 174, 277

Corvidæ................. 171

Coturnicops................. 96

Coucous........10,26, 150-152, 269

Courlans.................. 93

Courlis....86, 101, 106, 108, 112, 113

Cuwbird................. 177

Crane...................... 93

Crécerelles................... 142

Creeper..................... 239

Crocethia.................. 107

Crossbills................. 187, 188

Crow..................... 174

Cryptoglaux................. 146

Cuckoos... . . . . . . . . . . . . . . . 150-152

Curlews..........86, 106, 112, 113

Cyanocitta.................... 172

Cygnes (Cygninæ) . . . . . . . 68, 84, 85

Cypseli..................... 162

D.

Dafila................... 72

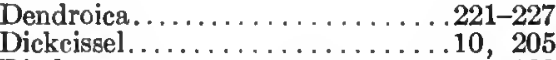

Dindons.................... 123

Dolichonyx................ 177

Dove.................... 125
Dovekie................... $\quad 50$

Dowitcher. . . . .............. 102

Dryobates................... 154

Ducs..................... 148, 268

Ducks.................. . 68-82

Dumetella.................... 234

Dunlin................. 106

\section{E.}

Eagles..............129, 137, 138

Echasses................... 101

Echassiers................ $11,86,87$

Ectopistes................... 124

Effraies...................... 144

Egret.................... 90

Eiders. . . . . . . . . . . . . . . . . . . . 78-80

Elanoldes..................... 130

Emerillon................131, 141

Empidonax...................168-170

Engou levents.........26, 159-161, 273

Eperviers. . 25, $129-132,142,150,264,266$

Ereunetes................... 107

Erismatura.................. 81

Erolia.................... 106

Eroliinæ.................. 102

Esterlette................... 58

Etourneaux.....28, 175-179, 278, 279

Euphagus................. 181

\section{F.}

Faisans..................... 118

Falcons. . . . . . . . . . . . . . . 139-142

Falcones.................... 129

Falconidø................. 139

Farlouse...............

Faucons.......... io, 25, i39'142, 266

Fauvettes....29, 216-232, 243, 291-296

Finch................... 184

Fleming, J.-H. . . . . . . . . . 5

Flicker................... 158

Florida................... 91

Flycatcher $\ldots \ldots \ldots \ldots \ldots \ldots \ldots \ldots \ldots \ldots$. . . . 1650

Foulques.................. 21, 98

Fous de Bassan. . . . . . . . . . . . . 20, 64

Fraterculinø................ 48

Fringillid $\ldots \ldots \ldots \ldots \ldots \ldots \ldots \ldots \ldots \ldots \ldots, \ldots \ldots \ldots$

Fulicinæ................... 98

Fuligulinæ.................. 74

Fulmars................ 61

G.

Galdwall................. 71

Gallinacés (Gallinæ)............. 117

Gallinago................... 102

Gallinules................. $3,96,97$

Gannet..................... 64 
PAGE

I.

PAGE

Garrulinx

172

Gavidæx...............

Geese...................... 82,83

Gelinottes.....118, 120, 122, 123, 262

Geothlypis................... 229

Glaucionetta................ 76

Glossaire..................... 249

Gnatcatcher............... 245

Gobe-mouches...............243, 245

Godwits.................. 108

Goélands..............20, 52-56, 254

Goglu............10, 28, 177, 277

Golden-eye................ 76

Goldfinch................. 190

Goosander................. 68

Goose. Voir Geese.

Goshawk.................... 133

Grackle. Voir Blackbird.

Grèbes...............21, 44, 45, 253

Green-head................. 70

Grièches...................211, 212

Grimpereaux........30,218, 239, 298

Grives. . 10, 30, 31, 228, 229, 234, 235, 245$247,295-297,300-301$

Gros-becs (Grosbeaks)..10, 28, 183, 184,

203, 204, 280, 280

Grouse.....................120, 123

Grues.................

Guillemots................21, $47-49$

Guiraca................... 204

Gulls................... $54-56$

Gyrfalcon............... 140

Ibididæ, Ibis.............. $\quad \mathbf{8 6}$

Icteria.................... 231

Icteridæ................... 176

Icterus................... 180

Index méthodique............ $\quad 31$

Ionornis.................... 97

Iridoprocne $\ldots \ldots \ldots \ldots \ldots \ldots \ldots, \quad 209$

\section{J.}

Jaegers.....................51, 52

Jaseurs..............29, 210, 289

Jays....................... 172, 173

Johnson, Claude E........... 5

Junco...................... 199

K.

Kakawi................. 77

Kildeer..................... 115

Kingbird................. 166

Kingfisher................ 152

Kinglets............... 244

Kite..................... 130

Kittiwake................ 53

Knot..................... 103

L.

Labbes................20,51, 52

Lagopèdes (Lagopus) .......... 10, 118, 122

Laniers. Voir Grièches.

Laniidre.................... 211

H.

Lanivireo.................. 214

Laridæ.................. 52

Hæmatopodidæ............... 117

Hializetus................. 137

Harelda.................... 77

Harfangs................. 149

Harlequin ................ 78

Harles.............. 20, 68, 69, 255

Hawks...........130-136, 141, 142, 161

Helmitheros.................. 218

Hennessey, Frank.............. 5

Herodii................... $\quad 87$

Herodiones.................. 86

Hérons..............22, 86-92, 258

Hesperiphona............... 183

Hiboux............25, 143-150, 268

Hierofalco..................... 140

Hirondelles. ..28, $162,206-209,287-289$

Hirundinidæ................. 206

Histrionicus................. 78

Hochequeues...............228, 233

Huard $\ldots \ldots \ldots \ldots \ldots \ldots \ldots \ldots \ldots, 46$

Huftriers....................... 117

Hummingbird................ 164

Hydrochelidon.................. 6

Hylocichla ....................246-248

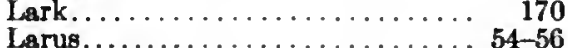

Limicols...................... 98

Limmodronus................. 102

Limosa..................... 108

Linnet................... 189

Linottes................... 182

Littérature ornithologique......... 14

Lobipes..................... 100

Longipennes................. 50

Iongueur des oiseaux, manière de les

mesurer................... 4

Longspur................... 191

Loons...................... 46,47

Lophodytes................ 69

Loriots...............10, 28 , iso, 279

Loxia..................... 187

M.

Macareux..............47, 48

Macoun, John et James M....... 4, 5

Macreuses..............20,80,81 
Macrochires...

Magpie.

Mainates.

Mallard.

Mangeur de maringouins........... 161

Mareca.........................

Margot.................... 64

Marila.................... 74-76

Marmette................. 48

Marsh hawk................. $\quad 130$

Martin................. 207 Bee. Voir Kingbird.

Martins-pêcheurs......26, 150, 152, 269

Martinets..........26, 159, 162, 273

Maubèches........23, 98, 101-111, 260

Mauve................... 56

Meadowlark................ $\quad 179$

Melanerpes................. 158

Meleagridæ............... 123

Melospiza.................., 199

Mergansers................... 68, 69

Merginæ................... 68

Mergules.................. 50

Merles...............234, 248, 302

Mésanges.........10,30, 241-243, 299

Micropalama................ 103

Micropodidæ................ 162

Migration..................... 10

Milans....................129, 130

Milouins....................... 74

Mimidæ (Mimus)............. 234

Mniotiltidæ.................. $\quad 216$

Mniotilta.................. 218

Mockingbird................. 234

Moineaux........10, $28,182,185,281$

Molothrus.................. 177

Moqueurs..................... 234

Morillone...................75, 78

Motacillidæ.................. 233

Moucherolles.....27, 165-170, 274, 275

Mouettes.................. 53

Moyac..................... 79

Murre.................... 48

Muscivora.................... 165

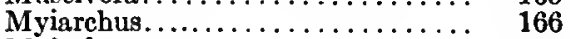

Myiochanes.................. 168

N.

Nageurs. Voir Palmipèdes.

Nannus... .................. $\quad 238$

Nettion................... 71

Nighthawk............... 161

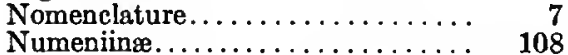

Numenius...................... 112-113

Nuthatches...............240, 241

Nuttallornis................, 167

Nyctales..................146, 147

Nyctanassa................. 92

Nyctea.................... 149

Nycticorax.................. 92
Oceanites, Oceanodroma......... 63

Odontophoridæ............... 118

Gnanthe...................... 248

Oidemia..................... 80

Oies.................21, 68, 81-83

Oiseaux blancs................. 191

chanteurs............. 170

criards................ 165

de marais............... 93,94

de proie.............126-151

de rivage............. 98-117

du type bécassine......... 101

râle........... 94

manière de mesurer les.... 4

mouches.......26, 159, 163, 164

moqueurs....... 234

nageurs. Voir Palmipedes.

percheurs..............27, 164

plongeurs................

rouges................. 184

Old-Squaw ou Oid Wife............. 77

Olor......................... 84

Oporornis................. 229

Orfraies. Voir Balbuzards.

Orioles. Voir Loriots.

Ortolan .................... 170

Oscines.................... 170

Osprey...................... 143

Otocoris.................. 170

Otus................... 148

Outarde............... 83

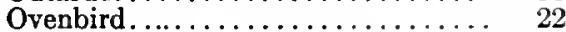

Owls.................... . 144-150

Oxyechus................ 115

Oyster-catcher.............. 117

\section{$\mathbf{P}$.}

Palmipèdes......20, 50, 60, 61, 64, 67

Paludicolæ..................., 93

Pandionidæ......................142, 143

Paridæ...................... 241

Partridge....................... 120

Passer..................... 185

Passerculus.................... 192

Passereaux..................... $\quad 182$

Passerella........................ 201

Passeres..................... 164

Passerherbulus.................. 194

Passerina................... 204

Pedicecetes................... 123

Pélicans (Pelecanidæ).......... 66

Pelidna....................... 106

Penthestes...................242, 243

Percheurs....................... 164

Perdrix.....................120, 122

Perisoreus....................... 173

Perroquet.................... 48

Pétrels.......................

Petrochelidon.................. 208

Pewee........................ 168 
Phalacrocoracida.

65

Phalaropes (Phalaropodidæ)...2i, 99,100

Phasiani.................. 118

Philohela.................. 102

Phlœotomus................ 157

Phoebe................... 167

Pica............... 172

Pics (Pici) . . . . 10, 27, 154-158, 270-272

Pieds d'oiseaux.............. 19-31

Pies...................172, 173

Pies-grièches. . . . 10, 29, 211, 212, 290

Pigeons..........25, 124, 141, 263

Pingouins.............21, 46-50

Pinicola................... 184

Pinsons.......10, 28, $182,184,192-202$ $206,281-286$

Pintail. . . . . . . . . . . . . . 72

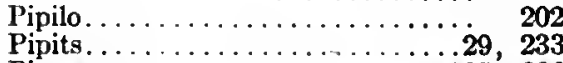

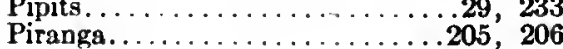

Pisobia................ 104, 105

Pivert $\ldots \ldots \ldots \ldots \ldots \ldots \ldots \ldots \ldots \ldots \ldots, 158$

Planesticus................ 248

Plautus................. 50

Plectrophanes (Plect rophenax). 10, 191, 282
Plegadis. . . . . . . . $8 \ldots \ldots \ldots \ldots \ldots$

Plongeons. ......... 19, 46, 47, 253

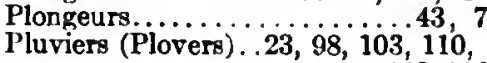

113-116, 261

Poacetes.................. 192

Polioptilina................ $\mathbf{2 4 5}$

Porzana................ 90

Poules.............98, 118, 122

Poulets de la mère Carey. . . . . . . . . 63

Procellariidæ................ 61

Progne................... 207

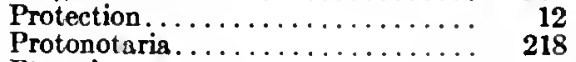

Ptarmigans............ 122

Puffin $\ldots \ldots \ldots \ldots \ldots \ldots \ldots \ldots \ldots,{ }_{61} 48$

Pygopodes................. 43

\section{Q.}

Quac.................... 92

Quails.......................... 118

Querquedula ................ 72

Quiscalus.................. 181

\section{R.}

Râles (Rails, Ralli).......23, 93-96, 259

Ramoneur................. 162

Raptores.................. 126-150

Ratita................. 43

Raven................... 173

Recurvirostridæ.............. 101

Redhead................... 74

Redpolls................... . 188,189

Redstart.................... 232

Regulins........................ 243

Remerciements.............. 5

Ring-neck................ 76

Riparia.................. 209

Rissa.................. 53

Robin.................. 248

Roitelets. . 10, 31, 236-238, 243, 244, 299

Rossignol. . . . . . . . . . . . . . . 199

Rouge-gorge bleu......... 10, 249, 302

\section{S.}

Sanderling................. 107

Sandpipers................ 103-111

Sapsucker.................. 156

Sarcelles................71, 72, 256

Sarcorbamphi.............. 127

Saunders, $\mathbf{W} . \mathbf{E} \ldots \ldots \ldots \ldots \ldots \ldots \ldots \ldots, \quad 5$

Saw-bill................. 68

Sayornis................... 167

Scaup...................... 75

Scolopacida................. 101

Scoters....................80, 81

Scotiaptex................... 146

Seiurus.................228, 229

Setophaga................... 231, 232

Sharp-tail, Nelson's............... 194

Shearwaters................. 62

Sheldrakes. Voir Harles.

Shoveller................. 72

Shrikes................... 212

Sialia.................... 249

Siffleurs.................71, 196

Siskin................... 190

Sittelles (Sittidæ). . 10, 30, 240, 241, 298

Sizerins..................188, 189

Snipe.................... 102

Somateria................. 78

Sora Rail..................... 98

South-southerly. Voir Old Squaw.

Sparrows...............185, 192-002

Spatula..................... 72

Sphyrapicus................ 156

Spinus...................... 190

Spizs...................... 205

Spizella.................. 197, 198

Spoonbill. Voir Shoveller.

Squatarola................... 114

Starlings.....................

Steganopodes................... 64

Steganopus.................... 100

Stelgidopteryx.................. 209

Stercoraires (Stercoriidx) . . . . . . 51, 52

Sternes (Sterninæ) .......52, $57-60,254$ zone d'habitat..........9, 10 migration (St. arctique)..... 11

Striges.................. 143

Strigid . $\ldots \ldots \ldots \ldots \ldots \ldots \ldots \ldots \ldots \ldots, 144$ 
U.

PAGE

Strix

145

Sturnella.

179

Sturninidæ

175

Sulidæe.

64

Surnia.

150

Swallows.

$208-209$

Swans..................84,85

Swifts..................... 162

Swimmers. Voir Palmipèdes.

Sylviidæe.

T.

Tangaras ('Tanagers). 28, 205, 206, 287

Tarins...................... 190

Teals.................... 71, 72

Telmatodytes................... 238

Terns..................... 57-60

Tétras (Tetraonidæ) ........24, 120, 262

Thrasher.................. 235

Thrushes...........228, 229, 245-247

Thryomanes................. 237

Thryothorus.................. 236

Tinnunculus................. 141

Tip-Ups..................... 101

Titmouse.................. 241

Totanus. . . . . . . . . . . . . . . . 108, 109

Totipalmés, palmipèdes.......... 19, 64

Tourne-pierre..................24, 116

Tourte..................... 124

Tourterelle................. 125, 263

Towhee................... 202

Toxostoma.................. 235

Traquet motteux............... 248

Tringa....................... 109

Tritri..................... 166

Trochili.................... 163

Troglodytes (Troglodytidæ) 30, 236-238, 297

Tryngites.................. 111

Tubinares................... 60

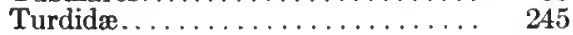

Turkey..................... 123

Turnstone.................. 116

Tympanuchus................ 122

Tyrannidæ.................. 165

Tyto...................... 144

Uria................... 48

V.

Vanneau gris............... 114

Vautours...............24, 127-129

Veery. Voir Wilson's Thrush...... 246

Vermivora................. 218-220

Vireos.......10, 29, 213-216, 290, 291

Vultures....................128, 129

W.

Warblers................... 218-232

Water-Thrushes............228, 229

Wavey. Voir Snow goose......... 82

Waxwings.................. 210

Wheatear................... 248

Whip-poor-will.................. 160

White-throat................. 196

Widgeon.................. 71

Willet................... 110

Wilsonia.................231, 232

Wilson's thrush............... 246

Woodcock.................. 102

Woodpeckers................. 154-157

Wrens.... . . . . . . . . . . . . 236-238

$\mathbf{X}$.

Xanthocephalus............. 178

\section{$\mathbf{Y}$}

Yellow-legs................108, 109

Yellow-throat............... 230

Z.

Zamelodia.................. 203

Zanaidura...................... 125

Zonotrichia...............195, 196 



UNIVERSITY OF ILLINOIS-UABANA

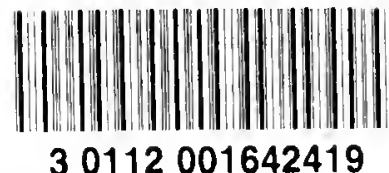

30112001642419 